LA-14358-PR

Progress Report

Approved for public release;

distribution is unlimited.

Groundwater Level Status Report for Fiscal Year 2007

Los Alamos National Laboratory 
Edited by Hector Hinojosa, Group IRM-CAS.

The two most recent reports in this unclassified series are LA-14292-PR and LA-14331-PR.

Los Alamos National Laboratory, an affirmative action/ equal opportunity employer, is operated by Los Alamos National Security, LLC, for the National Nuclear Security Administration of the U.S. Department of Energy under contract DE-AC52-06NA25396.

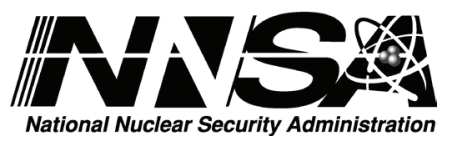

This report was prepared as an account of work sponsored by an agency of the U.S. Government. Neither Los Alamos National Security, LLC, the U.S. Government nor any agency thereof, nor any of their employees make any warranty, express or implied, or assume any legal liability or responsibility for the accuracy, completeness, or usefulness of any information, apparatus, product, or process disclosed, or represent that its use would not infringe privately owned rights. Reference herein to any specific commercial product, process, or service by trade name, trademark, manufacturer, or otherwise does not necessarily constitute or imply its endorsement, recommendation, or favoring by Los Alamos National Security, LLC, the U.S. Government, or any agency thereof. The views and opinions of authors expressed herein do not necessarily state or reflect those of Los Alamos National Security, LLC, the U.S. Government, or any agency thereof. Los Alamos National Laboratory strongly supports academic freedom and a researcher's right to publish; as an institution, however, the Laboratory does not endorse the viewpoint of a publication or guarantee its technical correctness. 
LA-14358-PR

Progress Report

Issued: March 2008

Groundwater Level Status Report for Fiscal Year 2007

Los Alamos National Laboratory

Shannon P. Allen

Richard J. Koch 



\section{Contents}

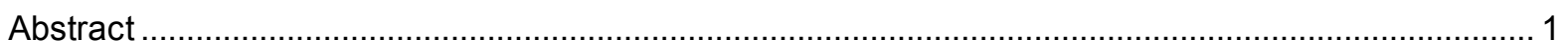

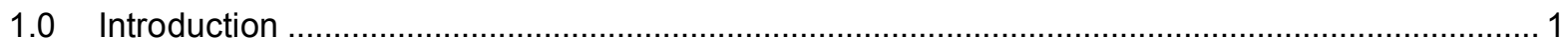

2.0 Description of Groundwater Level Data .......................................................................... 1

3.0 Groundwater Level Data from Regional Aquifer Wells ........................................................ 3

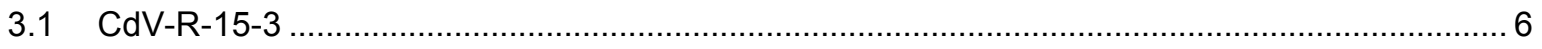

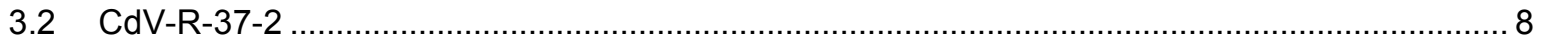

3.3 R-1 …

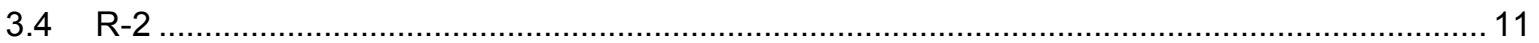

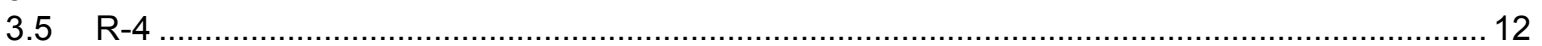

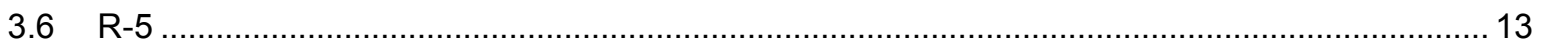

3.7 R-6

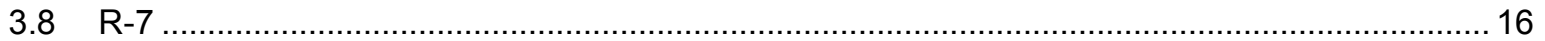

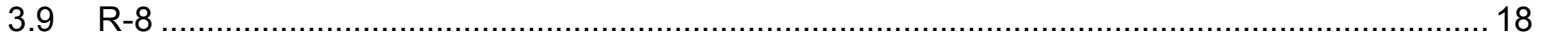

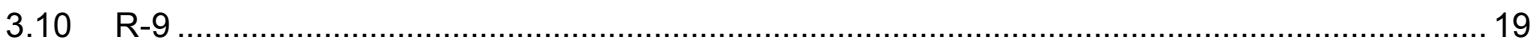

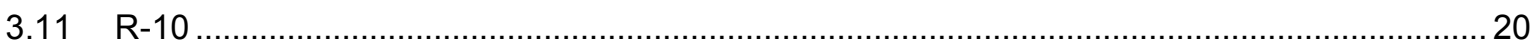

3.12 R-10a

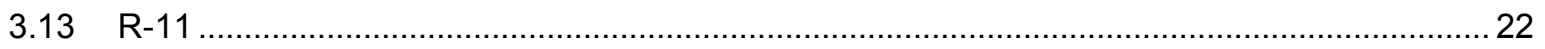

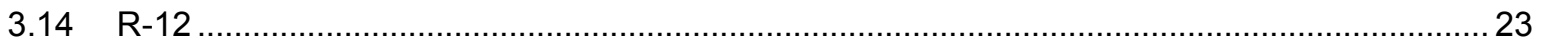

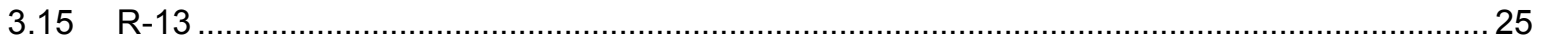

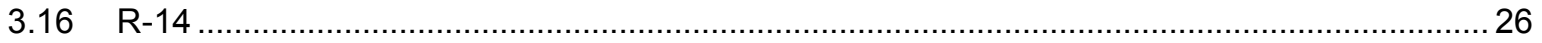

3.17 R-15

3.18 R-16

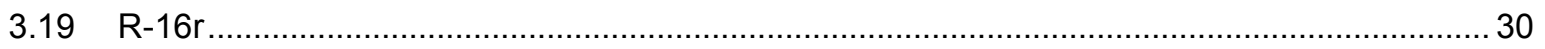

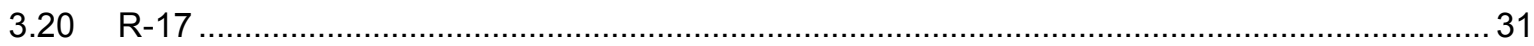

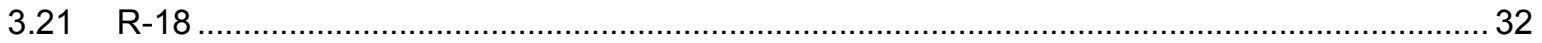

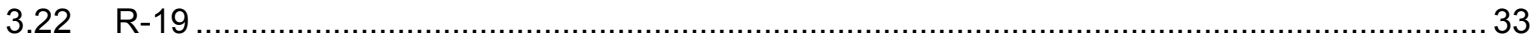

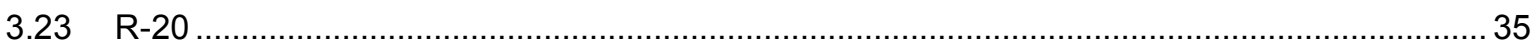

3.24 R-21

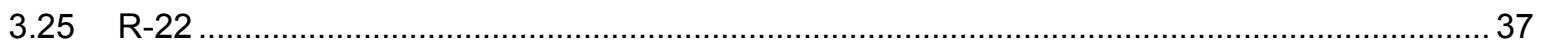

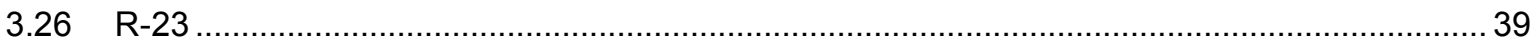

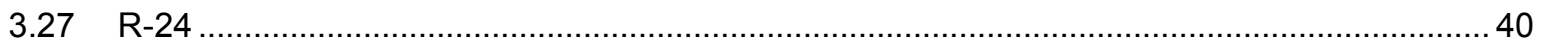

3.28 R-25

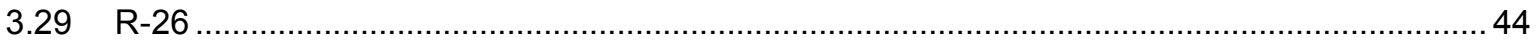

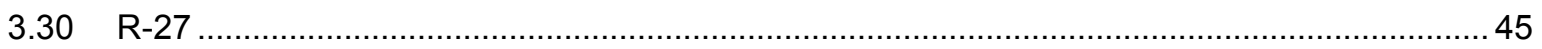

3.31 R-28

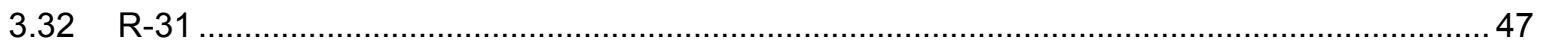

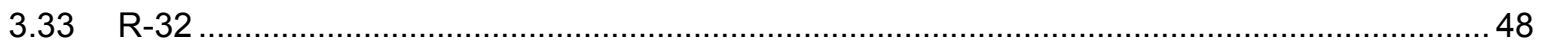

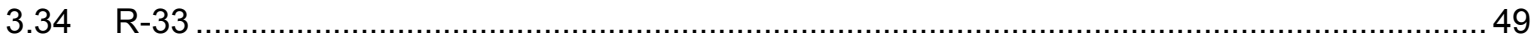

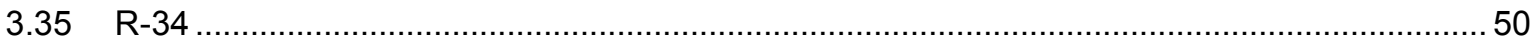

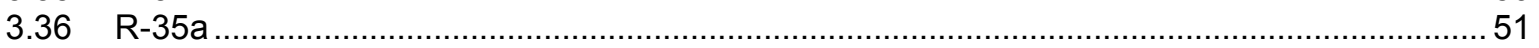

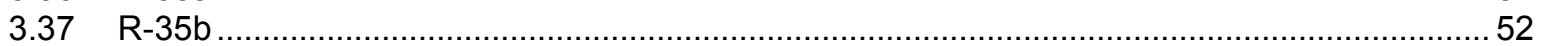

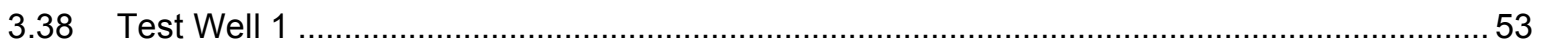

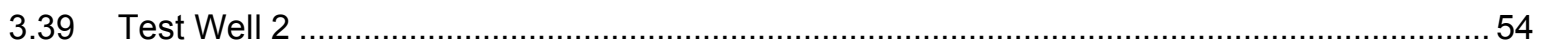

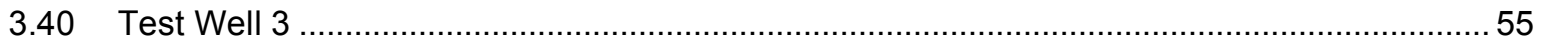

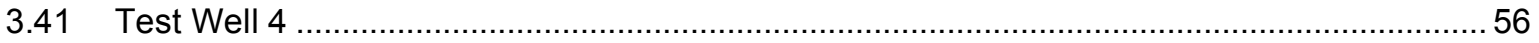

3.42 Test Well 8

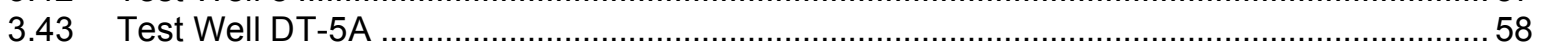

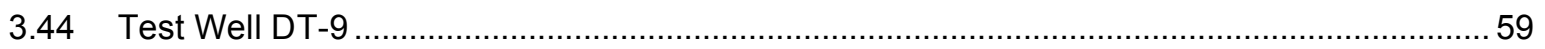

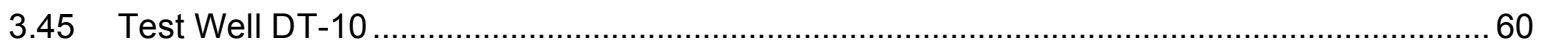

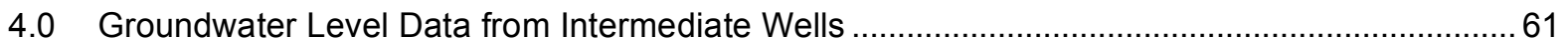

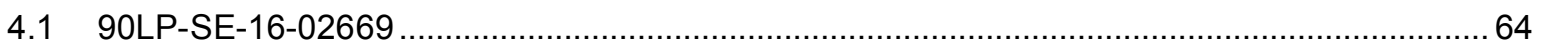

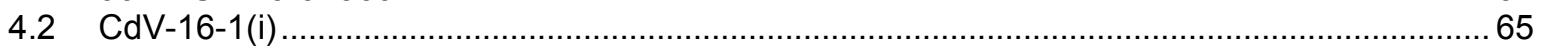




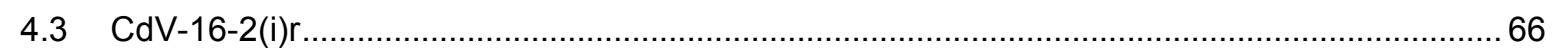

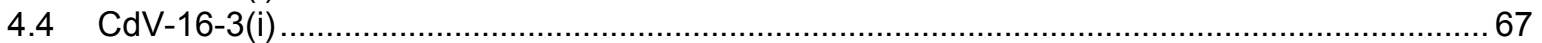

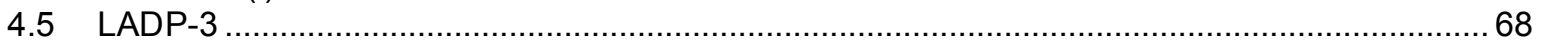

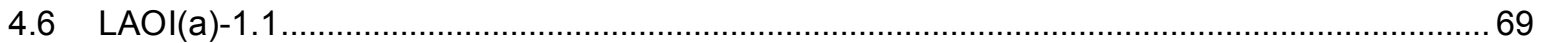

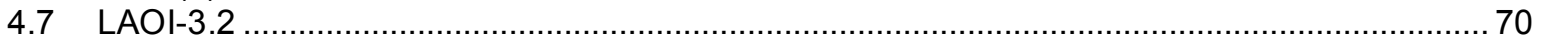

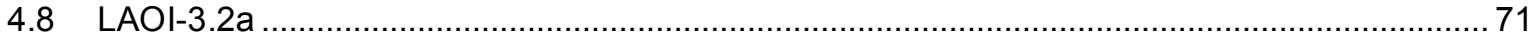

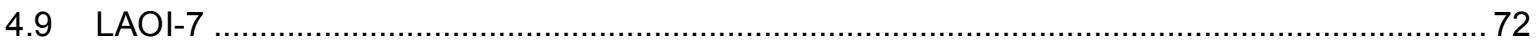

4.10 MCOBT-4.4

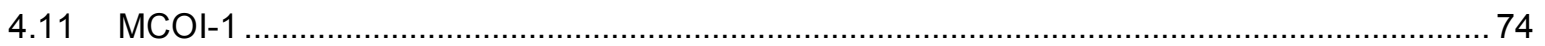

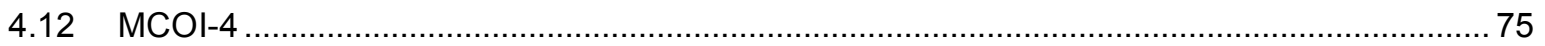

4.13 MCOI-5

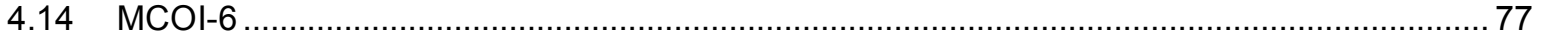

4.15 MCOI-8

4.16 MSC-16-02665

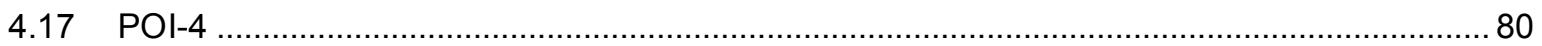

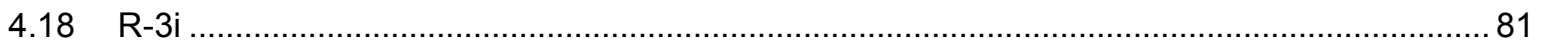

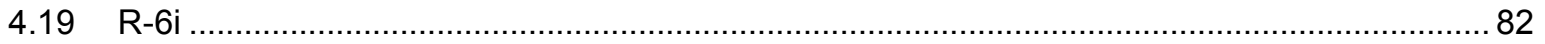

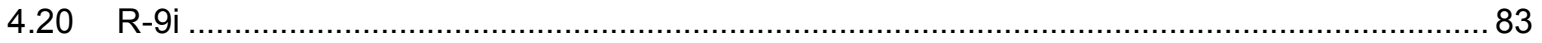

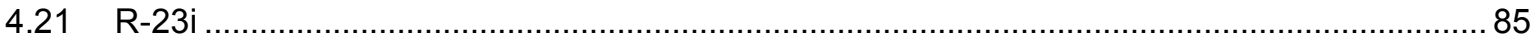

$4.22 \mathrm{SCl}-1$

4.23 Test Well 1A

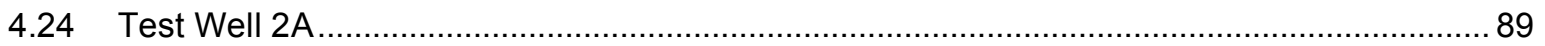

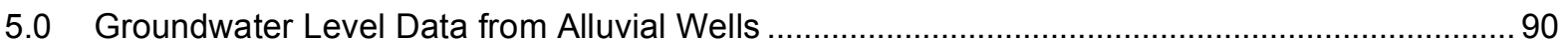

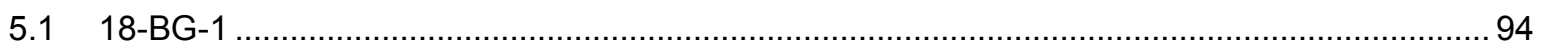

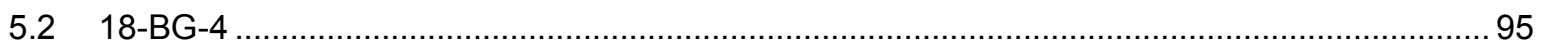

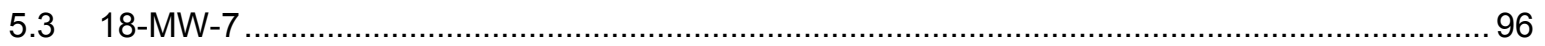

5.4 18-MW-8

5.5 18-MW-9

5.6 18-MW-11

5.7 18-MW-17

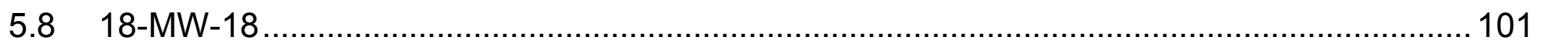

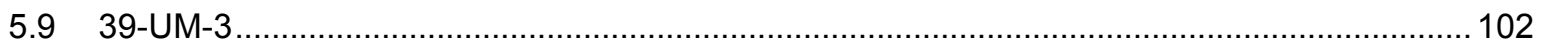

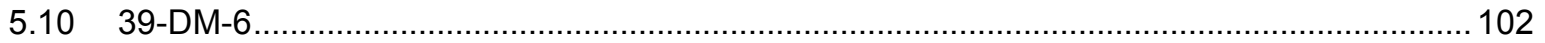

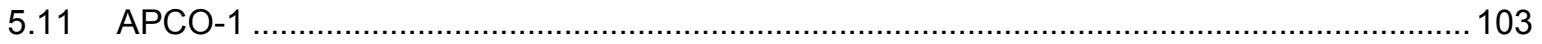

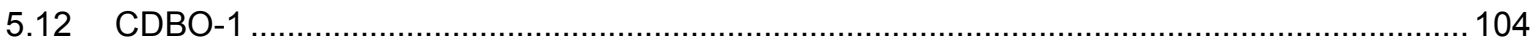

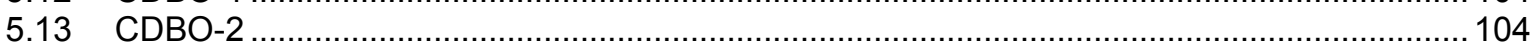

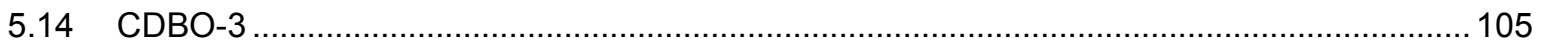

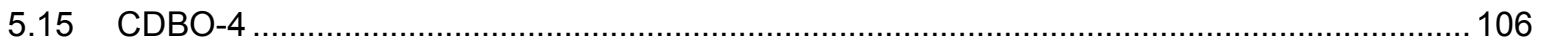

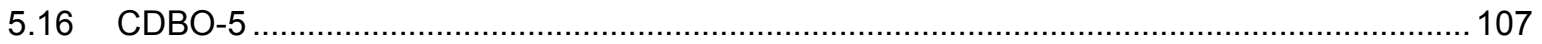

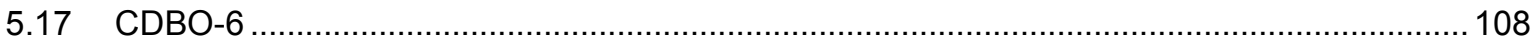

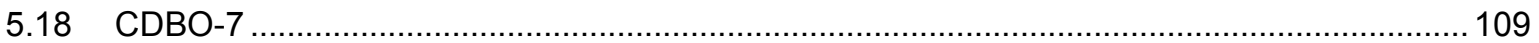

5.19 CDBO-8

5.20 CDBO-9

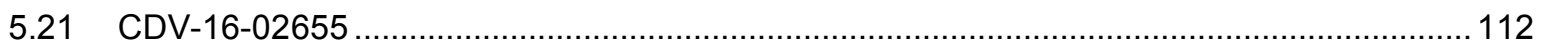

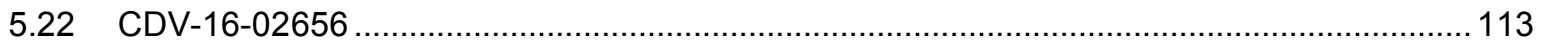

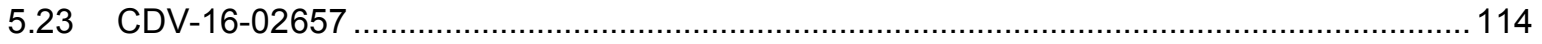

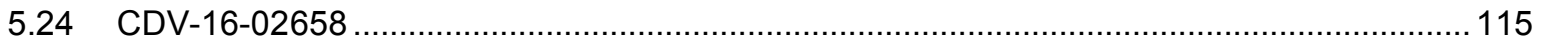

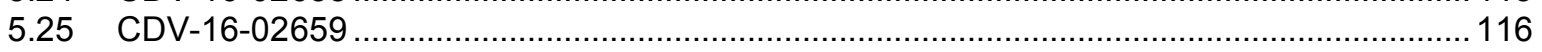

5.26 FCO-1

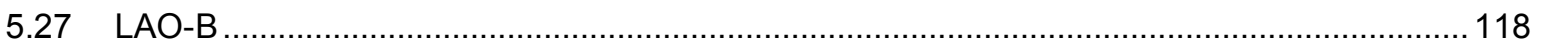

5.28 LAO-0.3

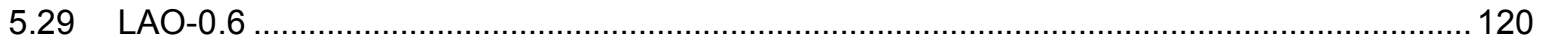

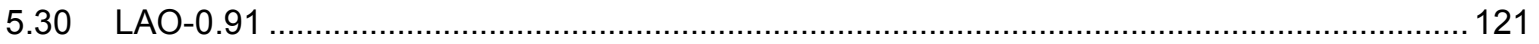

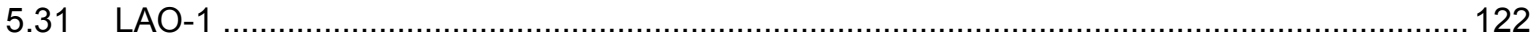

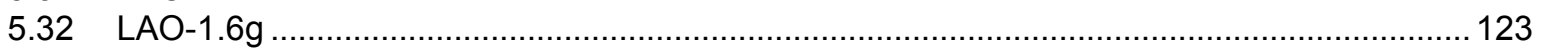




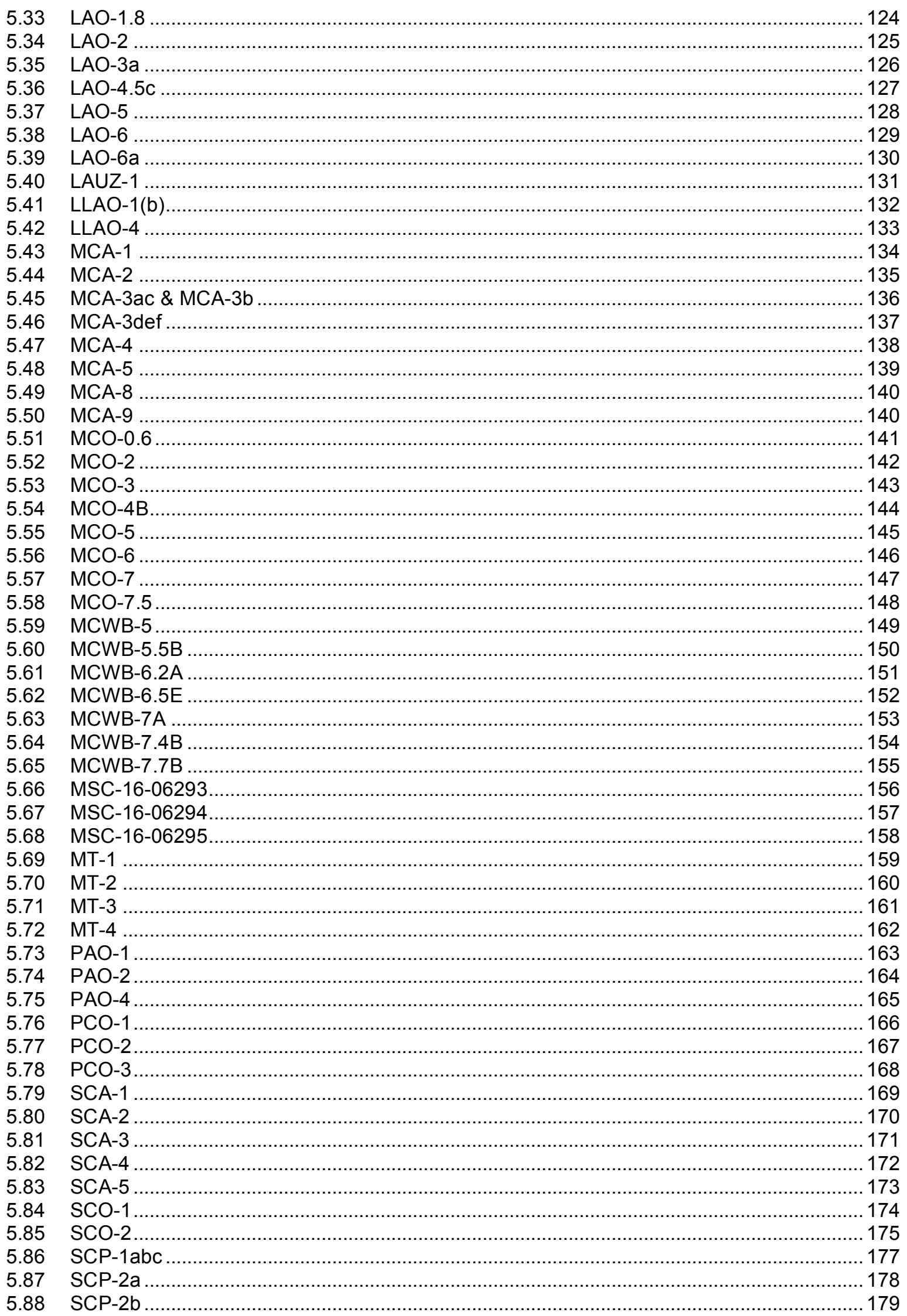




\begin{tabular}{|c|c|}
\hline 5.89 & TSCA-6 \\
\hline 5.90 & 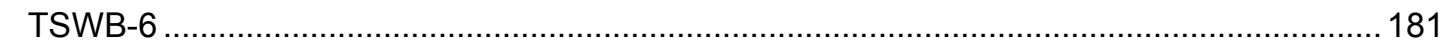 \\
\hline 5.91 & WCO-1.... \\
\hline 5.92 & WCO-2 \\
\hline 5.93 & WCO-3 \\
\hline 6.0 & roundwater Level Data from Water Supply Wells. \\
\hline 6.1 & G-2A \\
\hline 6.2 & .......... 189 \\
\hline 6.3 & ...... 190 \\
\hline 6.4 & 191 \\
\hline 6.5 & 192 \\
\hline 6.6 & O \\
\hline 6.7 & PM-1 \\
\hline 6.8 & PM-2 \\
\hline 6.9 & PM-3 \\
\hline 6.10 & 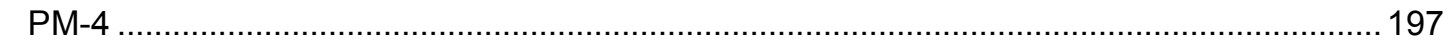 \\
\hline 6.11 & PM-5 \\
\hline
\end{tabular}

Appendix A Mean Annual Water Level at the Surface of the Regional Aquifer in LANL Monitoring Wells for 2007

Appendix B Summary of Transient Responses to Supply Well Pumping in LANL

Monitoring Wells

\section{List of Figures}

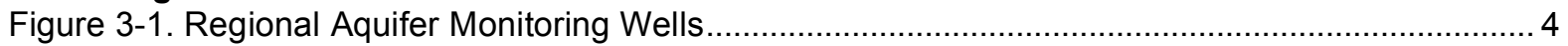

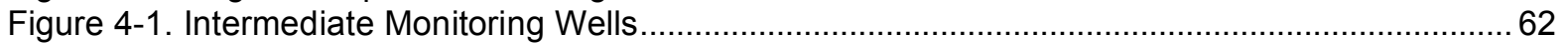

Figure 5-1. Alluvial Wells Monitored for Groundwater Levels in FY 2007 ....................................... 93

\section{List of Tables}

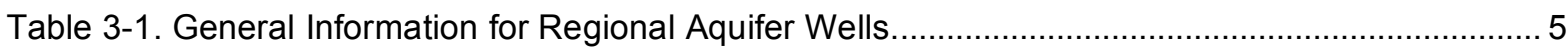

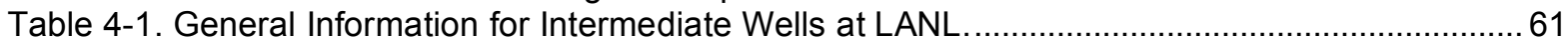

Table 4-2. Well Completion Information for Intermediate Wells and Screens..................................... 63

Table 5-1. Information and Location Data for Alluvial Aquifer Wells at LANL...................................... 90

Table 6-1. General Information for Los Alamos County Water Supply Wells.................................... 187 


\title{
Groundwater Level Status Report for Fiscal Year 2007 Los Alamos National Laboratory
}

by

Shannon P. Allen and Richard J. Koch

\begin{abstract}
The status of groundwater level monitoring at Los Alamos National Laboratory in Fiscal Year 2007 is provided in this report. The Groundwater Level Monitoring Project was instituted in 2005 to provide a framework for the collection and processing of quality controlled groundwater level data. This report summarizes groundwater level data for 166 monitoring wells, including 45 regional aquifer wells, 25 intermediate wells, and 96 alluvial wells, and 11 water supply wells. Pressure transducers were installed in 133 monitoring wells for continuous monitoring of groundwater levels. Time-series hydrographs of groundwater level data are presented along with pertinent construction and location information for each well.
\end{abstract}

\subsection{Introduction}

This report presents and describes groundwater level data obtained by Los Alamos National Laboratory (LANL) during Fiscal Year (FY) 2007 to provide regulatory compliance and to provide other programs at LANL with groundwater level data for modeling and data assessment.

By the end of September 2007, 45 regional aquifer monitoring wells, 25 intermediate wells, 92 alluvial wells at LANL, and 11 water supply wells were being monitored for groundwater levels. In addition, eight of the multiple completion regional aquifer wells monitored intermediate zones; however, the intermediate zones were dry in three of these wells, thus five of the multiple completion regional aquifer wells were used to monitor intermediate groundwater levels. In FY 2007, pressure transducers were installed in 39 regional aquifer wells and 19 intermediate wells; manual measurements were obtained from four intermediate wells (dry, monitored quarterly or annually). Transducers were installed in 75 alluvial wells during FY 2007 and 18 alluvial wells were monitored with quarterly manual measurements.

This report includes groundwater level data obtained during FY 2007 (October 1, 2006, through September 30,2007$)$ and, where available, historical data. The groundwater level data are presented on time-series graphs to provide a comprehensive representation of the groundwater level characteristics, to the extent possible with available data. For the alluvial wells, the first graph for each well represents the entire period of record, while the second graph represents FY 2007 to provide better representation of recent and seasonal changes.

\subsection{Description of Ground water Level Data}

The Groundwater Level Monitoring (GWLM) Project at LANL is conducted under the Quality Assurance Project Plan (QAPP) for Groundwater Level Monitoring (LANL 2006) to assure the quality of groundwater level data. The QAPP contains the work processes and the data quality objectives utilized in the GWLM Project.

Groundwater level data were collected during FY 2007 according to the criteria outlined in the Groundwater Level Monitoring Plan for 2007 (LANL 2007). Two types of groundwater level data were collected:

- Manual groundwater level measurements were taken in wells and in boreholes and

- Pressure transducers were used to measure groundwater levels in completed monitoring wells. 
Manual groundwater level measurements were obtained according to standard operating procedure ENV-SOP-202, Manual Groundwater Level Measurements. Transducer measurements were obtained according to procedure ENV-SOP-201, Pressure Transducer Installation, Removal, and Maintenance, and ENV-WQH-SOP-064, Westbay ${ }^{\circledR}$ Pressure Transducer Installation, Removal, and Maintenance.

Groundwater level data obtained both manually and with pressure transducers were reviewed and validated according to procedure ENV-WQH-SOP-062, Groundwater Level Data Processing, Review, and Validation. The groundwater level data are maintained in the LANL water quality database (WQDB); the data are accessible to the public on the internet at http://wqdbworld.lanl.gov.

Wells installed with pressure transducers had measurements collected at least hourly. Where possible, manual groundwater level measurements were obtained at least semi-annually to provide quality control for the transducer measurements. In the following sections, both manual measurements and transducer measurements are shown on the time-series hydrographs. Because hourly transducer measurements are too voluminous to reproduce for most hydrographs, mean daily groundwater levels are shown on the hydrographs in this report. Some monitoring wells have significant drawdown of the water level in the well when pumped during sampling events. Because pumping of the monitoring wells for sampling usually occurs over several hours, the mean daily water level value will not usually portray the full amount of drawdown experienced during pumping of a well. For this reason, mean daily water level data are not usually appropriate for determining monitoring well characteristics such as specific capacity.

Transducers that measure pressure head in wells typically have a measurement precision of $\pm 0.1 \%$ of the full-scale measurement capability. Thus, typical measurement accuracy for a 100-psi transducer is $0.23 \mathrm{ft}$, and for a $1000-p s i$ transducer is $2.31 \mathrm{ft}$. The higher-pressure-rated transducers are required in the deeper Westbay ${ }^{\circledR}$ installations where higher water pressures are encountered. Most shallow wells and deep wells not installed with the Westbay ${ }^{\circledR}$ or Barcad system are equipped with 30-psi transducers, with a measurement accuracy of $0.07 \mathrm{ft}$. A few of the shallow alluvial wells are equipped with 15-psi transducers. Manual groundwater level measurements typically have an accuracy of approximately $0.1 \mathrm{ft}$ per $100 \mathrm{ft}$ of measurement $(0.1 \%)$.

From 2000 through 2004, groundwater level data obtained during groundwater sampling of Westbay ${ }^{\circledR}$ wells was from a 1000-psi-rated transducer that had an accuracy of about $\pm 2.3 \mathrm{ft}$. In 2005 a new sampling transducer was obtained with a 500-psi rating, which has an accuracy of about $1.2 \mathrm{ft}$. This change in transducers is the cause for the apparent water level shift for sampling water levels in mid 2005 , as observed on many of the accompanying hydrograph plots for Westbay ${ }^{\circledR}$ wells. Also, the apparent scatter of water level data on the hydrographs from groundwater sampling of Westbay ${ }^{\circledR}$ wells is the result of the less accurate pressure transducers that are used for sampling.

In the following sections, acronyms used to describe groundwater level data include

GW data obtained from transducers during groundwater sampling events

Trans measurements from transducers installed in a well

MP Measurement Port identification in multiple completion wells.

Previous reports of groundwater level data at LANL were compiled for the regional aquifer test wells (TWs) by Koch et al. (2004) and for all wells in a submittal to the New Mexico Environment Department in January 2005 (LANL 2005). Groundwater levels in water supply wells at Los Alamos have been summarized in the series of water supply reports for Los Alamos, e.g., Koch and Rogers (2003). The previous reports in this series, Groundwater Level Status Report for 2005, was issued in May 2006 (Allen and Koch 2006), and Groundwater Level Status Report for Fiscal Year 2006 (Allen and Koch 2007), was issued in March 2007. 


\subsection{Groundwater Level Data from Regional Aquifer Wells}

Figure 3-1 shows the locations of the regional aquifer monitoring wells and water supply wells in the vicinity of LANL. Table 3-1 lists the regional aquifer monitoring wells that were monitored for groundwater levels in 2007. The location information was obtained from the WQDB at http://wadbworld.lanl.gov. Screen intervals and port depths for each well are shown in subsequent sections.

The Appendix A table lists the mean annual water level for 2007 for each well screen located at the top of the regional aquifer. The Appendix B table summarizes the transient responses observed in monitoring wells that result from supply well pumping at Los Alamos.

In the following sections reference is made to the barometric efficiency of some monitoring wells. Barometric efficiency is defined as the ratio of the water level change observed in a well divided by the concurrent atmospheric pressure change, expressed as a percentage. For a given change in atmospheric pressure, if the water in a well responds by an equal amount, the well is said to have $100 \%$ barometric efficiency; however, this type of response by the water in the well can occur only when the aquifer adjacent to the well does not experience the atmospheric pressure change. Thus, a well with a $100 \%$ barometric efficiency is installed into an aquifer that does not experience the atmospheric pressure fluctuations. 


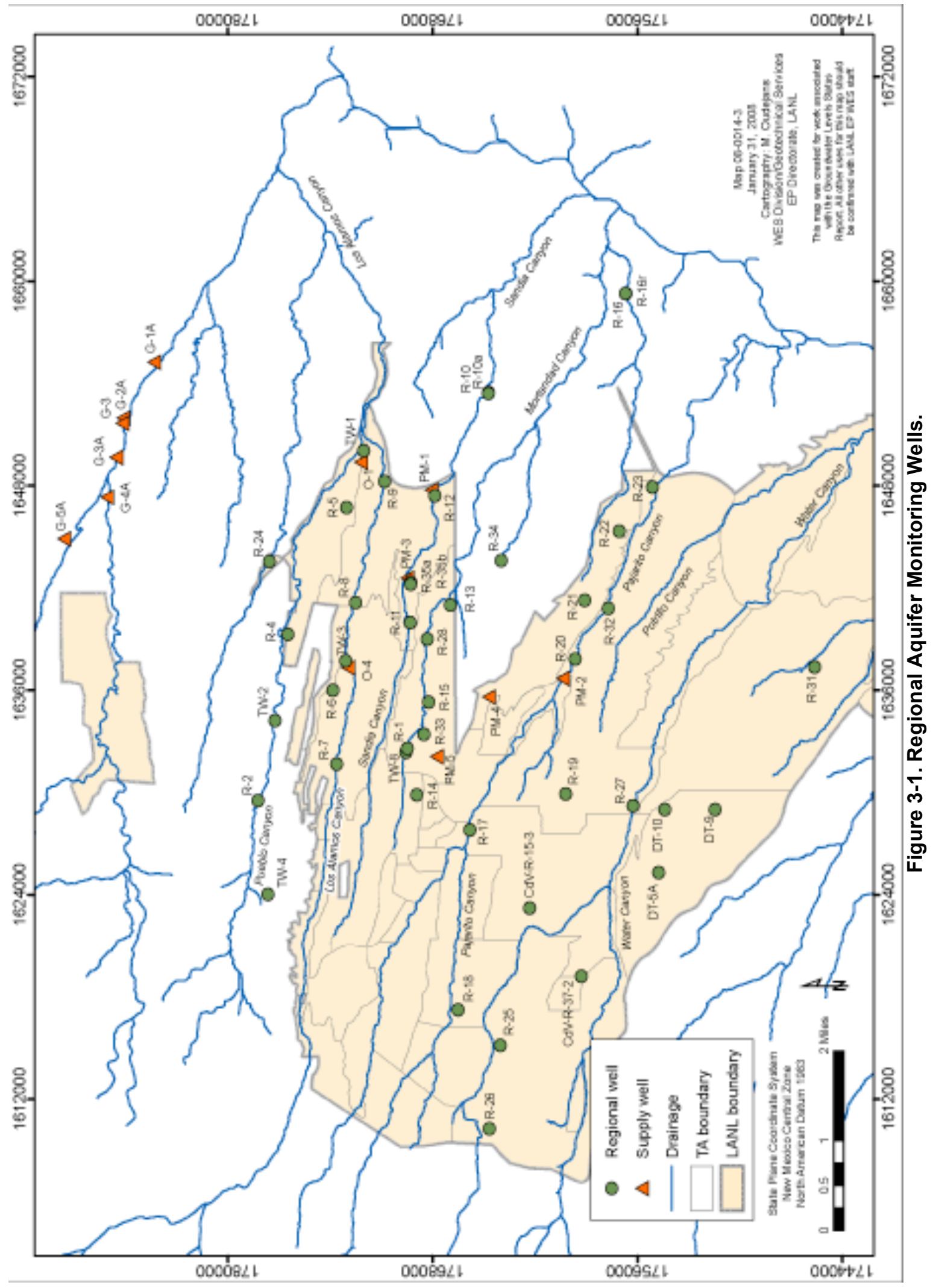


Table 3-1. General Information for Regional Aquifer Wells

\begin{tabular}{|c|c|c|c|c|c|}
\hline Well Name & $\begin{array}{c}\text { Date } \\
\text { Completed }\end{array}$ & $\begin{array}{l}\text { Completed } \\
\text { Depth (ft) }\end{array}$ & Easting (ft) & Northing (ft) & $\begin{array}{c}\text { Surface } \\
\text { Elevation (ft) }\end{array}$ \\
\hline CdV-R-15-3 & $05 / 03 / 00$ & 1722.0 & 1623222.30 & 1762347.50 & 7258.90 \\
\hline CdV-R-37-2 & $08 / 09 / 01$ & 1664.0 & 1619216.70 & 1759320.80 & 7330.60 \\
\hline R-1 & 11/10/03 & $\overline{1080.1}$ & 1632354.13 & 1769600.84 & 6881.21 \\
\hline $\mathrm{R}-2$ & $10 / 19 / 03$ & 943.3 & 1629519.57 & 1778281.56 & 6770.38 \\
\hline $\mathrm{R}-4$ & $09 / 28 / 03$ & 840.0 & 1639287.98 & 1776530.28 & 6577.49 \\
\hline$R-5$ & $05 / 22 / 01$ & 884.0 & 1646707.00 & 1773063.00 & 6472.60 \\
\hline $\mathrm{R}-6$ & $11 / 16 / 04$ & 1252.0 & 1636011.02 & 1773884.07 & 6995.80 \\
\hline R-7 & $01 / 20 / 01$ & 977.0 & 1631666.00 & 1773653.00 & 6779.20 \\
\hline R-8 & $01 / 28 / 02$ & 850.0 & 1641139.01 & 1772554.62 & 6544.74 \\
\hline $\mathrm{R}-9$ & $10 / 04 / 99$ & $\overline{758.0}$ & 1648236.50 & 1770847.10 & 6382.80 \\
\hline$R-10$ & $10 / 05 / 05$ & 1079.0 & 1653465.92 & 1764766.46 & 6362.31 \\
\hline$R-10 a$ & $08 / 18 / 05$ & 706.0 & 1653411.63 & 1764782.29 & 6363.74 \\
\hline$R-11$ & $10 / 08 / 04$ & 901.7 & 1639959.31 & 1769353.57 & 6673.72 \\
\hline $\mathrm{R}-12$ & $01 / 11 / 00$ & 886.0 & 1647424.20 & 1767913.40 & 6499.60 \\
\hline$R-13$ & $10 / 06 / 01$ & 1029.4 & 1640991.66 & 1766994.17 & 6673.05 \\
\hline $\mathrm{R}-14$ & $07 / 04 / 02$ & 1315.6 & 1629855.01 & 1768953.12 & 7062.08 \\
\hline$R-15$ & $09 / 21 / 99$ & 1030.6 & 1635308.60 & 1768272.50 & 6820.00 \\
\hline$R-16$ & $08 / 31 / 02$ & 1276.7 & 1659283.61 & 1756710.97 & 6256.87 \\
\hline R-16r & 10/11/05 & 631.4 & 1659289.39 & 1756730.68 & 6256.97 \\
\hline R-17 & $01 / 04 / 06$ & 1140.9 & 1627795.96 & 1765861.23 & 6921.51 \\
\hline$R-18$ & $12 / 14 / 04$ & 1405.0 & 1617254.37 & 1766545.47 & 7404.83 \\
\hline $\mathrm{R}-19$ & $03 / 30 / 00$ & 1877.4 & 1629918.40 & 1760252.10 & 7066.30 \\
\hline $\mathrm{R}-20$ & $09 / 08 / 02$ & 1353.3 & 1637835.40 & 1759694.51 & 6694.35 \\
\hline $\mathrm{R}-21$ & $11 / 20 / 02$ & 941.4 & 1641284.17 & 1759143.06 & 6656.24 \\
\hline $\mathrm{R}-22$ & $10 / 19 / 00$ & 1472.9 & 1645324.40 & 1757111.10 & 6650.50 \\
\hline$R-23$ & $10 / 02 / 02$ & 886.3 & 1647913.60 & 1755165.37 & 6527.75 \\
\hline R-24 & $09 / 02 / 05$ & 861.0 & 1643554.46 & 1777591.35 & 6547.38 \\
\hline R-25 & $09 / 28 / 00$ & 1934.7 & 1615178.42 & 1764060.50 & 7516.10 \\
\hline$R-26$ & $10 / 17 / 03$ & 1479.0 & 1610267.23 & 1764721.12 & 7641.69 \\
\hline $\mathrm{R}-27$ & $11 / 07 / 05$ & 878.7 & 1629230.52 & 1756296.28 & 6713.72 \\
\hline$R-28$ & $12 / 17 / 03$ & 980.3 & 1638988.73 & 1768358.57 & 6728.61 \\
\hline$R-31$ & $12 / 01 / 00$ & 1077.7 & 1637353.80 & 1745648.40 & 6362.50 \\
\hline R-32 & $08 / 09 / 02$ & 1002.0 & 1640797.67 & 1757730.25 & 6637.63 \\
\hline$R-33$ & $10 / 13 / 04$ & 1126.0 & 1633401.71 & 1768532.65 & 6853.33 \\
\hline$R-34$ & $08 / 20 / 04$ & 920.7 & 1643595.82 & 1764028.77 & 6629.99 \\
\hline R-35a & $06 / 21 / 07$ & 1086.2 & 1642321.701 & 1769316.232 & 6623.06 \\
\hline$R-35 b$ & $07 / 11 / 07$ & 872.2 & 1642229.877 & 1769328.368 & 6625.21 \\
\hline TW-1 & $01 / 03 / 50$ & 642.0 & 1650041.50 & 1772076.87 & 6369.19 \\
\hline TW-2 & $12 / 15 / 90$ & 834.0 & 1634231.12 & 1777267.87 & 6648.06 \\
\hline TW-3 & $11 / 20 / 49$ & 815.0 & 1637727.50 & 1773138.12 & 6626.90 \\
\hline TW-4 & $03 / 08 / 50$ & 1205.0 & 1624028.12 & 1777680.12 & 7244.56 \\
\hline TW-8 & $12 / 15 / 60$ & 1065.0 & 1632573.87 & 1769506.62 & 6873.50 \\
\hline DT-10 & $03 / 13 / 60$ & 1408.0 & 1628988.50 & 1754448.75 & 7019.90 \\
\hline DT-5A & $01 / 25 / 60$ & 1819.5 & 1625310.00 & 1754789.37 & 7143.86 \\
\hline DT-9 & $02 / 19 / 60$ & 1501.0 & 1628993.62 & 1751492.62 & 6935.00 \\
\hline
\end{tabular}




\subsection{CdV-R-15-3}

Location: CdV-R-15-3 is located on a mesa between upper Three-Mile Canyon and Cañon de Valle within the Cañon de Valle watershed.

Completion Type: Multiple completion, three screens in intermediate zones, three screens in regional zones.

Period of Record: Westbay ${ }^{\circledR}$ installed September 17, 2000; transducers installed March 1, 2001; intermittent data through 2007.

Remarks: The three intermediate screens have been dry since well installation. A transducer was never installed at screen 2 . Transducers monitoring dry screens 1 and 3 were removed in January 2006. Regional screens 4 and 5 have similar heads; screen 6 head is $35 \mathrm{ft}$ lower. Six $\mathrm{ft}$ of water appeared in screen 3 sump at MP3C October 2006; still present in October 2007. Screens 4 and 5 do not indicate a water level response to atmospheric pressure fluctuations; screen 6 indicates a $30 \%$ response to atmospheric pressure.

\begin{tabular}{|c|c|c|c|c|c|c|c|c|c|c|c|}
\hline \multirow[b]{2}{*}{ Zone } & \multicolumn{10}{|c|}{ Measurement and Sampling Ports in CDVR-15-3 } & \multirow[b]{2}{*}{ Comment } \\
\hline & $\begin{array}{c}\text { Screen } \\
\text { Top } \\
\text { Depth (ft) }\end{array}$ & $\begin{array}{l}\text { Screen } \\
\text { Bottom } \\
\text { Depth } \\
\text { (ft) }\end{array}$ & $\begin{array}{c}\text { Screen } \\
\text { Top } \\
\text { Elev (ft) }\end{array}$ & $\begin{array}{l}\text { Screen } \\
\text { Bottom } \\
\text { Elev (ft) }\end{array}$ & $\begin{array}{l}\text { Screen } \\
\text { Length } \\
\text { (ft) }\end{array}$ & Port & $\begin{array}{c}\text { Port } \\
\text { Depth (ft) }\end{array}$ & $\begin{array}{c}\text { Port } \\
\text { Elevation } \\
\text { (ft) }\end{array}$ & $\begin{array}{l}\text { Distance } \\
\text { from } \\
\text { Bottom of } \\
\text { Screen } \\
\text { (ft) }\end{array}$ & $\begin{array}{l}\text { Sump } \\
\text { Volume } \\
\text { (L) }\end{array}$ & \\
\hline \multirow{3}{*}{1} & \multirow{3}{*}{617.7} & \multirow{3}{*}{624.5} & \multirow{3}{*}{6641.2} & \multirow{3}{*}{6634.4} & \multirow{3}{*}{6.8} & MP1A & 624.3 & 6634.6 & 0.2 & & Within Screen, port dry \\
\hline & & & & & & PP1 & 629.7 & 6629.2 & -5.2 & 11.2 & Below Screen \\
\hline & & & & & & MP1B & 635.3 & 6623.6 & -10.8 & 23.4 & Below Screen, port dry \\
\hline \multirow{3}{*}{2} & \multirow{3}{*}{800.8} & \multirow{3}{*}{807.8} & \multirow{3}{*}{6458.1} & \multirow{3}{*}{6451.1} & \multirow{3}{*}{7.0} & MP2A & 807.3 & 6451.6 & 0.5 & & Within Screen, port dry \\
\hline & & & & & & PP2 & 812.6 & 6446.3 & -4.8 & 10.4 & Below Screen \\
\hline & & & & & & MP2B & 818.3 & 6440.6 & -10.5 & 22.7 & Below Screen \\
\hline \multirow{4}{*}{3} & \multirow{4}{*}{964.8} & \multirow{4}{*}{980.9} & \multirow{4}{*}{6294.1} & \multirow{4}{*}{6278.0} & \multirow{4}{*}{16.1} & MP3A & 969.0 & 6289.9 & 11.9 & & Within Screen, port dry \\
\hline & & & & & & MP3B & 979.3 & 6279.6 & 1.6 & & Within Screen, port dry \\
\hline & & & & & & PP3 & 984.7 & 6274.2 & -3.8 & 8.2 & Below Screen \\
\hline & & & & & & MP3C & 990.3 & 6268.6 & -9.4 & 20.3 & Below Screen, 6' water in sump \\
\hline \multirow{5}{*}{4} & \multirow{5}{*}{1235.1} & \multirow{5}{*}{1278.9} & \multirow{5}{*}{6023.8} & \multirow{5}{*}{5980.0} & \multirow{5}{*}{43.8} & MP4A & 1254.4 & 6004.5 & 24.5 & & Within Screen, Regional Aquifer \\
\hline & & & & & & PP4A & 1259.6 & 5999.3 & 19.3 & & Within Screen \\
\hline & & & & & & MP4B & 1275.1 & 5983.8 & 3.8 & & Within Screen \\
\hline & & & & & & PP4B & 1280.5 & 5978.4 & -1.6 & 3.5 & Below Screen \\
\hline & & & & & & MP4C & 1286.1 & 5972.8 & -7.2 & 15.6 & Below Screen \\
\hline \multirow{3}{*}{5} & \multirow{3}{*}{1348.4} & \multirow{3}{*}{1355.3} & \multirow{3}{*}{5910.5} & \multirow{3}{*}{5903.6} & & MP5A & 1350.1 & 5908.8 & 5.2 & & Within Screen \\
\hline & & & & & 6.9 & PP5 & 1355.4 & 5903.5 & -0.1 & 0.2 & Below Screen \\
\hline & & & & & & MP5B & 1361.1 & 5897.8 & -5.8 & 12.5 & Below Screen \\
\hline & & & & & & MP6A & 1640.1 & 5618.8 & 4.7 & & Within Screen \\
\hline 6 & 1637.9 & 1644.8 & 5621.0 & 5614.1 & 6.9 & PP6 & 1645.5 & 5613.4 & -0.7 & 1.5 & Below Screen \\
\hline & & & & & & MP6B & 1651.1 & 5607.8 & -6.3 & 13.6 & Below Screen \\
\hline
\end{tabular}

Note: CDV-R-15-3 Brass Cap Ground Elevation: $7258.9 \mathrm{ft}$; all measurements are from this elevation;

$\mathrm{MP}=$ Monitor Port; $\mathrm{PP}=$ Pump Port; Monitor Ports shown in bold are instrumented ports 

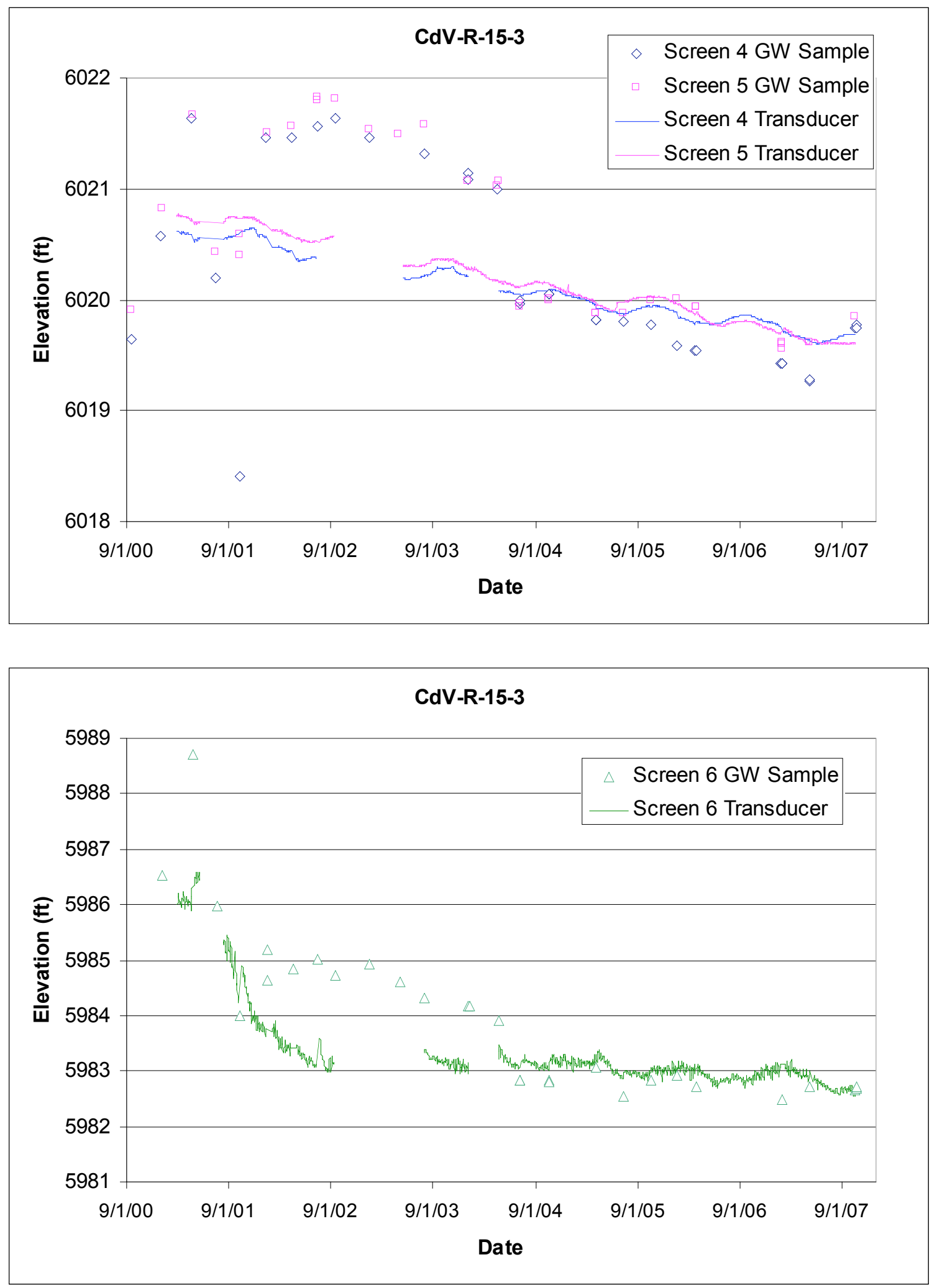


\subsection{CdV-R-37-2}

Location: CdV-R-37-2 is located on a mesa between Cañon de Valle and Water Canyon at Technical Area (TA) 37 in the Water Canyon watershed.

Completion Type: Multiple completion, one screen in an intermediate zone, three screens in regional zones.

Period of Record: Westbay ${ }^{\circledR}$ installed October 8, 2001; transducers installed August 8, 2003; data through 2007.

Remarks: The intermediate screen has been dry since well installation; the transducer at this screen was removed in January 2006. The three regional screens have similar heads that show downward gradient of about $1 \mathrm{ft}$ between each screen. The screens do not indicate a water level response to atmospheric pressure fluctuations.

\begin{tabular}{|c|c|c|c|c|c|c|c|c|c|c|c|}
\hline \multirow[b]{2}{*}{ Zone } & \multicolumn{10}{|c|}{ Measurement and Sampling Ports in CDV-R-37-2 } & \multirow[b]{2}{*}{ Comment } \\
\hline & $\begin{array}{c}\text { Screen } \\
\text { Top } \\
\text { Depth (ft) }\end{array}$ & $\begin{array}{l}\text { Screen } \\
\text { Bottom } \\
\text { Depth } \\
\text { (ft) }\end{array}$ & $\begin{array}{c}\text { Screen } \\
\text { Top } \\
\text { Elev (ft) }\end{array}$ & $\begin{array}{l}\text { Screen } \\
\text { Bottom } \\
\text { Elev (ft) }\end{array}$ & $\begin{array}{l}\text { Screen } \\
\text { Length } \\
\text { (ft) }\end{array}$ & Port & $\begin{array}{c}\text { Port } \\
\text { Depth (ft) }\end{array}$ & $\begin{array}{c}\text { Port } \\
\text { Elevation } \\
\text { (ft) }\end{array}$ & $\begin{array}{l}\text { Distance } \\
\text { from } \\
\text { Bottom of } \\
\text { Screen } \\
\text { (ft) }\end{array}$ & $\begin{array}{l}\text { Sump } \\
\text { Volume } \\
\text { (L) }\end{array}$ & \\
\hline \multirow{3}{*}{1} & \multirow{3}{*}{914.4} & \multirow{3}{*}{939.5} & \multirow{3}{*}{6416.2} & \multirow{3}{*}{6391.1} & \multirow{3}{*}{25.1} & MP1A & 934.9 & 6395.7 & 4.6 & & Within Screen, Intermediate (Dry) \\
\hline & & & & & & PP1 & 940.2 & 6390.4 & -0.7 & 1.5 & Below Screen \\
\hline & & & & & & MP1B & 945.9 & 6384.7 & -6.4 & 13.8 & Below Screen \\
\hline \multirow{3}{*}{2} & \multirow{3}{*}{1188.7} & \multirow{3}{*}{1213.8} & \multirow{3}{*}{6141.9} & \multirow{3}{*}{6116.8} & \multirow{3}{*}{25.1} & MP2A & 1200.3 & 6130.3 & 13.5 & & Within Screen, Regional Aquifer \\
\hline & & & & & & PP2 & 1205.7 & 6124.9 & 8.1 & & Within Screen \\
\hline & & & & & & MP2B & 1216.2 & 6114.4 & -2.4 & 5.2 & Below Screen \\
\hline \multirow{3}{*}{3} & \multirow{3}{*}{1353.7} & \multirow{3}{*}{1377.1} & \multirow{3}{*}{5976.9} & \multirow{3}{*}{5953.5} & \multirow{3}{*}{23.4} & MP3A & 1359.3 & 5971.3 & 17.8 & & Within Screen \\
\hline & & & & & & PP3 & 1365.0 & 5965.6 & 12.1 & & Within Screen \\
\hline & & & & & & MP3B & 1375.2 & 5955.4 & 1.9 & & Within Screen \\
\hline \multirow{3}{*}{4} & \multirow{3}{*}{1549.3} & \multirow{3}{*}{1556.0} & \multirow{3}{*}{5781.3} & \multirow{3}{*}{5774.6} & \multirow{3}{*}{6.7} & MP4A & 1550.6 & 5780.0 & 5.4 & & Within Screen \\
\hline & & & & & & PP4 & 1556.0 & 5774.6 & 0 & & Base of Screen \\
\hline & & & & & & MP4B & 1561.6 & 5769.0 & -5.6 & 12.1 & Below Screen \\
\hline
\end{tabular}

Note: CDV-R-37-2 Brass Cap Ground Elevation: $7330.6 \mathrm{ft}$; all measurements are from this elevation;

$\mathrm{MP}=$ Monitor Port; PP = Pump Port; Monitor Ports shown in bold are instrumented ports 


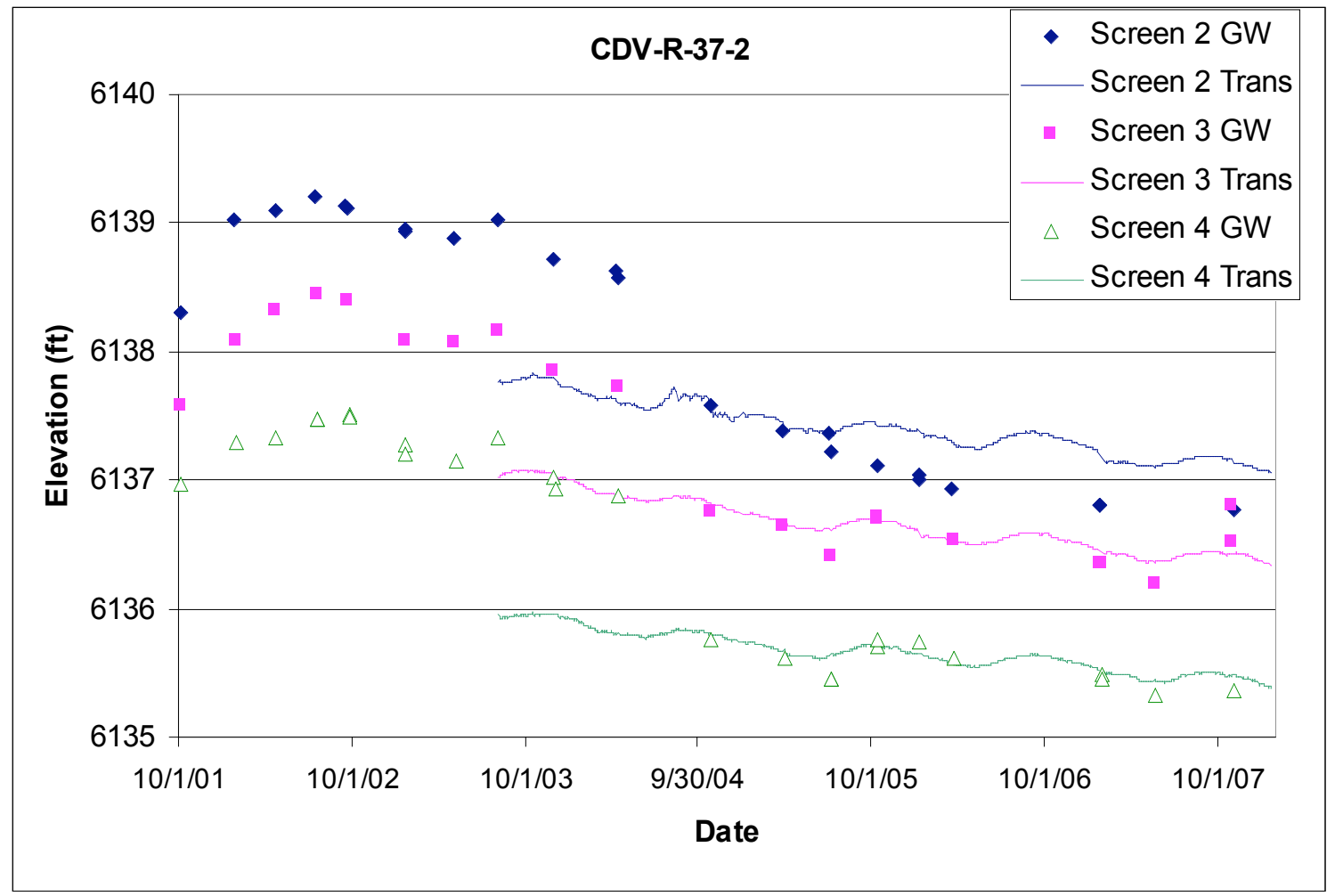




\section{$3.3 \quad$ R-1}

Location: R-1 is located in Mortandad Canyon about $220 \mathrm{ft}$ west of TW-8.

Completion Type: Single completion at the top of the regional aquifer. The top of the screen is about $29 \mathrm{ft}$ below the water table.

Period of Record: Well completed November 2003, transducer installed January 2005, transducer data through 2007.

Remarks: R-1 was completed to a depth of $1080.1 \mathrm{ft}$, about $80 \mathrm{ft}$ into the regional aquifer. The well is $100 \%$ barometrically efficient; the aquifer has no immediate response to atmospheric pressure fluctuations. The aquifer indicates a seasonal response to supply well pumping and primarily responds to pumping PM-5, and possibly to pumping PM-4.

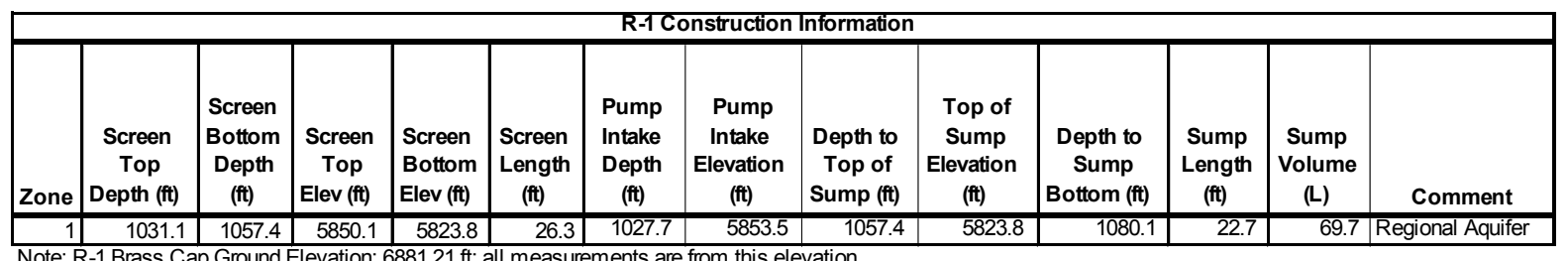

Note: R-1 Brass Cap Ground Elevation: $6881.21 \mathrm{ft}$; all measurements are from this elevation

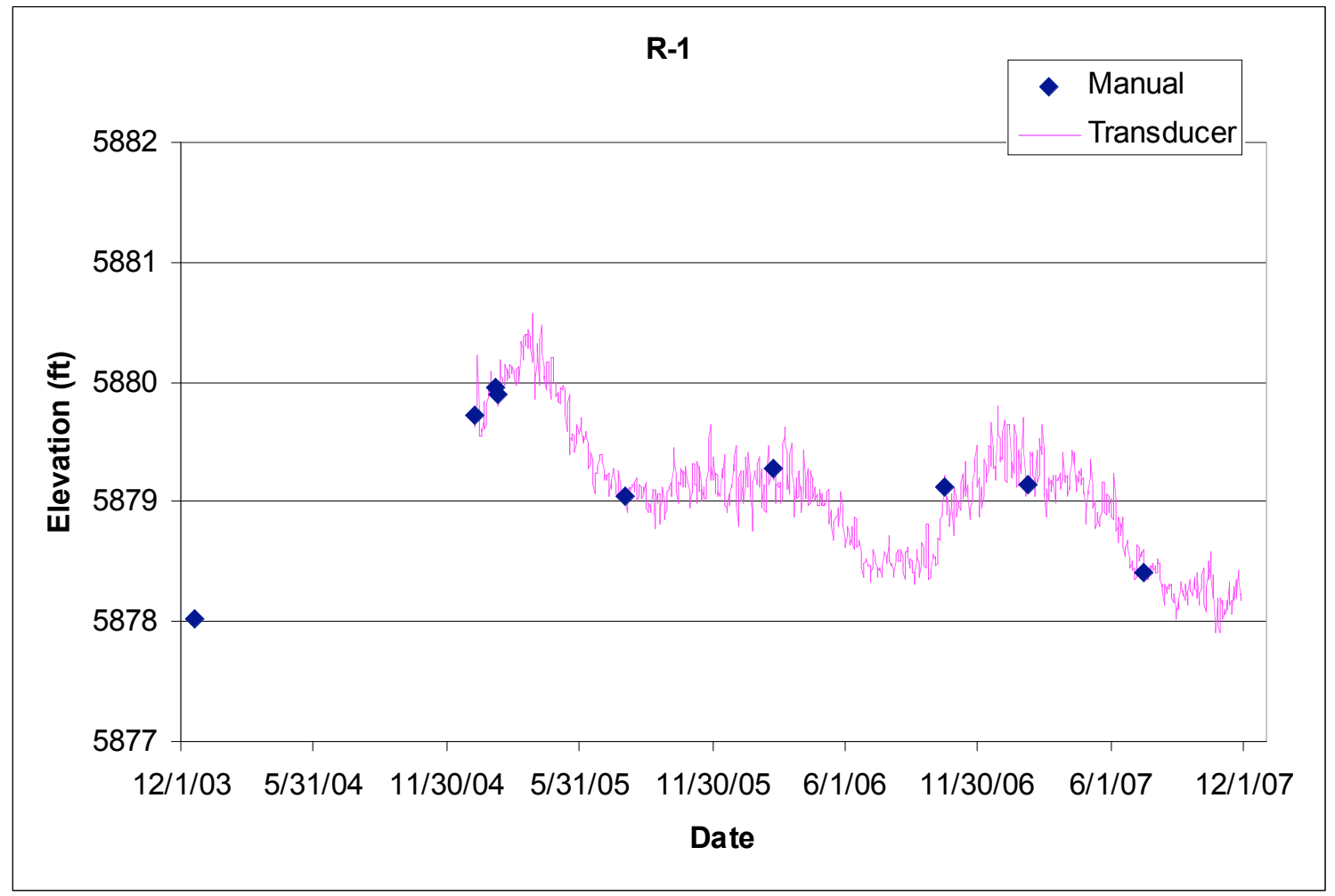




\section{$3.4 \quad \mathrm{R}-2$}

Location: R-2 is located in middle Pueblo Canyon between TW-4 and TW-2.

Completion Type: Single completion at the top of the regional aquifer. The top of the screen is about $8 \mathrm{ft}$ below the water table.

Period of Record: Well completed October 2003, transducer installed January 2005, transducer data through 2007.

Remarks: R-2 was completed to a depth of $943.3 \mathrm{ft}$, about $50 \mathrm{ft}$ into the regional aquifer. The well is $100 \%$ barometrically efficient; the aquifer has no immediate response to atmospheric pressure fluctuations. The well does not indicate a seasonal response to supply well pumping or an apparent response to pumping of any specific supply well.

\begin{tabular}{|c|c|c|c|c|c|c|c|c|c|c|c|c|c|}
\hline \multicolumn{14}{|c|}{ R-2 Construction Information } \\
\hline Zone & $\begin{array}{c}\text { Screen } \\
\text { Top } \\
\text { Depth (ft) }\end{array}$ & $\begin{array}{c}\text { Screen } \\
\text { Bottom } \\
\text { Depth } \\
\text { (ft) }\end{array}$ & $\begin{array}{c}\text { Screen } \\
\text { Top } \\
\text { Elev (ft) }\end{array}$ & $\begin{array}{l}\text { Screen } \\
\text { Bottom } \\
\text { Elev (ft) }\end{array}$ & $\begin{array}{c}\text { Screen } \\
\text { Length } \\
\text { (ft) }\end{array}$ & $\begin{array}{l}\text { Pump } \\
\text { Intake } \\
\text { Depth } \\
\text { (ft) }\end{array}$ & $\begin{array}{l}\text { Pump } \\
\text { Intake } \\
\text { Elevation } \\
\text { (ft) }\end{array}$ & $\begin{array}{c}\text { Depth to } \\
\text { Top of } \\
\text { Sump (ft) }\end{array}$ & $\begin{array}{c}\text { Top of } \\
\text { Sump } \\
\text { Elevation } \\
\text { (ft) }\end{array}$ & $\begin{array}{c}\text { Depth to } \\
\text { Sump } \\
\text { Bottom (ft) }\end{array}$ & $\begin{array}{l}\text { Sump } \\
\text { Length } \\
\text { (ft) }\end{array}$ & $\begin{array}{c}\text { Sump } \\
\text { Volume } \\
\text { (L) }\end{array}$ & Comment \\
\hline$\overline{1}$ & 906.4 & 929.6 & 5864.0 & 5840.8 & 23.2 & 917 & 5853.4 & 929.6 & 5840.8 & 943.3 & 13.7 & 42.1 & Regional Aquife \\
\hline
\end{tabular}

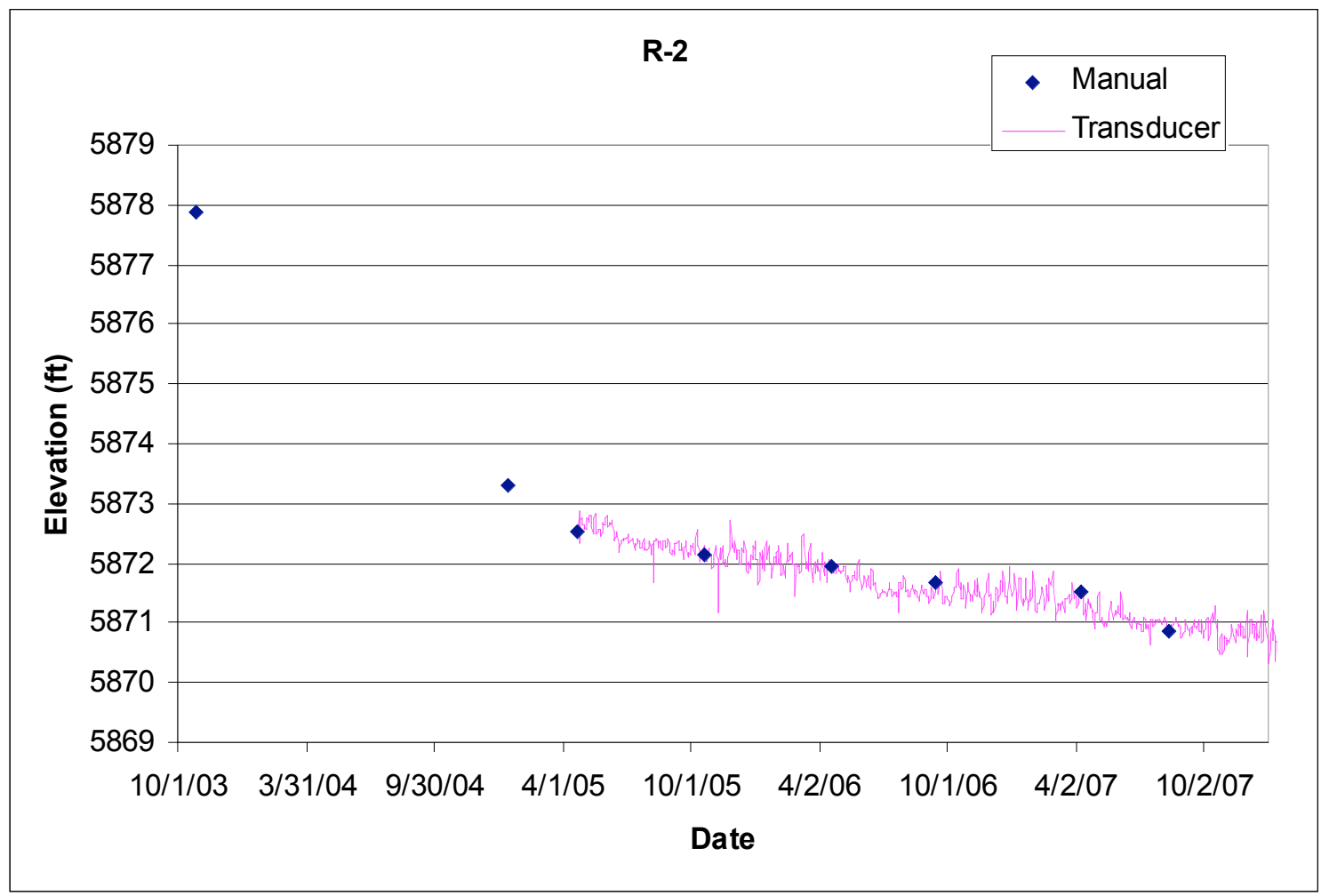




\section{$3.5 \quad \mathrm{R}-4$}

Location: R-4 is located in Pueblo Canyon upstream of the Bayo Sewage Treatment Plant.

Completion Type: Single completion at the top of the regional aquifer. The top of the screen is about $49 \mathrm{ft}$ below the piezometric water table in a confined zone.

Period of Record: Well completed September 2003, transducer installed January 2005, data through December 2007.

Remarks: R-4 was completed to a depth of $840 \mathrm{ft}$, about $90 \mathrm{ft}$ into the regional aquifer. The water level appears to respond in part to pumping of supply well PM-3. The well is $100 \%$ barometrically efficient; the aquifer has no immediate response to atmospheric pressure fluctuations. The aquifer indicates a seasonal response to supply well pumping and appears to respond primarily to pumping PM-3, and possibly to pumping at O-4 and the Guaje well field.

\begin{tabular}{|c|c|c|c|c|c|c|c|c|c|c|c|c|c|}
\hline \multicolumn{14}{|c|}{ R-4Construction Information } \\
\hline Zone & $\begin{array}{c}\text { Screen } \\
\text { Top } \\
\text { Depth (ft) }\end{array}$ & $\begin{array}{l}\text { Screen } \\
\text { Bottom } \\
\text { Depth } \\
\text { (ft) }\end{array}$ & $\begin{array}{c}\text { Screen } \\
\text { Top } \\
\text { Elev }(\mathrm{ft})\end{array}$ & $\begin{array}{l}\text { Screen } \\
\text { Bottom } \\
\text { Elev (ft) }\end{array}$ & $\begin{array}{l}\text { Screen } \\
\text { Length } \\
\text { (ft) }\end{array}$ & $\begin{array}{l}\text { Pump } \\
\text { Intake } \\
\text { Depth } \\
\text { (ft) }\end{array}$ & $\begin{array}{l}\text { Pump } \\
\text { Intake } \\
\text { Elevation } \\
\text { (ft) }\end{array}$ & $\begin{array}{l}\text { Depth to } \\
\text { Top of } \\
\text { Sump (ft) }\end{array}$ & $\begin{array}{l}\text { Top of } \\
\text { Sump } \\
\text { Elevation } \\
\text { (ft) }\end{array}$ & $\begin{array}{l}\text { Depth to } \\
\text { Sump } \\
\text { Bottom (ft) }\end{array}$ & $\begin{array}{l}\text { Sump } \\
\text { Length } \\
\text { (ft) }\end{array}$ & $\begin{array}{c}\text { Sump } \\
\text { Volume } \\
\text { (L) }\end{array}$ & Comment \\
\hline & 792.9 & 816 & 5784.6 & 5761.5 & 23.1 & 787.5 & 5790.0 & 816.0 & 5761.5 & 840 & 24.0 & 73.7 & Regional Aquifer \\
\hline
\end{tabular}

Note: R-4 Brass Cap Ground Elevation: $6577.49 \mathrm{ft}$; all measurements are from this elevation

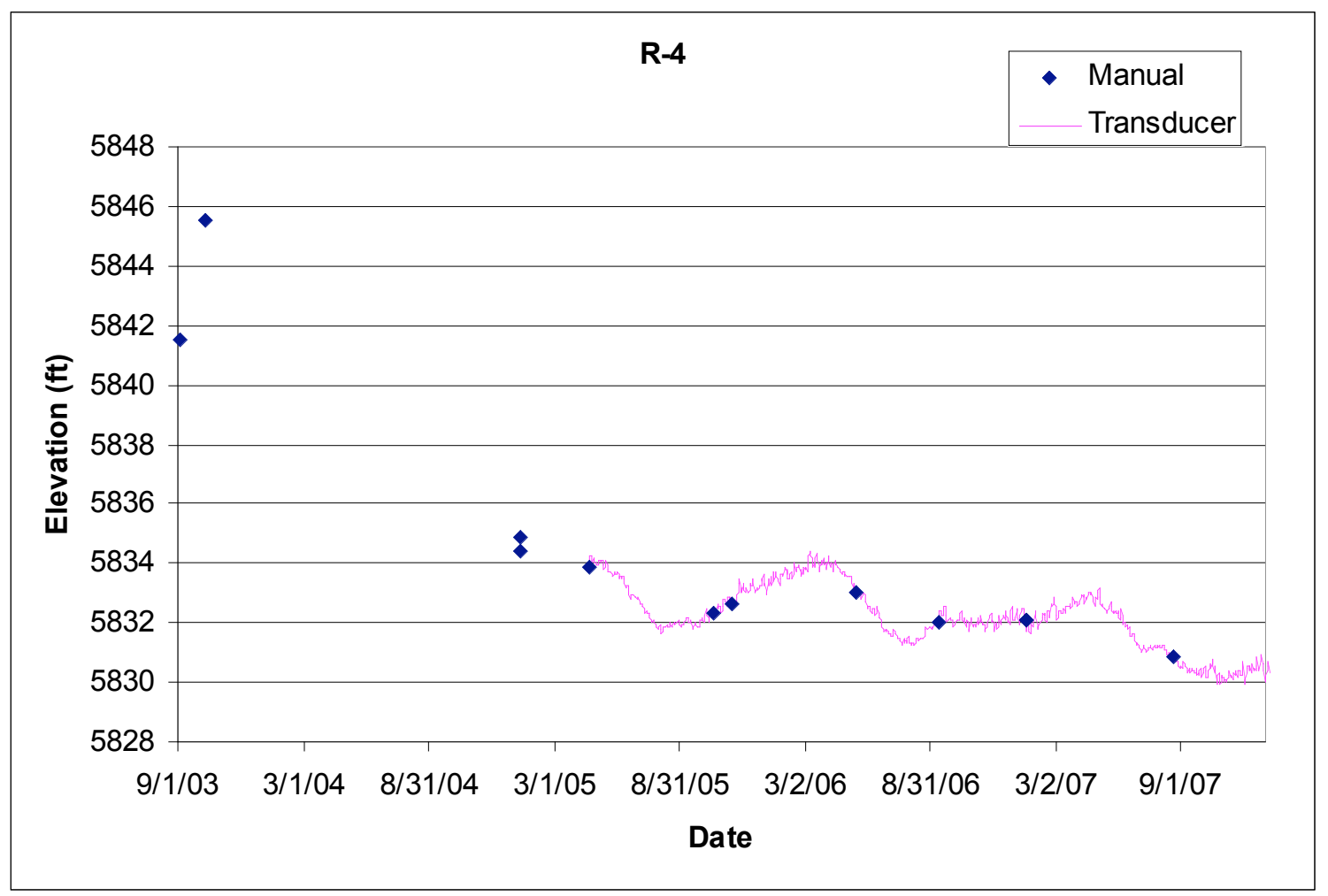




\section{$3.6 \quad \mathrm{R}-5$}

Location: R-5 is located in lower Pueblo Canyon upstream of supply well O-1.

Completion Type: Multiple completion, two screens in intermediate zones, two screens in regional zones.

Period of Record: Westbay ${ }^{\circledR}$ installed July 17, 2001, transducers installed December 17, 2001, and April 4, 2005, intermittent data through 2007.

Remarks: Screen 1 has been dry since well installation, although there is a small amount of water in the sump below screen 1 . The two regional screens have heads about 10 to $15 \mathrm{ft}$ apart. The water level at the top of the regional aquifer at screen 3 declined below port MP3A in 2001; samples are collected and groundwater level is monitored from port MP3B. The water level at screen 4 responds primarily to supply well pumping at PM-1, but screen 3 apparently shows little or no response. The R- 5 screens do not indicate a response to atmospheric pressure fluctuations.

\begin{tabular}{|c|c|c|c|c|c|c|c|c|c|c|c|}
\hline \multicolumn{12}{|c|}{ R-5 Port Data } \\
\hline Zone & $\begin{array}{l}\text { Screen } \\
\text { Top } \\
\text { Depth } \\
\text { (ft) }\end{array}$ & $\begin{array}{c}\text { Screen } \\
\text { Bottom } \\
\text { Depth } \\
\text { (ft) }\end{array}$ & $\begin{array}{c}\text { Screen } \\
\text { Top } \\
\text { Elev } \\
\text { (ft) }\end{array}$ & $\begin{array}{l}\text { Screen } \\
\text { Bottom } \\
\text { Elev (ft) }\end{array}$ & $\begin{array}{l}\text { Screen } \\
\text { Length } \\
\text { (ft) }\end{array}$ & Port & $\begin{array}{l}\text { Port } \\
\text { Depth } \\
\text { (ft) }\end{array}$ & $\begin{array}{c}\text { Port } \\
\text { Elevation } \\
\text { (ft) }\end{array}$ & $\begin{array}{l}\text { Distance } \\
\text { from } \\
\text { Bottom } \\
\text { of Screen } \\
\text { (ft) }\end{array}$ & $\begin{array}{c}\text { Sump } \\
\text { Volume } \\
\text { (L) }\end{array}$ & Comment \\
\hline \multirow{3}{*}{1} & \multirow{3}{*}{326.4} & \multirow{3}{*}{331.5} & \multirow{3}{*}{6146.2} & \multirow{3}{*}{6141.1} & \multirow{3}{*}{5.1} & MP1A & 329.5 & 6143.1 & 2.0 & & Within Screen, Intermediate Zone - Dry \\
\hline & & & & & & $\overline{P P 1}$ & 334.9 & 6137.7 & -3.4 & 9.8 & Below Screen \\
\hline & & & & & & MP1B & 350.4 & 6122.2 & -18.9 & 54.7 & Below Screen \\
\hline \multirow{3}{*}{2} & \multirow{3}{*}{372.8} & \multirow{3}{*}{388.8} & \multirow{3}{*}{6099.8} & \multirow{3}{*}{6083.8} & \multirow{3}{*}{16.0} & MP2A & 383.9 & 6088.7 & 4.9 & & Within Screen, Intermediate Zone \\
\hline & & & & & & PP2 & 388.8 & 6083.8 & 0.0 & 0.0 & At Bottom of Screen \\
\hline & & & & & & MP2B & 394.4 & 6078.2 & -5.6 & 16.2 & Below Screen \\
\hline \multirow{3}{*}{3} & \multirow{3}{*}{676.9} & \multirow{3}{*}{720.3} & \multirow{3}{*}{5795.7} & \multirow{3}{*}{5752.3} & \multirow{3}{*}{43.4} & MP3A & 695.1 & 5777.5 & 25.2 & & Within Screen, Regional Aquifer, Port Dry \\
\hline & & & & & & MP3B & 718.6 & 5754.0 & 1.7 & & Within Screen, MP for GW samples \\
\hline & & & & & & PP3 & 724.0 & 5748.6 & -3.7 & 10.7 & Below Screen \\
\hline \multirow{3}{*}{4} & \multirow{3}{*}{858.7} & \multirow{3}{*}{863.7} & \multirow{3}{*}{5613.9} & \multirow{3}{*}{5608.9} & \multirow{3}{*}{5.0} & MP4A & 860.9 & 5611.7 & 2.8 & & Within Screen \\
\hline & & & & & & PP4 & 866.3 & 5606.3 & -2.6 & 7.5 & Below Screen \\
\hline & & & & & & MP4B & 871.9 & 5600.7 & -8.2 & 23.7 & Below Screen \\
\hline
\end{tabular}

Note: R-5 Brass Cap Ground Elevation: $6472.6 \mathrm{ft}$; all measurements are from this elevation;

$\mathrm{MP}=$ Monitor Port; $\mathrm{PP}=$ Pump Port; Monitor Ports shown in bold are instrumented ports 

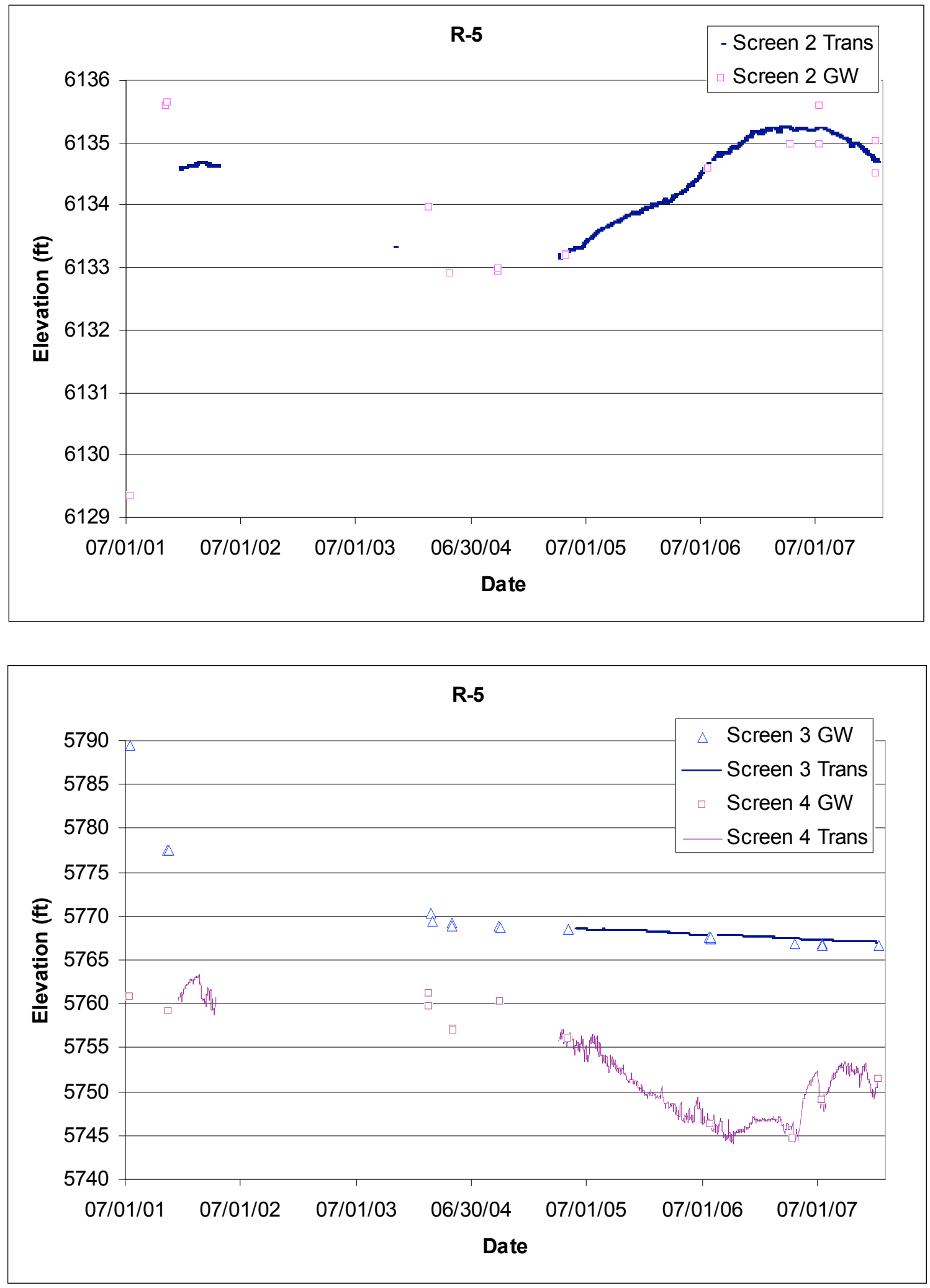


\section{$3.7 \quad$ R-6}

Location: R-6 is located at the east end of DP Mesa between DP Canyon and Los Alamos Canyon. Completion Type: Single completion at the top of the regional aquifer. The top of the screen is about $49 \mathrm{ft}$ below the water table.

Period of Record: Well completed November 2004, transducer installed December 2004, data through 2007.

Remarks: R- 6 was completed to a depth of $1252 \mathrm{ft}$, about $100 \mathrm{ft}$ into the regional aquifer. The well is $100 \%$ barometrically efficient; the aquifer has no immediate response to atmospheric pressure fluctuations.

\begin{tabular}{|c|c|c|c|c|c|c|c|c|c|c|c|c|c|}
\hline \multicolumn{14}{|c|}{ R-6 Construction Information } \\
\hline Zone & $\begin{array}{c}\text { Screen } \\
\text { Top } \\
\text { Depth (ft) }\end{array}$ & $\begin{array}{l}\text { Screen } \\
\text { Bottom } \\
\text { Depth } \\
\text { (ft) }\end{array}$ & $\begin{array}{c}\text { Screen } \\
\text { Top } \\
\text { Elev (ft) }\end{array}$ & $\begin{array}{l}\text { Screen } \\
\text { Bottom } \\
\text { Elev (ft) }\end{array}$ & $\begin{array}{l}\text { Screen } \\
\text { Length } \\
\text { (ft) }\end{array}$ & $\begin{array}{l}\text { Pump } \\
\text { Intake } \\
\text { Depth } \\
\text { (ft) }\end{array}$ & $\begin{array}{l}\text { Pump } \\
\text { Intake } \\
\text { Elevation } \\
\text { (ft) }\end{array}$ & $\begin{array}{l}\text { Depth to } \\
\text { Top of } \\
\text { Sump (ft) }\end{array}$ & $\begin{array}{c}\text { Top of } \\
\text { Sump } \\
\text { Elevation } \\
\text { (ft) }\end{array}$ & $\begin{array}{c}\text { Depth to } \\
\text { Sump } \\
\text { Bottom (ft) }\end{array}$ & $\begin{array}{l}\text { Sump } \\
\text { Length } \\
\text { (ft) }\end{array}$ & $\begin{array}{c}\text { Sump } \\
\text { Volume } \\
\text { (L) }\end{array}$ & Comment \\
\hline 1 & 1205.0 & 1228 & 5790.8 & 5767.8 & 23.0 & & 6995.8 & 1228.0 & 5767.8 & 1252 & 24.0 & 73.7 & Regional Aquifer \\
\hline
\end{tabular}

Note: Brass Cap Ground Elevation: $6995.8 \mathrm{ft}$; all measurements are from this elevation

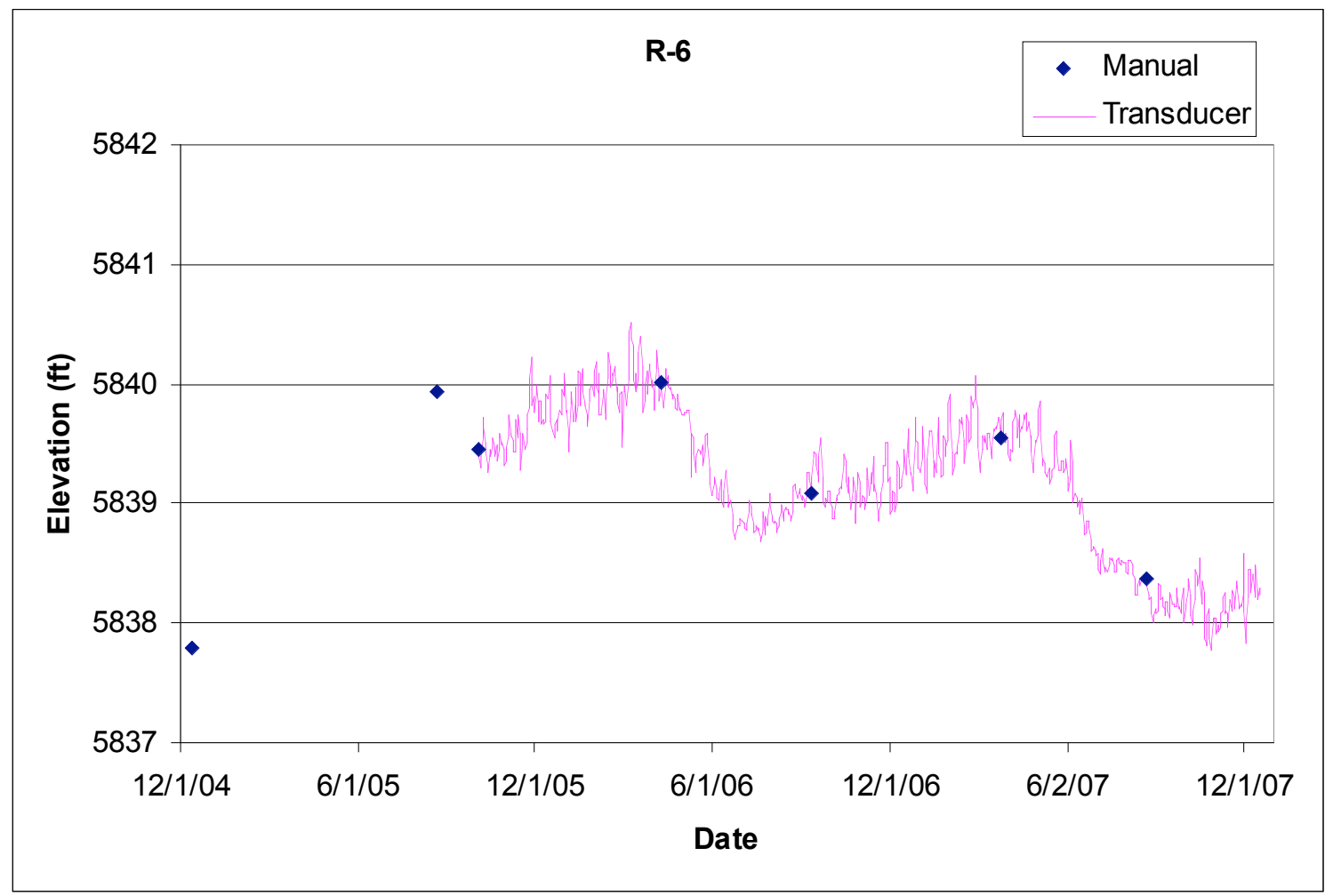




\section{$3.8 \quad \mathrm{R}-7$}

Location: R-7 is located in middle Los Alamos Canyon about 1 mile upstream of supply well O-4.

Completion Type: Multiple completion, two screens in intermediate zones, one screen at the top of the regional aquifer.

Period of Record: Westbay ${ }^{\circledR}$ installed February 25, 2001, transducers installed February 28, 2001, intermittent data through 2007.

Remarks: Initial transducer data from MP1A are not valid because transducer apparently did not connect properly to port. Port MP1A at intermediate screen 1 went dry during sampling December 18, 2003. In 2004, MP1B showed water at the bottom of screen 1, but since 2005 no water has been observed at screen 1 . The screen 2 intermediate zone has been dry since well installation. The groundwater at R-7 screens 1 and 3 does not indicate a response to atmospheric pressure fluctuations. The regional aquifer at screen 3 does not show a seasonal water level response and does not show a response to any pumping at any individual water supply well.

\begin{tabular}{|c|c|c|c|c|c|c|c|c|c|c|c|}
\hline \multicolumn{12}{|c|}{ R-7 Measurement and Sampling Ports } \\
\hline Zone & $\begin{array}{c}\text { Screen } \\
\text { Top } \\
\text { Depth (ft) }\end{array}$ & \begin{tabular}{|c|} 
Screen \\
Bottom \\
Depth \\
(ft)
\end{tabular} & $\begin{array}{c}\text { Screen } \\
\text { Top } \\
\text { Elev (ft) }\end{array}$ & $\begin{array}{l}\text { Screen } \\
\text { Bottom } \\
\text { Elev (ft) }\end{array}$ & $\begin{array}{l}\text { Screen } \\
\text { Length } \\
\text { (ft) }\end{array}$ & Port & $\begin{array}{c}\text { Port } \\
\text { Depth (ft) }\end{array}$ & $\begin{array}{c}\text { Port } \\
\text { Elevation } \\
\text { (ft) }\end{array}$ & $\begin{array}{c}\text { Distance } \\
\text { from } \\
\text { Bottom of } \\
\text { Screen (ft) }\end{array}$ & $\begin{array}{c}\text { Sump } \\
\text { Volume } \\
\text { (L) }\end{array}$ & Comment \\
\hline \multirow{3}{*}{1} & \multirow{3}{*}{363.2} & \multirow{3}{*}{379.2} & \multirow{3}{*}{6416.0} & \multirow{3}{*}{6400.0} & \multirow{3}{*}{16.0} & MP1A & 378.0 & 6401.2 & 1.2 & & Within screen, Intermediate Zone \\
\hline & & & & & & PP1 & 383.3 & 6395.9 & -4.1 & 8.9 & Below screen \\
\hline & & & & & & MP1B & 389.0 & 6390.2 & -9.8 & 21.2 & Below screen \\
\hline \multirow{3}{*}{2} & \multirow{3}{*}{730.4} & \multirow{3}{*}{746.4} & \multirow{3}{*}{6048.8} & \multirow{3}{*}{6032.8} & \multirow{3}{*}{16.0} & MP2A & 744.8 & 6034.4 & 1.6 & & Within screen, Intermediate Zone (Dry) \\
\hline & & & & & & PP2 & 750.1 & 6029.1 & -3.7 & 8.0 & Below screen \\
\hline & & & & & & MP2B & 755.8 & 6023.4 & -9.4 & 20.3 & Below screen \\
\hline \multirow{4}{*}{3} & \multirow{4}{*}{895.5} & \multirow{4}{*}{937.4} & \multirow{4}{*}{5883.7} & \multirow{4}{*}{5841.8} & \multirow{4}{*}{41.9} & MP3A & 915.1 & 5864.1 & 22.3 & & Within screen, Regional Aquifer \\
\hline & & & & & & MP3B & 935.3 & 5843.9 & 2.1 & & Within screen \\
\hline & & & & & & PP3 & 940.6 & 5838.6 & -3.2 & 6.9 & Below screen \\
\hline & & & & & & MP3C & 946.3 & 5832.9 & -8.9 & 19.2 & Below screen \\
\hline
\end{tabular}

Note: R-7 Brass Cap Ground Elevation: $6779.2 \mathrm{ft}$; all measurements are from this elevation;

$\mathrm{MP}=$ Monitor Port; PP = Pump Port; Monitor Ports shown in bold are instrumented ports

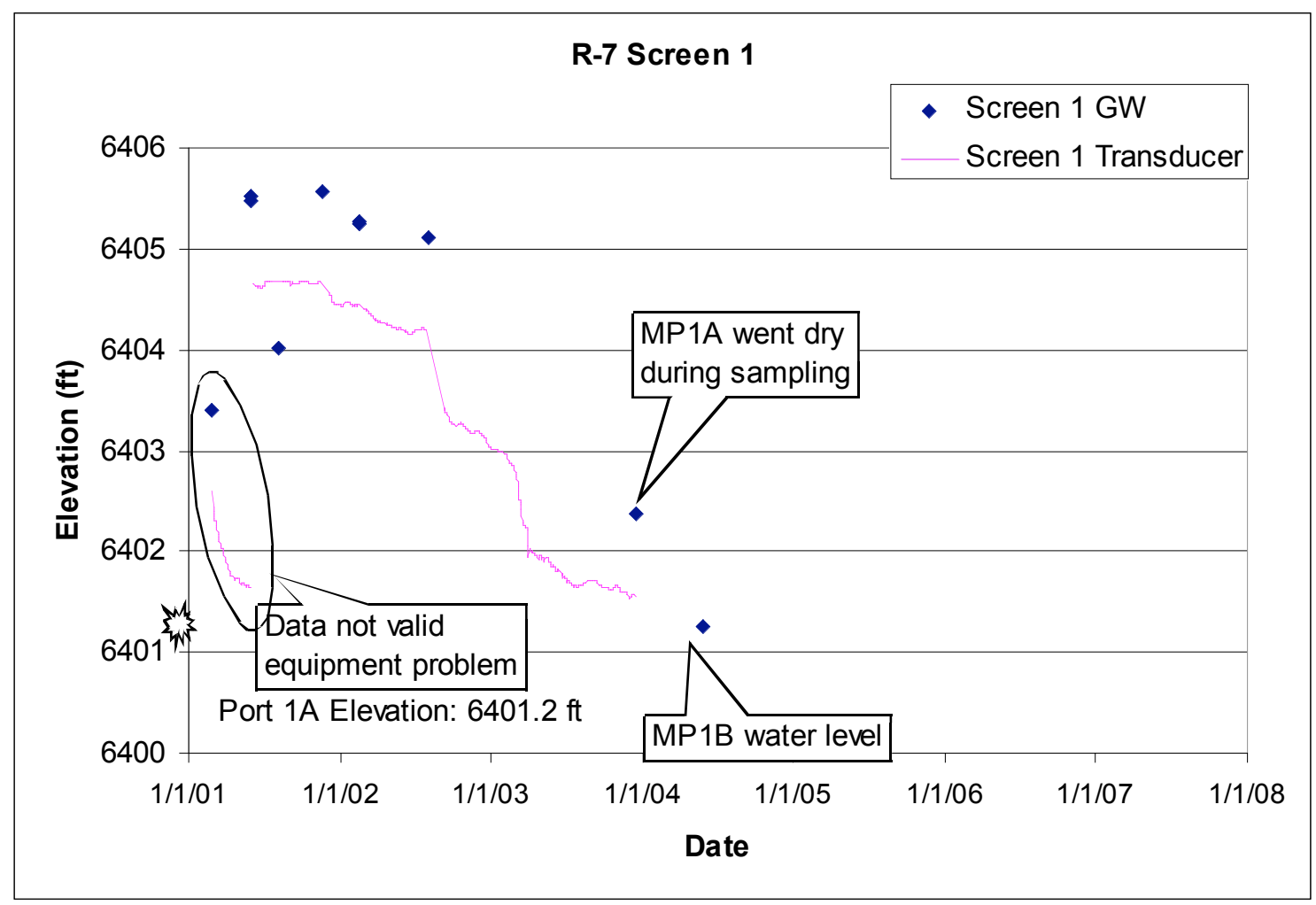




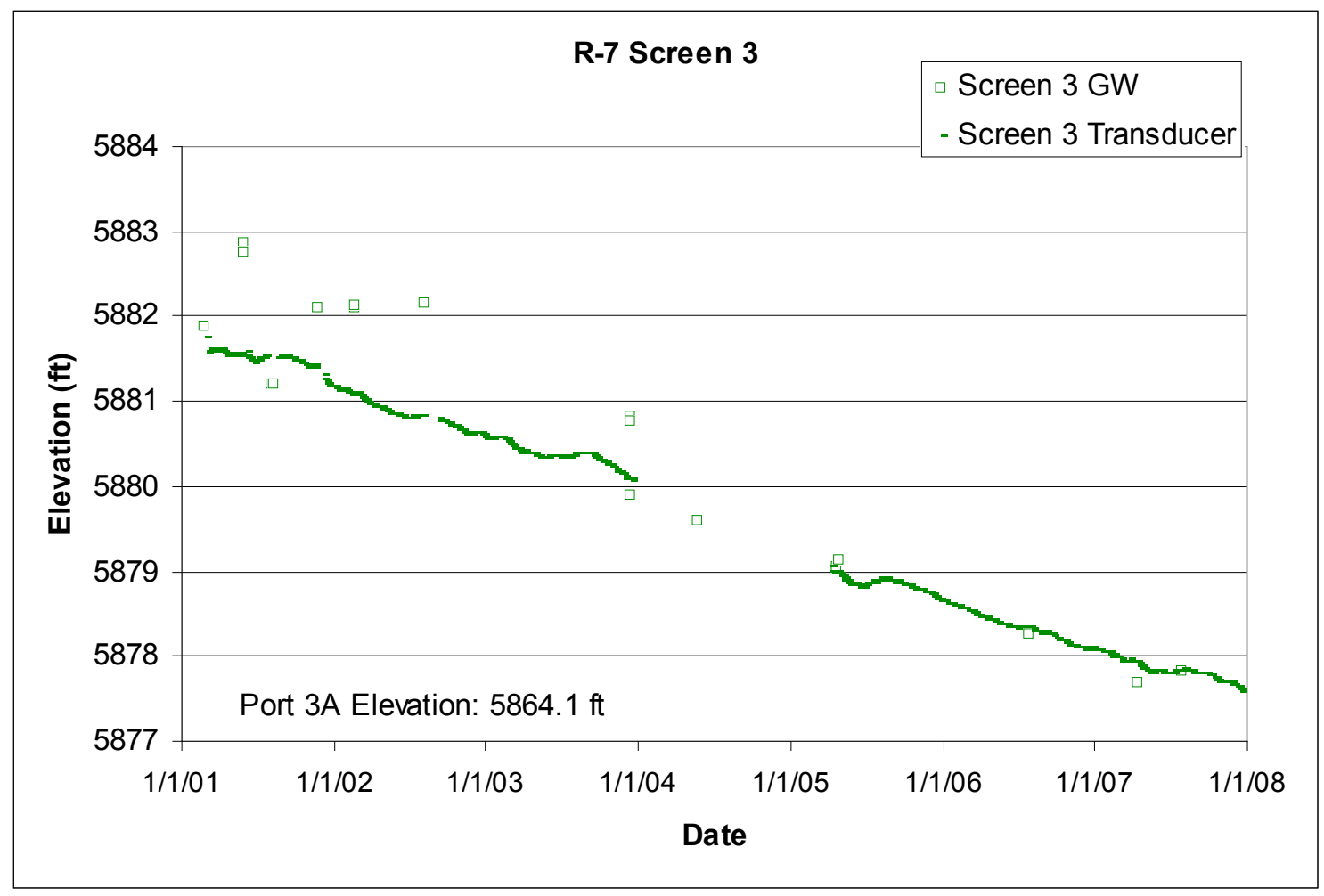




\section{$3.9 \quad$ R-8}

Location: R-8 is located in middle Los Alamos Canyon about 0.75 miles downstream of the confluence with DP Canyon and supply well O-4.

Completion Type: Multiple completion, two screens in the regional aquifer. The top of screen 1 is about $16 \mathrm{ft}$ below the water table.

Period of Record: Westbay ${ }^{\circledR}$ installed February 23, 2002, transducers installed April 7, 2005, data through 2007.

Remarks: Screens are $66 \mathrm{ft}$ apart, head in screen 2 about $20 \mathrm{ft}$ lower than screen 1 . The groundwater at both screens does not indicate a response to atmospheric pressure fluctuations, but the groundwater at both screens responds to pumping supply well PM-3.

\begin{tabular}{|c|c|c|c|c|c|c|c|c|c|c|c|}
\hline \multicolumn{12}{|c|}{ R-8 Port Data } \\
\hline Zone & \begin{tabular}{|c} 
Screen \\
Top \\
Depth (ft)
\end{tabular} & $\begin{array}{l}\text { Screen } \\
\text { Bottom } \\
\text { Depth (ft) }\end{array}$ & $\begin{array}{l}\text { Screen } \\
\text { Top Elev } \\
\text { (ft) }\end{array}$ & $\begin{array}{l}\text { Screen } \\
\text { Bottom } \\
\text { Elev (ft) }\end{array}$ & $\begin{array}{l}\text { Screen } \\
\text { Length } \\
\text { (ft) }\end{array}$ & Port & $\begin{array}{l}\text { Port } \\
\text { Depth } \\
\text { (ft) }\end{array}$ & \begin{tabular}{|c} 
Port \\
Elevation \\
(ft)
\end{tabular} & $\begin{array}{l}\text { from } \\
\text { Bottom } \\
\text { of } \\
\text { Screen } \\
\text { (ft) }\end{array}$ & $\begin{array}{l}\text { Sump } \\
\text { Volume } \\
\text { (L) }\end{array}$ & Comment \\
\hline \multirow{5}{*}{1} & \multirow{5}{*}{705.3} & \multirow{5}{*}{755.7} & \multirow{5}{*}{5839.4} & \multirow{5}{*}{5789.04} & \multirow{5}{*}{50.4} & $\overline{M P 1 A}$ & 711.1 & 5833.64 & 44.6 & & Regional Aquifer \\
\hline & & & & & & MP1B & 721.4 & 5823.34 & 34.3 & & Within Screen \\
\hline & & & & & & MP1C & 751.3 & 5793.44 & 4.4 & & Within Screen \\
\hline & & & & & & PP1 & 756.7 & 5788.04 & -1.0 & 2.2 & Below Screen \\
\hline & & & & & & MP1D & 762.3 & 5782.44 & -6.6 & 14.3 & Below Screen \\
\hline \multirow{3}{*}{2} & \multirow{3}{*}{821.3} & \multirow{3}{*}{828.0} & \multirow{3}{*}{5723.4} & \multirow{3}{*}{5716.74} & \multirow{3}{*}{6.7} & MP2A & 825.0 & 5719.74 & 3.0 & & Within Screen \\
\hline & & & & & & PP2 & 830.4 & 5714.34 & -2.4 & 5.2 & Below Screen \\
\hline & & & & & & MP2B & 836.0 & 5708.7 & -8.0 & 17.3 & Below Screen \\
\hline
\end{tabular}

Note: R-8 Brass Cap Ground Elevation: $6544.74 \mathrm{ft}$; all measurements are from this elevation;

$\mathrm{MP}=$ Monitor Port; PP = Pump Port; Monitor Ports shown in bold are instrumented ports

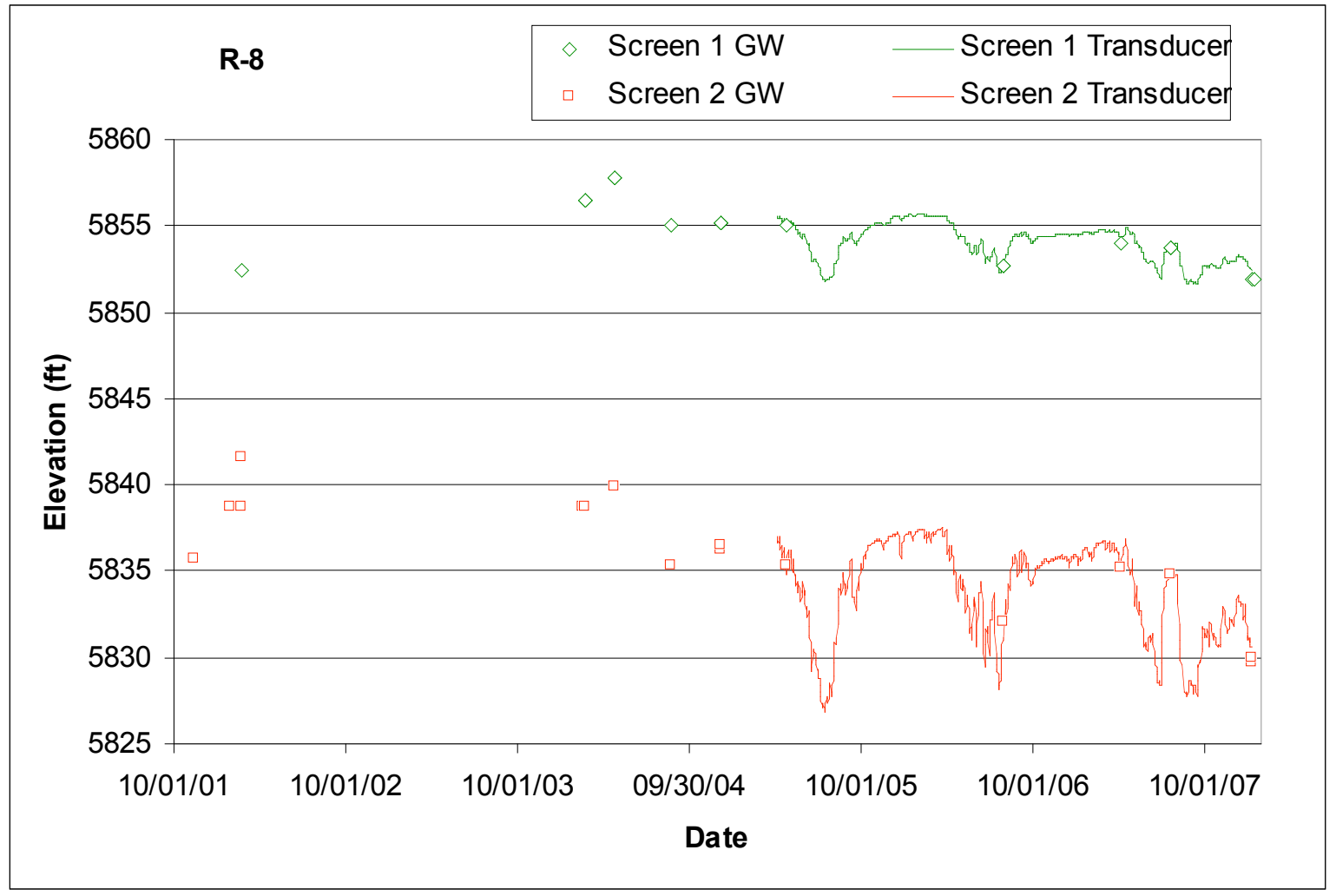




\subsection{R-9}

Location: R-9 is located in Los Alamos Canyon near the eastern LANL boundary.

Completion Type: Single completion at the top of the regional aquifer. The screen straddles the water table.

Period of Record: March 2, 1998, to August 12, 1998, in temporary well. Final well completed October 1999. Transducer installed April 5, 2005, data through 2007.

Remarks: R-9 was completed to a depth of $758 \mathrm{ft}$, about $70 \mathrm{ft}$ into the regional aquifer. The well is $100 \%$ barometrically efficient; the groundwater has no immediate response to atmospheric pressure fluctuations. However, the aquifer indicates a delayed $65 \%$ response to atmospheric pressure.

\begin{tabular}{|c|c|c|c|c|c|c|c|c|c|c|c|c|c|}
\hline \multicolumn{14}{|c|}{ R-9 Construction Information } \\
\hline Zone & $\begin{array}{c}\text { Screen } \\
\text { Top } \\
\text { Depth (ft) }\end{array}$ & $\begin{array}{c}\text { Screen } \\
\text { Bottom } \\
\text { Depth } \\
\text { (ft) }\end{array}$ & $\begin{array}{c}\text { Screen } \\
\text { Top } \\
\text { Elev (ft) }\end{array}$ & $\begin{array}{l}\text { Screen } \\
\text { Bottom } \\
\text { Elev (ft) }\end{array}$ & $\begin{array}{l}\text { Screen } \\
\text { Length } \\
\text { (ft) }\end{array}$ & $\begin{array}{l}\text { Pump } \\
\text { Intake } \\
\text { Depth } \\
\text { (ft) }\end{array}$ & $\begin{array}{c}\text { Pump } \\
\text { Intake } \\
\text { Elevation } \\
\text { (ft) }\end{array}$ & $\begin{array}{c}\text { Depth to } \\
\text { Top of } \\
\text { Sump (ft) }\end{array}$ & $\begin{array}{c}\text { Top of } \\
\text { Sump } \\
\text { Elevation } \\
\text { (ft) }\end{array}$ & $\begin{array}{l}\text { Depth } \\
\text { to } \\
\text { Sump } \\
\text { Bottom } \\
\text { (ft) }\end{array}$ & $\begin{array}{l}\text { Sump } \\
\text { Length } \\
\text { (ft) }\end{array}$ & $\begin{array}{c}\text { Sump } \\
\text { Volume } \\
\text { (L) }\end{array}$ & Comment \\
\hline 1 & 683.0 & 748.5 & 5699.8 & 5634.3 & 65.5 & 741.4 & 5641.4 & 748.5 & 5634.3 & 758 & 9.5 & 29.7 & Regional Aquifer \\
\hline
\end{tabular}

Note: Brass Cap Ground Elevation: 6382.80; all measurements are from this elevation

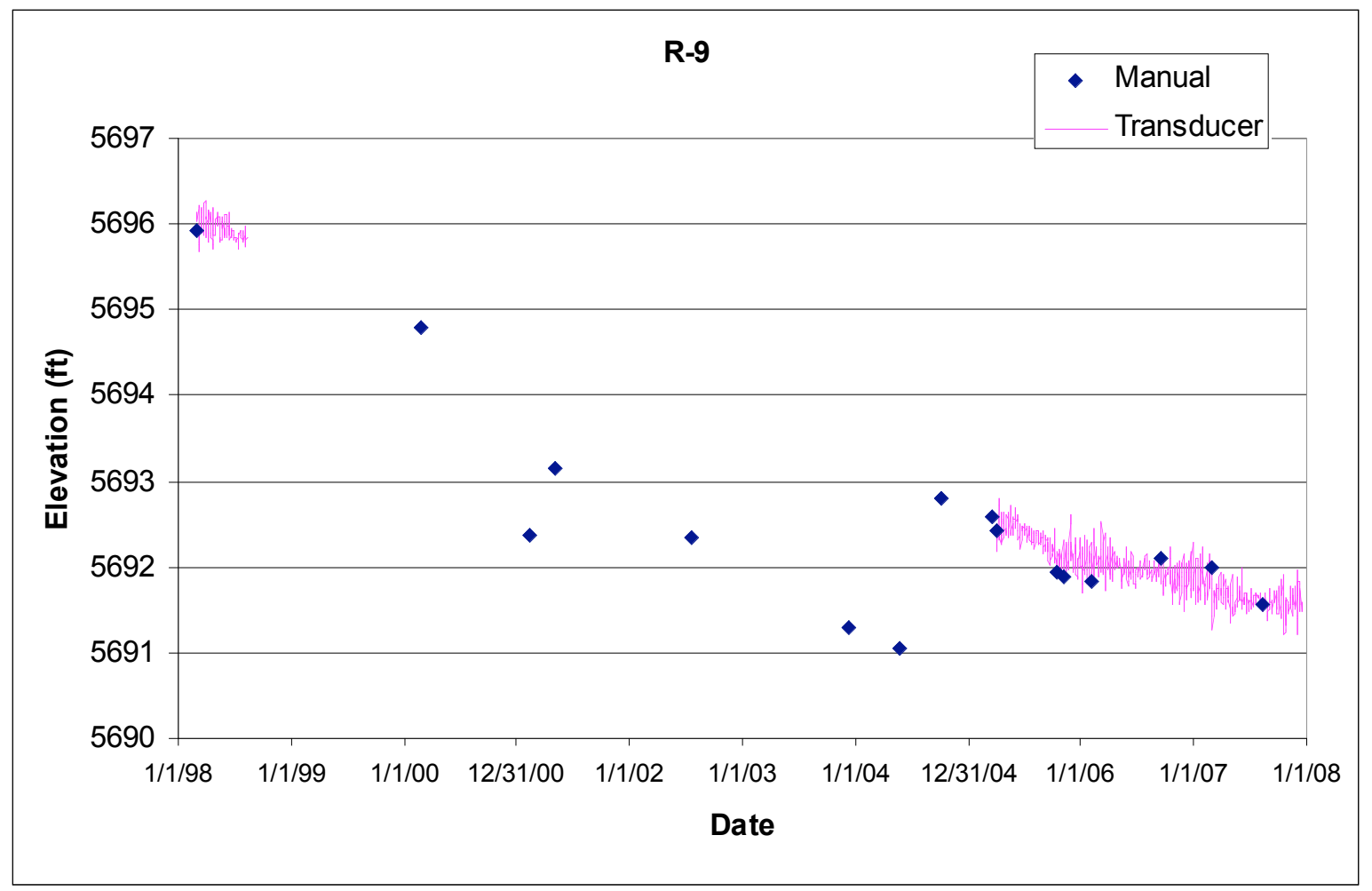




\subsection{R-10}

Location: R-10 is located in lower Sandia Canyon east of the LANL boundary.

Completion Type: Dual completion in two deeper zones within the regional aquifer. Baski packer and dual valve assembly with single submersible pump installed in May 2006.

Period of Record: Well completed October 2005, transducers installed July 26, 2006, data through 2007.

Remarks: R-10 screen 1 is $174 \mathrm{ft}$ deeper than the screen at R-10a; due to relatively low hydraulic conductivity of the formation between these screens, the head at R-10 screen 1 is $30 \mathrm{ft}$ lower than at R-10a. The screen 2 gage tube is plugged or constricted; water level data for 2006 and 2007 are not valid. In February 2007 several attempts to unplug the gage line were made without success. The screen 2 gage line is scheduled for repair in 2008. The groundwater at $\mathrm{R}-10$ screen 1 exhibits an immediate $55 \%$ response to atmospheric pressure fluctuations for a barometric efficiency of the well at screen 1 of $45 \%$. The groundwater at screen 1 responds to pumping at supply well PM-1.

\begin{tabular}{|c|c|c|c|c|c|c|c|c|c|c|c|c|c|}
\hline \multicolumn{14}{|c|}{ R-10 Construction Information } \\
\hline Zone & $\begin{array}{c}\text { Screen } \\
\text { Top } \\
\text { Depth (ft) }\end{array}$ & $\begin{array}{l}\text { Screen } \\
\text { Bottom } \\
\text { Depth } \\
\text { (ft) }\end{array}$ & $\begin{array}{c}\text { Screen } \\
\text { Top } \\
\text { Elev }(\mathrm{ft})\end{array}$ & $\begin{array}{l}\text { Screen } \\
\text { Bottom } \\
\text { Elev (ft) }\end{array}$ & $\begin{array}{l}\text { Screen } \\
\text { Length } \\
\text { (ft) }\end{array}$ & $\begin{array}{l}\text { Pump } \\
\text { Intake } \\
\text { Depth } \\
\text { (ft) }\end{array}$ & $\begin{array}{l}\text { Pump } \\
\text { Intake } \\
\text { Elevation } \\
\text { (ft) }\end{array}$ & $\begin{array}{l}\text { Depth to } \\
\text { Top of } \\
\text { Packerl } \\
\text { Sump (ft) }\end{array}$ & $\begin{array}{c}\text { Top of } \\
\text { Packerl } \\
\text { Sump } \\
\text { Elevation } \\
\text { (ft) }\end{array}$ & $\begin{array}{l}\text { Depth to } \\
\text { Sump } \\
\text { Bottom } \\
\text { (ft) }\end{array}$ & $\begin{array}{l}\text { Sump } \\
\text { Length } \\
\text { (ft) }\end{array}$ & $\begin{array}{c}\text { Sump } \\
\text { Volume } \\
\text { (L) }\end{array}$ & Comment \\
\hline 1 & 874.0 & 897.0 & 5488.3 & 5465.3 & 23.0 & 884.3 & 5478.0 & 905.2 & 5457.2 & 905.2 & 8.2 & 25.5 & Regional Aquife \\
\hline 2 & 1042.0 & 1065.0 & 5320.3 & 5297.3 & 23.0 & 1053.1 & 5309.2 & 1065.0 & 5297.3 & 1081.6 & 16.6 & 10.2 & Regional Aquife \\
\hline
\end{tabular}

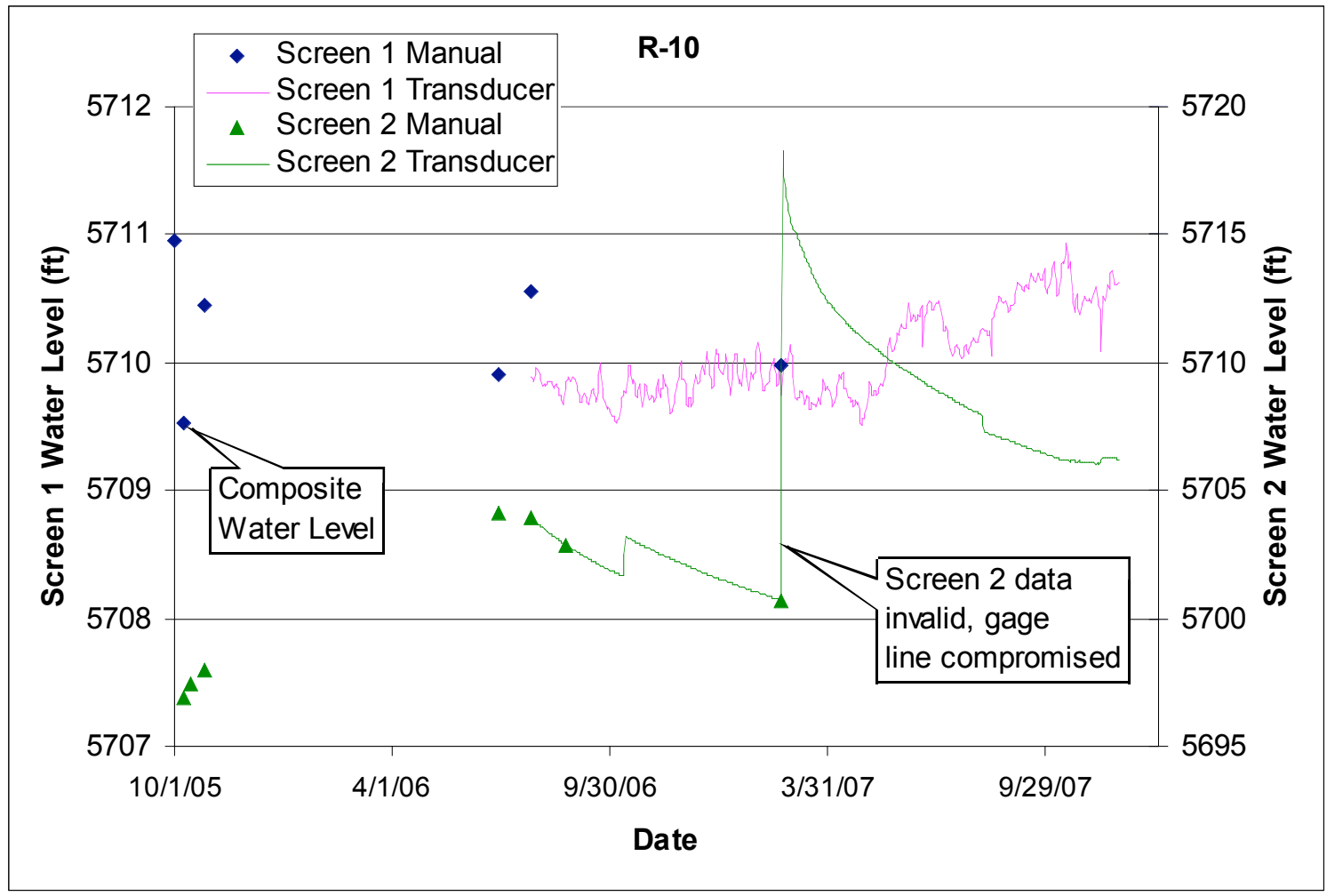




\subsection{R-10a}

Location: R-10a is located in lower Sandia Canyon east of the LANL boundary and adjacent to R-10. Completion Type: Single completion at the top of the regional aquifer. The top of the screen is about $67 \mathrm{ft}$ below the water table.

Period of Record: Well completed August 2005, transducer installed April 3, 2006, data through 2007. Remarks: The R-10a water level is about $30 \mathrm{ft}$ higher than at R-10 screen 1 . The groundwater at R10 a shows an immediate $58 \%$ response to atmospheric pressure fluctuations for a well barometric efficiency of $42 \%$. There is no apparent response to supply well pumping at R$10 \mathrm{a}$.

\begin{tabular}{|c|c|c|c|c|c|c|c|c|c|c|c|c|c|}
\hline \multicolumn{14}{|c|}{ R-10a Construction Information } \\
\hline Zone & $\begin{array}{c}\text { Screen } \\
\text { Top } \\
\text { Depth (ft) }\end{array}$ & $\begin{array}{c}\text { Screen } \\
\text { Bottom } \\
\text { Depth } \\
\text { (ft) }\end{array}$ & $\begin{array}{c}\text { Screen } \\
\text { Top } \\
\text { Elev (ft) }\end{array}$ & $\begin{array}{l}\text { Screen } \\
\text { Bottom } \\
\text { Elev (ft) }\end{array}$ & $\begin{array}{c}\text { Screen } \\
\text { Length } \\
\text { (ft) }\end{array}$ & $\begin{array}{l}\text { Pump } \\
\text { Intake } \\
\text { Depth } \\
\text { (ft) }\end{array}$ & $\begin{array}{l}\text { Pump } \\
\text { Intake } \\
\text { Elevation } \\
\text { (ft) }\end{array}$ & $\begin{array}{c}\text { Depth to } \\
\text { Top of } \\
\text { Sump (ft) }\end{array}$ & $\begin{array}{c}\text { Top of } \\
\text { Sump } \\
\text { Elevation } \\
\text { (ft) }\end{array}$ & $\begin{array}{l}\text { Depth to } \\
\text { Sump } \\
\text { Bottom (ft) }\end{array}$ & $\begin{array}{l}\text { Sump } \\
\text { Length } \\
\text { (ft) }\end{array}$ & $\begin{array}{c}\text { Sump } \\
\text { Volume } \\
\text { (L) }\end{array}$ & Comment \\
\hline 1 & 690.0 & 700 & 5673.7 & 5663.7 & 10.0 & 685.6 & 5678.1 & 700.0 & 5663.7 & 706 & 6.0 & 18.4 & Regional Aquife \\
\hline
\end{tabular}

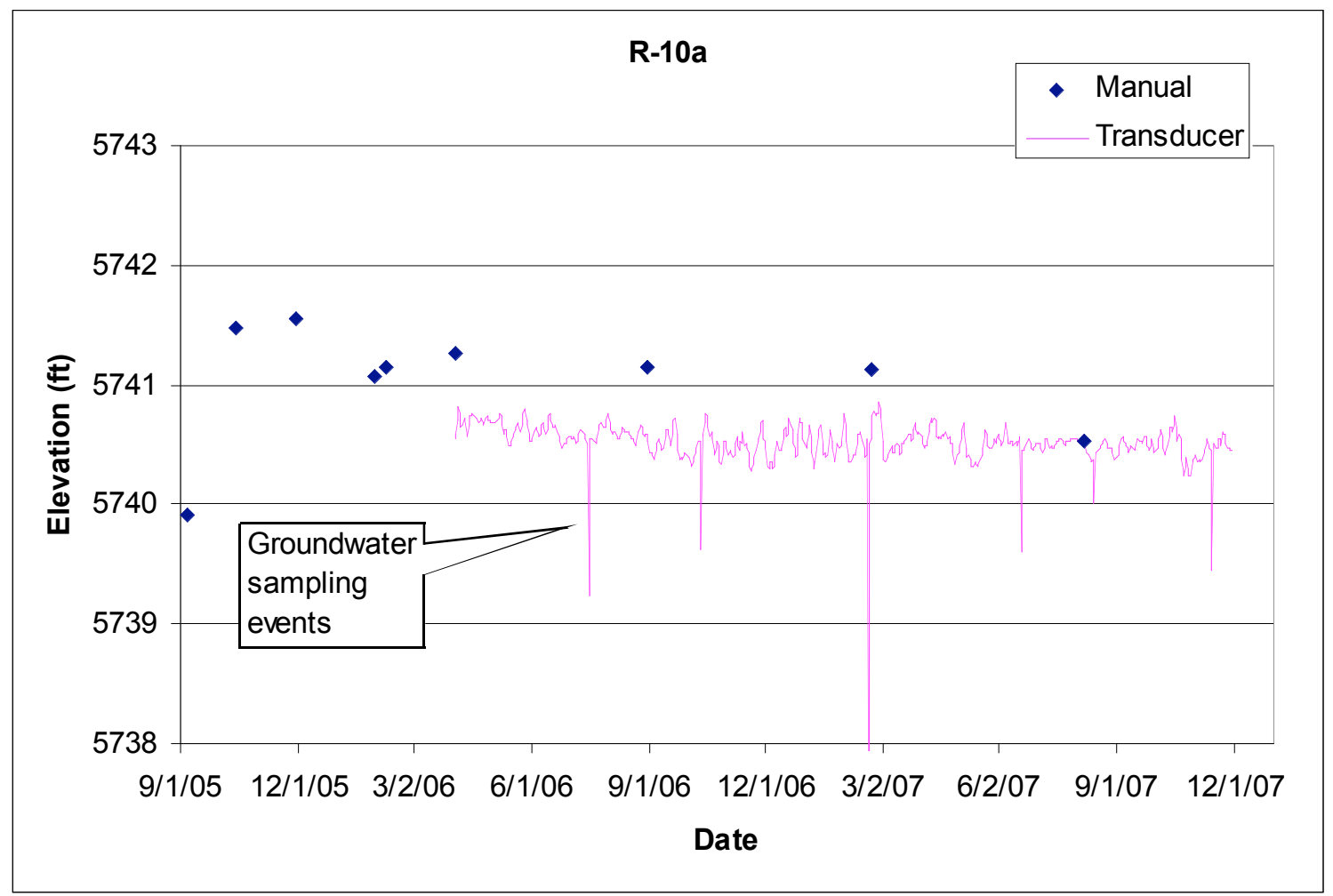




\subsection{R-11}

Location: R-11 is located in middle Sandia Canyon about 0.5 miles upstream of PM-3.

Completion Type: Single completion at the top of the regional aquifer. The top of the screen is about $20 \mathrm{ft}$ below the water table.

Period of Record: Transducer installed May 4, 2005; data through 2007.

Remarks: R-11 was completed in 2004 to a depth of $901.7 \mathrm{ft}$, about $66 \mathrm{ft}$ into the regional aquifer. The well is $100 \%$ barometrically efficient; the groundwater has no immediate response to atmospheric pressure fluctuations. However, the aquifer indicates a delayed response to atmospheric pressure. R-11 exhibits a seasonal response to supply well pumping but does not indicate a direct response to any specific supply well.

\begin{tabular}{|c|c|c|c|c|c|c|c|c|c|c|c|c|c|}
\hline \multicolumn{14}{|c|}{ R-11 Construction Information } \\
\hline Zone & $\begin{array}{c}\text { Screen } \\
\text { Top } \\
\text { Depth (ft) }\end{array}$ & $\begin{array}{c}\text { Screen } \\
\text { Bottom } \\
\text { Depth } \\
\text { (ft) }\end{array}$ & $\begin{array}{c}\text { Screen } \\
\text { Top } \\
\text { Elev (ft) }\end{array}$ & $\begin{array}{l}\text { Screen } \\
\text { Bottom } \\
\text { Elev (ft) }\end{array}$ & $\begin{array}{c}\text { Screen } \\
\text { Length } \\
\text { (ft) }\end{array}$ & $\begin{array}{l}\text { Pump } \\
\text { Intake } \\
\text { Depth } \\
\text { (ft) }\end{array}$ & $\begin{array}{l}\text { Pump } \\
\text { Intake } \\
\text { Elevation } \\
\text { (ft) }\end{array}$ & $\begin{array}{c}\text { Depth to } \\
\text { Top of } \\
\text { Sump (ft) }\end{array}$ & $\begin{array}{l}\text { Top of } \\
\text { Sump } \\
\text { Elevation } \\
\text { (ft) }\end{array}$ & $\begin{array}{c}\text { Depth to } \\
\text { Sump } \\
\text { Bottom (ft) }\end{array}$ & $\begin{array}{c}\text { Sump } \\
\text { Length } \\
\text { (ft) }\end{array}$ & $\begin{array}{c}\text { Sump } \\
\text { Volume } \\
\text { (L) }\end{array}$ & Comment \\
\hline 1 & 855.0 & 877.9 & 5818.7 & 5795.8 & 22.9 & 850 & 5823.7 & 877.9 & 5795.8 & 901.7 & 23.8 & 73.1 & Regional Aquife \\
\hline
\end{tabular}

Note: R-11 Brass Cap Ground Elevation: $6673.72 \mathrm{ft}$; all measurements are from this elevation

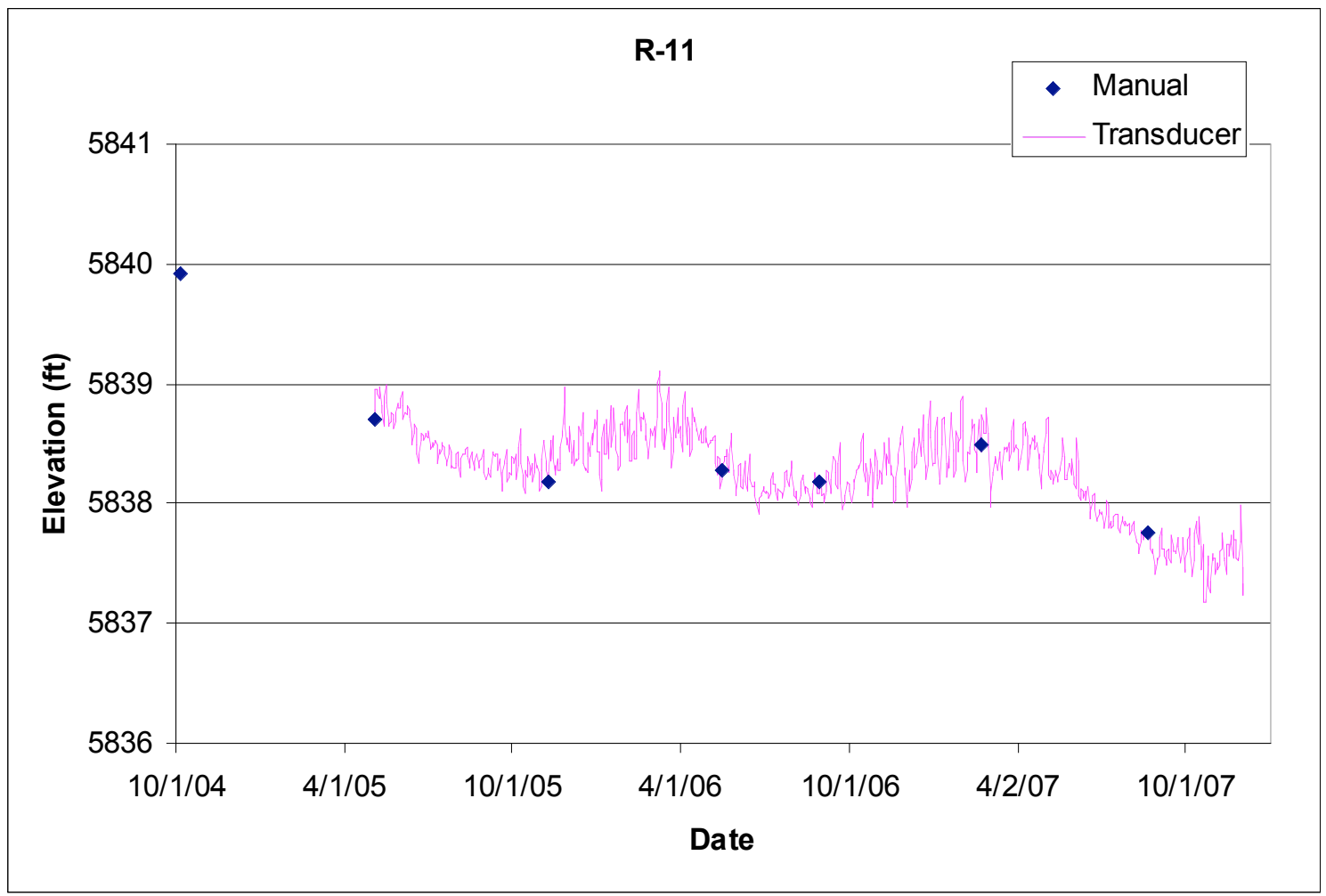




\subsection{R-12}

Location: R-12 is located in lower Sandia Canyon near State Route (SR) 4 and supply well PM-1.

Completion Type: Multiple completion, two screens in intermediate zones, one screen at the top of the regional aquifer until September 2006. Well recompleted as two intermediate screens on December 13, 2007.

Period of Record: Westbay ${ }^{\circledR}$ installed March 21, 2000, transducers installed December 14, 2000, intermittent data to September 21, 2006, when transducers were removed for removal of the Westbay ${ }^{\circledR}$ system for well rehabilitation. No water level data for FY 2007. Transducers were reinstalled at screens 1 and 2 on December 13, 2007.

Remarks: In December 2007, screen 3 was abandoned and a Baski packer and sampling system was installed at the two intermediate screens. Monitoring well R-36 is planned to replace R12 screen 3.

Intermediate screens 1 and 2 have similar head values about $380 \mathrm{ft}$ above the regional aquifer; intermediate screen 2 has a slightly higher head than screen 1. Intermediate screens 1 and 2 apparently responded to snowmelt runoff events in Los Alamos Canyon in 2001 and 2005; no data available during 2007.

The regional aquifer at screen 3 did not exhibit a seasonal response to supply well pumping, or a response to pumping of any specific supply well, including nearby supply well PM-1. There is no immediate response to atmospheric pressure fluctuations at any screen; however, screens 1, 2, and 3 exhibit a delayed response to atmospheric pressure fluctuations.

\begin{tabular}{|c|c|c|c|c|c|c|c|c|c|c|c|}
\hline \multicolumn{12}{|c|}{ R-12 Former Westbay Port Data } \\
\hline Zone & $\begin{array}{c}\text { Screen } \\
\text { Top } \\
\text { Depth (ft) }\end{array}$ & $\begin{array}{c}\text { Screen } \\
\text { Bottom } \\
\text { Depth (ft) }\end{array}$ & $\begin{array}{c}\text { Screen } \\
\text { Top Elev } \\
\text { (ft) }\end{array}$ & $\begin{array}{l}\text { Screen } \\
\text { Bottom } \\
\text { Elev (ft) }\end{array}$ & $\begin{array}{l}\text { Screen } \\
\text { Length } \\
\text { (ft) }\end{array}$ & Port & $\begin{array}{l}\text { Port } \\
\text { Depth } \\
\text { (ft) }\end{array}$ & $\begin{array}{c}\text { Port } \\
\text { Elevation } \\
\text { (ft) }\end{array}$ & $\begin{array}{l}\text { Distance } \\
\text { from Bottom } \\
\text { of Screen (ft) }\end{array}$ & $\begin{array}{c}\text { Sump } \\
\text { Volume } \\
\text { (L) }\end{array}$ & Comment \\
\hline \multirow{3}{*}{1} & \multirow{3}{*}{459.0} & \multirow{3}{*}{467.5} & \multirow{3}{*}{6040.6} & \multirow{3}{*}{6032.1} & \multirow{3}{*}{8.5} & MP1A & 468.1 & 6031.5 & -0.6 & 1.1 & Intermediate Zone, below screen \\
\hline & & & & & & PP1 & 473.5 & 6026.1 & -6.0 & 11.3 & Below screen \\
\hline & & & & & & MP1B & 479.1 & 6020.5 & -11.6 & 21.9 & Below screen \\
\hline \multirow{3}{*}{2} & \multirow{3}{*}{504.5} & \multirow{3}{*}{508.0} & \multirow{3}{*}{5995.1} & \multirow{3}{*}{5991.6} & \multirow{3}{*}{3.5} & MP2A & 507.0 & 5992.6 & 1.0 & & Intermediate Zone \\
\hline & & & & & & PP2 & 512.4 & 5987.2 & -4.4 & 8.3 & Below screen \\
\hline & & & & & & MP2B & 518.0 & 5981.6 & -10.0 & 18.9 & Below screen \\
\hline \multirow{5}{*}{3} & \multirow{5}{*}{801.0} & \multirow{5}{*}{839.0} & \multirow{5}{*}{5698.6} & \multirow{5}{*}{5660.6} & \multirow{5}{*}{38} & MP3A & 810.8 & 5688.8 & 28.2 & & Regional Aquifer \\
\hline & & & & & & PP3A & 816.2 & 5683.4 & 22.8 & & \\
\hline & & & & & & MP3B & 821.8 & 5677.8 & 17.2 & & \\
\hline & & & & & & PP3B & 827.2 & 5672.4 & 11.8 & & \\
\hline & & & & & & MP3C & 832.9 & 5666.7 & 6.1 & & \\
\hline
\end{tabular}

\begin{tabular}{|c|c|c|c|c|c|c|c|c|c|c|c|c|c|}
\hline \multicolumn{14}{|c|}{ R-12 Screen Data } \\
\hline Screen & $\begin{array}{c}\text { Screen } \\
\text { Top } \\
\text { Depth (ft) }\end{array}$ & $\begin{array}{c}\text { Screen } \\
\text { Bottom } \\
\text { Depth (ft) }\end{array}$ & $\begin{array}{l}\text { Screen } \\
\text { Top Elev } \\
\text { (ft) }\end{array}$ & $\begin{array}{l}\text { Screen } \\
\text { Bottom } \\
\text { Elev (ft) }\end{array}$ & $\begin{array}{l}\text { Screen } \\
\text { Length } \\
\text { (ft) }\end{array}$ & $\begin{array}{l}\text { Pump } \\
\text { Intake } \\
\text { Depth } \\
\text { (ft) }\end{array}$ & $\begin{array}{l}\text { Pump } \\
\text { Intake } \\
\text { Elevation } \\
\text { (ft) }\end{array}$ & $\begin{array}{l}\text { Depth to } \\
\text { Top of } \\
\text { Packer/ } \\
\text { Sump (ft) }\end{array}$ & $\begin{array}{l}\text { Packerl } \\
\text { Sump } \\
\text { Elevation } \\
\text { (ft) }\end{array}$ & $\begin{array}{l}\text { to } \\
\text { to } \\
\text { Sump } \\
\text { Bottom } \\
\text { (ft) }\end{array}$ & $\begin{array}{l}\text { Sump } \\
\text { Length } \\
\text { (ft) }\end{array}$ & $\begin{array}{c}\text { Sump } \\
\text { Volume } \\
\text { (L) }\end{array}$ & Comment \\
\hline 1 & 459.0 & 467.5 & 6040.6 & 6032.1 & 8.5 & 465.0 & 6034.6 & 470.7 & 6028.9 & 470.7 & 3.2 & 9.1 & Intermediate Zone \\
\hline 2 & 504.5 & 508.0 & 5995.1 & 5991.6 & 3.5 & 501.1 & 5998.5 & 508.0 & 5991.6 & 540.8 & 32.8 & 93.7 & Intermediate Zone \\
\hline 3 & 801.0 & 839.0 & 5698.6 & 5660.6 & 38 & & & & & & & & Plugged and abandoned \\
\hline
\end{tabular}



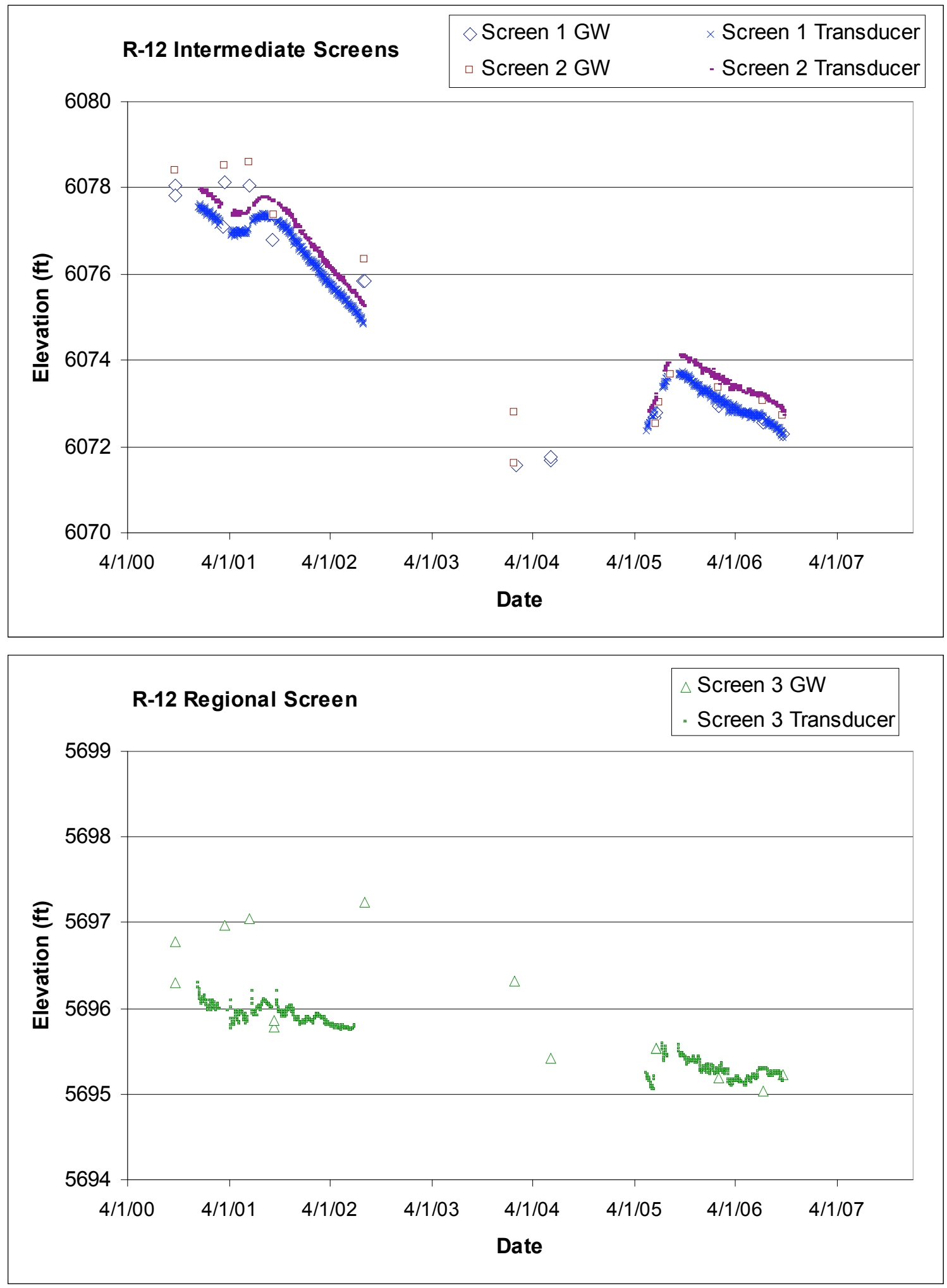


\subsection{R-13}

Location: R-13 is located in lower Mortandad Canyon at the LANL boundary.

Completion Type: Single completion at the top of the regional aquifer. The top of the screen is about $122 \mathrm{ft}$ below the water table.

Period of Record: Well completed October 2001, transducer installed January 3, 2005, data through 2007.

Remarks: R-13 was completed to a depth of $1029.4 \mathrm{ft}$, about $200 \mathrm{ft}$ into the regional aquifer. The well is $100 \%$ barometrically efficient; the groundwater has no immediate response to atmospheric pressure fluctuations. However, the aquifer indicates a delayed $30 \%$ response to atmospheric pressure. $\mathrm{R}-13$ exhibits a seasonal response to supply well pumping and responds primarily to pumping at PM-4 (McLin 2006) and possibly to PM-2 and PM-5, but apparently does not respond significantly to pumping at nearby supply well PM-3.

\begin{tabular}{|c|c|c|c|c|c|c|c|c|c|c|c|c|c|}
\hline \multicolumn{14}{|c|}{ R-13 Construction Information } \\
\hline Zone & $\begin{array}{c}\text { Screen } \\
\text { Top } \\
\text { Depth (ft) }\end{array}$ & $\begin{array}{l}\text { Screen } \\
\text { Bottom } \\
\text { Depth } \\
\text { (ft) }\end{array}$ & $\begin{array}{c}\text { Screen } \\
\text { Top } \\
\text { Elev (ft) }\end{array}$ & $\begin{array}{l}\text { Screen } \\
\text { Bottom } \\
\text { Elev (ft) }\end{array}$ & $\begin{array}{l}\text { Screen } \\
\text { Length } \\
\text { (ft) }\end{array}$ & $\begin{array}{l}\text { Pump } \\
\text { Intake } \\
\text { Depth } \\
\text { (ft) }\end{array}$ & $\begin{array}{l}\text { Pump } \\
\text { Intake } \\
\text { Elevation } \\
\text { (ft) }\end{array}$ & $\begin{array}{c}\text { Depth to } \\
\text { Top of } \\
\text { Sump (ft) }\end{array}$ & $\begin{array}{c}\text { Top of } \\
\text { Sump } \\
\text { Elevation } \\
\text { (ft) }\end{array}$ & $\begin{array}{l}\text { Depth to } \\
\text { Sump } \\
\text { Bottom (ft) }\end{array}$ & $\begin{array}{l}\text { Sump } \\
\text { Length } \\
\text { (ft) }\end{array}$ & $\begin{array}{c}\text { Sump } \\
\text { Volume } \\
\text { (L) }\end{array}$ & Comment \\
\hline 1 & 958.3 & 1018.7 & 5714.8 & 5654.4 & 60.4 & 933.0 & 5740.1 & 1018.7 & 5654.4 & 1029.4 & 10.7 & 33.5 & Regional Aquifer \\
\hline
\end{tabular}

Note: R-13 Brass Cap Ground Elevation: $6673.05 \mathrm{ft}$; all measurements are from this elevation

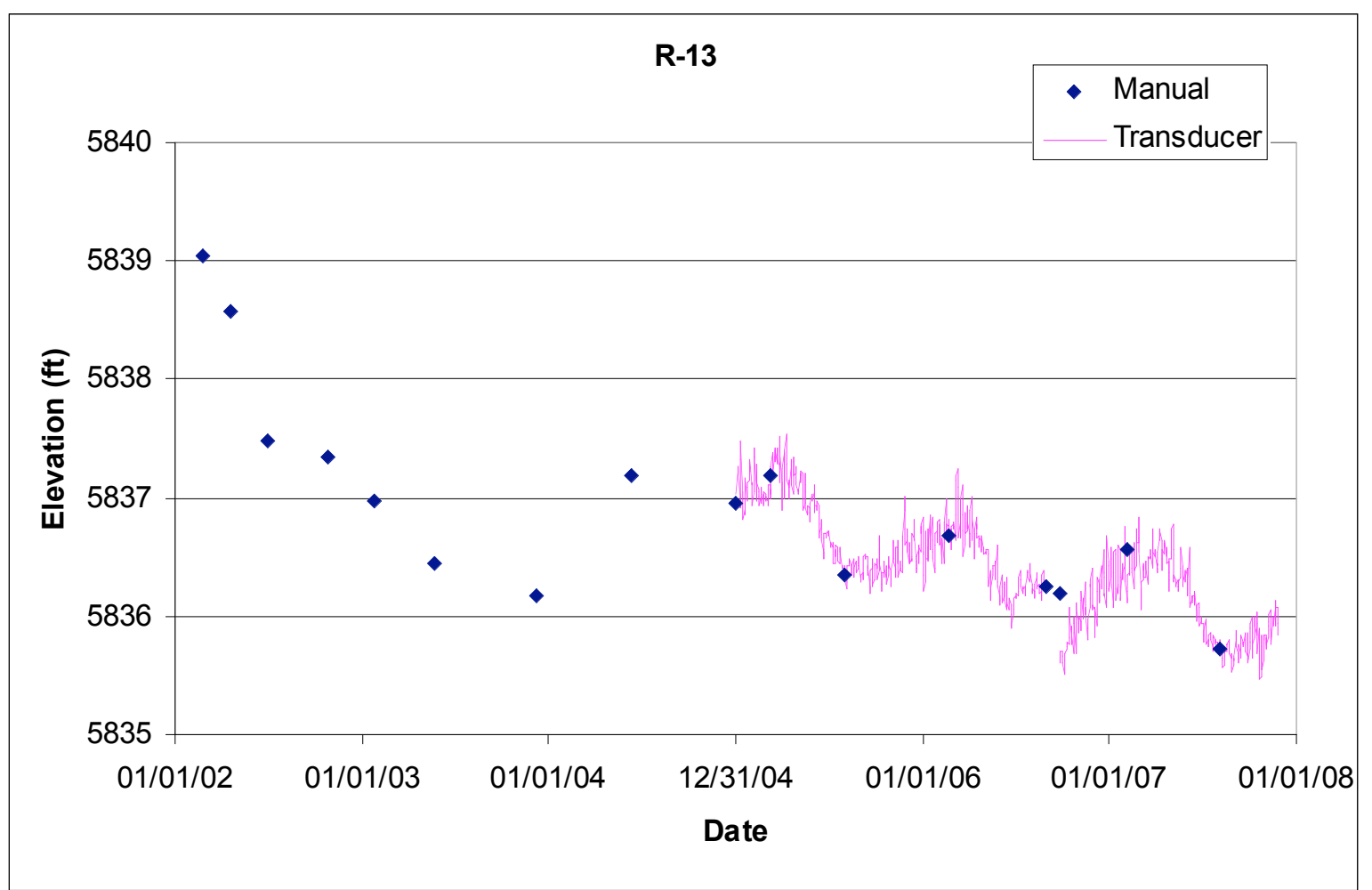




\subsection{R-14}

Location: R-14 is located in upper Ten Site Canyon about 0.5 miles upgradient of supply well PM-5.

Completion Type: Multiple completion, two screens in the regional aquifer. The top of screen 1 is about $22 \mathrm{ft}$ below the water table.

Period of Record: Westbay ${ }^{\circledR}$ installed November 23, 2002, transducers installed December 14, 2004 , intermittent data through 2007.

Remarks: Screens are $53 \mathrm{ft}$ apart; heads in screens are within $0.5 \mathrm{ft}$ of each other. The aquifer shows no response to atmospheric pressure fluctuations. The water level responds primarily to pumping supply well PM-5.

\begin{tabular}{|c|c|c|c|c|c|c|c|c|c|c|c|}
\hline \multicolumn{12}{|c|}{ R-14 Port Data } \\
\hline Zone & $\begin{array}{l}\text { Screen } \\
\text { Top } \\
\text { Depth } \\
\text { (ft) }\end{array}$ & $\begin{array}{c}\text { Screen } \\
\text { Bottom } \\
\text { Depth } \\
\text { (ft) }\end{array}$ & $\begin{array}{c}\text { Screen } \\
\text { Top Elev } \\
\text { (ft) }\end{array}$ & $\begin{array}{l}\text { Screen } \\
\text { Bottom } \\
\text { Elev (ft) }\end{array}$ & $\begin{array}{l}\text { Screen } \\
\text { Length } \\
\text { (ft) }\end{array}$ & Port & $\begin{array}{c}\text { Port } \\
\text { Depth (ft) }\end{array}$ & $\begin{array}{c}\text { Port } \\
\text { Elevation } \\
\text { (ft) }\end{array}$ & $\begin{array}{l}\text { Distance } \\
\text { from } \\
\text { Bottom } \\
\text { of Screen } \\
\text { (ft) }\end{array}$ & $\begin{array}{c}\text { Sump } \\
\text { Volume } \\
\text { (L) }\end{array}$ & Comment \\
\hline \multirow{4}{*}{1} & \multirow{4}{*}{1200.6} & \multirow{4}{*}{1233.2} & \multirow{4}{*}{5861.48} & \multirow{4}{*}{5828.88} & \multirow{4}{*}{32.6} & MP1A & 1204.5 & 5857.58 & 28.7 & & Within Screen, Regional aquifer \\
\hline & & & & & & MP1B & 1229.6 & 5832.48 & 3.6 & & Within Screen \\
\hline & & & & & & PP1 & 1234.9 & 5827.18 & -1.7 & 3.7 & Below Screen \\
\hline & & & & & & MP1C & 1240.6 & 5821.48 & -7.4 & 16.0 & Below Screen \\
\hline \multirow{3}{*}{2} & \multirow{3}{*}{1286.5} & \multirow{3}{*}{1293.1} & \multirow{3}{*}{5775.58} & \multirow{3}{*}{5768.98} & \multirow{3}{*}{6.6} & MP2A & 1288.5 & 5773.58 & 4.6 & & Within Screen \\
\hline & & & & & & PP2 & 1293.8 & 5768.28 & -0.7 & 1.5 & Below Screen \\
\hline & & & & & & MP2B & 1299.5 & 5762.58 & -6.4 & 13.8 & Below Screen \\
\hline
\end{tabular}

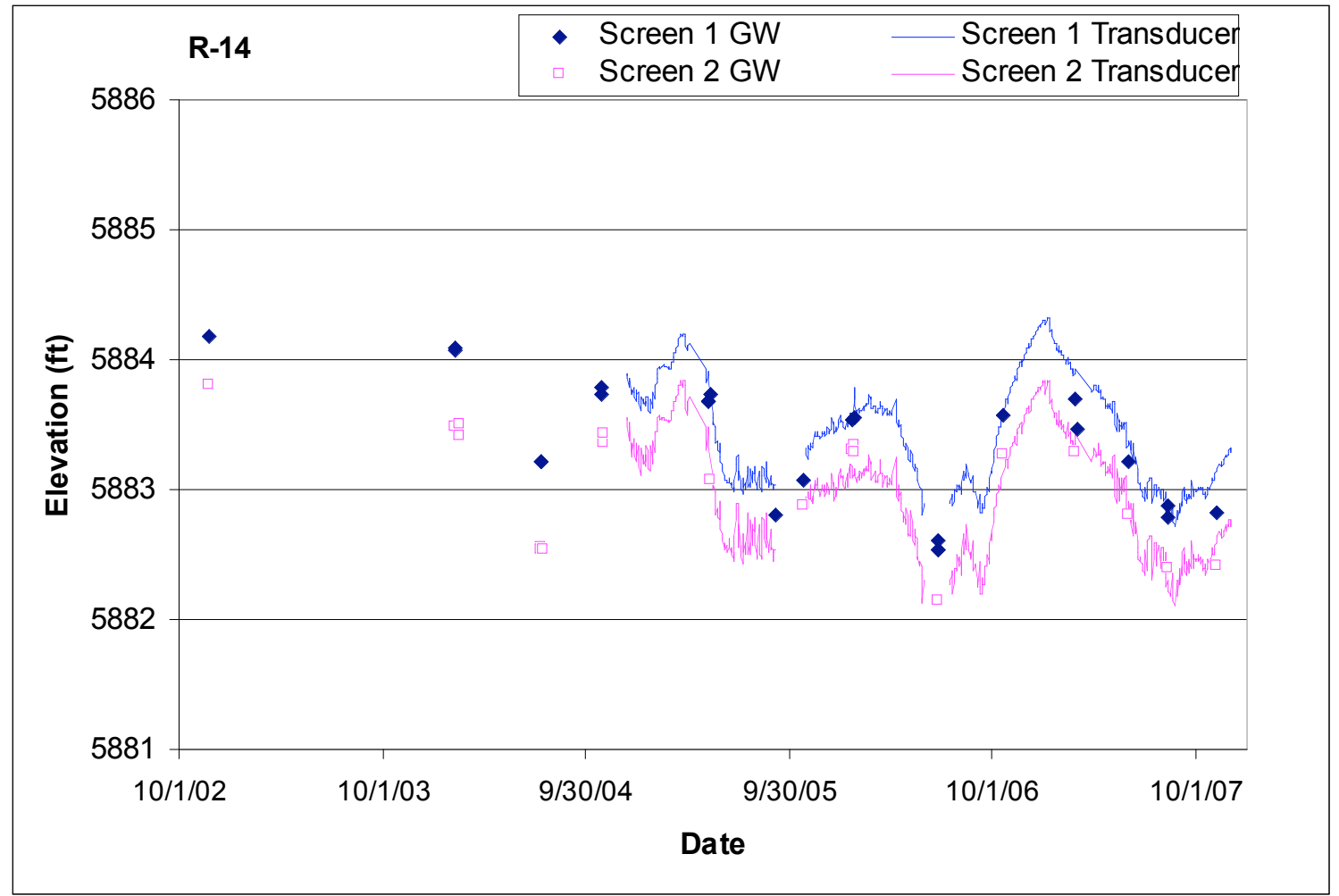




\subsection{R-15}

Location: R-15 is located in lower Mortandad Canyon downstream of the sediment traps.

Completion Type: Single completion at the top of the regional aquifer. The screen straddles the water table.

Period of Record: Well completed September 1999, transducer installed December 23, 2004, transducer data through 2007.

Remarks: R-15 was completed in 1999 to a depth of $1030.6 \mathrm{ft}$, about $140 \mathrm{ft}$ into the regional aquifer. The well is $100 \%$ barometrically efficient; the aquifer does not respond to atmospheric pressure fluctuations. The water level responds to pumping supply wells PM-4 and PM-5.

\begin{tabular}{|c|c|c|c|c|c|c|c|c|c|c|c|c|c|}
\hline \multicolumn{14}{|c|}{ R-15 Construction Information } \\
\hline Zone & $\begin{array}{c}\text { Screen } \\
\text { Top } \\
\text { Depth (ft) }\end{array}$ & $\begin{array}{l}\text { Screen } \\
\text { Bottom } \\
\text { Depth } \\
\text { (ft) }\end{array}$ & $\begin{array}{c}\text { Screen } \\
\text { Top } \\
\text { Elev (ft) }\end{array}$ & $\begin{array}{l}\text { Screen } \\
\text { Bottom } \\
\text { Elev (ft) }\end{array}$ & $\begin{array}{l}\text { Screen } \\
\text { Length } \\
\text { (ft) }\end{array}$ & $\begin{array}{l}\text { Pump } \\
\text { Intake } \\
\text { Depth } \\
\text { (ft) }\end{array}$ & $\begin{array}{l}\text { Pump } \\
\text { Intake } \\
\text { Elevation } \\
\text { (ft) }\end{array}$ & $\begin{array}{c}\text { Depth to } \\
\text { Top of } \\
\text { Sump (ft) }\end{array}$ & $\begin{array}{l}\text { Top of } \\
\text { Sump } \\
\text { Elevation } \\
\text { (ft) }\end{array}$ & $\begin{array}{c}\text { Depth to } \\
\text { Sump } \\
\text { Bottom (ft) }\end{array}$ & $\begin{array}{l}\text { Sump } \\
\text { Length } \\
\text { (ft) }\end{array}$ & $\begin{array}{c}\text { Sump } \\
\text { Volume } \\
\text { (L) }\end{array}$ & Comment \\
\hline 1 & 958.6 & 1020.3 & 5861.4 & 5799.7 & 61.7 & 1015.6 & 5804.4 & 1020.3 & 5799.7 & 1030.6 & 10.3 & 39.8 & Regional Aquife \\
\hline
\end{tabular}

Note: R-15 Brass Cap Ground Elevation: $6820.0 \mathrm{ft}$; all measurements are from this elevation

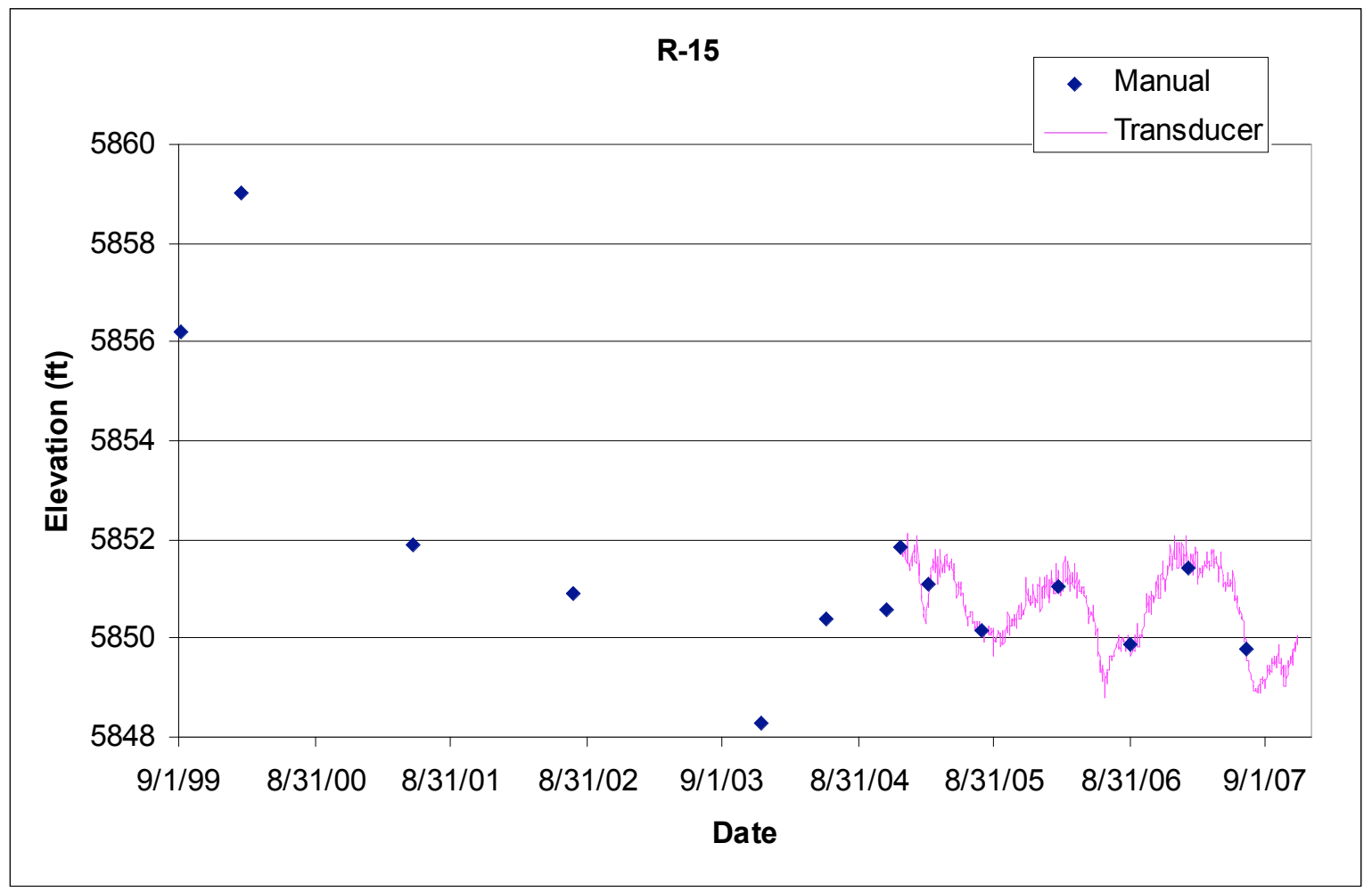




\subsection{R-16}

Location: R-16 is located northeast of White Rock in lower Cañada del Buey near the confluence with lower Mortandad Canyon.

Completion Type: Multiple completion, four screens in the regional aquifer, screen 1 is blocked by casing and is not useable.

Period of Record: Westbay ${ }^{\circledR}$ installed December 14, 2002, transducers installed June 16, 2005, transducer data to July 12,2006 , when the Westbay ${ }^{\circledR}$ system was removed for additional screen development. The Westbay ${ }^{\circledR}$ system was reinstalled and transducers were reinstalled October 18, 2006. Transducer data extends through 2007.

Remarks: Screens 2 and 3 are about $144 \mathrm{ft}$ apart with a head difference of over $80 \mathrm{ft}$. Screens 3 and 4 show similar water level trends. The aquifer response to atmospheric pressure declines downward from screen 2 to screen 4 , from $68 \%$ at screen 2 to $57 \%$ at screen 4 .

\begin{tabular}{|c|c|c|c|c|c|c|c|c|c|c|c|}
\hline \multicolumn{12}{|c|}{ R-16 Port Data } \\
\hline Zone & $\begin{array}{l}\text { Screen } \\
\text { Top } \\
\text { Depth (ft) }\end{array}$ & $\begin{array}{l}\text { Screen } \\
\text { Bottom } \\
\text { Depth (ft) }\end{array}$ & $\begin{array}{l}\text { Screen } \\
\text { Top Elev } \\
\text { (ft) }\end{array}$ & $\begin{array}{l}\text { Screen } \\
\text { Bottom } \\
\text { Elev (ft) }\end{array}$ & $\begin{array}{l}\text { Screen } \\
\text { Length } \\
\text { (ft) }\end{array}$ & Port & $\begin{array}{c}\text { Port } \\
\text { Depth (ft) }\end{array}$ & $\begin{array}{c}\text { Port } \\
\text { Elevation } \\
\text { (ft) }\end{array}$ & $\begin{array}{l}\text { Distance } \\
\text { from } \\
\text { Bottom } \\
\text { of Screen } \\
\text { (ft) }\end{array}$ & $\begin{array}{c}\text { Sump } \\
\text { Volume } \\
\text { (L) }\end{array}$ & Comment \\
\hline \multirow{3}{*}{1} & \multirow{3}{*}{641.0} & \multirow{3}{*}{648.6} & \multirow{3}{*}{5615.9} & \multirow{3}{*}{5608.3} & \multirow{3}{*}{7.6} & MP1A & & & & & Screen \#1 is \\
\hline & & & & & & PP1 & & & & 0.0 & behind 11-in. dia. \\
\hline & & & & & & MP1B & & & & 0.0 & steel casing, Regional Aquifer \\
\hline \multirow{3}{*}{2} & \multirow{3}{*}{863.4} & \multirow{3}{*}{870.9} & \multirow{3}{*}{5393.5} & \multirow{3}{*}{5386.0} & \multirow{3}{*}{7.5} & MP2A & 866.1 & 5390.8 & 4.8 & & Within Screen \\
\hline & & & & & & PP2 & 871.4 & 5385.5 & -0.5 & 1.1 & Below Screen \\
\hline & & & & & & MP2B & 877.1 & 5379.8 & -6.2 & 13.4 & Below Screen \\
\hline \multirow{3}{*}{3} & \multirow{3}{*}{1014.8} & \multirow{3}{*}{1022.4} & \multirow{3}{*}{5242.1} & \multirow{3}{*}{5234.5} & \multirow{3}{*}{7.6} & MP3A & 1018.4 & 5238.5 & 4.0 & & Within Screen \\
\hline & & & & & & MP3B & 1023.8 & 5233.1 & -1.4 & 3.0 & Below Screen \\
\hline & & & & & & PP3 & 1029.4 & 5227.5 & -7.0 & 15.1 & Below Screen \\
\hline \multirow{3}{*}{4} & \multirow{3}{*}{1237.0} & \multirow{3}{*}{1244.6} & \multirow{3}{*}{5019.9} & \multirow{3}{*}{5012.3} & \multirow{3}{*}{7.6} & MP4A & 1238.0 & 5018.9 & 6.6 & & Within Screen \\
\hline & & & & & & PP4 & 1243.4 & 5013.5 & 1.2 & & Within Screen \\
\hline & & & & & & MP4B & 1249.0 & 5007.9 & -4.4 & 9.5 & Below Screen \\
\hline
\end{tabular}

Brass Cap Elevation: $6256.87 \mathrm{ft}$; all measurements are from this elevation;

$\mathrm{MP}=$ measurement port, $\mathrm{PP}=$ pumping port

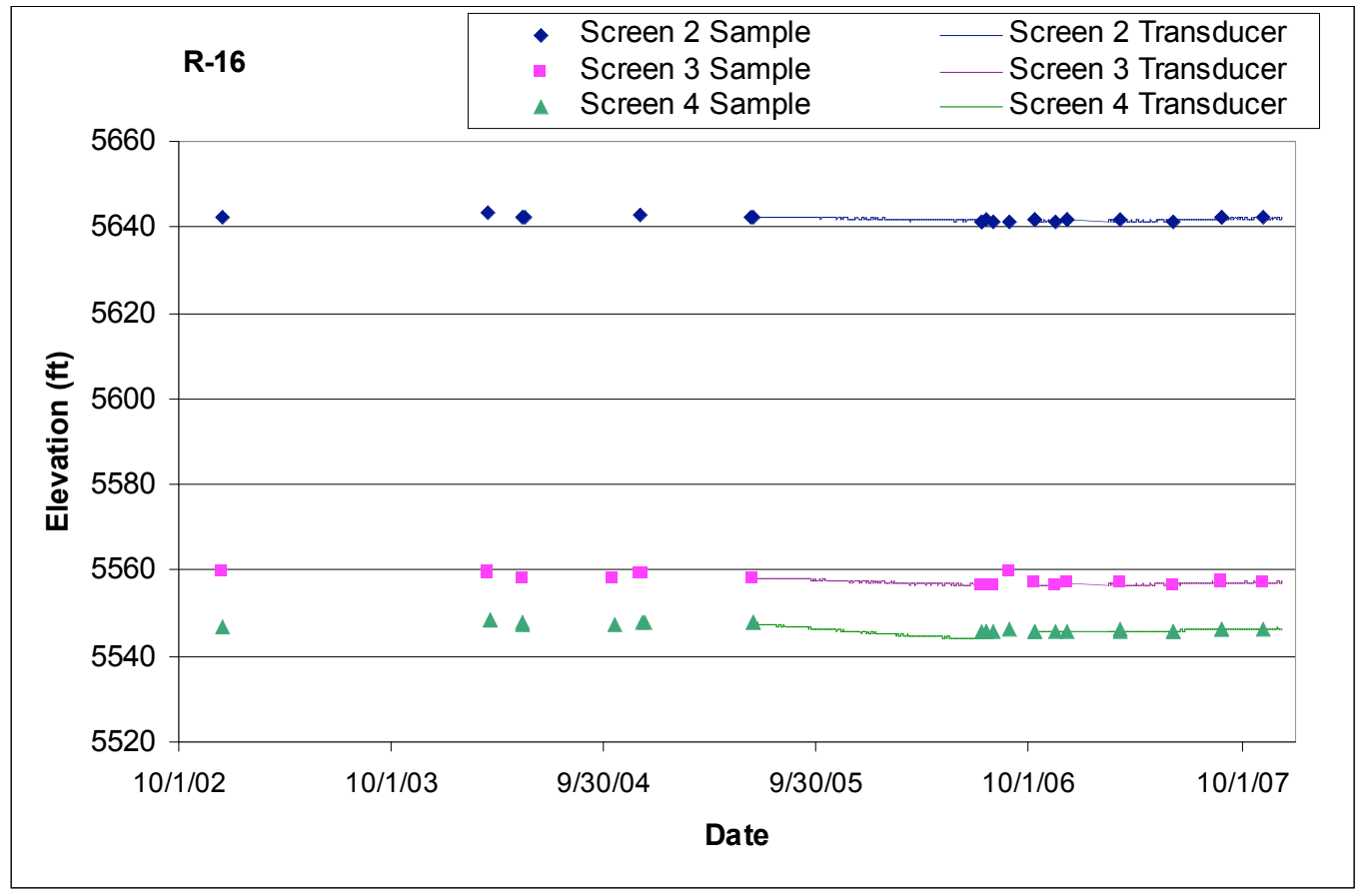



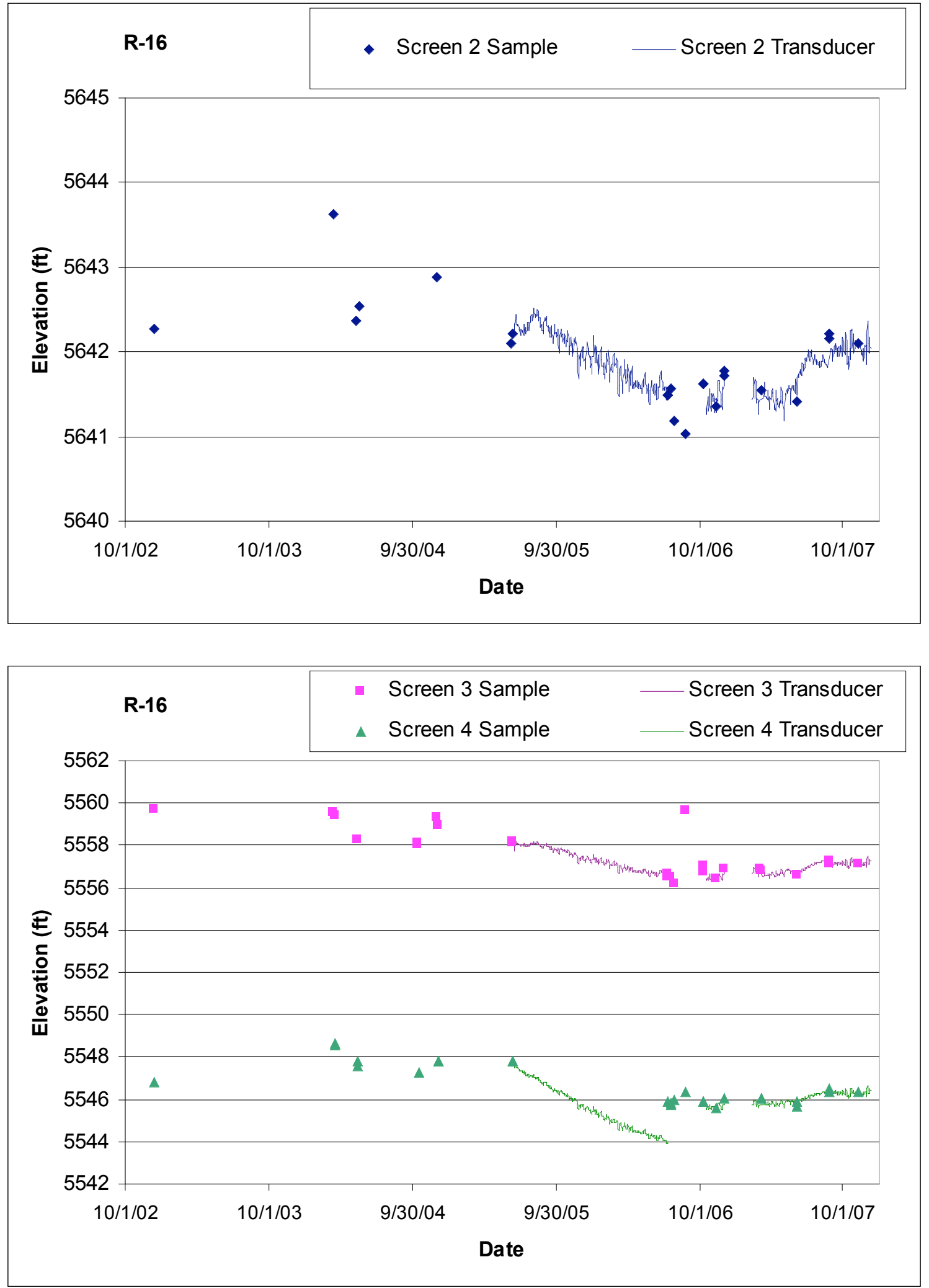


\subsection{R-16r}

Location: R-16r is located northeast of White Rock adjacent to R-16 in lower Cañada del Buey near the confluence with lower Mortandad Canyon.

Completion Type: Single completion at the top of the regional aquifer. Provides data for the top of the regional aquifer in place of $\mathrm{R}-16$ screen 1 , which is blocked by casing and is not useable. The top of the screen is about $36 \mathrm{ft}$ below the water table.

Period of Record: Well completed October 11, 2005, transducers installed February 21, 2006, data through 2007.

Remarks: R-16r water level at the top of the regional aquifer about $50 \mathrm{ft}$ higher than the water level at $\mathrm{R}-16$ screen 2, which is located about $250 \mathrm{ft}$ lower than $\mathrm{R}-16 \mathrm{r}$ screen. The well is $90 \%$ barometrically efficient; the aquifer indicates a $10 \%$ response to atmospheric pressure. Additionally, the aquifer shows a delayed response to atmospheric pressure fluctuations.

\begin{tabular}{|c|c|c|c|c|c|c|c|c|c|c|c|c|c|}
\hline \multicolumn{14}{|c|}{ R-16r Construction Information } \\
\hline Zone & $\begin{array}{c}\text { Screen } \\
\text { Top } \\
\text { Depth (ft) }\end{array}$ & $\begin{array}{l}\text { Screen } \\
\text { Bottom } \\
\text { Depth } \\
\text { (ft) }\end{array}$ & $\begin{array}{c}\text { Screen } \\
\text { Top } \\
\text { Elev (ft) }\end{array}$ & $\begin{array}{l}\text { Screen } \\
\text { Bottom } \\
\text { Elev (ft) }\end{array}$ & $\begin{array}{l}\text { Screen } \\
\text { Length } \\
\text { (ft) }\end{array}$ & $\begin{array}{l}\text { Pump } \\
\text { Intake } \\
\text { Depth } \\
\text { (ft) }\end{array}$ & $\begin{array}{l}\text { Pump } \\
\text { Intake } \\
\text { Elevation } \\
\text { (ft) }\end{array}$ & $\begin{array}{c}\text { Depth to } \\
\text { Top of } \\
\text { Sump (ft) }\end{array}$ & $\begin{array}{c}\text { Top of } \\
\text { Sump } \\
\text { Elevation } \\
\text { (ft) }\end{array}$ & $\begin{array}{l}\text { Depth to } \\
\text { Sump } \\
\text { Bottom (ft) }\end{array}$ & $\begin{array}{l}\text { Sump } \\
\text { Length } \\
\text { (ft) }\end{array}$ & $\begin{array}{l}\text { Sump } \\
\text { Volume } \\
\text { (L) }\end{array}$ & Comment \\
\hline 1 & 600.0 & 617.6 & 5657.0 & 5639.4 & 17.6 & 596.6 & 5660.4 & 617.6 & 5639.4 & 631.4 & 13.8 & 42.4 & Regional Aquifer \\
\hline
\end{tabular}

Note: Brass Cap Ground Elevation: $6256.97 \mathrm{ft}$; all measurements are from this elevation

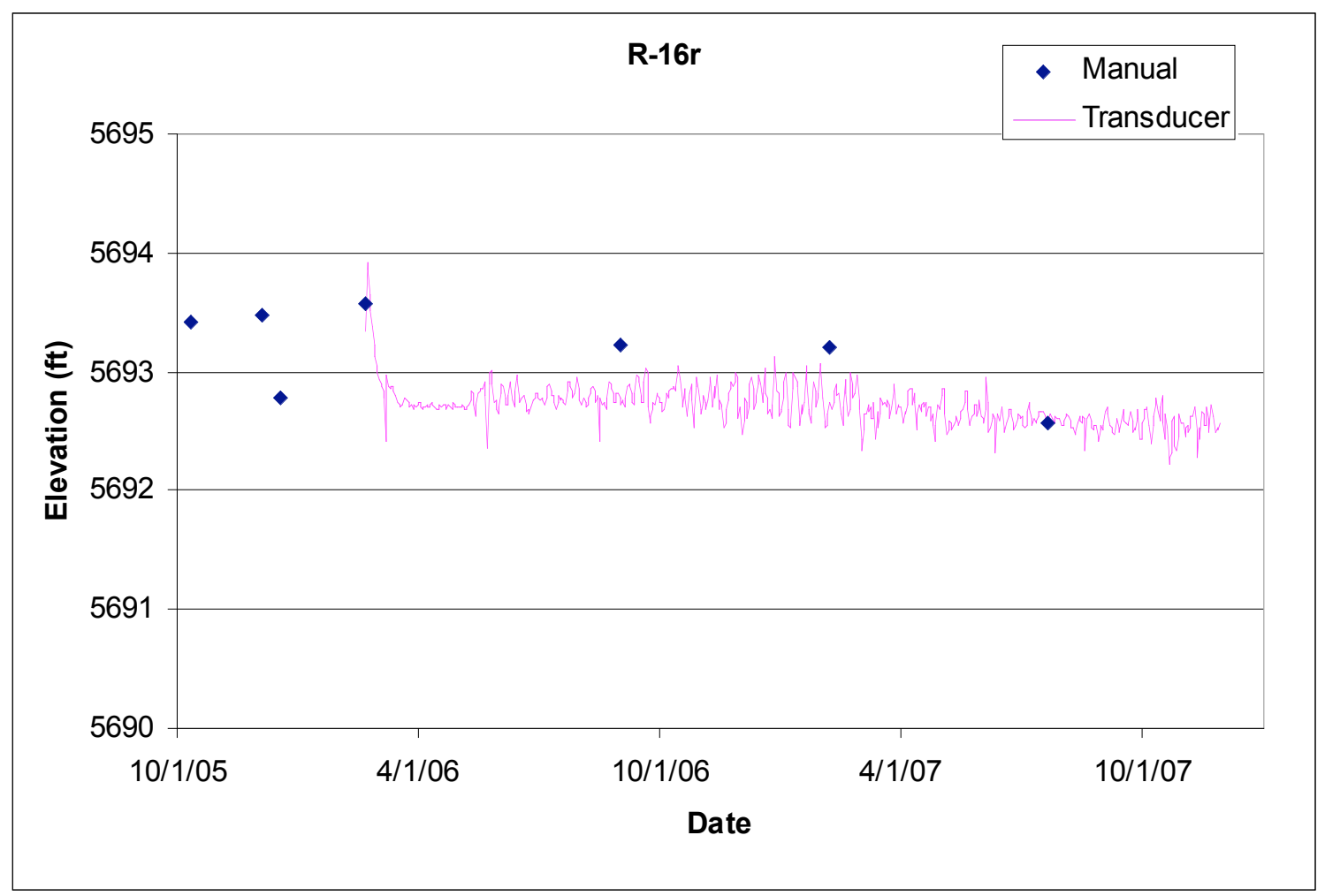




\subsection{R-17}

Location: R-17 is located in middle Pajarito Canyon below the confluence with Two-Mile Canyon and about 1 mile southwest of supply well PM-5.

Completion Type: Dual completion within the regional aquifer with a Baski packer and valve system and single submersible pump. The top of screen 1 is located about $20 \mathrm{ft}$ below the water table.

Period of Record: Completed January 4, 2006, transducers installed December 12, 2006, transducer data through 2007.

Remarks: R-17 was completed to a depth of $1140.9 \mathrm{ft}$, about $100 \mathrm{ft}$ into the regional aquifer. Screen 1 is $100 \%$ barometrically efficient; the aquifer does not show a response to atmospheric pressure fluctuations. Screen 2 is $90 \%$ barometrically efficient. Both screens show a seasonal response to supply well pumping; screen 2 shows a small response to pumping nearby supply well PM-5.

\begin{tabular}{|c|c|c|c|c|c|c|c|c|c|c|c|c|c|}
\hline \multicolumn{14}{|c|}{ R-17 Construction Information } \\
\hline Zone & $\begin{array}{c}\text { Screen } \\
\text { Top } \\
\text { Depth (ft) }\end{array}$ & $\begin{array}{c}\text { Screen } \\
\text { Bottom } \\
\text { Depth } \\
\text { (ft) }\end{array}$ & $\begin{array}{c}\text { Screen } \\
\text { Top } \\
\text { Elev (ft) }\end{array}$ & $\begin{array}{l}\text { Screen } \\
\text { Bottom } \\
\text { Elev (ft) }\end{array}$ & $\begin{array}{c}\text { Screen } \\
\text { Length } \\
\text { (ft) }\end{array}$ & $\begin{array}{l}\text { Pump } \\
\text { Intake } \\
\text { Depth } \\
\text { (ft) }\end{array}$ & $\begin{array}{l}\text { Pump } \\
\text { Intake } \\
\text { Elevation } \\
\text { (ft) }\end{array}$ & $\begin{array}{l}\text { Depth to } \\
\text { Top of } \\
\text { Packerl } \\
\text { Sump (ft) }\end{array}$ & $\begin{array}{c}\text { Top of } \\
\text { Packerl } \\
\text { Sump } \\
\text { Elevation } \\
\text { (ft) }\end{array}$ & $\begin{array}{c}\text { Depth to } \\
\text { Sump } \\
\text { Bottom (ft) }\end{array}$ & $\begin{array}{l}\text { Sump } \\
\text { Length } \\
\text { (ft) }\end{array}$ & $\begin{array}{c}\text { Sump } \\
\text { Volume } \\
\text { (L) }\end{array}$ & Comment \\
\hline 1 & 1057.0 & 1080.0 & 5864.5 & 5841.5 & 23.0 & 1089.6 & 5831.9 & 1101.2 & 5820.4 & 1101.2 & 21.1 & 66.1 & Regional Aquife \\
\hline 2 & 1124.0 & 1134.0 & 5797.5 & 5787.5 & 10.0 & 1128.6 & 5792.9 & 1134.0 & 5787.5 & 1140.9 & 6.9 & 21.6 & Regional Aquife \\
\hline
\end{tabular}

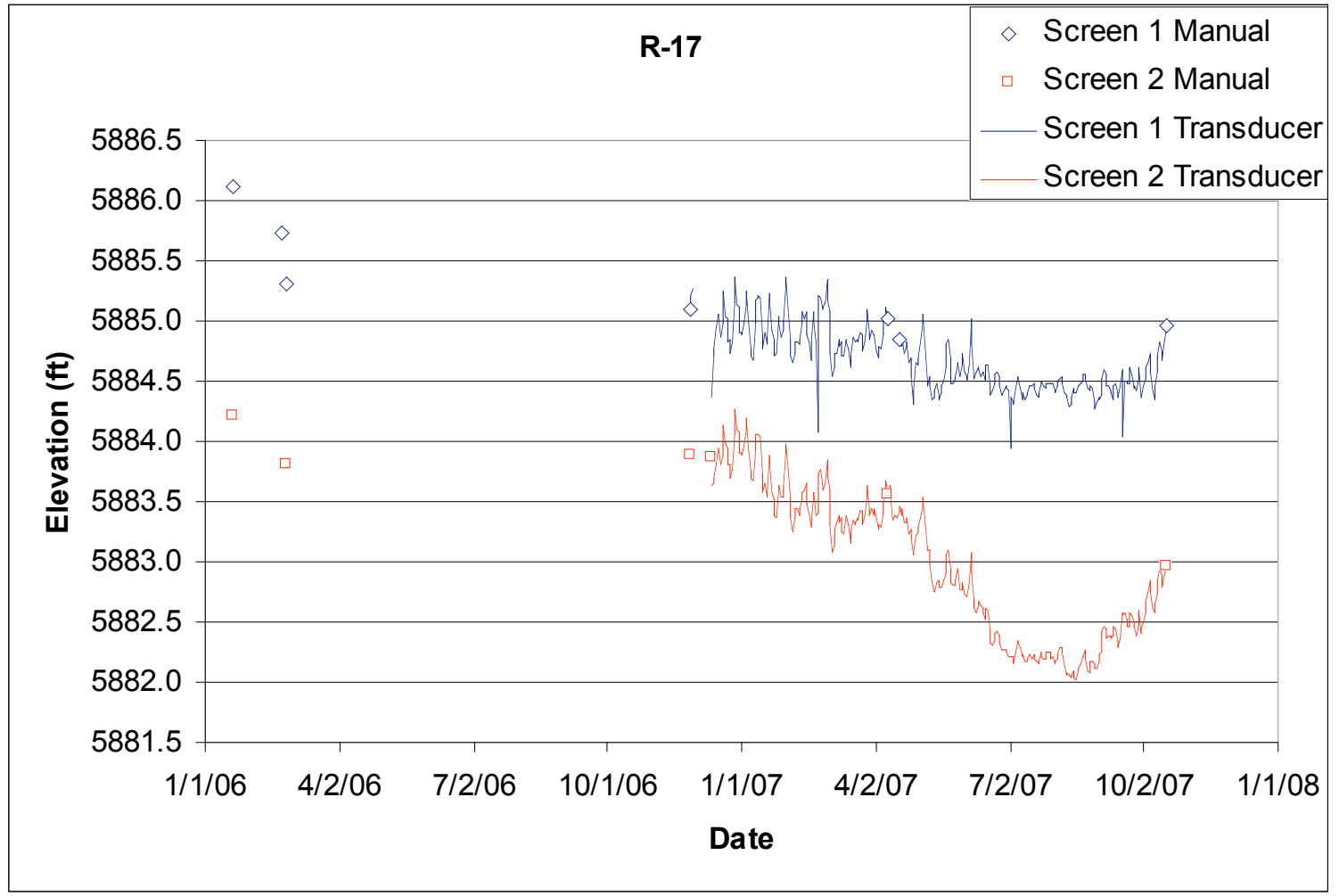




\subsection{R-18}

Location: R-18 is located on a mesa at TA-14 between Pajarito Canyon and Cañon de Valle, about $3000 \mathrm{ft}$ northeast of R-25.

Completion Type: Single completion at the top of the regional aquifer. The top of the screen is about $70 \mathrm{ft}$ bellow the water table.

Period of Record: Completed December 12, 2004, transducer installed October 11, 2005, transducer data through 2007.

Remarks: R-18 was completed to a depth of $1405 \mathrm{ft}$, about $118 \mathrm{ft}$ into the regional aquifer. The well is $100 \%$ barometrically efficient; the aquifer does not indicate a response to atmospheric pressure fluctuations. There is no apparent response to supply well pumping.

\begin{tabular}{|c|c|c|c|c|c|c|c|c|c|c|c|c|c|}
\hline \multirow[b]{2}{*}{ Zone } & \multicolumn{13}{|c|}{ R-18 Construction Information } \\
\hline & $\begin{array}{c}\text { Screen } \\
\text { Top } \\
\text { Depth (ft) }\end{array}$ & $\begin{array}{c}\text { Screen } \\
\text { Bottom } \\
\text { Depth } \\
\text { (ft) }\end{array}$ & $\begin{array}{c}\text { Screen } \\
\text { Top } \\
\text { Elev (ft) }\end{array}$ & $\begin{array}{l}\text { Screen } \\
\text { Bottom } \\
\text { Elev (ft) }\end{array}$ & $\begin{array}{c}\text { Screen } \\
\text { Length } \\
\text { (ft) }\end{array}$ & $\begin{array}{l}\text { Pump } \\
\text { Intake } \\
\text { Depth } \\
\text { (ft) }\end{array}$ & $\begin{array}{c}\text { Pump } \\
\text { Intake } \\
\text { Elevation } \\
\text { (ft) }\end{array}$ & $\begin{array}{c}\text { Depth to } \\
\text { Top of } \\
\text { Sump (ft) }\end{array}$ & $\begin{array}{l}\text { Top of } \\
\text { Sump } \\
\text { Elevation } \\
\text { (ft) }\end{array}$ & $\begin{array}{c}\text { Depth to } \\
\text { Sump } \\
\text { Bottom (ft) }\end{array}$ & $\begin{array}{l}\text { Sump } \\
\text { Length } \\
\text { (ft) }\end{array}$ & $\begin{array}{c}\text { Sump } \\
\text { Volume } \\
\text { (L) }\end{array}$ & Comment \\
\hline 1 & 1358.0 & 1381 & 6046.8 & 6023.8 & 23.0 & 1353 & 6051.8 & 1381.0 & 6023.8 & 1405 & 24.0 & 75.1 & Regional Aquifer \\
\hline
\end{tabular}

Note: Brass Cap Ground Elevation: $7404.83 \mathrm{ft}$; all measurements are from this elevation

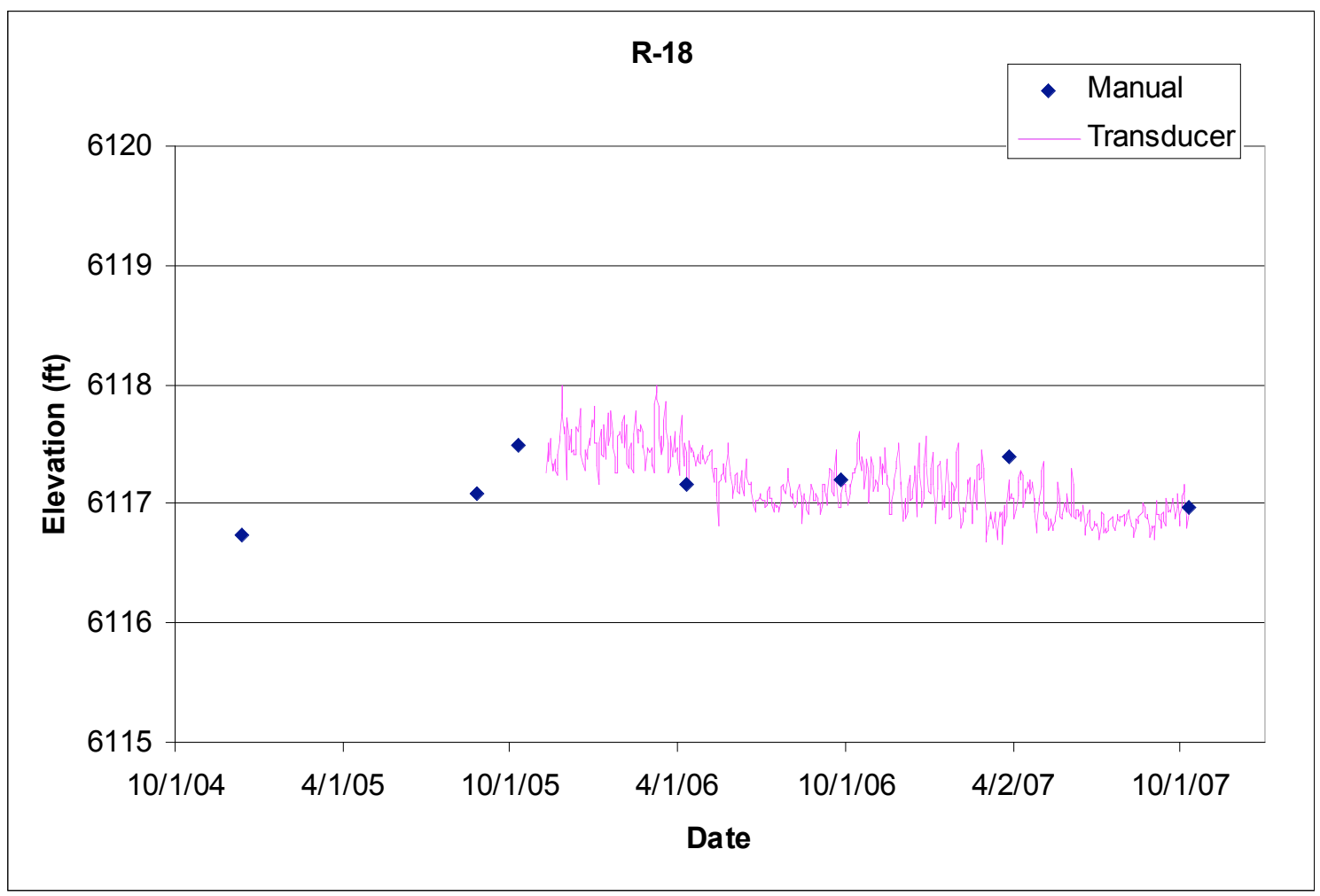




\section{$3.22 \quad R-19$}

Location: R-19 is located on a mesa south of Three-Mile Canyon about 1.2 miles west of supply well PM-2.

Completion Type: Multiple completion, two screens in intermediate zones, and five screens in the regional aquifer. Screen 3 straddles the regional water table.

Period of Record: Westbay ${ }^{\circledR}$ installed September 11, 2000, transducers installed June 04, 2002 , equipment problems occurred within two weeks. Transducers reinstalled December 10, 2004; transducer data to June 25, 2007, when the transducer string cable failed. Quarterly sampling data are available through 2007.

Remarks: Screen 1 has been dry since Westbay ${ }^{\circledR}$ installation. Screen 3 at the top of the regional aquifer does not show a response to atmospheric pressure fluctuations, but the deeper screens 4 through 7 indicate $40 \%$ to $50 \%$ response. The deeper screens ( 4 through 7 ) in the regional aquifer respond to supply well pumping at PM-2 and PM-4 and possibly to PM-5.

\begin{tabular}{|c|c|c|c|c|c|c|c|c|c|c|c|}
\hline \multicolumn{12}{|c|}{ R-19 Port Data } \\
\hline Zone & $\begin{array}{c}\text { Screen } \\
\text { Top } \\
\text { Depth (ft) }\end{array}$ & \begin{tabular}{|c|} 
Screen \\
Bottom \\
Depth \\
(ft)
\end{tabular} & $\begin{array}{c}\text { Screen } \\
\text { Top } \\
\text { Elev (ft) }\end{array}$ & $\begin{array}{l}\text { Screen } \\
\text { Bottom } \\
\text { Elev (ft) }\end{array}$ & $\begin{array}{l}\text { Screen } \\
\text { Length } \\
\text { (ft) }\end{array}$ & Port & $\begin{array}{c}\text { Port } \\
\text { Depth (ft) }\end{array}$ & $\begin{array}{c}\text { Port } \\
\text { Elevation } \\
\text { (ft) }\end{array}$ & $\begin{array}{c}\begin{array}{c}\text { Distance } \\
\text { from }\end{array} \\
\text { Bottom of } \\
\text { Screen (ft) }\end{array}$ & $\begin{array}{c}\text { Sump } \\
\text { Volume } \\
\text { (L) }\end{array}$ & Comment \\
\hline \multirow{3}{*}{1} & \multirow{3}{*}{827.2} & \multirow{3}{*}{843.6} & \multirow{3}{*}{6239.1} & \multirow{3}{*}{6222.7} & \multirow{3}{*}{16.4} & MP1A & 844.2 & 6222.1 & -0.6 & 1.3 & Below Screen, Intermediate \\
\hline & & & & & & PP1 & 849.6 & 6216.7 & -6 & 13.0 & Below Screen \\
\hline & & & & & & MP1B & 855.2 & 6211.1 & -11.6 & 25.1 & Below Screen \\
\hline \multirow{3}{*}{2} & \multirow{3}{*}{893.3} & \multirow{3}{*}{909.6} & \multirow{3}{*}{6173.0} & \multirow{3}{*}{6156.7} & \multirow{3}{*}{16.3} & MP2A & 909.3 & 6157.0 & 0.3 & & Within Screen Intermediate \\
\hline & & & & & & PP2 & 914.7 & 6151.6 & -5.1 & 11.0 & Below Screen \\
\hline & & & & & & MP2B & 920.3 & 6146.0 & -10.7 & 23.1 & Below Screen \\
\hline \multirow{4}{*}{3} & \multirow{4}{*}{1171.4} & \multirow{4}{*}{1215.4} & \multirow{4}{*}{5894.9} & \multirow{4}{*}{5850.9} & \multirow{4}{*}{44.0} & MP3A & 1190.7 & 5875.6 & 24.7 & & Within Screen, Regional Aquifer \\
\hline & & & & & & PP3 & 1196.1 & 5870.2 & 19.3 & & Within Screen \\
\hline & & & & & & MP3B & 1201.7 & 5864.6 & 13.7 & & Within Screen \\
\hline & & & & & & MP3C & 1212.8 & 5853.5 & 2.6 & & Within Screen \\
\hline \multirow{3}{*}{4} & \multirow{3}{*}{1410.2} & \multirow{3}{*}{1417.4} & \multirow{3}{*}{5656.1} & \multirow{3}{*}{5648.9} & \multirow{3}{*}{7.2} & MP4A & 1412.9 & 5653.4 & 4.5 & & Within Screen \\
\hline & & & & & & PP4 & 1418.3 & 5648.0 & -0.9 & 1.9 & Below Screen \\
\hline & & & & & & MP4B & 1423.9 & 5642.4 & -6.5 & 14.1 & Below Screen \\
\hline \multirow{3}{*}{5} & \multirow{3}{*}{1582.6} & \multirow{3}{*}{1589.8} & \multirow{3}{*}{5483.7} & \multirow{3}{*}{5476.5} & \multirow{3}{*}{7.2} & MP5A & 1586.1 & 5480.2 & 3.7 & & Within Screen \\
\hline & & & & & & PP5 & 1591.5 & 5474.8 & -1.7 & 3.7 & Below Screen \\
\hline & & & & & & MP5B & 1597.1 & 5469.2 & -7.3 & 15.8 & Below Screen \\
\hline & & & & & & MP6A & 1730.1 & 5336.2 & 3.8 & & Within Screen \\
\hline 6 & 1726.8 & 1733.9 & 5339.5 & 5332.4 & 7.1 & PP6 & 1735.4 & 5330.9 & -1.5 & 3.2 & Below Screen \\
\hline & & & & & & MP6B & 1741.1 & 5325.2 & -7.2 & 15.6 & Below Screen \\
\hline & & & & & & MP7A & 1834.7 & 5231.6 & 4.8 & & Within Screen \\
\hline 7 & 1832.4 & 1839.5 & 5233.9 & 5226.8 & 7.1 & PP7 & 1840.0 & 5226.3 & -0.5 & 1.1 & Below Screen \\
\hline & & & & & & MP7B & 1845.7 & 5220.6 & -6.2 & 13.4 & Below Screen \\
\hline
\end{tabular}

Note: R-19 Brass Cap Ground Elevation: $7066.3 \mathrm{ft}$; all measurements are from this elevation;

$\mathrm{MP}=$ Monitor Port; PP = Pump Port; Monitor Ports shown in bold are instrumented ports 

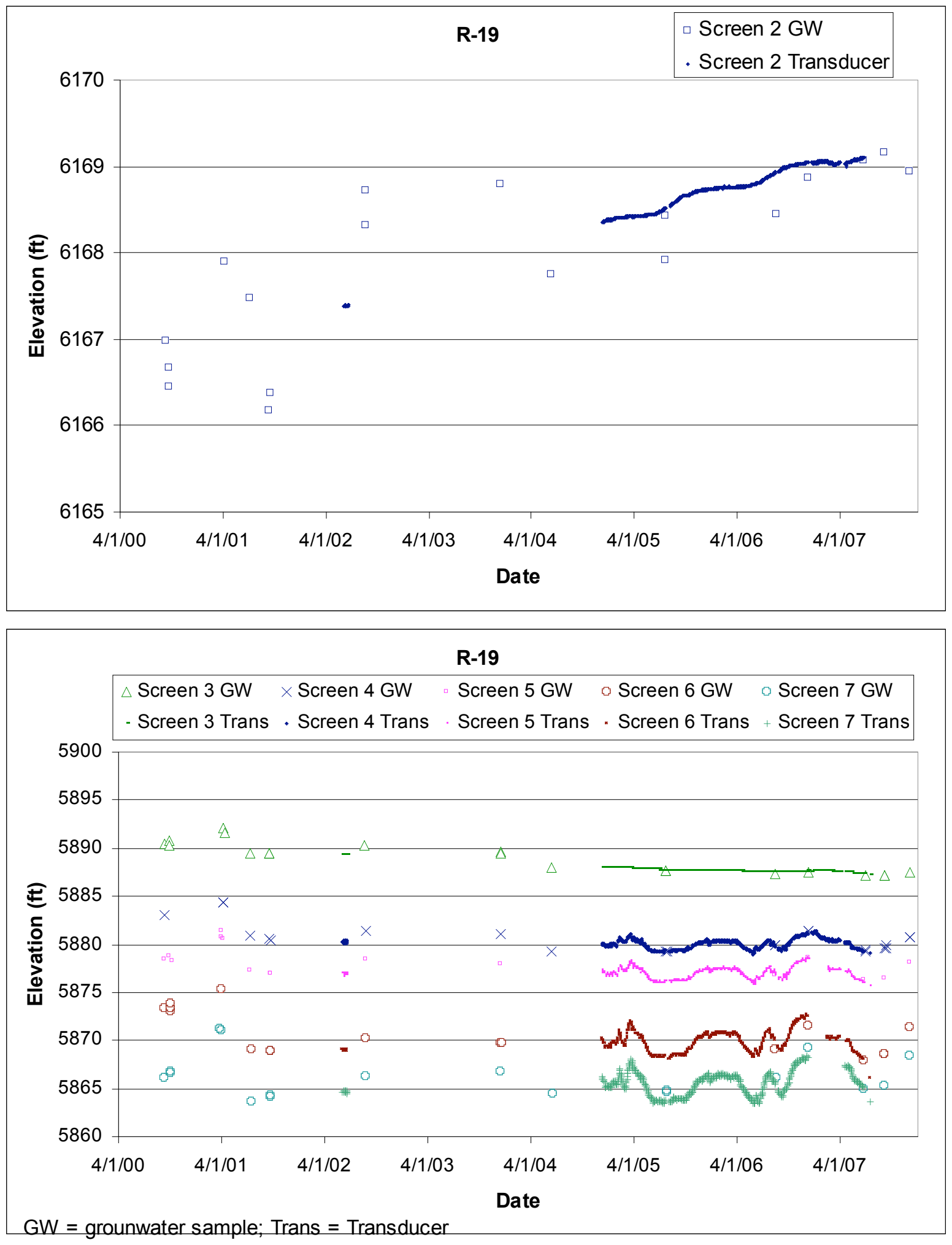


\subsection{R-20}

Location: R-20 is located in lower Pajarito Canyon about $1300 \mathrm{ft}$ east of supply well PM-2.

Completion Type: Multiple completion, originally three screens in the regional aquifer. Screen 3 was abandoned in 2007, leaving 2 screens in the regional aquifer. The top of screen 1 is about 76 $\mathrm{ft}$ below the regional water table.

Period of Record: Westbay ${ }^{\circledR}$ installed January 18, 2003, transducers installed March 26, 2003, intermittent transducer data to June 1,2006 , when the Westbay ${ }^{\circledR}$ system was removed. No water level data in the last half of 2006 and in 2007 during well rehabilitation.

Remarks: Screen 1 shows no response to atmospheric pressure fluctuations. Screen 3 responds to supply well pumping at PM-2 and PM-4. The shallower screens 1 and 2 show a muted response to pumping.

\begin{tabular}{|c|c|c|c|c|c|c|c|c|c|c|c|}
\hline \multicolumn{12}{|c|}{ Measurement and Sampling Ports in R-20 } \\
\hline Zone & $\begin{array}{c}\text { Screen } \\
\text { Top } \\
\text { Depth (ft) }\end{array}$ & $\begin{array}{l}\text { Screen } \\
\text { Bottom } \\
\text { Depth } \\
\text { (ft) }\end{array}$ & $\begin{array}{c}\text { Screen } \\
\text { Top } \\
\text { Elev (ft) }\end{array}$ & $\begin{array}{l}\text { Screen } \\
\text { Bottom } \\
\text { Elev (ft) }\end{array}$ & $\begin{array}{c}\text { Screen } \\
\text { Length } \\
\text { (ft) }\end{array}$ & Port & $\begin{array}{c}\text { Port } \\
\text { Depth (ft) }\end{array}$ & $\begin{array}{c}\text { Port } \\
\text { Elevation } \\
\text { (ft) }\end{array}$ & $\begin{array}{c}\begin{array}{c}\text { Distance } \\
\text { from }\end{array} \\
\text { Bottom of } \\
\text { Screen (ft) }\end{array}$ & $\begin{array}{c}\text { Sump } \\
\text { Volume } \\
\text { (L) }\end{array}$ & Comment \\
\hline \multirow{3}{*}{1} & \multirow{3}{*}{904.6} & \multirow{3}{*}{912.2} & \multirow{3}{*}{5789.8} & \multirow{3}{*}{5782.2} & \multirow{3}{*}{7.6} & MP1A & 907.0 & 5787.4 & 5.2 & & Within Screen, Regional Aquifer \\
\hline & & & & & & PP1 & 912.4 & 5782.0 & -0.2 & 0.4 & Below Screen \\
\hline & & & & & & MP1B & 918.0 & 5776.4 & -5.8 & 12.5 & Below Screen \\
\hline \multirow{3}{*}{2} & \multirow{3}{*}{1147.1} & \multirow{3}{*}{1154.7} & \multirow{3}{*}{5547.3} & \multirow{3}{*}{5539.7} & \multirow{3}{*}{7.6} & MP2A & 1149.7 & 5544.7 & 5 & & Within Screen \\
\hline & & & & & & PP2 & 1155.0 & 5539.4 & -0.3 & 0.6 & Below Screen \\
\hline & & & & & & MP2B & 1160.7 & 5533.7 & -6.0 & 13.0 & Below Screen \\
\hline \multirow{3}{*}{3} & \multirow{3}{*}{1328.8} & \multirow{3}{*}{1336.5} & \multirow{3}{*}{5365.6} & \multirow{3}{*}{5357.9} & \multirow{3}{*}{7.7} & MIP3A & 1330.0 & 5364.4 & 6.5 & & Within Screen \\
\hline & & & & & & PP3 & 1335.4 & 5359.0 & 1.1 & & Within Screen \\
\hline & & & & & & MP3B & 1341.0 & 5353.4 & -4.5 & $\overline{9.7}$ & Below Screen \\
\hline
\end{tabular}

Note: R-20 Brass Cap Ground Elevation: $6694.35 \mathrm{ft}$; all measurements are from this elevation;

$\mathrm{MP}=$ Monitor Port; PP = Pump Port; Monitor Ports shown in bold are instrumented ports

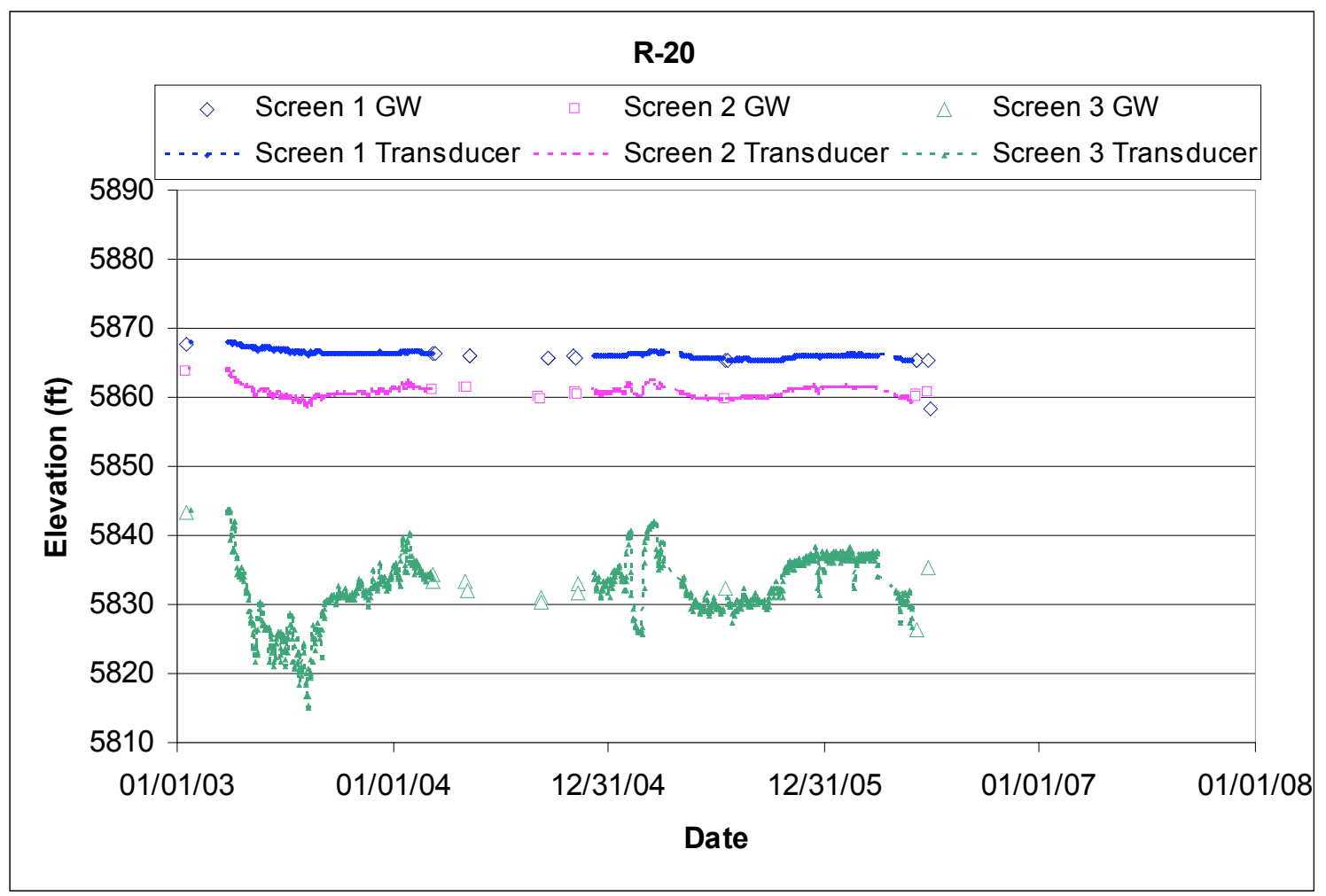




\subsection{R-21}

Location: R-21 is located in Cañada del Buey north of TA-54.

Completion Type: Single completion at the top of the regional aquifer. The top of the screen is about $87 \mathrm{ft}$ below the water table.

Period of Record: Well completed November 2002, transducer installed May 23, 2005, transducer data through 2007.

Remarks: R-21 installed to a depth of $941.4 \mathrm{ft}$, about $140 \mathrm{ft}$ into the regional aquifer. The well is $100 \%$ barometrically efficient; the aquifer does not respond to atmospheric pressure fluctuations. The well responds to pumping of PM-2 and to another well or combination of wells.

\begin{tabular}{|c|c|c|c|c|c|c|c|c|c|c|c|c|c|}
\hline \multicolumn{14}{|c|}{ R-21 Construction Information } \\
\hline Zone & $\begin{array}{c}\text { Screen } \\
\text { Top } \\
\text { Depth (ft) }\end{array}$ & $\begin{array}{l}\text { Screen } \\
\text { Bottom } \\
\text { Depth } \\
\text { (ft) }\end{array}$ & $\begin{array}{c}\text { Screen } \\
\text { Top } \\
\text { Elev (ft) }\end{array}$ & $\begin{array}{l}\text { Screen } \\
\text { Bottom } \\
\text { Elev (ft) }\end{array}$ & $\begin{array}{l}\text { Screen } \\
\text { Length } \\
\text { (ft) }\end{array}$ & $\begin{array}{l}\text { Pump } \\
\text { Intake } \\
\text { Depth } \\
\text { (ft) }\end{array}$ & $\begin{array}{l}\text { Pump } \\
\text { Intake } \\
\text { Elevation } \\
\text { (ft) }\end{array}$ & $\begin{array}{c}\text { Depth to } \\
\text { Top of } \\
\text { Sump (ft) }\end{array}$ & $\begin{array}{l}\text { Top of } \\
\text { Sump } \\
\text { Elevation } \\
\text { (ft) }\end{array}$ & $\begin{array}{l}\text { Depth to } \\
\text { Sump } \\
\text { Bottom (ft) }\end{array}$ & $\begin{array}{l}\text { Sump } \\
\text { Length } \\
\text { (ft) }\end{array}$ & $\begin{array}{l}\text { Sump } \\
\text { Volume } \\
\text { (L) }\end{array}$ & Comment \\
\hline 1 & 888.8 & 906.8 & 5767.4 & 5749.4 & 18.0 & 861 & 5795.2 & 906.8 & 5749.4 & 941.4 & 34.6 & 192.4 & Regional Aquifer \\
\hline
\end{tabular}

Note: R-21 Brass Cap Ground Elevation: $6656.24 \mathrm{ft}$; all measurements are from this elevation

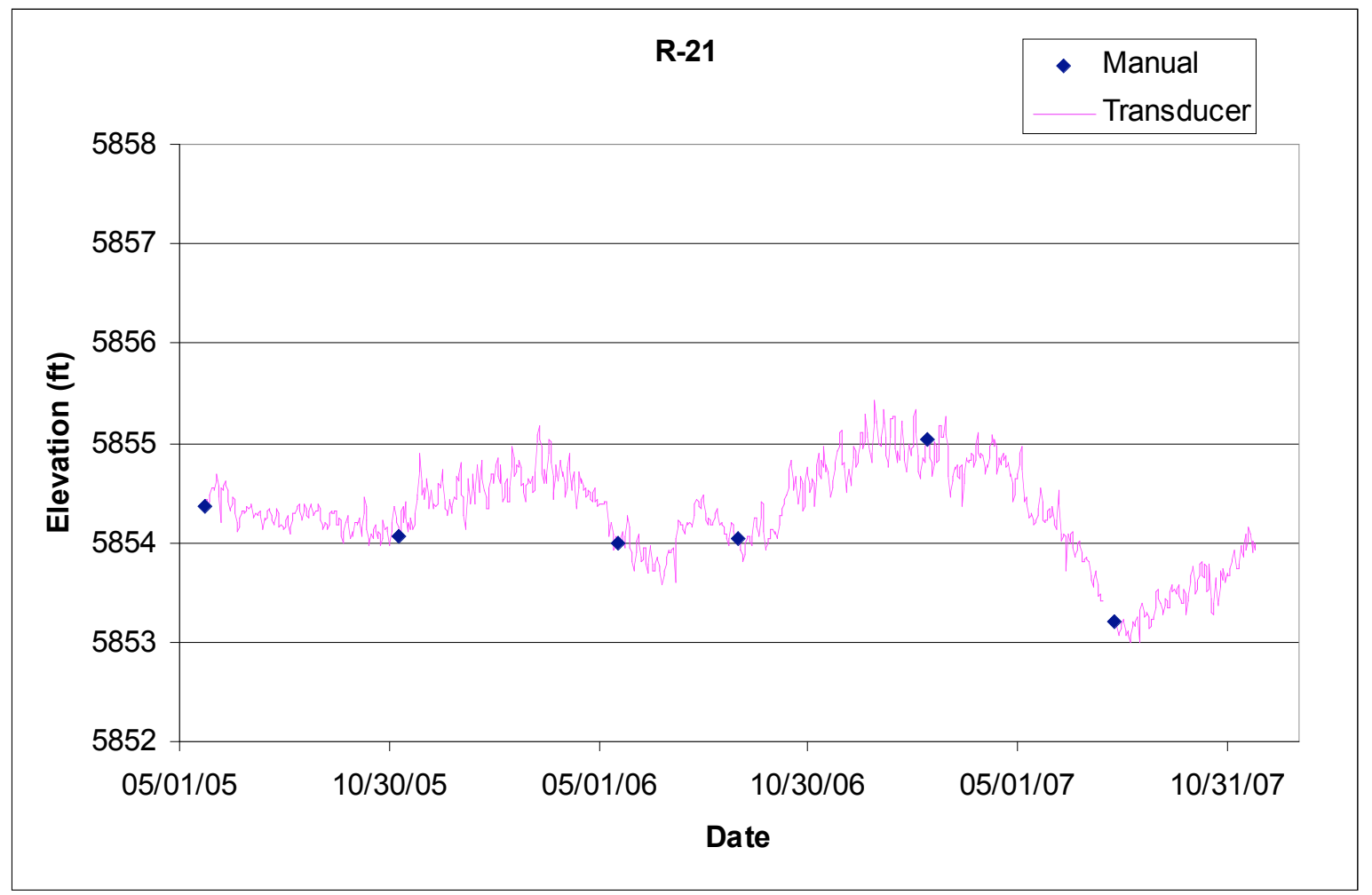




\section{$3.25 \quad \mathrm{R}-22$}

Location: R-22 is located at the east end of Mesita del Buey, east of TA-54.

Completion Type: Multiple completion, five screens in the regional aquifer. Screen 1 straddles the water table.

Period of Record: Westbay ${ }^{\circledR}$ installed December 11, 2000, transducers installed March 26, 2003, intermittent transducer data through 2007.

Remarks: Screens 1 and 2 have similar head values about $6 \mathrm{ft}$ apart. Screens 3, 4, and 5 have similar heads within $6 \mathrm{ft}$ of each other, but about $60 \mathrm{ft}$ lower than screens 1 and 2 . Screens 4 and 5 have nearly identical head values. The R-22 screens do not show an immediate response to atmospheric pressure fluctuations, but show a delayed response ranging from $20 \%$ to $100 \%$. The deeper screens 2 through 5 appear to have a small response to supply well pumping, primarily at PM-2, but may also respond to other wells.

\begin{tabular}{|c|c|c|c|c|c|c|c|c|c|c|c|}
\hline \multicolumn{12}{|c|}{ R-22 Measurement and Sampling Ports } \\
\hline Zone & $\begin{array}{c}\text { Screen } \\
\text { Top } \\
\text { Depth (ft) }\end{array}$ & $\begin{array}{l}\text { Screen } \\
\text { Bottom } \\
\text { Depth } \\
\text { (ft) }\end{array}$ & $\begin{array}{c}\text { Screen } \\
\text { Top } \\
\text { Elev (ft) }\end{array}$ & $\begin{array}{l}\text { Screen } \\
\text { Bottom } \\
\text { Elev (ft) }\end{array}$ & $\begin{array}{l}\text { Screen } \\
\text { Length } \\
\text { (ft) }\end{array}$ & Port & $\begin{array}{c}\text { Port } \\
\text { Depth (ft) }\end{array}$ & $\begin{array}{c}\text { Port } \\
\text { Elevation } \\
\text { (ft) }\end{array}$ & $\begin{array}{l}\text { Distance } \\
\text { from } \\
\text { Bottom of } \\
\text { Screen (ft) }\end{array}$ & $\begin{array}{c}\text { Sump } \\
\text { Volume } \\
\text { (L) }\end{array}$ & Comment \\
\hline \multirow{3}{*}{1} & \multirow{3}{*}{872.3} & \multirow{3}{*}{914.2} & \multirow{3}{*}{5778.2} & \multirow{3}{*}{5736.3} & \multirow{3}{*}{41.9} & MP1A & $\overline{907.1}$ & 5743.4 & 7.1 & & Within Screen, Regional Aquifer \\
\hline & & & & & & PP1 & 912.4 & 5738.1 & 1.8 & & Within Screen \\
\hline & & & & & & MP1B & 918.1 & 5732.4 & -3.9 & 8.4 & Below Screen \\
\hline \multirow{3}{*}{2} & \multirow{3}{*}{947.0} & \multirow{3}{*}{988.9} & \multirow{3}{*}{5703.5} & \multirow{3}{*}{5661.6} & \multirow{3}{*}{41.9} & MP2A & 962.8 & 5687.7 & 26.1 & & Within Screen \\
\hline & & & & & & PP2 & 967.7 & 5682.8 & 21.2 & & Within Screen \\
\hline & & & & & & MP2B & 973.4 & 5677.1 & 15.5 & & Within Screen \\
\hline \multirow{3}{*}{3} & \multirow{3}{*}{1272.2} & \multirow{3}{*}{1278.9} & \multirow{3}{*}{5378.3} & \multirow{3}{*}{5371.6} & \multirow{3}{*}{6.7} & MP3A & 1273.5 & 5377.0 & 5.4 & & Within Screen \\
\hline & & & & & & PP3 & 1278.9 & 5371.6 & 0 & & Within Screen \\
\hline & & & & & & MP3B & 1284.5 & 5366.0 & -5.6 & 12.1 & Below Screen \\
\hline \multirow{3}{*}{4} & \multirow{3}{*}{1378.2} & \multirow{3}{*}{1384.9} & \multirow{3}{*}{5272.3} & \multirow{3}{*}{5265.6} & \multirow{3}{*}{6.7} & MP4A & 1378.0 & 5272.5 & 6.9 & & Above Screen \\
\hline & & & & & & PP4 & 1383.4 & 5267.1 & 1.5 & & Within Screen \\
\hline & & & & & & MP4B & 1389.1 & 5261.4 & -4.2 & 9.1 & Below Screen \\
\hline \multirow{3}{*}{5} & \multirow{3}{*}{1447.3} & \multirow{3}{*}{1452.3} & \multirow{3}{*}{5203.2} & \multirow{3}{*}{5198.2} & \multirow{3}{*}{5.0} & MP5A & 1448.2 & 5202.3 & 4.1 & & Within Screen \\
\hline & & & & & & PP5 & 1453.6 & 5196.9 & -1.3 & 2.8 & Below Screen \\
\hline & & & & & & MP5B & 1459.2 & 5191.3 & -6.9 & 14.9 & Below Screen \\
\hline
\end{tabular}

Note: R-22 Brass Cap Ground Elevation: $6650.5 \mathrm{ft}$; all measurements are from this elevation;

$\mathrm{MP}=$ Monitor Port; PP = Pump Port; Monitor Ports shown in bold are instrumented ports 

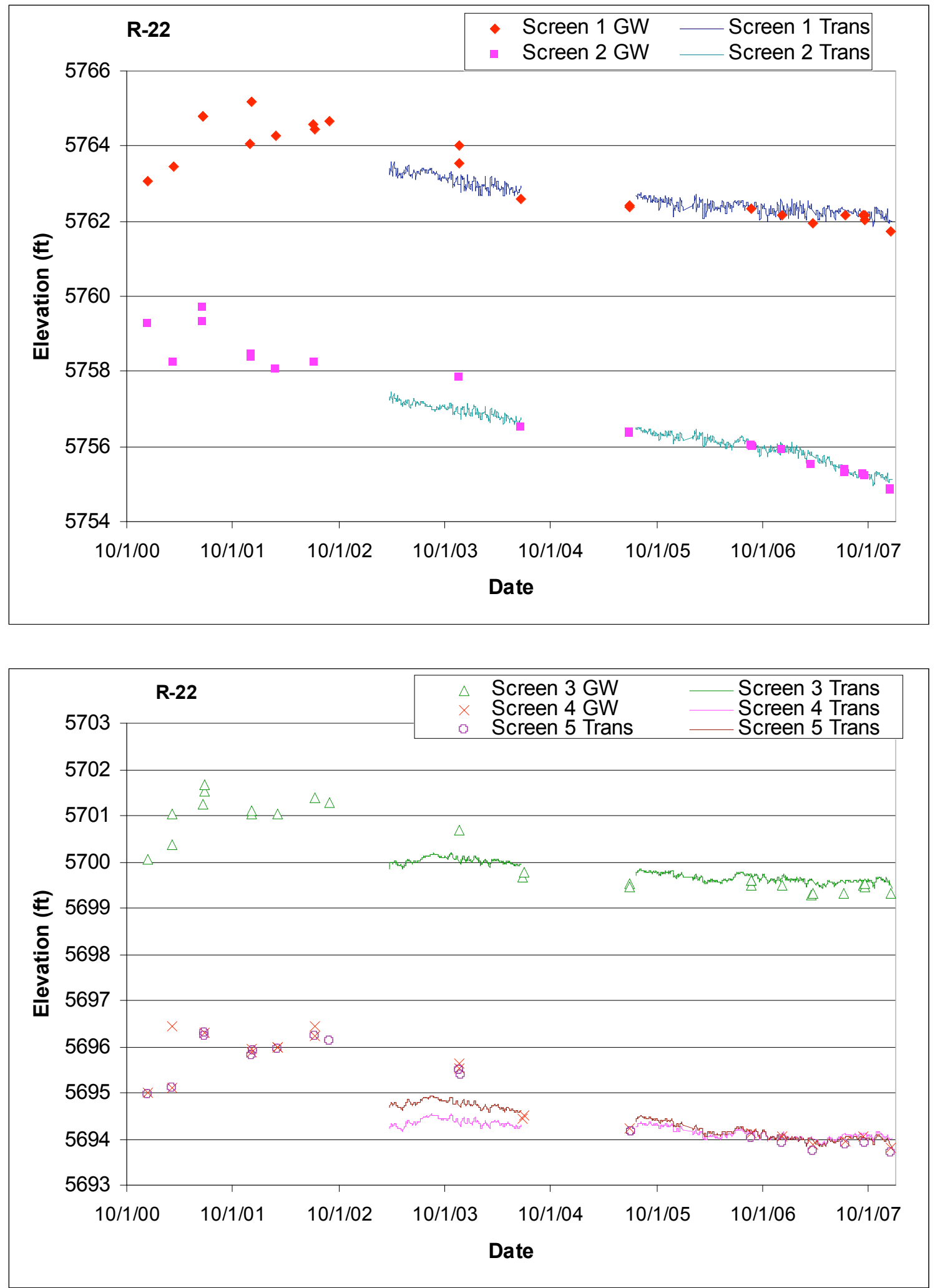


\section{$3.26 \quad R-23$}

Location: R-23 is located in lower Pajarito Canyon near SR-4 and the eastern LANL boundary.

Completion Type: Single completion at the top of the regional aquifer. The screen straddles the water table.

Period of Record: Well completed October 2002, transducer installed June 20, 2005, transducer data through 2007.

Remarks: R-23 was installed to a depth of $886.3 \mathrm{ft}$, about $60 \mathrm{ft}$ into the regional aquifer. The well is $100 \%$ barometrically efficient; the aquifer has no immediate response to atmospheric pressure fluctuations, however, the aquifer has a delayed response to atmospheric pressure. There is no apparent response to pumping the PM well field or the Buckman well field at R-

23.

\begin{tabular}{|c|c|c|c|c|c|c|c|c|c|c|c|c|c|}
\hline \multicolumn{14}{|c|}{ R-23 Construction Information } \\
\hline Zone & $\begin{array}{c}\text { Screen } \\
\text { Top } \\
\text { Depth (ft) }\end{array}$ & $\begin{array}{l}\text { Screen } \\
\text { Bottom } \\
\text { Depth } \\
\text { (ft) }\end{array}$ & $\begin{array}{c}\text { Screen } \\
\text { Top } \\
\text { Elev (ft) }\end{array}$ & $\begin{array}{l}\text { Screen } \\
\text { Bottom } \\
\text { Elev (ft) }\end{array}$ & $\begin{array}{c}\text { Screen } \\
\text { Length } \\
\text { (ft) }\end{array}$ & $\begin{array}{l}\text { Pump } \\
\text { Intake } \\
\text { Depth } \\
\text { (ft) }\end{array}$ & $\begin{array}{l}\text { Pump } \\
\text { Intake } \\
\text { Elevation } \\
\text { (ft) }\end{array}$ & $\begin{array}{c}\text { Depth to } \\
\text { Top of } \\
\text { Sump (ft) }\end{array}$ & $\begin{array}{l}\text { Top of } \\
\text { Sump } \\
\text { Elevation } \\
\text { (ft) }\end{array}$ & $\begin{array}{l}\text { Depth to } \\
\text { Sump } \\
\text { Bottom (ft) }\end{array}$ & $\begin{array}{c}\text { Sump } \\
\text { Length } \\
\text { (ft) }\end{array}$ & $\begin{array}{c}\text { Sump } \\
\text { Volume } \\
\text { (L) }\end{array}$ & Comment \\
\hline 1 & 816.0 & 873.2 & 5711.8 & 5654.6 & 57.2 & 870.7 & 5657.1 & 873.2 & 5654.6 & 886.3 & 13.1 & 41.0 & Regional Aquifer \\
\hline
\end{tabular}

Note: R-23 Brass Cap Ground Elevation: $6527.75 \mathrm{ft}$; all measurements are from this elevation

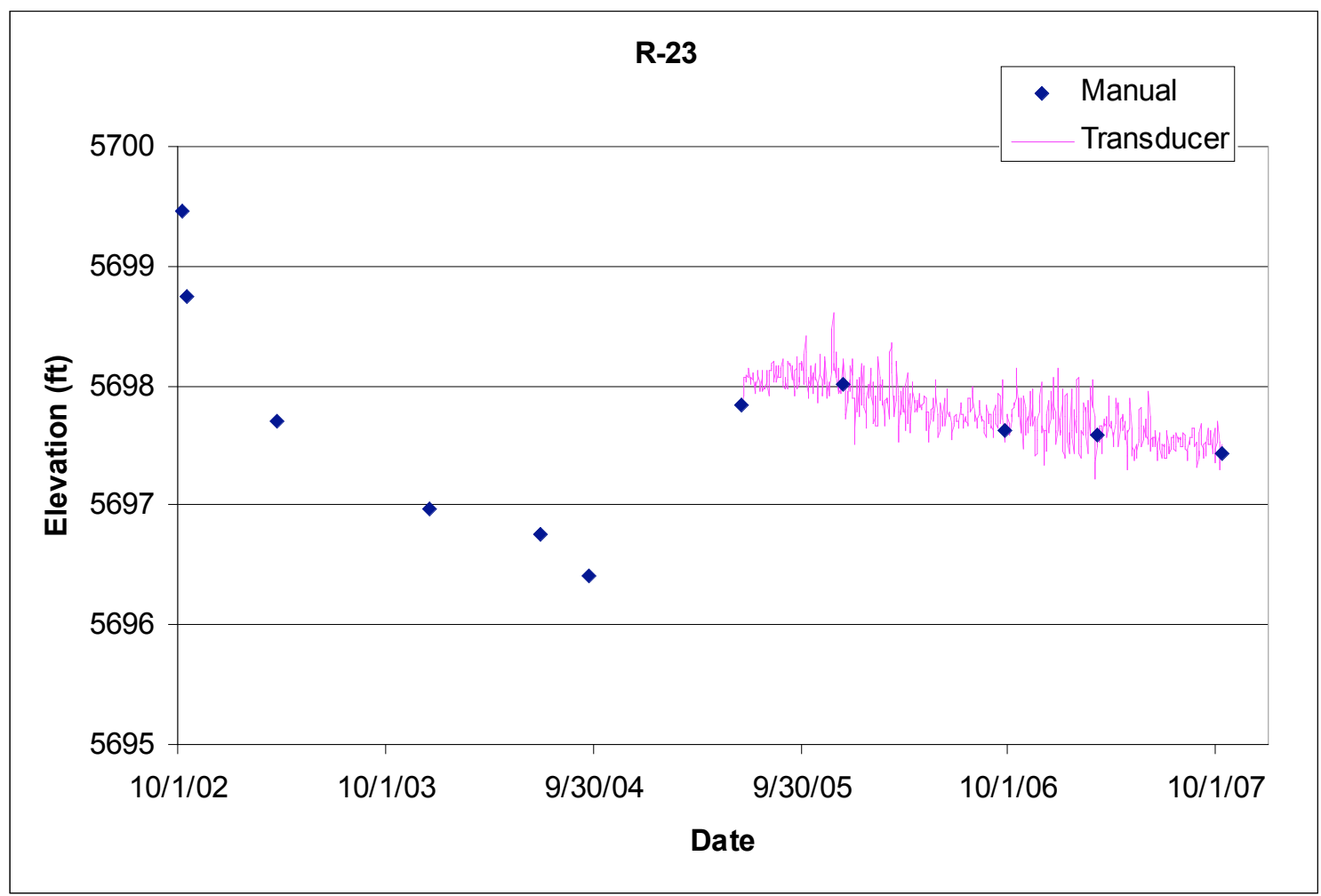




\section{$3.27 \quad \mathrm{R}-24$}

Location: R-24 is located in Bayo Canyon north of the Bayo Sewage Treatment Plant.

Completion Type: Single completion at the top of the regional aquifer. The top of the screen is in a confined zone about $110 \mathrm{ft}$ below the water table.

Period of Record: Well completed September 2005, transducer installed March 1, 2006, data through 2007.

Remarks: R-24 installed to a depth of $861 \mathrm{ft}$, about $150 \mathrm{ft}$ into the regional aquifer. The well is $100 \%$ barometrically efficient; the aquifer does not respond to atmospheric pressure fluctuations. The water level responds primarily to pumping at supply well PM-3 but may also respond to pumping the Guaje well field and supply well O-4.

\begin{tabular}{|c|c|c|c|c|c|c|c|c|c|c|c|c|c|}
\hline \multicolumn{14}{|c|}{ R-24 Construction Information } \\
\hline Zone & $\begin{array}{c}\text { Screen } \\
\text { Top } \\
\text { Depth (ft) }\end{array}$ & $\begin{array}{c}\text { Screen } \\
\text { Bottom } \\
\text { Depth } \\
\text { (ft) }\end{array}$ & $\begin{array}{c}\text { Screen } \\
\text { Top } \\
\text { Elev (ft) }\end{array}$ & $\begin{array}{l}\text { Screen } \\
\text { Bottom } \\
\text { Elev (ft) }\end{array}$ & $\begin{array}{c}\text { Screen } \\
\text { Length } \\
\text { (ft) }\end{array}$ & $\begin{array}{l}\text { Pump } \\
\text { Intake } \\
\text { Depth } \\
\text { (ft) }\end{array}$ & $\begin{array}{c}\text { Pump } \\
\text { Intake } \\
\text { Elevation } \\
\text { (ft) }\end{array}$ & $\begin{array}{c}\text { Depth to } \\
\text { Top of } \\
\text { Sump (ft) }\end{array}$ & $\begin{array}{c}\text { Top of } \\
\text { Sump } \\
\text { Elevation } \\
\text { (ft) }\end{array}$ & $\begin{array}{l}\text { Depth to } \\
\text { Sump } \\
\text { Bottom (ft) }\end{array}$ & $\begin{array}{c}\text { Sump } \\
\text { Length } \\
\text { (ft) }\end{array}$ & $\begin{array}{c}\text { Sump } \\
\text { Volume } \\
\text { (L) }\end{array}$ & Comment \\
\hline 1 & 825.0 & 848 & 5722.4 & 5699.4 & 23.0 & 818.7 & 5728.7 & 848.0 & 5699.4 & 861 & 13.0 & 40.7 & Regional Aquifer \\
\hline
\end{tabular}

Note: R-24 Brass Cap Ground Elevation: $6547.38 \mathrm{ft}$; all measurements are from this elevation

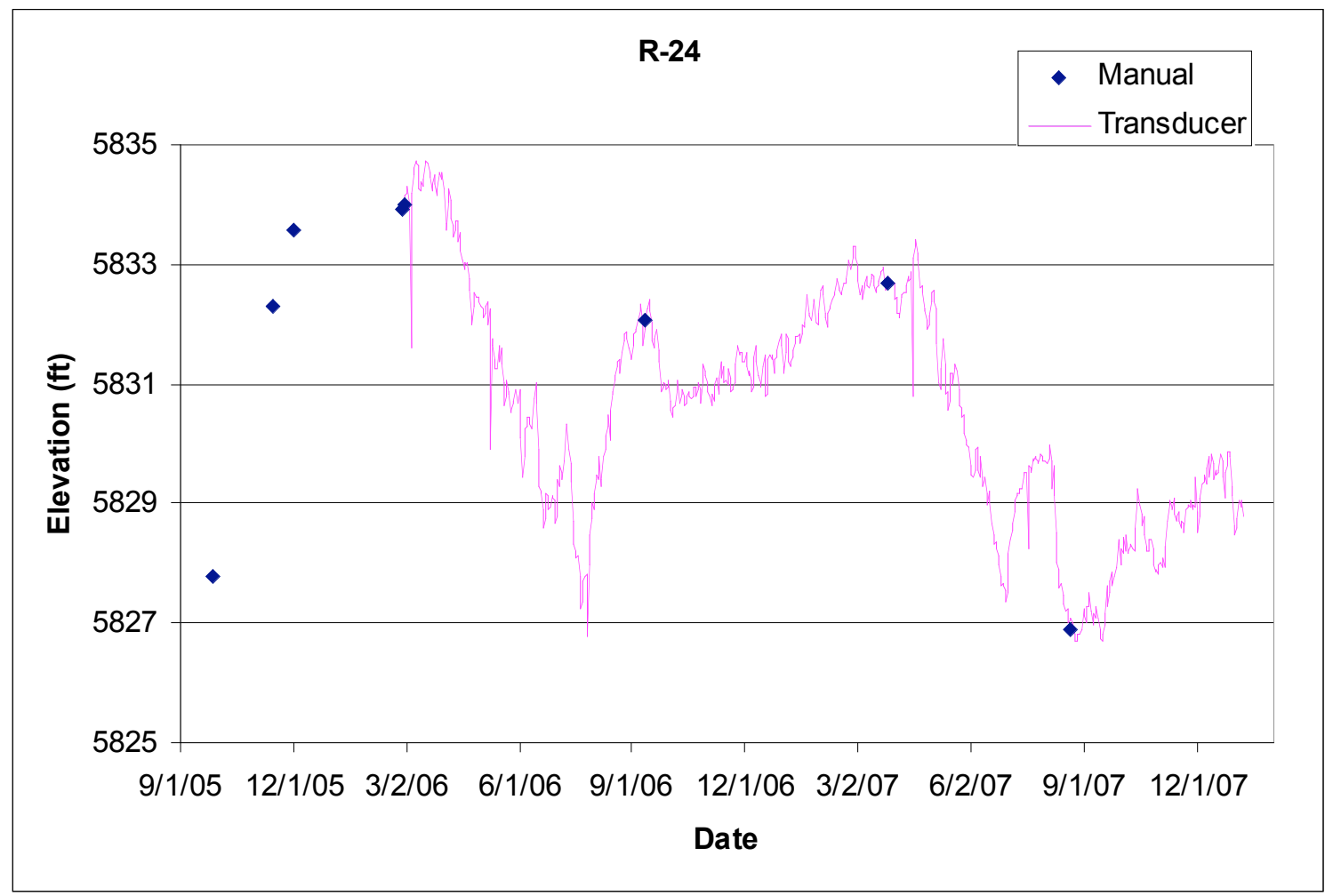




\subsection{R-25}

Location: R-25 is located at TA-16 within the Cañon de Valle watershed.

Completion Type: Multiple completion, four screens in intermediate zones, and five screens in the regional aquifer. Screens 3 and 9 were damaged during installation and are not reliable for water level monitoring. Screen 5 straddles the regional water table.

Period of Record: Westbay ${ }^{\circledR}$ installed October 3, 2000, transducers installed February 26, 2001, and between sampling events through 2002. Transducers installed again June 2, 2005; data through 2007.

Remarks: Recurring problems with the transducer cables from 2001 to 2005 caused loss of data. The transducer cables were rebuilt in 2005. Screens 1 and 2 appear to be in the same intermediate zone. Screen 4 appears to be in a separate intermediate zone. The water level at screen 5 , the top of the regional aquifer, declines during low flow sampling and recovers slowly. There is no significant response to atmospheric pressure at any of the screens. Intermediate screens 1, 2, and 4 responded to snowmelt runoff in 2005 and 2007. The regional aquifer screens do not indicate an apparent response to supply well pumping.

\begin{tabular}{|c|c|c|c|c|c|c|c|c|c|c|c|c|}
\hline \multicolumn{13}{|c|}{ Measurement and Sampling Ports in R-25 } \\
\hline $\begin{array}{c}\text { Screen } \\
\text { Zone }\end{array}$ & $\begin{array}{c}\text { Screen } \\
\text { Top } \\
\text { Depth (ft) }\end{array}$ & \begin{tabular}{|c} 
screen \\
Bottom \\
Depth \\
(ft)
\end{tabular} & $\begin{array}{c}\text { Screen } \\
\text { Top Elev } \\
\text { (ft) }\end{array}$ & $\begin{array}{l}\text { Screen } \\
\text { Bottom } \\
\text { Elev (ft) }\end{array}$ & $\begin{array}{l}\text { Screen } \\
\text { Length } \\
\text { (ft) }\end{array}$ & Port & $\begin{array}{c}\text { Port } \\
\text { Depth (ft) }\end{array}$ & $\begin{array}{c}\text { Port } \\
\text { Elevation } \\
\text { (tt) }\end{array}$ & \begin{tabular}{|c|} 
Distance \\
from \\
Bottom of \\
Screen (ft)
\end{tabular} & \begin{tabular}{|c} 
Sump \\
Volume \\
above \\
Port (L)
\end{tabular} & \begin{tabular}{|c} 
Sump \\
Volume \\
Total (L)
\end{tabular} & Comment \\
\hline \multirow{3}{*}{1} & \multirow{3}{*}{737.6} & \multirow{3}{*}{758.4} & \multirow{3}{*}{6778.5} & \multirow{3}{*}{6757.7} & \multirow{3}{*}{20.8} & MP1A & 754.8 & 6761.3 & 3.6 & & & Within Screen, Intermediate Zone \\
\hline & & & & & & PP1 & 760.1 & 6756.0 & -1.7 & 4.9 & & Below Screen \\
\hline & & & & & & MP1B & 765.8 & 6750.3 & -7.4 & 21.4 & 31.9 & Below Screen \\
\hline \multirow{3}{*}{2} & \multirow{3}{*}{882.6} & \multirow{3}{*}{893.4} & \multirow{3}{*}{6633.5} & \multirow{3}{*}{6622.7} & \multirow{3}{*}{10.8} & MP2A & 891.8 & 6624.3 & 1.6 & & & Within Screen, Intermediate Zone \\
\hline & & & & & & PP2 & 897.2 & 6618.9 & -3.8 & 11.0 & & Below Screen \\
\hline & & & & & & MP2B & 902.8 & 6613.3 & -9.4 & 27.2 & 37.9 & Below Screen \\
\hline \multirow{3}{*}{3} & \multirow{3}{*}{1054.6} & \multirow{3}{*}{1064.6} & \multirow{3}{*}{6461.5} & \multirow{3}{*}{6451.5} & \multirow{3}{*}{10.0} & MP3A & 1063.4 & 6452.7 & 1.2 & & & Within Screen, Intermediate Zone \\
\hline & & & & & & PP3 & 1068.8 & 6447.3 & -4.2 & 12.2 & & Below Screen, Screen Damaged \\
\hline & & & & & & MP3B & 1084.2 & 6431.9 & -19.6 & 56.8 & 72.4 & Below Screen \\
\hline \multirow{3}{*}{4} & \multirow{3}{*}{1184.6} & \multirow{3}{*}{1194.6} & \multirow{3}{*}{6331.5} & \multirow{3}{*}{6321.5} & \multirow{3}{*}{10.0} & MP4A & 1192.4 & 6323.7 & 2.2 & & & Within Screen, Intermediate Zone \\
\hline & & & & & & PP4 & 1197.8 & 6318.3 & -3.2 & 9.3 & & Below Screen \\
\hline & & & & & & MP4B & 1203.4 & 6312.7 & -8.8 & 25.5 & 36.5 & Below Screen \\
\hline \multirow{3}{*}{5} & \multirow{3}{*}{1294.7} & \multirow{3}{*}{1304.7} & \multirow{3}{*}{6221.4} & & & MP5A & 1303.4 & 6212.7 & 1.3 & & & Within Screen, Regional Aquifer \\
\hline & & & & 6211.4 & 10.0 & PP5 & 1308.8 & 6207.3 & -4.1 & 11.9 & & Below Screen \\
\hline & & & & & & MP5B & 1314.4 & 6201.7 & -9.7 & 28.1 & 39.1 & Below Screen \\
\hline & & & & & & MP6A & 1406.3 & 6109.8 & 8.4 & & & Within Screen \\
\hline 6 & 1404.7 & 1414.7 & 6111.4 & 6101.4 & 10.0 & PP6 & 1411.7 & 6104.4 & 3 & & & Within Screen \\
\hline & & & & & & MP6B & 1417.3 & 6098.8 & -2.6 & 7.5 & 18.5 & Below Screen \\
\hline & & & & & & MP7A & 1606.0 & 5910.1 & 8.7 & & & Within Screen \\
\hline 7 & 1604.7 & 1614.7 & 5911.4 & 5901.4 & 10.0 & PP7 & 1611.4 & 5904.7 & 3.3 & & & Within Screen \\
\hline & & & & & & MP7B & 1617.1 & 5899.0 & -2.4 & 7.0 & 17.7 & Below Screen \\
\hline & & & & & & MP8A & 1796.0 & 5720.1 & 8.7 & & & Within Screen \\
\hline 8 & 1794.7 & 1804.7 & 5721.4 & 5711.4 & 10.0 & PP8 & 1801.4 & 5714.7 & 3.3 & & & Within Screen \\
\hline & & & & & & MP8B & 1807.0 & 5709.1 & -2.3 & 6.7 & 17.4 & Below Screen \\
\hline 9 & 1894.7 & 1904.7 & $\overline{5621.4}$ & $\overline{5611.4}$ & 10.0 & MP9 & 1825.1 & 5691.0 & 79.6 & & & Screen 9 blocked by sediment \\
\hline
\end{tabular}

Note: R-25 Brass Cap Ground Elevation: $7516.1 \mathrm{ft}$; all measurements are from this elevation;

$\mathrm{MP}=$ Monitor Port; PP = Pump Port; Monitor Ports shown in bold are instrumented ports 

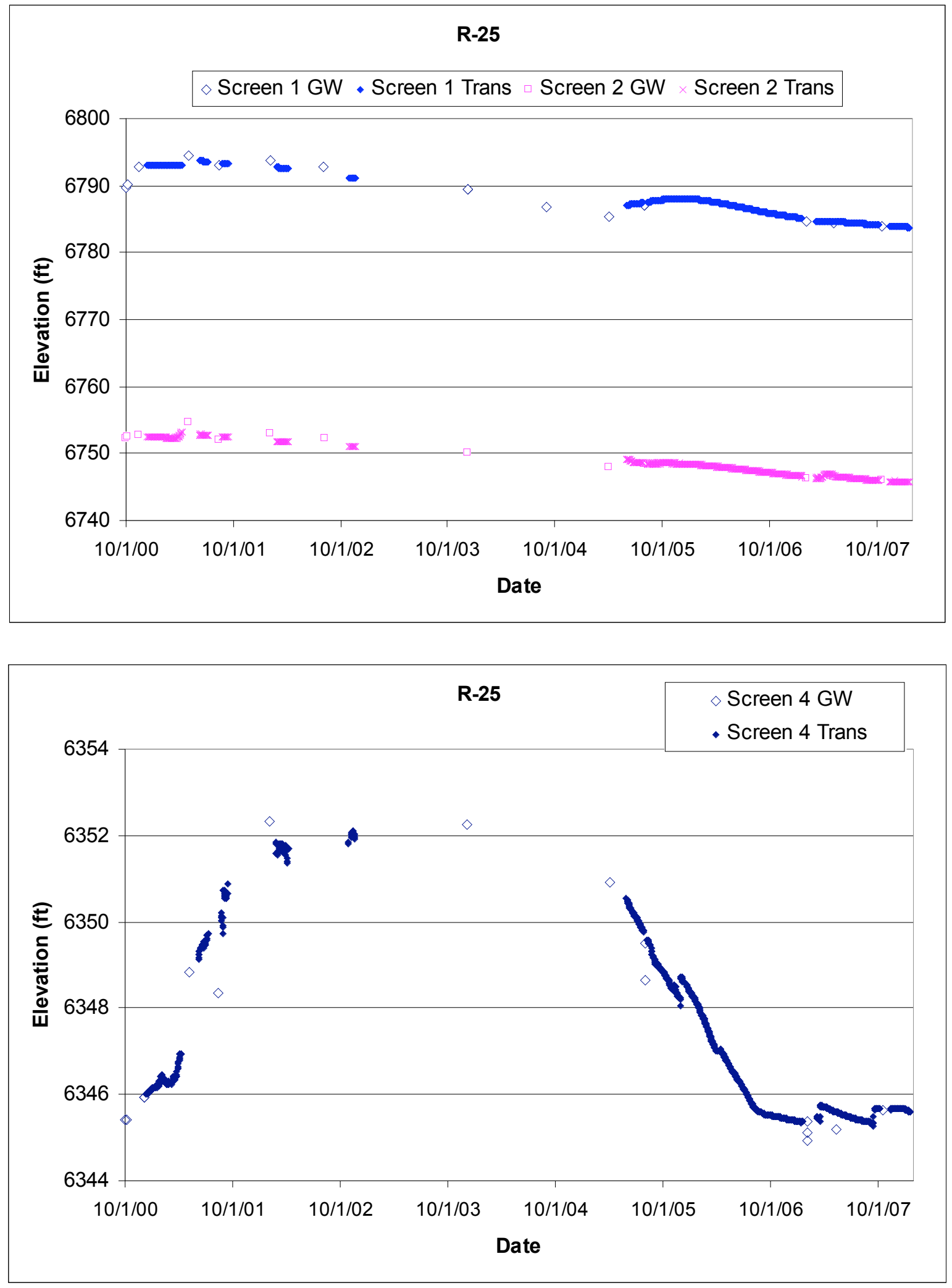


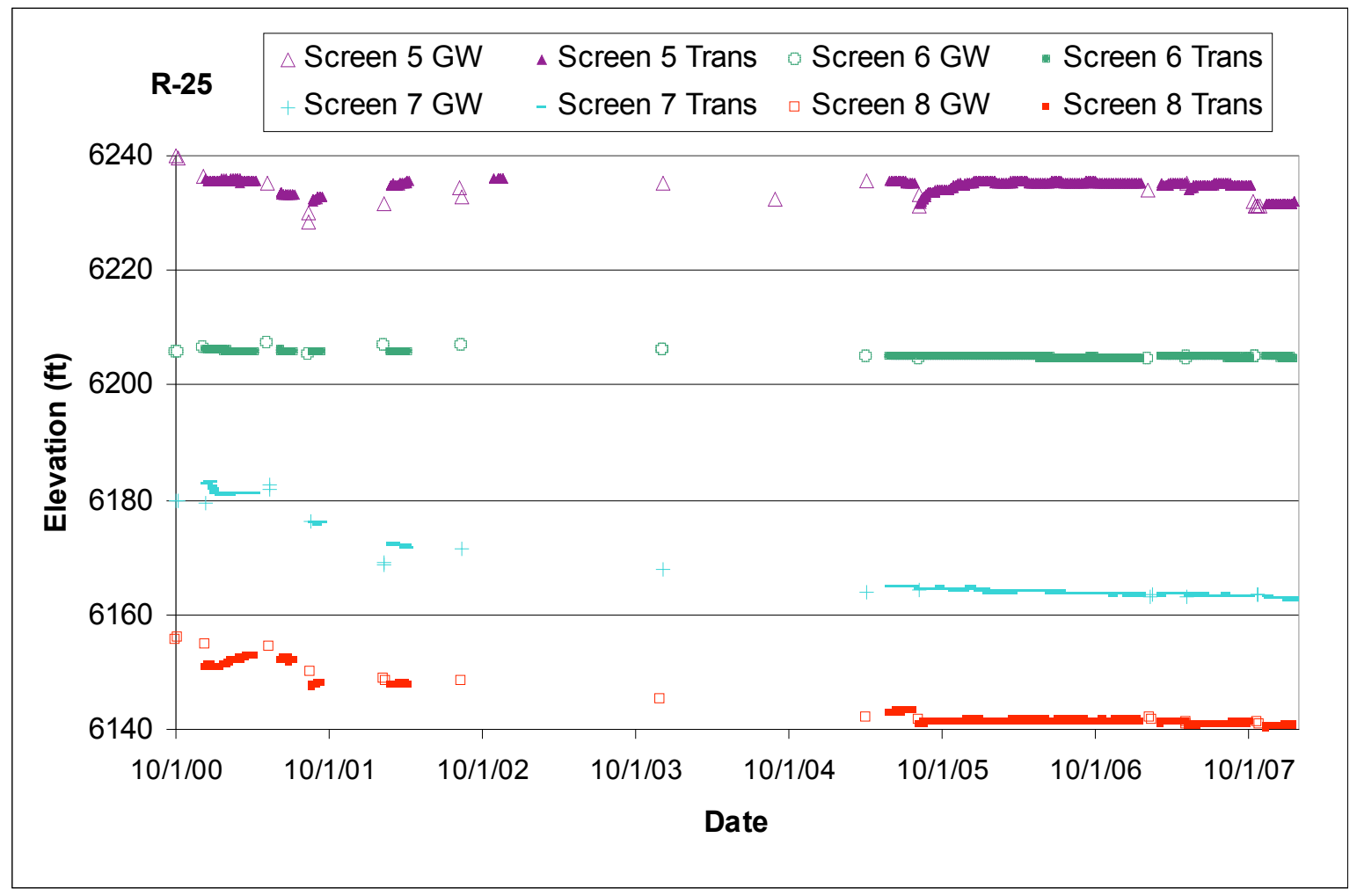




\subsection{R-26}

Location: R-26 is located at the western LANL boundary between Cañon de Valle and Water Canyon. Completion Type: Multiple completion, screen 1 is in an intermediate zone, and screen 2 is within the regional aquifer. The top of screen 2 is about $319 \mathrm{ft}$ below the regional water table.

Period of Record: Westbay ${ }^{\circledR}$ installed July 18, 2004, transducers installed July 29, 2005, transducer data through 2007.

Remarks: Screen 2 is in a tight zone or improperly completed zone. Sampling attempts at MP2A caused plugging of the port and sampler with bentonite; the transducers were installed in the B ports on November 3, 2005; water level data from screen 2 at port MP2B appear valid with some questions as to validity pending additional data and review. There is no apparent response to supply well pumping at R-26.

\begin{tabular}{|c|c|c|c|c|c|c|c|c|c|c|c|}
\hline \multicolumn{12}{|c|}{ R-26 Measurement and Sampling Ports } \\
\hline Zone & $\begin{array}{l}\text { Screen } \\
\text { Top } \\
\text { Depth } \\
\text { (ft) }\end{array}$ & $\begin{array}{l}\text { Screen } \\
\text { Bottom } \\
\text { Depth } \\
\text { (ft) }\end{array}$ & $\begin{array}{c}\text { Screen } \\
\text { Top Elev } \\
\text { (ft) }\end{array}$ & $\begin{array}{l}\text { Screen } \\
\text { Bottom } \\
\text { Elev (ft) }\end{array}$ & $\begin{array}{l}\text { Screen } \\
\text { Length } \\
\text { (ft) }\end{array}$ & Port & $\begin{array}{c}\text { Port } \\
\text { Depth (ft) }\end{array}$ & $\begin{array}{c}\text { Port } \\
\text { Elevation } \\
\text { (ft) }\end{array}$ & $\begin{array}{l}\text { Distance } \\
\text { from } \\
\text { Bottom of } \\
\text { Screen (ft) }\end{array}$ & $\begin{array}{c}\text { Sump } \\
\text { Volume } \\
\text { (L) }\end{array}$ & Comment \\
\hline \multirow{3}{*}{1} & \multirow{3}{*}{651.8} & \multirow{3}{*}{669.9} & \multirow{3}{*}{6989.9} & \multirow{3}{*}{6971.8} & \multirow{3}{*}{18.1} & MP1A & 659.3 & 6982.4 & 10.6 & & Within Screen, Intermediate \\
\hline & & & & & & PP1 & 664.7 & 6977.0 & 5.2 & & Within Screen \\
\hline & & & & & & MP1B & 670.3 & 6971.4 & -0.4 & 0.8 & Below Screen \\
\hline \multirow{3}{*}{2} & \multirow{3}{*}{1421.8} & \multirow{3}{*}{1445.0} & \multirow{3}{*}{6219.9} & \multirow{3}{*}{6196.7} & \multirow{3}{*}{23.2} & MP2A & 1427.0 & 6214.7 & 18.0 & & Within Screen Regional Aquifer \\
\hline & & & & & & PP2 & 1432.4 & 6209.3 & 12.6 & & Within Screen \\
\hline & & & & & & MP2B & 1438 & 6203.7 & 7.0 & & Within Screen \\
\hline
\end{tabular}

Note: R-26 Brass Cap Ground Elevation: $7641.69 \mathrm{ft}$; all measurements are from this elevation;

$\mathrm{MP}=$ Monitor Port; $\mathrm{PP}=$ Pump Port; Monitor Ports shown in bold are instrumented ports
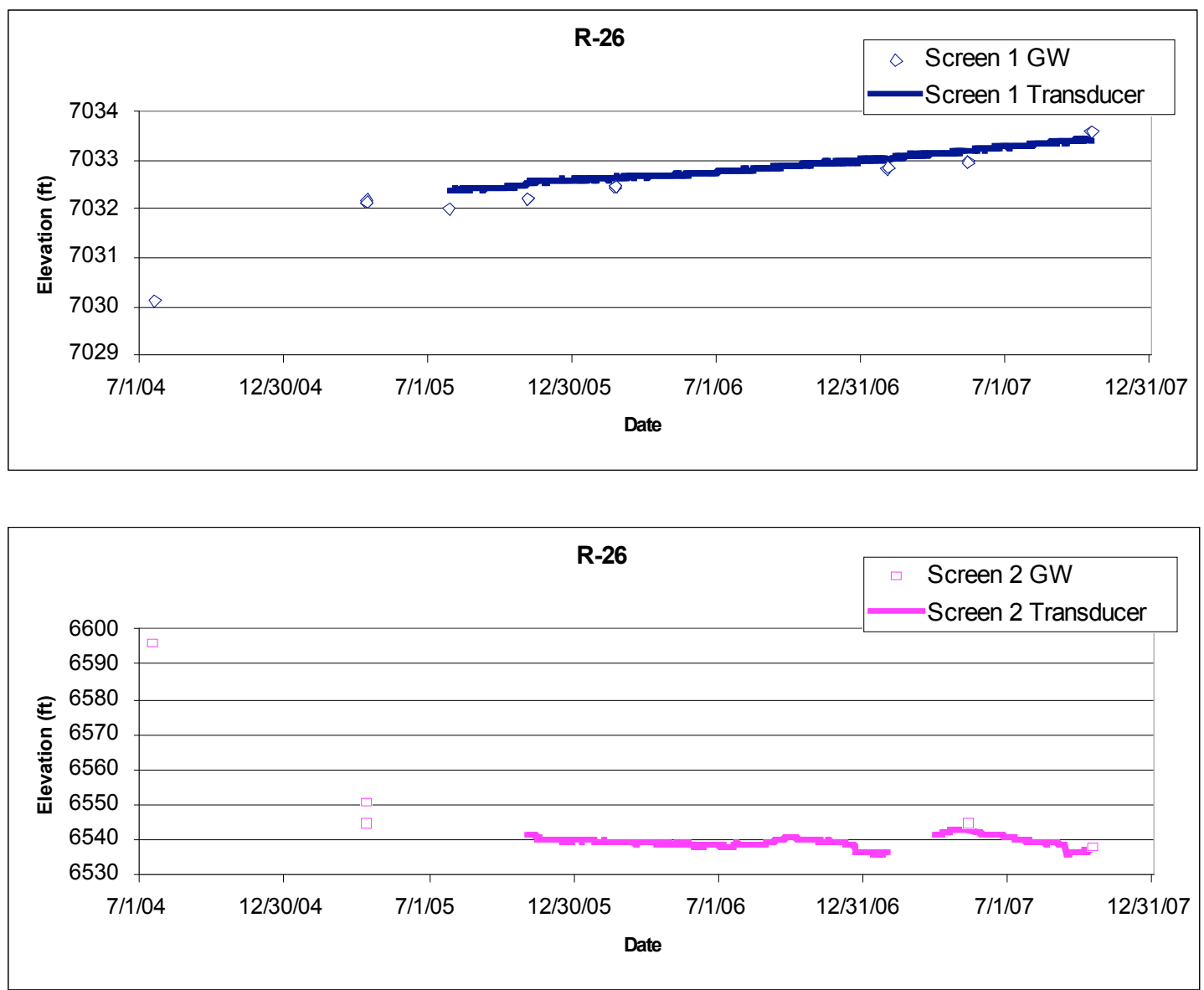


\section{$3.30 \quad$ R-27}

Location: R-27 is located in middle Water Canyon about 0.35 miles north of DT-10.

Completion Type: Single completion at the top of the regional aquifer in Puye fanglomerates. The top of the screen is about $38 \mathrm{ft}$ below the water table.

Period of Record: Well completed November 2005, transducer installed September 29, 2006, transducer data through 2007.

Remarks: R-27 is installed to a depth of $878.7 \mathrm{ft}$, about $60 \mathrm{ft}$ into the regional aquifer. The well is $100 \%$ barometrically efficient; the aquifer does not show a response to atmospheric pressure fluctuations. Although there is about a $0.3-\mathrm{ft}$ seasonal response, there is no apparent response to a specific supply well.

\begin{tabular}{|c|c|c|c|c|c|c|c|c|c|c|c|c|c|}
\hline \multirow[b]{2}{*}{ Zone } & \multicolumn{13}{|c|}{ R-27 Construction Information } \\
\hline & $\begin{array}{c}\text { Screen } \\
\text { Top } \\
\text { Depth (ft) }\end{array}$ & $\begin{array}{c}\text { Screen } \\
\text { Bottom } \\
\text { Depth } \\
\text { (ft) }\end{array}$ & $\begin{array}{c}\text { Screen } \\
\text { Top } \\
\text { Elev (ft) }\end{array}$ & $\begin{array}{l}\text { Screen } \\
\text { Bottom } \\
\text { Elev (ft) }\end{array}$ & $\begin{array}{l}\text { Screen } \\
\text { Length } \\
\text { (ft) }\end{array}$ & $\begin{array}{l}\text { Pump } \\
\text { Intake } \\
\text { Depth } \\
\text { (ft) }\end{array}$ & $\begin{array}{c}\text { Pump } \\
\text { Intake } \\
\text { Elevation } \\
\text { (ft) }\end{array}$ & $\begin{array}{c}\text { Depth to } \\
\text { Top of } \\
\text { Sump (ft) }\end{array}$ & $\begin{array}{l}\text { Top of } \\
\text { Sump } \\
\text { Elevation } \\
\text { (ft) }\end{array}$ & $\begin{array}{c}\text { Depth to } \\
\text { Sump } \\
\text { Bottom (ft) }\end{array}$ & $\begin{array}{c}\text { Sump } \\
\text { Length } \\
\text { (ft) }\end{array}$ & $\begin{array}{l}\text { Sump } \\
\text { Volume } \\
\text { (L) }\end{array}$ & Comment \\
\hline 1 & 852.0 & 875.0 & 5861.7 & 5838.7 & 23.0 & 847 & 5866.7 & 875.0 & 5838.7 & 878.7 & 3.7 & 11.6 & Regional Aquifer \\
\hline
\end{tabular}

Note: Brass Cap Ground Elevation: $6713.72 \mathrm{ft}$; all measurements are from this elevation

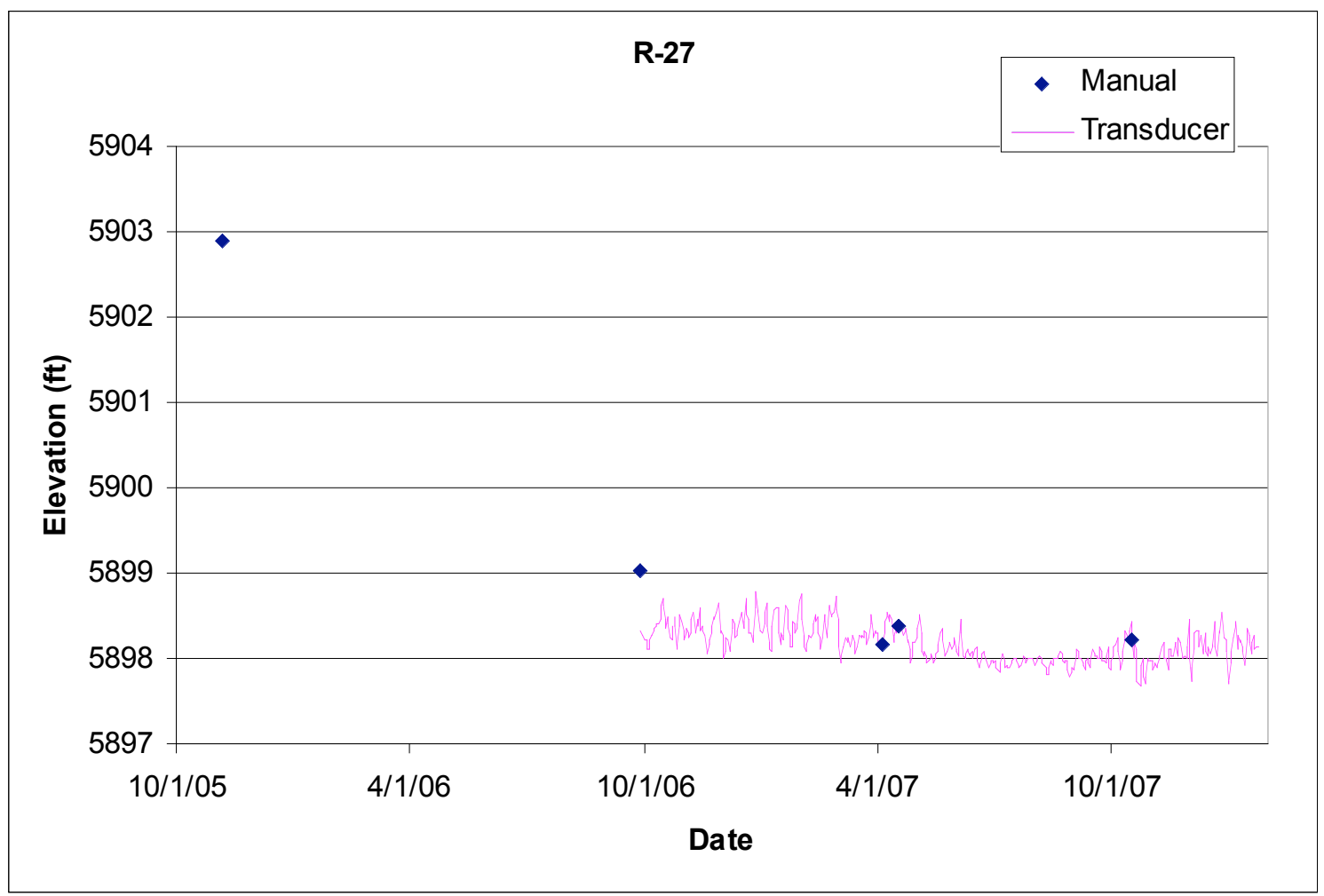




\subsection{R-28}

Location: R-28 is located in lower Mortandad Canyon about $1400 \mathrm{ft}$ southwest of R-11 in Sandia Canyon.

Completion Type: Single completion at the top of the regional aquifer. The top of the screen is about $45 \mathrm{ft}$ below the water table.

Period of Record: Well completed December 2003, transducer installed January 7, 2005, data through 2007.

Remarks: R-28 installed to a depth of $980.3 \mathrm{ft}$, about $100 \mathrm{ft}$ into the regional aquifer. The well is $100 \%$ barometrically efficient; the aquifer does not respond to atmospheric pressure fluctuations. R28 exhibits a seasonal response to supply well pumping and responds primarily to pumping at PM-4 and PM-2 and possibly to PM-5, but apparently does not respond significantly to pumping at nearby supply well PM-3.

\begin{tabular}{|c|c|c|c|c|c|c|c|c|c|c|c|c|c|}
\hline \multicolumn{14}{|c|}{ R-28 Construction Information } \\
\hline Zone & $\begin{array}{c}\text { Screen } \\
\text { Top } \\
\text { Depth (ft) }\end{array}$ & $\begin{array}{l}\text { Screen } \\
\text { Bottom } \\
\text { Depth } \\
\text { (ft) }\end{array}$ & $\begin{array}{c}\text { Screen } \\
\text { Top } \\
\text { Elev (ft) }\end{array}$ & $\begin{array}{l}\text { Screen } \\
\text { Bottom } \\
\text { Elev (ft) }\end{array}$ & $\begin{array}{l}\text { Screen } \\
\text { Length } \\
\text { (ft) }\end{array}$ & $\begin{array}{l}\text { Pump } \\
\text { Intake } \\
\text { Depth } \\
\text { (ft) }\end{array}$ & $\begin{array}{l}\text { Pump } \\
\text { Intake } \\
\text { Elevation } \\
\text { (ft) }\end{array}$ & $\begin{array}{c}\text { Depth to } \\
\text { Top of } \\
\text { Sump (ft) }\end{array}$ & $\begin{array}{l}\text { Top of } \\
\text { Sump } \\
\text { Elevation } \\
\text { (ft) }\end{array}$ & $\begin{array}{l}\text { Depth to } \\
\text { Sump } \\
\text { Bottom (ft) }\end{array}$ & $\begin{array}{l}\text { Sump } \\
\text { Length } \\
\text { (ft) }\end{array}$ & $\begin{array}{l}\text { Sump } \\
\text { Volume } \\
\text { (L) }\end{array}$ & Comment \\
\hline & 934.3 & 958.1 & 5794.3 & 5770.5 & 23.8 & 929.6 & 5799.0 & 958.1 & 5770.5 & 980.3 & 22.2 & 68.2 & Regional Aquifer \\
\hline
\end{tabular}

Note: R-28 Brass Cap Ground Elevation: $6728.61 \mathrm{ft}$; all measurements are from this elevation

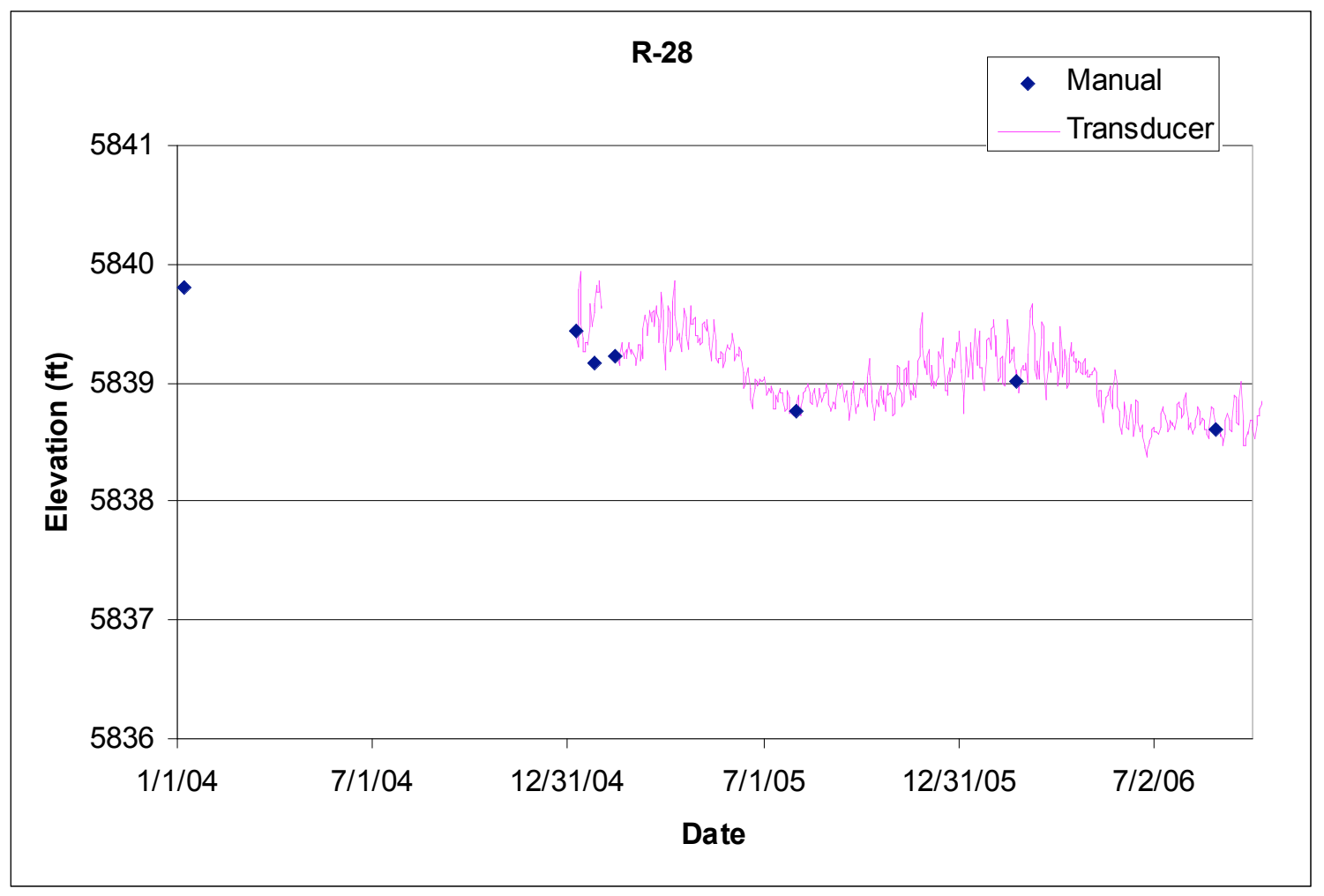




\subsection{R-31}

Location: R-31 is located in the southern part of LANL in the north Ancho Canyon tributary.

Completion Type: Multiple completion, one screen in an intermediate zone, and four screens in the regional aquifer. The intermediate screen has been dry since Westbay ${ }^{\circledR}$ installation.

Period of Record: Westbay ${ }^{\circledR}$ installed April 7, 2000, transducers installed May 4, 2000, transducer data through 2007.

Remarks: Screen 5 has the highest head values, followed by screen 4 and screen 2; screen 3 has the lowest head values. Screens 2 and 3 have $80 \%$ and $100 \%$ response to atmospheric pressure fluctuations, respectively, while screens 3 and 4 have about $45 \%$ response. Screens 4 and 5 show seasonal responses to supply well pumping that coincide with the non-pumping water levels at PM-2.

\begin{tabular}{|c|c|c|c|c|c|c|c|c|c|c|c|}
\hline \multicolumn{12}{|c|}{ R-31 Port Data } \\
\hline Zone & $\begin{array}{c}\text { Screen } \\
\text { Top } \\
\text { Depth (ft) }\end{array}$ & $\begin{array}{c}\text { Screen } \\
\text { Bottom } \\
\text { Depth (ft) }\end{array}$ & $\begin{array}{c}\text { Screen } \\
\text { Top Elev } \\
\text { (ft) }\end{array}$ & $\begin{array}{l}\text { Screen } \\
\text { Bottom } \\
\text { Elev (ft) }\end{array}$ & $\begin{array}{l}\text { Screen } \\
\text { Length } \\
\text { (ft) }\end{array}$ & Port & $\begin{array}{l}\text { Port } \\
\text { Depth } \\
\text { (ft) }\end{array}$ & $\begin{array}{c}\text { Port } \\
\text { Elevation } \\
\text { (ft) }\end{array}$ & $\begin{array}{c}\text { Distance from } \\
\text { Bottom of } \\
\text { Screen (ft) }\end{array}$ & $\begin{array}{c}\text { Sump } \\
\text { Volume } \\
\text { (L) }\end{array}$ & Comment \\
\hline \multirow{3}{*}{1} & \multirow{3}{*}{439.1} & \multirow{3}{*}{454.4} & \multirow{3}{*}{5923.4} & \multirow{3}{*}{5908.1} & \multirow{3}{*}{15.3} & MP1A & 453.8 & 5908.7 & 0.6 & & Intermediate Zone (Dry) \\
\hline & & & & & & PP1 & 459.2 & 5903.3 & -4.8 & 13.9 & Below screen \\
\hline & & & & & & MP1B & 464.8 & 5897.7 & -10.4 & 30.1 & Below screen \\
\hline \multirow{4}{*}{2} & \multirow{4}{*}{515.0} & \multirow{4}{*}{545.7} & \multirow{4}{*}{5847.5} & \multirow{4}{*}{5816.8} & \multirow{4}{*}{30.7} & MP2A & 532.2 & 5830.3 & 13.5 & & Top of Regional Aquifer \\
\hline & & & & & & MP2B & 542.5 & 5820.0 & 3.2 & & \\
\hline & & & & & & PP2 & 547.9 & 5814.6 & -2.2 & 6.4 & Below screen \\
\hline & & & & & & MP2C & 553.5 & 5809.0 & -7.8 & 22.6 & Below screen \\
\hline \multirow{3}{*}{3} & \multirow{3}{*}{666.3} & \multirow{3}{*}{676.3} & \multirow{3}{*}{5696.2} & \multirow{3}{*}{5686.2} & \multirow{3}{*}{10} & MP3A & 670.3 & 5692.2 & 6.0 & & \\
\hline & & & & & & PP3 & 675.6 & 5686.9 & 0.7 & & \\
\hline & & & & & & MP3B & 681.3 & 5681.2 & -5.0 & 14.5 & Below screen \\
\hline \multirow{3}{*}{4} & \multirow{3}{*}{826.6} & \multirow{3}{*}{836.6} & \multirow{3}{*}{5535.9} & \multirow{3}{*}{5525.9} & \multirow{3}{*}{10} & MP4A & 830.9 & 5531.6 & 5.7 & & \\
\hline & & & & & & PP4 & 836.3 & 5526.2 & 0.3 & & \\
\hline & & & & & & MP4B & 841.9 & 5520.6 & -5.3 & 15.3 & Below screen \\
\hline \multirow{3}{*}{5} & \multirow{3}{*}{1007.1} & \multirow{3}{*}{1017.1} & \multirow{3}{*}{5355.4} & \multirow{3}{*}{5345.4} & \multirow{3}{*}{10} & MP5A & 1011.3 & 5351.2 & 5.8 & & \\
\hline & & & & & & PP5 & 1016.7 & 5345.8 & 0.4 & & \\
\hline & & & & & & MP5B & 1022.3 & 5340.2 & -5.2 & 15.1 & Below screen \\
\hline
\end{tabular}

Brass Cap Elevation: $6362.5 \mathrm{ft}$; all measurements are from this elevation;

$\mathrm{MP}=$ measurement port; $\mathrm{PP}=$ pumping port

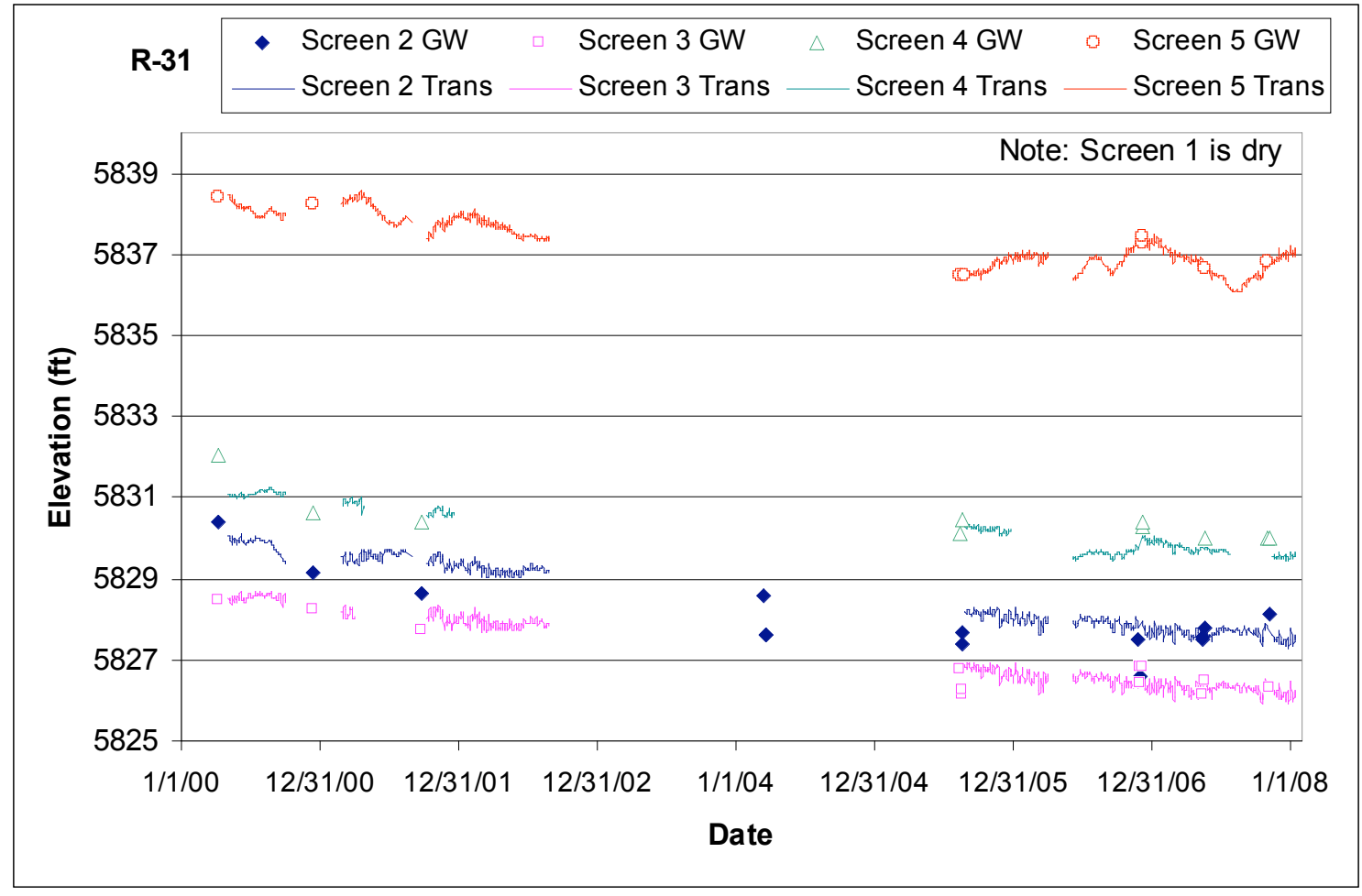




\subsection{R-32}

Location: R-32 is located in lower Pajarito Canyon about 1 mile east of supply well PM-2.

Completion Type: Multiple completion, three screens in the regional aquifer. The top of screen 1 is about $90 \mathrm{ft}$ below the water table.

Period of Record: Westbay ${ }^{\circledR}$ installed December 14, 2002, transducers installed January 21, 2003 , transducer data through August 2007. The Westbay system was removed on September 18, 2007, and the well was rehabilitated to a single completion well at screen 1 in September 2007. A submersible pump was installed in December 2007 and a new transducer was installed at screen 1 in February 2008.

Remarks: Screens 2 and 3 have nearly identical head values and respond to pumping supply wells PM-2 and PM-4. Screen 1 apparently responded to long-term pumping of PM-4 in 2003, but vaguely to aquifer test pumping at PM-2 in 2004 and PM-4 in 2005. Screens 2 and 3 responded to the PM-2 aquifer test in January 2003 (McLin 2005), to the PM-4 aquifer test in January 2005 (McLin 2006), and to PM-4 pumping in June 2006 and July 2007.

\begin{tabular}{|c|c|c|c|c|c|c|c|c|c|c|c|}
\hline \multicolumn{12}{|c|}{ R-32 Port Data } \\
\hline Screen & $\begin{array}{l}\text { Screen } \\
\text { Top } \\
\text { Depth } \\
\text { (ft) }\end{array}$ & $\begin{array}{l}\text { Screen } \\
\text { Bottom } \\
\text { Depth } \\
\text { (ft) }\end{array}$ & $\begin{array}{l}\text { Screen } \\
\text { Top } \\
\text { Elev } \\
\text { (ft) }\end{array}$ & $\begin{array}{l}\text { Screen } \\
\text { Bottom } \\
\text { Elev (ft) }\end{array}$ & $\begin{array}{c}\text { Screen } \\
\text { Length } \\
\text { (ft) }\end{array}$ & Port & $\begin{array}{l}\text { Port } \\
\text { Depth } \\
\text { (ft) }\end{array}$ & $\begin{array}{c}\text { Port } \\
\text { Elevation } \\
\text { (ft) }\end{array}$ & $\begin{array}{l}\text { Distance } \\
\text { from } \\
\text { Bottom } \\
\text { of Screen } \\
\text { (ft) }\end{array}$ & $\begin{array}{c}\text { Sump } \\
\text { Volume } \\
\text { (L) }\end{array}$ & Comment \\
\hline \multirow{3}{*}{1} & \multirow{3}{*}{867.5} & \multirow{3}{*}{875.2} & \multirow{3}{*}{5770.1} & \multirow{3}{*}{5762.4} & \multirow{3}{*}{7.7} & MP1A & 870.9 & 5766.7 & 4.3 & & Within Screen, Regional Aquifer \\
\hline & & & & & & PP1 & 876.3 & 5761.3 & -1.1 & 2.4 & Below Screen \\
\hline & & & & & & MP1B & 881.9 & 5755.7 & -6.7 & 14.5 & Below Screen \\
\hline 2 & 931.8 & 934.9 & 5705.8 & 5702.7 & 3.1 & MP2 & 933.1 & 5704.5 & 1.8 & & Screen abandoned 9/07 \\
\hline \multirow{3}{*}{3} & \multirow{3}{*}{972.9} & \multirow{3}{*}{980.6} & \multirow{3}{*}{5657.0} & \multirow{3}{*}{5657.0} & \multirow{3}{*}{7.7} & MP3A & 976.0 & 5661.6 & 4.6 & & Screen abandoned 9/07 \\
\hline & & & & & & PP3 & 981.4 & 5656.2 & -0.8 & 1.7 & Below Screen \\
\hline & & & & & & MP3B & 987.1 & 5650.5 & -6.5 & 14.1 & Below Screen \\
\hline
\end{tabular}

Note: R-32 Brass Cap Ground Elevation: $6637.63 \mathrm{ft}$; all measurements are from this elevation;

$\mathrm{MP}=$ Monitor Port; PP = Pump Port; Monitor Ports shown in bold are instrumented ports

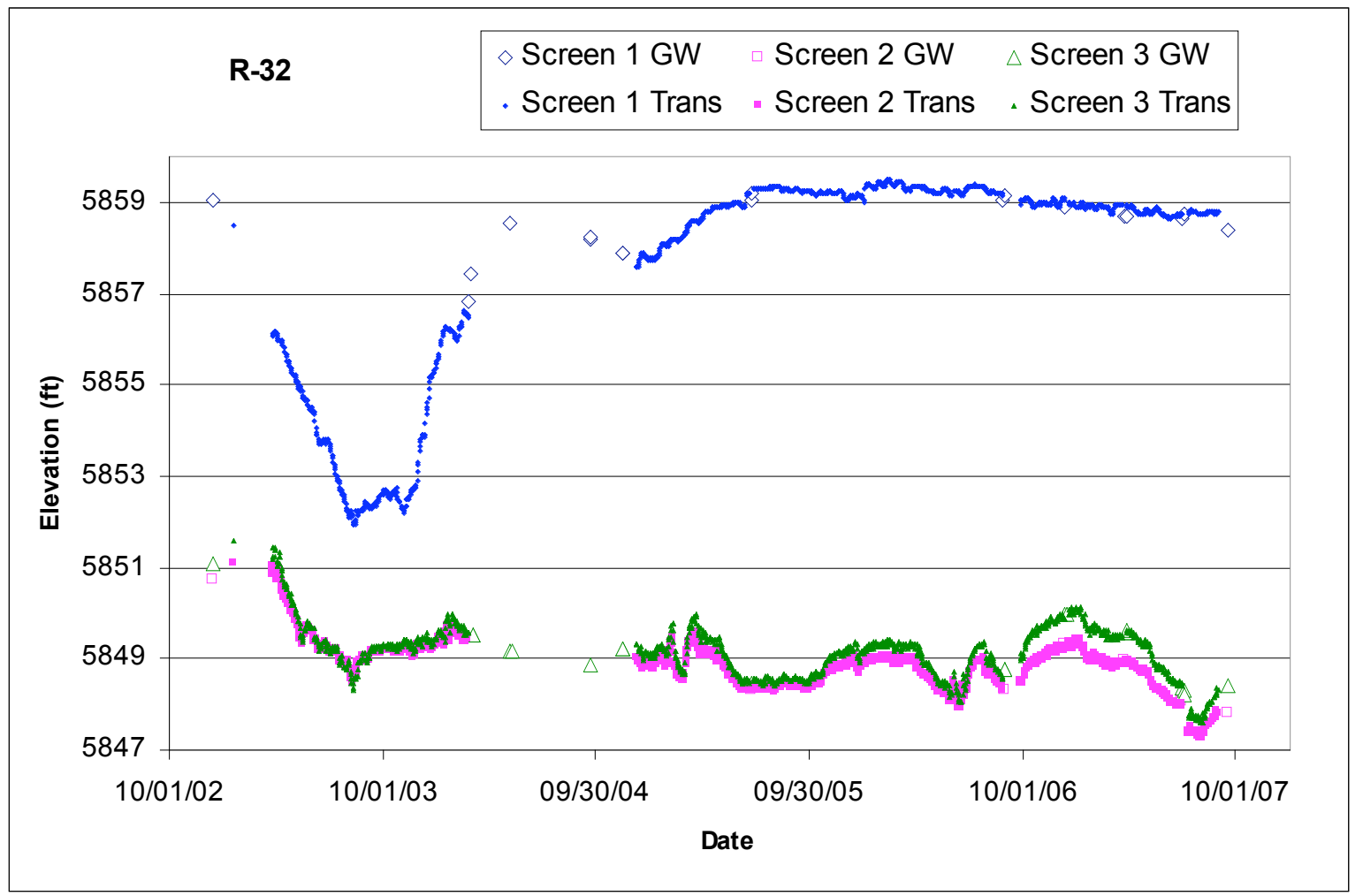




\section{$3.34 \quad R-33$}

Location: R-33 is located in lower Ten Site Canyon about $1500 \mathrm{ft}$ northeast of supply well PM-5.

Completion Type: Dual completion in the regional aquifer. Screen 1 is at the water table.

Period of Record: Well completed October 2004, transducers installed February 2005 but equipment problems hindered data collection. Transducers calibrated and the packer inflated in August 2006 and again in October 2006 with nitrogen bottle to maintain packer pressure. Water level data for screen 2 from October 24, 2006, to November 8, 2007; data for screen 1 end December 5, 2006.

Remarks: R-33 screen 1 installed at the top of the regional aquifer at a depth of about $1020 \mathrm{ft}$, and screen 2 within the regional aquifer to a depth of $1126 \mathrm{ft}$, about $140 \mathrm{ft}$ into the regional aquifer. Equipment problems occurred until October 2006 when transducers and packer equipment became fully operational. The screen 1 transducer equipment failed December 5 , 2006, no data for screen 1 after that time. The transducer equipment was removed from the well on November 8, 2007, in preparation for removing the Barcad sampling system from the well. The water level at screen 2 responds primarily to pumping of supply well PM- 5 and also to pumping of PM-4.

\begin{tabular}{|c|c|c|c|c|c|c|c|c|c|c|c|c|c|}
\hline \multirow[b]{2}{*}{ Zone } & \multicolumn{12}{|c|}{ R-33 Construction Information } & \multirow[b]{2}{*}{ Comment } \\
\hline & $\begin{array}{c}\text { Screen } \\
\text { Top Depth } \\
\text { (ft) }\end{array}$ & $\begin{array}{l}\text { Screen } \\
\text { Bottom } \\
\text { Depth } \\
\text { (ft) }\end{array}$ & $\begin{array}{c}\text { Screen } \\
\text { Top } \\
\text { Elev (ft) }\end{array}$ & $\begin{array}{l}\text { Screen } \\
\text { Bottom } \\
\text { Elev (ft) }\end{array}$ & $\begin{array}{l}\text { Screen } \\
\text { Length } \\
\text { (ft) }\end{array}$ & $\begin{array}{l}\text { Pump } \\
\text { Intake } \\
\text { Depth } \\
\text { (ft) }\end{array}$ & $\begin{array}{c}\text { Pump } \\
\text { Intake } \\
\text { Elevation } \\
\text { (ft) }\end{array}$ & $\begin{array}{l}\text { Depth to } \\
\text { Top of } \\
\text { Packerl } \\
\text { Sump (ft) }\end{array}$ & $\begin{array}{l}\text { Packerl } \\
\text { Sump } \\
\text { Elevation } \\
\text { (ft) }\end{array}$ & $\begin{array}{l}\text { Depth to } \\
\text { Sump } \\
\text { Bottom } \\
\text { (ft) }\end{array}$ & $\begin{array}{c}\text { Sump } \\
\text { Length } \\
\text { (ft) }\end{array}$ & $\begin{array}{c}\text { Sump } \\
\text { Volume } \\
\text { (L) }\end{array}$ & \\
\hline 1 & 995.5 & 1018.5 & 5857.8 & 5834.8 & 23.0 & 1011.9 & 5841.4 & 1080.8 & 5772.5 & 1080.8 & 62.3 & 194.8 & Regional Aquifer \\
\hline 2 & 1112.4 & 1122.3 & 5740.9 & 5731.0 & 9.9 & 1114.9 & 5738.4 & 1122.3 & 5731.0 & 1126.0 & 3.7 & 2.0 & Regional Aquifer \\
\hline
\end{tabular}

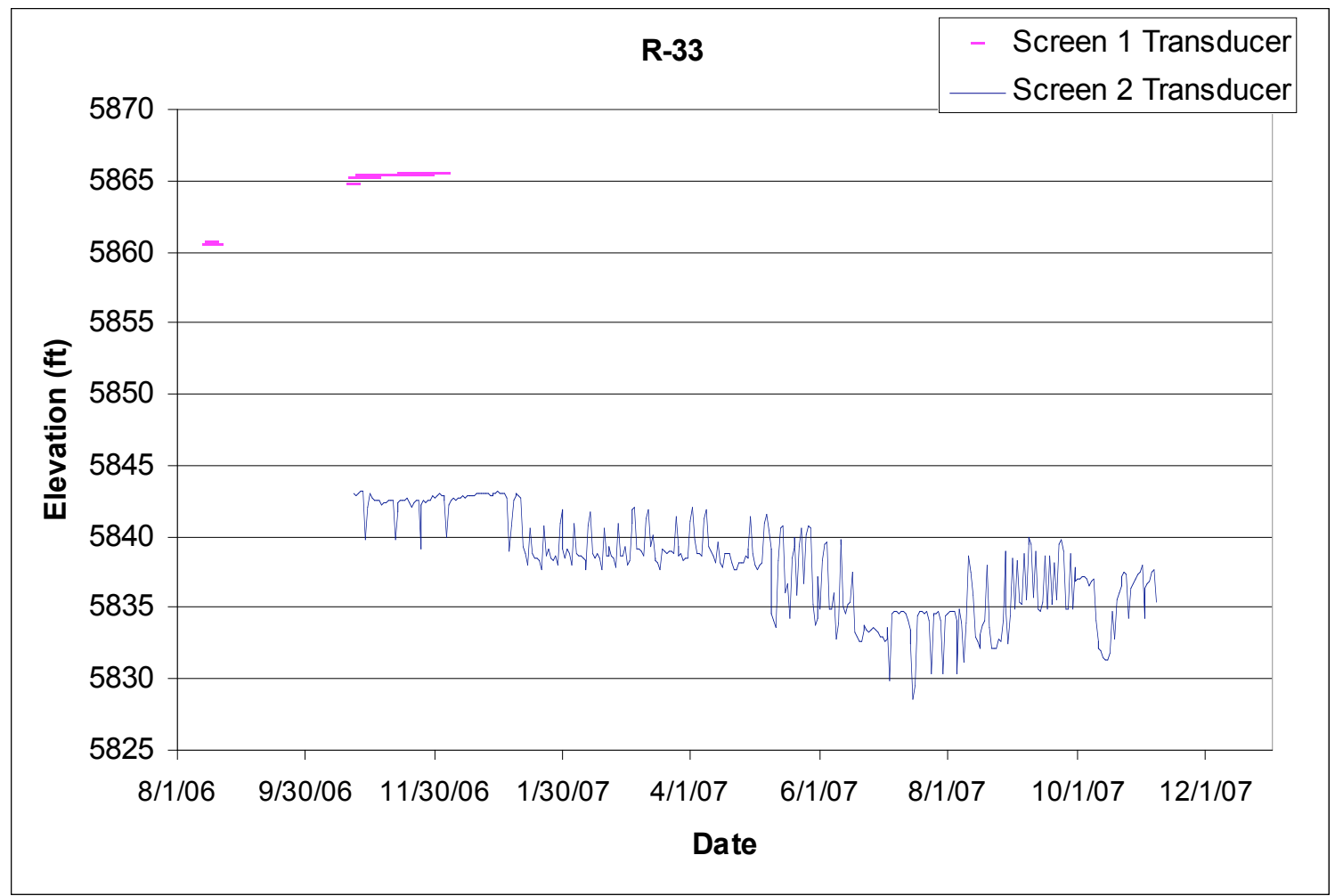




\section{$3.35 \quad$ R-34}

Location: R-34 is located in Cedro Canyon on San Ildefonso land east of LANL.

Completion Type: Single completion in the regional aquifer. The top of the screen is about $90 \mathrm{ft}$ below the water table.

Period of Record: Well completed August 2004, transducer installed January 2005, water level data through 2007.

Remarks: R-34 installed at the top of the regional aquifer at a depth of $920.7 \mathrm{ft}$, about $110 \mathrm{ft}$ into the regional aquifer. The well is $100 \%$ barometrically efficient; the aquifer does not respond to atmospheric pressure fluctuations. R-34 exhibits a seasonal response to supply well pumping but does not indicate a response to any specific supply well.

\begin{tabular}{|c|c|c|c|c|c|c|c|c|c|c|c|c|c|}
\hline \multirow[b]{2}{*}{ Zone } & \multicolumn{13}{|c|}{ R-34 Construction Information } \\
\hline & $\begin{array}{c}\text { Screen } \\
\text { Top } \\
\text { Depth (ft) }\end{array}$ & $\begin{array}{l}\text { Screen } \\
\text { Bottom } \\
\text { Depth } \\
\text { (ft) }\end{array}$ & $\begin{array}{c}\text { Screen } \\
\text { Top } \\
\text { Elev (ft) }\end{array}$ & $\begin{array}{l}\text { Screen } \\
\text { Bottom } \\
\text { Elev (ft) }\end{array}$ & $\begin{array}{c}\text { Screen } \\
\text { Length } \\
\text { (ft) }\end{array}$ & $\begin{array}{l}\text { Pump } \\
\text { Intake } \\
\text { Depth } \\
\text { (ft) }\end{array}$ & $\begin{array}{l}\text { Pump } \\
\text { Intake } \\
\text { Elevation } \\
\text { (ft) }\end{array}$ & $\begin{array}{l}\text { Depth to } \\
\text { Top of } \\
\text { Sump (ft) }\end{array}$ & $\begin{array}{c}\text { Top of } \\
\text { Sump } \\
\text { Elevation } \\
\text { (ft) }\end{array}$ & $\begin{array}{l}\text { Depth to } \\
\text { Sump } \\
\text { Bottom (ft) }\end{array}$ & $\begin{array}{l}\text { Sump } \\
\text { Length } \\
\text { (ft) }\end{array}$ & $\begin{array}{l}\text { Sump } \\
\text { Volume } \\
\text { (L) }\end{array}$ & Comment \\
\hline 1 & 883.7 & 906.6 & 5746.3 & 5723.4 & 22.9 & 881.6 & 5748.4 & 906.6 & 5723.4 & 920.7 & 14.1 & 44.1 & Regional Aquifer \\
\hline
\end{tabular}

Note: R-34 Brass Cap Ground Elevation: $6629.99 \mathrm{ft}$; all measurements are from this elevation

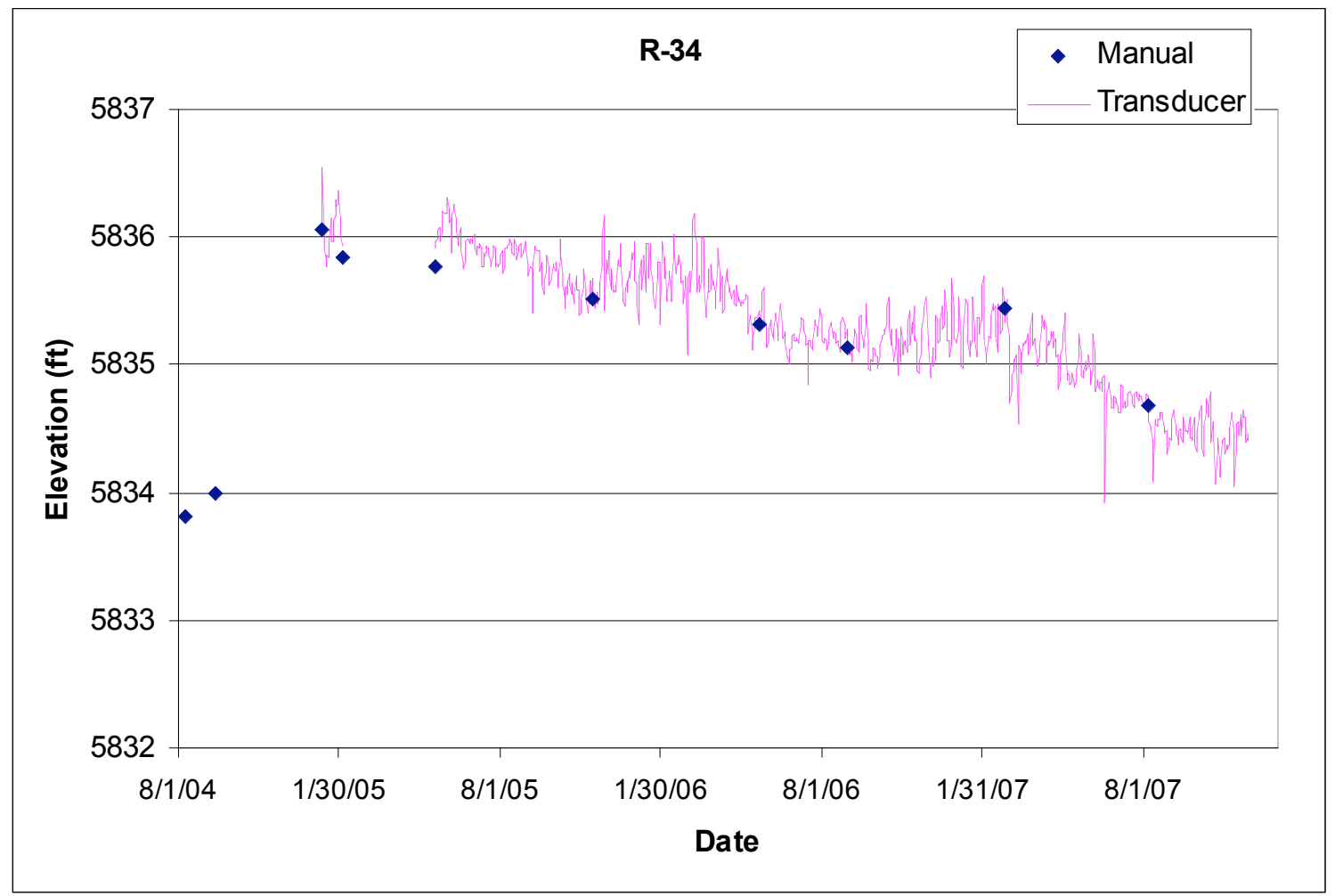




\subsection{R-35a}

Location: R-35a is located in Sandia Canyon about $340 \mathrm{ft}$ southwest of supply well PM-3.

Completion Type: Single completion in the regional aquifer. The top of the screen is about $220 \mathrm{ft}$ below the water table at the same elevation as the top of the PM-3 screen.

Period of Record: Well completed June 2007, transducer installed August 3, 2007; water level data through 2007.

Remarks: R-35a installed at a depth of $1082.2 \mathrm{ft}$, about $290 \mathrm{ft}$ into the regional aquifer. R-35a responds primarily to pumping supply well $P M-3$, about $3 \mathrm{ft}$ daily, but also shows a response to pumping supply well O-4. When the well was completed, the static water level at R-35a was about $7 \mathrm{ft}$ lower than at nearby monitoring well R-35b.

\begin{tabular}{|c|c|c|c|c|c|c|c|c|c|c|c|c|c|}
\hline \multicolumn{14}{|c|}{ R-35a Construction Information } \\
\hline Zone & $\begin{array}{c}\text { Screen } \\
\text { Top } \\
\text { Depth (ft) }\end{array}$ & $\begin{array}{c}\text { Screen } \\
\text { Bottom } \\
\text { Depth } \\
\text { (ft) }\end{array}$ & $\begin{array}{l}\text { Screen } \\
\text { Top } \\
\text { Elev (ft) }\end{array}$ & $\begin{array}{l}\text { Screen } \\
\text { Bottom } \\
\text { Elev (ft) }\end{array}$ & $\begin{array}{l}\text { Screen } \\
\text { Length } \\
\text { (ft) }\end{array}$ & $\begin{array}{l}\text { Pump } \\
\text { Intake } \\
\text { Depth } \\
\text { (ft) }\end{array}$ & $\begin{array}{c}\text { Pump } \\
\text { Intake } \\
\text { Elevation } \\
\text { (ft) }\end{array}$ & $\begin{array}{l}\text { Depth to } \\
\text { Top of } \\
\text { Sump (ft) }\end{array}$ & $\begin{array}{c}\text { Top of } \\
\text { Sump } \\
\text { Elevation } \\
\text { (ft) }\end{array}$ & $\begin{array}{l}\text { Depth to } \\
\text { Sump } \\
\text { Bottom (ft) }\end{array}$ & $\begin{array}{l}\text { Sump } \\
\text { Length } \\
\text { (ft) }\end{array}$ & $\begin{array}{c}\text { Sump } \\
\text { Volume } \\
\text { (L) }\end{array}$ & Comment \\
\hline 1 & 1013.1 & 1062.2 & 5610.0 & 5560.9 & 49.1 & 998.3 & 5624.8 & 1062.2 & 5560.9 & 1086.2 & 24.0 & 75.1 & Regional Aquifer \\
\hline
\end{tabular}

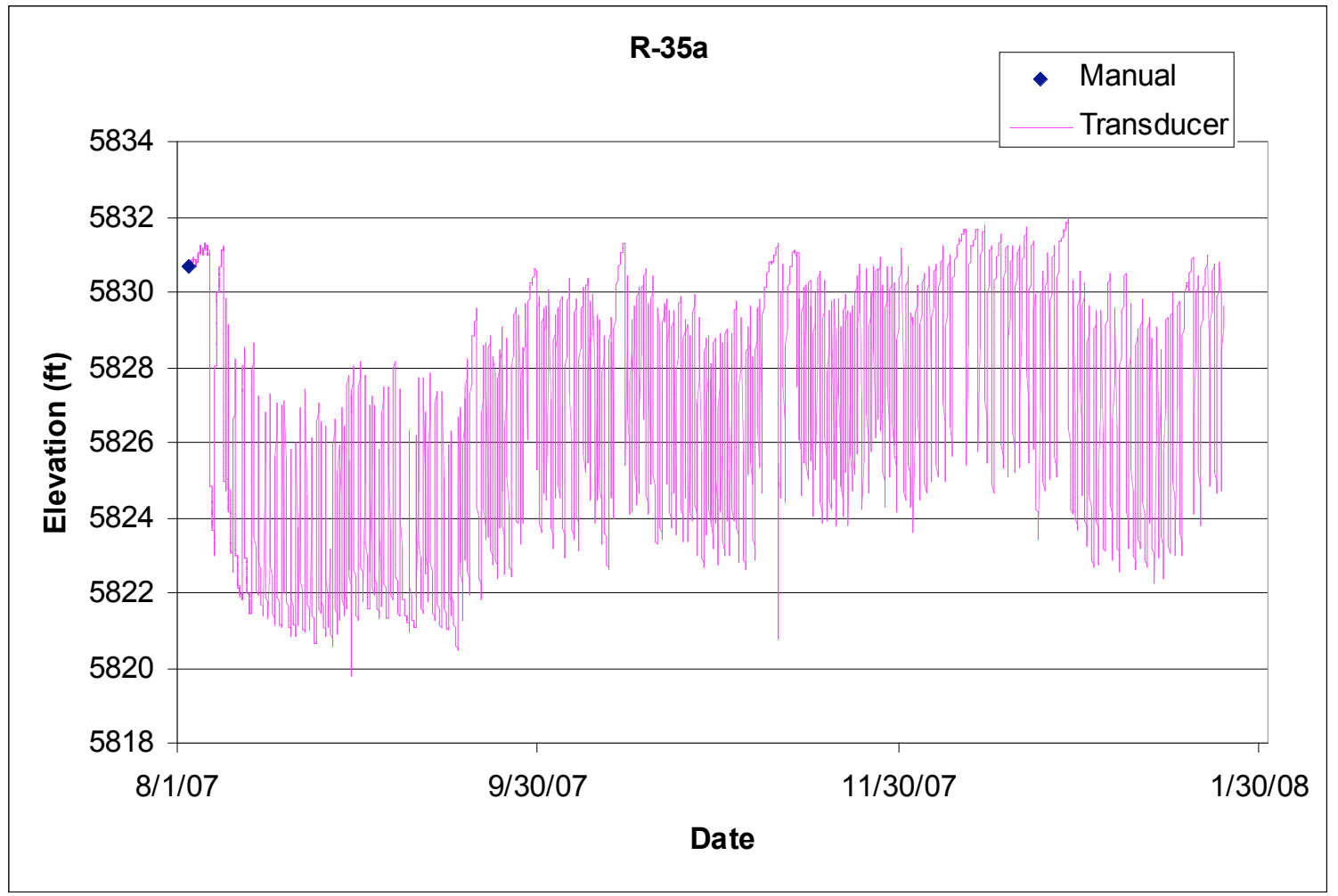

Note: Time series graph shows hourly data 


\subsection{R-35b}

Location: R-35b is located in Sandia Canyon about $90 \mathrm{ft}$ west of R-35a.

Completion Type: Single completion at the top of the regional aquifer. The top of the screen was about $37 \mathrm{ft}$ below the water table when the well was installed.

Period of Record: Well completed July 2007, transducer installed August 3, 2007; water level data through 2007.

Remarks: R-35b installed near the top of the regional aquifer at a depth of $872.2 \mathrm{ft}$, about $80 \mathrm{ft}$ into the regional aquifer. The well is $100 \%$ barometrically efficient; the aquifer does not respond to atmospheric pressure fluctuations. $\mathrm{R}-35 \mathrm{~b}$ does not indicate a response to pumping of nearby well PM-3.

\begin{tabular}{|c|c|c|c|c|c|c|c|c|c|c|c|c|c|}
\hline \multicolumn{14}{|c|}{ R-35b Construction Information } \\
\hline Zone & $\begin{array}{c}\text { Screen } \\
\text { Top } \\
\text { Depth (ft) }\end{array}$ & $\begin{array}{l}\text { Screen } \\
\text { Bottom } \\
\text { Depth } \\
\text { (ft) }\end{array}$ & $\begin{array}{c}\text { Screen } \\
\text { Top } \\
\text { Elev (ft) }\end{array}$ & $\begin{array}{l}\text { Screen } \\
\text { Bottom } \\
\text { Elev (ft) }\end{array}$ & $\begin{array}{c}\text { Screen } \\
\text { Length } \\
\text { (ft) }\end{array}$ & $\begin{array}{l}\text { Pump } \\
\text { Intake } \\
\text { Depth } \\
\text { (ft) }\end{array}$ & $\begin{array}{c}\text { Pump } \\
\text { Intake } \\
\text { Elevation } \\
\text { (ft) }\end{array}$ & $\begin{array}{c}\text { Depth to } \\
\text { Top of } \\
\text { Sump (ft) }\end{array}$ & $\begin{array}{c}\text { Top of } \\
\text { Sump } \\
\text { Elevation } \\
\text { (ft) }\end{array}$ & $\begin{array}{l}\text { Depth to } \\
\text { Sump } \\
\text { Bottom (ft) }\end{array}$ & $\begin{array}{l}\text { Sump } \\
\text { Length } \\
\text { (ft) }\end{array}$ & $\begin{array}{c}\text { Sump } \\
\text { Volume } \\
\text { (L) }\end{array}$ & Comment \\
\hline 1 & 825.4 & 848.5 & 5799.8 & 5776.7 & 23.1 & 832.7 & 5792.5 & 848.5 & 5776.7 & 872.2 & 23.7 & 74.1 & Regional Aquifer \\
\hline
\end{tabular}

Note: Brass Cap Ground Elevation: $6625.21 \mathrm{ft}$ all measurements are from this elevation

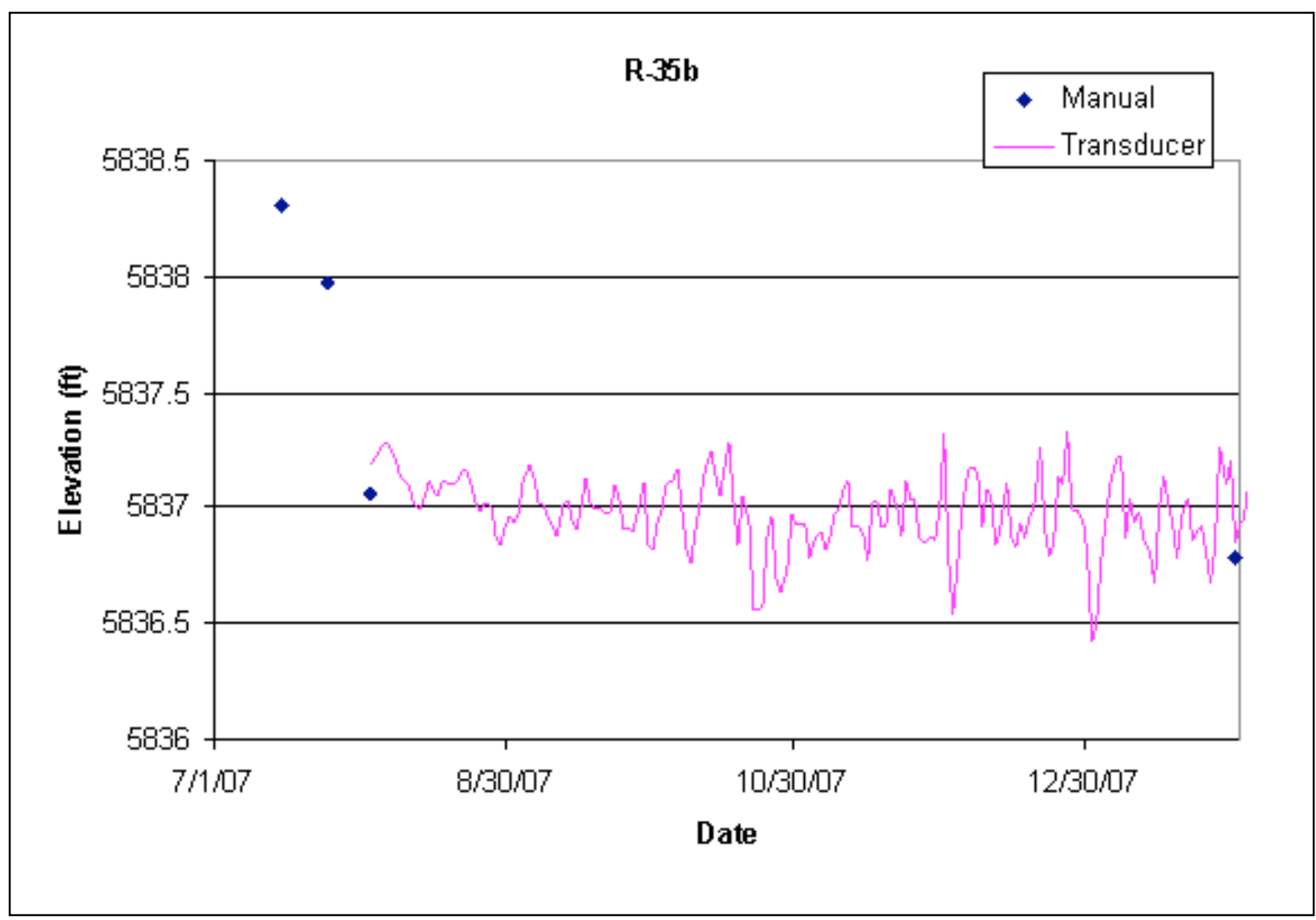




\subsection{Test Well 1}

Location: TW-1 is located in lower Pueblo Canyon downstream of supply well O-1.

Completion Type: Single completion within the regional aquifer. The top of the screen was about 120 $\mathrm{ft}$ below the water level in 2006.

Period of Record: Well completed January 1950, transducer installed January 23, 1992, intermittent water level data to February 6, 2006, when the transducer was removed for well plugging and abandonment.

Remarks: TW-1 installed in the regional aquifer at a depth of $642 \mathrm{ft}$, about $100 \mathrm{ft}$ into the regional aquifer. Water level in TW-1 is recharged locally by surface water from Pueblo Canyon (Koch and Rogers 2003) and does not correlate with the water level of surrounding regional aquifer wells.

\begin{tabular}{|c|c|c|c|c|c|c|c|c|c|c|c|c|c|}
\hline \multicolumn{14}{|c|}{ TW-1 Construction Information } \\
\hline Zone & $\begin{array}{c}\text { Screen } \\
\text { Top } \\
\text { Depth (ft) }\end{array}$ & $\begin{array}{l}\text { Screen } \\
\text { Bottom } \\
\text { Depth } \\
\text { (ft) }\end{array}$ & $\begin{array}{c}\text { Screen } \\
\text { Top } \\
\text { Elev (ft) }\end{array}$ & $\begin{array}{l}\text { Screen } \\
\text { Bottom } \\
\text { Elev (ft) }\end{array}$ & $\begin{array}{l}\text { Screen } \\
\text { Length } \\
\text { (ft) }\end{array}$ & $\begin{array}{c}\text { Pump } \\
\text { Intake } \\
\text { Depth } \\
\text { (ft) }\end{array}$ & $\begin{array}{l}\text { Pump } \\
\text { Intake } \\
\text { Elevation } \\
\text { (ft) }\end{array}$ & $\begin{array}{l}\text { Depth to } \\
\text { Top of } \\
\text { Sump (ft) }\end{array}$ & $\begin{array}{l}\text { Top of } \\
\text { Sump } \\
\text { Elevation } \\
\text { (ft) }\end{array}$ & $\begin{array}{l}\text { Depth to } \\
\text { Sump } \\
\text { Bottom (ft) }\end{array}$ & $\begin{array}{l}\text { Sump } \\
\text { Length } \\
\text { (ft) }\end{array}$ & $\begin{array}{c}\text { Sump } \\
\text { Volume } \\
\text { (L) }\end{array}$ & Comment \\
\hline 1 & 632.0 & 642 & 5737.2 & 5727.2 & 10.0 & & & 642.0 & 5727.2 & 642 & 0.0 & 0.0 & Regional Aquifer \\
\hline
\end{tabular}

Note: TW-1 Ground Elevation: $6369.19 \mathrm{ft}$; all measurements are from this elevation

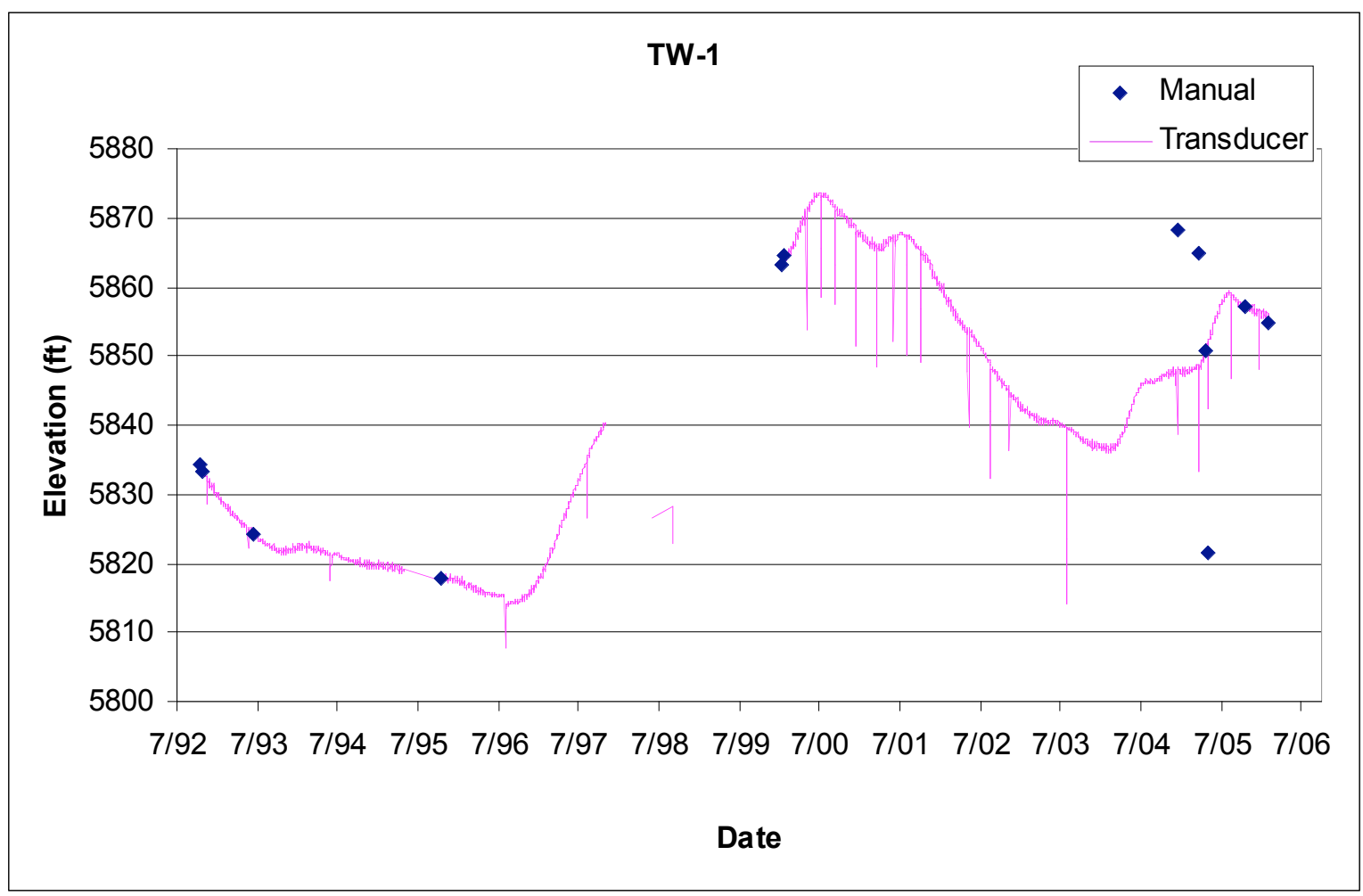




\subsection{Test Well 2}

Location: TW-2 is located in middle Pueblo Canyon.

Completion Type: Single completion at the top of the regional aquifer.

Period of Record: Well originally drilled in 1949, recompleted in 1990. Transducer installed June 1993; data to January 1996. Transducer reinstalled January 2000; transducer data to March 2005.

Remarks: TW-2 completed at the top of the regional aquifer at a depth of $834 \mathrm{ft}$, about $35 \mathrm{ft}$ into the regional aquifer. The transducer failed in November 2000, transducer data since then are questionable. A manual measurement attempt in March 2005 resulted in the measurement tape stuck in the well. Thus, transducer water level data since November 2000 are not valid with respect to elevation, but are shown for reference and character information only.

\begin{tabular}{|c|c|c|c|c|c|c|c|c|c|c|c|c|c|}
\hline \multicolumn{14}{|c|}{ Test Well 2 Construction Information } \\
\hline Zone & $\begin{array}{c}\text { Screen } \\
\text { Top } \\
\text { Depth (ft) }\end{array}$ & $\begin{array}{c}\text { Screen } \\
\text { Bottom } \\
\text { Depth } \\
\text { (ft) }\end{array}$ & $\begin{array}{c}\text { Screen } \\
\text { Top } \\
\text { Elev (ft) }\end{array}$ & $\begin{array}{l}\text { Screen } \\
\text { Bottom } \\
\text { Elev (ft) }\end{array}$ & $\begin{array}{l}\text { Screen } \\
\text { Length } \\
\text { (ft) }\end{array}$ & $\begin{array}{c}\text { Pump } \\
\text { Intake } \\
\text { Depth } \\
\text { (ft) }\end{array}$ & $\begin{array}{l}\text { Pump } \\
\text { Intake } \\
\text { Elevation } \\
\text { (ft) }\end{array}$ & $\begin{array}{l}\text { Depth to } \\
\text { Top of } \\
\text { Sump (ft) }\end{array}$ & $\begin{array}{c}\text { Top of } \\
\text { Sump } \\
\text { Elevation } \\
\text { (ft) }\end{array}$ & $\begin{array}{l}\text { Depth to } \\
\text { Sump } \\
\text { Bottom (ft) }\end{array}$ & $\begin{array}{l}\text { Sump } \\
\text { Length } \\
\text { (ft) }\end{array}$ & $\begin{array}{l}\text { Sump } \\
\text { Volume } \\
\text { (L) }\end{array}$ & Comment \\
\hline 1 & 768.0 & 824 & 5880.1 & 5824.1 & 56.0 & & & 824.0 & 5824.1 & 834 & 10.0 & 55.6 & Regional Aquifer \\
\hline
\end{tabular}

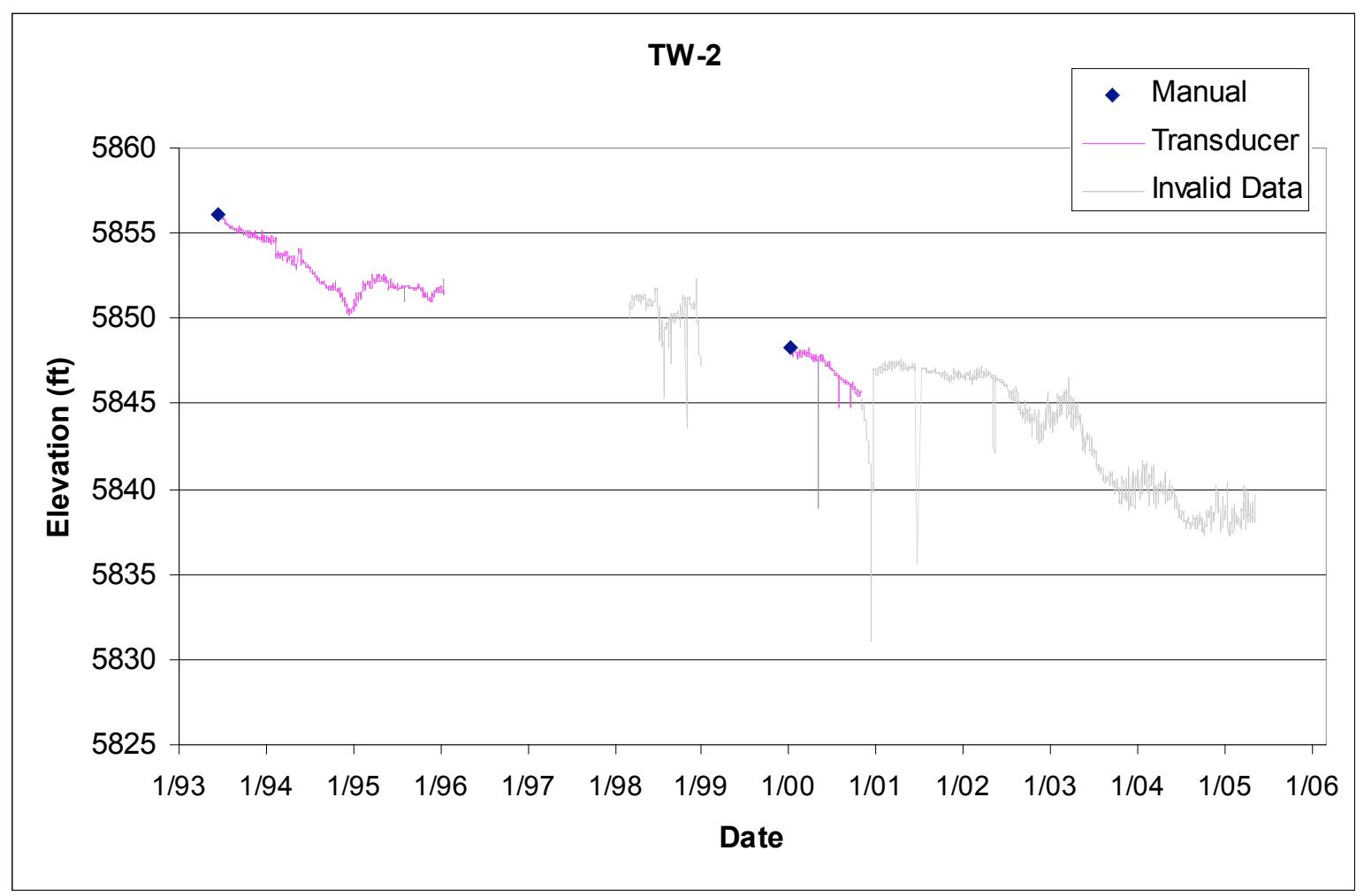




\subsection{Test Well 3}

Location: TW-3 is located in middle Los Alamos Canyon at confluence with DP Canyon.

Completion Type: Single completion at the top of the regional aquifer.

Period of Record: Well drilled in 1949, transducer installed November 1992, intermittent data to February 2006.

Remarks: TW-3 completed at the top of the regional aquifer at a depth of $815 \mathrm{ft}$, about $30 \mathrm{ft}$ into the regional aquifer. Transducer removed February 9, 2006, in preparation for well plugging and abandonment.

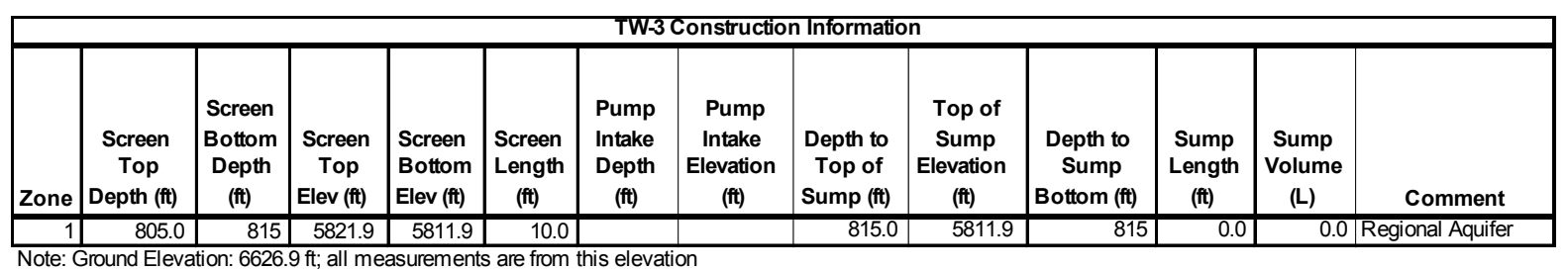

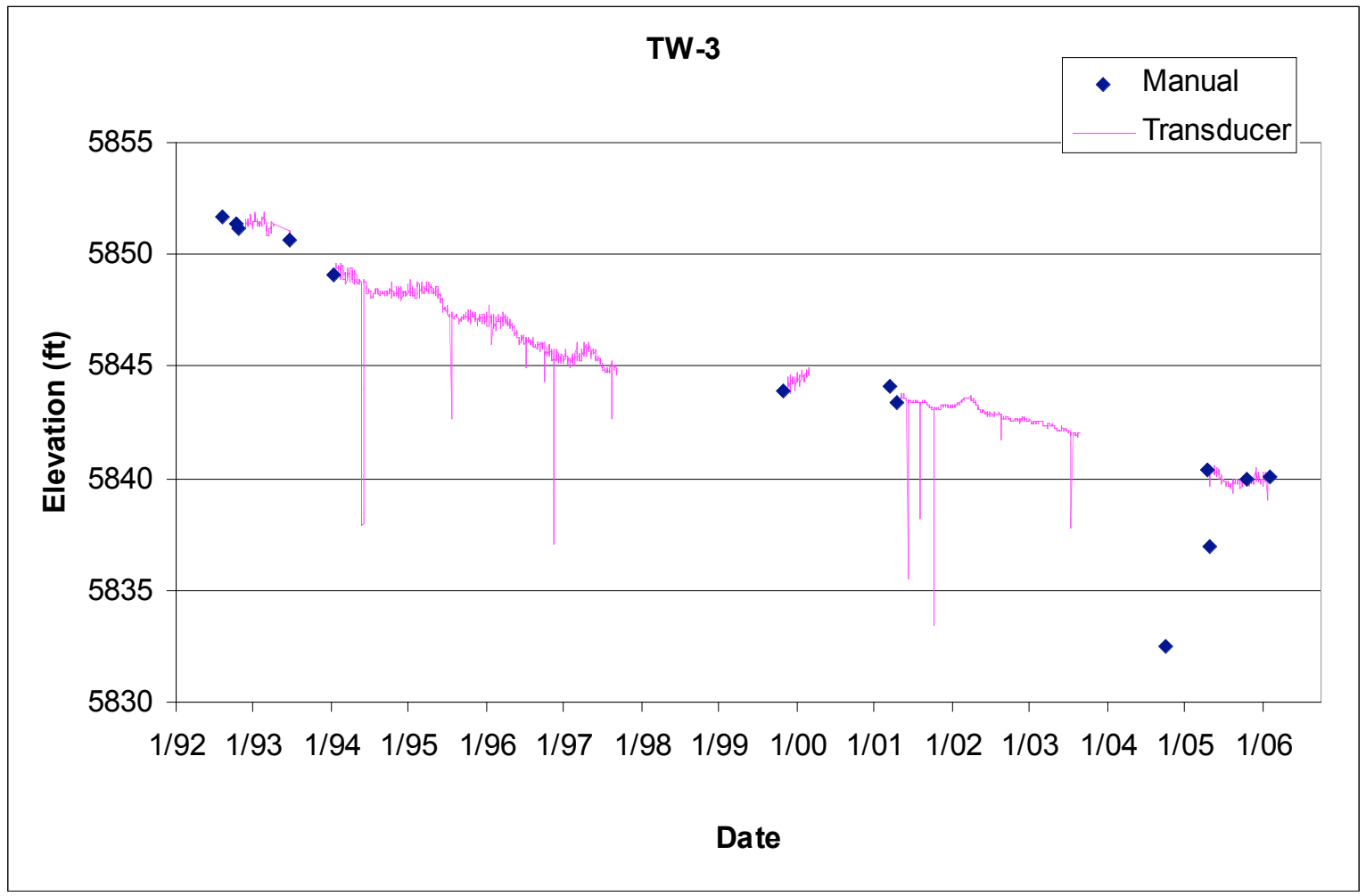




\subsection{Test Well 4}

Location: TW-4 is located east of Acid Canyon in upper Pueblo Canyon.

Completion Type: Single completion at the top of the regional aquifer.

Period of Record: Well drilled in 1950, transducer installed June 1993 but problems occurred with the transducer. Transducer reinstalled July 1997, intermittent data to February 8, 2006.

Remarks: Completed at the top of the regional aquifer at a depth of $1205 \mathrm{ft}$, about $30 \mathrm{ft}$ into the regional aquifer. Transducer removed February 8, 2006, in preparation for well plugging and abandonment.

\begin{tabular}{|c|c|c|c|c|c|c|c|c|c|c|c|c|c|}
\hline \multicolumn{14}{|c|}{ TW-4 Construction Information } \\
\hline Zone & $\begin{array}{c}\text { Screen } \\
\text { Top } \\
\text { Depth (ft) }\end{array}$ & $\begin{array}{l}\text { Screen } \\
\text { Bottom } \\
\text { Depth } \\
\text { (ft) }\end{array}$ & $\begin{array}{c}\text { Screen } \\
\text { Top } \\
\text { Elev (ft) }\end{array}$ & $\begin{array}{l}\text { Screen } \\
\text { Bottom } \\
\text { Elev (ft) }\end{array}$ & $\begin{array}{l}\text { Screen } \\
\text { Length } \\
\text { (ft) }\end{array}$ & $\begin{array}{c}\text { Pump } \\
\text { Intake } \\
\text { Depth } \\
\text { (ft) }\end{array}$ & $\begin{array}{l}\text { Pump } \\
\text { Intake } \\
\text { Elevation } \\
\text { (ft) }\end{array}$ & $\begin{array}{c}\text { Depth to } \\
\text { Top of } \\
\text { Sump (ft) }\end{array}$ & $\begin{array}{l}\text { Top of } \\
\text { Sump } \\
\text { Elevation } \\
\text { (ft) }\end{array}$ & $\begin{array}{l}\text { Depth to } \\
\text { Sump } \\
\text { Bottom (ft) }\end{array}$ & $\begin{array}{l}\text { Sump } \\
\text { Length } \\
\text { (ft) }\end{array}$ & $\begin{array}{c}\text { Sump } \\
\text { Volume } \\
\text { (L) }\end{array}$ & Comment \\
\hline 1 & 1195.0 & 1205 & 6049.6 & 6039.6 & 10.0 & & & 1205.0 & 6039.6 & 1205 & 0.0 & 0.0 & Regional Aquifer \\
\hline
\end{tabular}

Note: TW-4 Ground Elevation: $7244.56 \mathrm{ft}$; all measurements are from this elevation

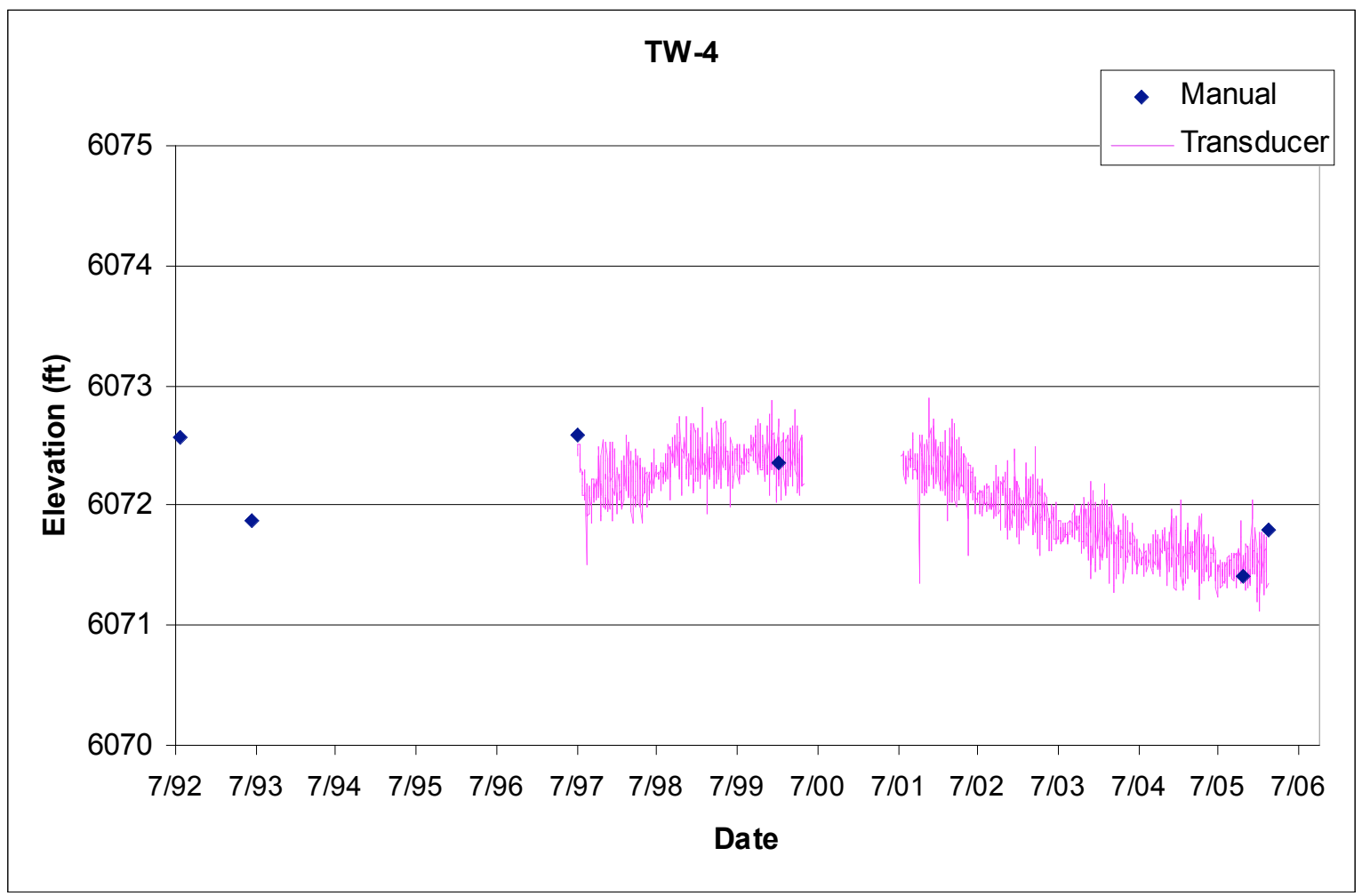




\subsection{Test Well 8}

Location: TW-8 is located in middle Mortandad Canyon about $220 \mathrm{ft}$ east of R-1, which was drilled to replace TW-8.

Completion Type: Single completion at the top of the regional aquifer. The screen straddles the water table.

Period of Record: Well drilled in 1960, transducer installed June 1993, transducer data to March 1997. Transducer reinstalled January 2000; intermittent data through 2007.

Remarks: TW-8 completed at the top of the regional aquifer at a depth of $1065 \mathrm{ft}$, about $70 \mathrm{ft}$ into the regional aquifer. The well is nearly $100 \%$ barometrically efficient; the aquifer has no response to atmospheric pressure fluctuations. The aquifer indicates a seasonal response to supply well pumping and primarily responds to pumping PM-5 and possibly to pumping PM-4.

\begin{tabular}{|c|c|c|c|c|c|c|c|c|c|c|c|c|c|}
\hline \multicolumn{14}{|c|}{ TW-8 Construction Information } \\
\hline Zone & $\begin{array}{c}\text { Screen } \\
\text { Top } \\
\text { Depth (ft) }\end{array}$ & $\begin{array}{c}\text { Screen } \\
\text { Bottom } \\
\text { Depth } \\
\text { (ft) }\end{array}$ & $\begin{array}{c}\text { Screen } \\
\text { Top } \\
\text { Elev (ft) }\end{array}$ & $\begin{array}{l}\text { Screen } \\
\text { Bottom } \\
\text { Elev (ft) }\end{array}$ & $\begin{array}{l}\text { Screen } \\
\text { Length } \\
\text { (ft) }\end{array}$ & $\begin{array}{l}\text { Pump } \\
\text { Intake } \\
\text { Depth } \\
\text { (ft) }\end{array}$ & $\begin{array}{l}\text { Pump } \\
\text { Intake } \\
\text { Elevation } \\
\text { (ft) }\end{array}$ & $\begin{array}{l}\text { Depth to } \\
\text { Top of } \\
\text { Sump (ft) }\end{array}$ & $\begin{array}{c}\text { Top of } \\
\text { Sump } \\
\text { Elevation } \\
\text { (ft) }\end{array}$ & $\begin{array}{l}\text { Depth to } \\
\text { Sump } \\
\text { Bottom (ft) }\end{array}$ & $\begin{array}{l}\text { Sump } \\
\text { Length } \\
\text { (ft) }\end{array}$ & $\begin{array}{c}\text { Sump } \\
\text { Volume } \\
\text { (L) }\end{array}$ & Comment \\
\hline 1 & 953.0 & 1065 & 5920.5 & 5808.5 & 112.0 & & & 1065.0 & 5808.5 & 1065 & 0.0 & 0.0 & Regional Aquifer \\
\hline
\end{tabular}

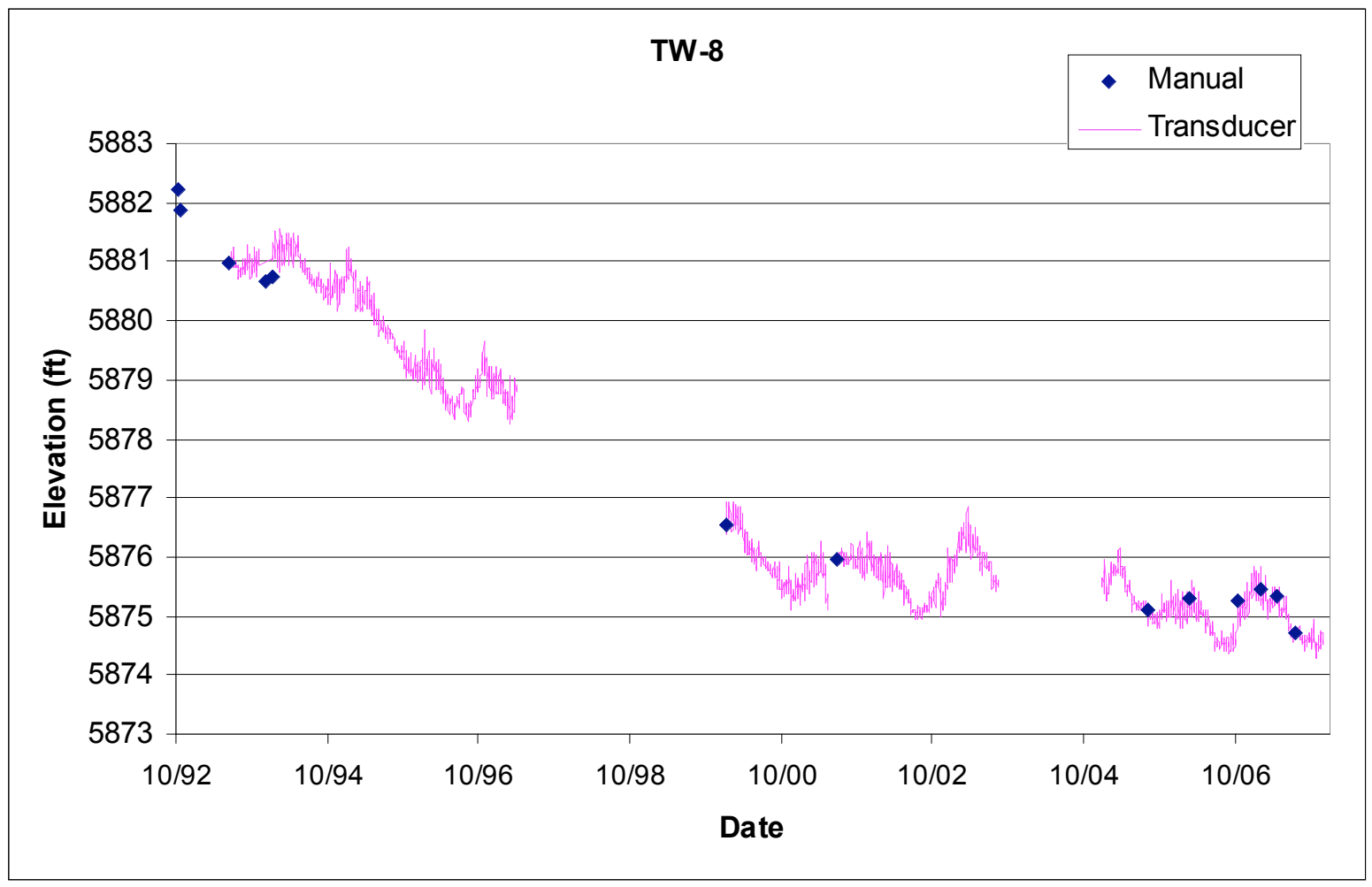




\subsection{Test Well DT-5A}

Location: DT-5A is located at TA-49 near the southern boundary of LANL.

Completion Type: Single completion at the top of the regional aquifer. The screen straddles the water table.

Period of Record: Well drilled in 1960, transducer installed June 1993, data to September 1996.

Transducer reinstalled January 2000 but equipment problems occurred. Transducer reinstalled April 2001; data through 2007.

Remarks: DT-5A completed at the top of the regional aquifer at a depth of $1819.5 \mathrm{ft}$, about $650 \mathrm{ft}$ into the regional aquifer. The transducer malfunctioned in September 2005, reinstalled January 2006. The well is $100 \%$ barometrically efficient; the aquifer does not respond immediately to atmospheric pressure fluctuations but shows a delayed response. The long-term water level shows a decline of about $0.2 \mathrm{ft} / \mathrm{yr}$, likely in response to supply well pumping.

\begin{tabular}{|c|c|c|c|c|c|c|c|c|c|c|c|c|c|}
\hline \multicolumn{14}{|c|}{ TestWellDT-5AConstruction Information } \\
\hline Zone & $\begin{array}{c}\text { Screen } \\
\text { Top } \\
\text { Depth (ft) }\end{array}$ & $\begin{array}{c}\text { Screen } \\
\text { Bottom } \\
\text { Depth } \\
\text { (ft) }\end{array}$ & $\begin{array}{c}\text { Screen } \\
\text { Top } \\
\text { Elev }(\mathrm{ft})\end{array}$ & $\begin{array}{l}\text { Screen } \\
\text { Bottom } \\
\text { Elev (ft) }\end{array}$ & $\begin{array}{l}\text { Screen } \\
\text { Length } \\
\text { (ft) }\end{array}$ & $\begin{array}{l}\text { Pump } \\
\text { Intake } \\
\text { Depth } \\
\text { (ft) }\end{array}$ & $\begin{array}{l}\text { Pump } \\
\text { Intake } \\
\text { Elevation } \\
\text { (ft) }\end{array}$ & $\begin{array}{c}\text { Depth to } \\
\text { Top of } \\
\text { Sump (ft) }\end{array}$ & $\begin{array}{c}\text { Top of } \\
\text { Sump } \\
\text { Elevation } \\
\text { (ft) }\end{array}$ & $\begin{array}{l}\text { Depth to } \\
\text { Sump } \\
\text { Bottom (ft) }\end{array}$ & $\begin{array}{l}\text { Sump } \\
\text { Length } \\
\text { (ft) }\end{array}$ & $\begin{array}{l}\text { Sump } \\
\text { Volume } \\
\text { (L) }\end{array}$ & Comment \\
\hline 1 & 1171.5 & 1788.5 & 5972.4 & 5355.4 & 617.0 & & & 1788.5 & 5355.4 & 1819.5 & 31.0 & 306.4 & Regional Aquifer \\
\hline
\end{tabular}

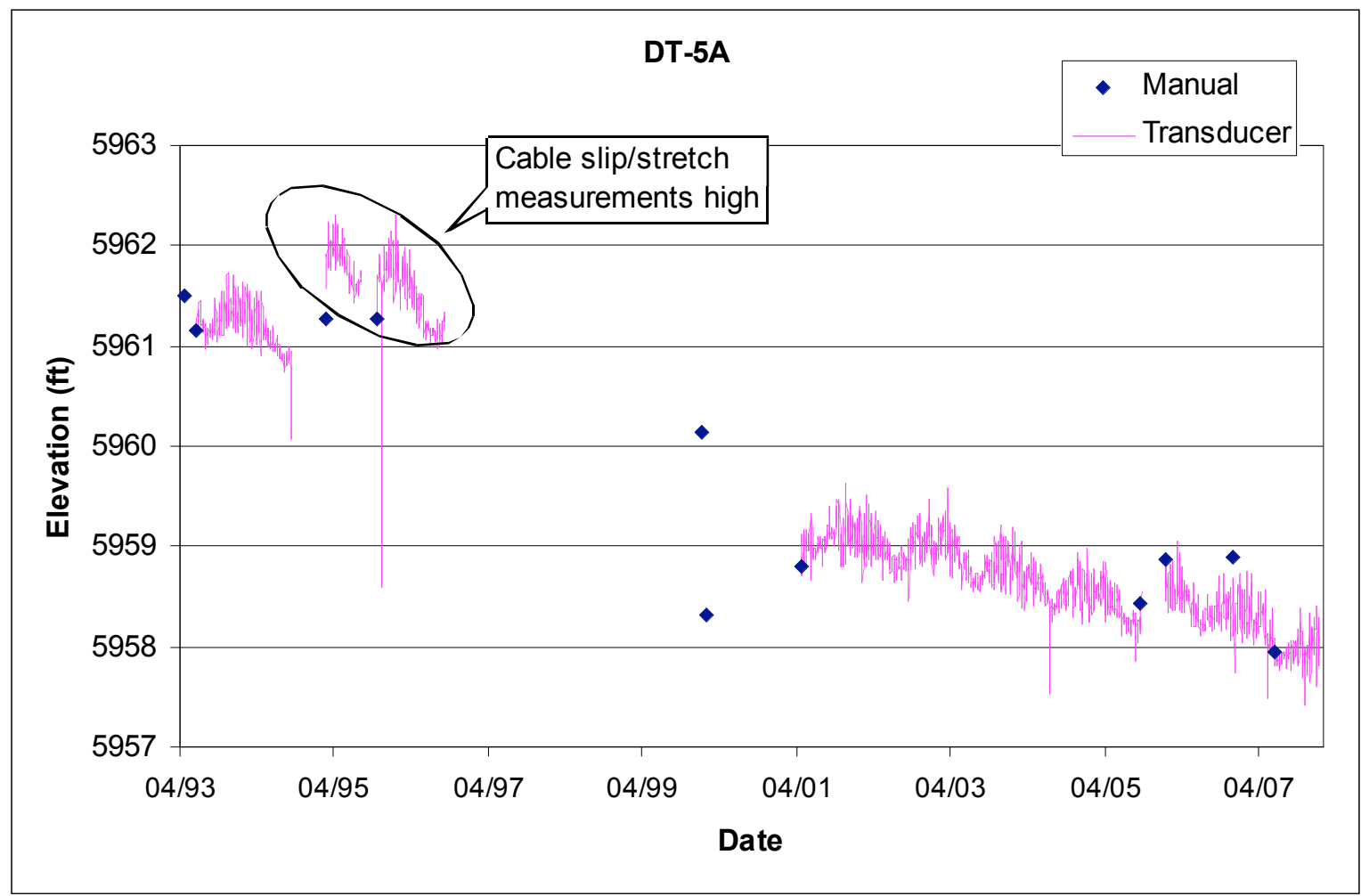




\subsection{Test Well DT-9}

Location: DT-9 is located at TA-49 near the southern LANL boundary.

Completion Type: Single completion at the top of the regional aquifer. The screen straddles the water table.

Period of Record: Well drilled in 1960, transducer installed November 1992, intermittent data to July 2002. Transducer reinstalled June 2005, data through 2007.

Remarks: DT-9 completed at the top of the regional aquifer at a depth of $1501 \mathrm{ft}$, about $500 \mathrm{ft}$ into the regional aquifer. The well is $100 \%$ barometrically efficient; the aquifer does not respond immediately to atmospheric pressure fluctuations but shows a delayed response. The aquifer shows a long-term decline of about $0.35 \mathrm{ft} / \mathrm{yr}$, likely associated with supply well pumping.

\begin{tabular}{|c|c|c|c|c|c|c|c|c|c|c|c|c|c|}
\hline \multicolumn{14}{|c|}{ Test WellDT-9 Construction Information } \\
\hline Zone & $\begin{array}{c}\text { Screen } \\
\text { Top } \\
\text { Depth (ft) }\end{array}$ & $\begin{array}{c}\text { Screen } \\
\text { Bottom } \\
\text { Depth } \\
\text { (ft) }\end{array}$ & $\begin{array}{l}\text { Screen } \\
\text { Top } \\
\text { Elev (ft) }\end{array}$ & $\begin{array}{l}\text { Screen } \\
\text { Bottom } \\
\text { Elev (ft) }\end{array}$ & $\begin{array}{c}\text { Screen } \\
\text { Length } \\
\text { (ft) }\end{array}$ & $\begin{array}{c}\text { Pump } \\
\text { Intake } \\
\text { Depth } \\
\text { (ft) }\end{array}$ & $\begin{array}{l}\text { Pump } \\
\text { Intake } \\
\text { Elevation } \\
\text { (ft) }\end{array}$ & $\begin{array}{c}\text { Depth to } \\
\text { Top of } \\
\text { Sump (ft) }\end{array}$ & $\begin{array}{c}\text { Top of } \\
\text { Sump } \\
\text { Elevation } \\
\text { (ft) }\end{array}$ & $\begin{array}{c}\text { Depth to } \\
\text { Sump } \\
\text { Bottom (ft) }\end{array}$ & $\begin{array}{c}\text { Sump } \\
\text { Length } \\
\text { (ft) }\end{array}$ & $\begin{array}{c}\text { Sump } \\
\text { Volume } \\
\text { (L) }\end{array}$ & Comment \\
\hline 1 & 1040.0 & 1501 & 5895.0 & 5434.0 & 461.0 & & 6935.0 & 1501.0 & 5434.0 & 1501 & 0.0 & 0.0 & Regional Aquifer \\
\hline
\end{tabular}

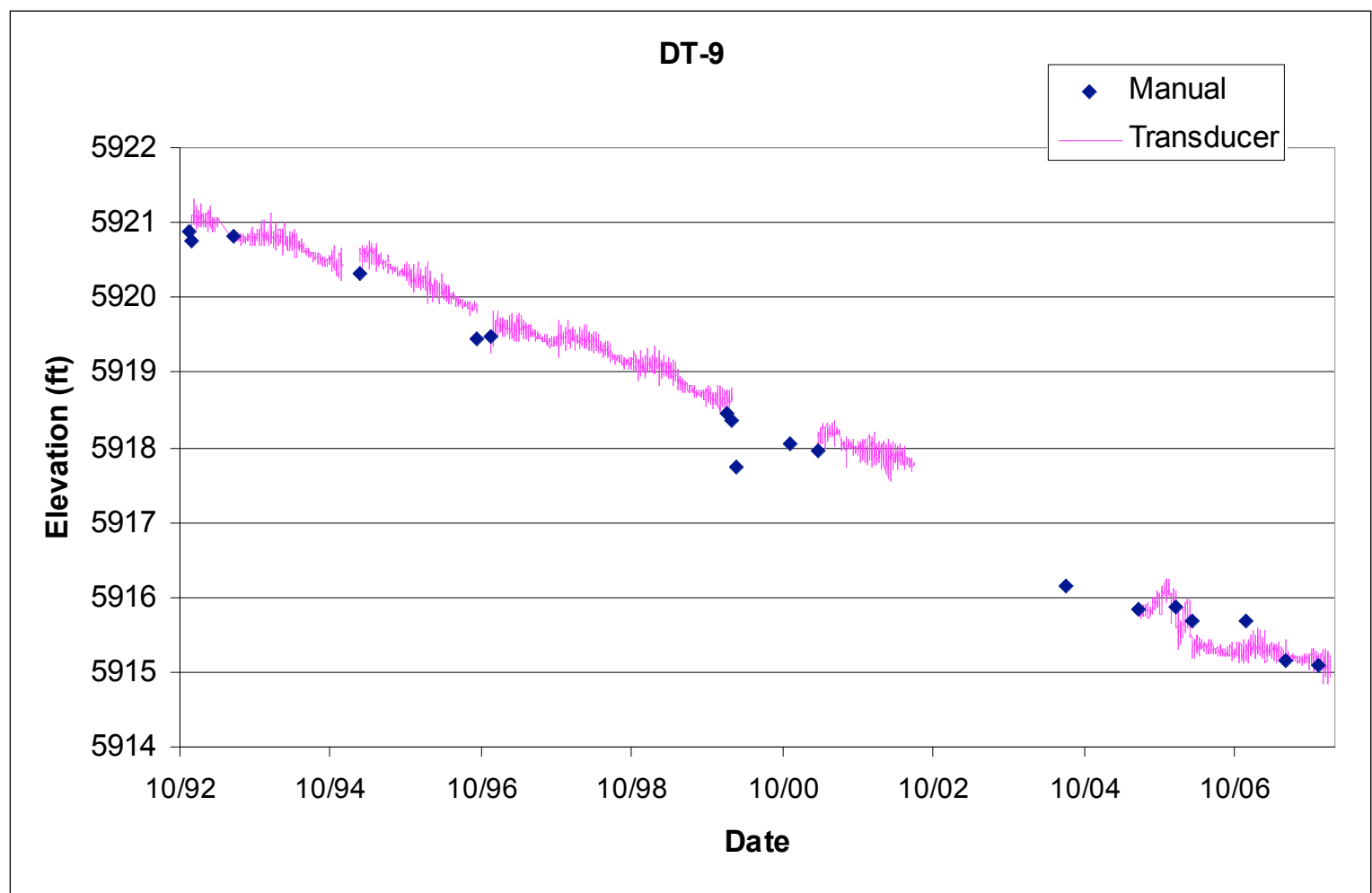




\subsection{Test Well DT-10}

Location: DT-10 is located at TA-49 near the southern LANL boundary.

Completion Type: Single completion at the top of the regional aquifer. The screen straddles the water table.

Period of Record: Well drilled in 1960, transducer installed June 1993 and again in November 1996 and June 2005. Transducer equipment failed June 2006, new transducer installed January 2007; data through 2007.

Remarks: DT-10 completed at the top of the regional aquifer at a depth of $1408 \mathrm{ft}$, about $300 \mathrm{ft}$ into the regional aquifer. The well is about $70 \%$ barometrically efficient; the aquifer shows a $30 \%$ response to atmospheric pressure fluctuations. The aquifer exhibits a long-term water level decline of about $0.32 \mathrm{ft} / \mathrm{yr}$, likely associated with supply well pumping.

\begin{tabular}{|c|c|c|c|c|c|c|c|c|c|c|c|c|c|}
\hline \multicolumn{14}{|c|}{ Test Well DT-10 Construction Information } \\
\hline Zone & $\begin{array}{c}\text { Screen } \\
\text { Top } \\
\text { Depth (ft) }\end{array}$ & $\begin{array}{l}\text { Screen } \\
\text { Bottom } \\
\text { Depth } \\
\text { (ft) }\end{array}$ & $\begin{array}{c}\text { Screen } \\
\text { Top } \\
\text { Elev (ft) }\end{array}$ & $\begin{array}{l}\text { Screen } \\
\text { Bottom } \\
\text { Elev (ft) }\end{array}$ & $\begin{array}{l}\text { Screen } \\
\text { Length } \\
\text { (ft) }\end{array}$ & $\begin{array}{c}\text { Pump } \\
\text { Intake } \\
\text { Depth } \\
\text { (ft) }\end{array}$ & $\begin{array}{l}\text { Pump } \\
\text { Intake } \\
\text { Elevation } \\
\text { (ft) }\end{array}$ & $\begin{array}{c}\text { Depth to } \\
\text { Top of } \\
\text { Sump (ft) }\end{array}$ & $\begin{array}{c}\text { Top of } \\
\text { Sump } \\
\text { Elevation } \\
\text { (ft) }\end{array}$ & $\begin{array}{l}\text { Depth to } \\
\text { Sump } \\
\text { Bottom (ft) }\end{array}$ & $\begin{array}{l}\text { Sump } \\
\text { Length } \\
\text { (ft) }\end{array}$ & $\begin{array}{l}\text { Sump } \\
\text { Volume } \\
\text { (L) }\end{array}$ & Comment \\
\hline 1 & 1080.0 & 1408.0 & 5939.9 & 5611.9 & 328.0 & & & 1408.0 & 5611.9 & 1408.0 & 0.0 & 0.0 & Regional Aquifer \\
\hline
\end{tabular}

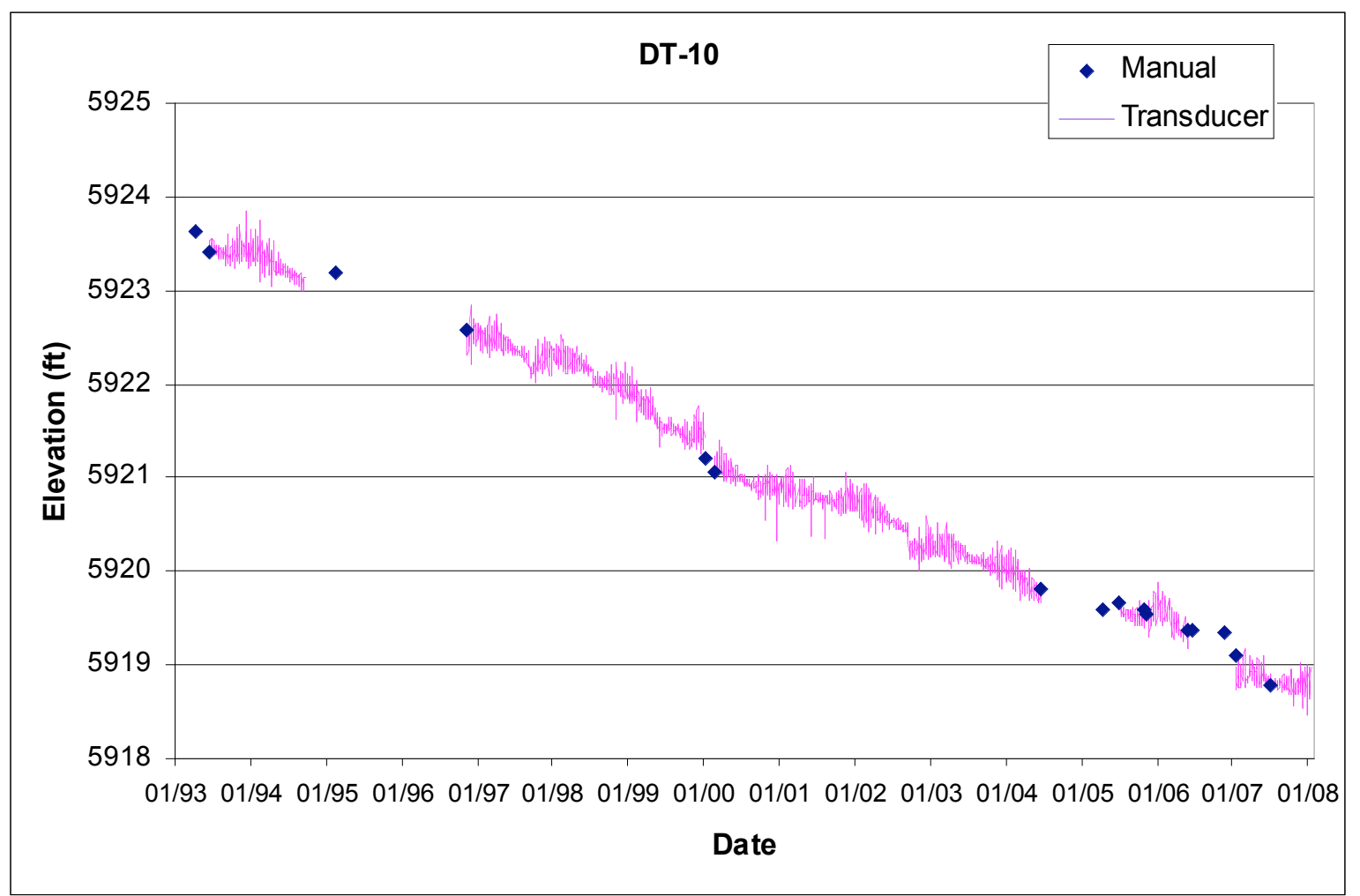




\subsection{Groundwater Level Data from Intermediate Wells}

Table 4-1 lists the monitoring wells that specifically monitor intermediate groundwater at LANL and includes the well name, completed depth, surveyed location coordinates, and the date of completion. Table 4-2 lists the well construction information for the intermediate wells and for regional aquifer wells that have intermediate screens. The table includes information for the depth to the top and bottom of screens, screen casing size, geologic formation where the screen is completed, and whether the well/screen contains intermediate groundwater. The hydrographs for intermediate zones in the multiple completion regional aquifer wells are shown in the previous section.

Figure 4-1 shows the locations of the intermediate wells and regional wells that monitor intermediate groundwater. (Note that multiple completion regional wells that do not contain intermediate groundwater, such as CdV-R-15-3, CdV-R-37-2, and R-31, are not shown in Figure 4-1 because the intermediate screens in these wells are dry.)

Table 4-1. General Information for Intermediate Wells at LANL

\begin{tabular}{|l|r|r|r|r|r|}
\hline \multicolumn{1}{|c|}{ Well Name } & $\begin{array}{c}\text { Date } \\
\text { Completed }\end{array}$ & $\begin{array}{c}\text { Completed } \\
\text { Depth (ft) }\end{array}$ & Easting (ft) & Northing (ft) & $\begin{array}{c}\text { Surface } \\
\text { Elevation (ft) }\end{array}$ \\
\hline 90LP-SE-16-02669 & $3 / 15 / 1998$ & 163.4 & 1612152.57 & 1763749.00 & 7583.26 \\
\hline CdV-16-1(i) & $11 / 9 / 2003$ & 657.8 & 1615078.20 & 1764415.20 & 7382.17 \\
\hline CdV-16-2(i) & $12 / 8 / 2003$ & 1037.1 & 1616741.20 & 1764237.20 & 7457.11 \\
\hline CdV-16-2(i)r & $7 / 30 / 2005$ & 863.2 & 1616673.24 & 1764219.40 & 7456.67 \\
\hline CdV-16-3(i) & $1 / 23 / 2004$ & Open Hole & 1615981.60 & 1762434.90 & 7486.4 \\
\hline LADP-3 & $12 / 17 / 1993$ & 326.0 & 1632989.00 & 1773469.10 & 6756.70 \\
\hline LAOI(a)-1.1 & $10 / 28 / 1994$ & 309.8 & 1629427.38 & 1773924.51 & 6835.20 \\
\hline LAOI-3.2 & $5 / 1 / 2005$ & 165.0 & 1637642.10 & 1773066.93 & 6622.60 \\
\hline LAOI-3.2a & $1 / 20 / 2006$ & 194.1 & 1637619.97 & 1773100.91 & 6624.43 \\
\hline LAOI-7 & $9 / 21 / 2005$ & 264.9 & 1644788.53 & 1771584.11 & 6458.35 \\
\hline MCOBT-4.4 & $6 / 30 / 2001$ & 545.0 & 1634196.30 & 1768513.00 & 6836.18 \\
\hline MCOI-1 & $1 / 9 / 2005$ & 825.6 & 1628044.51 & 1769957.39 & 7106.20 \\
\hline MCOI-4 & $11 / 6 / 2004$ & 525.7 & 1634128.53 & 1768542.01 & 6837.20 \\
\hline MCOI-5 & $10 / 25 / 2004$ & 699.0 & 1635247.94 & 1768300.46 & 6819.70 \\
\hline MCOI-6 & $1 / 13 / 2005$ & 713.2 & 1635345.65 & 1768428.06 & 6811.10 \\
\hline MCOI-8 & $1 / 7 / 2005$ & 675.0 & 1633329.74 & 1769214.40 & 6859.20 \\
\hline MSC-16-02665 & $10 / 23 / 1997$ & 124.0 & 1614427.59 & 1762530.55 & 7516.92 \\
\hline POI-4 & $5 / 1 / 1996$ & 176.5 & 1649432.46 & 1772587.08 & 6372.29 \\
\hline R-3i & $8 / 16 / 2005$ & 220.3 & 1649196.5 & 1772599.2 & 6390.15 \\
\hline R-6i & $12 / 20 / 2004$ & 615.0 & 1635992.34 & 1773889.89 & 6996.90 \\
\hline R-9i & $3 / 10 / 2000$ & 309.9 & 1648202.70 & 1770837.80 & 6383.20 \\
\hline R-23i & $11 / 10 / 2005$ & 550.7 & 1647898.02 & 1755148.04 & 6527.88 \\
\hline SCI-1 & $10 / 7 / 2006$ & 377.9 & 1636822.9 & 1770298.2 & 6738.27 \\
\hline TW-1A & $1 / 11 / 1950$ & 225.0 & 1650056.87 & 1772065.87 & 6369.28 \\
\hline TW-2A & $2 / 7 / 1950$ & 133.0 & 1634184.87 & 1777288.12 & 6650.40 \\
\hline
\end{tabular}




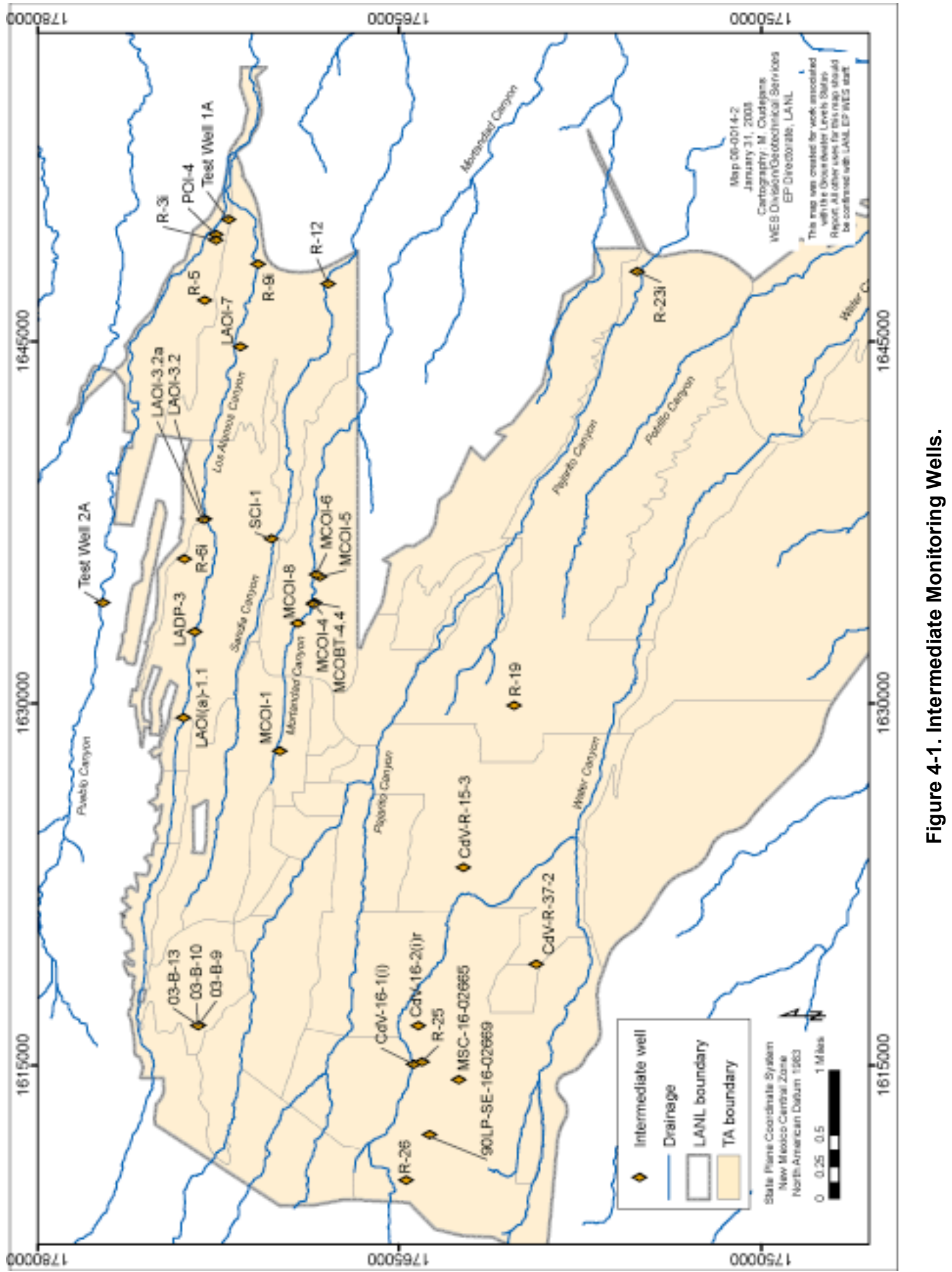


Table 4-2. Well Completion Information for Intermediate Wells and Screens

\begin{tabular}{|c|c|c|c|c|c|c|c|}
\hline Well Name & Screen Common Name & $\begin{array}{c}\text { Screen } \\
\text { Material }\end{array}$ & $\begin{array}{l}\text { Top of } \\
\text { Screen } \\
\text { (ft) }\end{array}$ & $\begin{array}{c}\text { Bottom } \\
\text { of } \\
\text { Screen } \\
\text { (ft) }\end{array}$ & $\begin{array}{l}\text { Screen } \\
\text { Inside } \\
\text { Diameter } \\
\text { (in.) }\end{array}$ & $\begin{array}{c}\text { Geologic } \\
\text { Unit }\end{array}$ & Comment \\
\hline 90LP-SE-16-02669 & 16-02669 Screen \#1 & PVC & 131.5 & 162.5 & 2.00 & Qbt3 & Dry \\
\hline $\mathrm{CdV}-16-1(\mathrm{i})$ & CdV-16-1(i) Screen \#1 & SS304 & 624.0 & 634.0 & 4.50 & Qbo & \\
\hline CdV-16-2(i) & CdV-16-2(i) Screen \#1 & SS304 & 850.2 & 867.8 & 4.46 & $\mathrm{Tp}$ & Dry \\
\hline CdV-16-2(i) & CdV-16-2(i) Screen \#2 & SS304 & 992.0 & 1015.2 & 4.46 & $T p$ & Dry \\
\hline CdV-16-2(i)r & CdV-16-2(i)r Screen \#1 & SS304 & 850.0 & 859.7 & 4.46 & Tpf & \\
\hline CdV-16-3(i) & Open Borehole & NA & NA & $\mathrm{NA}$ & $\mathrm{NA}$ & $\mathrm{Tt}$ & Regional Aquifer? \\
\hline CdV-R-15-3 & CdV-R-15-3 Screen 1 & SS312 & 617.7 & 624.5 & 4.50 & Qbo & Dry \\
\hline CdV-R-15-3 & CdV-R-15-3 Screen 2 & SS312 & 800.8 & 807.8 & 4.50 & $\mathrm{Tp}$ & Dry \\
\hline CdV-R-15-3 & CdV-R-15-3 Screen 3 & SS312 & 964.8 & 980.9 & 4.50 & $\mathrm{~Tb}$ & Dry \\
\hline CdV-R-37-2 & CdV-R37-2 Screen \#1 & SS304 & 914.4 & 939.5 & 4.50 & $\overline{T p}$ & Dry \\
\hline LADP-3 & LADP-3 Screen \#1 & PVC & 316.0 & 325.0 & 3.00 & Qbog & \\
\hline LAOI(A)-1.1 & LAOI(A)-1.1 Screen \#1 & PVC & 295.2 & 305.0 & 3.00 & Qbog & \\
\hline LAOI-3.2 & LAOI-3 Screen \#1 & PVC & 153.3 & 162.8 & 4.46 & $\mathrm{~Tb}$ & \\
\hline LAOI-3.2a & LAOI-3a Screen \#1 & SS304 & 181.4 & 191.0 & 3.10 & Tpf & \\
\hline LAOI-7 & LAOI-7 Screen \#1 & SS304 & 240.0 & 259.6 & 3.00 & Tb4 & \\
\hline MCOBT-4.4 & MCOBT4.4 Screen \#1 & SS304 & 485.4 & 524.0 & 4.50 & Tpf & \\
\hline MCOI-1 & MCOI-1 Screen \#1 & SS & 815.0 & 825.5 & 1.10 & Tpf & \\
\hline MCOI-4 & MCOI-4 Screen \#1 & PVC & 498.9 & 522.0 & 4.50 & Tpf & \\
\hline MCOI-5 & MCOI-5 Screen \#1 & PVC & 689.0 & 699.0 & 4.50 & $\mathrm{~Tb}$ & \\
\hline MCOI-6 & MCOI-6 Screen \#1 & PVC & 686.0 & 708.3 & 4.50 & $\mathrm{~Tb}$ & \\
\hline MCOI-8 & MCOI-6 Screen \#1 & PVC & 665.0 & 675.0 & 4.46 & $\mathrm{~Tb}$ & \\
\hline MSC-16-02665 & $16-02665$ Screen \#1 & PVC & 93.5 & 123.5 & 2.00 & Qbt3 & Usually dry \\
\hline POI-4 & POI-4 Screen \#1 & PVC & 159.0 & 174.0 & 4.00 & Tb4 & \\
\hline R-3i & R-3i Screen \#1 & PVC & 215.2 & 220.0 & 2.00 & Tb4 & \\
\hline $\mathrm{R}-12$ & R-12 Screen \#1 & SS304 & 459.0 & 467.5 & 4.50 & $\mathrm{~Tb}$ & \\
\hline $\mathrm{R}-12$ & R-12 Screen \#2 & SS304 & 504.5 & 508.0 & 4.50 & $\mathrm{Tp}$ & \\
\hline R-19 & R-19 Screen \#1 & SS304 & 827.2 & 843.6 & 4.50 & Qbog & Dry \\
\hline R-19 & R-19 Screen \#2 & SS304 & 893.3 & 909.6 & 4.50 & $\mathrm{Tp}$ & \\
\hline R-23i & R-23i Screen \#1 & SS304 & 400.3 & 420.0 & 2.10 & $\mathrm{~Tb} 4$ & \\
\hline$R-23 i$ & R-23i Screen \#2 & SS304 & 470.2 & 480.1 & 4.50 & Tb4 & \\
\hline R-23i & R-23i Screen \#3 & SS304 & 524.0 & 547.0 & 4.50 & $\mathrm{~Tb} 4$ & \\
\hline R-25 & R-25 Screen \#1 & SS304 & 737.6 & 758.4 & 5.17 & Qbo & \\
\hline $\mathrm{R}-25$ & R-25 Screen \#2 & SS304 & 882.6 & 893.4 & 5.17 & $\mathrm{Tp}$ & \\
\hline $\mathrm{R}-25$ & R-25 Screen \#3 damaged & SS304 & 1054.6 & 1064.6 & 5.17 & $T p$ & Dry \\
\hline R-25 & R-25 Screen \#4 & SS304 & 1184.6 & 1194.6 & 5.17 & $T p$ & \\
\hline $\mathrm{R}-26$ & R-26 Screen \#1 (Upper) & SS304 & 643.0 & 662.0 & 4.50 & Qct & \\
\hline R-26 PZ-1 & R-26 Piezometer Screen \#1 & PVC & 230.0 & 250.0 & & Qbt3 & Dry \\
\hline R-26 PZ-2 & R-26 Piezometer Screen \#2 & PVC & 150.0 & 180.0 & & Qbt3 & Dry \\
\hline R-31 & R-31 Screen \#1 & SS304 & 439.1 & 454.4 & 4.50 & $\mathrm{~Tb}$ & Dry \\
\hline $\mathrm{R}-5$ & R-5 Screen \#1 & SS304 & 326.4 & 331.5 & 4.50 & $T p$ & Dry \\
\hline $\mathrm{R}-5$ & R-5 Screen \#2 & SS304 & 372.8 & 388.8 & 4.50 & $T p$ & \\
\hline R-6i & R-6i Screen \#1 & SS304 & 602.0 & 612.0 & 4.46 & Tpf & \\
\hline R-7 & R-7 Screen \#1 & SS304 & 363.2 & 379.2 & 4.50 & $\mathrm{Tp}$ & Dry since 2005 \\
\hline R-7 & R-7 Screen \#2 & SS304 & 730.4 & 746.4 & 4.50 & $\overline{T p}$ & Dry \\
\hline R-9i & R-9i Screen \#1 & SS304 & 189.1 & 199.5 & 5.00 & $\mathrm{~Tb}$ & \\
\hline$R-9 i$ & R-9i Screen \#2 & SS304 & 269.6 & 280.3 & 5.00 & $\mathrm{~Tb}$ & \\
\hline $\mathrm{SCl}-1$ & SCl-1 Screen \#1 & PVC & 358.4 & 377.9 & 3.80 & Tpf & \\
\hline Test Well 1A & TW-1A Screen \#1 & CS & 215.0 & 225.0 & 6.00 & $\mathrm{~Tb}$ & \\
\hline Test Well 2A & TW-2A Screen \#1a & CS & 123.0 & 133.0 & 6.00 & $\mathrm{Tp}$ & \\
\hline
\end{tabular}

Note: $\mathrm{SS}=$ stainless steel, $\mathrm{PVC}=$ polyvinyl chloride, $\mathrm{Qbo}=$ Otowi Member of the Bandelier Tuff, $\mathrm{Tp}=$ Puye Formation, Qbog = Guaje Pumice member of the Bandelier Tuff, Tpf $=$ fluvial facies of the Puye Formation, $\mathrm{Tb}=$ undifferentiated basalt, Tb4 = Cerros del Rio Basaltic Rocks; Qbt3 = Unit 3 of the Tshirege Member of the Bandelier Tuff, Tt = Tschicoma Formation (dacite). 
The following sections include additional port and construction information for single and multiple completion intermediate wells at LANL. Time-series groundwater level data are shown for each well.

\subsection{LP-SE-16-02669}

Location: 90LP-SE-16-02669 is located at TA-16 downgradient of the 90LP Pond.

Completion Type: Single completion in an intermediate zone in Unit 3 of the Bandelier Tuff.

Period of Record: Well drilled in March 1998, periodic measurements through 2007.

Remarks: The borehole contained water at the completion of drilling, but since completion of the well no water has been observed in the well; the well was last checked March 16, 2007.

\begin{tabular}{|c|c|c|c|c|c|c|c|c|c|c|c|c|c|}
\hline \multirow[b]{2}{*}{ Zone } & \multicolumn{13}{|c|}{ 90LP-SE-16-02669 Construction Information } \\
\hline & $\begin{array}{c}\text { Screen } \\
\text { Top } \\
\text { Depth (ft) }\end{array}$ & $\begin{array}{c}\text { Screen } \\
\text { Bottom } \\
\text { Depth } \\
\text { (ft) }\end{array}$ & $\begin{array}{l}\text { Screen } \\
\text { Top } \\
\text { Elev (ft) }\end{array}$ & $\begin{array}{l}\text { Screen } \\
\text { Bottom } \\
\text { Elev (ft) }\end{array}$ & $\begin{array}{l}\text { Screen } \\
\text { Length } \\
\text { (ft) }\end{array}$ & $\begin{array}{l}\text { Pump } \\
\text { Intake } \\
\text { Depth } \\
\text { (ft) }\end{array}$ & $\begin{array}{l}\text { Pump } \\
\text { Intake } \\
\text { Elevation } \\
\text { (ft) }\end{array}$ & $\begin{array}{l}\text { Depth to } \\
\text { Top of } \\
\text { Sump (ft) }\end{array}$ & $\begin{array}{l}\text { Top of } \\
\text { Sump } \\
\text { Elevation } \\
\text { (ft) }\end{array}$ & $\begin{array}{l}\text { Depth to } \\
\text { Sump } \\
\text { Bottom (ft) }\end{array}$ & $\begin{array}{l}\text { Sump } \\
\text { Length } \\
\text { (ft) }\end{array}$ & $\begin{array}{l}\text { Sump } \\
\text { Volume } \\
\text { (L) }\end{array}$ & Comment \\
\hline & 131.5 & 163.0 & 7451.8 & 7420.3 & 31.5 & & & 163.0 & 7420.3 & 163.4 & 0.4 & 0.2 & termediate Zone \\
\hline
\end{tabular}




\subsection{CdV-16-1(i)}

Location: CdV-16-1(i) is located at TA-16 downgradient of the TA-6-260 outfall.

Completion Type: Single completion in an intermediate zone.

Period of Record: Well drilled in 2003. Transducer installed September 2005; data through 2007.

Remarks: Well completed in an intermediate zone in the Otowi Member of the Tshirege Formation; the water level is about $50 \mathrm{ft}$ above the top of the screen. The well is $100 \%$ barometrically efficient; the groundwater does not respond to atmospheric pressure fluctuations. The intermediate groundwater rose about $1 \mathrm{ft}$ in response to snowmelt runoff in the spring of 2007.

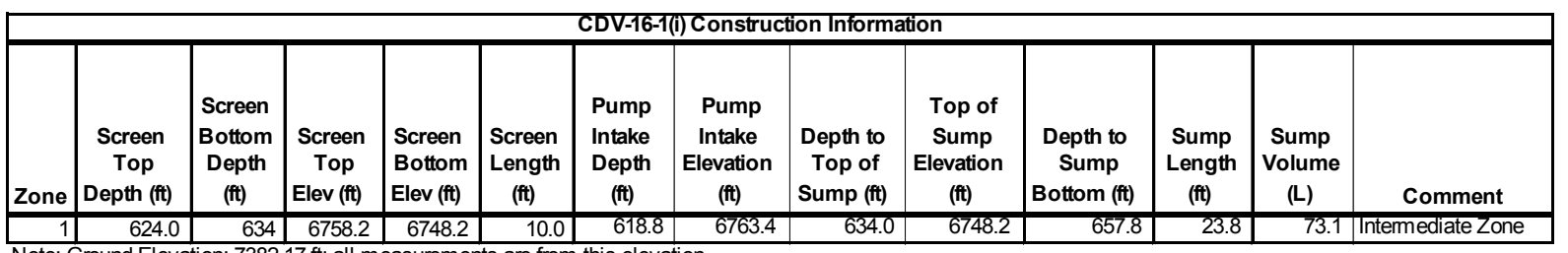

Note: Ground Elevation: $7382.17 \mathrm{ft}$; all measurements are from this elevation

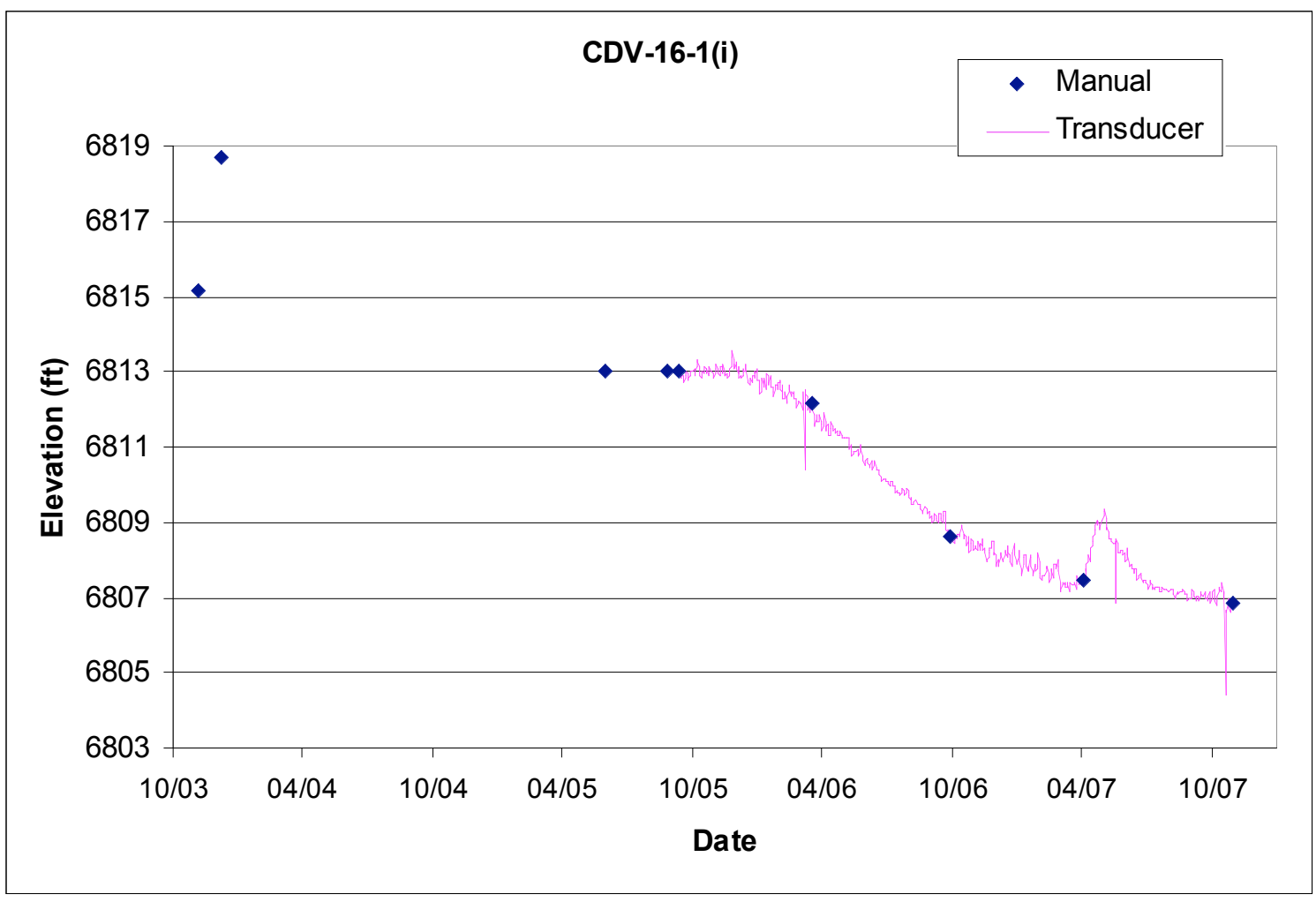




\subsection{CdV-16-2(i)r}

Location: CdV-16-2(i)r is located at TA-16 downgradient of the TA-6-260 outfall.

Completion Type: Single completion in intermediate zone in the Puye Formation.

Period of Record: Well completed in July 2005, periodic manual measurements in 2005. A transducer was installed February 16, 2006; data through 2007.

Remarks: Well replaces CdV-16-2(i). The water level is about $20 \mathrm{ft}$ above bottom of screen. The well is about $90 \%$ barometrically efficient. The groundwater did not indicate a response to snowmelt runoff in 2007.

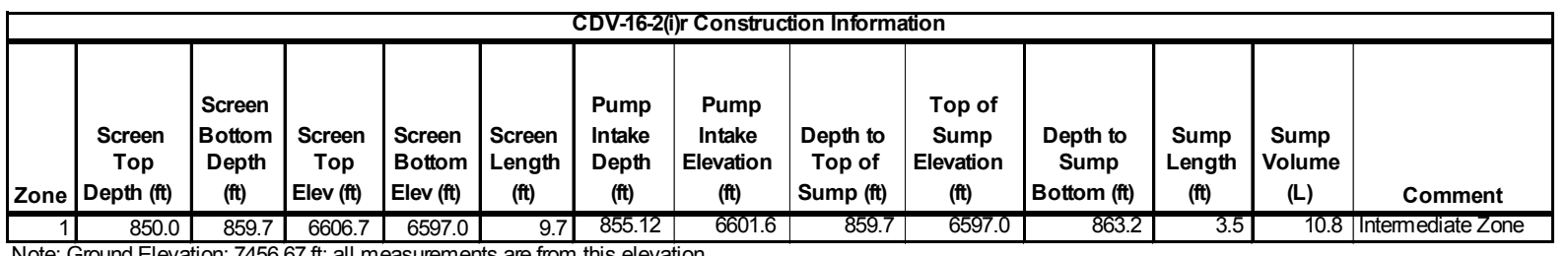

Note: Ground Elevation: $7456.67 \mathrm{ft}$; all measurements are from this elevation

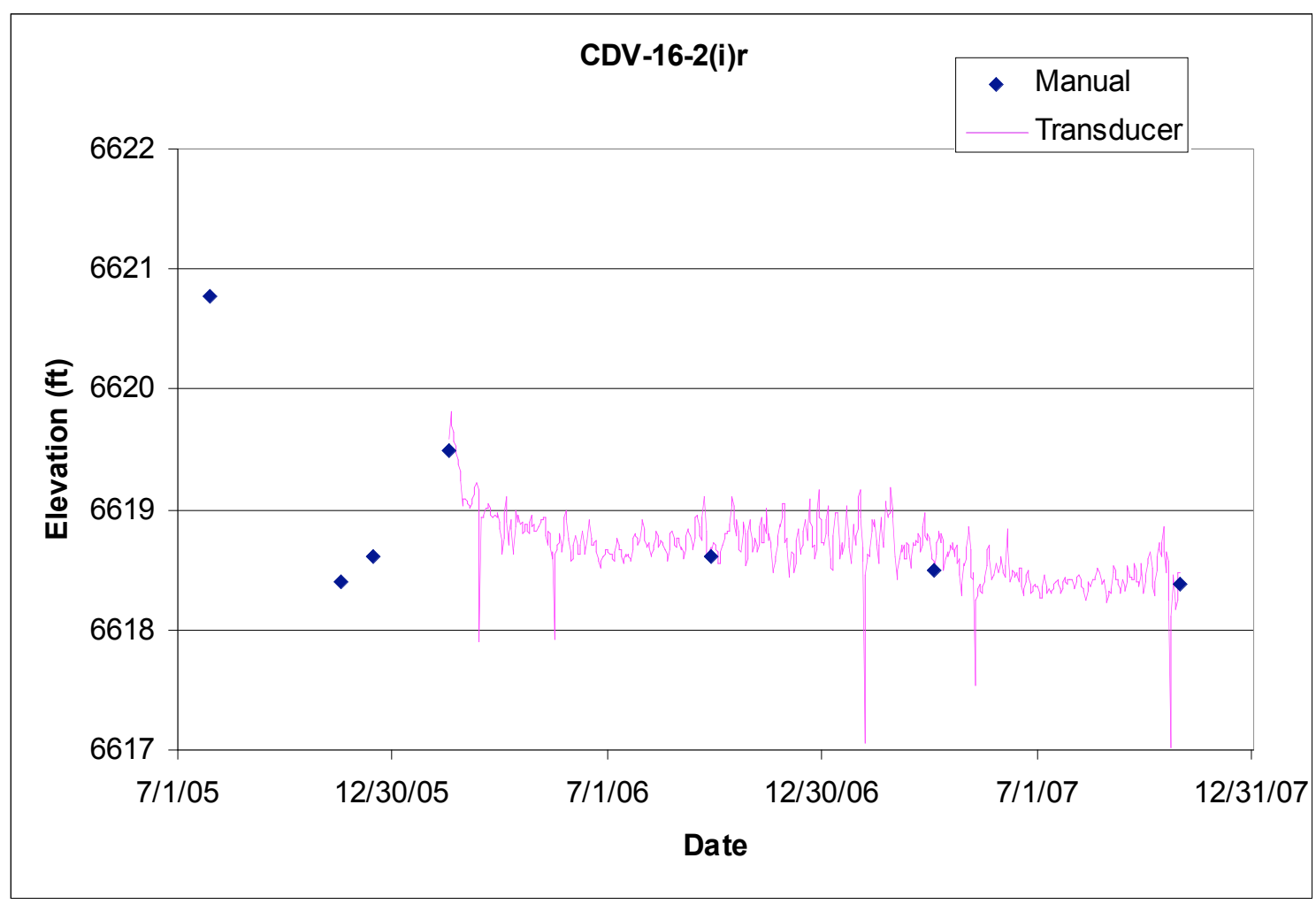




\subsection{CdV-16-3(i)}

Location: CdV-16-3(i) is located at TA-16 downgradient of the TA-6-260 outfall.

Completion Type: Open borehole, well not installed.

Period of Record: Well drilled in 2004, periodic manual measurements through 2005. Well monitoring ceased in 2005.

Remarks: Water not present in borehole when drilling was completed. Since January 2004, the borehole has contained about $40 \mathrm{ft}$ of water. Note that the water level is similar to the top of the regional aquifer at CdV-R-37-2.

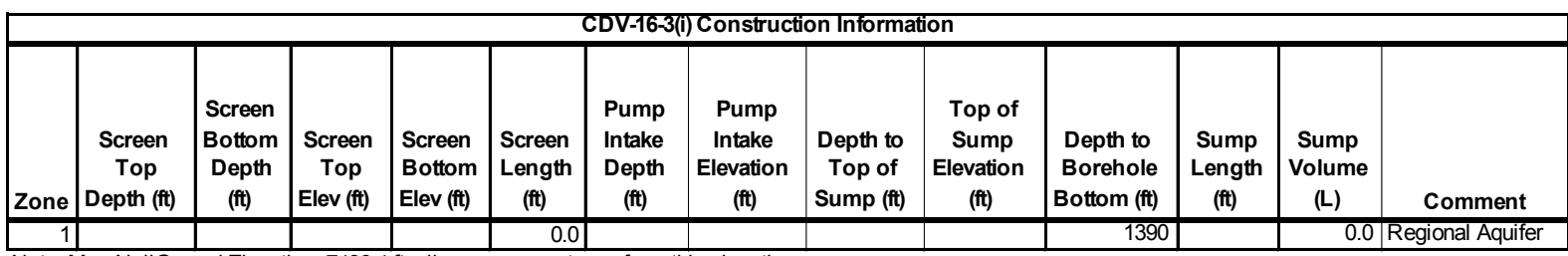

Note: Mag NailGround Elevation: $7486.4 \mathrm{ft}$; all measurements are from this elevation;

Well not completed, open borehole with surface pad and protective casing

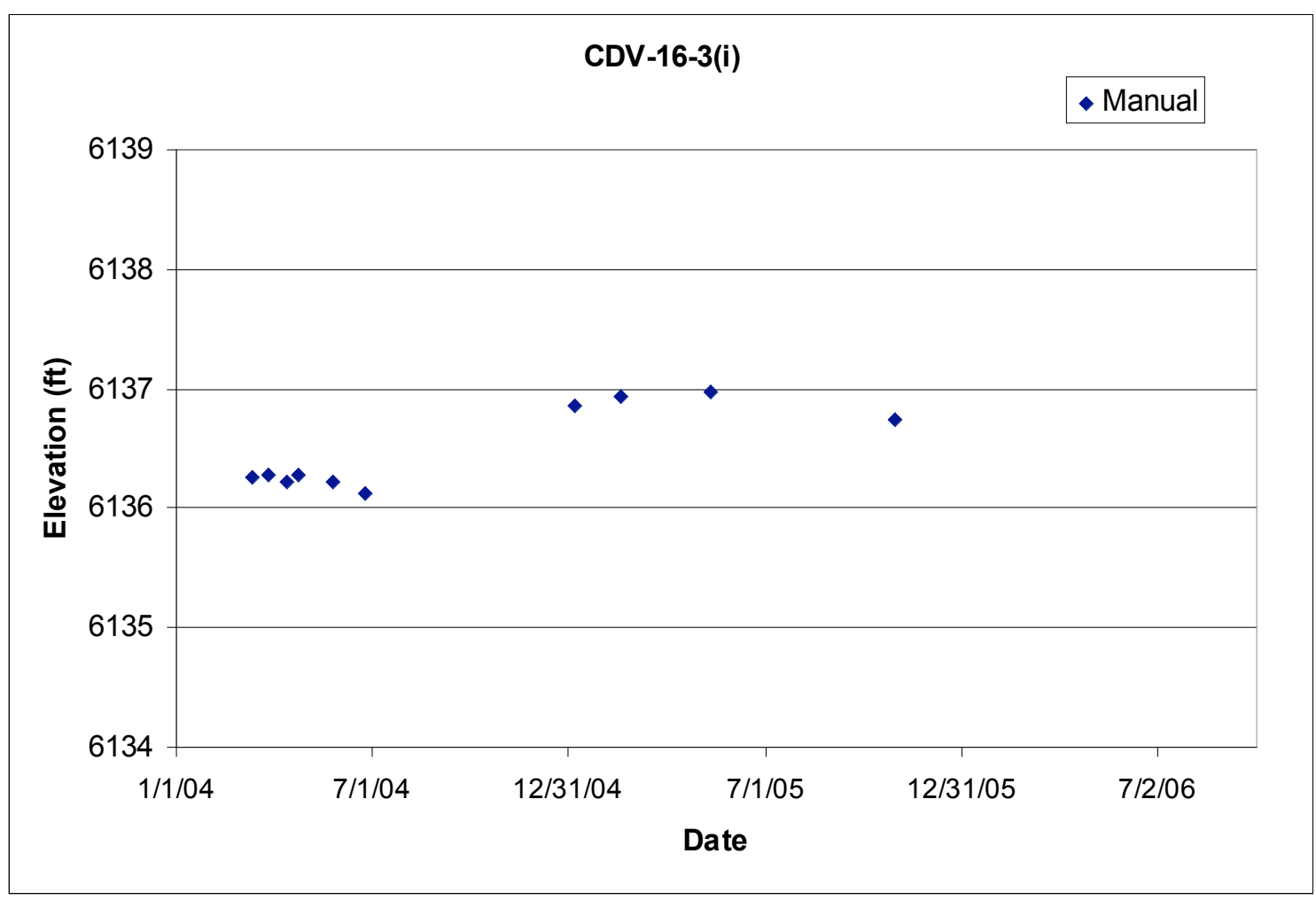




\subsection{LADP-3}

Location: LADP-3 is located in middle Los Alamos Canyon upstream of DP Canyon.

Completion Type: Single completion in an intermediate zone in the Guaje Pumice bed.

Period of Record: Well drilled in 1993. Transducer first installed May 1994, reinstalled in May 2005, intermittent transducer data through 2007.

Remarks: No manual measurement available for April 2002 transducer installation, data from April 2002 to November 2003 questionable. The water level declined below the transducer in April 2006 and recovered in November 2006. The well is $100 \%$ barometrically efficient; the groundwater does not respond to atmospheric pressure fluctuations. The groundwater did not indicate a response to snowmelt runoff in 2007.

\begin{tabular}{|c|c|c|c|c|c|c|c|c|c|c|c|c|c|}
\hline \multicolumn{14}{|c|}{ LADP-3 Construction Information } \\
\hline Zone & $\begin{array}{c}\text { Screen } \\
\text { Top } \\
\text { Depth (ft) }\end{array}$ & $\begin{array}{c}\text { Screen } \\
\text { Bottom } \\
\text { Depth } \\
\text { (ft) }\end{array}$ & $\begin{array}{c}\text { Screen } \\
\text { Top } \\
\text { Elev (ft) }\end{array}$ & $\begin{array}{l}\text { Screen } \\
\text { Bottom } \\
\text { Elev (ft) }\end{array}$ & $\begin{array}{l}\text { Screen } \\
\text { Length } \\
\text { (ft) }\end{array}$ & $\begin{array}{c}\text { Pump } \\
\text { Intake } \\
\text { Depth } \\
\text { (ft) }\end{array}$ & $\begin{array}{l}\text { Pump } \\
\text { Intake } \\
\text { Elevation } \\
\text { (ft) }\end{array}$ & $\begin{array}{c}\text { Depth to } \\
\text { Top of } \\
\text { Sump (ft) }\end{array}$ & $\begin{array}{l}\text { Top of } \\
\text { Sump } \\
\text { Elevation } \\
\text { (ft) }\end{array}$ & $\begin{array}{l}\text { Depth to } \\
\text { Sump } \\
\text { Bottom (ft) }\end{array}$ & $\begin{array}{l}\text { Sump } \\
\text { Length } \\
\text { (ft) }\end{array}$ & $\begin{array}{c}\text { Sump } \\
\text { Volume } \\
\text { (L) }\end{array}$ & Comment \\
\hline 1 & 316.0 & 326 & 6440.7 & 6430.7 & 10.0 & & & 326.0 & 6430.7 & 326 & 0.0 & 0.0 & Intermediate Zone \\
\hline
\end{tabular}

\section{LADP-3}

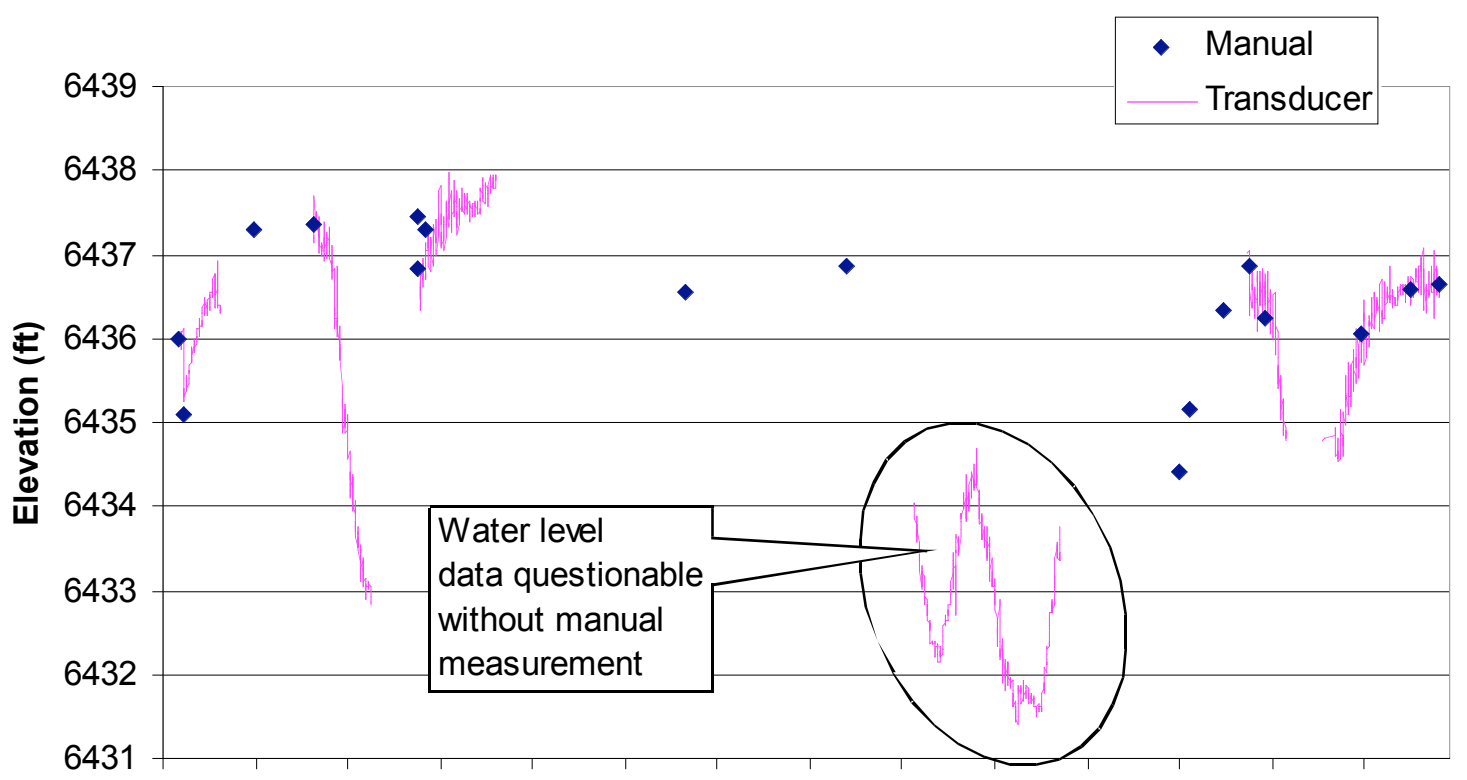

03/94 03/95 02/96 02/97 03/98 03/99 02/00 02/01 03/02 03/03 02/04 02/05 03/06

Date 


\subsection{LAOI(a)-1.1}

Location: LAOI(a)-1.1 is located in middle Los Alamos Canyon downstream of TA-2 and TA-41. Completion Type: Single completion in an intermediate zone in the Guaje Pumice bed.

Period of Record: Well drilled in 1994. Transducer initially installed June 1997, reinstalled in April 2005; transducer data through 2007.

Remarks: The well is $100 \%$ barometrically efficient; the groundwater does not respond to atmospheric pressure fluctuations. The groundwater did not indicate a response to snowmelt runoff in 2007.

\begin{tabular}{|c|c|c|c|c|c|c|c|c|c|c|c|c|c|}
\hline \multicolumn{14}{|c|}{ LAOI(A)-1.1 Construction Information } \\
\hline Zone & $\begin{array}{c}\text { Screen } \\
\text { Top } \\
\text { Depth (ft) }\end{array}$ & $\begin{array}{l}\text { Screen } \\
\text { Bottom } \\
\text { Depth } \\
\text { (ft) }\end{array}$ & $\begin{array}{c}\text { Screen } \\
\text { Top } \\
\text { Elev (ft) }\end{array}$ & $\begin{array}{l}\text { Screen } \\
\text { Bottom } \\
\text { Elev (ft) }\end{array}$ & $\begin{array}{l}\text { Screen } \\
\text { Length } \\
\text { (ft) }\end{array}$ & $\begin{array}{l}\text { Pump } \\
\text { Intake } \\
\text { Depth } \\
\text { (ft) }\end{array}$ & $\begin{array}{l}\text { Pump } \\
\text { Intake } \\
\text { Elevation } \\
\text { (ft) }\end{array}$ & $\begin{array}{c}\text { Depth to } \\
\text { Top of } \\
\text { Sump (ft) }\end{array}$ & $\begin{array}{l}\text { Top of } \\
\text { Sump } \\
\text { Elevation } \\
\text { (ft) }\end{array}$ & $\begin{array}{c}\text { Depth to } \\
\text { Sump } \\
\text { Bottom (ft) }\end{array}$ & $\begin{array}{l}\text { Sump } \\
\text { Length } \\
\text { (ft) }\end{array}$ & $\begin{array}{l}\text { Sump } \\
\text { Volume } \\
\text { (L) }\end{array}$ & Comment \\
\hline & 295.2 & 305 & 6540.0 & 6530.2 & 9.8 & & 6835.2 & 305.0 & 6530.2 & 309.8 & 4.8 & 6.7 & Intermediate Zone \\
\hline
\end{tabular}

Note: LAOI(A)-1.1 Ground Elevation: $6835.2 \mathrm{ft}$; all measurements are from this elevation

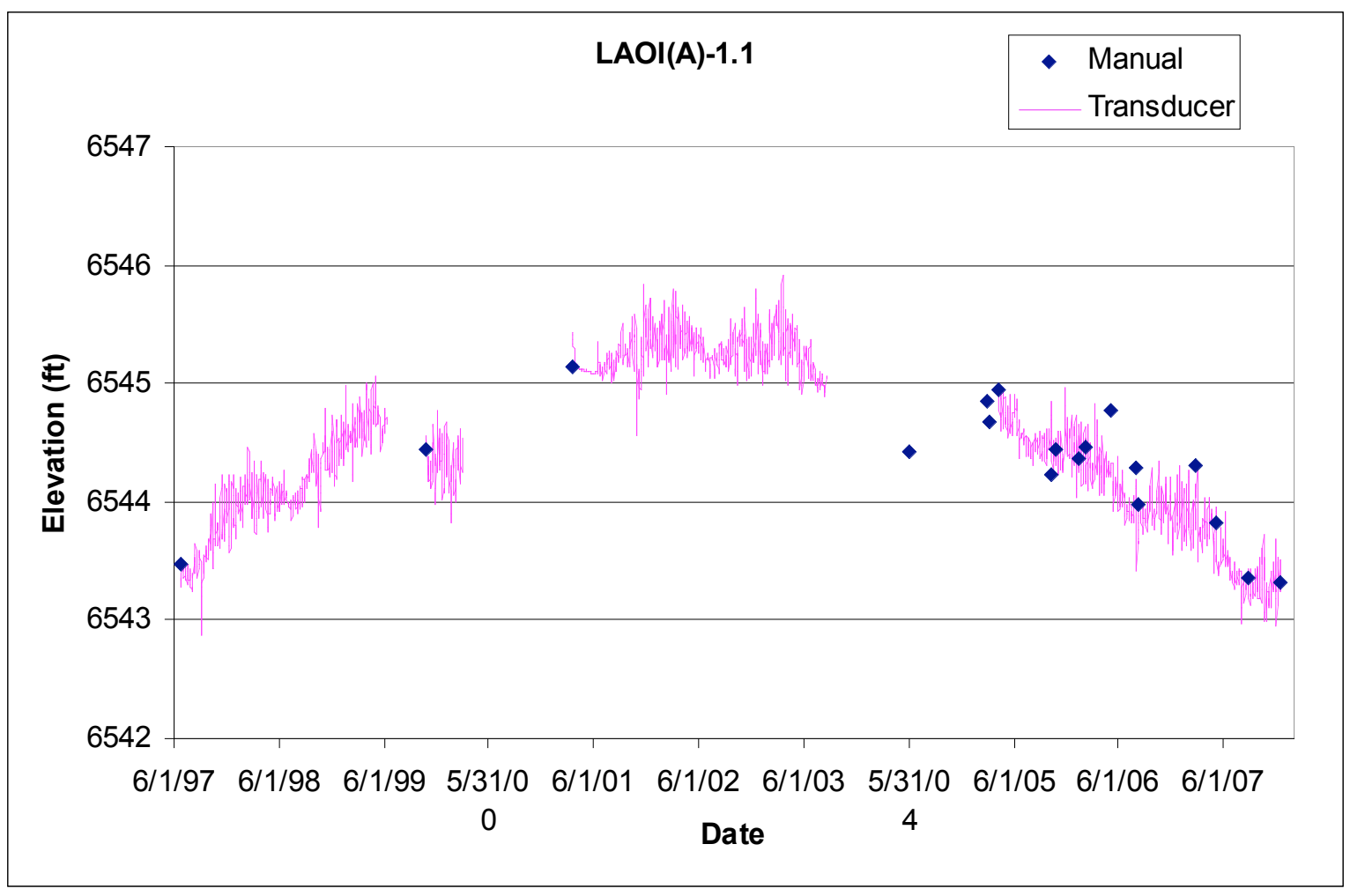




\subsection{LAOI-3.2}

Location: LAOI-3.2 is located in middle Los Alamos Canyon at the confluence with DP Canyon.

Completion Type: Single completion in an intermediate zone in basalt.

Period of Record: Well completed in May 2005. Transducer installed September 2005; transducer data through 2007.

Remarks: The transducer was removed in October 2005 for pump installation. The transducer was reinstalled in November 2005 after the pump was installed. The water level declined below the level of the transducer for a time during pumping of the well in December 2005. The well is $100 \%$ barometrically efficient; the groundwater does not respond to atmospheric pressure fluctuations. The groundwater did not indicate a response to snowmelt runoff in 2007.

\begin{tabular}{|c|c|c|c|c|c|c|c|c|c|c|c|c|c|}
\hline \multicolumn{14}{|c|}{ LAOI-3.2 Construction Information } \\
\hline Zone & $\begin{array}{l}\text { Screen } \\
\text { Top } \\
\text { Depth (ft) }\end{array}$ & $\begin{array}{l}\text { Screen } \\
\text { Bottom } \\
\text { Depth } \\
\text { (ft) }\end{array}$ & $\begin{array}{c}\text { Screen } \\
\text { Top } \\
\text { Elev (ft) }\end{array}$ & $\begin{array}{l}\text { Screen } \\
\text { Bottom } \\
\text { Elev (ft) }\end{array}$ & $\begin{array}{l}\text { Screen } \\
\text { Length } \\
\text { (ft) }\end{array}$ & $\begin{array}{l}\text { Pump } \\
\text { Intake } \\
\text { Depth } \\
\text { (ft) }\end{array}$ & $\begin{array}{c}\text { Pump } \\
\text { Intake } \\
\text { Elevation } \\
\text { (ft) }\end{array}$ & $\begin{array}{l}\text { Depth to } \\
\text { Top of } \\
\text { Sump (ft) }\end{array}$ & $\begin{array}{l}\text { Top of } \\
\text { Sump } \\
\text { Elevation } \\
\text { (ft) }\end{array}$ & $\begin{array}{l}\text { Depth to } \\
\text { Sump } \\
\text { Bottom (ft) }\end{array}$ & $\begin{array}{l}\text { Sump } \\
\text { Length } \\
\text { (ft) }\end{array}$ & $\begin{array}{c}\text { Sump } \\
\text { Volume } \\
\text { (L) }\end{array}$ & Comment \\
\hline 1 & 153.3 & 162.8 & 6469.3 & 6459.8 & 9.5 & 159.3 & 6463.3 & 162.8 & 6459.8 & 165 & 2.2 & 1.5 & Intermediate Zone \\
\hline
\end{tabular}

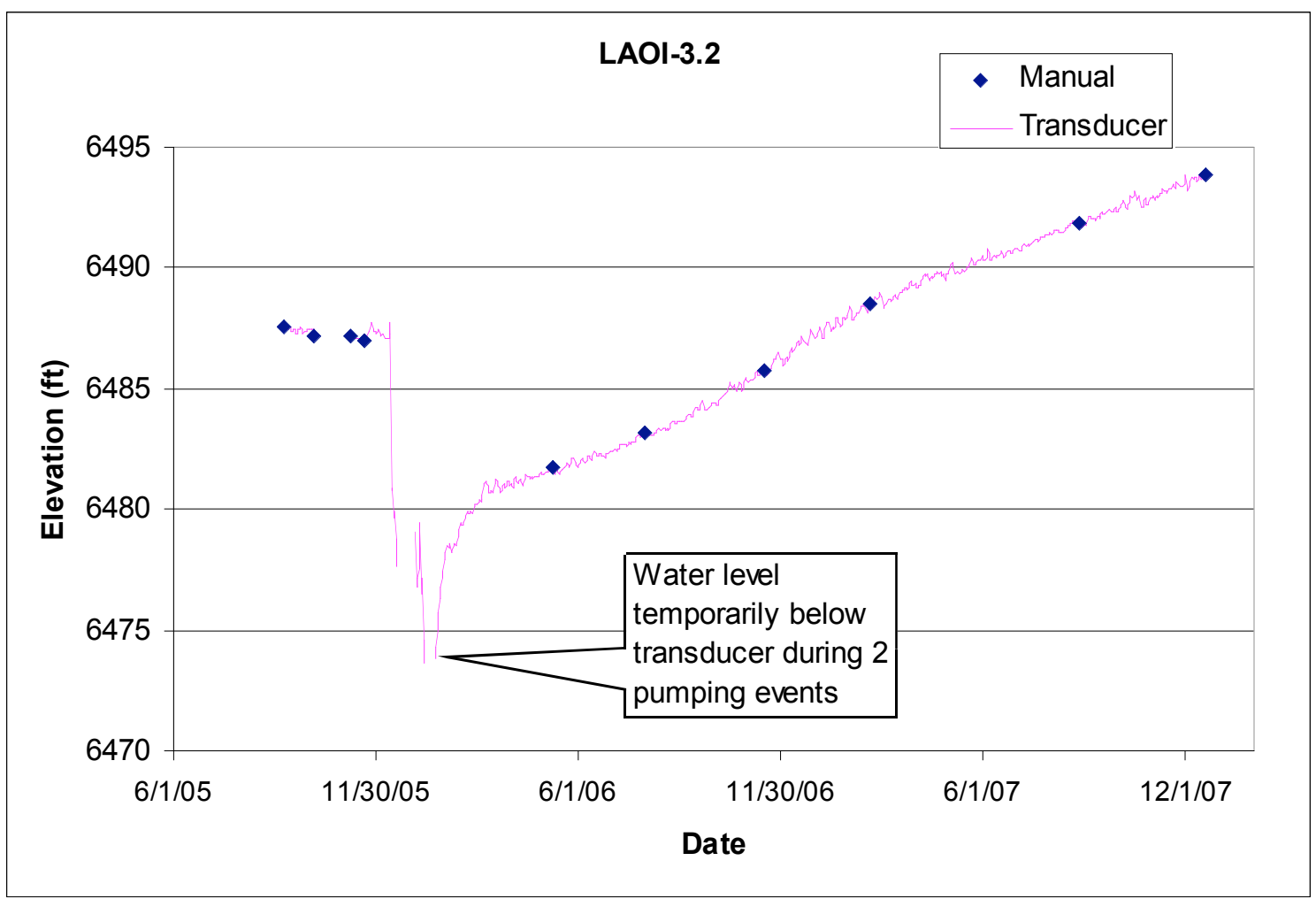




\subsection{LAOI-3.2a}

Location: LAOI-3.2a is located in middle Los Alamos Canyon near the confluence with DP Canyon and about $50 \mathrm{ft}$ northwest of LAOI-3.2.

Completion Type: Single completion in an intermediate zone in Puye fanglomerate.

Period of Record: Well completed in January 2006. Transducer installed August 2006; transducer data through 2007.

Remarks: The water level is about $6 \mathrm{ft}$ above the bottom of the screen. The well is about $50 \%$ barometrically efficient. The groundwater did not indicate a response to snowmelt runoff in 2007.

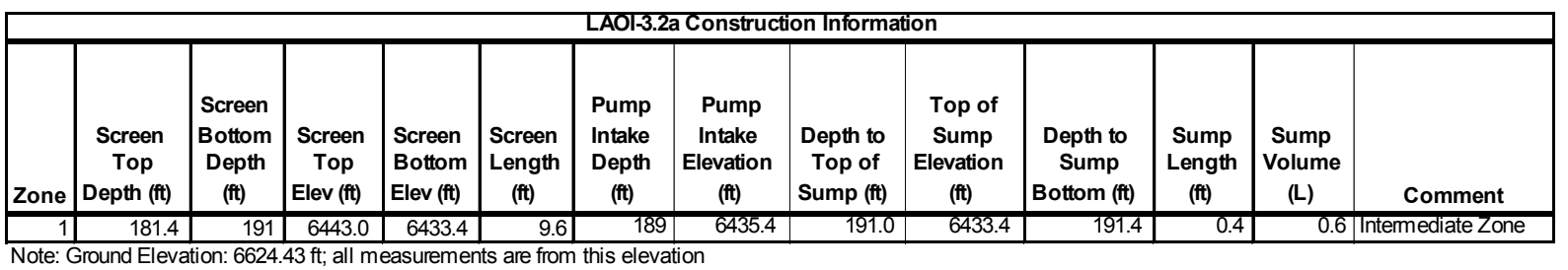

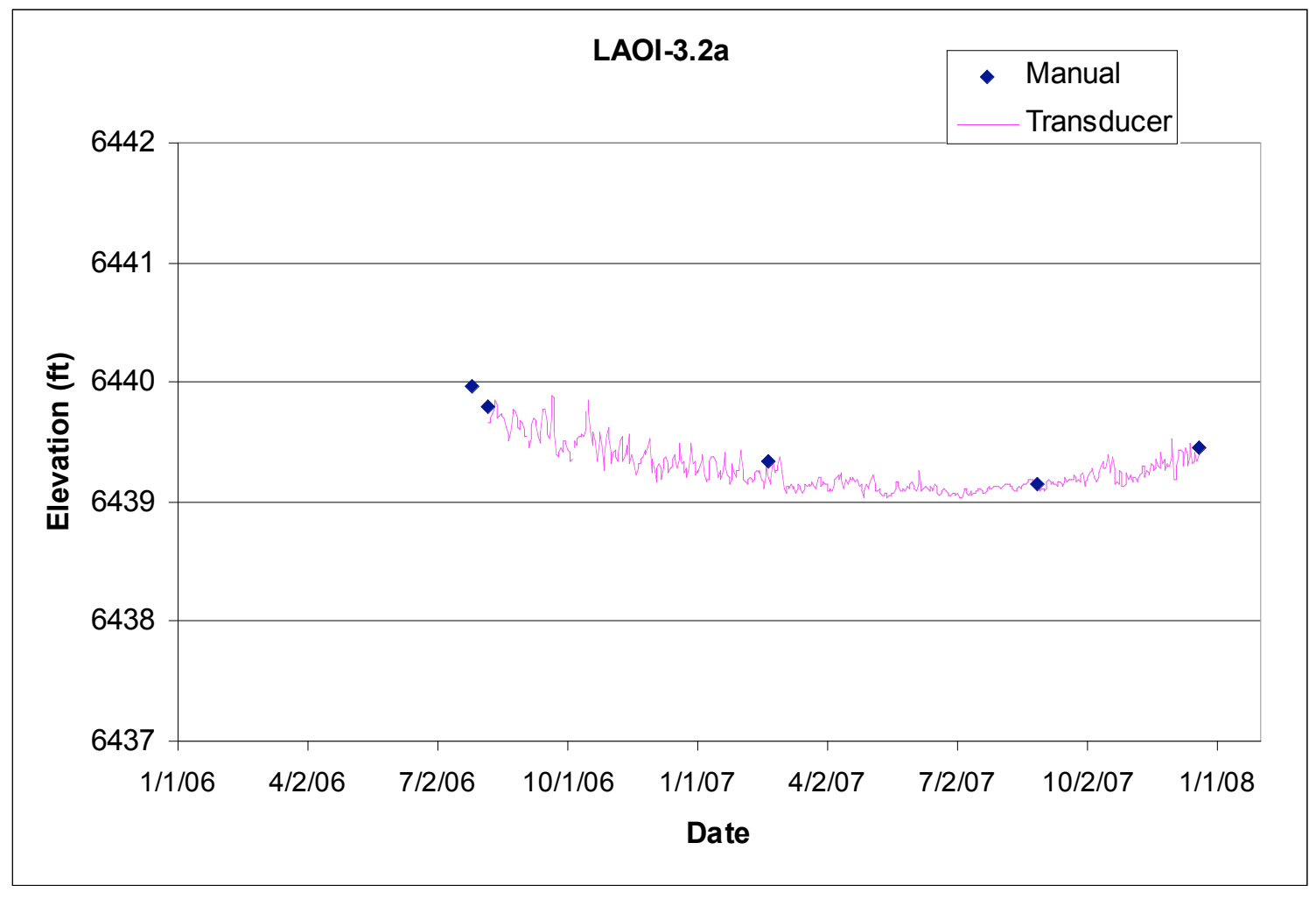




\section{$4.9 \quad$ LAOI-7}

Location: LAOI-7 is located in middle Los Alamos Canyon about 0.75 miles upstream of R-9i.

Completion Type: Single completion in an intermediate zone in basalt.

Period of Record: Well completed in September 2005, transducer installed May 2006, data through 2007.

Remarks: The well has an estimated 18\% barometric efficiency (Kleinfelder 2006a); the groundwater shows a delayed, partial response to atmospheric pressure fluctuations. The groundwater rose about $11 \mathrm{ft}$ in response to snowmelt runoff in 2007.

\begin{tabular}{|c|c|c|c|c|c|c|c|c|c|c|c|c|c|}
\hline \multicolumn{14}{|c|}{ LAOI-7 Construction Information } \\
\hline Zone & $\begin{array}{c}\text { Screen } \\
\text { Top } \\
\text { Depth (ft) }\end{array}$ & $\begin{array}{c}\text { Screen } \\
\text { Bottom } \\
\text { Depth } \\
\text { (ft) }\end{array}$ & $\begin{array}{c}\text { Screen } \\
\text { Top } \\
\text { Elev (ft) }\end{array}$ & $\begin{array}{l}\text { Screen } \\
\text { Bottom } \\
\text { Elev (ft) }\end{array}$ & $\begin{array}{l}\text { Screen } \\
\text { Length } \\
\text { (ft) }\end{array}$ & $\begin{array}{l}\text { Pump } \\
\text { Intake } \\
\text { Depth } \\
\text { (ft) }\end{array}$ & $\begin{array}{l}\text { Pump } \\
\text { Intake } \\
\text { Elevation } \\
\text { (ft) }\end{array}$ & $\begin{array}{c}\text { Depth to } \\
\text { Top of } \\
\text { Sump (ft) }\end{array}$ & $\begin{array}{c}\text { Top of } \\
\text { Sump } \\
\text { Elevation } \\
\text { (ft) }\end{array}$ & $\begin{array}{l}\text { Depth to } \\
\text { Sump } \\
\text { Bottom (ft) }\end{array}$ & $\begin{array}{l}\text { Sump } \\
\text { Length } \\
\text { (ft) }\end{array}$ & $\begin{array}{l}\text { Sump } \\
\text { Volume } \\
\text { (L) }\end{array}$ & Comment \\
\hline 1 & 240.0 & 259.6 & 6218.4 & 6198.8 & 19.6 & 240 & 6218.4 & 259.6 & 6198.8 & 264.9 & 5.3 & 7.4 & Intermediate Zone \\
\hline
\end{tabular}

Note: Brass Cap Elevation: $6458.35 \mathrm{ft}$; all measurements are from this elevation

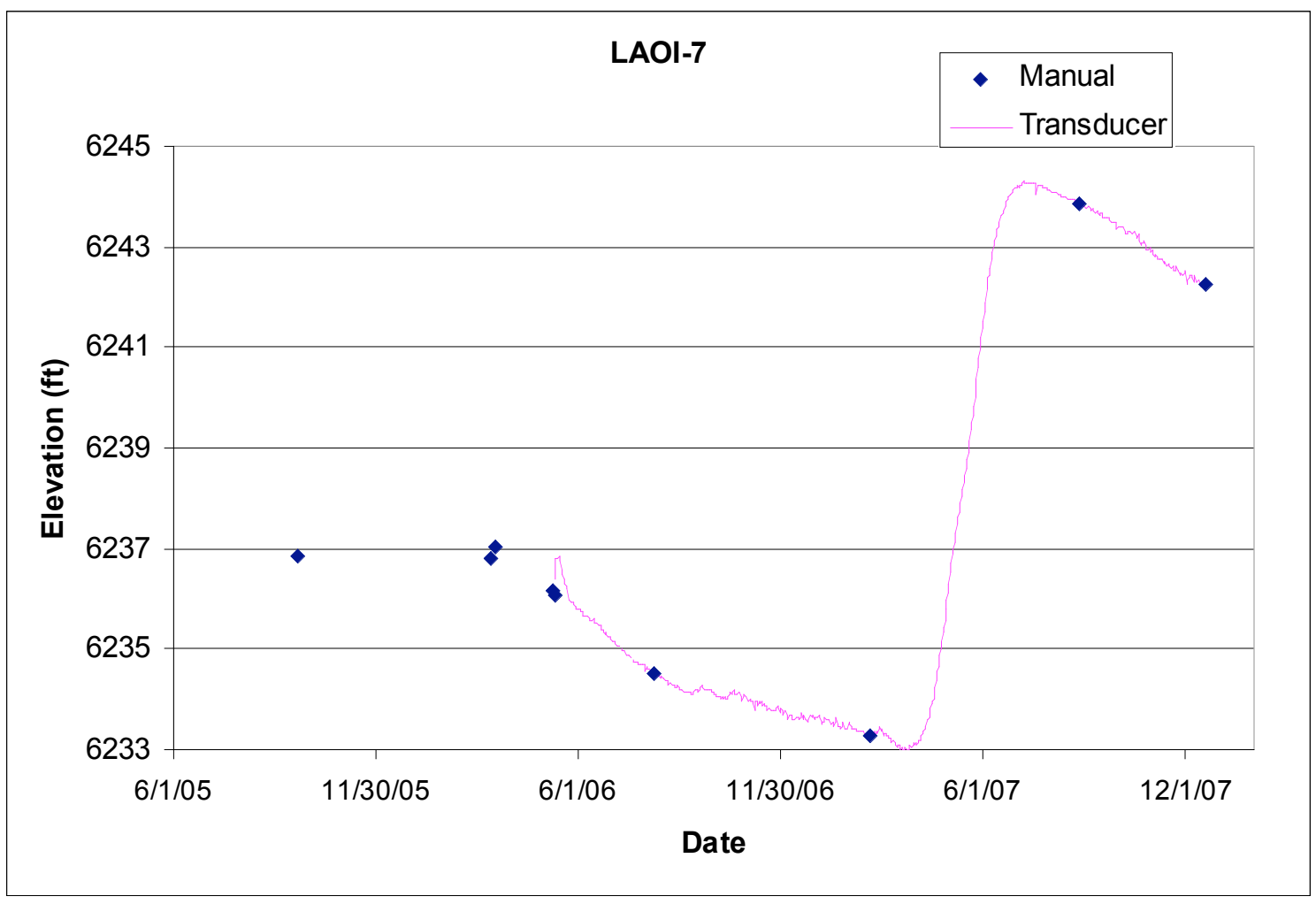




\subsection{0 МСОВТ-4.4}

Location: MCOBT-4.4 is located in lower Mortandad Canyon near the confluence with Ten Site Canyon.

Completion Type: Single completion at the base of the Puye Formation fanglomerate member and the top of basalt.

Period of Record: Well completed in June 2001, transducer installed July 2002, data through 2007.

Remarks: MCOI-4 is located about $70 \mathrm{ft}$ west of MCOBT-4.4. The bottom of the transducer gage tube is located above the pump and about $1.2 \mathrm{ft}$ above the bottom of the screen. The water level declined below the gage tube for portions of 2006 and most of 2007. The water level appeared to remain about $1 \mathrm{ft}$ above the bottom of the screen.

\begin{tabular}{|c|c|c|c|c|c|c|c|c|c|c|c|c|c|}
\hline \multirow[b]{2}{*}{ Zone } & \multicolumn{12}{|c|}{ MСОВТ-4.4 Construction Information } & \\
\hline & $\begin{array}{c}\text { Screen } \\
\text { Top } \\
\text { Depth (ft) }\end{array}$ & $\begin{array}{l}\text { Screen } \\
\text { Bottom } \\
\text { Depth } \\
\text { (ft) }\end{array}$ & $\begin{array}{l}\text { Screen } \\
\text { Top } \\
\text { Elev (ft) }\end{array}$ & $\begin{array}{l}\text { Screen } \\
\text { Bottom } \\
\text { Elev (ft) }\end{array}$ & $\begin{array}{l}\text { Screen } \\
\text { Length } \\
\text { (ft) }\end{array}$ & $\begin{array}{l}\text { Pump } \\
\text { Intake } \\
\text { Depth } \\
\text { (ft) }\end{array}$ & $\begin{array}{l}\text { Pump } \\
\text { Intake } \\
\text { Elevation } \\
\text { (ft) }\end{array}$ & $\begin{array}{l}\text { Depth to } \\
\text { Top of } \\
\text { Sump (ft) }\end{array}$ & $\begin{array}{c}\text { Top of } \\
\text { Sump } \\
\text { Elevation } \\
\text { (ft) }\end{array}$ & $\begin{array}{l}\text { Depth to } \\
\text { Sump } \\
\text { Bottom (ft) }\end{array}$ & $\begin{array}{l}\text { Sump } \\
\text { Length } \\
\text { (ft) }\end{array}$ & $\begin{array}{c}\text { Sump } \\
\text { Volume } \\
\text { (L) }\end{array}$ & Comment \\
\hline 1 & 485.4 & 524 & 6350.8 & 6312.2 & 38.6 & 524 & 6312.2 & 524.0 & 6312.2 & 545 & 21.0 & 64.5 & Intermediate Zone \\
\hline
\end{tabular}

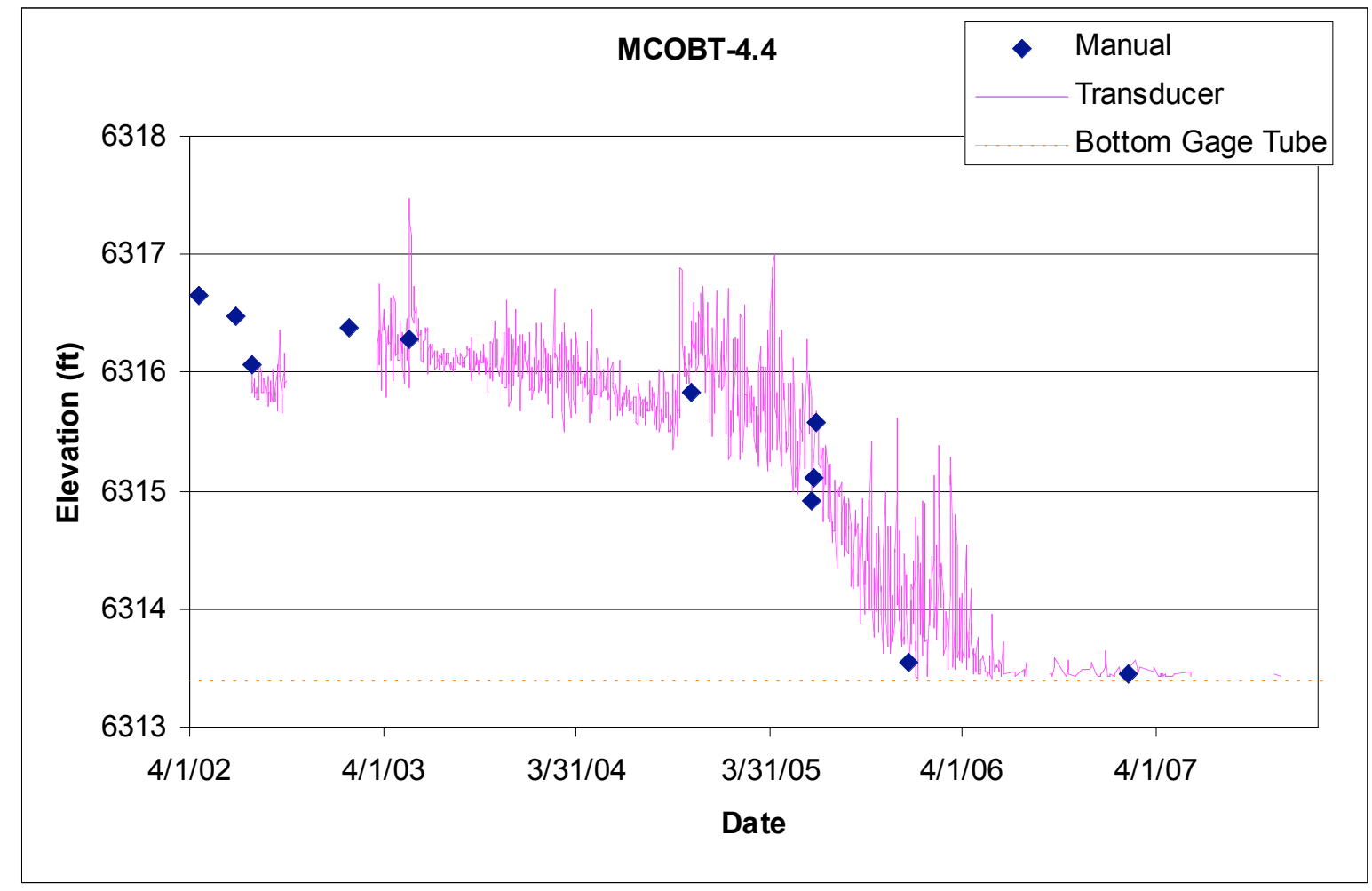




\subsection{MCOI-1}

Location: MCOI-1 is located adjacent to upper Mortandad Canyon below the confluence with Effluent Canyon.

Completion Type: Single completion in the Puye Formation.

Period of Record: Well completed in January 2005. Periodic manual checks for water through 2006.

Remarks: MCOI-1 was dry when completed and has not contained water during periodic checks.

Soundings for water throughout 2006 and 2007 have been dry with a total depth of about 814 $\mathrm{ft}$ below ground surface, encountering sand at total depth. This total depth is above the screen; thus it appears that the well screen in the 1-in.-diameter PVC may have parted from the tubing or has been somehow damaged, potentially rendering the well inoperative.

\begin{tabular}{|c|c|c|c|c|c|c|c|c|c|c|c|c|c|}
\hline \multicolumn{14}{|c|}{ MCOI-1 Construction Information } \\
\hline Zone & $\begin{array}{c}\text { Screen } \\
\text { Top } \\
\text { Depth (ft) }\end{array}$ & \begin{tabular}{|c} 
Screen \\
Bottom \\
Depth \\
(ft)
\end{tabular} & $\begin{array}{c}\text { Screen } \\
\text { Top } \\
\text { Elev (ft) }\end{array}$ & $\begin{array}{l}\text { Screen } \\
\text { Bottom } \\
\text { Elev (ft) }\end{array}$ & $\begin{array}{l}\text { Screen } \\
\text { Length } \\
\text { (ft) }\end{array}$ & $\begin{array}{c}\text { Pump } \\
\text { Intake } \\
\text { Depth } \\
\text { (ft) }\end{array}$ & $\begin{array}{c}\text { Pump } \\
\text { Intake } \\
\text { Elevation } \\
\text { (ft) }\end{array}$ & $\begin{array}{l}\text { Depth to } \\
\text { Top of } \\
\text { Sump (ft) }\end{array}$ & $\begin{array}{c}\text { Top of } \\
\text { Sump } \\
\text { Elevation } \\
\text { (ft) }\end{array}$ & $\begin{array}{c}\text { Depth to } \\
\text { Sump } \\
\text { Bottom (ft) }\end{array}$ & $\begin{array}{l}\text { Sump } \\
\text { Length } \\
\text { (ft) }\end{array}$ & $\begin{array}{l}\text { Sump } \\
\text { Volume } \\
\text { (L) }\end{array}$ & Comment \\
\hline 1 & 815.0 & 825.5 & 6291.2 & 6280.8 & 10.5 & & 7106.2 & 825.5 & 6280.8 & 825.58 & 0.1 & 0.0 & Intermediate Zone \\
\hline
\end{tabular}

Note: Ground Elevation: $7106.20 \mathrm{ft}$; all measurements are from this elevation 


\subsection{MCOI-4}

Location: MCOI-4 is located in lower Mortandad Canyon near the confluence with Ten Site Canyon and about $65 \mathrm{ft}$ upstream of MCOBT-4.4.

Completion Type: Single completion at the base of the Puye Formation fanglomerate member and the top of basalt.

Period of Record: Well completed in November 2004, transducer installed October 2005, data through 2007.

Remarks: Since 2006, the water level in MCOI-4 is 2 to $3 \mathrm{ft}$ higher than in adjacent well MCOBT-4.4.

\begin{tabular}{|c|c|c|c|c|c|c|c|c|c|c|c|c|c|}
\hline \multicolumn{14}{|c|}{ MCOI-4 Construction Information } \\
\hline Zone & $\begin{array}{c}\text { Screen } \\
\text { Top } \\
\text { Depth (ft) }\end{array}$ & $\begin{array}{c}\text { Screen } \\
\text { Bottom } \\
\text { Depth } \\
\text { (ft) }\end{array}$ & $\begin{array}{c}\text { Screen } \\
\text { Top } \\
\text { Elev (ft) }\end{array}$ & $\begin{array}{l}\text { Screen } \\
\text { Bottom } \\
\text { Elev (ft) }\end{array}$ & $\begin{array}{l}\text { Screen } \\
\text { Length } \\
\text { (ft) }\end{array}$ & $\begin{array}{c}\text { Pump } \\
\text { Intake } \\
\text { Depth } \\
\text { (ft) }\end{array}$ & $\begin{array}{l}\text { Pump } \\
\text { Intake } \\
\text { Elevation } \\
\text { (ft) }\end{array}$ & $\begin{array}{l}\text { Depth to } \\
\text { Top of } \\
\text { Sump (ft) }\end{array}$ & $\begin{array}{l}\text { Top of } \\
\text { Sump } \\
\text { Elevation } \\
\text { (ft) }\end{array}$ & $\begin{array}{l}\text { Depth to } \\
\text { Sump } \\
\text { Bottom (ft) }\end{array}$ & $\begin{array}{l}\text { Sump } \\
\text { Length } \\
\text { (ft) }\end{array}$ & $\begin{array}{c}\text { Sump } \\
\text { Volume } \\
\text { (L) }\end{array}$ & Comment \\
\hline 1 & 499.0 & 522.0 & 6338.2 & 6315.2 & 23.0 & & 6837.2 & 522.0 & 6315.2 & 525.7 & 3.7 & 11.6 & Intermediate Zone \\
\hline
\end{tabular}

Note: Ground Elevation: $6837.20 \mathrm{ft}$; all measurements are from this elevation

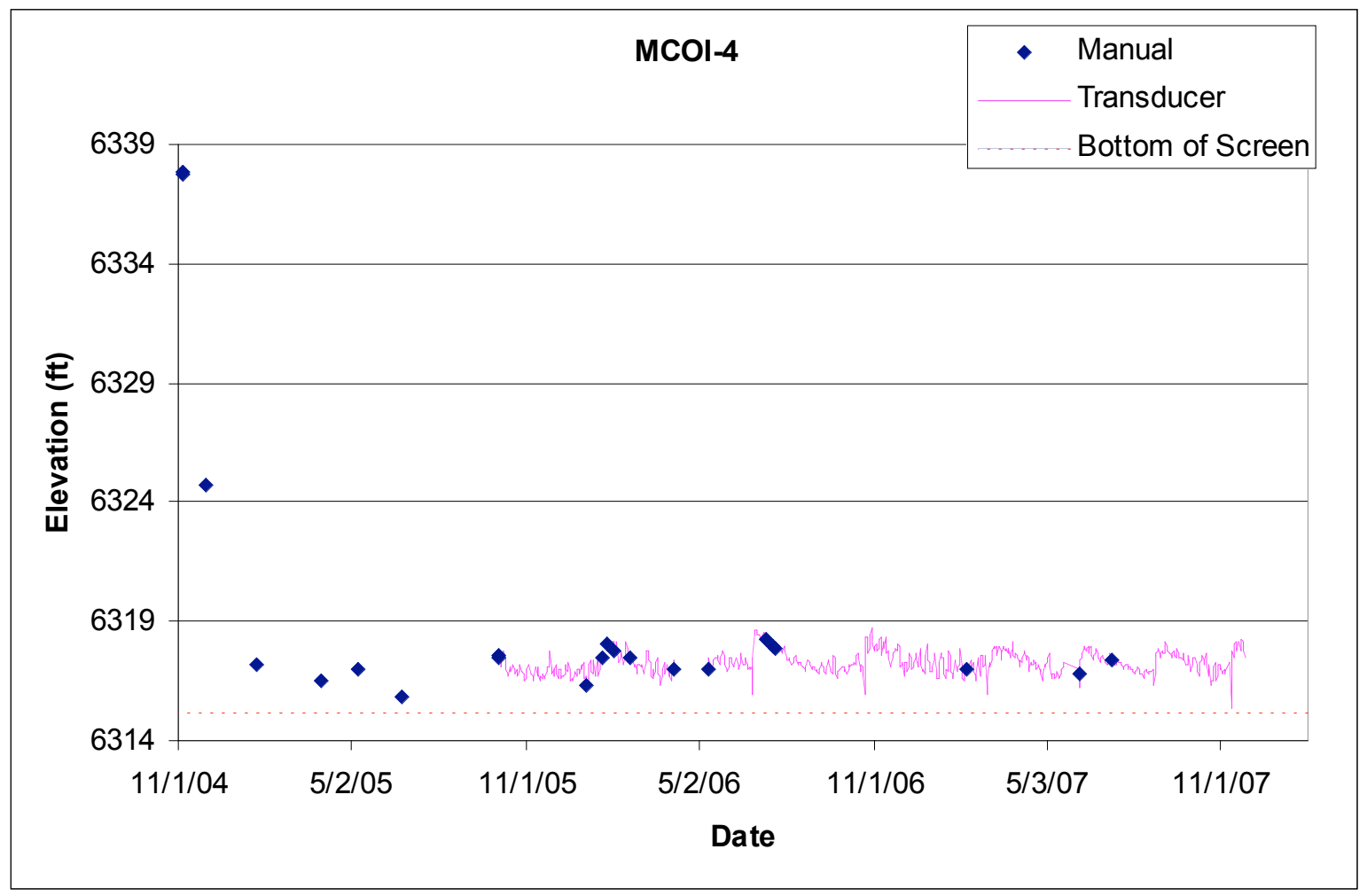




\subsection{MCOI-5}

Location: MCOI-5 is located in lower Mortandad Canyon near regional aquifer well R-15.

Completion Type: Single completion in Cerros del Rio basalt.

Period of Record: Well completed in October 2004, transducer installed August 2005, data through 2007.

Remarks: The transducer was removed for bailing sampling in 2005. A dedicated submersible pump was installed March 2006. The intermediate groundwater has a delayed response to atmospheric pressure fluctuations.

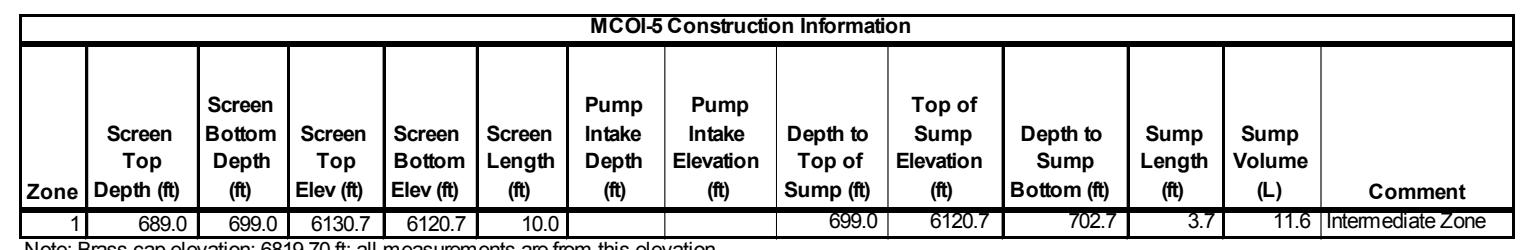

Note: Brass cap elevation: $6819.70 \mathrm{ft}$; all measurements are from this elevation

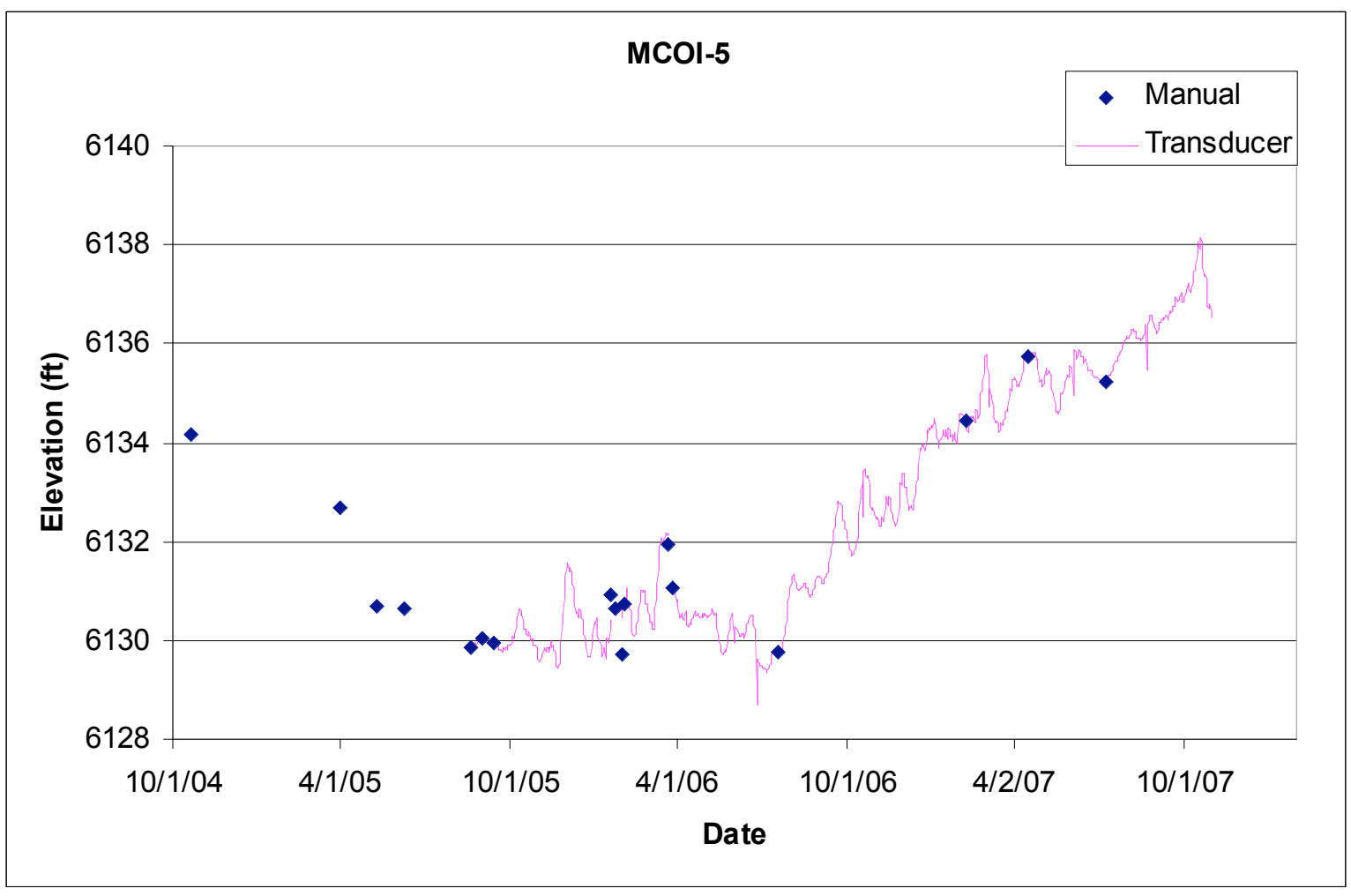




\subsection{MCOI-6}

Location: MCOI-6 is located in lower Mortandad Canyon about $160 \mathrm{ft}$ northeast of MCOI-5.

Completion Type: Single completion in Cerros del Rio basalt.

Period of Record: Well completed in January 2005, transducer installed August 2005, data through 2007.

Remarks: The groundwater level is about $20 \mathrm{ft}$ above the top of the screen and 17 to $18 \mathrm{ft}$ higher than at MCOI-5. The intermediate groundwater has a delayed response to atmospheric pressure fluctuations.

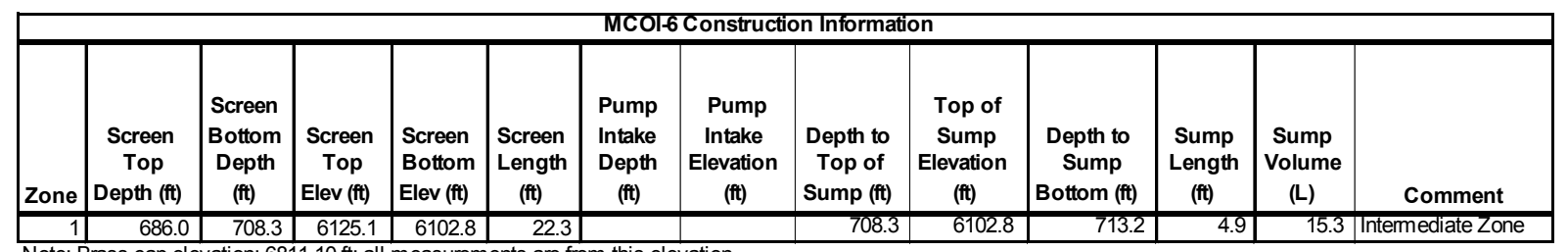

Note: Brass cap elevation: $6811.10 \mathrm{ft}$; all measurements are from this elevation

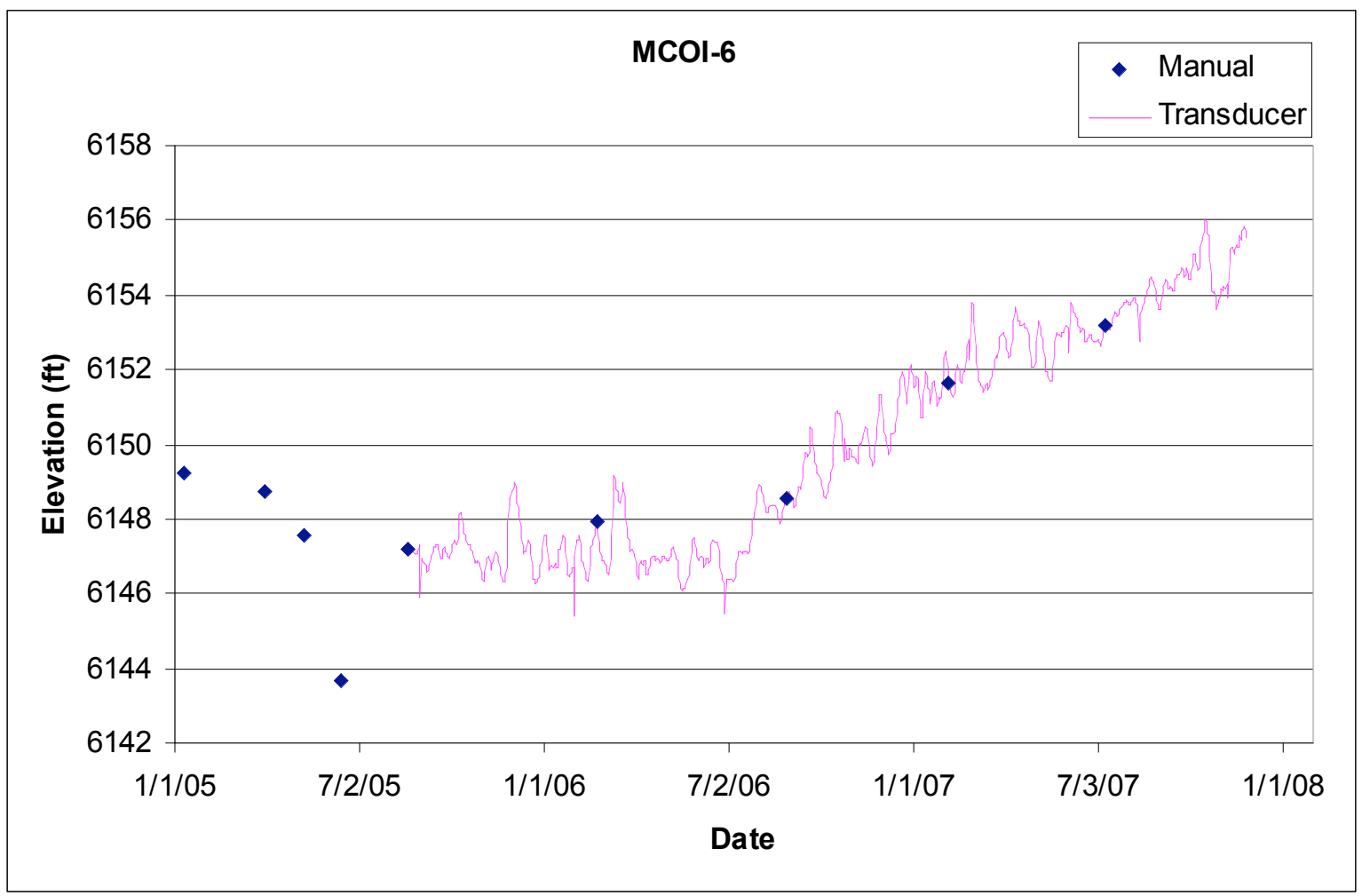




\section{$4.15 \mathrm{MCOI}-8$}

Location: MCOI-8 is located in lower Mortandad Canyon above the confluence with Ten Site Canyon. Completion Type: Single completion in Cerros del Rio basalt.

Period of Record: Well completed in January 2005, transducer installed August 2005, data through 2007.

Remarks: Since well completion, water has been measured in the sump of the well; thus data are not valid groundwater level data.

\begin{tabular}{|c|c|c|c|c|c|c|c|c|c|c|c|c|c|}
\hline \multicolumn{14}{|c|}{ MCOI-8 Construction Information } \\
\hline Zone & $\begin{array}{c}\text { Screen } \\
\text { Top } \\
\text { Depth (ft) }\end{array}$ & $\begin{array}{c}\text { Screen } \\
\text { Bottom } \\
\text { Depth } \\
\text { (ft) }\end{array}$ & $\begin{array}{c}\text { Screen } \\
\text { Top } \\
\text { Elev (ft) }\end{array}$ & $\begin{array}{l}\text { Screen } \\
\text { Bottom } \\
\text { Elev (ft) }\end{array}$ & $\begin{array}{c}\text { Screen } \\
\text { Length } \\
\text { (ft) }\end{array}$ & $\begin{array}{l}\text { Pump } \\
\text { Intake } \\
\text { Depth } \\
\text { (ft) }\end{array}$ & $\begin{array}{l}\text { Pump } \\
\text { Intake } \\
\text { Elevation } \\
\text { (ft) }\end{array}$ & $\begin{array}{c}\text { Depth to } \\
\text { Top of } \\
\text { Sump (ft) }\end{array}$ & $\begin{array}{c}\text { Top of } \\
\text { Sump } \\
\text { Elevation } \\
\text { (ft) }\end{array}$ & $\begin{array}{l}\text { Depth to } \\
\text { Sump } \\
\text { Bottom (ft) }\end{array}$ & $\begin{array}{l}\text { Sump } \\
\text { Length } \\
\text { (ft) }\end{array}$ & $\begin{array}{c}\text { Sump } \\
\text { Volume } \\
\text { (L) }\end{array}$ & Comment \\
\hline 1 & 665.0 & 675.0 & 6194.2 & 6184.2 & 10.0 & & & 675.0 & 6184.2 & 678.6 & 3.6 & 11.4 & Intermediate Zone \\
\hline
\end{tabular}

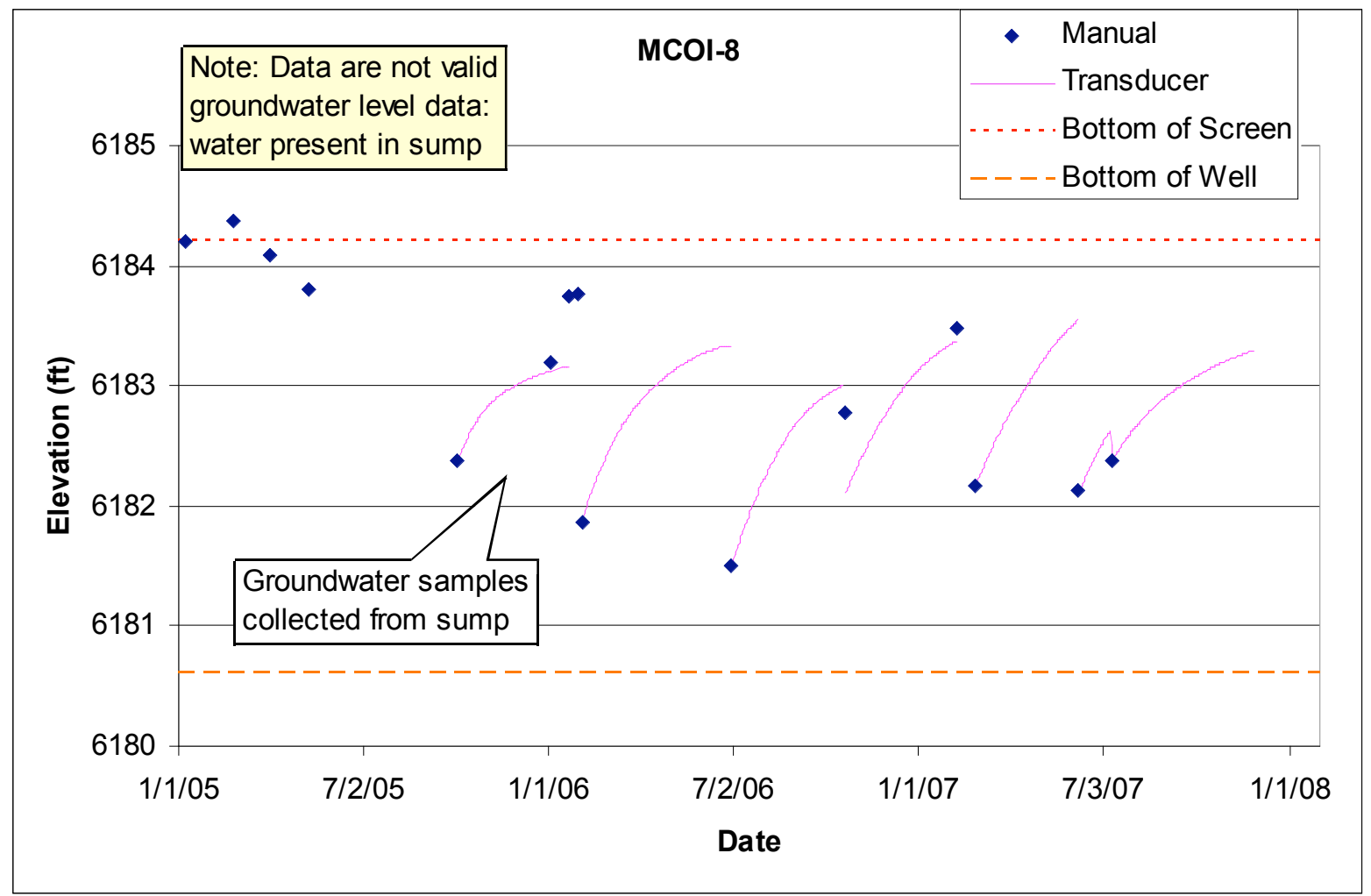




\subsection{MSC-16-02665}

Location: MSC-16-02665 is located at TA-16 at the head of Martin Spring Canyon (S-Site Canyon) about $1500 \mathrm{ft}$ west of CdV-16-3(i) and about $700 \mathrm{ft}$ northwest of Martin Spring.

Completion Type: Single completion in Unit 3 of the Bandelier tuff.

Period of Record: Well completed in October 1997, no transducer has been installed, periodic manual measurements through March 2007.

Remarks: MSC-16-02665 has usually been dry; water has been observed in the well after heavy precipitation periods and snowmelt runoff (LANL 2003, p. 4-58). The well was dry when checked in 2005, 2006, and 2007.
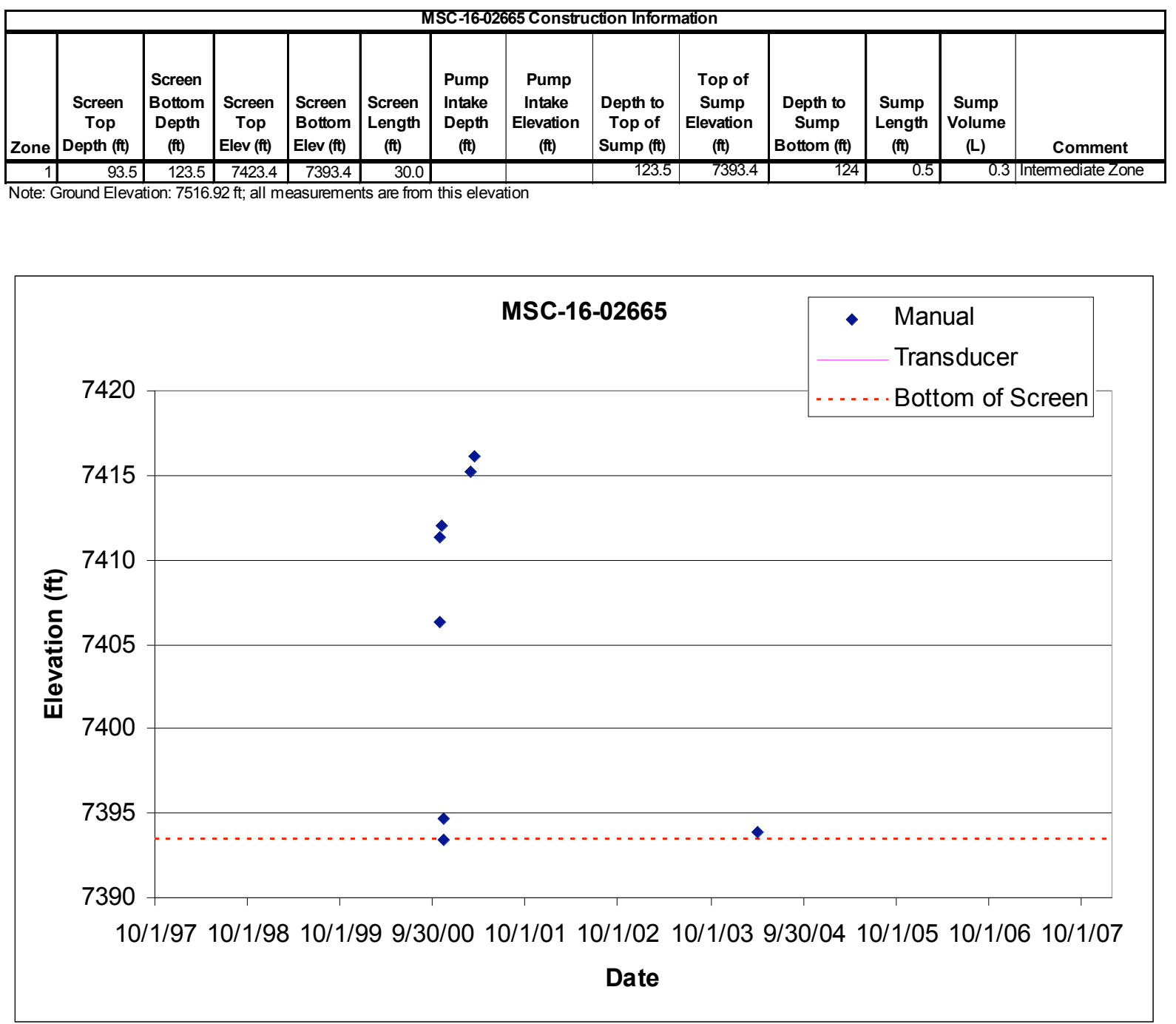


\subsection{POI-4}

Location: POI-4 is located in lower Pueblo Canyon about $800 \mathrm{ft}$ upstream of TW-1 and about $370 \mathrm{ft}$ north of supply well O-1.

Completion Type: Single completion in Cerros del Rio basalt.

Period of Record: Well completed in 1996, transducer installed April 2001 and again in April 2005, intermittent data through 2007.

Remarks: The well is $100 \%$ barometrically efficient; the intermediate groundwater displays a delayed response to atmospheric pressure fluctuations. The intermediate groundwater shows a seasonal water level fluctuation, generally lower in the summer and higher in the winter.
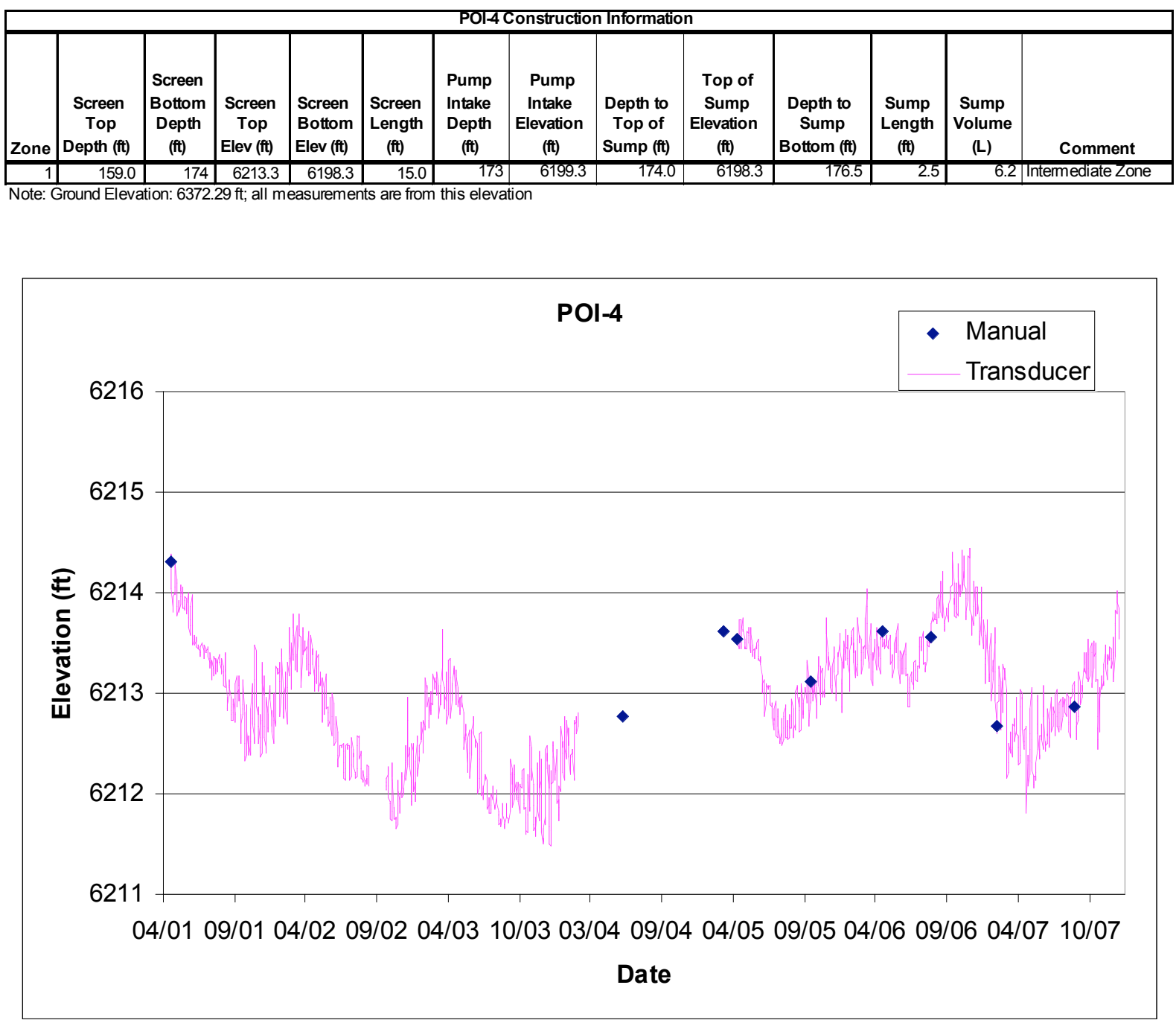


\subsection{R-3i}

Location: R-3i is located in lower Pueblo Canyon about $240 \mathrm{ft}$ west of intermediate well POI-4 and about $425 \mathrm{ft}$ northwest of supply well O-1.

Completion Type: Single completion in the Cerros del Rio basalt.

Period of Record: Well completed August 2005, transducer installed April 2007, data through 2007.

Remarks: The well is $100 \%$ barometrically efficient; the groundwater does not respond to atmospheric pressure fluctuations. The groundwater level rose about $4 \mathrm{ft}$ in the fall of 2007, but did not show a significant response to snowmelt runoff in 2007 . The intermediate groundwater appears to show a seasonal water level fluctuation similar to POI-4, but is about $15 \mathrm{ft}$ lower than the water level at POI-4.

\begin{tabular}{|c|c|c|c|c|c|c|c|c|c|c|c|c|c|}
\hline \multicolumn{14}{|c|}{ R-3Construction Information } \\
\hline Zone & $\begin{array}{l}\text { Screen } \\
\text { Top } \\
\text { Depth (ft) }\end{array}$ & $\begin{array}{l}\text { Screen } \\
\text { Bottom } \\
\text { Depth } \\
\text { (ft) }\end{array}$ & $\begin{array}{c}\text { Screen } \\
\text { Top } \\
\text { Elev (ft) }\end{array}$ & $\begin{array}{l}\text { Screen } \\
\text { Bottom } \\
\text { Elev (ft) }\end{array}$ & $\begin{array}{l}\text { Screen } \\
\text { Length } \\
\text { (ft) }\end{array}$ & $\begin{array}{l}\text { Pump } \\
\text { Intake } \\
\text { Depth } \\
\text { (ft) }\end{array}$ & $\begin{array}{c}\text { Pump } \\
\text { Intake } \\
\text { Elevation } \\
\text { (ft) }\end{array}$ & $\begin{array}{l}\text { Depth to } \\
\text { Top of } \\
\text { Sump (ft) }\end{array}$ & $\begin{array}{c}\text { Top of } \\
\text { Sump } \\
\text { Elevation } \\
\text { (ft) }\end{array}$ & $\begin{array}{l}\text { Depth to } \\
\text { Sump } \\
\text { Bottom (ft) }\end{array}$ & $\begin{array}{l}\text { Sump } \\
\text { Length } \\
\text { (ft) }\end{array}$ & $\begin{array}{c}\text { Sump } \\
\text { Volume } \\
\text { (L) }\end{array}$ & Comment \\
\hline 1 & 215.2 & 220 & 6175.0 & 6170.2 & 4.8 & & & 220.0 & 6170.2 & 220.34 & 0.3 & 0.2 & Intermediate Zone \\
\hline
\end{tabular}

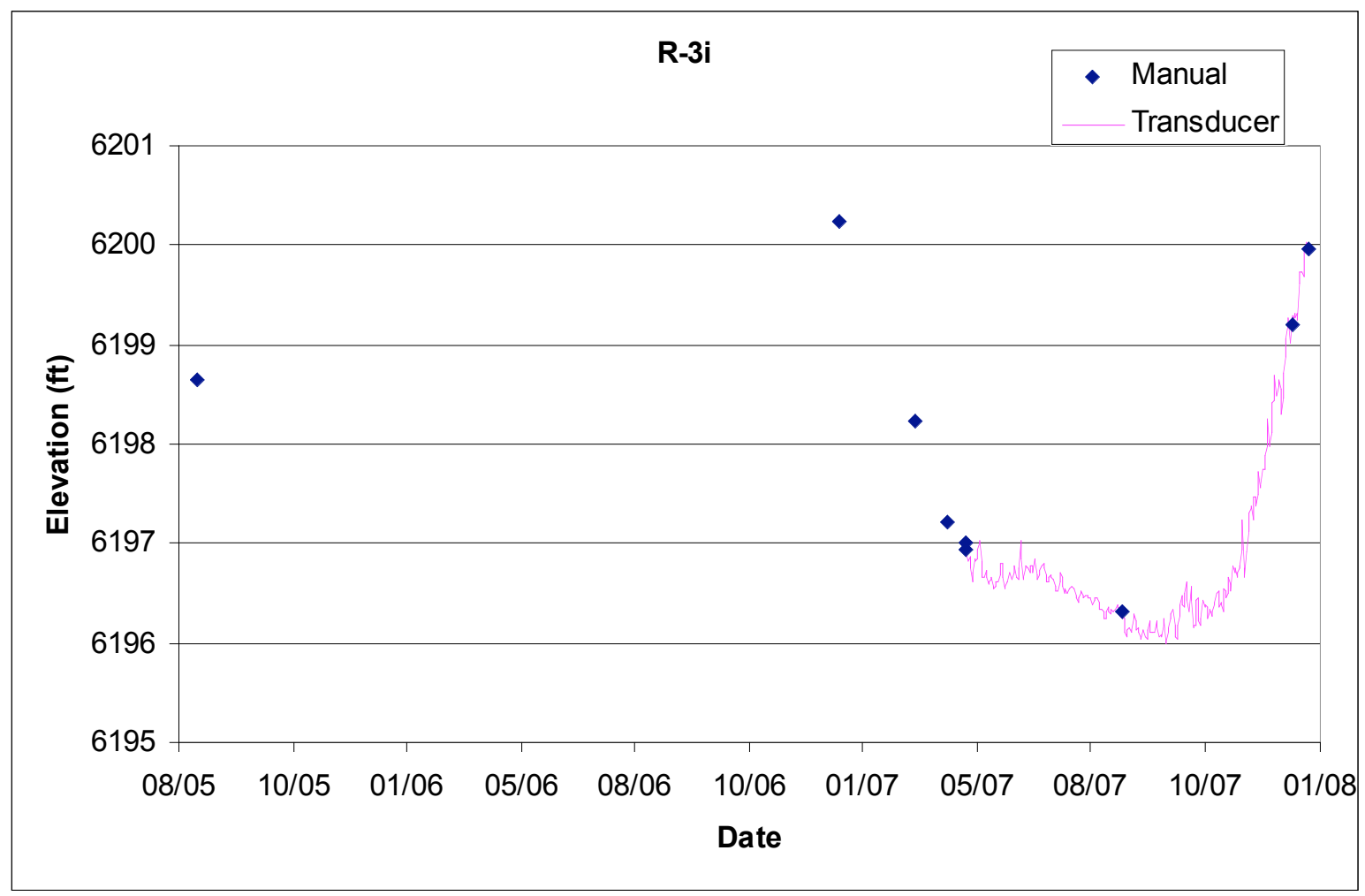




\subsection{R-6i}

Location: R-6i is located at the eastern extent of DP Mesa near the confluence of DP Canyon and Los Alamos Canyon and adjacent to regional aquifer monitoring well R-6.

Completion Type: Single completion in the Puye Formation fanglomerate member.

Period of Record: Well completed December 2004, transducer installed October 2005, data through 2007.

Remarks: The well is $100 \%$ barometrically efficient; the groundwater does not respond to atmospheric pressure fluctuations. The groundwater did not respond to snowmelt runoff in 2007.

\begin{tabular}{|c|c|c|c|c|c|c|c|c|c|c|c|c|c|}
\hline \multicolumn{14}{|c|}{ R-6i Construction Information } \\
\hline Zone & $\begin{array}{c}\text { Screen } \\
\text { Top } \\
\text { Depth (ft) }\end{array}$ & $\begin{array}{l}\text { Screen } \\
\text { Bottom } \\
\text { Depth } \\
\text { (ft) }\end{array}$ & $\begin{array}{c}\text { Screen } \\
\text { Top } \\
\text { Elev (ft) }\end{array}$ & $\begin{array}{l}\text { Screen } \\
\text { Bottom } \\
\text { Elev (ft) }\end{array}$ & $\begin{array}{l}\text { Screen } \\
\text { Length } \\
\text { (ft) }\end{array}$ & $\begin{array}{l}\text { Pump } \\
\text { Intake } \\
\text { Depth } \\
\text { (ft) }\end{array}$ & $\begin{array}{l}\text { Pump } \\
\text { Intake } \\
\text { Elevation } \\
\text { (ft) }\end{array}$ & $\begin{array}{c}\text { Depth to } \\
\text { Top of } \\
\text { Sump (ft) }\end{array}$ & $\begin{array}{l}\text { Top of } \\
\text { Sump } \\
\text { Elevation } \\
\text { (ft) }\end{array}$ & $\begin{array}{l}\text { Depth to } \\
\text { Sump } \\
\text { Bottom (ft) }\end{array}$ & $\begin{array}{c}\text { Sump } \\
\text { Length } \\
\text { (ft) }\end{array}$ & $\begin{array}{c}\text { Sump } \\
\text { Volume } \\
\text { (L) }\end{array}$ & Comment \\
\hline 1 & 602.0 & 612 & 6394.9 & 6384.9 & 10.0 & 609.0 & 6387.9 & 612.0 & 6384.9 & 615 & 3.0 & 9.2 & Regional Aquifer \\
\hline
\end{tabular}

Note: Brass Cap Ground Elevation: $6996.9 \mathrm{ft}$; all depths are from this elevation

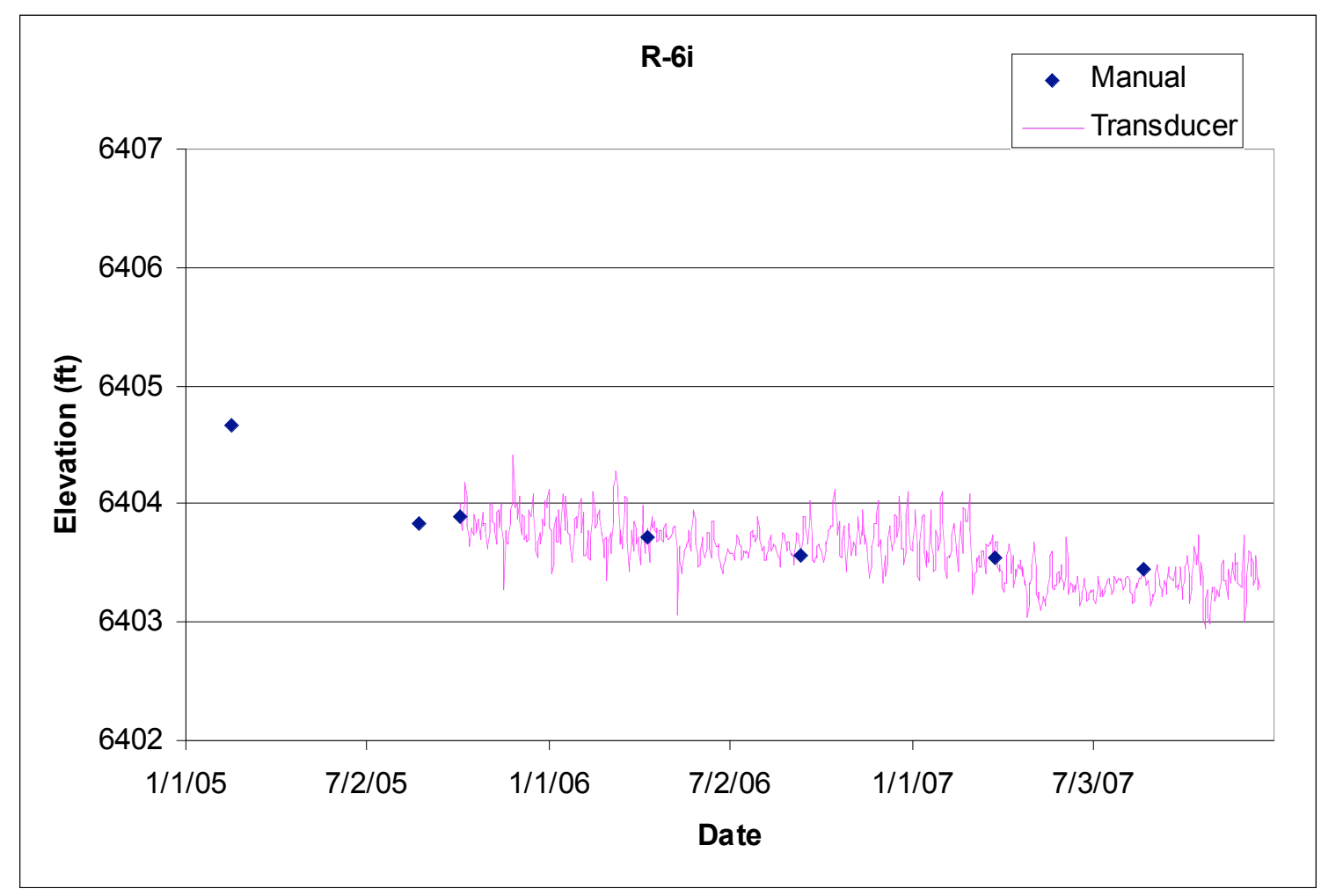




\subsection{R-9i}

Location: R-9i is located in Los Alamos Canyon near the eastern LANL boundary and adjacent to R-9. Completion Type: Dual Westbay ${ }^{\circledR}$ completion; both screens in Cerros del Rio basalt.

Period of Record: Well completed March 2000, transducers installed March 2001, intermittent data through 2007.

Remarks: The screens are about $70 \mathrm{ft}$ apart and the heads in the two intermediate zones are about $110 \mathrm{ft}$ apart. The water level at screen 1 is about $40 \mathrm{ft}$ above the top of the screen; the water level at screen 2 is about $15 \mathrm{ft}$ above the top of the screen. Groundwater at screen 1 appears to be recharged from large runoff events in lower Los Alamos Canyon; the water level responded to snowmelt runoff in 2001, 2005, and 2007, and to large storm runoff events in the summer of 2006. In the spring of 2007 the water level at screen 1 rose about $16 \mathrm{ft}$ in response to snowmelt runoff, while the water level at screen 2 rose about $2 \mathrm{ft}$.

\begin{tabular}{|c|c|c|c|c|c|c|c|c|c|c|c|}
\hline \multicolumn{12}{|c|}{ R-9i Port Data } \\
\hline Zone & $\begin{array}{l}\text { Screen } \\
\text { Top } \\
\text { Depth (ft) }\end{array}$ & $\begin{array}{l}\text { Screen } \\
\text { Bottom } \\
\text { Depth (ft) }\end{array}$ & $\begin{array}{c}\text { Screen } \\
\text { Top Elev } \\
\text { (ft) }\end{array}$ & $\begin{array}{l}\text { Screen } \\
\text { Bottom } \\
\text { Elev (ft) }\end{array}$ & $\begin{array}{l}\text { Screen } \\
\text { Length } \\
\text { (ft) }\end{array}$ & Port & $\begin{array}{c}\text { Port } \\
\text { Depth (ft) }\end{array}$ & $\begin{array}{c}\text { Port } \\
\text { Elevation } \\
\text { (ft) }\end{array}$ & $\begin{array}{l}\text { Distance } \\
\text { from } \\
\text { Bottom } \\
\text { of Screen } \\
\text { (ft) }\end{array}$ & $\begin{array}{c}\text { Sump } \\
\text { Volume } \\
\text { (L) }\end{array}$ & Comment \\
\hline \multirow{3}{*}{1} & \multirow{3}{*}{189.1} & \multirow{3}{*}{199.5} & \multirow{3}{*}{6194.1} & \multirow{3}{*}{6183.7} & \multirow{3}{*}{10.4} & MP1A & 198.8 & 6184.4 & 0.7 & & Intermediate Zone \\
\hline & & & & & & PP1 & 204.1 & 6179.1 & -4.6 & 13.3 & Below screen \\
\hline & & & & & & MP1B & 209.8 & 6173.4 & -10.3 & 29.8 & Below screen \\
\hline \multirow{3}{*}{2} & \multirow{3}{*}{269.6} & \multirow{3}{*}{280.3} & \multirow{3}{*}{6113.6} & \multirow{3}{*}{6102.9} & \multirow{3}{*}{10.7} & MP2A & 278.8 & 6104.4 & 1.5 & & Intermediate Zone \\
\hline & & & & & & PP2 & 284.1 & 6099.1 & -3.8 & 11.0 & Below screen \\
\hline & & & & & & MP2B & 289.8 & 6093.4 & -9.5 & 27.5 & Below screen \\
\hline
\end{tabular}

Note: Brass Cap Elevation is $6383.2 \mathrm{ft}$; all measurements are from this elevation;

MP = Monitoring Port, $\mathrm{PP}=$ Pumping Port; Ports shown in Bold are instrumented with transducers

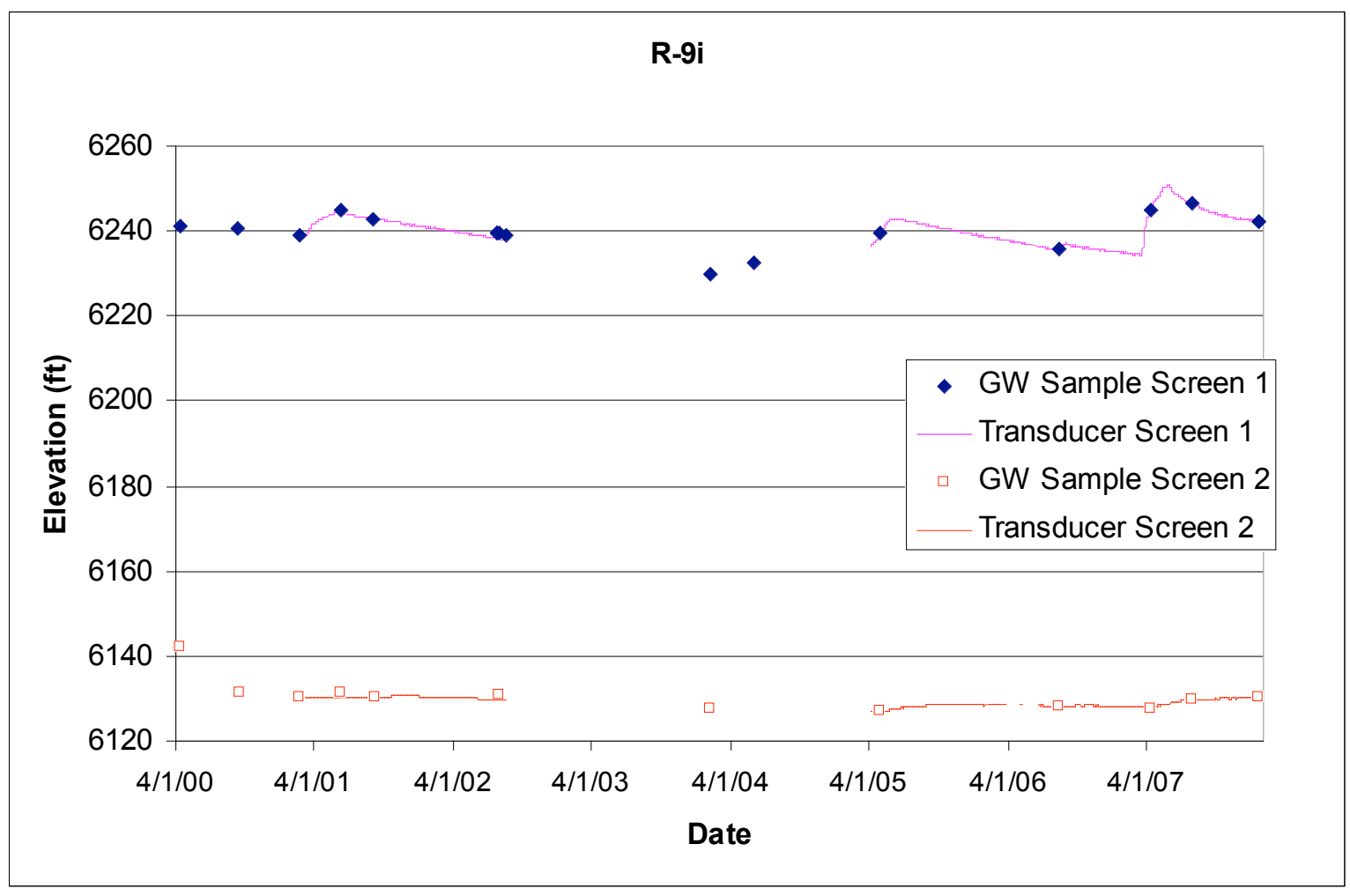




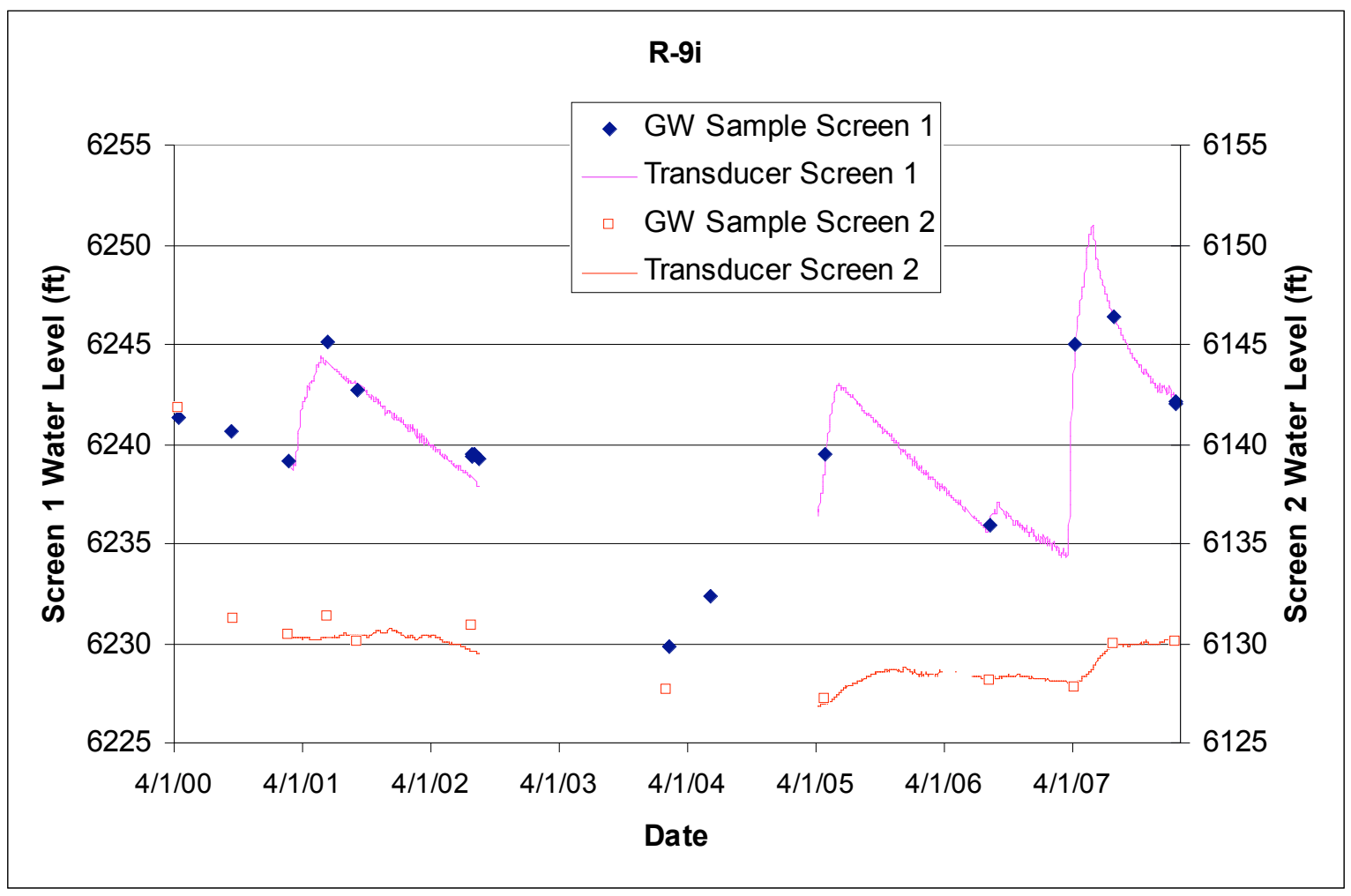




\subsection{R-23i}

Location: R-23i is located in lower Pajarito Canyon near SR-4 and adjacent to regional well R-23.

Completion Type: Multiple completion, three screens, screen 1 is in a 2.1-in.-diameter piezometer and screens 2 and 3 are in a 4 -in.-diameter well. A Baski packer and dual pump sampling system was installed at screens 2 and 3 in December 2006. All screens are in Cerros del Rio basalt.

Period of Record: Well completed November 2005, periodic manual measurements at screen 1. Transducers installed at screens 2 and 3 in December 2006, data through 2007.

Remarks: The water levels at screens 2 and 3 are about $9 \mathrm{ft}$ apart; the water level at screen 1 is about $44 \mathrm{ft}$ higher than screen 2 . The screen 3 gage tubing through the packer has shown occasional partial plugging, but water levels in the tubing appear to be representative of screen 2.

\begin{tabular}{|c|c|c|c|c|c|c|c|c|c|c|c|c|c|}
\hline \multicolumn{14}{|c|}{ R-23i Construction Information } \\
\hline Zone & $\begin{array}{c}\text { Screen } \\
\text { Top } \\
\text { Depth (ft) }\end{array}$ & $\begin{array}{c}\text { Screen } \\
\text { Bottom } \\
\text { Depth } \\
\text { (ft) }\end{array}$ & $\begin{array}{c}\text { Screen } \\
\text { Top } \\
\text { Elev (ft) }\end{array}$ & $\begin{array}{l}\text { Screen } \\
\text { Bottom } \\
\text { Elev (ft) }\end{array}$ & $\begin{array}{l}\text { Screen } \\
\text { Length } \\
\text { (ft) }\end{array}$ & $\begin{array}{l}\text { Pump } \\
\text { Intake } \\
\text { Depth } \\
\text { (ft) }\end{array}$ & $\begin{array}{l}\text { Pump } \\
\text { Intake } \\
\text { Elevation } \\
\text { (ft) }\end{array}$ & $\begin{array}{l}\text { Depth to } \\
\text { Top of } \\
\text { Packerl } \\
\text { Sump (ft) }\end{array}$ & $\begin{array}{c}\text { Top of } \\
\text { Sump } \\
\text { Elevation } \\
\text { (ft) }\end{array}$ & \begin{tabular}{|} 
Depth to \\
Sump \\
Bottom (ft)
\end{tabular} & $\begin{array}{l}\text { Sump } \\
\text { Length } \\
\text { (ft) }\end{array}$ & $\begin{array}{c}\text { Sump } \\
\text { Volume } \\
\text { (L) }\end{array}$ & Comment \\
\hline 1 & 400.3 & 420.0 & 6127.6 & 6107.9 & 19.7 & & & 420.0 & 6107.9 & 425.3 & 5.3 & 16.6 & Intermediate \\
\hline 2 & 470.2 & 480.1 & 6057.7 & 6047.8 & 9.9 & 477.1 & 6050.8 & 495.3 & 6032.5 & 495.3 & 0.0 & 0.0 & Intermediate \\
\hline 3 & 524.0 & 547.0 & 6003.9 & 5980.9 & 23.0 & 516.7 & 6011.2 & 547.0 & 5980.9 & 550.7 & 3.7 & 11.6 & Intermediate \\
\hline
\end{tabular}

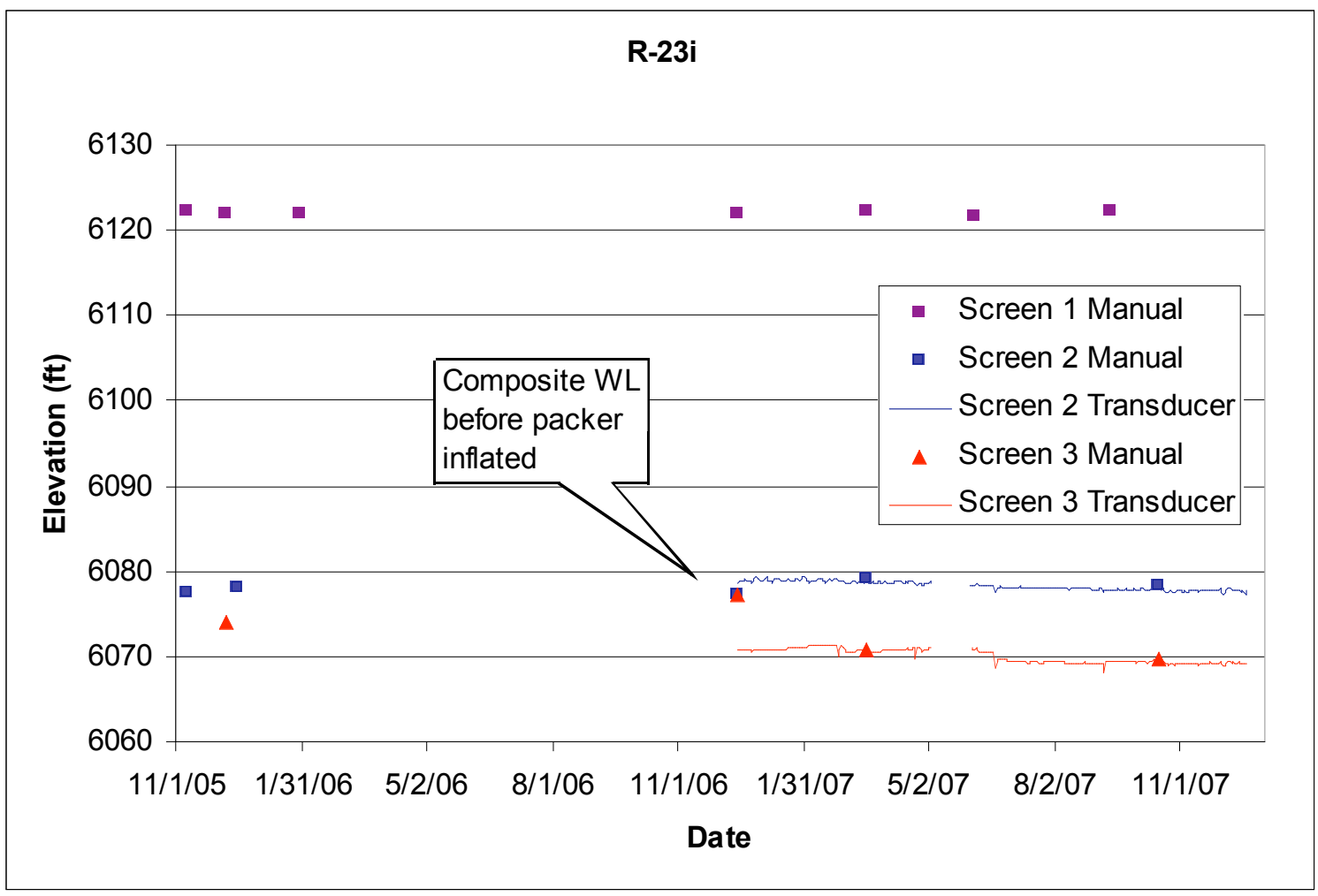




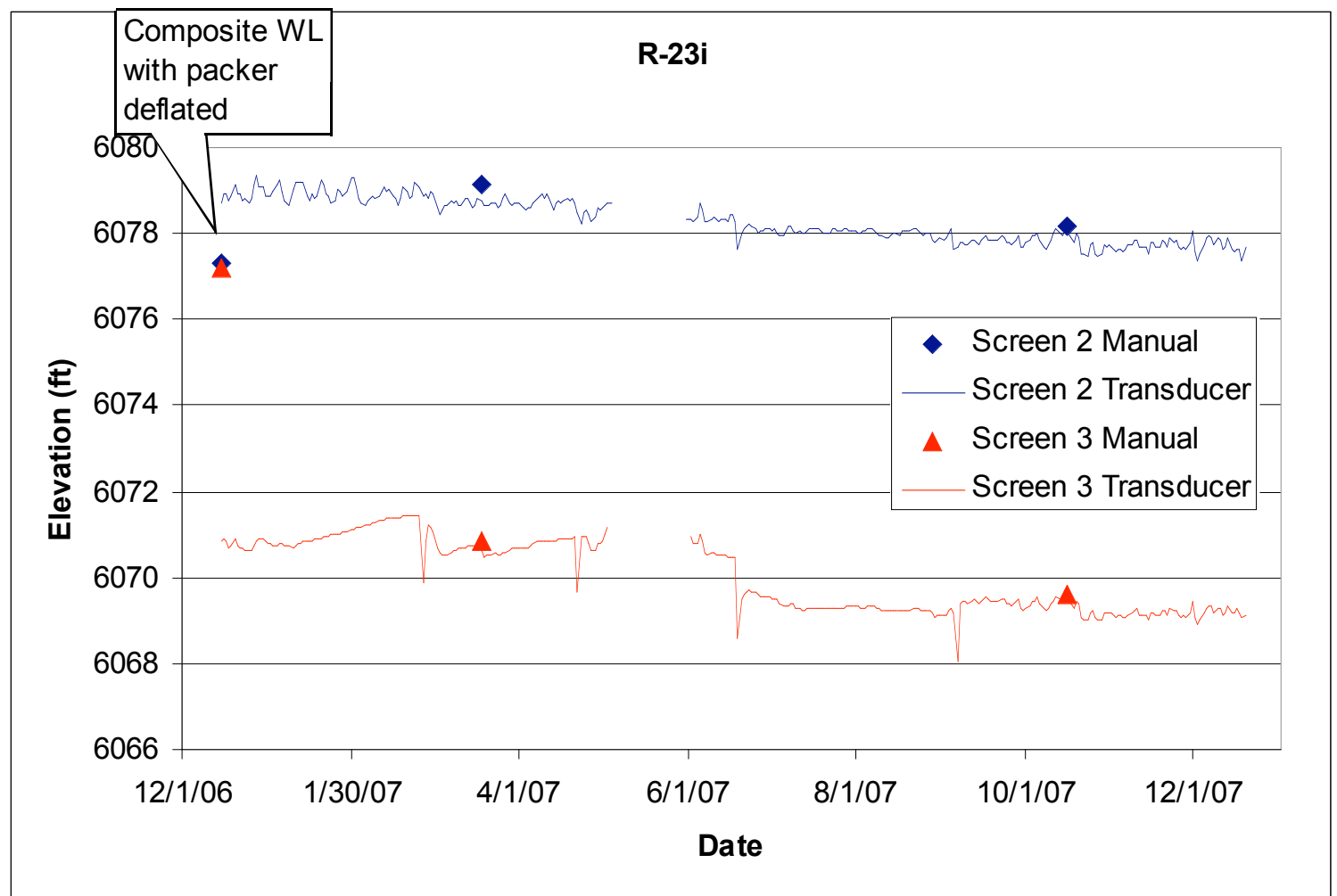




\section{$4.22 \mathrm{SCl}-1$}

Location: SCl-1 is located in Sandia Canyon between intermediate wells LAOI-3.2 in Los Alamos Canyon to the north and MCOI-6 in Mortandad Canyon to the southwest.

Completion Type: Single completion in the Puye Formation fanglomerate member; the screen is located above the Cerros del Rio basalt.

Period of Record: Well completed October 2006, transducer installed in February 2007, data through 2007.

Remarks: Originally drilled as core hole SCC-1, completed as intermediate well and named SCl-1. The well is immediately $100 \%$ barometrically efficient; additionally, the groundwater shows a delayed response to atmospheric pressure fluctuations.

\begin{tabular}{|c|c|c|c|c|c|c|c|c|c|c|c|c|c|}
\hline \multicolumn{14}{|c|}{ SCI-1 Construction Information } \\
\hline Zone & $\begin{array}{c}\text { Screen } \\
\text { Top } \\
\text { Depth (ft) }\end{array}$ & $\begin{array}{l}\text { Screen } \\
\text { Bottom } \\
\text { Depth } \\
\text { (ft) }\end{array}$ & $\begin{array}{c}\text { Screen } \\
\text { Top } \\
\text { Elev }(\mathrm{ft})\end{array}$ & $\begin{array}{l}\text { Screen } \\
\text { Bottom } \\
\text { Elev (ft) }\end{array}$ & $\begin{array}{l}\text { Screen } \\
\text { Length } \\
\text { (ft) }\end{array}$ & $\begin{array}{c}\text { Pump } \\
\text { Intake } \\
\text { Depth } \\
\text { (ft) }\end{array}$ & $\begin{array}{l}\text { Pump } \\
\text { Intake } \\
\text { Elevation } \\
\text { (ft) }\end{array}$ & $\begin{array}{c}\text { Depth to } \\
\text { Top of } \\
\text { Sump (ft) }\end{array}$ & $\begin{array}{c}\text { Top of } \\
\text { Sump } \\
\text { Elevation } \\
\text { (ft) }\end{array}$ & $\begin{array}{c}\text { Depth to } \\
\text { Sump } \\
\text { Bottom (ft) }\end{array}$ & $\begin{array}{l}\text { Sump } \\
\text { Length } \\
\text { (ft) }\end{array}$ & $\begin{array}{c}\text { Sump } \\
\text { Volume } \\
\text { (L) }\end{array}$ & Comment \\
\hline 1 & 358.4 & 377.9 & 6379.9 & 6360.4 & 19.5 & & & 377.9 & 6360.4 & 377.9 & 0.0 & 0.0 & Intermediate Zone \\
\hline
\end{tabular}

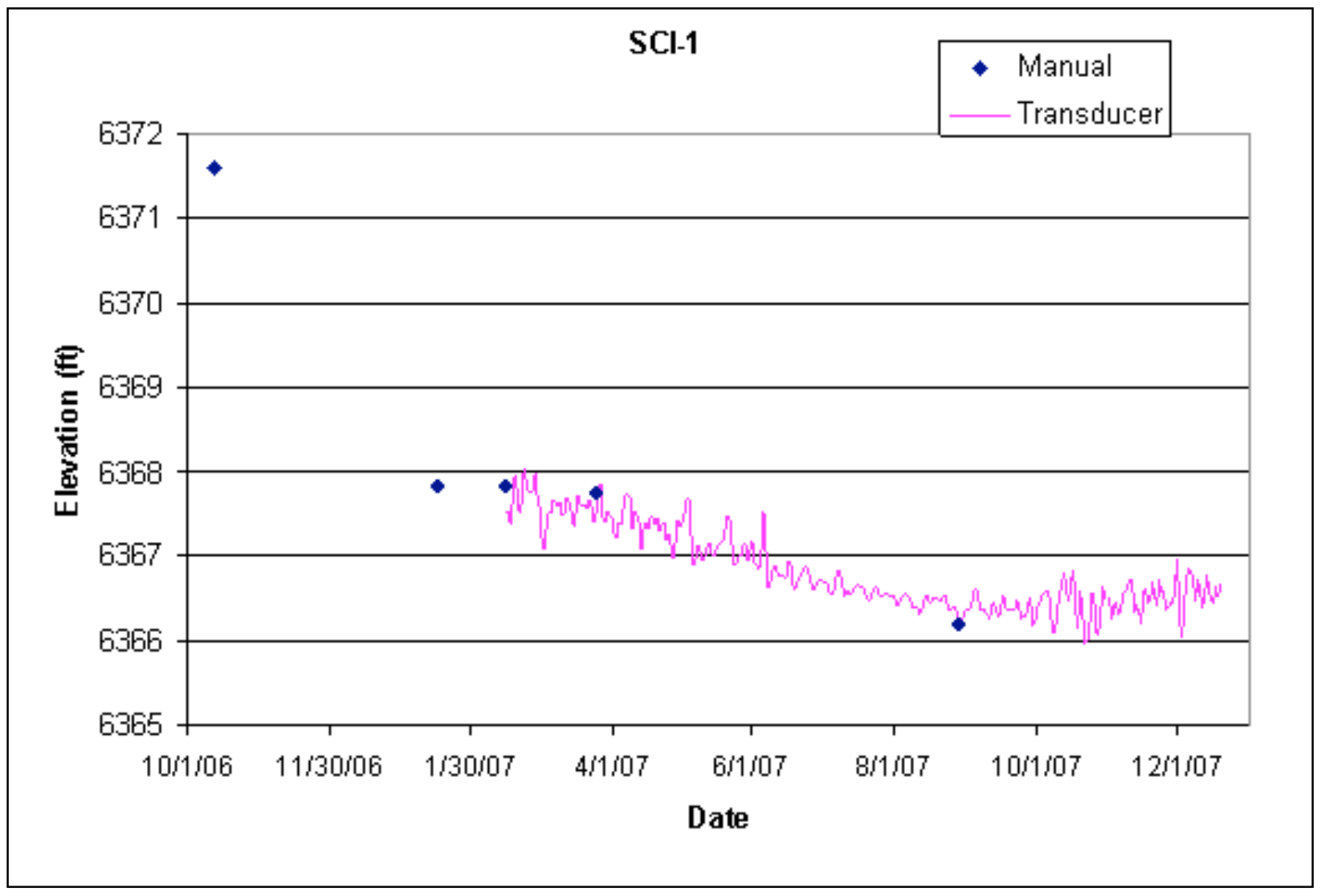




\subsection{Test Well 1A}

Location: TW-1A is located in lower Pueblo Canyon adjacent to TW-1.

Completion Type: Single completion in Cerros del Rio basalt.

Period of Record: Well completed in 1950, transducer installed June 1993, intermittent data to April 2005 when problems were encountered with equipment and the transducer was removed from the well.

Remarks: The wellhead equipment was removed from the well in February 2006 in preparation for plugging and abandonment of the well. The well is not planned for further monitoring.

\begin{tabular}{|c|c|c|c|c|c|c|c|c|c|c|c|c|c|}
\hline \multicolumn{14}{|c|}{ TW-1A Construction Information } \\
\hline Zone & $\begin{array}{c}\text { Screen } \\
\text { Top } \\
\text { Depth (ft) }\end{array}$ & $\begin{array}{c}\text { Screen } \\
\text { Bottom } \\
\text { Depth } \\
\text { (ft) }\end{array}$ & $\begin{array}{c}\text { Screen } \\
\text { Top } \\
\text { Elev (ft) }\end{array}$ & $\begin{array}{l}\text { Screen } \\
\text { Bottom } \\
\text { Elev (ft) }\end{array}$ & $\begin{array}{l}\text { Screen } \\
\text { Length } \\
\text { (ft) }\end{array}$ & $\begin{array}{c}\text { Pump } \\
\text { Intake } \\
\text { Depth } \\
\text { (ft) }\end{array}$ & $\begin{array}{l}\text { Pump } \\
\text { Intake } \\
\text { Elevation } \\
\text { (ft) }\end{array}$ & $\begin{array}{c}\text { Depth to } \\
\text { Top of } \\
\text { Sump (ft) }\end{array}$ & $\begin{array}{l}\text { Top of } \\
\text { Sump } \\
\text { Elevation } \\
\text { (ft) }\end{array}$ & $\begin{array}{l}\text { Depth to } \\
\text { Sump } \\
\text { Bottom (ft) }\end{array}$ & $\begin{array}{l}\text { Sump } \\
\text { Length } \\
\text { (ft) }\end{array}$ & $\begin{array}{c}\text { Sump } \\
\text { Volume } \\
\text { (L) }\end{array}$ & Comment \\
\hline 1 & 215.0 & 225 & 6154.3 & 6144.3 & 10.0 & & 6369.3 & 225.0 & 6144.3 & 225 & 0.0 & 0.0 & Intermediate Zone \\
\hline
\end{tabular}

Note: TW-1A Ground Elevation: $6369.28 \mathrm{ft}$; all depths are from this elevation

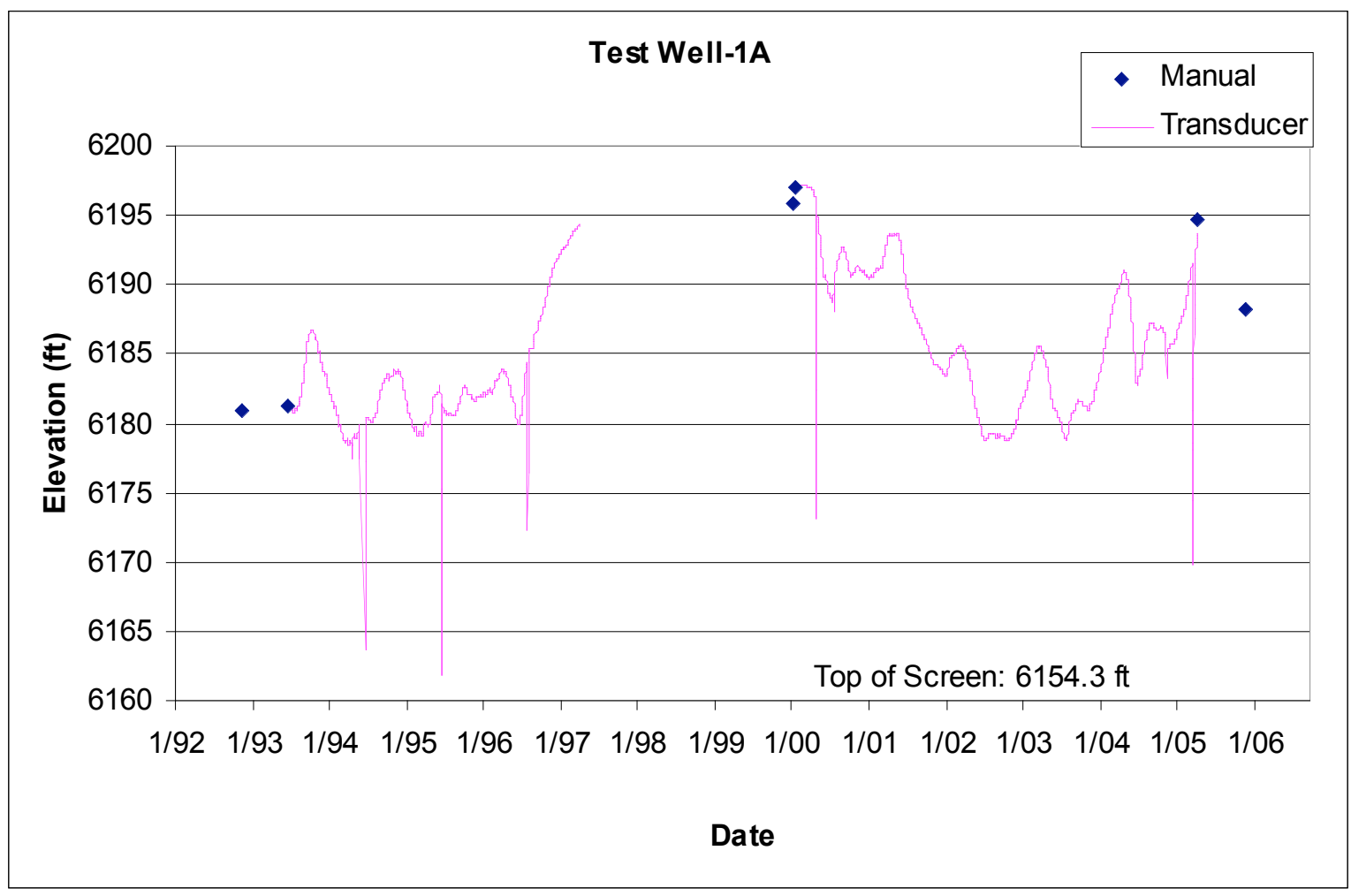




\subsection{Test Well 2A}

Location: TW-2A is located in middle Pueblo Canyon adjacent to TW-2.

Completion Type: Single completion in the Puye Formation.

Period of Record: Well completed in 1950, transducer installed January 1994 but equipment problems preclude data until 1995; intermittent data through 2007.

Remarks: Recent pumping of TW-2A when the water level is below $6535 \mathrm{ft}$ has shown slow recovery of the intermediate groundwater.

\begin{tabular}{|c|c|c|c|c|c|c|c|c|c|c|c|c|c|}
\hline \multicolumn{14}{|c|}{ TW-2A Construction Information } \\
\hline Zone & $\begin{array}{c}\text { Screen } \\
\text { Top } \\
\text { Depth (ft) }\end{array}$ & $\begin{array}{c}\text { Screen } \\
\text { Bottom } \\
\text { Depth } \\
\text { (ft) }\end{array}$ & $\begin{array}{c}\text { Screen } \\
\text { Top } \\
\text { Elev (ft) }\end{array}$ & $\begin{array}{l}\text { Screen } \\
\text { Bottom } \\
\text { Elev (ft) }\end{array}$ & $\begin{array}{c}\text { Screen } \\
\text { Length } \\
\text { (ft) }\end{array}$ & $\begin{array}{c}\text { Pump } \\
\text { Intake } \\
\text { Depth } \\
\text { (ft) }\end{array}$ & $\begin{array}{l}\text { Pump } \\
\text { Intake } \\
\text { Elevation } \\
\text { (ft) }\end{array}$ & $\begin{array}{c}\text { Depth to } \\
\text { Top of } \\
\text { Sump (ft) }\end{array}$ & $\begin{array}{c}\text { Top of } \\
\text { Sump } \\
\text { Elevation } \\
\text { (ft) }\end{array}$ & $\begin{array}{c}\text { Depth to } \\
\text { Sump } \\
\text { Bottom (ft) }\end{array}$ & $\begin{array}{c}\text { Sump } \\
\text { Length } \\
\text { (ft) }\end{array}$ & $\begin{array}{l}\text { Sump } \\
\text { Volume } \\
\text { (L) }\end{array}$ & Comment \\
\hline 1 & 123.0 & 133.0 & 6527.4 & 6517.4 & 10.0 & 130 & 6520.4 & 133.0 & 6517.4 & 133 & 0.0 & 0.0 & Intermediate Zone \\
\hline
\end{tabular}

Note: TW-2A Ground Elevation: $6650.4 \mathrm{ft}$; all measurements are from this elevation

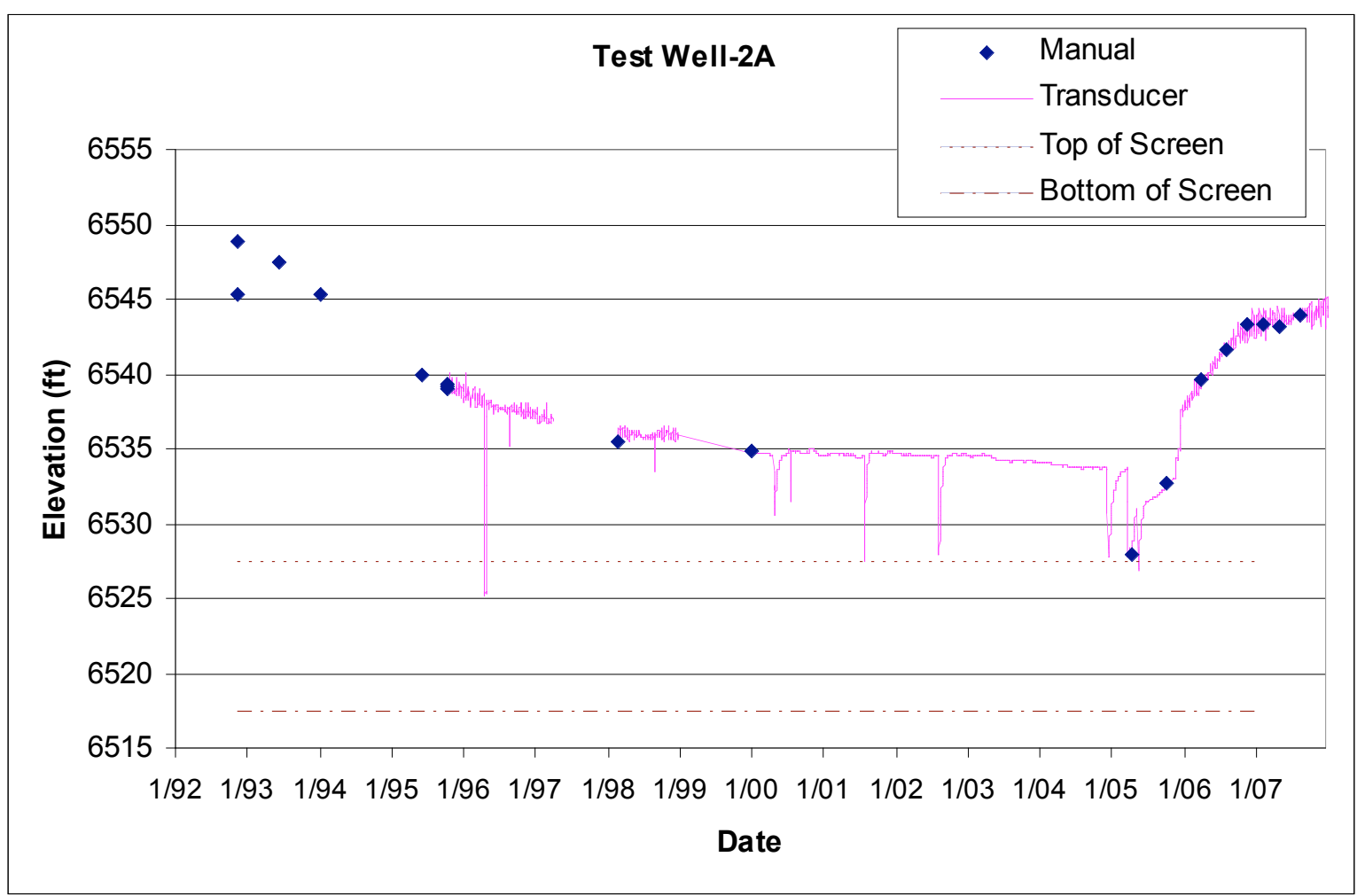




\subsection{Groundwater Level Data from Alluvial Wells}

Table 5-1 lists the alluvial wells that were monitored for groundwater levels in FY 2007. The table provides the well name, date of completion, well depth, surveyed location coordinates, ground surface elevation, and the screen top and bottom depths for each well. Figure 5-1 shows the locations of the wells. In the following alluvial groundwater sections, the first graph for each well represents the entire period of record, while the second graph represents groundwater level data from FY 2007. Alluvial groundwater levels respond to snowmelt runoff, storm runoff, and, in some canyons, effluent discharges. Some alluvial wells have been historically dry and do not show a seasonal response to precipitation and runoff.

Table 5-1. Information and Location Data for Alluvial Aquifer Wells at LANL

\begin{tabular}{|c|c|c|c|c|c|c|c|}
\hline Well Name & $\begin{array}{c}\text { Date } \\
\text { Completed } \\
\end{array}$ & $\begin{array}{c}\text { Completed } \\
\text { Depth (ft) }\end{array}$ & Easting (ft) & $\begin{array}{c}\text { Northing } \\
\text { (ft) }\end{array}$ & $\begin{array}{c}\text { Surface } \\
\text { Elevation } \\
\text { (ft) }\end{array}$ & $\begin{array}{c}\text { Screen } \\
\text { Top } \\
\text { Depth } \\
\text { (ft) } \\
\end{array}$ & $\begin{array}{c}\text { Screen } \\
\text { Bottom } \\
\text { Depth } \\
\text { (ft) }\end{array}$ \\
\hline 18-BG-1 & $8 / 1 / 1994$ & 35 & 1634152.90 & 1762575.36 & 6776.45 & 10 & 35 \\
\hline 18-BG-4 & 2/18/1998 & 6.5 & 1633510.00 & 1760760.00 & 6768 & 2.5 & 6.5 \\
\hline 18-MW-7 & $7 / 6 / 1994$ & 30 & 1634846.28 & 1761791.52 & 6755.5 & 10 & 30 \\
\hline 18-MW-8 & $8 / 4 / 1994$ & 37.9 & 1634714.26 & 1760658.14 & 6747.79 & 8 & 38 \\
\hline 18-MW-9 & $7 / 21 / 1994$ & 21 & 1635949.81 & 1760893.56 & 6732.91 & 6 & 31 \\
\hline 18-MW-11 & $8 / 11 / 1994$ & 47 & 1636001.69 & 1761139.83 & 6740.13 & 27 & 47 \\
\hline 18-MW-17 & $8 / 1 / 1995$ & 22 & 1637778.20 & 1759717.10 & 6695.2 & 12 & 22 \\
\hline 18-MW-18 & $7 / 31 / 1995$ & 23 & 1639925.00 & 1758247.20 & 6654.7 & 12.5 & 23 \\
\hline 39-UM-3 & $8 / 15 / 1994$ & 54 & 1637032.00 & 1747663.00 & 6394.2 & 44 & 54 \\
\hline 39-DM-6 & $8 / 15 / 1994$ & 60 & 1637094.00 & 1747228.00 & 6384.57 & 50 & 60 \\
\hline APCO-1 & $8 / 15 / 1990$ & 19.7 & 1649209.62 & 1773020.12 & 6367.53 & 4.7 & 14.7 \\
\hline CDBO-1 & 4/17/1985 & 13 & 1637968.59 & 1760943.96 & 6757.6 & 5.1 & 13.1 \\
\hline CDBO-2 & $4 / 18 / 1985$ & 18 & 1638119.02 & 1761103.11 & 6758.2 & 5.9 & 17.9 \\
\hline CDBO-3 & 4/18/1985 & 12 & 1640677.11 & 1759611.02 & 6670.2 & 4.4 & 12.4 \\
\hline CDBO-4 & $4 / 18 / 1985$ & 12 & 1645474.90 & 1758546.90 & 6564.5 & 4.1 & 12.1 \\
\hline CDBO-5 & $6 / 1 / 1992$ & 17 & 1633583.37 & 1765818.37 & 6879.01 & 7 & 17 \\
\hline CDBO-6 & $6 / 1 / 1992$ & 49 & 1636209.25 & 1764759.75 & 6817.2 & 34 & 44 \\
\hline CDBO-7 & $6 / 1 / 1992$ & 44 & 1637400.00 & 1763301.00 & 6771.81 & 29 & 39 \\
\hline CDBO-8 & $6 / 1 / 1992$ & 23 & 1639294.00 & 1762366.00 & 6722.47 & 3 & 13 \\
\hline CDBO-9 & $6 / 1 / 1992$ & 34 & 1642119.12 & 1759702.87 & 6633 & 19 & 29 \\
\hline $\begin{array}{l}\text { CDV-16- } \\
02655\end{array}$ & 9/27/1997 & 7.6 & 1611299.09 & 1764153.13 & 7583.7 & 2.3 & 7.3 \\
\hline $\begin{array}{l}\text { CDV-16- } \\
02656\end{array}$ & $11 / 5 / 1997$ & 8.2 & 1613634.46 & 1764932.79 & 7443.18 & 3 & 8 \\
\hline $\begin{array}{l}\text { CDV-16- } \\
02657\end{array}$ & $10 / 10 / 1997$ & 5.7 & 1613813.19 & 1764850.10 & 7433.25 & 0.4 & 5.4 \\
\hline $\begin{array}{l}\text { CDV-16- } \\
02658\end{array}$ & 9/16/1997 & 7.2 & 1615071.38 & 1764469.56 & 7375.6 & 1.9 & 6.9 \\
\hline $\begin{array}{l}\text { CDV-16- } \\
02659\end{array}$ & 9/8/1997 & 7 & 1616712.08 & 1765035.06 & 7300.5 & 1.7 & 6.7 \\
\hline FCO-1 & $8 / 22 / 1989$ & 12.4 & 1642409.00 & 1751177.00 & 6509.24 & 2.4 & 12.4 \\
\hline LAO-B & $4 / 28 / 1994$ & 27.2 & 1615148.80 & 1775170.40 & 7323.59 & 11.84 & 26.84 \\
\hline LAO-0.3 & $5 / 17 / 1994$ & 11.25 & 1624799.00 & 1774511.60 & 6968.13 & 5.9 & 10.9 \\
\hline LAO-0.6 & $5 / 6 / 1994$ & 13.35 & 1626748.10 & 1774332.90 & 6910.74 & 8 & 13 \\
\hline LAO-0.91 & $5 / 12 / 1994$ & 14.85 & 1628654.30 & 1774207.00 & 6861.74 & 9.5 & 14.5 \\
\hline
\end{tabular}


Table 5-1. Information and Location Data for Alluvial Aquifer Wells at LANL (cont.)

\begin{tabular}{|c|c|c|c|c|c|c|c|}
\hline Well Name & $\begin{array}{c}\text { Date } \\
\text { Completed }\end{array}$ & $\begin{array}{c}\text { Completed } \\
\text { Depth (ft) }\end{array}$ & Easting (ft) & $\begin{array}{c}\text { Northing } \\
(\mathrm{ft})\end{array}$ & $\begin{array}{c}\text { Surface } \\
\text { Elevation } \\
\text { (ft) }\end{array}$ & $\begin{array}{c}\text { Screen } \\
\text { Top } \\
\text { Depth } \\
\text { (ft) } \\
\end{array}$ & $\begin{array}{c}\text { Screen } \\
\text { Bottom } \\
\text { Depth } \\
\text { (ft) }\end{array}$ \\
\hline LAO-1 & $2 / 1 / 1996$ & 28 & 1629395.00 & 1773956.37 & 6836.24 & 8 & 28 \\
\hline LAO-1.6g & $3 / 20 / 1996$ & 30.82 & 1636083.42 & 1772557.63 & 6658.01 & 10.47 & 25.47 \\
\hline LAO-1.8 & $4 / 15 / 1969$ & 18 & 1635446.25 & 1772661.37 & 6680 & 8 & 18 \\
\hline LAO-2 & $2 / 1 / 1996$ & 32 & 1637607.75 & 1773095.87 & 6592.97 & 7 & 32 \\
\hline LAO-3a & 9/14/1989 & 14.7 & 1637980.87 & 1773099.75 & 6579.4 & 4.7 & 14.7 \\
\hline LAO-4.5c & $11 / 1 / 1989$ & 23.3 & 1643547.37 & 1772076.50 & 6457.63 & 13.3 & 23.3 \\
\hline LAO-5 & $2 / 15 / 1966$ & 25 & 1646202.25 & 1771424.12 & 6398.3 & 5 & 25 \\
\hline LAO-6 & $2 / 1 / 1966$ & 16 & 1646222.12 & 1771329.50 & 6395.3 & 6 & 16 \\
\hline LAO-6a & $8 / 1 / 1989$ & 14.2 & 1646221.62 & 1771344.00 & 6395.88 & 4.2 & 14.2 \\
\hline LAUZ-1 & & 10.55 & 1633435.13 & 1774809.81 & 7032.42 & 5.35 & 10.35 \\
\hline LLAO-1b & $7 / 16 / 1997$ & 24.17 & 1659738.70 & 1772381.65 & 5850.34 & 11.32 & 21.32 \\
\hline LLAO-4 & 9/30/1996 & 18.1 & 1671820.23 & 1774468.01 & 5515.46 & 5.24 & 15.24 \\
\hline MCA-1 & $1 / 24 / 2005$ & 5.9 & 1626586.50 & 1770410.77 & 7070.6 & 2.4 & 5.4 \\
\hline MCA-2 & $10 / 24 / 2004$ & 65 & 1634097.23 & 1768585.88 & 6837.2 & 45 & 60 \\
\hline MCA-3a & $11 / 29 / 2004$ & 43.4 & 1633586.89 & 1769068.28 & 6853 & 42.9 & 43.4 \\
\hline MCA-3c & $11 / 30 / 2004$ & 43.4 & 1633586.89 & 1769068.28 & 6853 & 38.4 & 38.9 \\
\hline MCA-3b & $11 / 22 / 2004$ & 42.1 & 1633589.40 & 1769069.84 & 6852.9 & 40.9 & 41.4 \\
\hline MCA-3d & $12 / 2 / 2004$ & 55.8 & 1634886.02 & 1768523.27 & 6819.8 & 54.6 & 55.1 \\
\hline MCA-3e & $12 / 2 / 2004$ & 55.8 & 1634886.02 & 1768523.27 & 6819.8 & 51 & 51.5 \\
\hline MCA-3f & $12 / 2 / 2004$ & 55.8 & 1634886.02 & 1768523.27 & 6819.8 & 47.5 & 48 \\
\hline MCA-4 & $2 / 1 / 2005$ & 6 & 1625945.50 & 1770129.55 & 7135.1 & 3.3 & 5.3 \\
\hline MCA-5 & $2 / 1 / 2005$ & 5.4 & 1627354.17 & 1770233.59 & 7053.8 & 1.75 & 5.75 \\
\hline MCA-8 & 9/29/2004 & 86.3 & 1641325.48 & 1767372.92 & 6668.8 & 66 & 81 \\
\hline MCA-9 & $12 / 4 / 2004$ & 113 & 1641470.29 & 1767393.22 & 6671.7 & 92.8 & 107.8 \\
\hline MCO-0.6 & $2 / 25 / 1999$ & 3.1 & 1623987.80 & 1771179.50 & 7188.28 & 1.05 & 3.05 \\
\hline MCO-2 & $11 / 1 / 1960$ & 9 & 1625919.25 & 1770135.12 & 7136.6 & 2 & 9 \\
\hline MCO-3 & $3 / 1 / 1967$ & 12 & 1627362.50 & 1770236.75 & 7052.6 & 2 & 12 \\
\hline MCO-4B & $8 / 1 / 1990$ & 33.9 & 1632036.37 & 1769697.00 & 6886.75 & 8.9 & 28.9 \\
\hline MCO-5 & $10 / 1 / 1960$ & 46 & 1632466.12 & 1769538.00 & 6875.66 & 21 & 46 \\
\hline MCO-6 & $3 / 1 / 1974$ & 47 & 1633635.37 & 1769012.75 & 6849.48 & 27 & 47 \\
\hline MCO-7 & $10 / 1 / 1960$ & 69 & 1634517.87 & 1768509.87 & 6827.31 & 39 & 69 \\
\hline MCO-7.5 & $4 / 1 / 1974$ & 60 & 1635454.87 & 1768440.50 & 6808.88 & 35 & 60 \\
\hline MCWB-5 & $12 / 6 / 1994$ & 33 & 1632578.31 & 1769484.60 & 6876.22 & 17 & 27 \\
\hline MCWB-5.5B & $12 / 22 / 1994$ & 37.5 & 1633420.54 & 1769125.78 & 6856.89 & 22.5 & 32.5 \\
\hline MCWB-6.2A & $12 / 7 / 1994$ & 45.5 & 1633754.49 & 1768968.15 & 6848.29 & 30.5 & 40.5 \\
\hline MCWB-6.5E & $12 / 21 / 1994$ & 50 & 1633833.36 & 1768583.81 & 6843.8 & 35 & 45 \\
\hline MCWB-7A & $12 / 9 / 1994$ & 52 & 1634356.62 & 1768551.02 & 6831.17 & 37 & 47 \\
\hline MCWB-7.4B & $12 / 13 / 1994$ & 70 & 1635287.73 & 1768407.84 & 6813.07 & 45 & 65 \\
\hline MCWB-7.7B & $12 / 20 / 1994$ & 70 & 1635921.84 & 1768517.26 & 6798.97 & 55 & 65 \\
\hline $\begin{array}{l}\text { MSC-16- } \\
06293\end{array}$ & $1 / 27 / 2000$ & 7.3 & 1615809.67 & 1761331.78 & 7370.79 & 2 & 7 \\
\hline $\begin{array}{l}\text { MSC-16- } \\
06294\end{array}$ & $1 / 26 / 2000$ & 7.6 & 1617848.17 & 1761298.78 & 7288.44 & 2.5 & 7.3 \\
\hline $\begin{array}{l}\text { MSC-16- } \\
06295\end{array}$ & $1 / 31 / 2000$ & 6.9 & 1618630.67 & 1761004.78 & 7257.03 & 1.5 & 6.5 \\
\hline
\end{tabular}


Table 5-1. Information and Location Data for Alluvial Aquifer Wells at LANL (cont.)

\begin{tabular}{|c|c|c|c|c|c|c|c|}
\hline Well Name & $\begin{array}{c}\text { Date } \\
\text { Completed }\end{array}$ & $\begin{array}{l}\text { Completed } \\
\text { Depth (ft) }\end{array}$ & Easting (ft) & $\begin{array}{c}\text { Northing } \\
\text { (ft) }\end{array}$ & $\begin{array}{c}\text { Surface } \\
\text { Elevation } \\
\text { (ft) }\end{array}$ & $\begin{array}{c}\text { Screen } \\
\text { Top } \\
\text { Depth } \\
\text { (ft) } \\
\end{array}$ & $\begin{array}{c}\text { Screen } \\
\text { Bottom } \\
\text { Depth } \\
\text { (ft) }\end{array}$ \\
\hline MT-1 & $11 / 1 / 1988$ & 69 & 1635262.86 & 1768493.96 & 6811.63 & 39 & 59 \\
\hline MT-2 & 11/1/1988 & 64 & 1636019.79 & 1768544.59 & 6796.2 & 44 & 64 \\
\hline MT-3 & $11 / 1 / 1988$ & 74 & 1635980.95 & 1768657.83 & 6796.65 & 44 & 64 \\
\hline MT-4 & $11 / 1 / 1988$ & 74 & 1636558.75 & 1768634.37 & 6783.59 & 54 & 64 \\
\hline PAO-1 & $10 / 30 / 1998$ & 13.74 & 1624165.85 & 1778988.72 & 6954.97 & 5.89 & 10.89 \\
\hline PAO-2 & $11 / 2 / 1998$ & 13.91 & 1625040.90 & 1778710.00 & 6930.98 & 6.06 & 11.06 \\
\hline PAO-4 & $7 / 24 / 1997$ & 9.82 & 1646090.28 & 1775098.35 & 6437.37 & 1.97 & 6.97 \\
\hline PCO-1 & $6 / 30 / 1985$ & 12 & 1637919.25 & 1759990.62 & 6687 & 4 & 12 \\
\hline PCO-2 & $6 / 30 / 1985$ & 9.5 & 1641700.37 & 1757442.75 & 6618.3 & 1.5 & 9.5 \\
\hline PCO-3 & $6 / 30 / 1985$ & 17.7 & 1646088.62 & 1755489.37 & 6546.3 & 5.7 & 17.7 \\
\hline SCA-1 & $8 / 25 / 2006$ & 2.1 & 1622482.45 & 1773264.59 & 7211.22 & 1.3 & 1.9 \\
\hline SCA-2 & $8 / 24 / 2006$ & 15.6 & 1636115.02 & 1770282.78 & 6749.08 & 10.3 & 15 \\
\hline SCA-3 & 9/9/2006 & 32.6 & 1637200.75 & 1769917.84 & 6723.22 & 27.6 & 32 \\
\hline SCA-4 & 9/10/2006 & 42 & 1638261.23 & 1769566.62 & 6703.58 & 37 & 41.5 \\
\hline SCA-5 & 9/11/2006 & 64.9 & 1639878.28 & 1769725.47 & 6669.02 & 55 & 64.4 \\
\hline SCO-1 & $8 / 15 / 1989$ & 19.3 & 1642297.62 & 1769502.25 & 6618.67 & 9.3 & 19.3 \\
\hline SCO-2 & $8 / 16 / 1989$ & 19.4 & 1647259.00 & 1767864.00 & 6500.67 & 9.4 & 19.4 \\
\hline SCP-1a & $9 / 12 / 2006$ & 41.8 & 1638254.68 & 1769567.80 & 6703.65 & 41.2 & 41.7 \\
\hline SCP-1b & $9 / 12 / 2006$ & 41.8 & 1638254.68 & 1769567.80 & 6703.65 & 39.4 & 39.9 \\
\hline SCP-1C & $9 / 12 / 2006$ & 41.8 & 1638254.68 & 1769567.80 & 6703.65 & 37.8 & 38.3 \\
\hline SCP-2a & $9 / 13 / 2006$ & 45.1 & 1637209.65 & 1769911.26 & 6722.95 & 44.5 & 45 \\
\hline SCP-2b & $9 / 12 / 2006$ & 50.1 & 1637205.05 & 1769914.53 & 6723.11 & 49.5 & 50 \\
\hline TSCA-6 & $11 / 9 / 2004$ & 21.3 & 1632954.60 & 1768471.44 & 6863.2 & 16.2 & 20.9 \\
\hline TSWB-6 & $12 / 21 / 1994$ & 40 & 1633383.09 & 1768490.75 & 6853.21 & 25 & 35 \\
\hline WCO-1 & 10/31/1989 & 34.4 & 1632758.75 & 1755069.12 & 6616.41 & 24.4 & 34.4 \\
\hline WCO-2 & 10/26/1989 & 23.5 & 1636870.37 & 1753228.37 & 6524.57 & 13.5 & 23.5 \\
\hline WCO-3 & $10 / 25 / 1989$ & 12.4 & 1640212.50 & 1750620.25 & 6436.43 & 7.4 & 12.4 \\
\hline
\end{tabular}




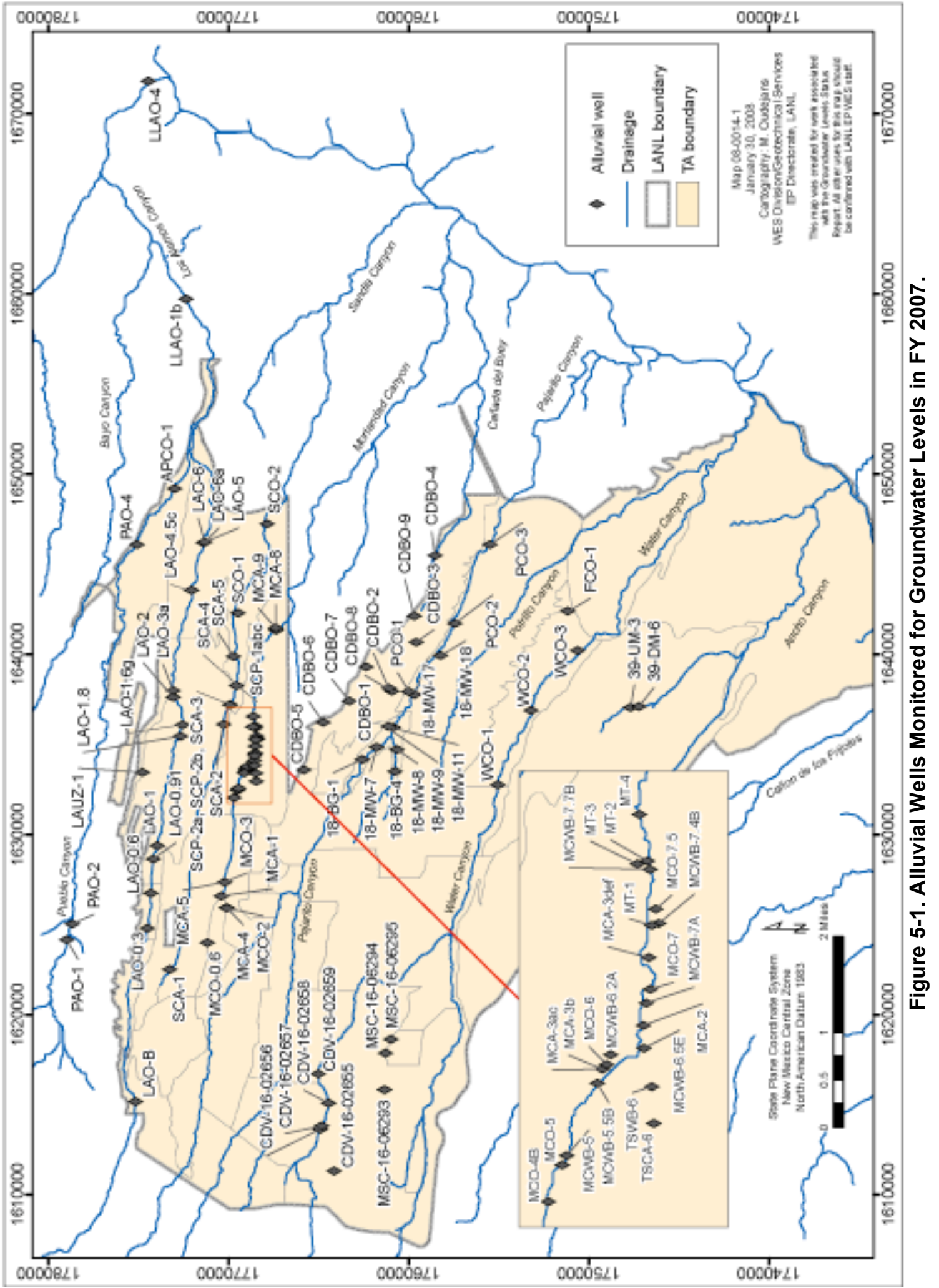




\subsection{8-BG-1}

Location: Pajarito Canyon, about 0.4 miles west of the TA-18 facilities.

Period of Record: August 1, 1994, through September 30, 2007

Remarks: A pressure transducer was installed in 18-BG-1 on August 16, 2005. The screen bottom elevation is $6741.45 \mathrm{ft}$.
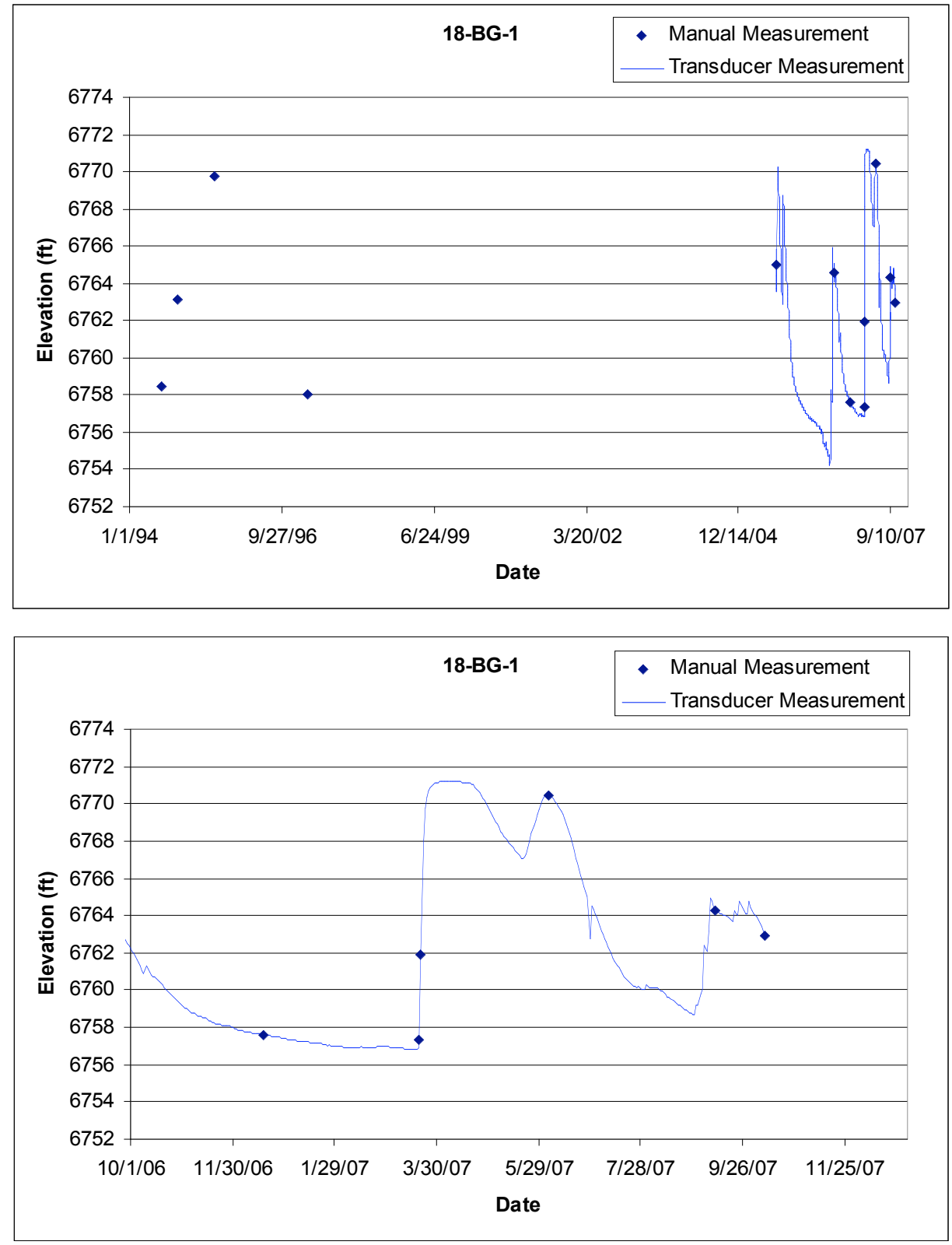


\subsection{8-BG-4}

Location: Three-Mile Canyon, about 0.3 miles west of the TA-18 facilities.

Period of Record: February 18, 1998, through September 30, 2007

Remarks: A pressure transducer was installed on August 18, 2005.
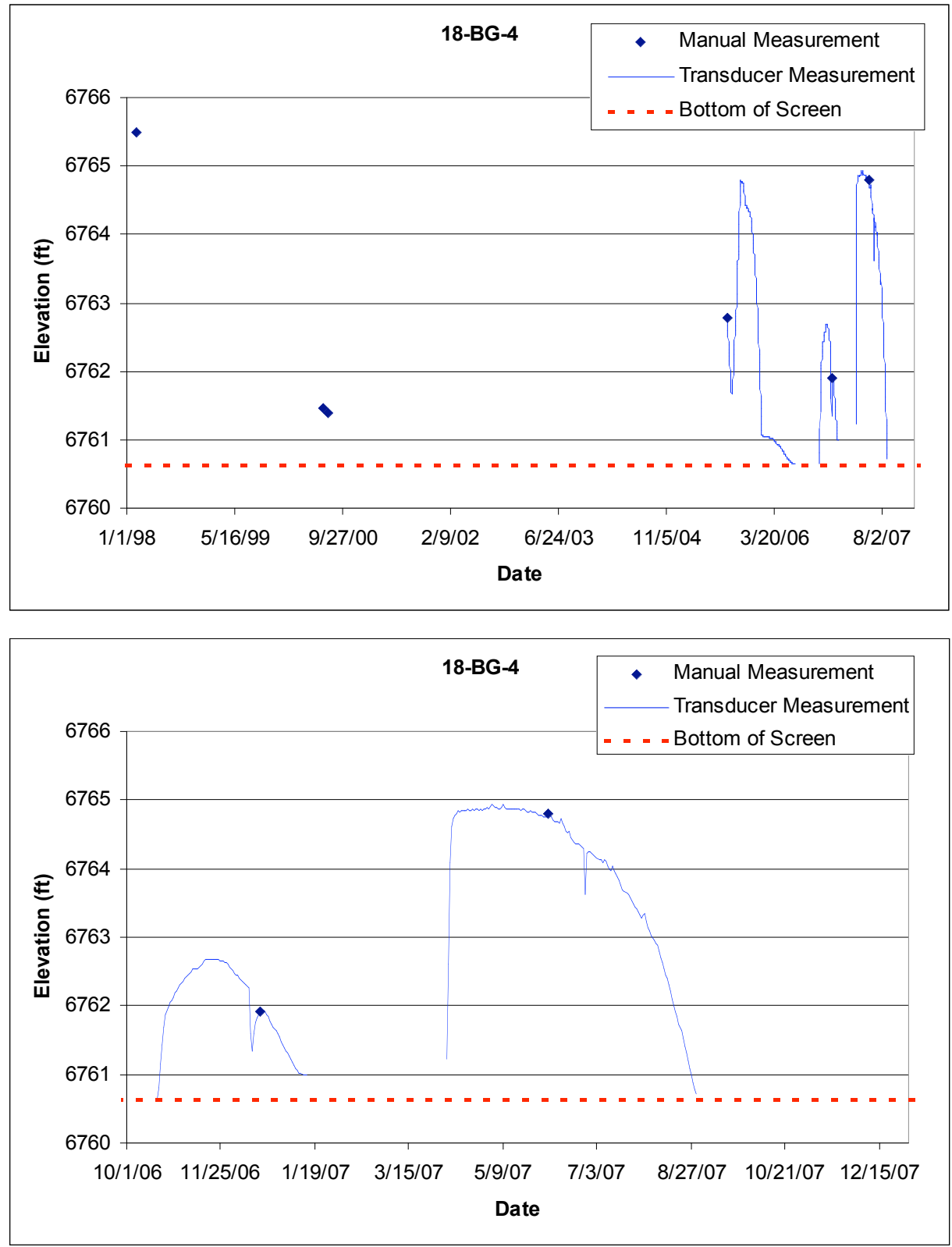


\section{$5.3 \quad 18-M W-7$}

Location: Pajarito Canyon, about 0.2 miles west of the TA-18 facilities.

Period of Record: July 6, 1994, through September 30, 2007

Remarks: A pressure transducer was installed in 18-MW-7 on August 18, 2005. Screen bottom elevation is $6725.5 \mathrm{ft}$. The transducer was removed on December 18, 2006, monitoring of this location was transferred to $18-\mathrm{MW}-11$.
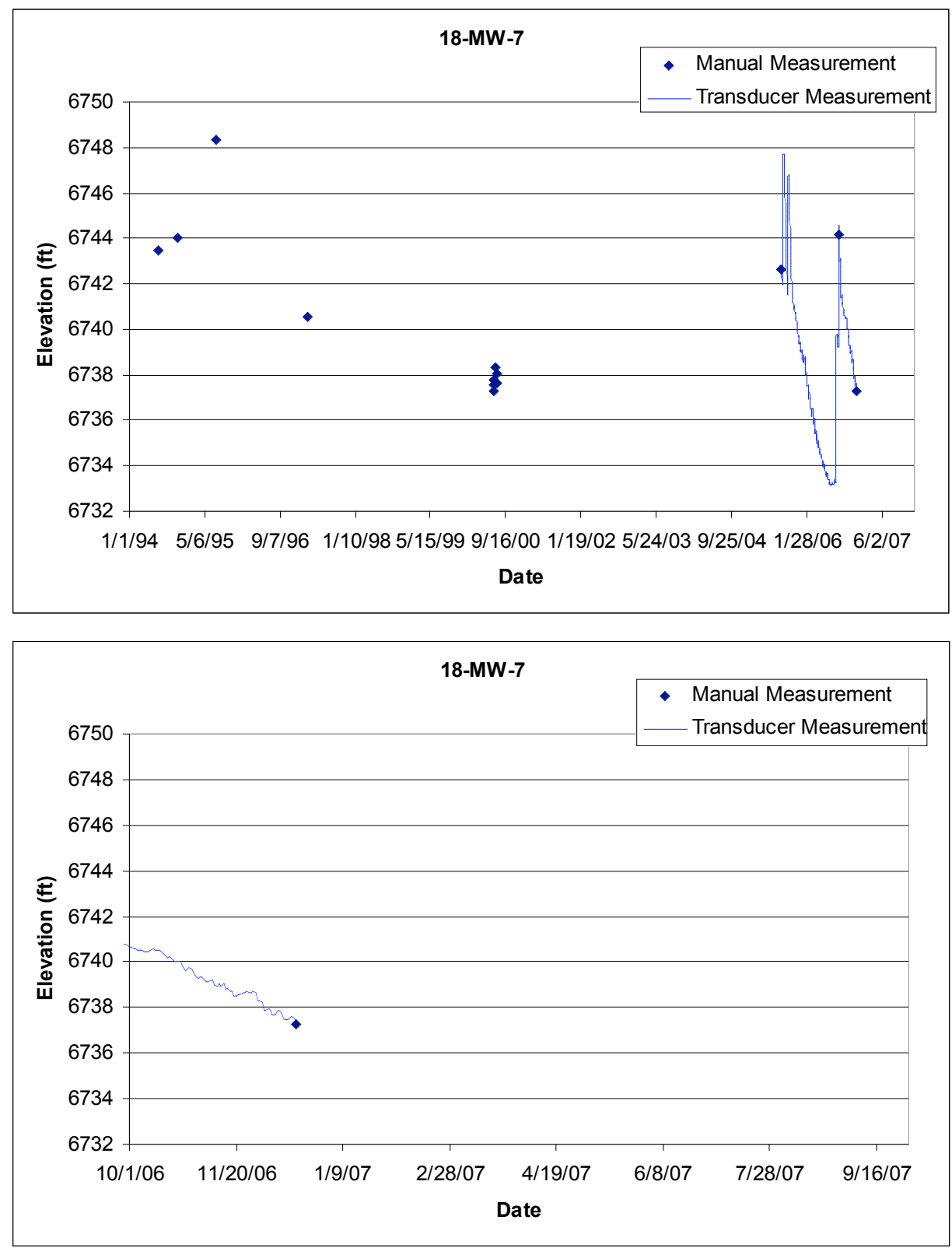


\section{$5.4 \quad 18-M W-8$}

Location: Three-Mile Canyon, about 0.1 miles west of the TA-18 facilities.

Period of Record: September 15, 1994, through September 30, 2007

Remarks: A pressure transducer was installed in 18-MW-8 on August 18, 2005. Screen bottom elevation is $6709.8 \mathrm{ft}$.
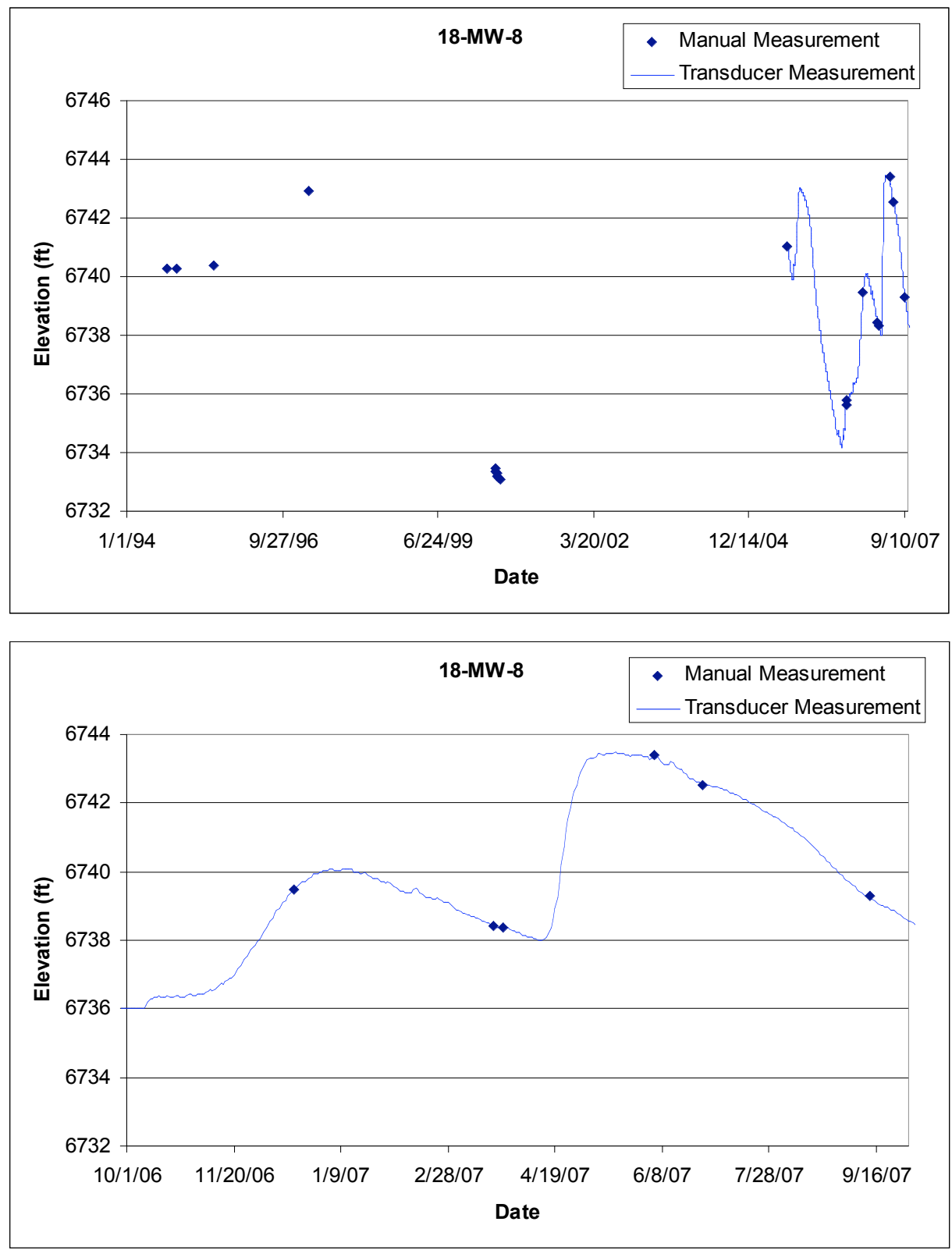


\section{$5.5 \quad 18-M W-9$}

Location: Pajarito Canyon, directly south of the main guard gate to TA-18.

Period of Record: July 21, 1994, through September 30, 2007

Remarks: A pressure transducer was installed in 18-MW-9 on August 18, 2005. Screen bottom elevation is $6711.9 \mathrm{ft}$.
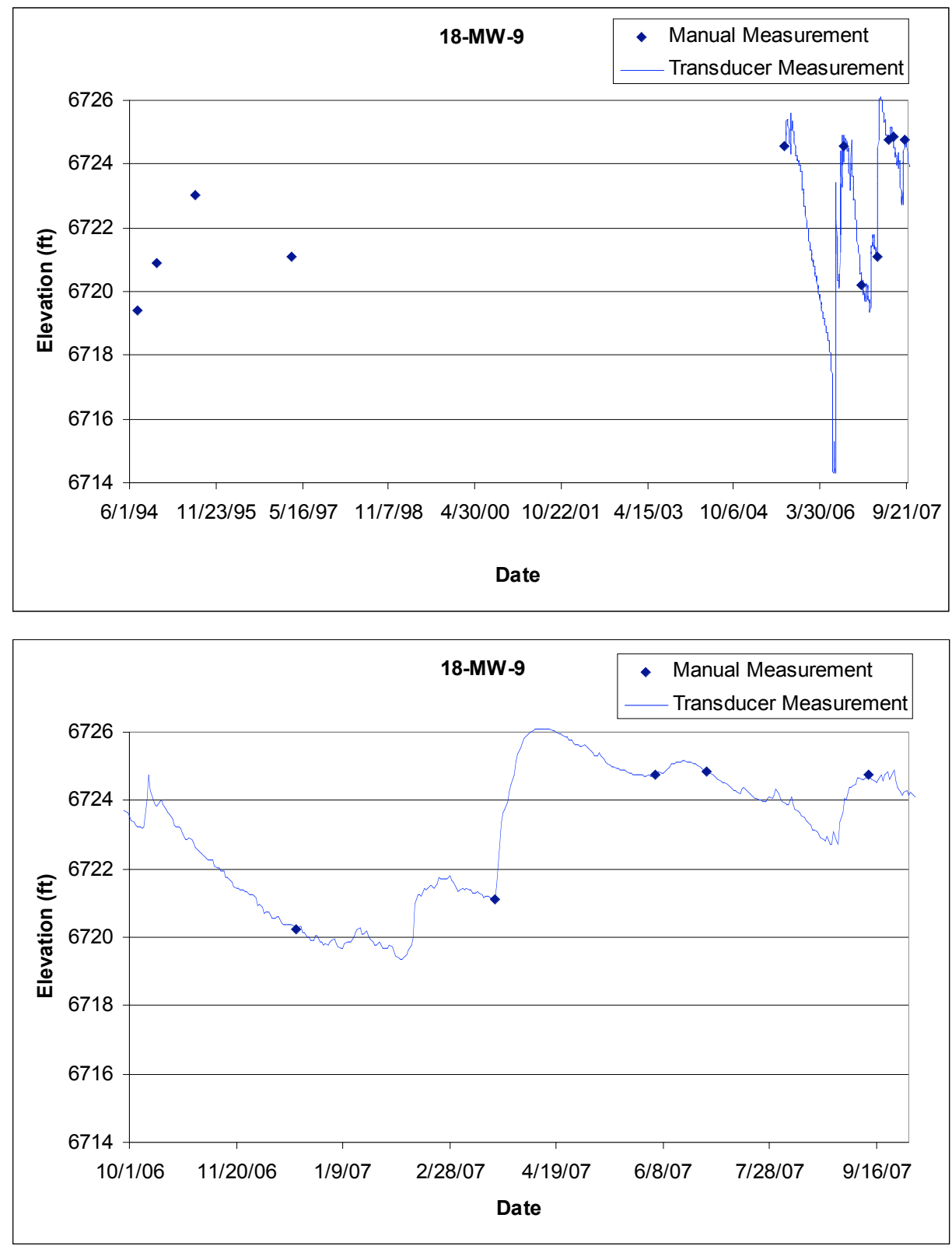


\subsection{8-MW-11}

Location: Pajarito Canyon, approximately $200 \mathrm{ft}$ north of $18-\mathrm{MW}-9$ in the TA-18 parking lot. Period of Record: August 29, 2006, through September 30, 2007

Remarks: None.
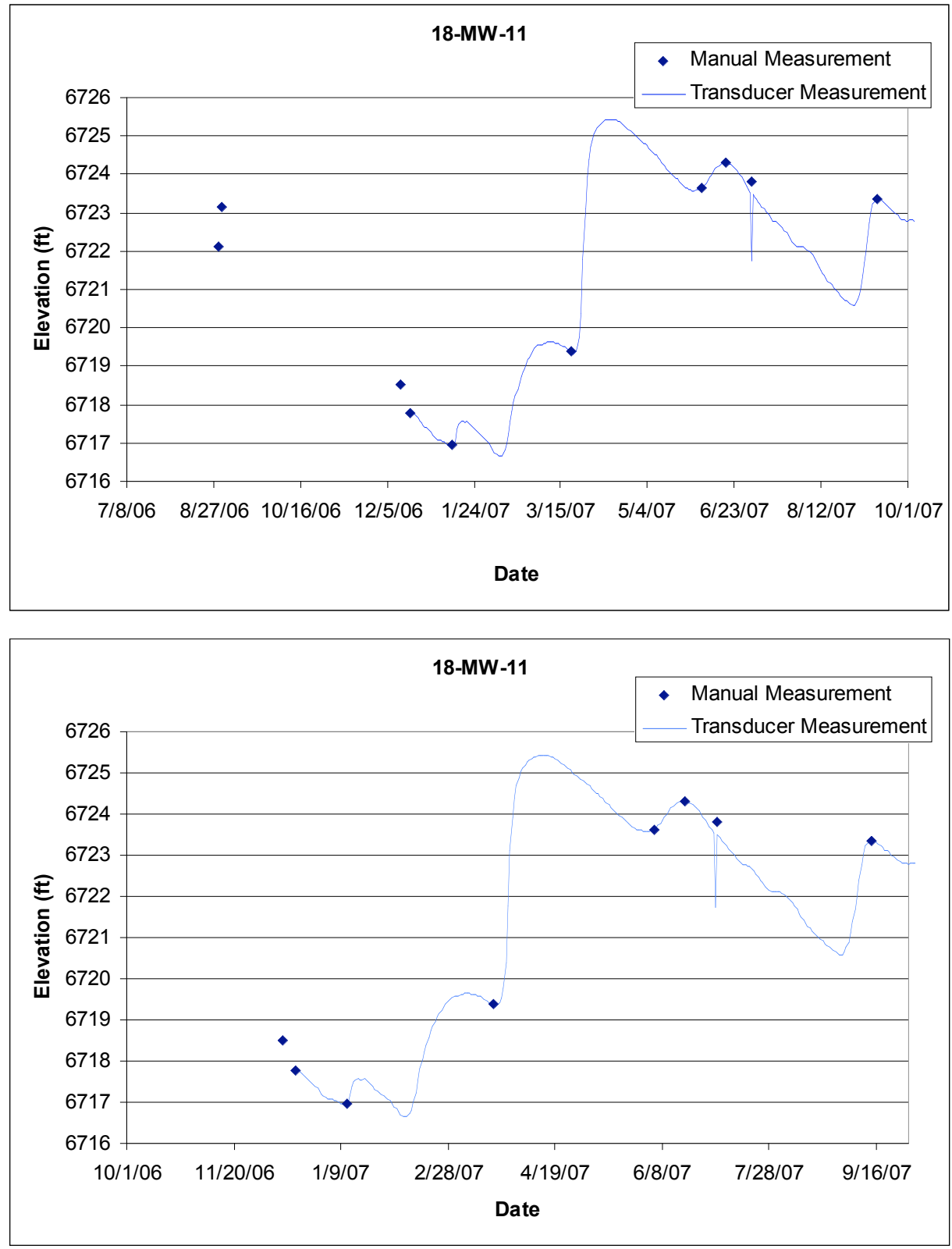


\section{$5.7 \quad 18-\mathrm{MW}-17$}

Location: Pajarito Canyon, east of TA-18, approximately $50 \mathrm{ft}$ west of R-20.

Period of Record: August 1, 1995, through September 30, 2007

Remarks: A pressure transducer was installed in 18-MW-17 on June 9, 2005. Screen bottom elevation is $6673.2 \mathrm{ft}$. The pressure transducer is installed above the top of the bladder pump. Due to the 2-in. well construction, the transducer does not fit below the pump. The water drops below the transducer sensor when the water level declines below $6678.4 \mathrm{ft}$.
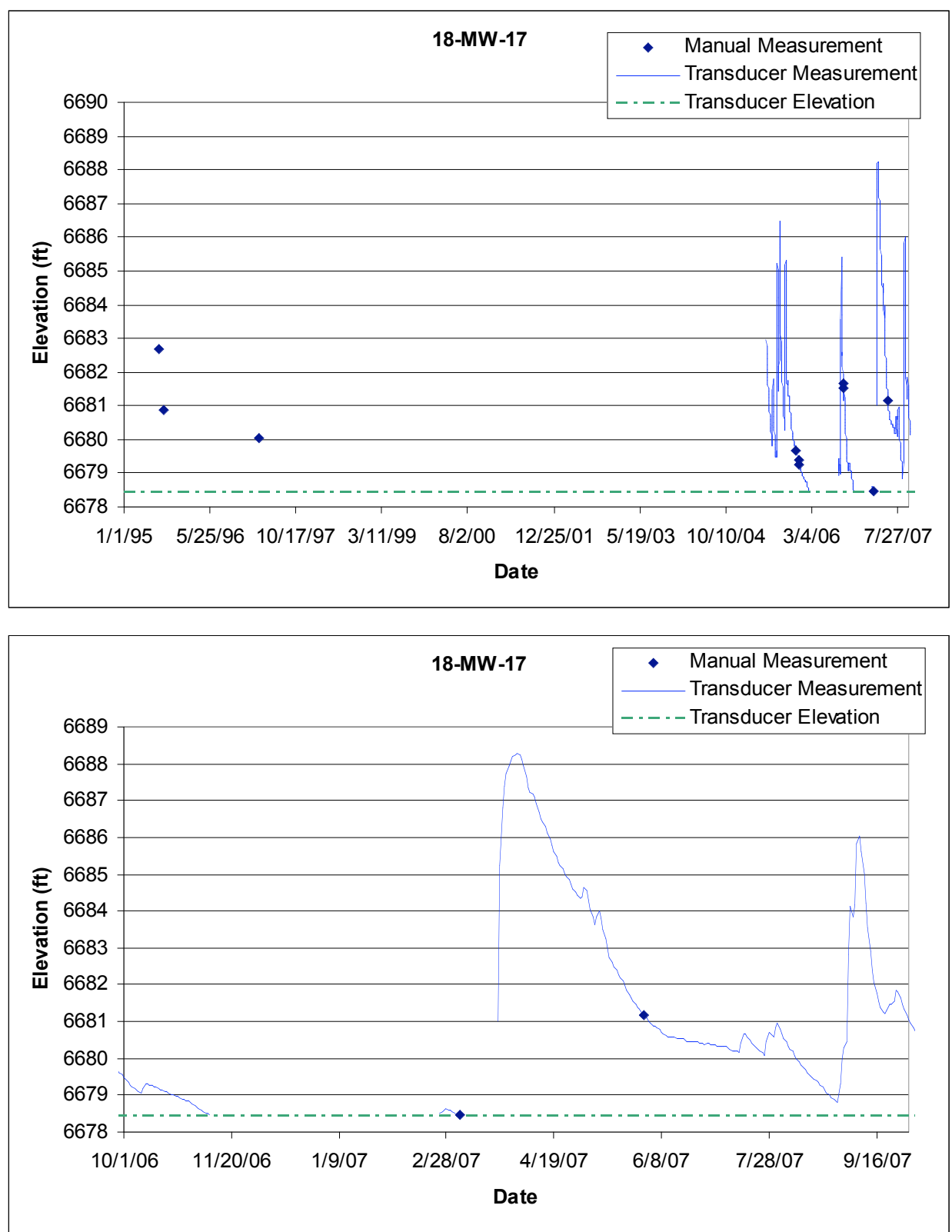


\section{$5.8 \quad 18-M W-18$}

Location: Alluvial well 18-MW-18 is located in Pajarito Canyon, approximately $1000 \mathrm{ft}$ east of 18-MW17.

Period of Record: July 31, 1995, through September 25, 2007

Remarks: A pressure transducer was installed in 18-MW-18 on June 9, 2005. Screen bottom elevation is $6631.7 \mathrm{ft}$. The transducer battery died on September 25, 2007, and was replaced on October 10, 2007.
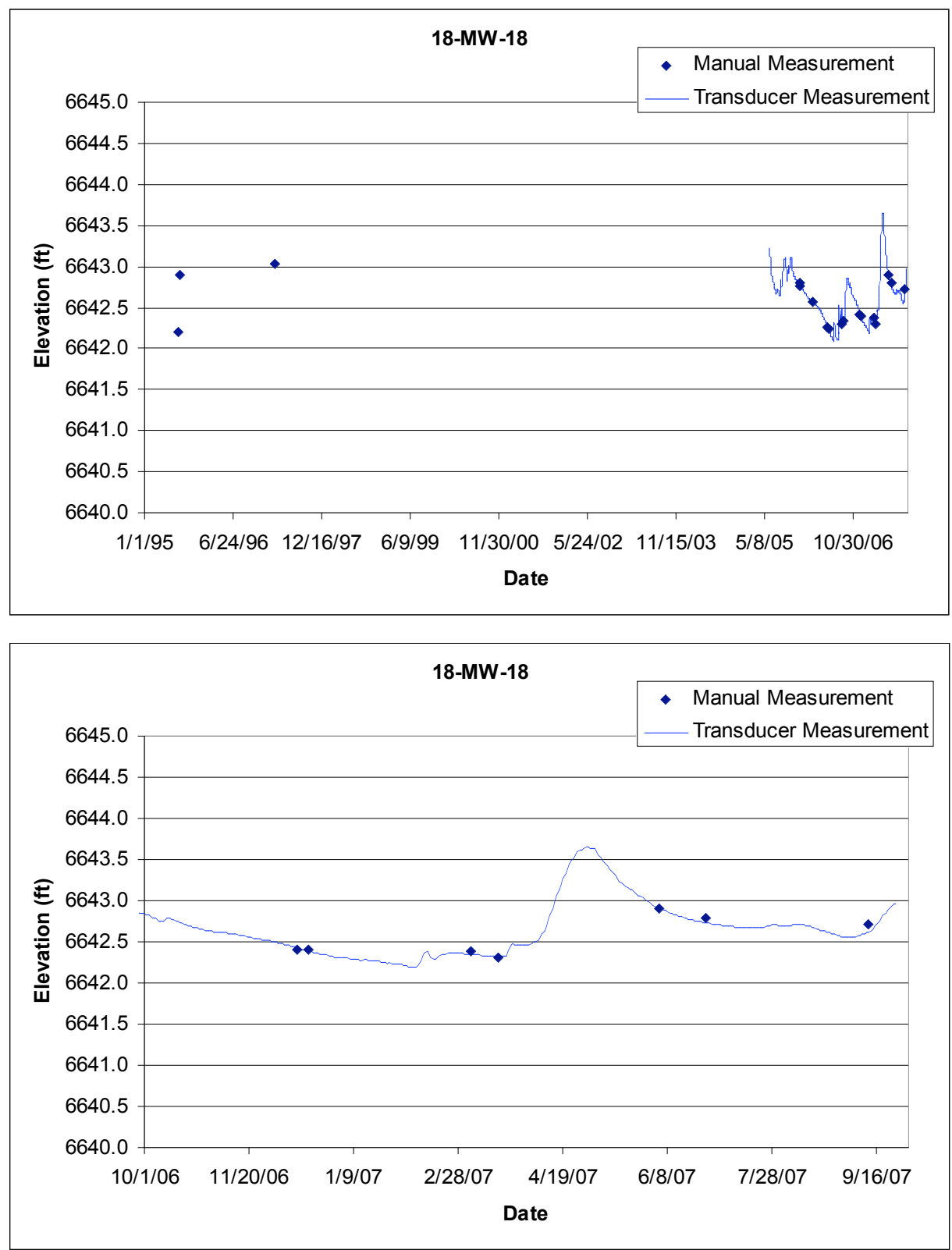


\section{$5.9 \quad 39-U M-3$}

Location: Ancho Canyon, TA-39, approximately $2100 \mathrm{ft}$ north of regional well R-31.

Period of Record: March 9, 2006, through September 5, 2007

Remarks: Well was dry during quarterly manual measurements.

\begin{tabular}{|c|c|c|c|}
\hline Well Name & Date Time & Water Level (ft) & Comments \\
\hline 39-UM-3 & 3/9/2006 10:00:00 AM & & Dry \\
\hline 39-UM-3 & 6/13/2006 7:07:00 AM & & Dry, $\mathrm{TD}=57.81 \mathrm{ft}$ \\
\hline 39-UM-3 & 9/7/2006 1:06:00 PM & & Dry \\
\hline 39-UM-3 & 11/30/2006 2:10:00 PM & & Dry \\
\hline 39-UM-3 & 12/12/2006 12:44:00 PM & & Dry \\
\hline 39-UM-3 & 3/15/2007 12:34:00 PM & & Dry \\
\hline 39-UM-3 & 5/10/2007 2:30:00 PM & & Dry \\
\hline 39-UM-3 & 6/6/2007 9:01:00 AM & & Dry \\
\hline 39-UM-3 & 9/5/2007 11:42:00 AM & & Dry \\
\hline
\end{tabular}

\subsection{9-DM-6}

Location: Ancho Canyon, TA-39, approximately $1600 \mathrm{ft}$ north of regional well R-31.

Period of Record: March 9, 2006, through September 5, 2007

Remarks: Well was dry during quarterly manual measurements.

\begin{tabular}{|c|c|c|c|}
\hline Well Name & Date Time & Water Level (ft) & Comments \\
\hline 39-DM-6 & 3/9/2006 10:28:00 AM & & Dry \\
\hline 39-DM-6 & 6/13/2006 7:25:00 AM & & Dry, TD $=60.19 \mathrm{ft}$ \\
\hline 39-DM-6 & 9/7/2006 1:03:00 PM & & Dry \\
\hline 39-DM-6 & 11/30/2006 2:16:00 PM & & Dry \\
\hline 39-DM-6 & 12/12/2006 12:37:00 PM & & Dry \\
\hline 39-DM-6 & 3/15/2007 12:28:00 PM & & Dry \\
\hline 39-DM-6 & 5/10/2007 2:50:00 PM & & Dry \\
\hline 39-DM-6 & 6/6/2007 8:55:00 AM & & Dry \\
\hline 39-DM-6 & 9/5/2007 11:46:00 AM & & Dry \\
\hline
\end{tabular}




\subsection{APCO-1}

Location: Alluvial well APCO-1 is located in lower Pueblo Canyon.

Period of Record: August 17, 1990, through September 30, 2007

Remarks: A pressure transducer was installed in APCO-1 February 17, 1993, through June 17, 1993, January 11, 1994, through November 9, 1994, and May 9, 2005. Screen bottom elevation is $6352.83 \mathrm{ft}$.
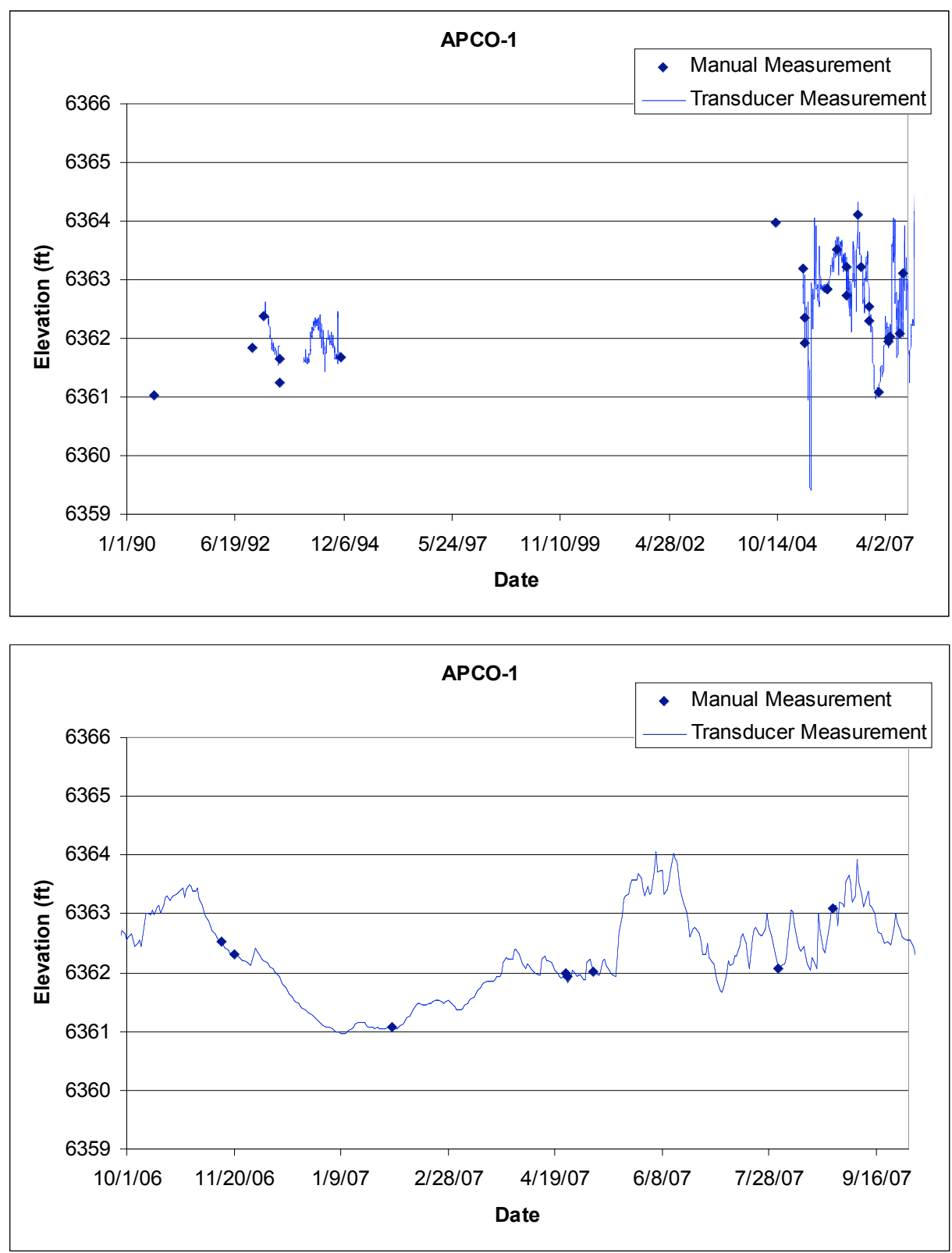


\subsection{CDBO-1}

Location: Alluvial well CDBO-1 is located in Cañada del Buey, approximately $1320 \mathrm{ft}$ north of regional well R-20.

Period of Record: March 8, 2006, through September 10, 2007

Remarks: Well was dry during quarterly measurements.

\begin{tabular}{|l||r|l||l|}
\hline Well Name & \multicolumn{1}{|c|}{ Date Time } & Water Level (ft) & Comments \\
\hline CDBO-1 & $3 / 8 / 2006$ 2:53:00 PM & & Dry \\
\hline CDBO-1 & $6 / 26 / 2006$ 9:58:00 AM & & Dry, TD = 14.41 ft \\
\hline CDBO-1 & $9 / 6 / 2006$ 9:44:00 AM & Dry \\
\hline CDBO-1 & $9 / 27 / 200611: 15: 00$ AM & & Dry \\
\hline CDBO-1 & $12 / 8 / 200610: 03: 00$ AM & Dry \\
\hline CDBO-1 & $2 / 22 / 2007$ 7:37:00 AM & Dry \\
\hline CDBO-1 & $3 / 19 / 200710: 40: 00$ AM & & Dry \\
\hline CDBO-1 & $6 / 5 / 2007$ 8:33:00 AM & Dry \\
\hline CDBO-1 & $6 / 11 / 200712: 10: 00$ PM & Dry \\
\hline CDBO-1 & $6 / 11 / 200712: 20: 00$ PM & Dry \\
\hline CDBO-1 & $9 / 10 / 200712: 42: 00$ PM & Dry \\
\hline
\end{tabular}

\subsection{CDBO-2}

Location: Alluvial well CDBO-2 is located in Cañada del Buey, approximately $260 \mathrm{ft}$ northeast of CDBO-1.

Period of Record: March 8, 2006, through September 10, 2007

Remarks: Well was dry during quarterly measurements.

\begin{tabular}{|l||r|l||l|}
\hline Well Name & \multicolumn{1}{|c|}{ Date Time } & Water Level (ft) & \multicolumn{1}{|c|}{ Comments } \\
\hline CDBO-2 & $3 / 8 / 2006$ 11:08:00 AM & & Dry \\
\hline CDBO-2 & $6 / 26 / 2006$ 10:05:00 AM & & Dry, TD = 19.24 ft \\
\hline CDBO-2 & $9 / 6 / 2006$ 9:52:00 AM & Dry \\
\hline CDBO-2 & $9 / 27 / 2006$ 11:25:00 AM & Dry \\
\hline CDBO-2 & $12 / 8 / 2006$ 9:53:00 AM & Dry \\
\hline CDBO-2 & $2 / 22 / 2007$ 7:45:00 AM & Dry \\
\hline CDBO-2 & $3 / 19 / 200710: 36: 00$ AM & Dry \\
\hline CDBO-2 & $6 / 5 / 2007$ 8:39:00 AM & Dry \\
\hline CDBO-2 & $6 / 11 / 200712: 14: 00 \mathrm{PM}$ & Dry \\
\hline CDBO-2 & $6 / 11 / 200712: 35: 00 \mathrm{PM}$ & Dry \\
\hline CDBO-2 & $9 / 10 / 200712: 51: 00 \mathrm{PM}$ & Dry \\
\hline
\end{tabular}




\subsection{CDBO-3}

Location: Alluvial well CDBO-3 is located in Cañada del Buey, approximately $630 \mathrm{ft}$ northwest of regional well R-21.

Period of Record: December 6, 2005, through September 10, 2007

Remarks: Well was dry during quarterly measurements.

\begin{tabular}{|c|c|c|c|}
\hline Well Name & Date Time & Water Level (ft) & Comments \\
\hline CDBO-3 & 12/6/2005 1:00:00 PM & & Dry \\
\hline CDBO-3 & 3/8/2006 10:06:00 AM & & Dry \\
\hline CDBO-3 & 6/26/2006 9:27:00 AM & & Dry, $T D=13.53 \mathrm{ft}$ \\
\hline CDBO-3 & 9/6/2006 9:19:00 AM & & Dry \\
\hline CDBO-3 & 9/27/2006 11:00:00 AM & & Dry \\
\hline CDBO-3 & 12/8/2006 10:23:00 AM & & Dry \\
\hline CDBO-3 & $2 / 22 / 2007$ 8:03:00 AM & & Dry \\
\hline CDBO-3 & 3/19/2007 10:59:00 AM & & Dry \\
\hline CDBO-3 & 6/5/2007 10:29:00 AM & & Dry \\
\hline CDBO-3 & 6/11/2007 12:00:00 PM & & Dry \\
\hline CDBO-3 & 6/11/2007 12:55:00 PM & & Dry \\
\hline CDBO-3 & 9/10/2007 1:16:00 PM & & Dry \\
\hline
\end{tabular}




\subsection{CDBO-4}

Location: Alluvial well CDBO-4 is located in Cañada del Buey, approximately $1600 \mathrm{ft}$ north of regional well R-22.

Period of Record: December 7, 2005, through September 10, 2007

Remarks: Well was dry during quarterly measurements.

\begin{tabular}{|l||r|l||l|}
\hline Well Name & Date Time & Water Level (ft) & Comments \\
\hline CDBO-4 & $12 / 7 / 2005$ 1:50:00 PM & & Dry \\
\hline CDBO-4 & $3 / 8 / 2006$ 9:24:00 AM & & Dry \\
\hline CDBO-4 & $6 / 26 / 2006$ 9:12:00 AM & Dry, TD = 13.42 ft \\
\hline CDBO-4 & $9 / 6 / 2006$ 8:58:00 AM & Dry \\
\hline CDBO-4 & $10 / 2 / 2006$ 2:43:00 PM & Dry \\
\hline CDBO-4 & $12 / 8 / 200612: 41: 00$ PM & Dry \\
\hline CDBO-4 & $2 / 22 / 2007$ 9:33:00 AM & Dry \\
\hline CDBO-4 & $3 / 19 / 200712: 20: 00$ PM & Dry \\
\hline CDBO-4 & $6 / 5 / 200710: 48: 00$ AM & Dry \\
\hline CDBO-4 & $6 / 11 / 200712: 35: 00 ~ P M$ & Dry \\
\hline CDBO-4 & $6 / 11 / 20071: 15: 00$ PM & Dry \\
\hline CDBO-4 & $9 / 10 / 20071: 46: 00$ PM & Dry \\
\hline
\end{tabular}




\subsection{CDBO-5}

Location: Alluvial well CDBO- 5 is located in Cañada del Buey, approximately 0.5 miles westnorthwest of CDBO-6.

Period of Record: December 7, 2005, through September 11, 2007

Remarks: Well was dry during quarterly measurements in FY 2007.

\begin{tabular}{|l|r|r||l|}
\hline Well Name & \multicolumn{1}{|c|}{ Date Time } & Water Level (ft) & Comments \\
\hline CDBO-5 & $12 / 7 / 2005$ 10:20:00 AM & & Dry \\
\hline CDBO-5 & $3 / 8 / 2006$ 12:23:00 PM & 6861.24 & Water is in sump \\
\hline CDBO-5 & $6 / 26 / 2006$ 12:24:00 PM & 6861.23 & Water is in sump \\
\hline CDBO-5 & $9 / 6 / 2006$ 11:34:00 AM & 6861.33 & Water is in sump \\
\hline CDBO-5 & $10 / 2 / 200611: 15: 00 \mathrm{AM}$ & & Dry \\
\hline CDBO-5 & $12 / 8 / 200611: 01: 00 \mathrm{AM}$ & & Dry \\
\hline CDBO-5 & $2 / 22 / 2007$ 8:35:00 AM & & Dry \\
\hline CDBO-5 & $3 / 19 / 200711: 30: 00 \mathrm{AM}$ & & Dry \\
\hline CDBO-5 & $6 / 5 / 2007$ 9:50:00 AM & & Dry \\
\hline CDBO-5 & $9 / 11 / 2007$ 9:23:00 AM & & Dry \\
\hline
\end{tabular}




\subsection{CDBO-6}

Location: Alluvial well CDBO-6 is located in Cañada del Buey, approximately $420 \mathrm{ft}$ east of production well PM-4.

Period of Record: June 1, 1992, through September 30, 2007

Remarks: The pressure transducer was installed above the pump at an elevation of $6776.83 \mathrm{ft}$ until April 30, 2007, when the pump was removed from the well. Transducer data before April 30, 2007 , do not represent water level elevations below $6776.83 \mathrm{ft}$. The screen bottom elevation is $6773.2 \mathrm{ft}$.
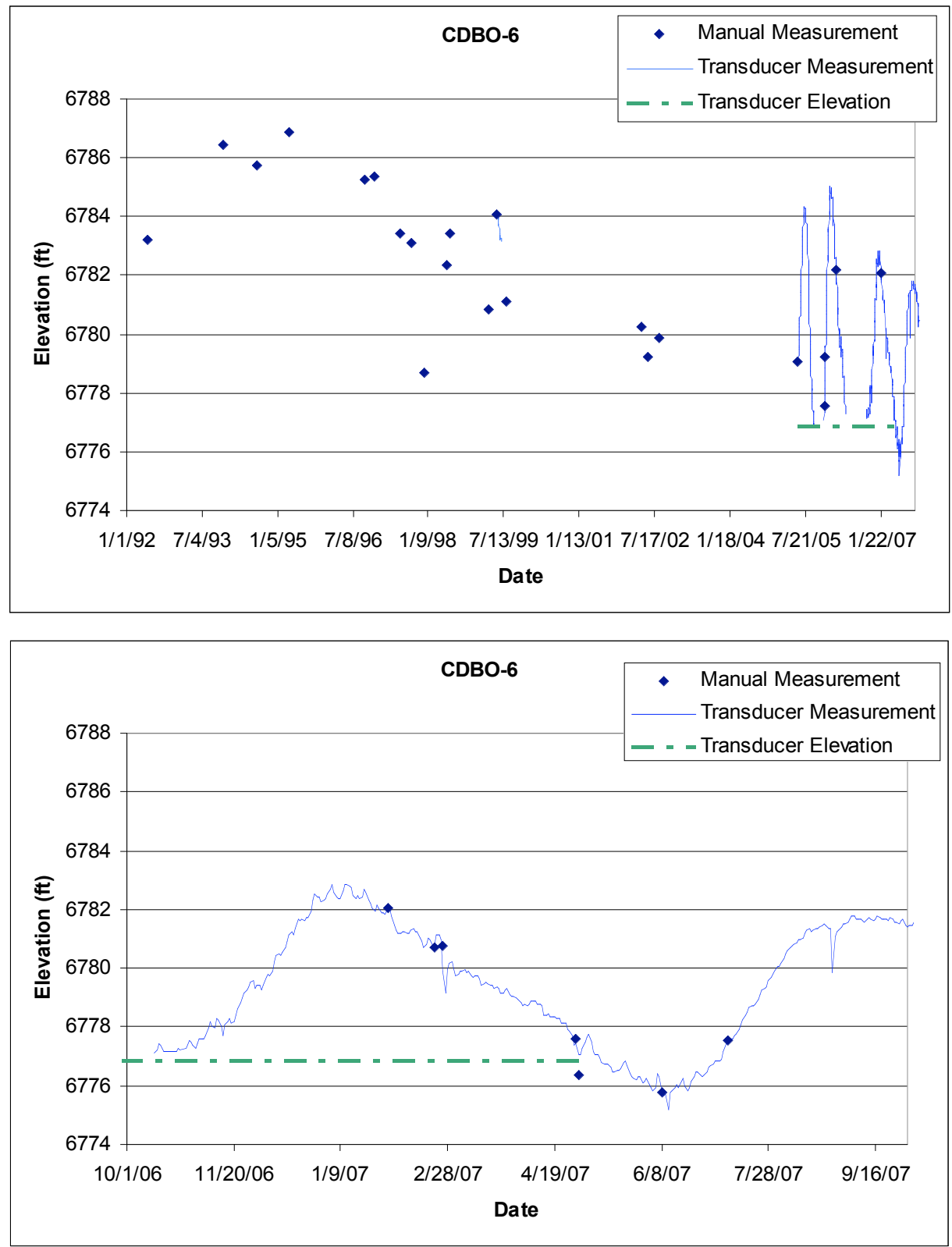


\subsection{CDBO-7}

Location: Alluvial well CDBO-7 is located in Cañada del Buey, approximately 0.3 miles southeast of CDBO-6.

Period of Record: June 1,1992, through September 30, 2007

Remarks: Alluvial well CDBO-7 had a bladder pump installed in a 2-in. casing until April 2, 2007. The pressure transducer was set above the pump at an elevation of $6737.14 \mathrm{ft}$. The transducer was lowered in the well after removal of the pump on April 2, 2007. Data before April 2, 2007, do not represent water levels below $6737.14 \mathrm{ft}$. The water level declined below the screen in late June 2007 . The screen bottom elevation is $6732.8 \mathrm{ft}$.
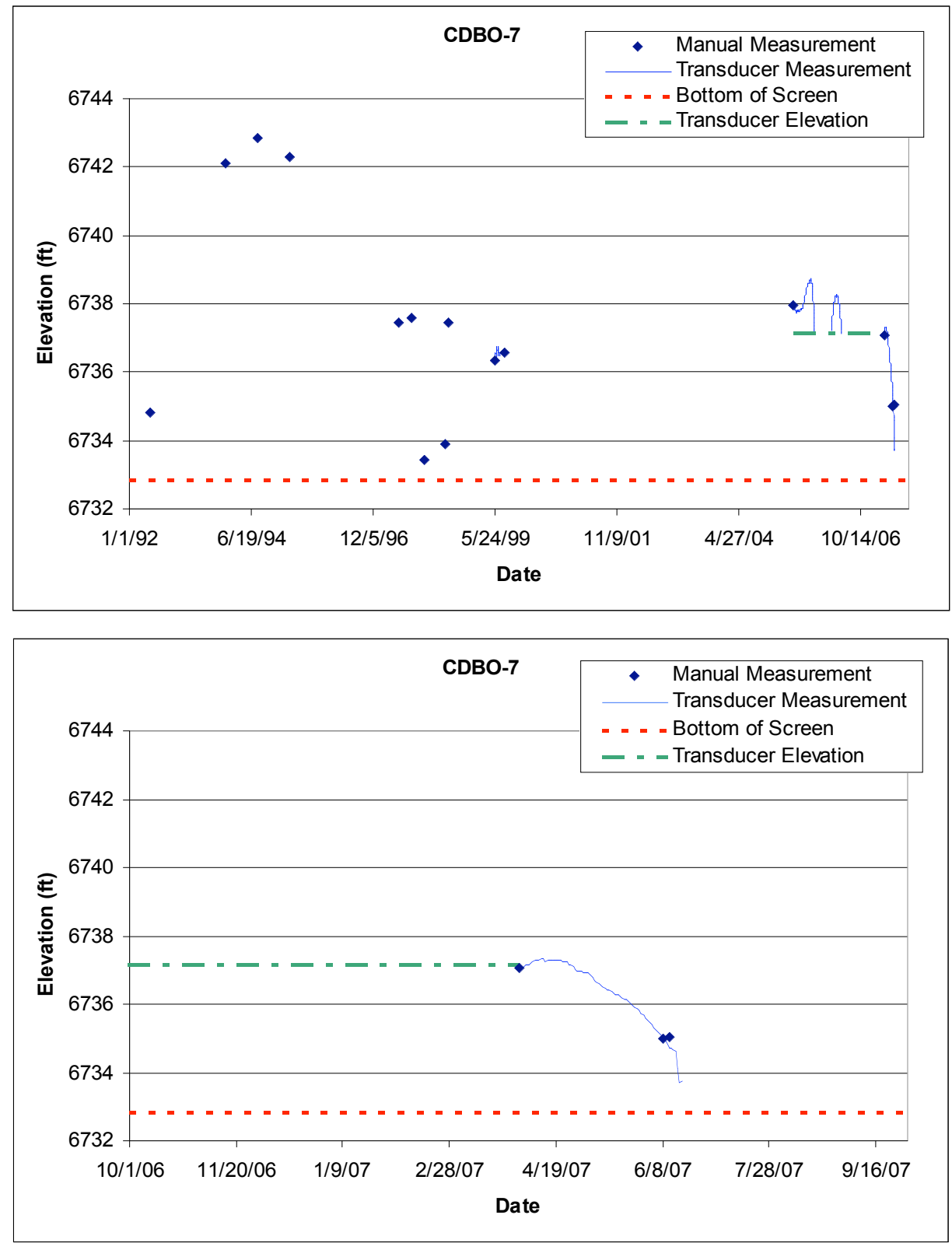


\subsection{CDBO-8}

Location: Alluvial well CDBO-8 is located in Cañada del Buey, approximately 0.4 miles southeast of CDBO-7.

Period of Record: July 2, 2001, through September 10, 2007

Remarks: Well was dry during quarterly measurements.

\begin{tabular}{|c|c|c|c|}
\hline Well Name & Date Time & Water Level (ft) & Comments \\
\hline CDBO-8 & 7/2/2001 12:00:00 PM & & Dry \\
\hline CDBO-8 & 8/22/2001 12:00:00 PM & & Dry \\
\hline CDBO-8 & 10/18/2001 12:00:00 PM & & Dry \\
\hline CDBO-8 & 4/16/2002 12:00:00 PM & & Dry \\
\hline CDBO-8 & 8/27/2002 12:00:00 PM & & Dry \\
\hline CDBO-8 & 11/15/2002 12:00:00 PM & & Dry \\
\hline CDBO-8 & 2/19/2003 12:00:00 PM & & Dry \\
\hline CDBO-8 & 2/19/2003 12:00:00 PM & & Dry \\
\hline CDBO-8 & 12/7/2005 1:30:00 PM & & Dry \\
\hline CDBO-8 & 3/8/2006 2:51:00 PM & & Dry \\
\hline CDBO-8 & 6/26/2006 10:53:00 AM & & Dry, $T D=25.66 \mathrm{ft}$ \\
\hline CDBO-8 & 9/7/2006 8:14:00 AM & & Dry \\
\hline CDBO-8 & 10/2/2006 12:48:00 PM & & Dry \\
\hline CDBO-8 & 12/8/2006 12:59:00 PM & & Dry \\
\hline CDBO-8 & 2/22/2007 9:10:00 AM & & Dry \\
\hline CDBO-8 & 3/19/2007 11:59:00 AM & & Dry \\
\hline CDBO-8 & 6/5/2007 9:11:00 AM & & Dry \\
\hline CDBO-8 & 6/8/2007 11:50:00 AM & & Dry \\
\hline CDBO-8 & 9/10/2007 1:25:00 PM & & Dry \\
\hline
\end{tabular}




\subsection{CDBO-9}

Location: Alluvial well CDBO-9 is located in Cañada del Buey, approximately 0.7 miles southeast of CDBO-8.

Period of Record: July 2, 2001, through September 10, 2007

Remarks: Well was dry during quarterly measurements.

\begin{tabular}{|c|c|c|c|}
\hline Well Name & Date Time & Water Level (ft) & Comments \\
\hline CDBO-9 & 7/2/2001 12:00:00 PM & & Dry \\
\hline CDBO-9 & 8/22/2001 12:00:00 PM & & Dry \\
\hline CDBO-9 & 10/18/2001 12:00:00 PM & & Dry \\
\hline CDBO-9 & 4/16/2002 12:00:00 PM & & Dry \\
\hline CDBO-9 & 8/27/2002 12:00:00 PM & & Dry \\
\hline CDBO-9 & 11/15/2002 12:00:00 PM & & Dry \\
\hline CDBO-9 & 2/19/2003 12:00:00 PM & & Dry \\
\hline CDBO-9 & 6/3/2003 12:00:00 PM & & Dry \\
\hline CDBO-9 & 12/6/2005 1:45:00 PM & & Dry \\
\hline CDBO-9 & 3/8/2006 2:42:00 PM & & Dry \\
\hline CDBO-9 & 6/26/2006 10:34:00 AM & & Dry, $T D=33.58 \mathrm{ft}$ \\
\hline CDBO-9 & 9/6/2006 10:27:00 AM & & Dry \\
\hline CDBO-9 & 10/2/2006 1:10:00 PM & & Dry \\
\hline CDBO-9 & 12/8/2006 1:14:00 PM & & Dry \\
\hline CDBO-9 & 2/22/2007 9:23:00 AM & & Dry \\
\hline CDBO-9 & 3/19/2007 12:10:00 PM & & Dry \\
\hline CDBO-9 & 6/5/2007 9:00:00 AM & & Dry \\
\hline CDBO-9 & 6/8/2007 12:05:00 PM & & Dry \\
\hline CDBO-9 & 9/10/2007 1:36:00 PM & & Dry \\
\hline
\end{tabular}




\subsection{CDV-16-02655}

Location: Westernmost upper Cañon de Valle in TA-16, approximately $800 \mathrm{ft}$ east of Anchor Ranch Road.

Period of Record: May 15, 1998, through September 30, 2007.

Remarks: None.
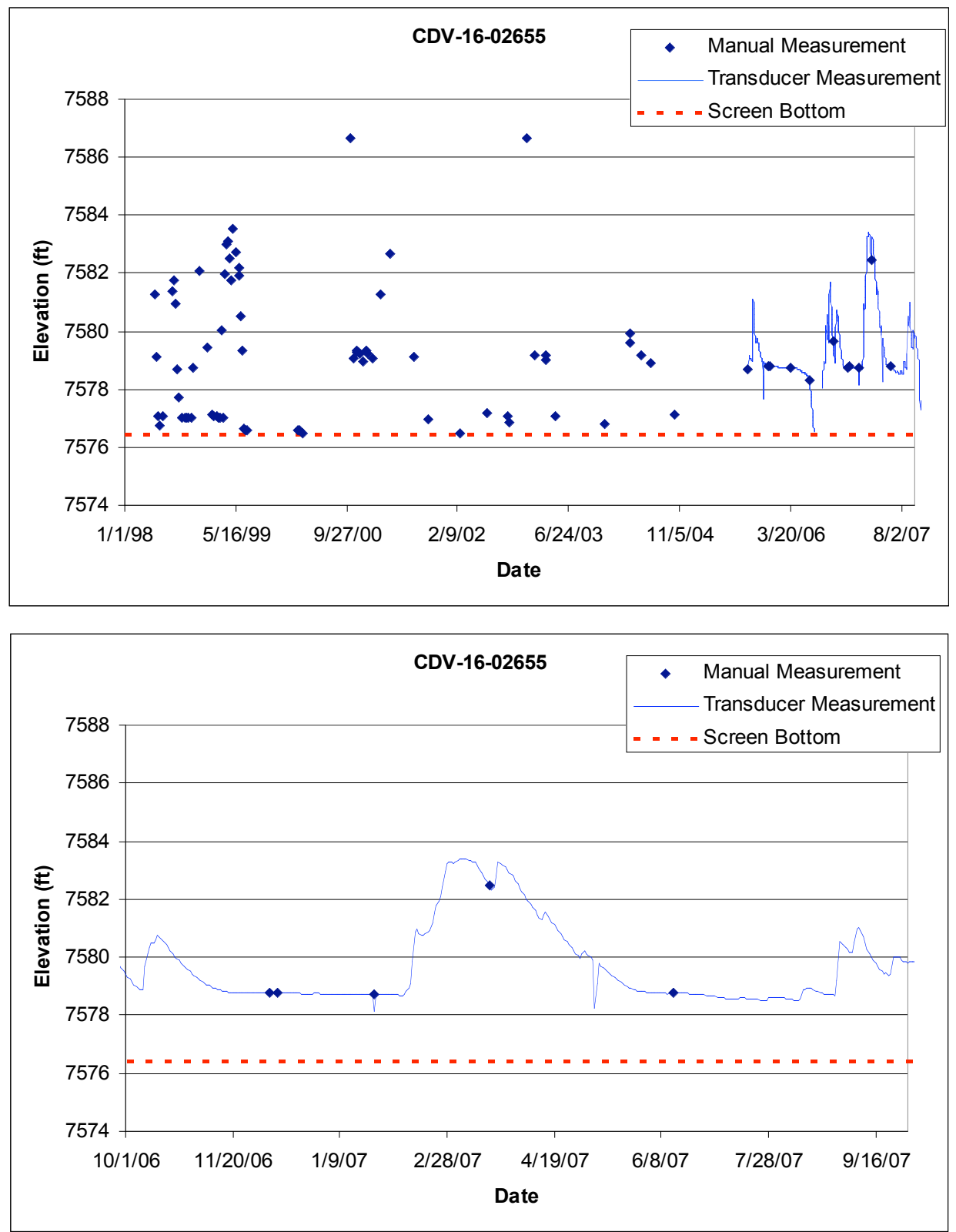


\subsection{CDV-16-02656}

Location: Upper Cañon de Valle at northern boundary of TA-16.

Period of Record: May 15, 1998, through September 30, 2007

Remarks: None.
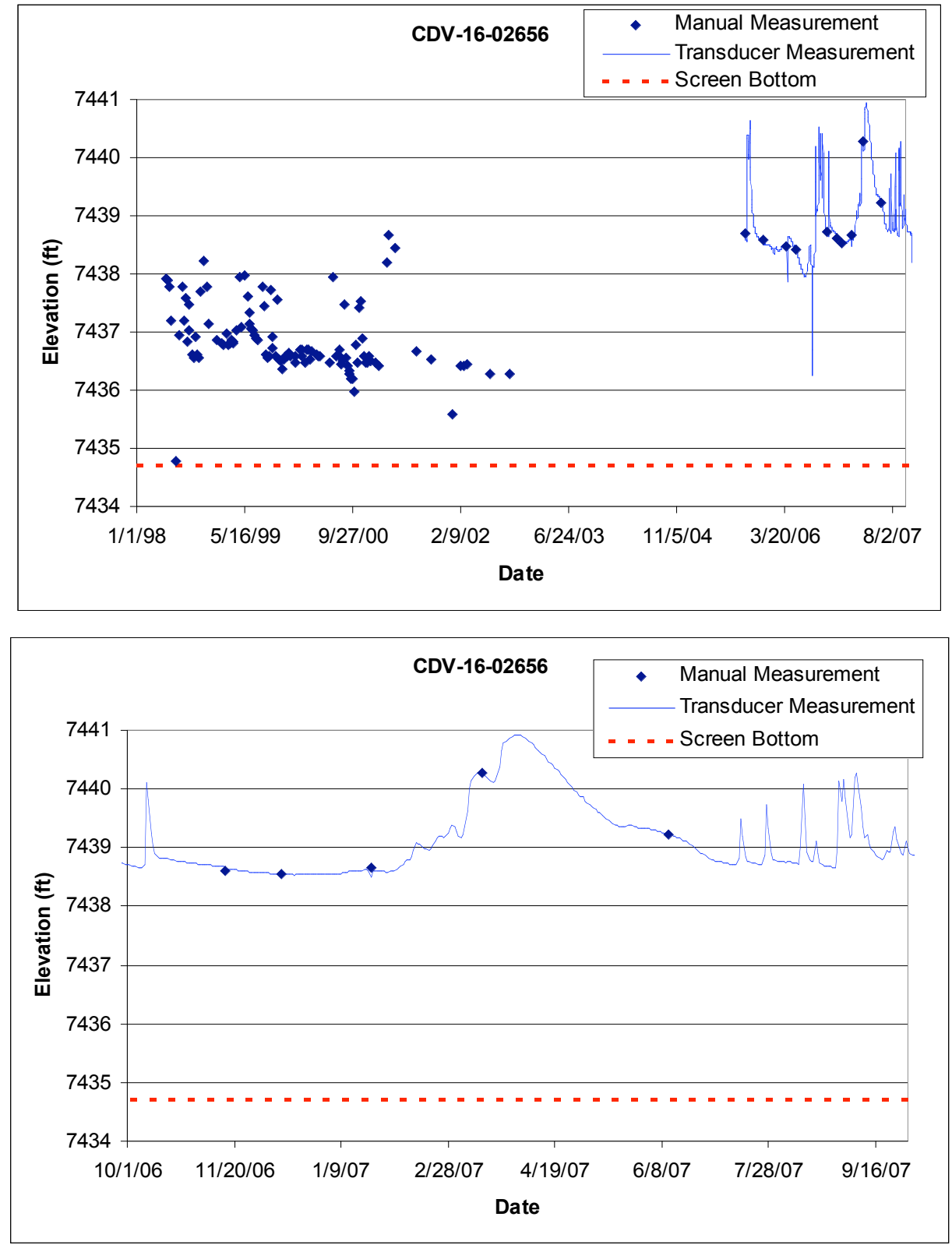


\subsection{CDV-16-02657}

Location: Upper Cañon de Valle at northern boundary of TA-16, approximately $200 \mathrm{ft}$ east-southeast of well CDV-16-02656.

Period of Record: May 15, 1998, through September 30, 2007

Remarks: CDV-16-02657 was bailed dry during sampling events on January 29, 2007, May 10, 2007, and May 11, 2007.
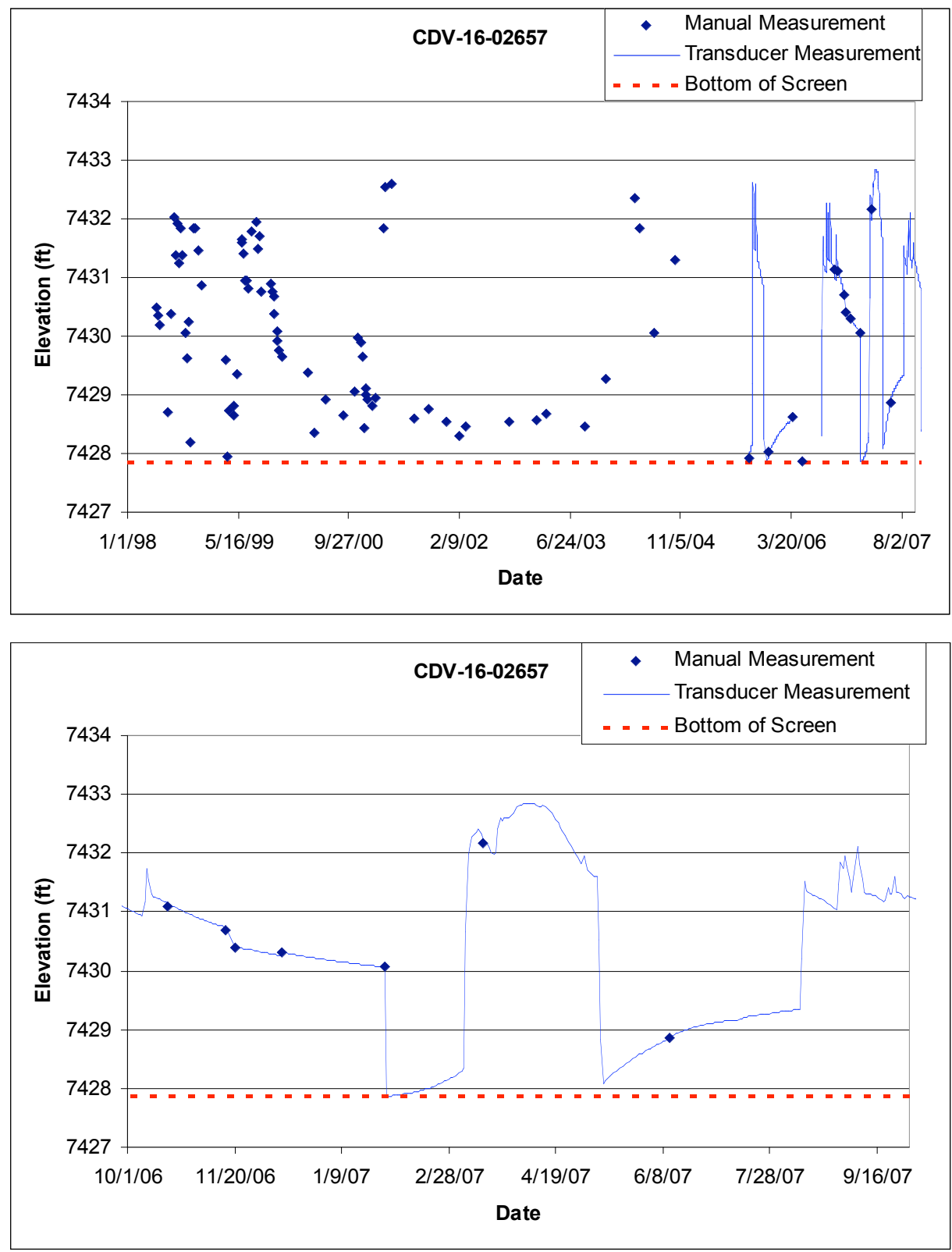


\subsection{CDV-16-02658}

Location: Upper Cañon de Valle at northern boundary of TA-16, approximately $200 \mathrm{ft}$ east-southeast of well CDV-16-02657 and approximately $800 \mathrm{ft}$ east-southeast of Burning Ground Spring.

Period of Record: September 15, 1997, through September 30, 2007

Remarks: Screen bottom elevation is $7368.26 \mathrm{ft}$.
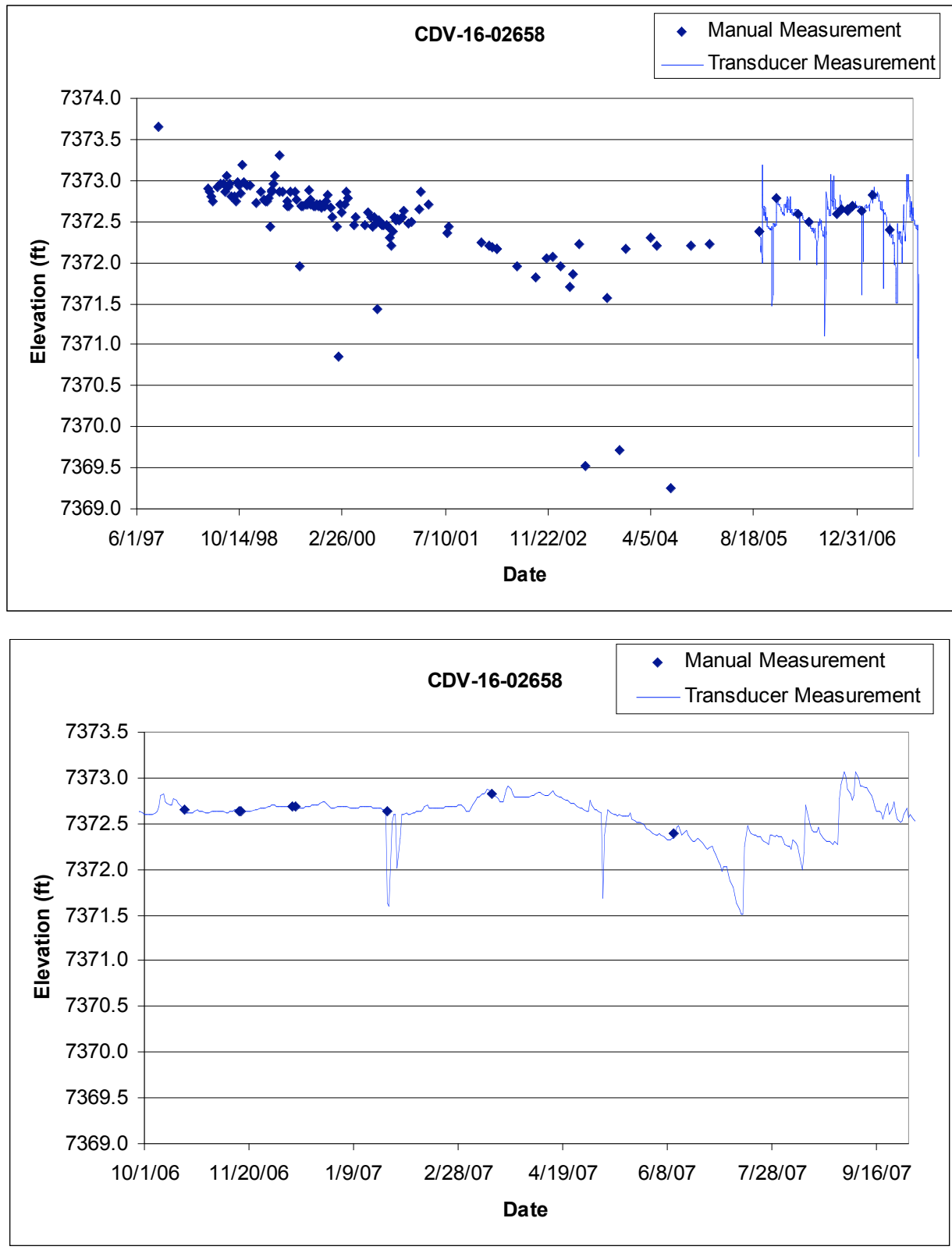


\subsection{CDV-16-02659}

Location: Upper Cañon de Valle at northern boundary of TA-16, approximately $1800 \mathrm{ft}$ east-northeast of well CDV-16-02657.

Period of Record: September 17, 1997, through September 30, 2007

Remarks: Screen bottom elevation is $7293.32 \mathrm{ft}$.
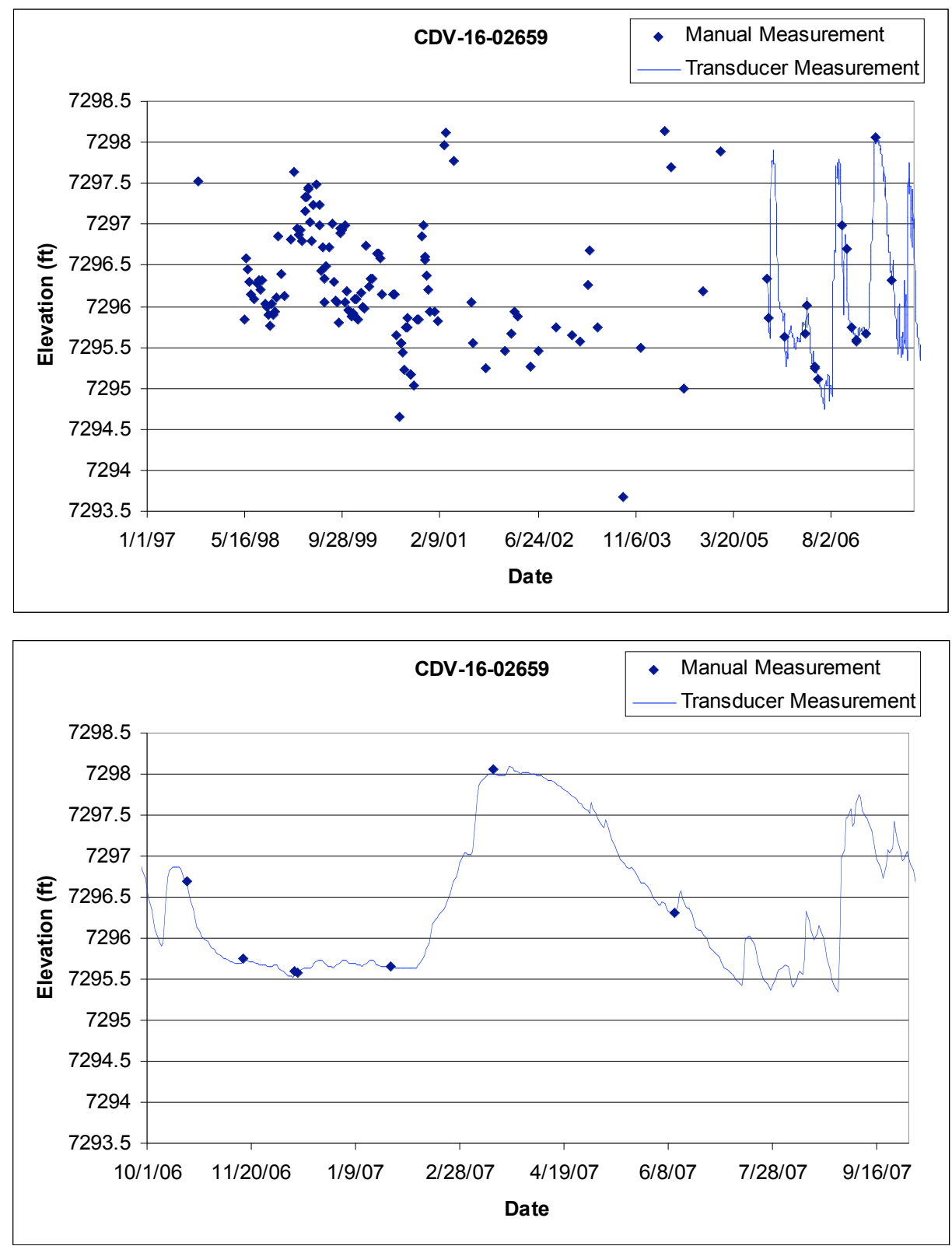


\subsection{FCO-1}

Location: Fence Canyon, approximately 0.1 miles northwest of SR-4.

Period of Record: June 9, 1997, through September 05, 2007

Remarks: Well has been dry during all measuring events.

\begin{tabular}{|c|c|c|c|}
\hline Well Name & Date Time & Water Levels (ft) & Comments \\
\hline FCO-1 & 6/9/1997 12:00:00 PM & & Dry \\
\hline FCO-1 & 10/13/1997 12:00:00 PM & & Dry \\
\hline FCO-1 & 3/25/1998 12:00:00 PM & & Dry \\
\hline FCO-1 & 5/29/1998 12:00:00 PM & & Dry \\
\hline FCO-1 & 7/28/1998 12:00:00 PM & & Dry \\
\hline FCO-1 & 3/3/1999 12:00:00 PM & & Dry \\
\hline FCO-1 & 6/23/1999 12:00:00 PM & & Dry \\
\hline FCO-1 & 8/30/1999 12:00:00 PM & & Dry \\
\hline FCO-1 & 11/15/1999 12:00:00 PM & & Dry \\
\hline FCO-1 & 3/26/2000 12:00:00 PM & & Dry \\
\hline FCO-1 & 5/16/2000 12:00:00 PM & & Dry \\
\hline FCO-1 & 8/30/2000 12:00:00 PM & & Dry \\
\hline FCO-1 & 10/8/2000 12:00:00 PM & & Dry \\
\hline FCO-1 & 4/16/2002 12:00:00 PM & & Dry \\
\hline FCO-1 & 8/19/2002 12:00:00 PM & & Dry \\
\hline FCO-1 & 11/13/2002 12:00:00 PM & & Dry \\
\hline FCO-1 & 2/19/2003 12:00:00 PM & & Dry \\
\hline FCO-1 & 5/18/2003 12:00:00 PM & & Dry \\
\hline FCO-1 & 4/7/2004 12:00:00 PM & & Dry \\
\hline FCO-1 & 9/14/2005 10:26:00 AM & & Dry \\
\hline FCO-1 & 6/23/2006 1:08:00 PM & & Dry, $T D=13.50 \mathrm{ft}$ \\
\hline FCO-1 & 9/8/2006 9:21:00 AM & & Dry \\
\hline FCO-1 & 12/15/2006 10:20:00 AM & & Dry \\
\hline FCO-1 & 1/24/2007 10:45:00 AM & & Dry \\
\hline FCO-1 & 3/15/2007 10:55:00 AM & & Dry \\
\hline FCO-1 & 5/24/2007 2:37:00 PM & & Dry \\
\hline FCO-1 & 6/6/2007 9:28:00 AM & & Dry \\
\hline FCO-1 & 9/5/2007 1:25:00 PM & & Dry \\
\hline
\end{tabular}




\subsection{LAO-B}

Location: Upper Los Alamos Canyon, approximately $3000 \mathrm{ft}$ west of the Diamond Drive bridge.

Period of Record: April 28, 1994, through September 30, 2007

Remarks: Screen bottom elevation is $7296.8 \mathrm{ft}$; some of the manual measurements in the upper chart were taken during sampling events and are not representative of static water level elevations.
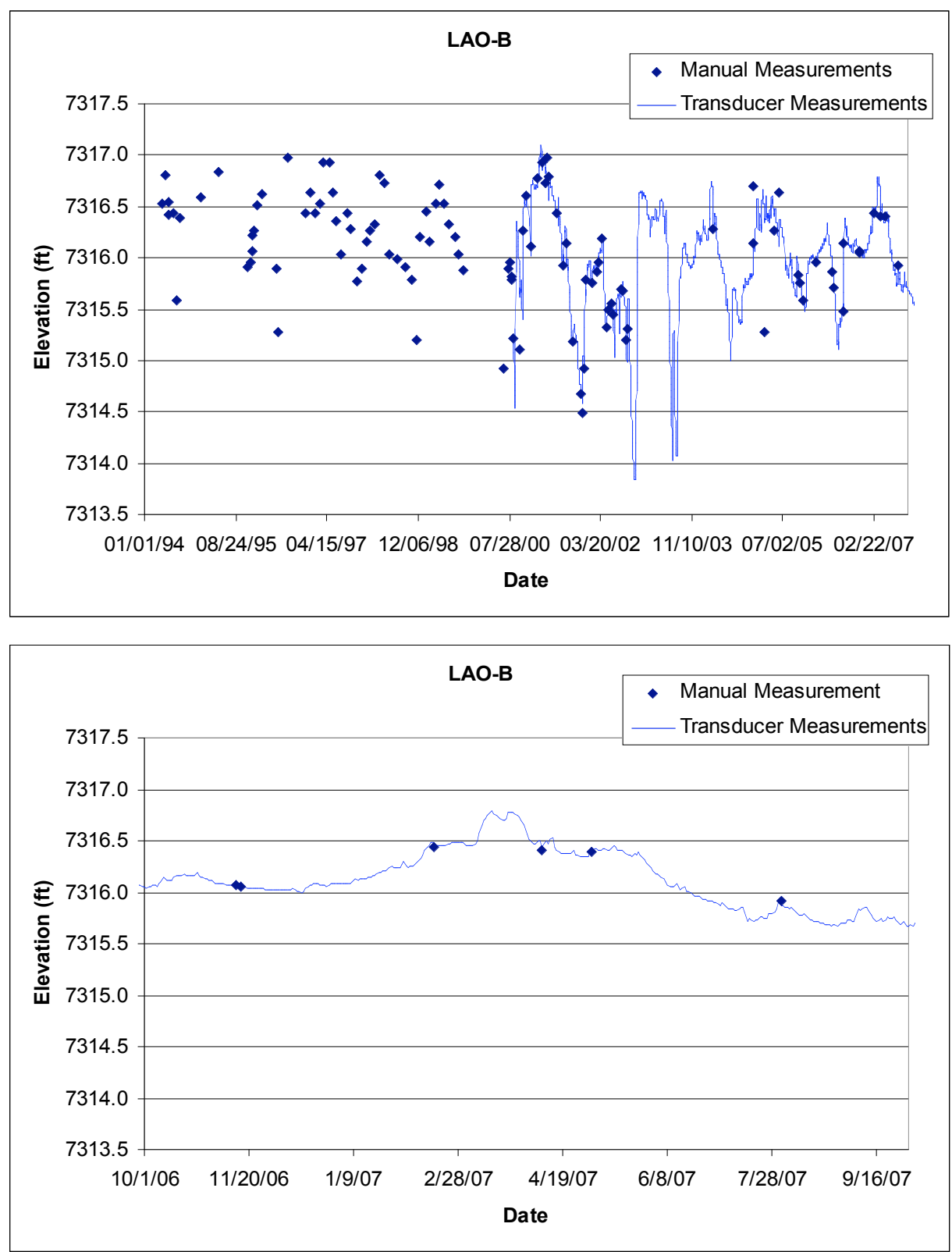


\subsection{LAO-0.3}

Location: Upper Los Alamos Canyon, approximately $5700 \mathrm{ft}$ east of the Diamond Drive bridge.

Period of Record: June 1, 1994, through September 30, 2007

Remarks: Transducer readings were not valid from July 7, 2005, through October 12, 2005. The pressure sensor was in the mud at the bottom of the well.
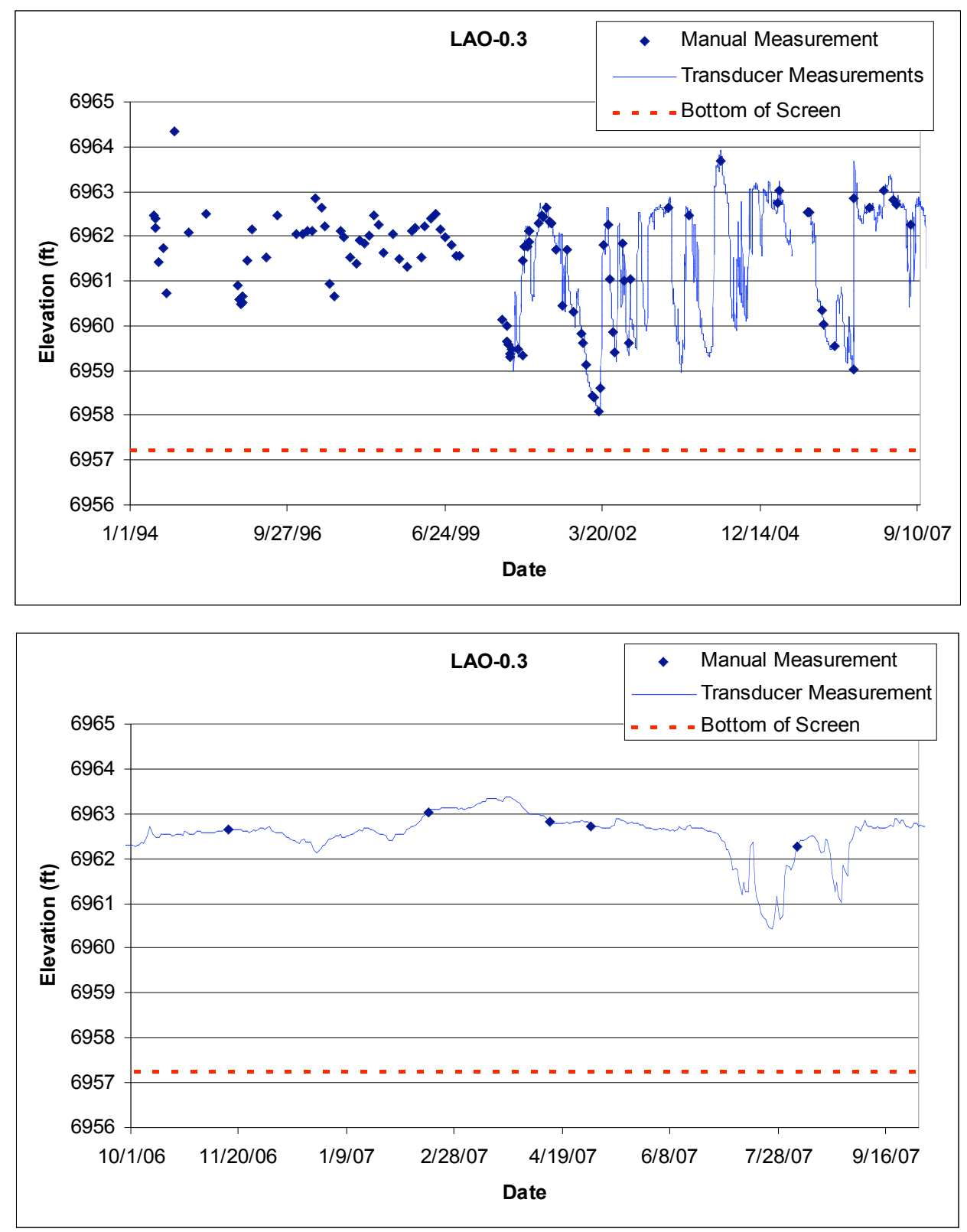


\subsection{LAO-0.6}

Location: Los Alamos Canyon, approximately $7500 \mathrm{ft}$ east of the Diamond Drive bridge.

Period of Record: May 6, 1994, through September 30, 2007

Remarks: Screen bottom elevation is $6897.34 \mathrm{ft}$.
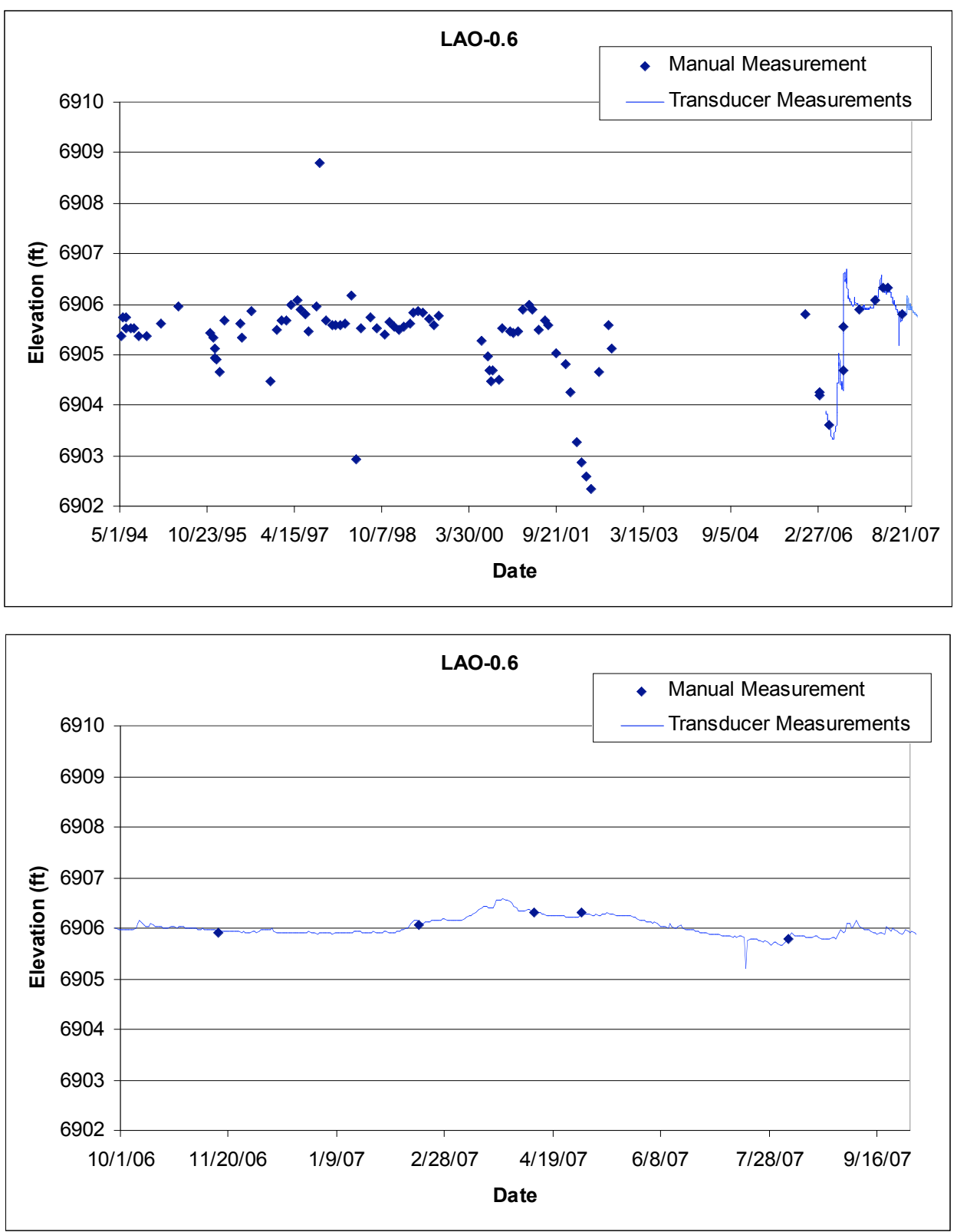


\subsection{LAO-0.91}

Location: Los Alamos Canyon approximately 10,200 ft east of the Diamond Drive bridge and approximately $100 \mathrm{ft}$ east of the eastern boundary of TA-2.

Period of Record: June 1, 1994, through September 30, 2007

Remarks: The transducer was removed on February 21, 2007. No further water level monitoring at LAO-0.91 is planned.
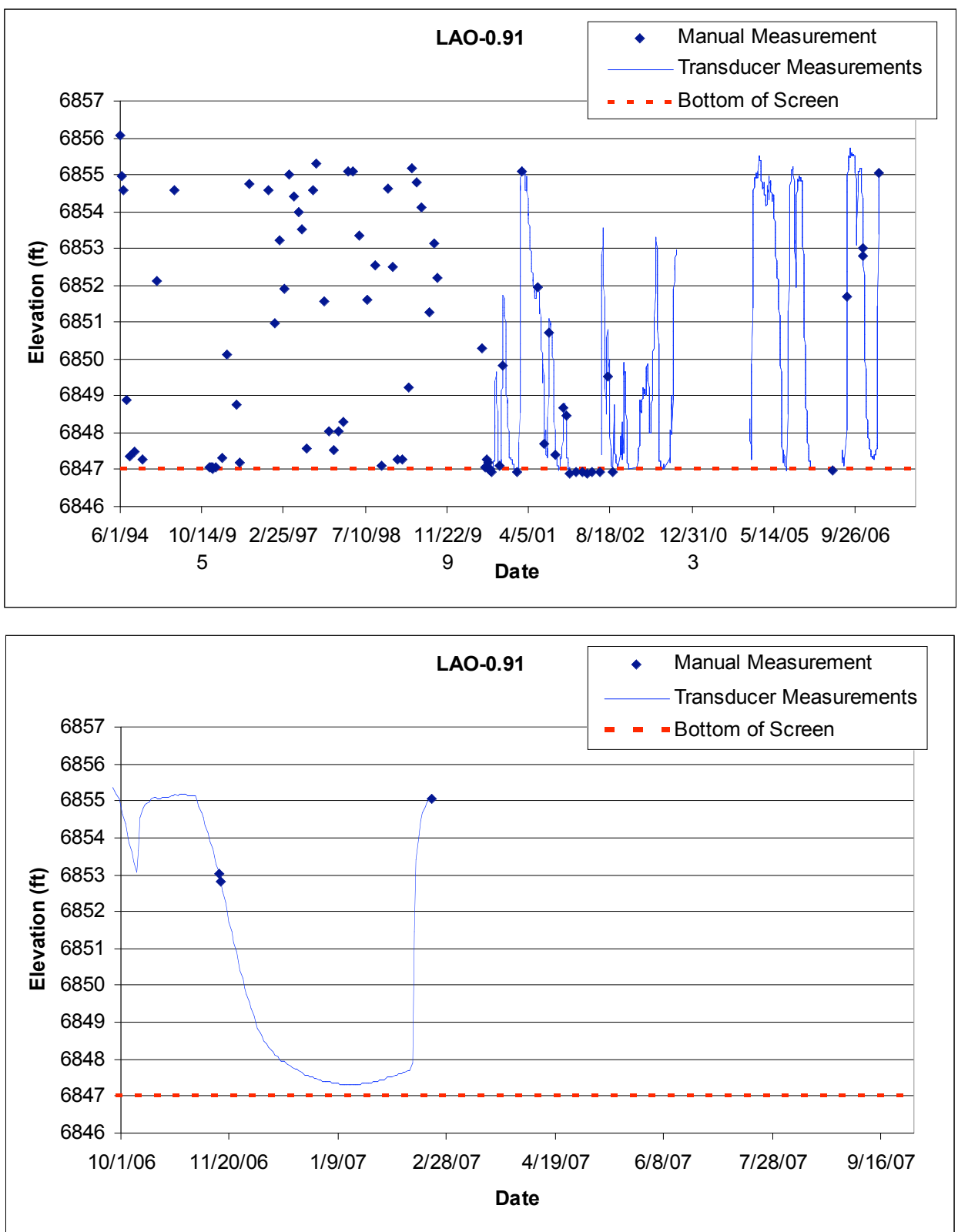


\subsection{LAO-1}

Location: Los Alamos Canyon, near the eastern border of TA-2.

Period of Record: February 15, 1966, through September 30, 2007

Remarks: LAO-1 is a 2-in.-diameter well with a dedicated bladder pump. The transducer is sitting on top of the pump. Screen bottom elevation is $6808.24 \mathrm{ft}$.
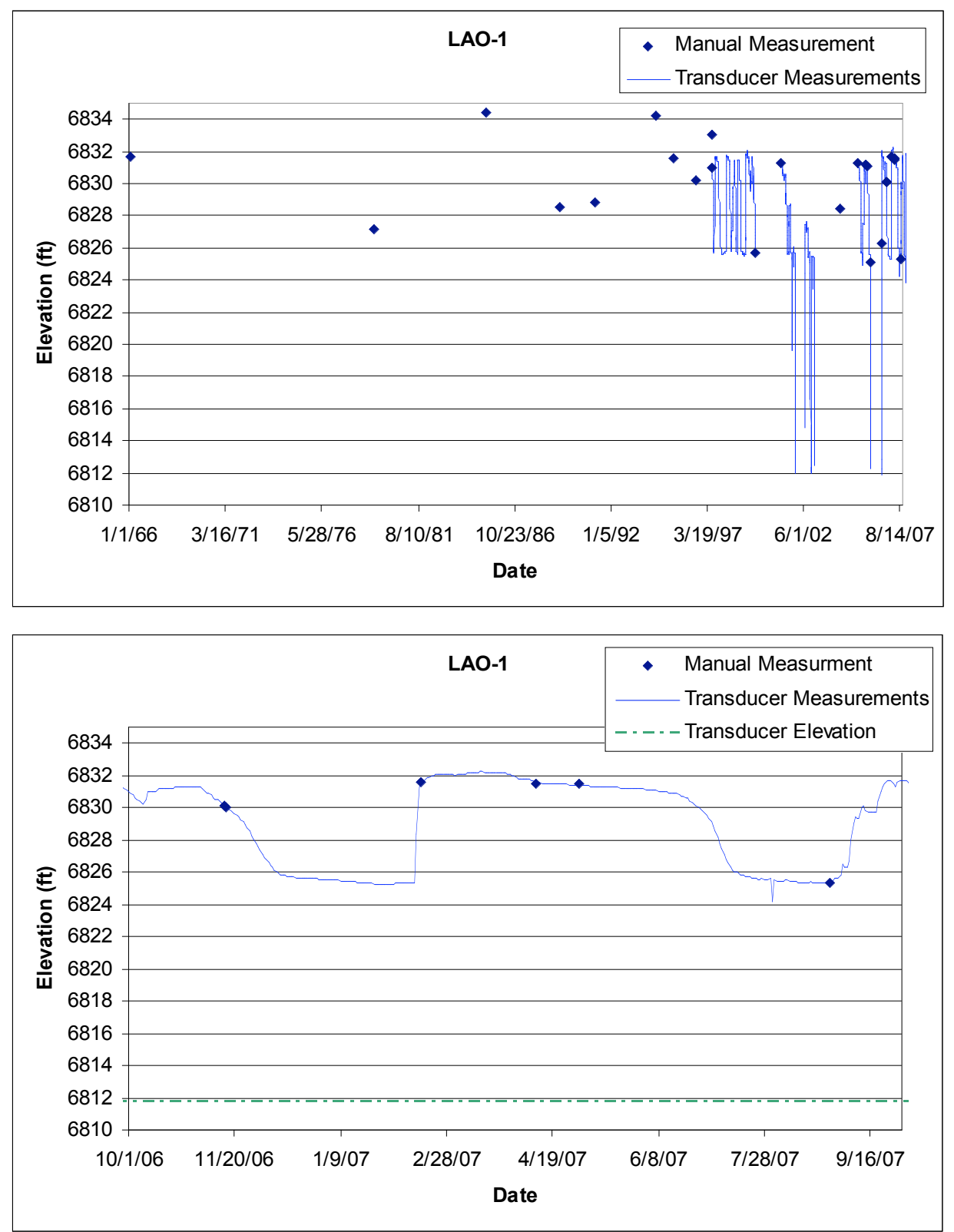


\subsection{LAO-1.6g}

Location: Los Alamos Canyon, approximately $400 \mathrm{ft}$ west of the confluence with DP Canyon. Period of Record: November 22, 1996, through September 30, 2007

Remarks: None.
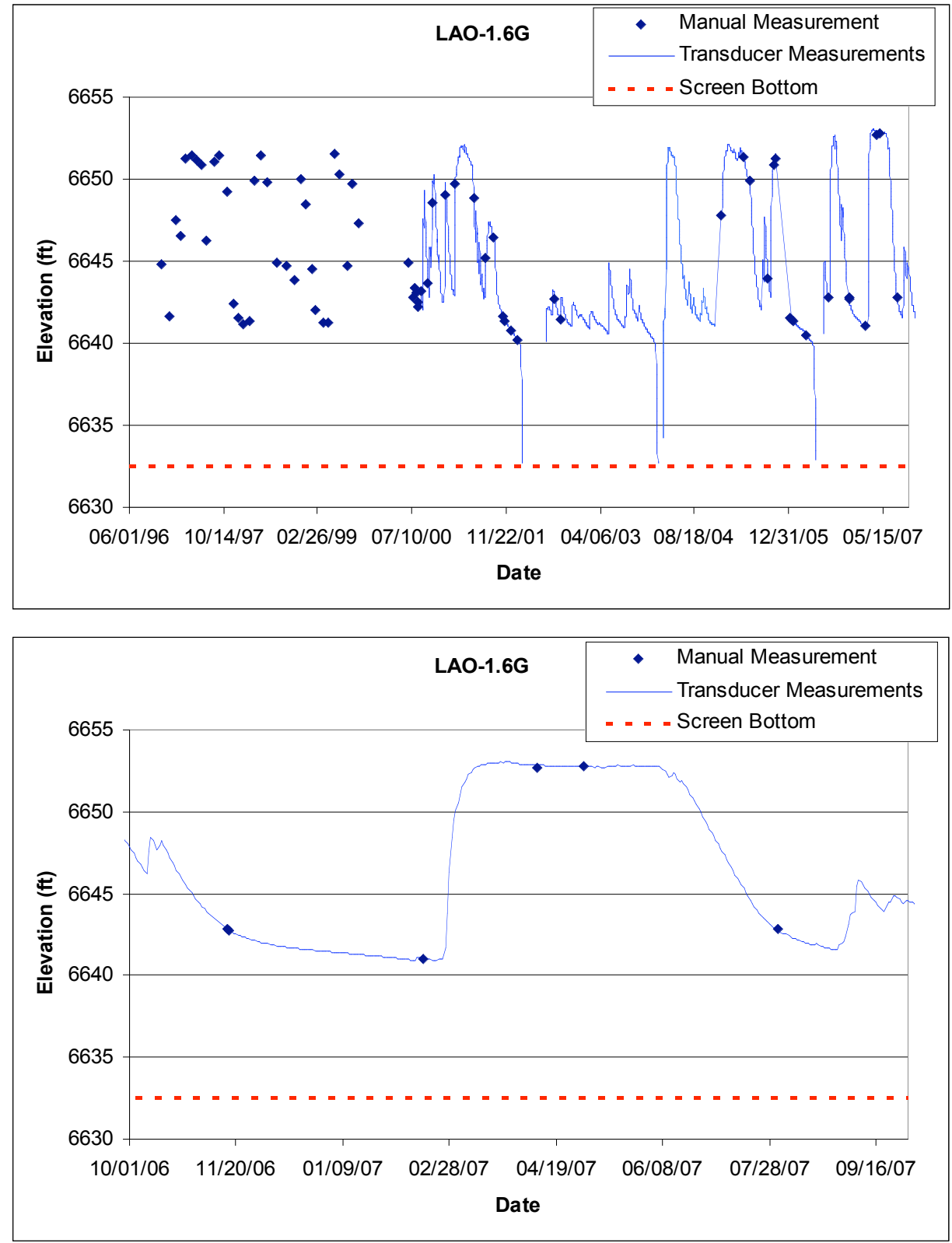


\subsection{LAO-1.8}

Location: Los Alamos Canyon, approximately $650 \mathrm{ft}$ west of LAO-1.6g.

Period of Record: January 8, 2001, through September 30, 2007

Remarks: None.
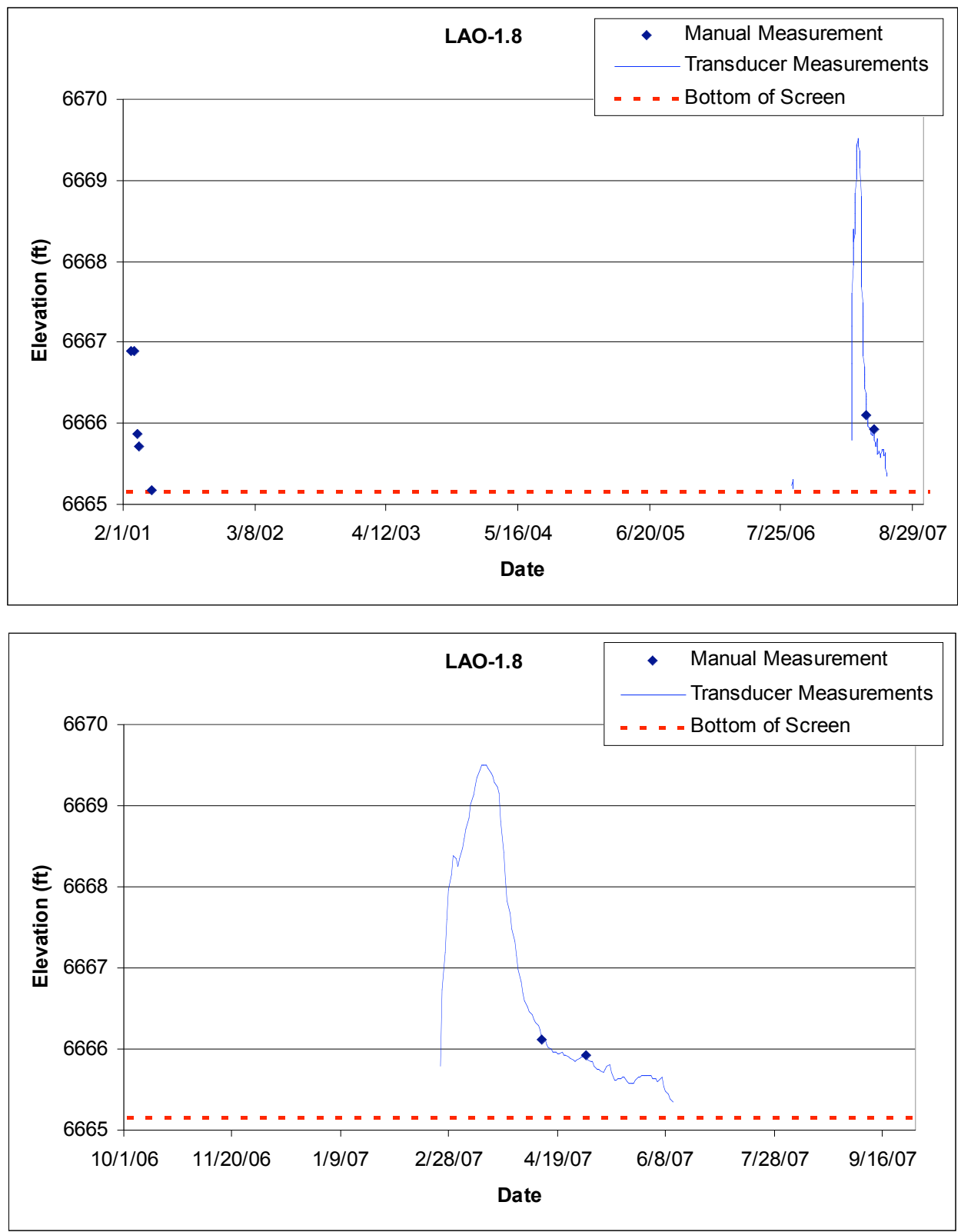


\subsection{LAO-2}

Location: Los Alamos Canyon, approximately $75 \mathrm{ft}$ north of the confluence with DP Canyon.

Period of Record: February 1, 1966, through September 30, 2007

Remarks: Screen bottom elevation $6560.97 \mathrm{ft}$. LAO-2 is a 2-in.-diameter well with a bladder pump. The transducer is installed above the top of the pump. The transducer sensor elevation is $6563.88 \mathrm{ft}$. Water level elevations below $6563.88 \mathrm{ft}$ are not represented by transducer data.
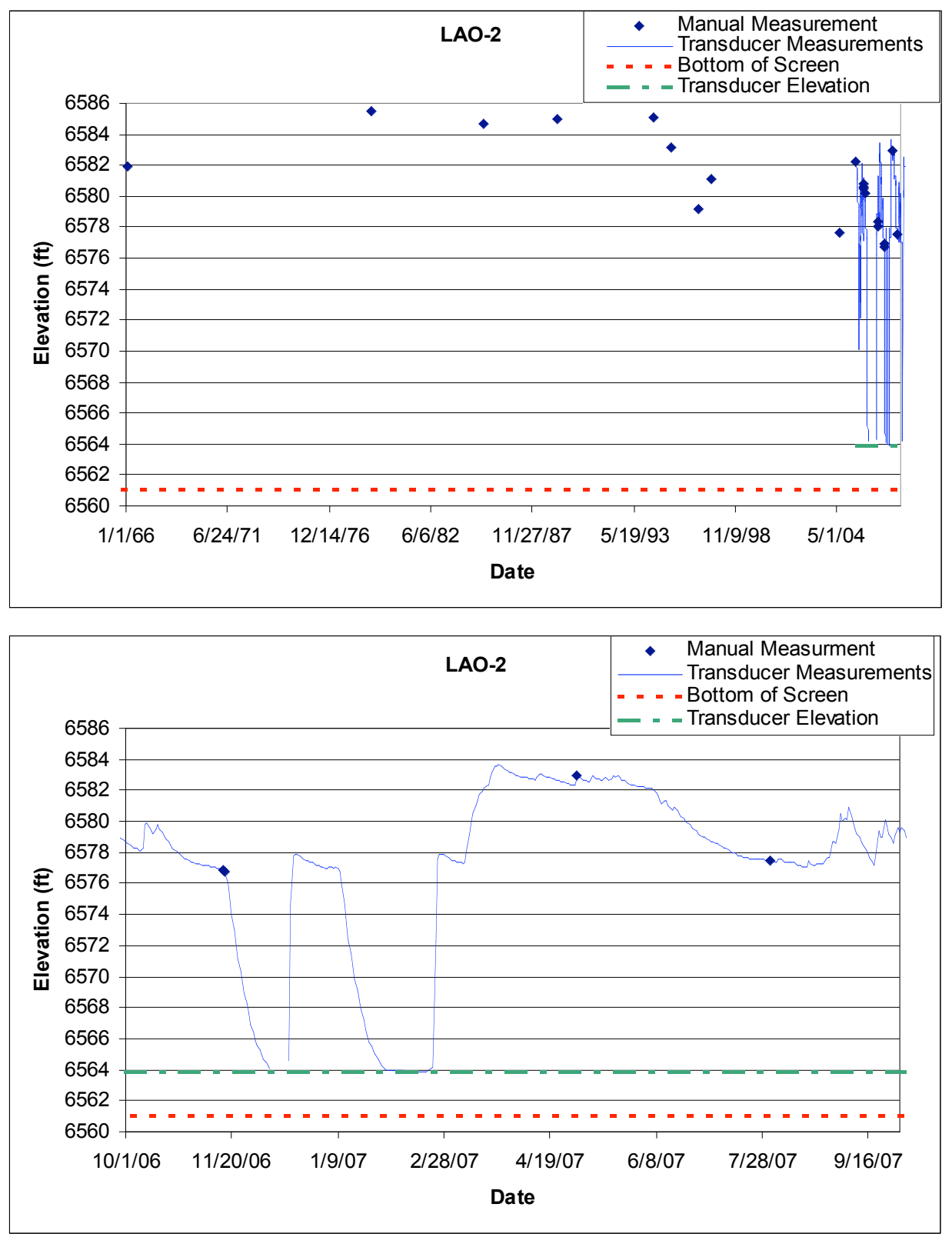


\subsection{LAO-3a}

Location: Los Alamos Canyon, approximately $1000 \mathrm{ft}$ east of the confluence with DP Canyon.

Period of Record: September 15,1989, through September 30, 2007

Remarks: Screen bottom elevation is $6564.7 \mathrm{ft}$. The transducer batteries died on March 29, 2007. No data were collected from March 29, 2007, to May 3, 2007.
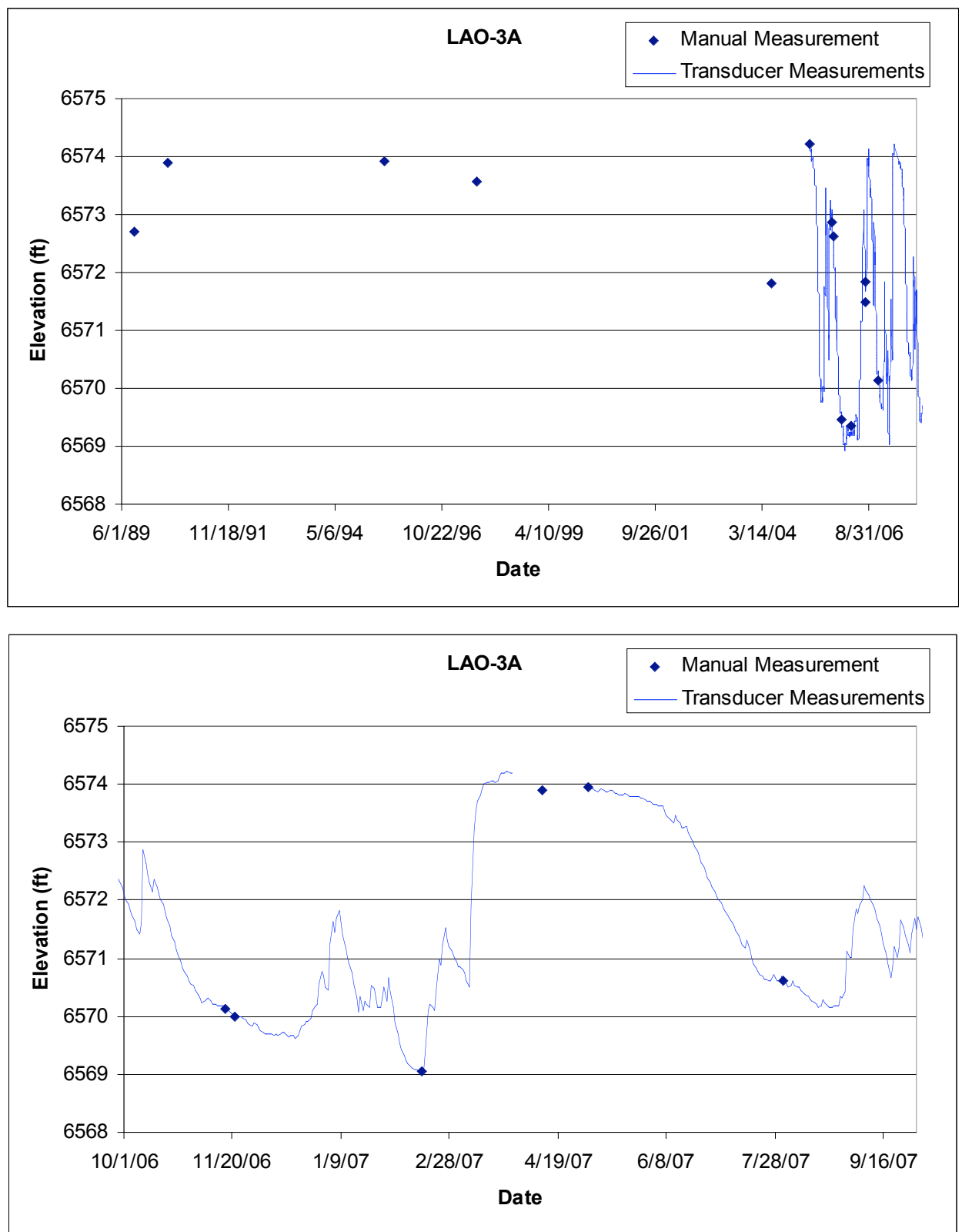


\subsection{LAO- $4.5 \mathrm{c}$}

Location: Los Alamos Canyon, approximately 1.25 miles east of the confluence with DP Canyon.

Period of Record: November 22, 1989, through September 30, 2007

Remarks: Screen bottom elevation is $6434.33 \mathrm{ft}$. The elevation of the transducer is $6438.34 \mathrm{ft}$ and is sitting on top of the bladder pump. The transducer batteries died on February 27, 2007, and were replaced on May 3, 2007. No transducer data were collected from February 27, 2007, to May 3, 2007.
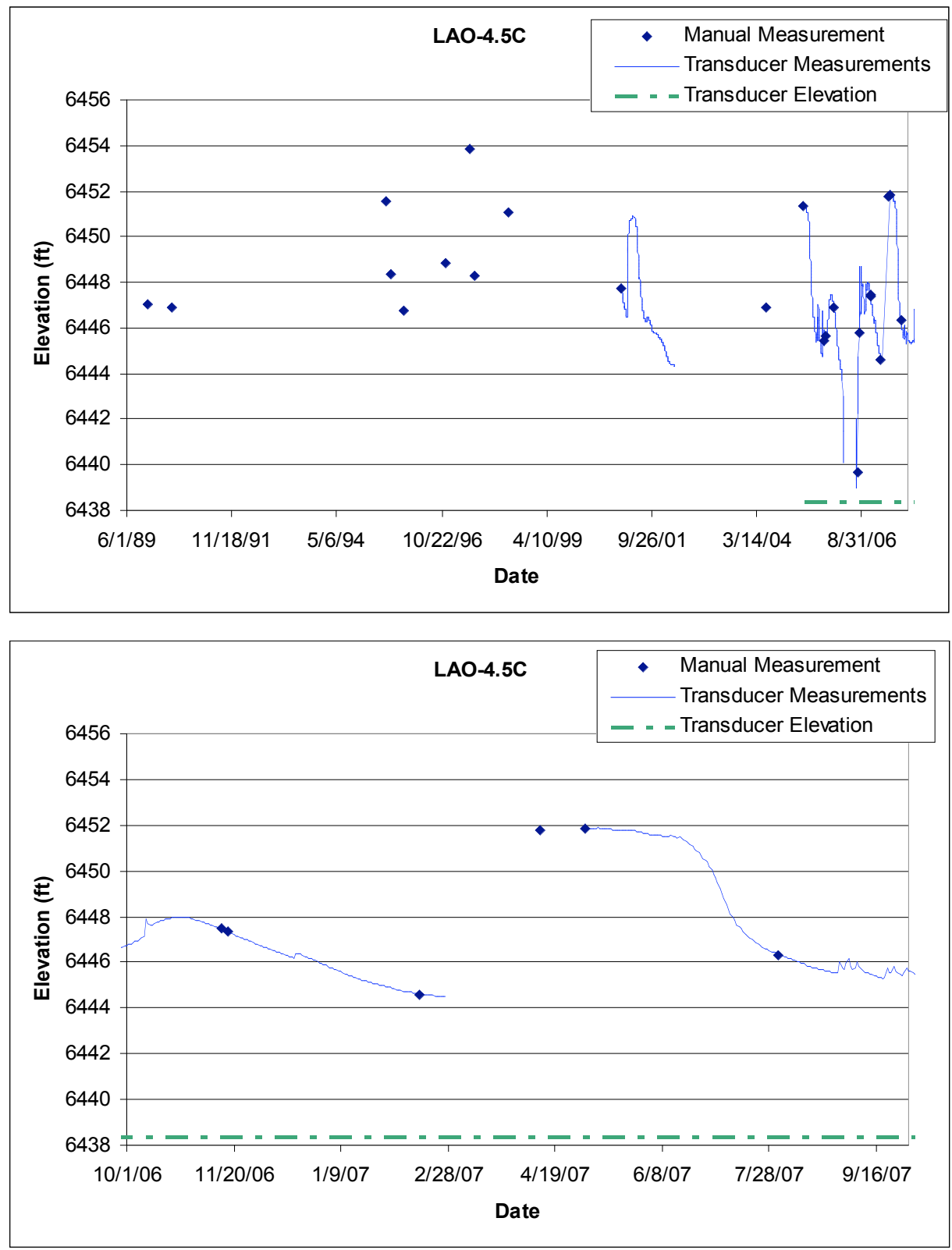


\subsection{LAO-5}

Location: Los Alamos Canyon, approximately 1 mile west of SR-4.

Period of Record: December 14, 2005, through September 5, 2007

Remarks: LAO- 5 is not installed with a pressure transducer and is measured manually on a quarterly schedule. The bottom of the screen is $6368.3 \mathrm{ft}$.

\begin{tabular}{|l||r|r||l|}
\hline Well Name & \multicolumn{1}{|c|}{ Date Time } & Water Level (ft) & \multicolumn{1}{|c|}{ Comments } \\
\hline LAO-5 & $12 / 14 / 2005$ 2:10:00 PM & & Dry \\
\hline LAO-5 & $3 / 14 / 2006$ 11:00:00 AM & 6380.35 & \\
\hline LAO-5 & $6 / 13 / 2006$ 9:24:00 AM & & Dry, TD = 23.28 ft \\
\hline LAO-5 & $8 / 3 / 2006$ 8:26:00 AM & & Dry \\
\hline LAO-5 & $9 / 7 / 2006$ 9:35:00 AM & & Dry \\
\hline LAO-5 & $8 / 2 / 200612: 45: 00$ PM & & Dry \\
\hline LAO-5 & $12 / 8 / 2006$ 2:28:00 PM & 6386.15 & \\
\hline LAO-5 & $3 / 13 / 20071: 45: 00$ PM & & Dry \\
\hline LAO-5 & $6 / 7 / 200710: 19: 00$ AM & 6388.45 & \\
\hline LAO-5 & $8 / 3 / 2007$ 3:50:00 PM & 6386.33 & GW sample \\
\hline LAO-5 & $9 / 5 / 2007$ 3:06:00 PM & 6380.46 & \\
\hline
\end{tabular}

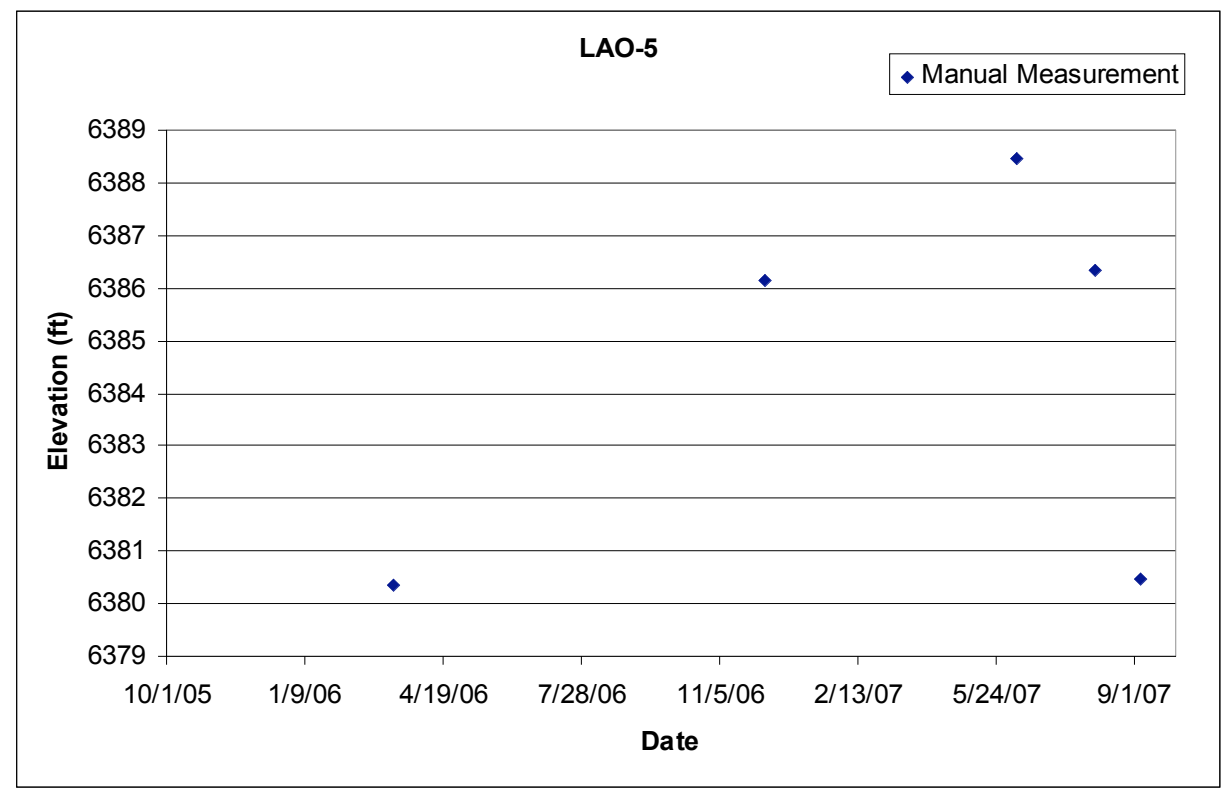




\subsection{LAO-6}

Location: Los Alamos Canyon, approximately 1 mile west of SR-4.

Period of Record: June 26, 1995, through September 5, 2007

Remarks: LAO-6 is measured quarterly; the bottom of the screen is $6379.3 \mathrm{ft}$.

\begin{tabular}{|l|r|r||l|}
\hline Well Name & \multicolumn{1}{|c|}{ Date Time } & Water Level (ft) & \multicolumn{1}{|c|}{ Comments } \\
\hline LAO-6 & $6 / 26 / 199512: 00: 00 \mathrm{PM}$ & 6385.6 & \\
\hline LAO-6 & $8 / 8 / 199512: 00: 00 \mathrm{PM}$ & 6385.1 & \\
\hline LAO-6 & $12 / 7 / 199512: 00: 00 \mathrm{PM}$ & 6383.5 & \\
\hline LAO-6 & $3 / 14 / 200611: 05: 00 \mathrm{AM}$ & & Dry \\
\hline LAO-6 & $6 / 13 / 2006$ 8:54:00 AM & & Dry, TD = 16.45 ft \\
\hline LAO-6 & $4 / 19 / 200611: 34: 00 \mathrm{AM}$ & & Dry to top of pump. \\
\hline LAO-6 & $9 / 7 / 2006$ 9:21:00 AM & & Dry \\
\hline LAO-6 & $7 / 27 / 200611: 40: 00 \mathrm{AM}$ & & Dry \\
\hline LAO-6 & $12 / 8 / 2006$ 2:36:00 PM & & Dry \\
\hline LAO-6 & $3 / 13 / 2007$ 1:00:00 PM & & Dry \\
\hline LAO-6 & $6 / 7 / 200710: 04: 00 \mathrm{AM}$ & 6383.47 & \\
\hline LAO-6 & $9 / 5 / 2007$ 2:54:00 PM & & Dry \\
\hline
\end{tabular}

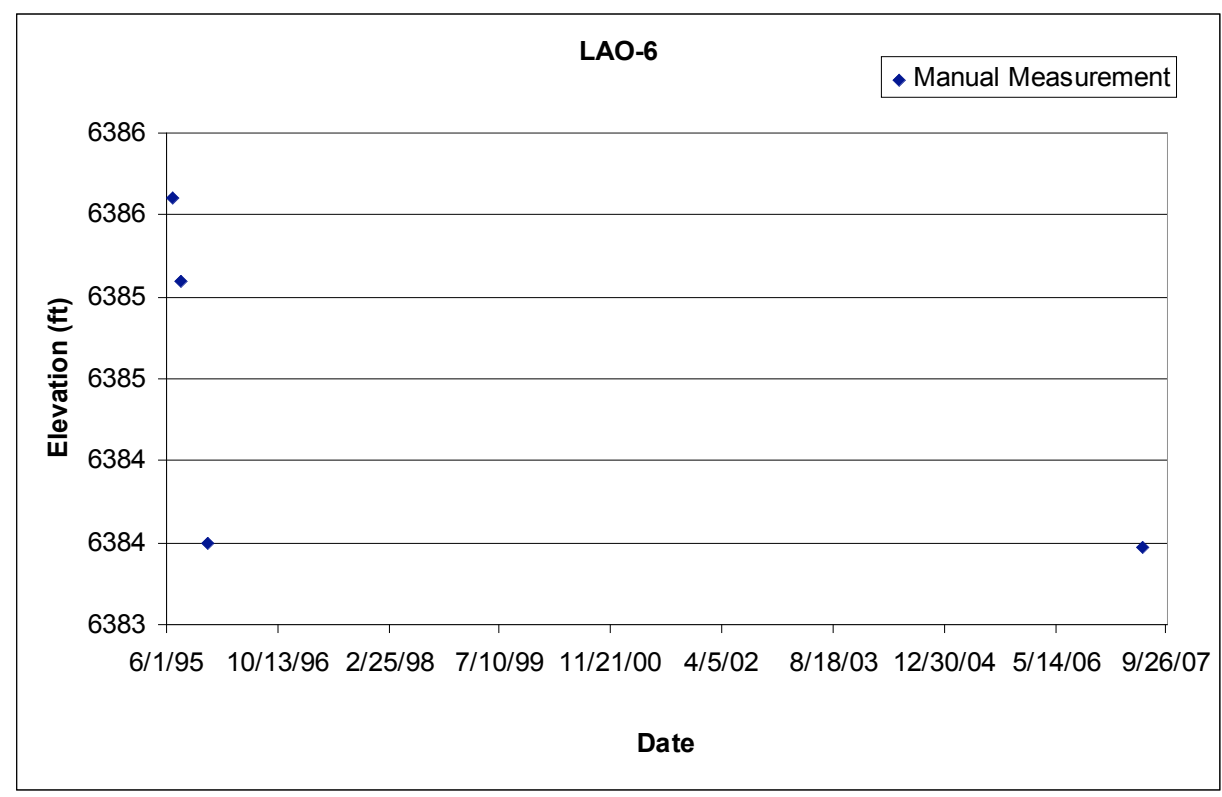




\subsection{LAO-6a}

Location: Los Alamos Canyon, approximately 1 mile west of SR-4.

Period of Record: August 17, 1989, through September 30, 2007

Remarks: LAO-6a was predominantly dry in FY 2007; water was in the well March 19, 2007, to July 29, 2007.
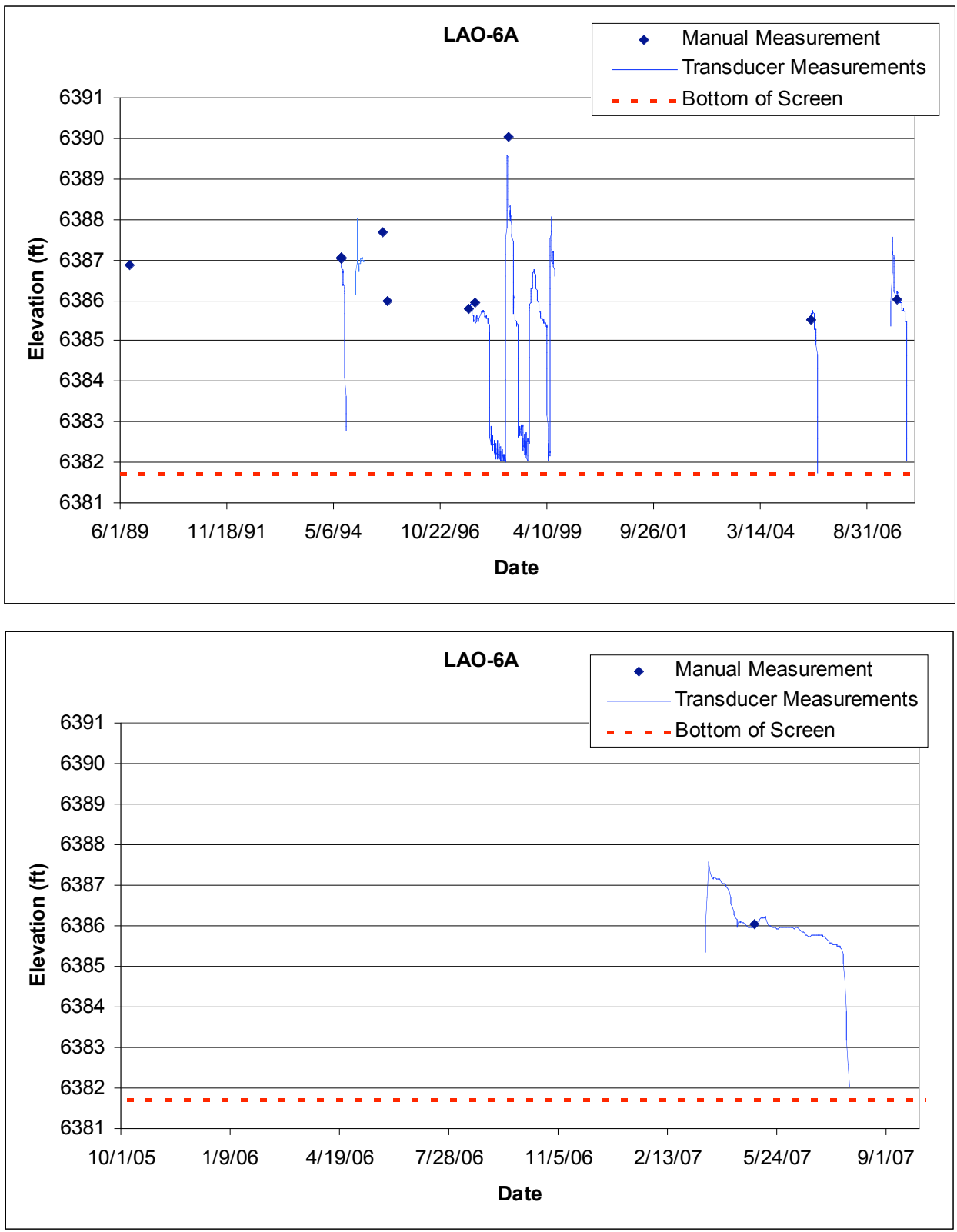


\subsection{LAUZ-1}

Location: DP Canyon, north of TA-21.

Period of Record: August 20, 1997, through September 30, 2007

Remarks: Screen bottom elevation is $7022.1 \mathrm{ft}$.
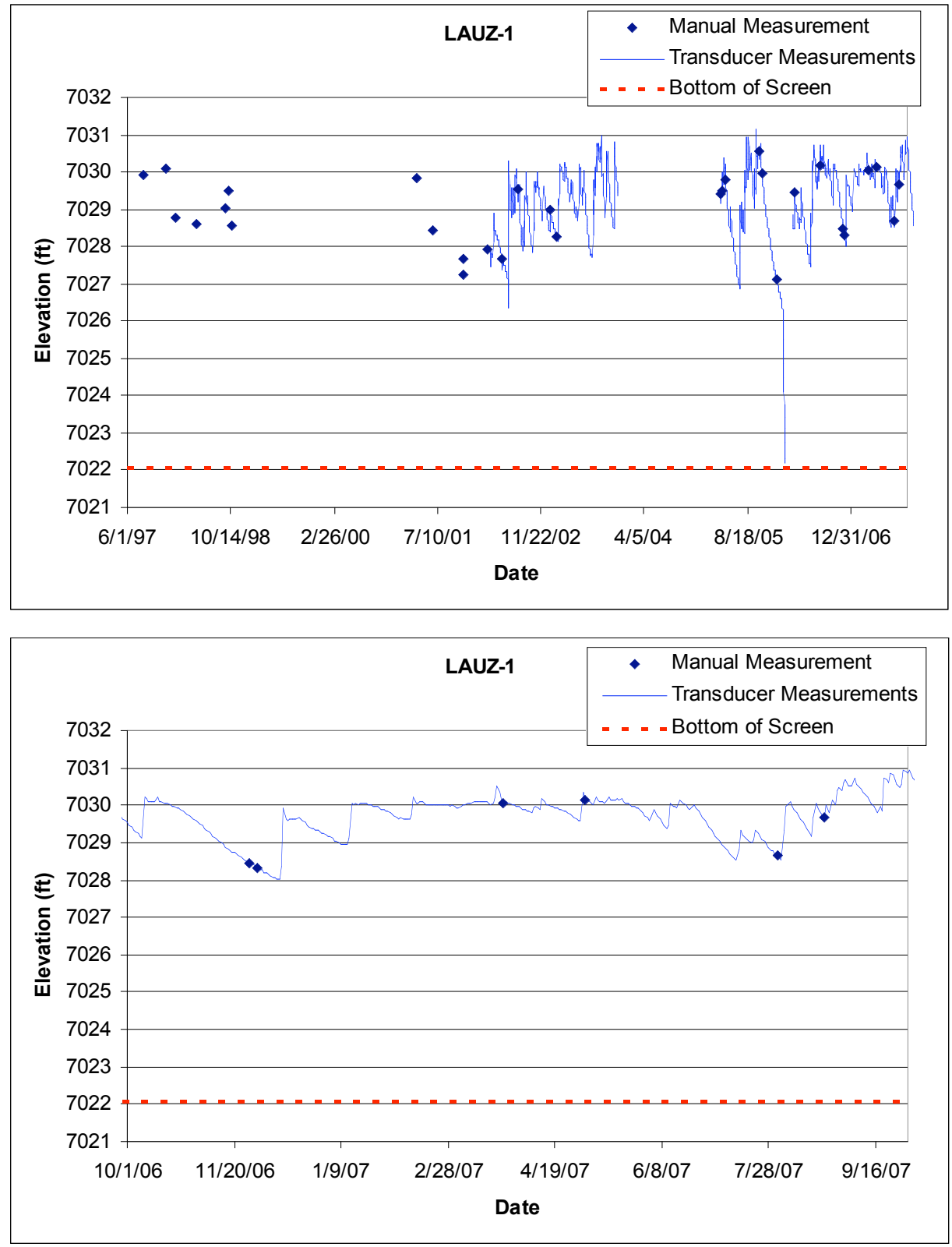


\section{$5.41 \operatorname{LLAO}-1(\mathrm{~b})$}

Location: Lower Los Alamos Canyon, approximately $3000 \mathrm{ft}$ southwest of Totavi, San Ildefonso.

Period of Record: August 27, 1997, through September 30, 2007

Remarks: None.
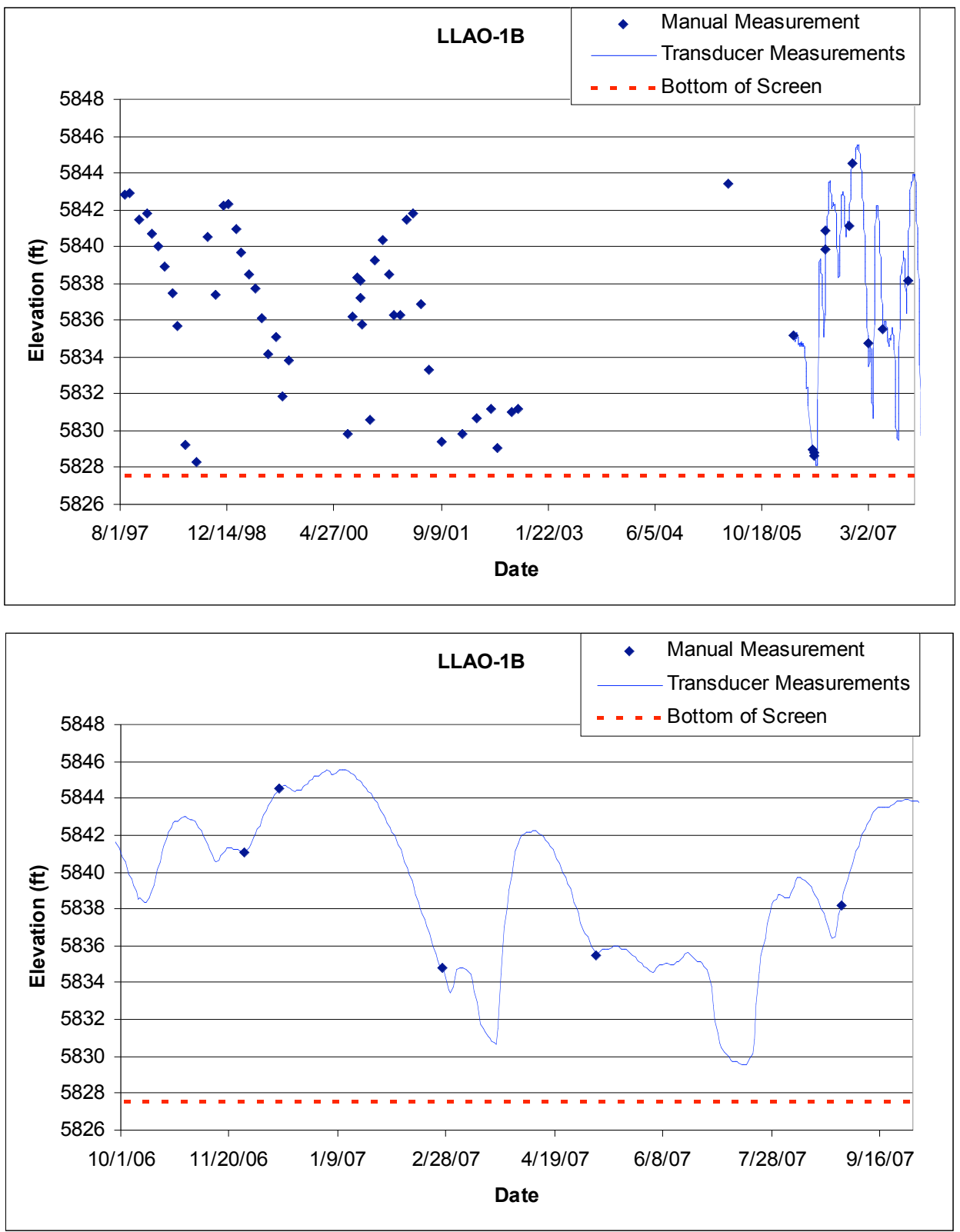


\subsection{LLAO-4}

Location: Lower Los Alamos Canyon, approximately $700 \mathrm{ft}$ northwest of the Rio Grande at SR-502.

Period of Record: November 22, 1996, through September 30, 2007

Remarks: Screen bottom elevation is $5499.97 \mathrm{ft}$.
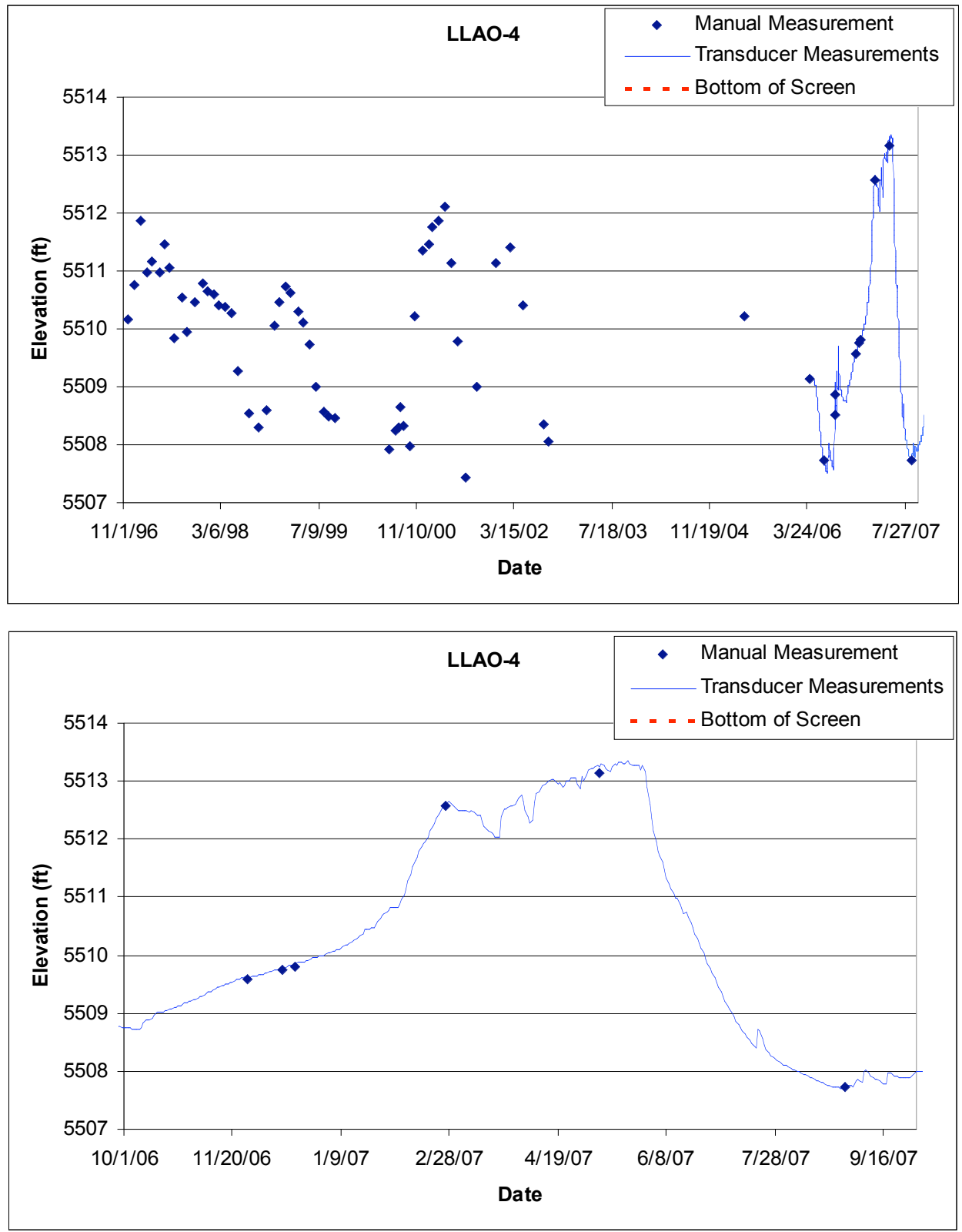


\subsection{MCA-1}

Location: Upper Mortandad Canyon, approximately $700 \mathrm{ft}$ northeast of the TA-50 outfall. Period of Record: April 20, 2005, through September 30, 2007

Remarks: None.
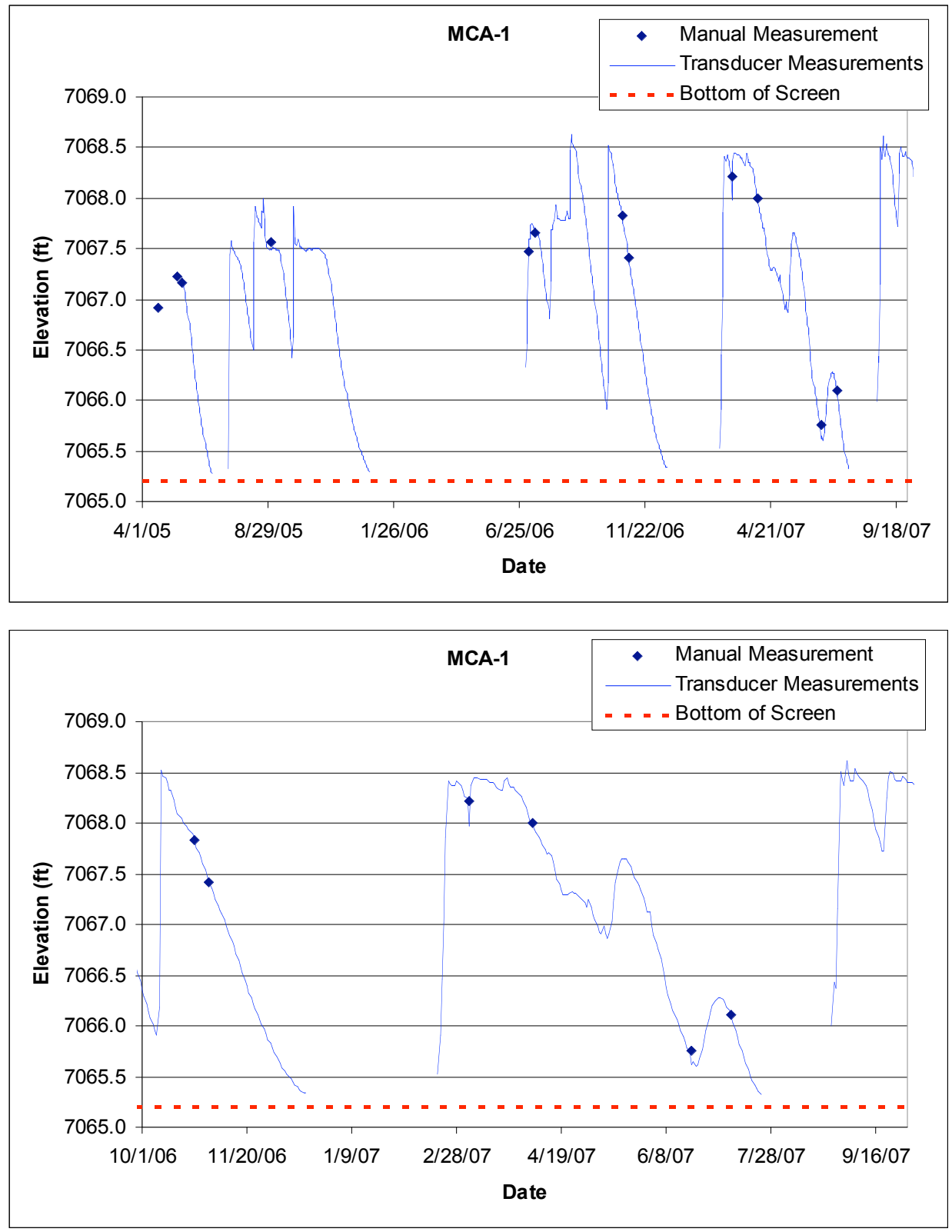


\subsection{MCA-2}

Location: Mortandad Canyon, approximately $400 \mathrm{ft}$ up canyon of the upper sediment trap. Period of Record: May 18, 2005, through September 30, 2007

Remarks: Screen bottom elevation is $6777.2 \mathrm{ft}$.
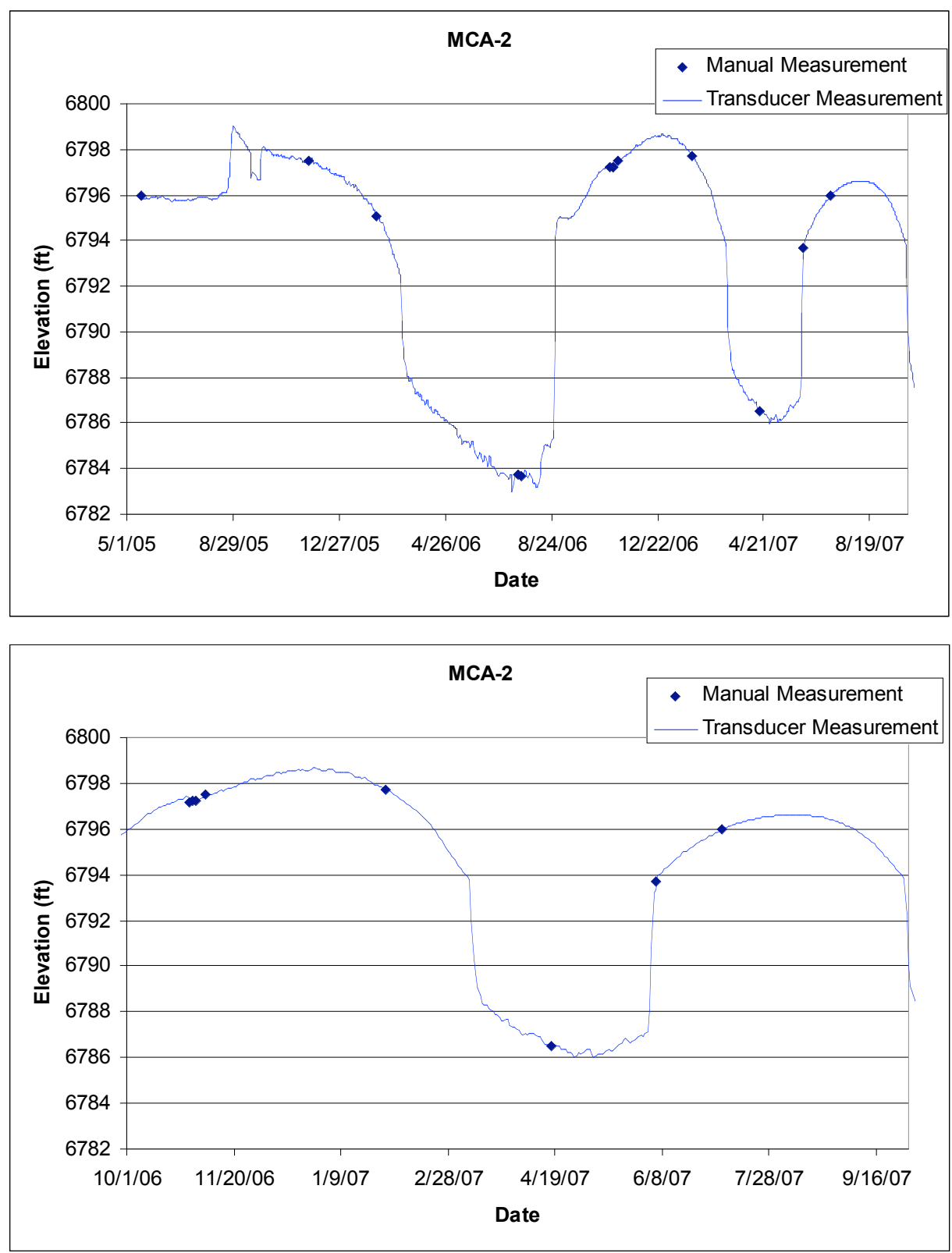


\subsection{MCA-3ac \& MCA-3b}

Location: Middle Mortandad Canyon

Period of Record: MCA-3ac November 30, 2004, through September 30, 2007. MCA-3b November 22, 2004, through September 30, 2007.

Remarks: MCA-3ac is a nested piezometer located about $5 \mathrm{ft}$ south of MCA-3b. Valid manual measurements in 1-in. PVC casing were not possible with transducer installed until 2006 when a small-diameter water level tape was purchased.
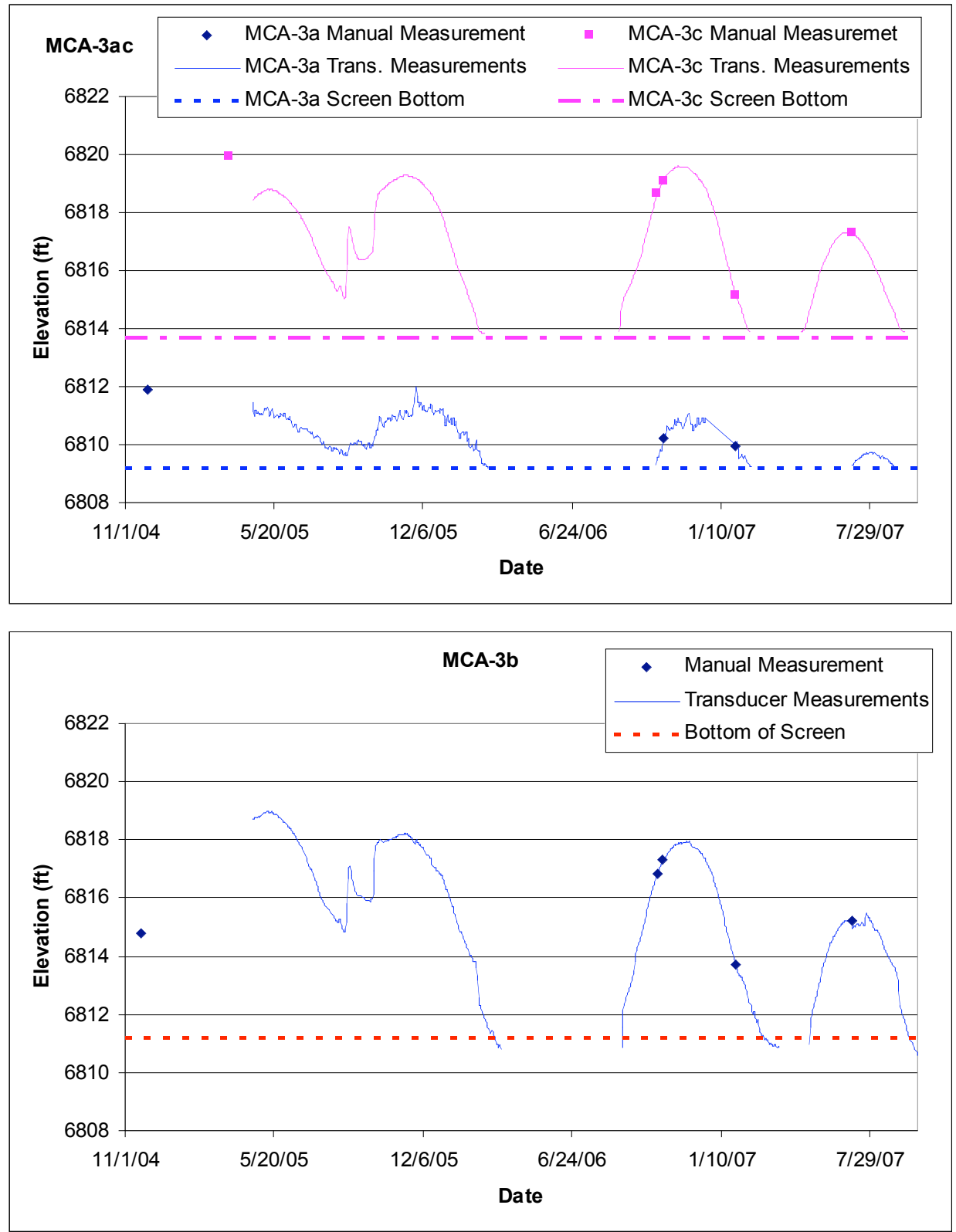


\subsection{MCA-3def}

Location: Middle Mortandad Canyon, south of sediment traps.

Period of Record: MCA-3d March 25, 2005, through September 30, 2007; MCA-3e March 30, 2005, through September 30, 2007; MCA-3f April 5, 2005, through September 30, 2007.

Remarks: MCA-3def is a nested piezometer. Screen bottom elevations: MCA-3d $6764.4 \mathrm{ft}, \mathrm{MCA}-3 \mathrm{e}$ $6768.0 \mathrm{ft}, \mathrm{MCA}-3 \mathrm{f} 6771.5 \mathrm{ft}$. Pressure transducers in MCA-3e and MCA-3f malfunctioned December 7, 2005, to October 16, 2006, no valid data available. Transducers in MCA-3e and MCA-3f were replaced on October 16, 2006.
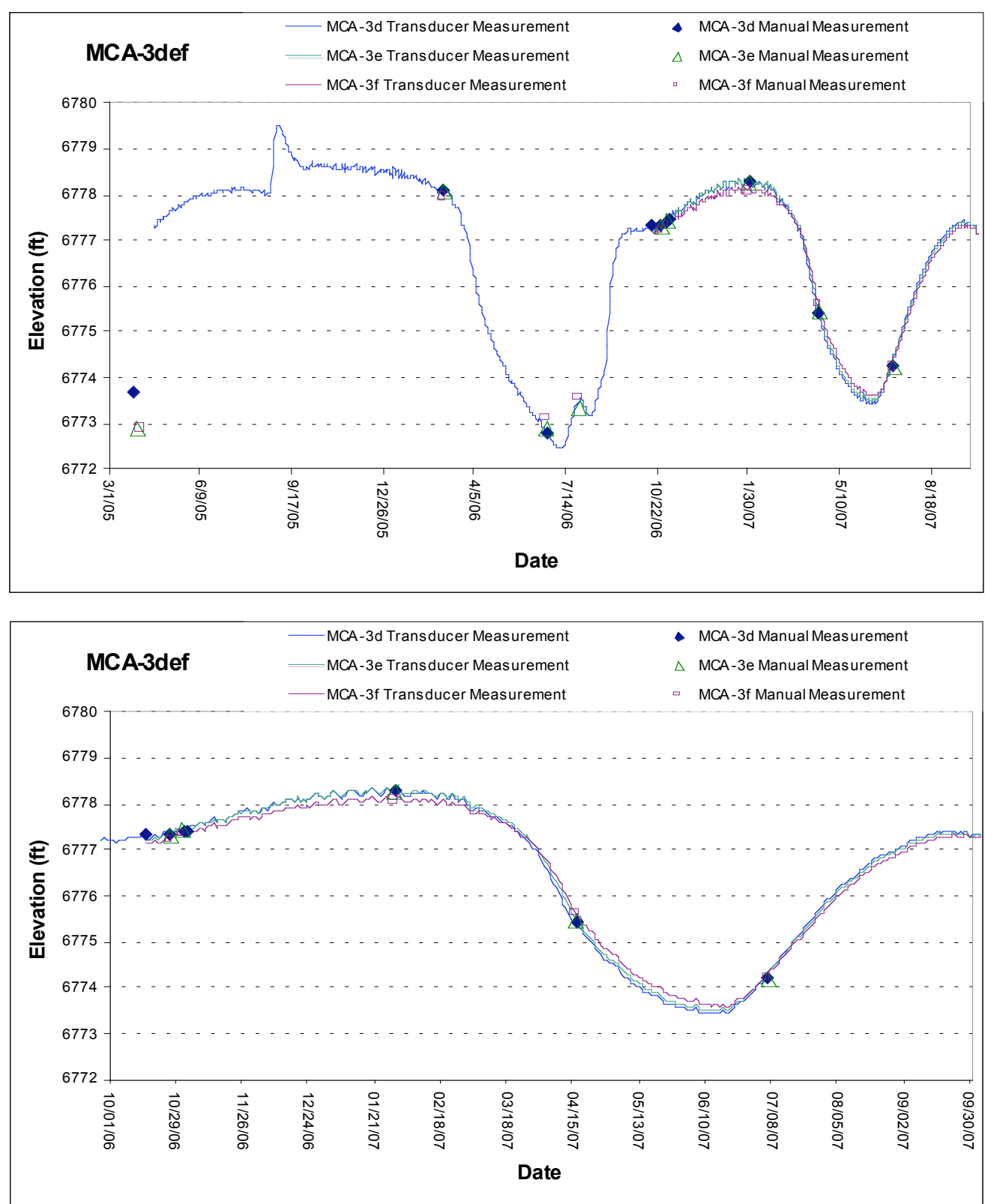


\subsection{MCA-4}

Location: Upper Effluent Canyon, approximately $200 \mathrm{ft}$ west of TA-50 outfall.

Period of Record: April 18, 2005, through September 30, 2007

Remarks: Screen bottom elevation is $7129.8 \mathrm{ft}$.
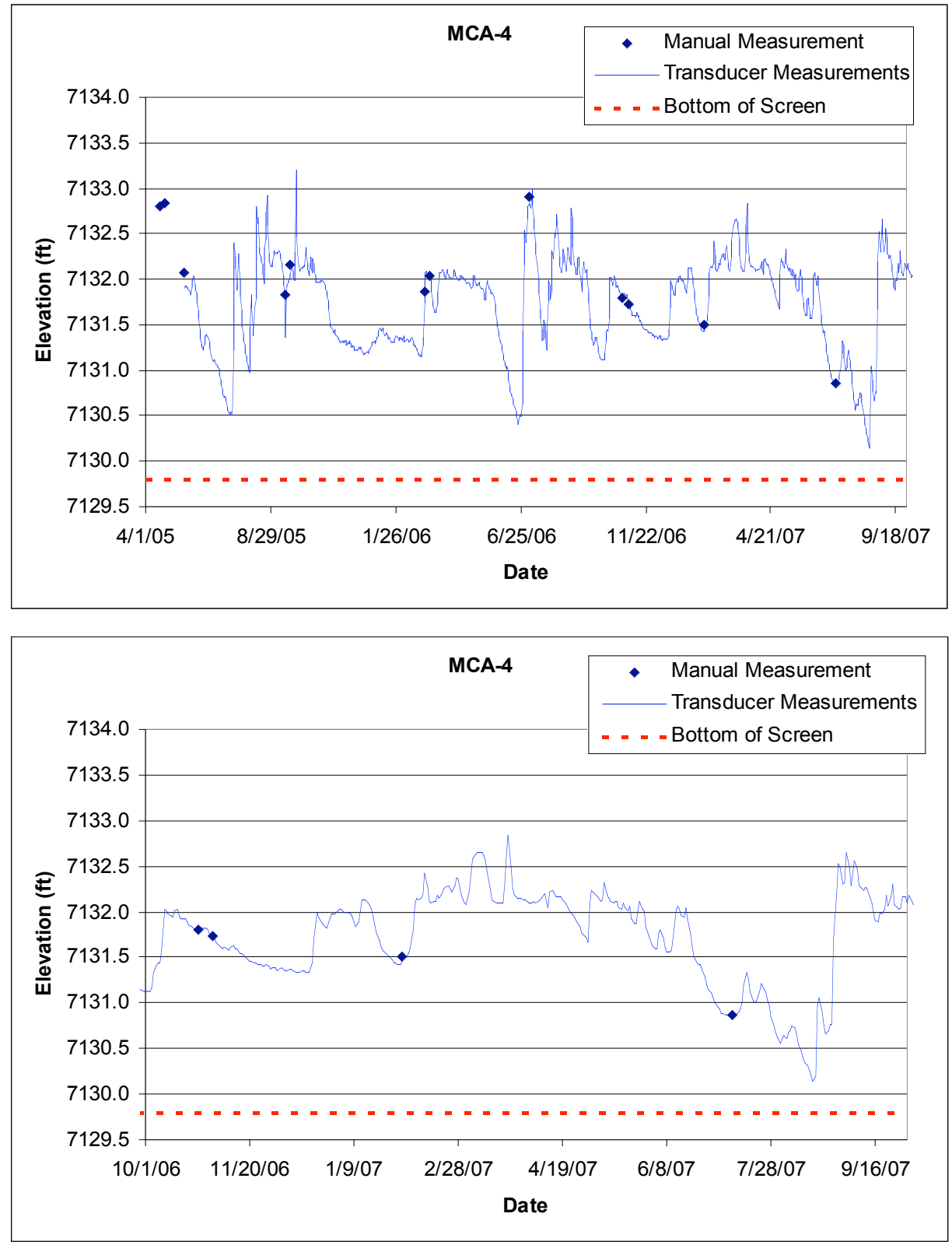


\subsection{MCA-5}

Location: Upper Mortandad Canyon, approximately $1250 \mathrm{ft}$ downstream of TA-50 outfall. Period of Record: April 25, 2005, through September 30, 2007

Remarks: Well is intermittently dry.
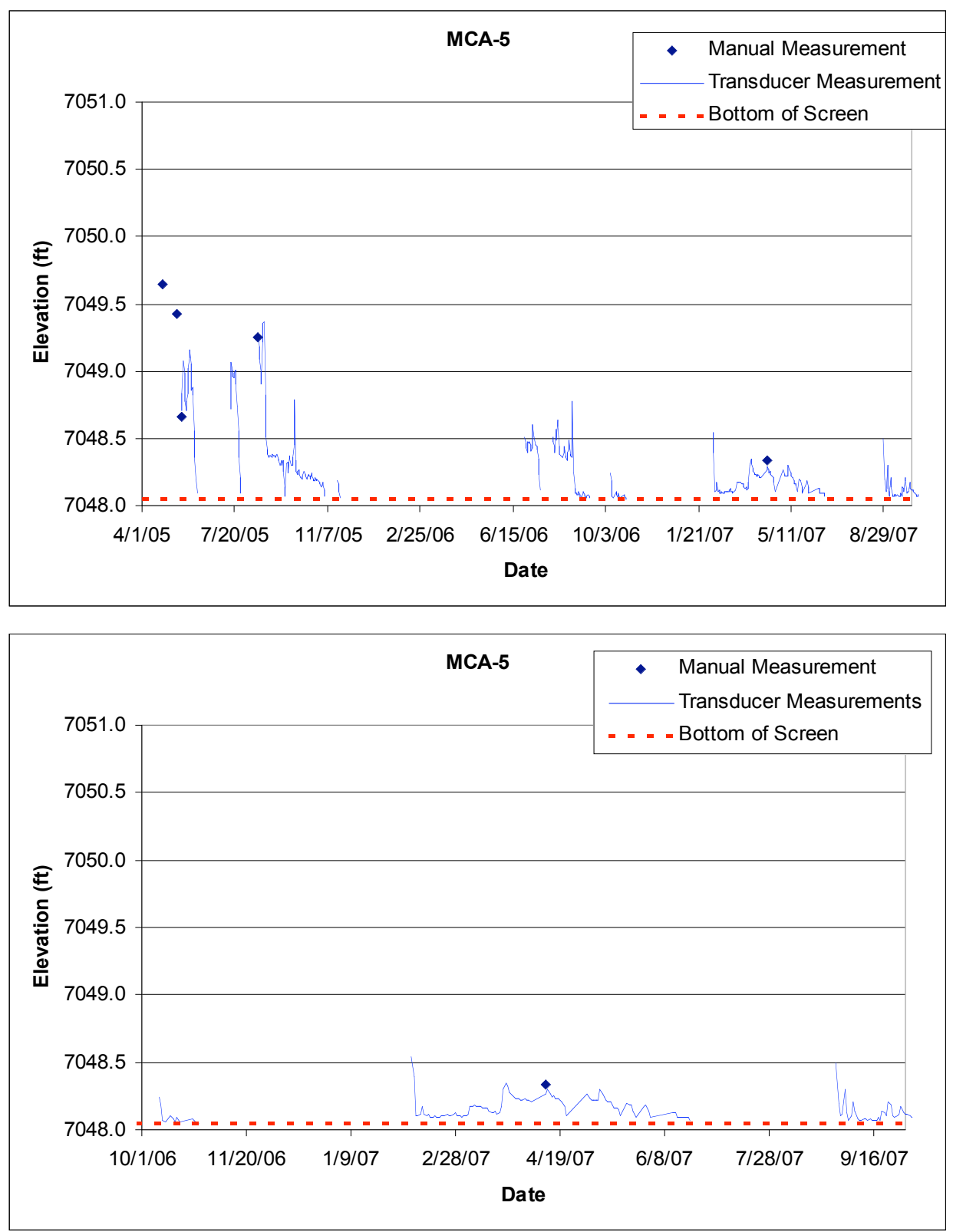


\subsection{MCA-8}

Location: Lower Mortandad Canyon.

Period of Record: October 3, 2005, through November 29, 2007

Remarks: No valid water level data exist for this well. Water has occurred only in the sump since completion on September 29, 2004.

\begin{tabular}{|l|r|r||l|}
\hline Well Name & Date Time & Water Level (ft) & Comments \\
\hline MCA-8 & $10 / 3 / 2005$ 9:50:00 AM & 6582.43 & Install transducer \\
\hline MCA-8 & $1 / 4 / 200612: 27: 00 \mathrm{PM}$ & 6583.52 & Water is in the sump \\
\hline MCA-8 & $4 / 13 / 2006$ 10:29:00 AM & 6584.09 & Water is in the sump \\
\hline MCA-8 & $7 / 18 / 200612: 26: 00 \mathrm{PM}$ & 6584.14 & Water is in the sump \\
\hline MCA-8 & $10 / 30 / 20061: 12: 00 \mathrm{PM}$ & 6584.17 & Water is in the sump \\
\hline MCA-8 & $9 / 5 / 20061: 03: 00 \mathrm{PM}$ & 6584.16 & Water is in the sump \\
\hline MCA-8 & $1 / 26 / 2007$ 9:10:00 AM & 6584.12 & Water is in the sump \\
\hline MCA-8 & $4 / 12 / 2007$ 9:25:00 AM & 6584.11 & Water is in the sump \\
\hline MCA-8 & $7 / 3 / 200711: 05: 00 \mathrm{AM}$ & 6584.1 & Water is in the sump \\
\hline MCA-8 & $11 / 29 / 20072: 12: 00 \mathrm{PM}$ & 6584.11 & Water is in the sump \\
\hline
\end{tabular}

\subsection{MCA-9}

Location: Lower Mortandad Canyon.

Period of Record: August 17, 2005, through November 29, 2007

Remarks: No valid water level data exist for this well. Water has occurred only in the sump since completion on December 4, 2004.

\begin{tabular}{|l|r||r||l|}
\hline Well Name & \multicolumn{1}{|c|}{ Date Time } & Water Level (ft) & Comments \\
\hline MCA-9 & $8 / 17 / 2005$ 11:52:00 AM & 6558.59 & Install transducer Dry \\
\hline MCA-9 & $2 / 23 / 2006$ 2:23:00 PM & 6558.76 & Water is in the sump \\
\hline MCA-9 & $7 / 18 / 2006$ 12:07:00 PM & 6558.76 & Water is in the sump \\
\hline MCA-9 & $5 / 16 / 2006$ 12:26:00 PM & 6558.74 & Water is in the sump \\
\hline MCA-9 & $5 / 16 / 20061: 01: 00$ PM & 6558.77 & Water is in the sump \\
\hline MCA-9 & $10 / 30 / 200612: 31: 00$ PM & 6558.95 & Water is in the sump \\
\hline MCA-9 & $10 / 31 / 2006$ 9:57:00 AM & 6558.72 & Water is in the sump \\
\hline MCA-9 & $1 / 26 / 2007$ 9:34:00 AM & 6559.13 & Water is in the sump \\
\hline MCA-9 & $4 / 12 / 2007$ 9:07:00 AM & 6559.42 & Water is in the sump \\
\hline MCA-9 & $7 / 3 / 200710: 45: 00$ AM & 6559.46 & Water is in the sump \\
\hline MCA-9 & $11 / 29 / 20073: 38: 00$ PM & 6559.35 & Water is in the sump \\
\hline
\end{tabular}




\subsection{MCO-0.6}

Location: Upper Mortandad Canyon, north of TA-48.

Period of Record: March 31, 1999, through September 30, 2007

Remarks: None.
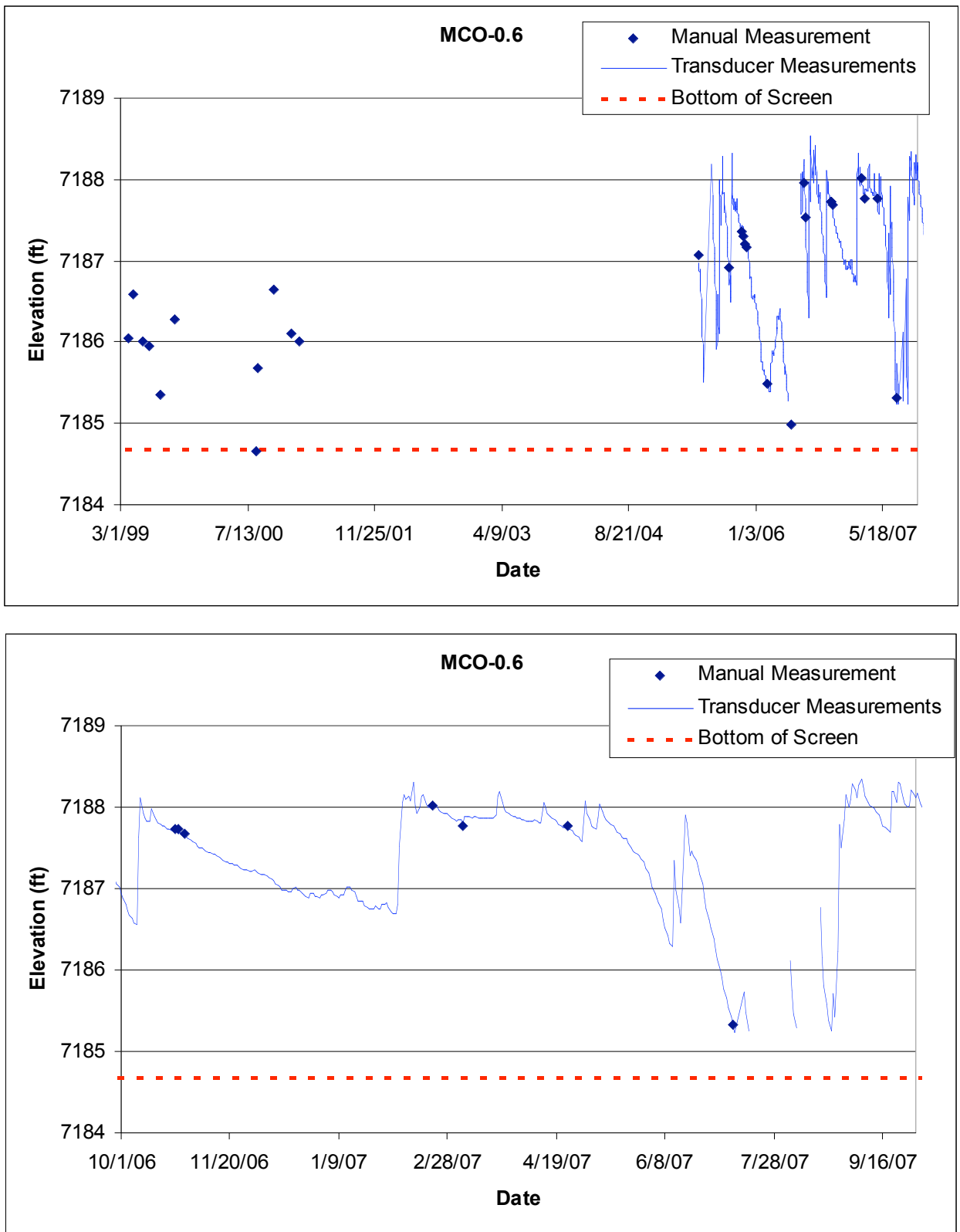


\subsection{MCO-2}

Location: Upper Effluent Canyon, approximately $200 \mathrm{ft}$ west of TA-50 outfall.

Period of Record: November 1, 1960, through September 30, 2007

Remarks: The transducer was sitting on top of the bladder pump in a 2-in.-diameter well at an elevation of $7133.8 \mathrm{ft}$ until April 12, 2007. The pump was removed from the well on April 12, 2007, to allow for more complete water level monitoring by lowering the transducer.
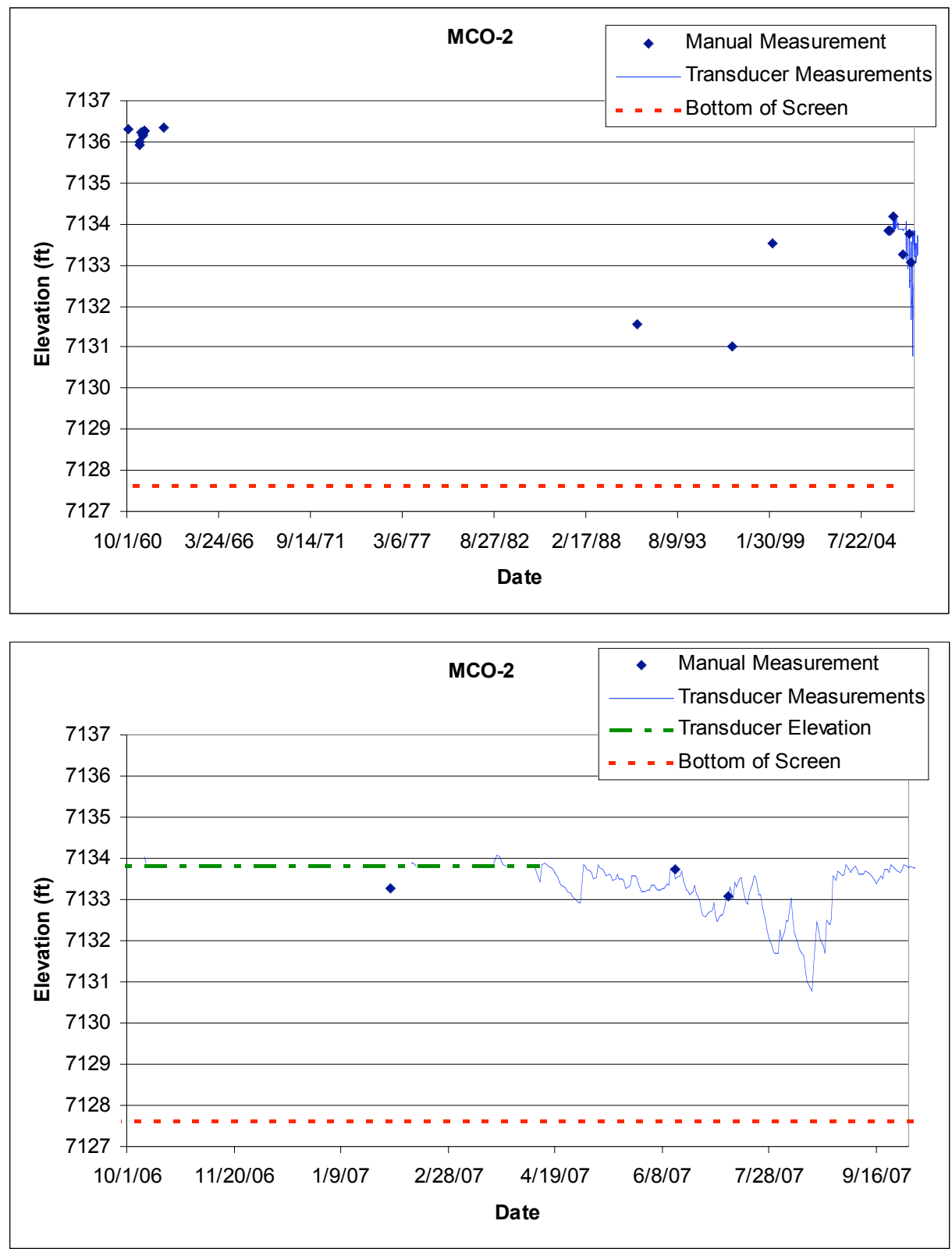


\subsection{MCO-3}

Location: Upper Mortandad Canyon, approximately $1250 \mathrm{ft}$ downstream of TA-50 outfall. Period of Record: March 27, 1961, through June 20, 2007.

Remarks: Bottom of screen elevation is $7040.72 \mathrm{ft}$.

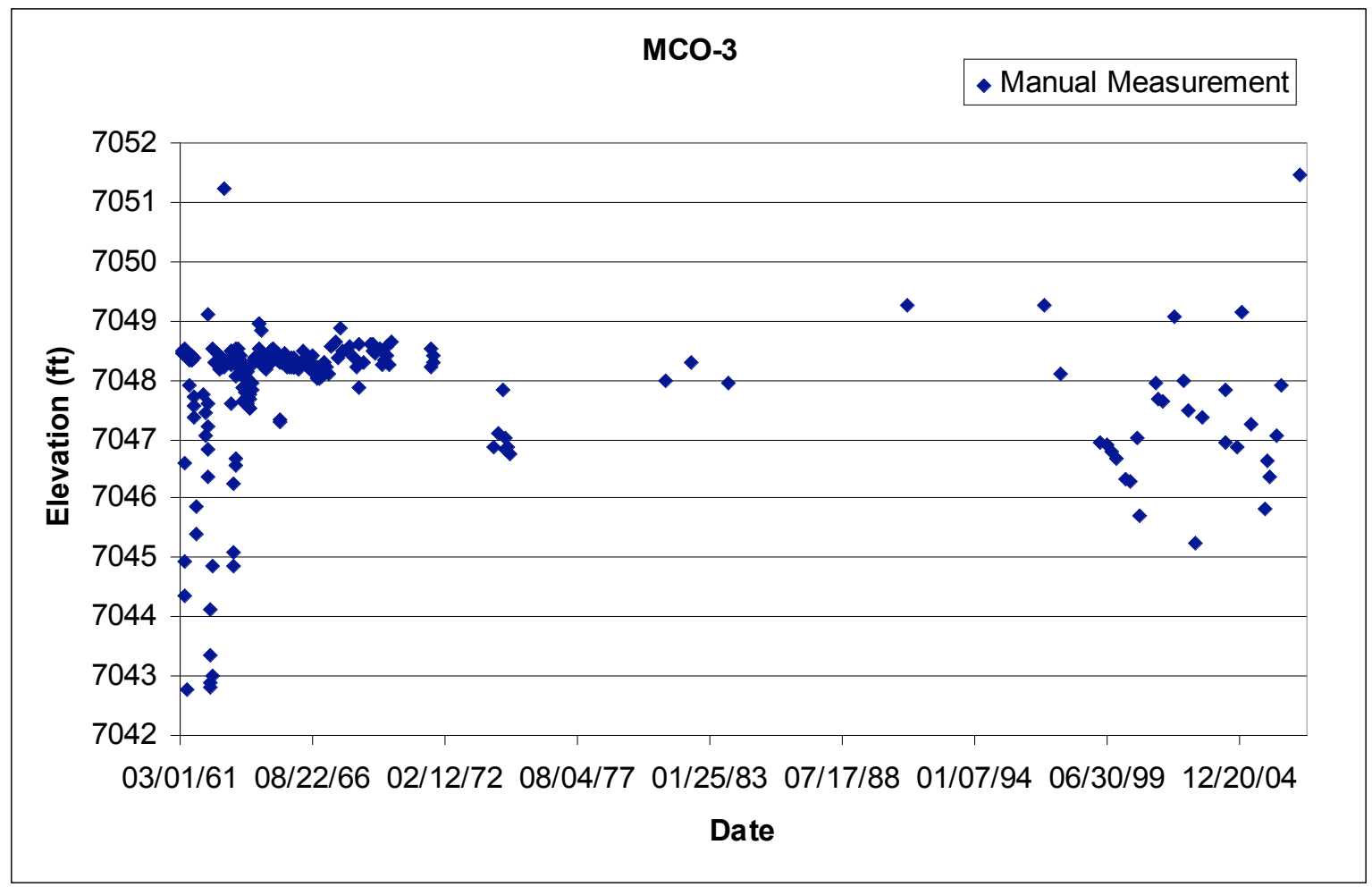




\subsection{MCO-4B}

Location: Middle Mortandad Canyon, approximately $3000 \mathrm{ft}$ up canyon from sediment traps.

Period of Record: August 21, 1990, through September 30, 2007

Remarks: Screen bottom elevation is $6857.9 \mathrm{ft}$.
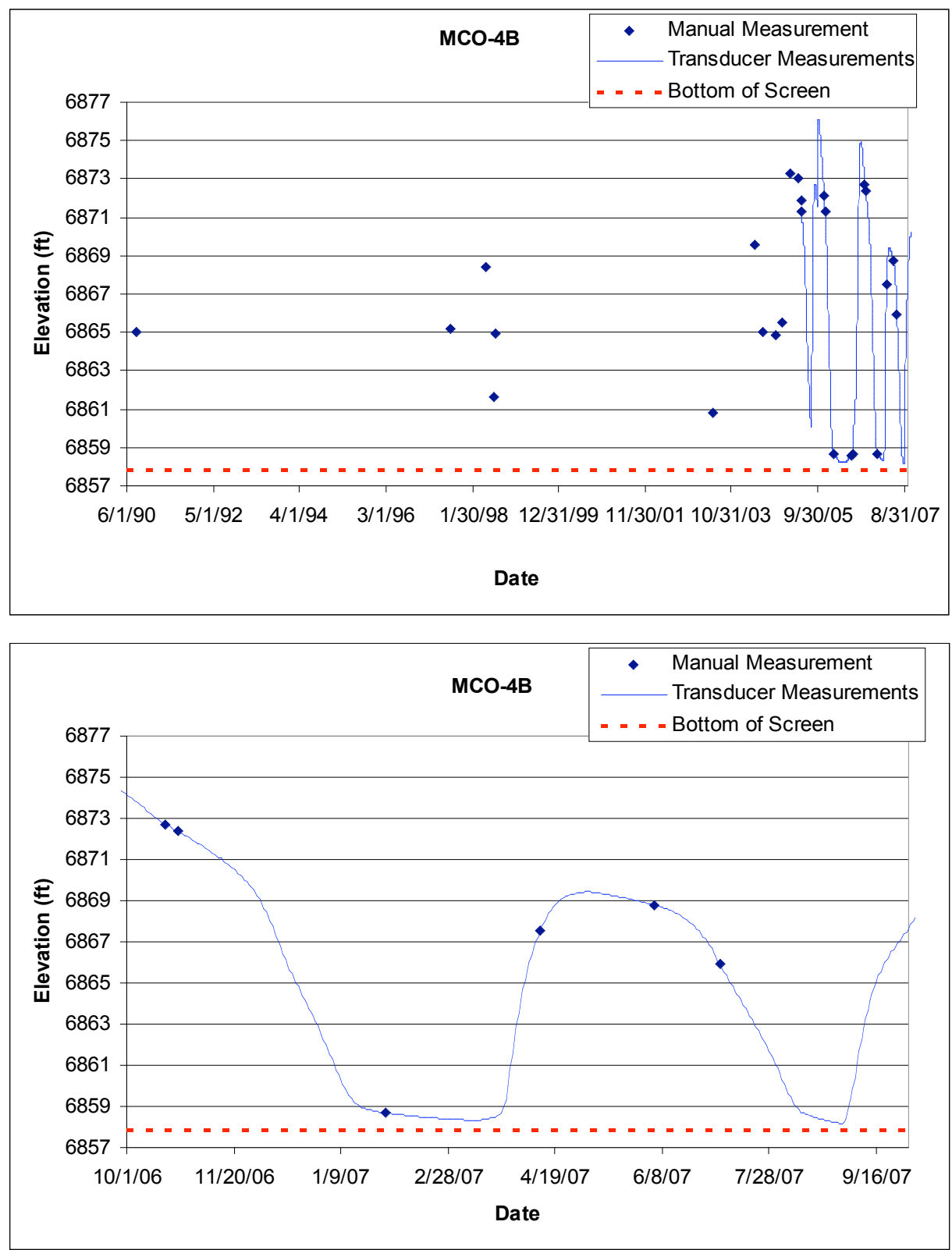


\subsection{MCO-5}

Location: Middle Mortandad Canyon, approximately $2300 \mathrm{ft}$ up canyon from sediment traps.

Period of Record: October 1, 1960, through September 30, 2007

Remarks: Bottom of screen elevation is $6829.66 \mathrm{ft}$.
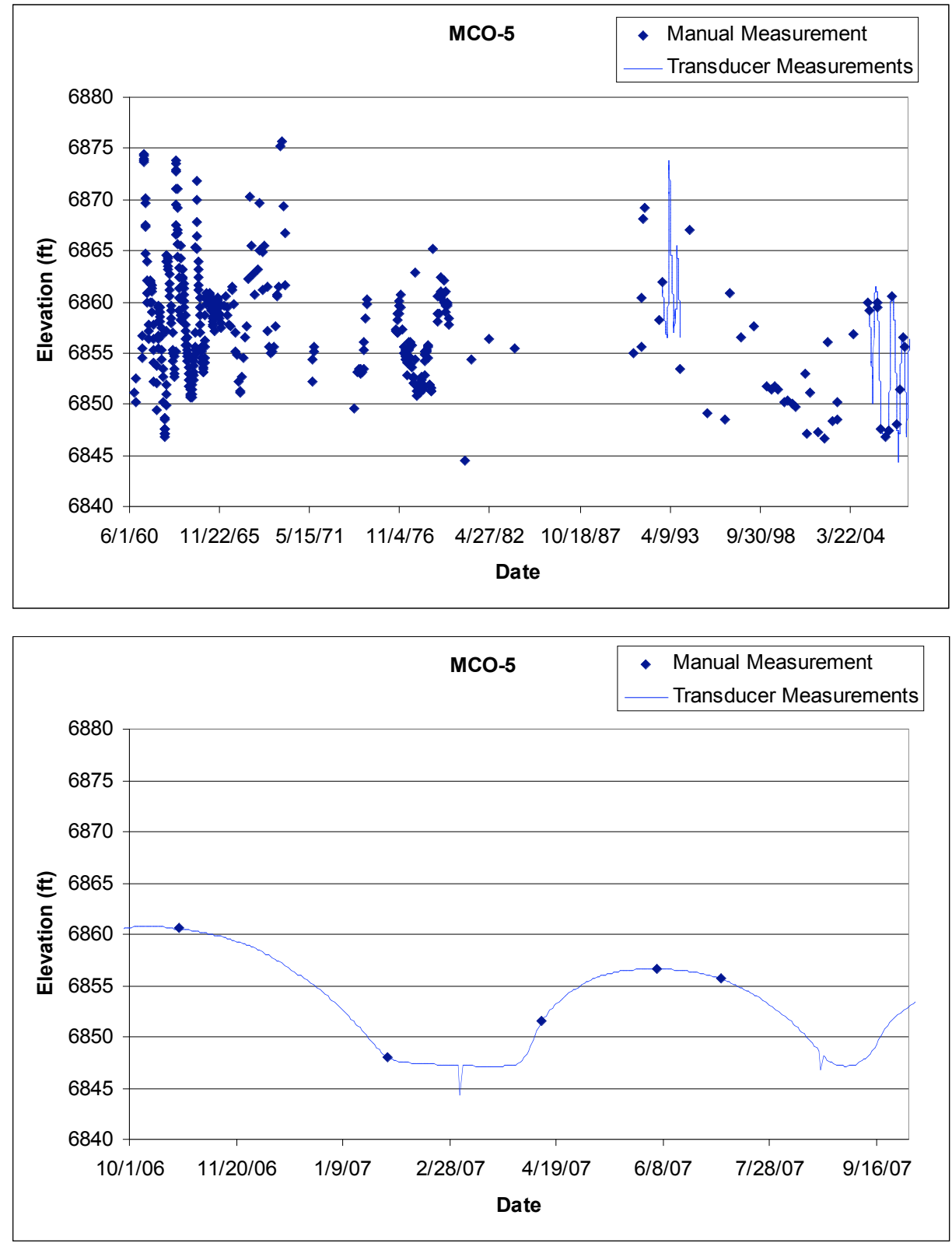


\subsection{MCO-6}

Location: Middle Mortandad Canyon, approximately 0.25 miles east of MCO-5.

Period of Record: August 25, 1961, through September 30, 2007

Remarks: Bottom of screen elevation is $6802.5 \mathrm{ft}$.
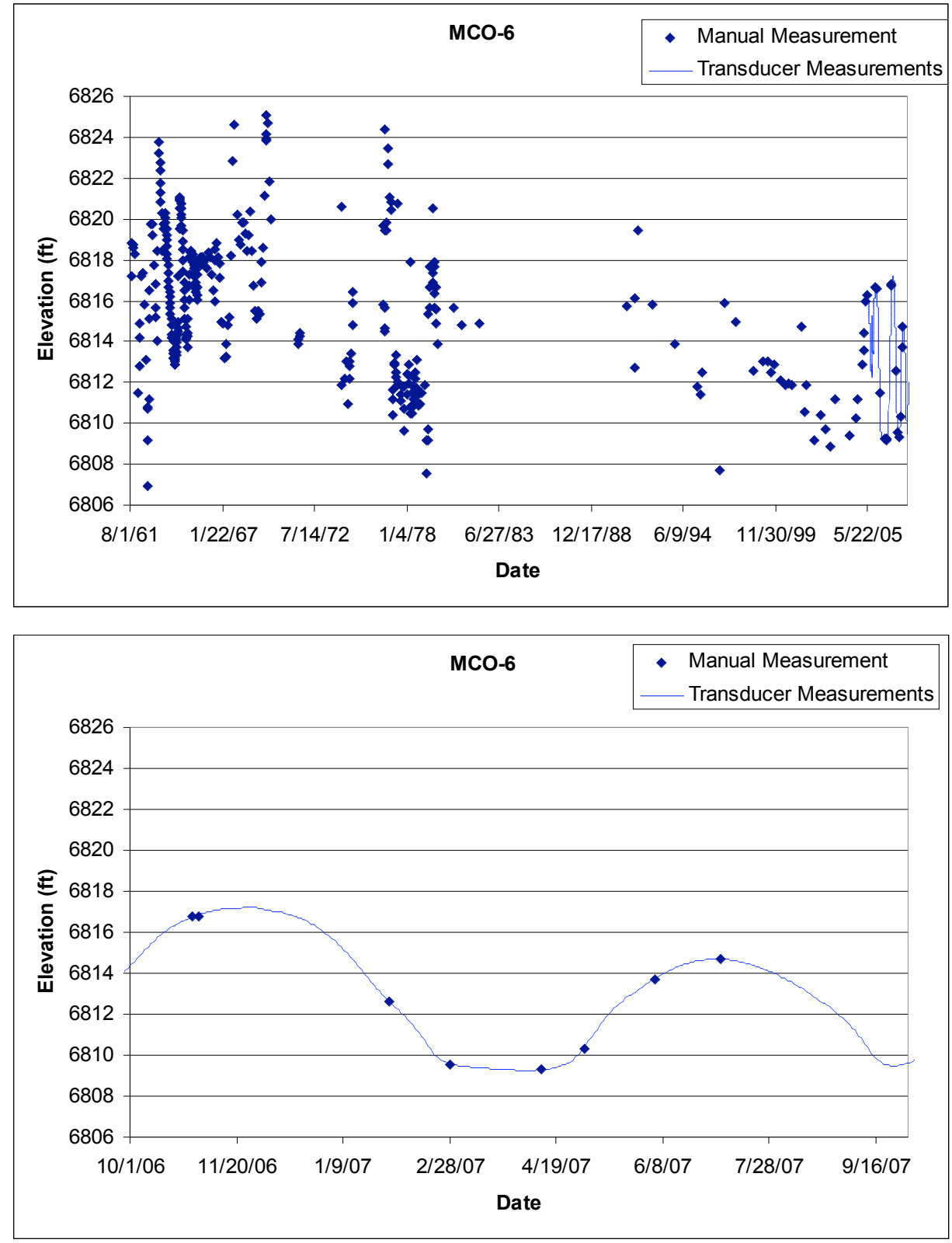


\subsection{MCO-7}

Location: Middle Mortandad Canyon, approximately 0.2 miles east of MCO-6.

Period of Record: October 1, 1960, through September 30, 2007

Remarks: Bottom of screen elevation is $6758.31 \mathrm{ft}$.
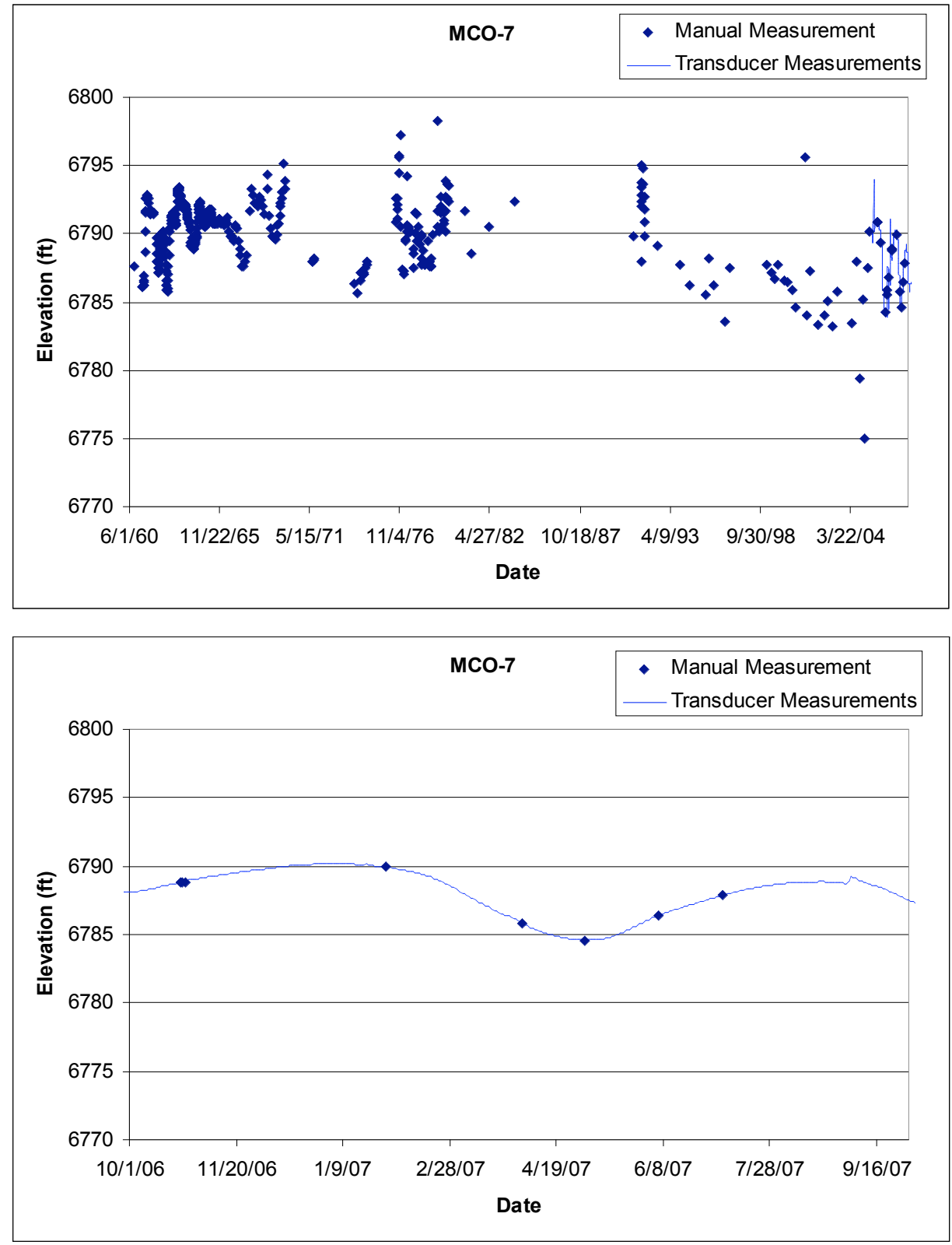


\subsection{MCO-7.5}

Location: Middle Mortandad Canyon, approximately 0.2 miles east of MCO-7.

Period of Record: November 1, 1961, through September 30, 2007

Remarks: Bottom of screen elevation is $6748.88 \mathrm{ft}$.
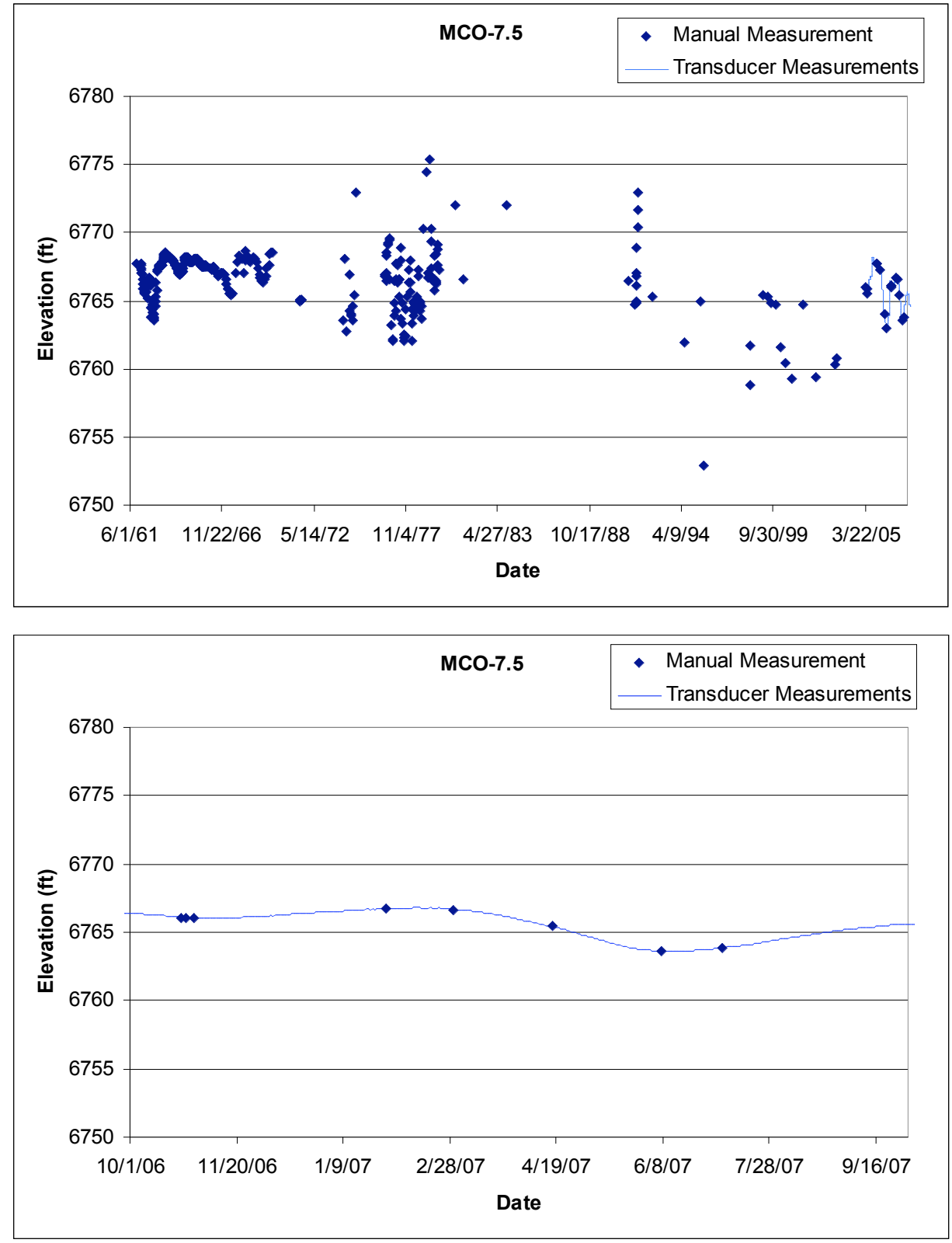


\subsection{MCWB-5}

Location: Middle Mortandad Canyon, up canyon from the sediment traps.

Period of Record: January 9, 1995, through September 30, 2007

Remarks: None.
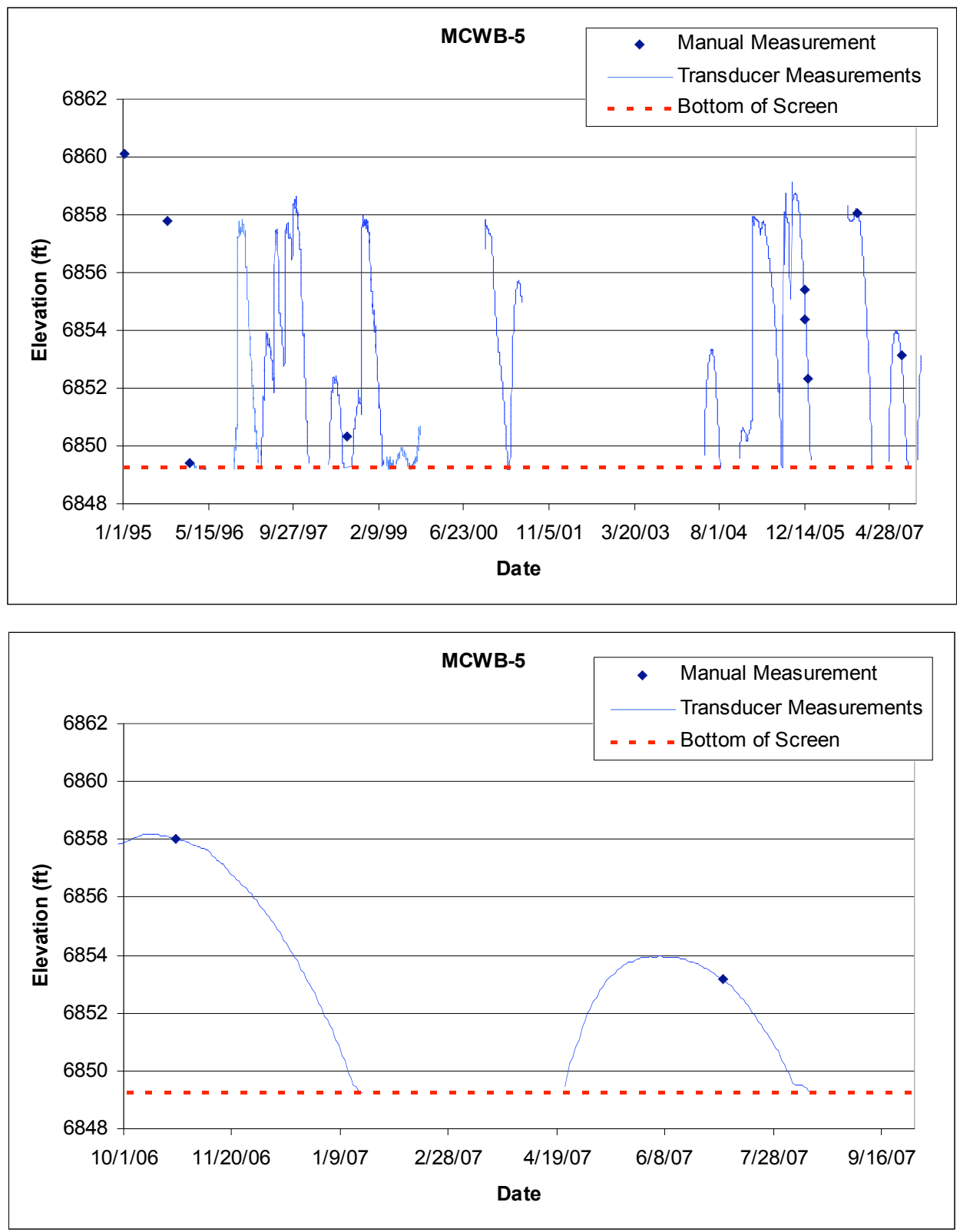


\subsection{MCWB-5.5B}

Location: Middle Mortandad Canyon, up canyon from sediment traps.

Period of Record: January 9, 1995, through September 30, 2007

Remarks: Water in sump appears to respond to groundwater level fluctuations.
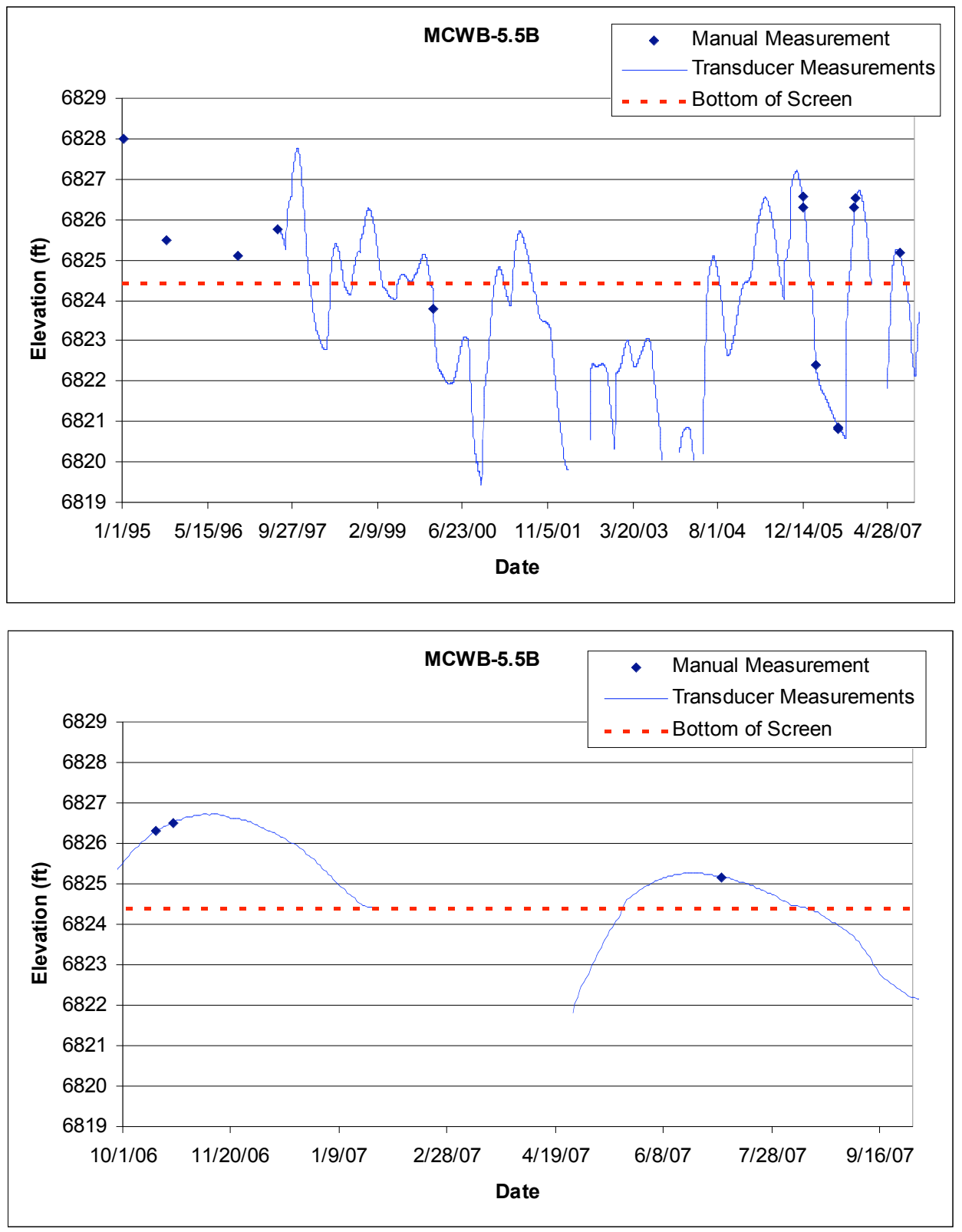


\subsection{MCWB-6.2A}

Location: Middle Mortandad Canyon, up canyon from sediment traps.

Period of Record: January 9, 1995, through September 30, 2007

Remarks: Water in the sump appears to respond to groundwater level fluctuations.
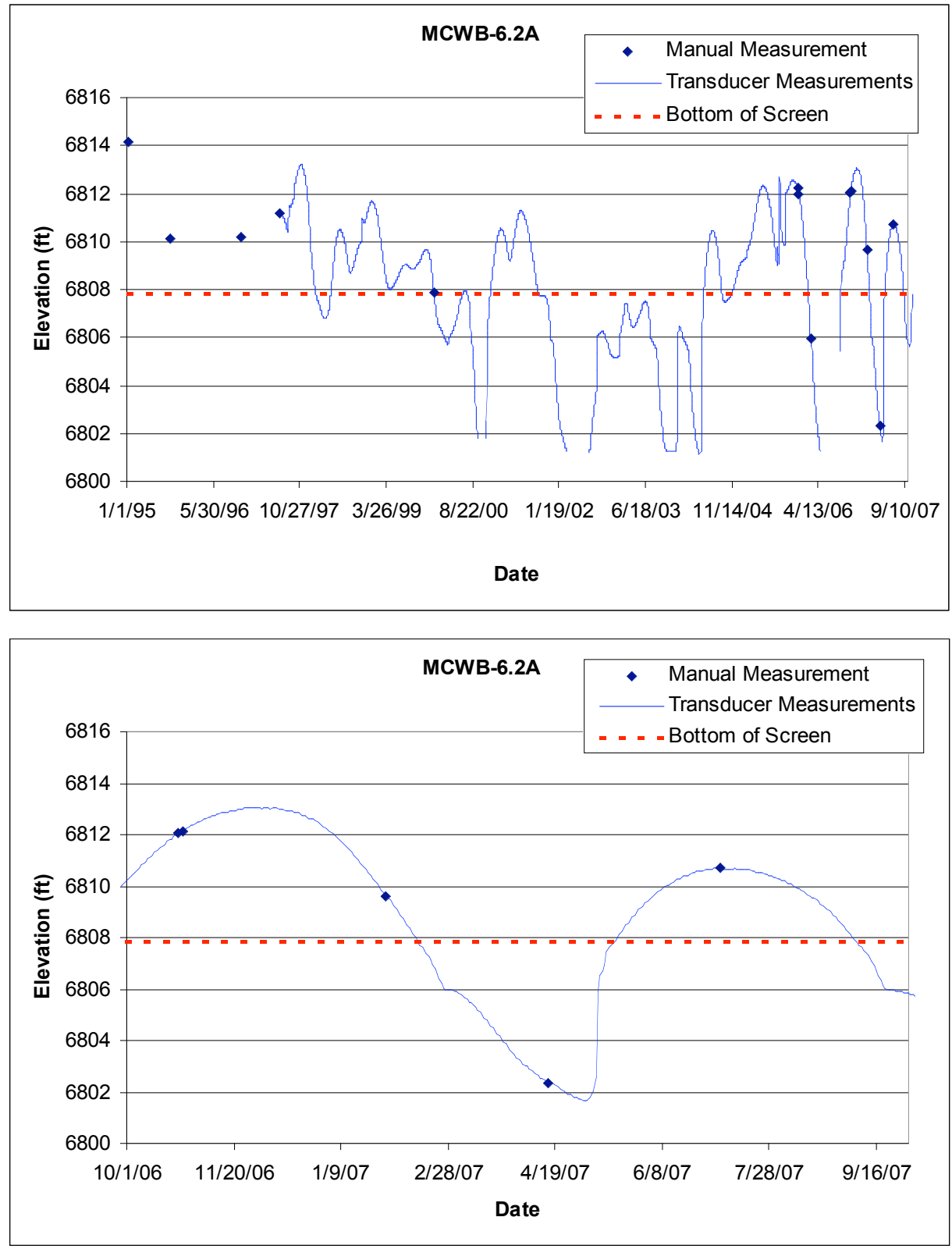


\subsection{MCWB-6.5E}

Location: Middle Mortandad Canyon, up canyon of the sediment traps.

Period of Record: January 9, 1995, through September 30, 2007

Remarks: Water in sump appears to respond to groundwater level fluctuations.
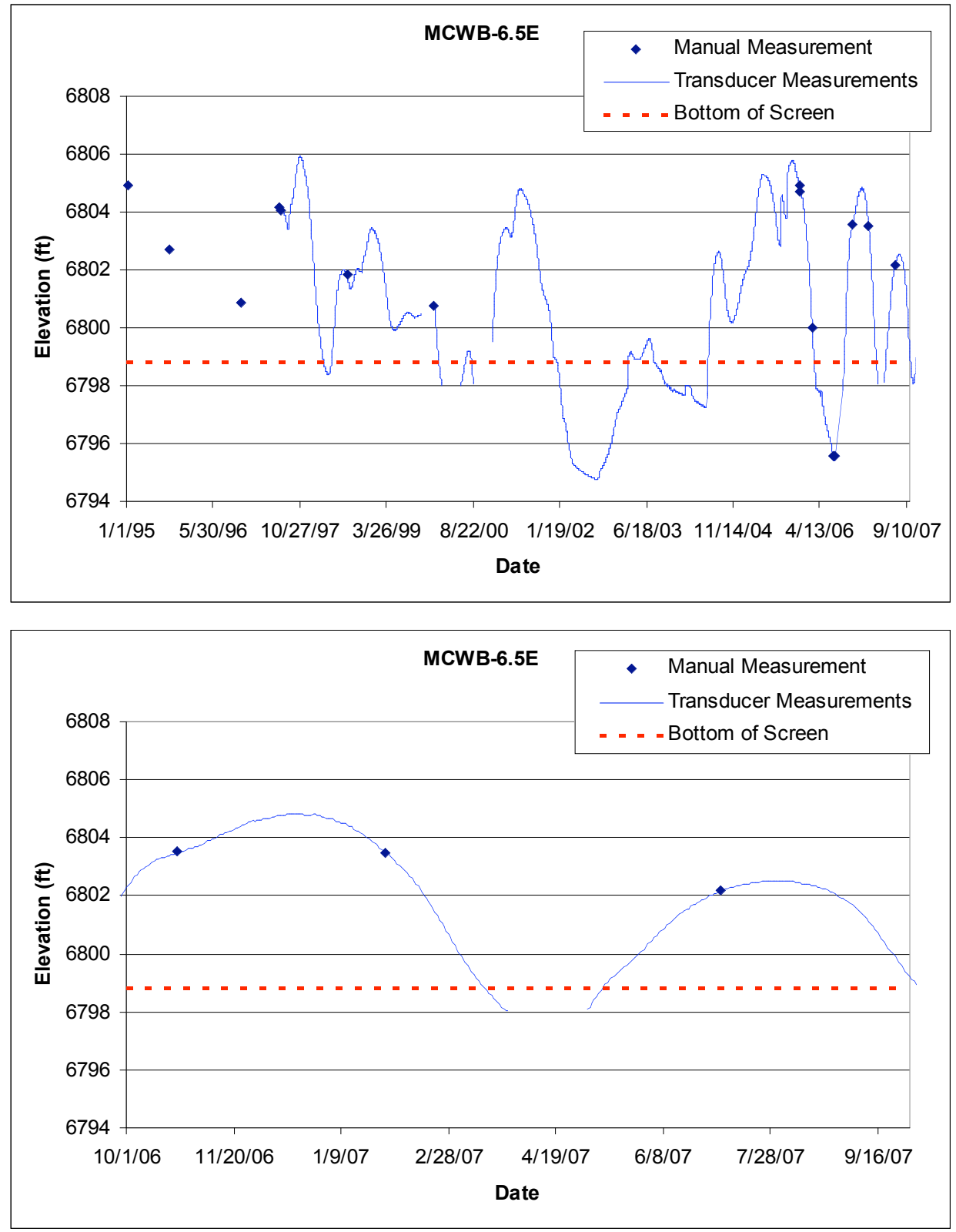


\subsection{MCWB-7A}

Location: Middle Mortandad Canyon, near sediment traps.

Period of Record: January 9, 1995, through September 30, 2007

Remarks: Water in sump appears to respond to groundwater level fluctuations.
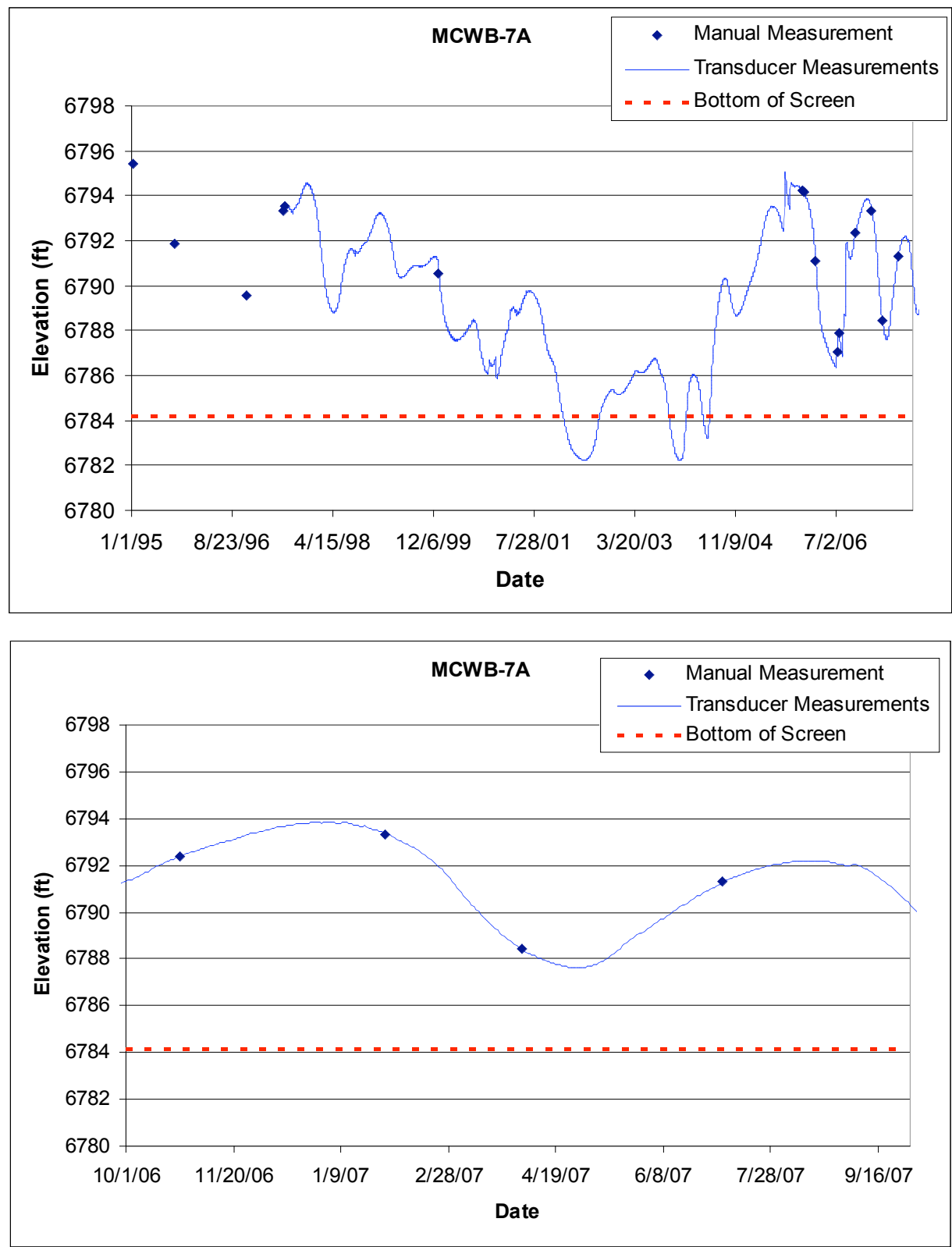


\subsection{MCWB-7.4B}

Location: Middle Mortandad Canyon, down canyon from sediment traps.

Period of Record: January 9, 1995, through September 30, 2007

Remarks: None.
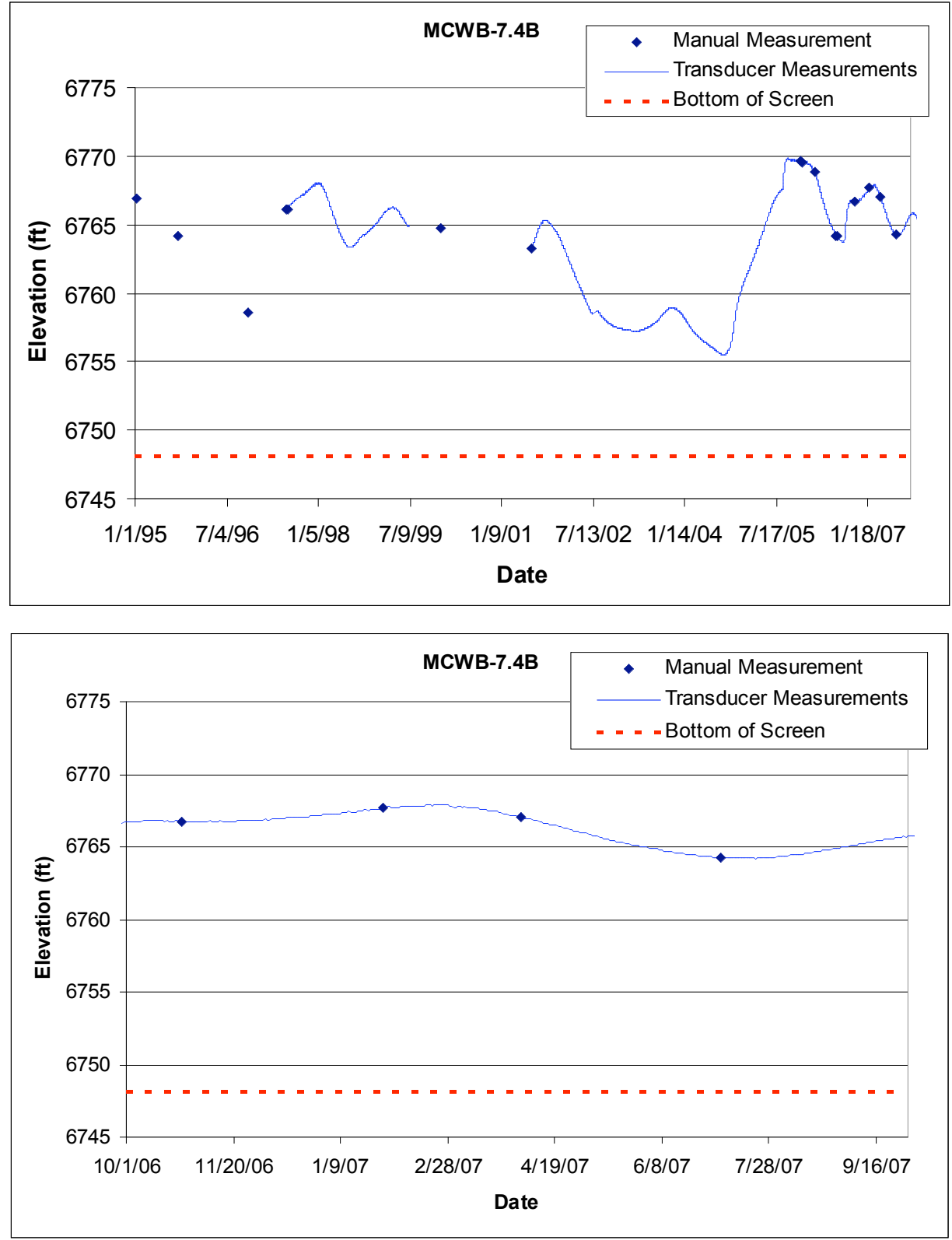


\subsection{MCWB-7.7B}

Location: Middle Mortandad Canyon, down canyon from sediment traps.

Period of Record: January 9, 1995, through September 30, 2007

Remarks: None.
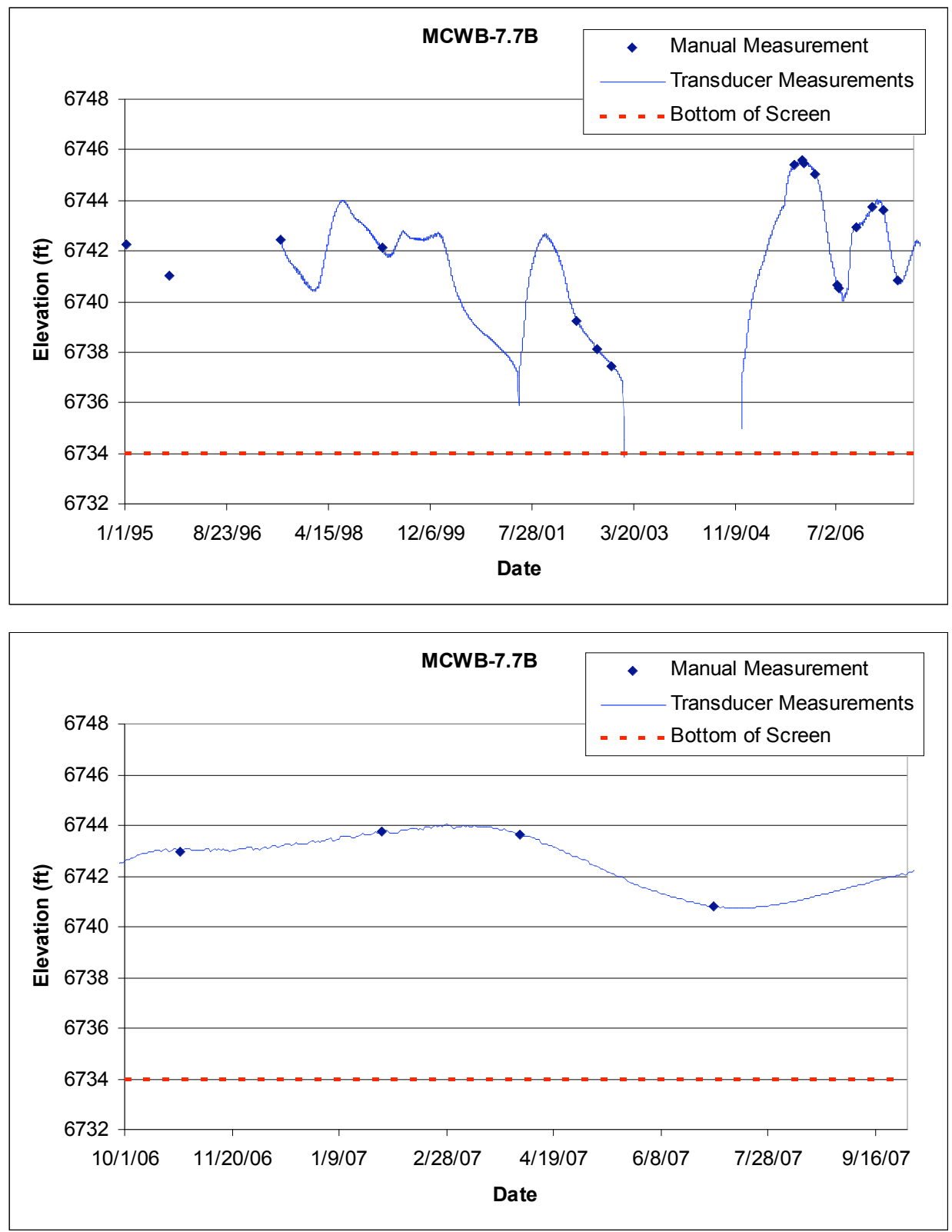


\subsection{MSC-16-06293}

Location: Martin Spring Canyon, about 1600 feet down drainage from Martin Spring outlet.

Period of Record: November 6, 2000, through September 30, 2007

Remarks: MSC-16-06293 was dry from November 7, 2006, to February 14, 2007, and May 3, 2007 , to August 29, 2007.
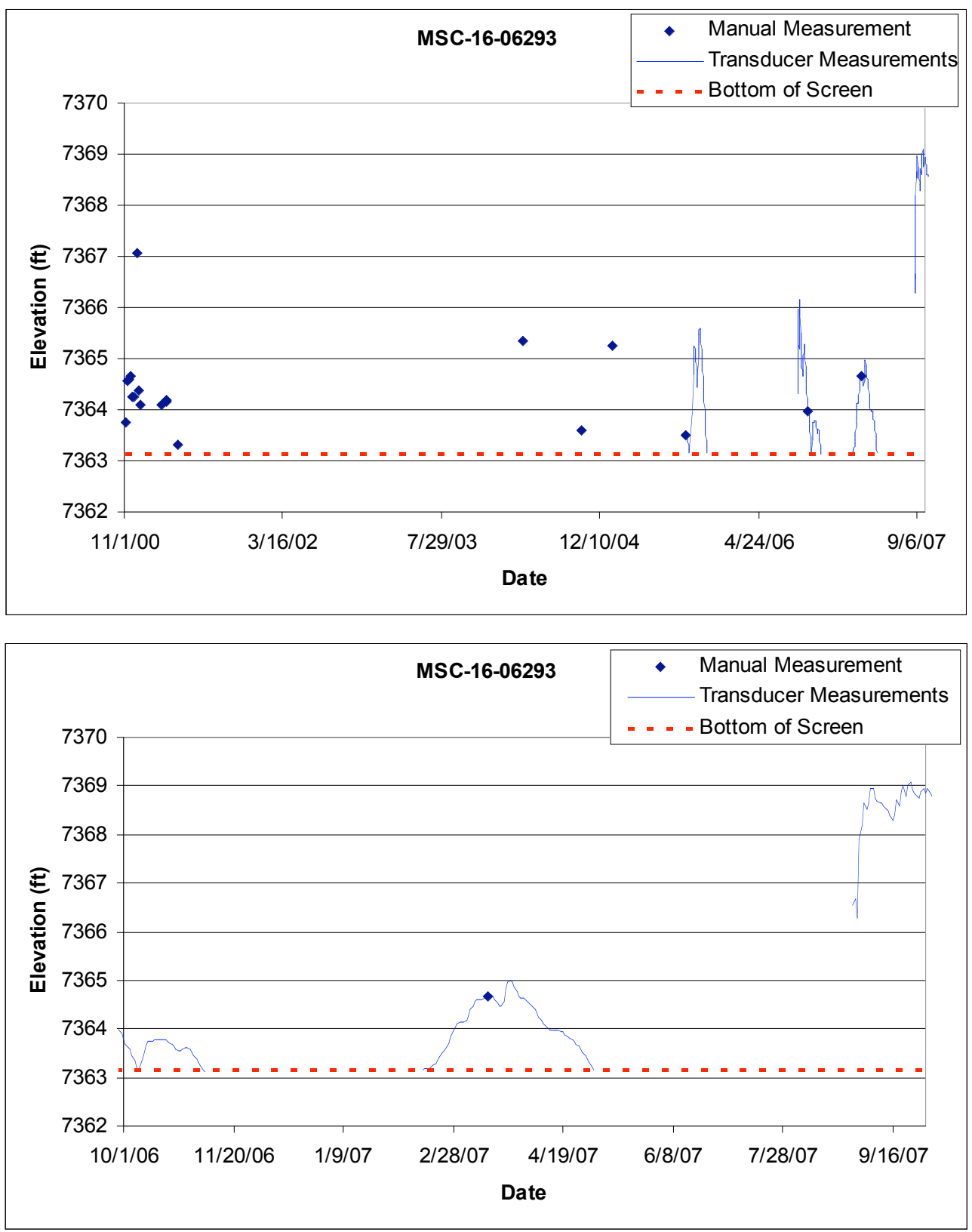


\subsection{MSC-16-06294}

Location: Martin Spring Canyon, about $1600 \mathrm{ft}$ upstream of the K-site wetlands.

Period of Record: November 6, 2000, through September 30, 2007

Remarks: The water was in the sump July 3, 2007, to July 14, 2007.
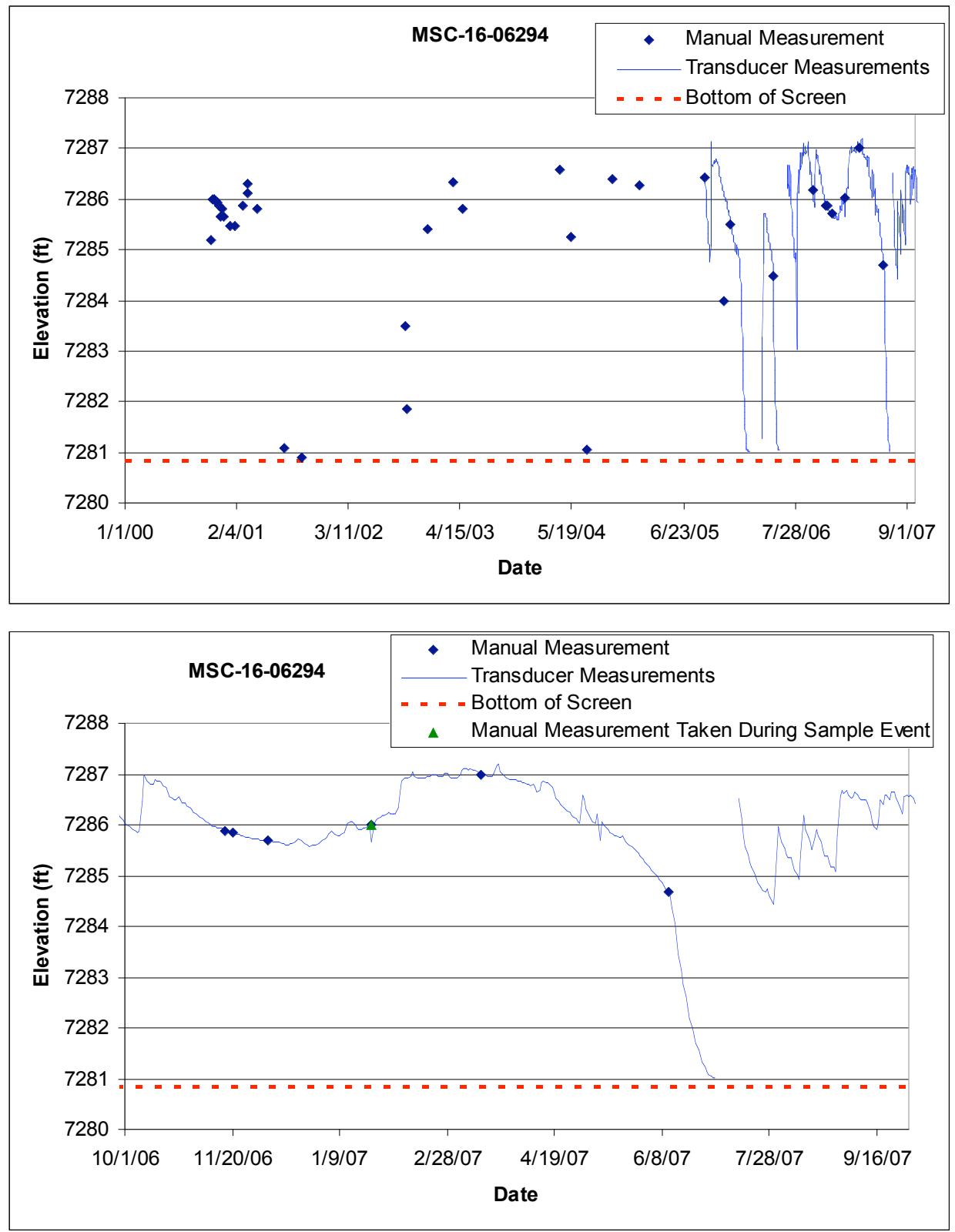


\subsection{MSC-16-06295}

Location: Martin Spring Canyon, just downstream of the K-site wetlands and north of the TA-11 drop tower.

Period of Record: March 10, 2000, through September 30, 2007

Remarks: None.
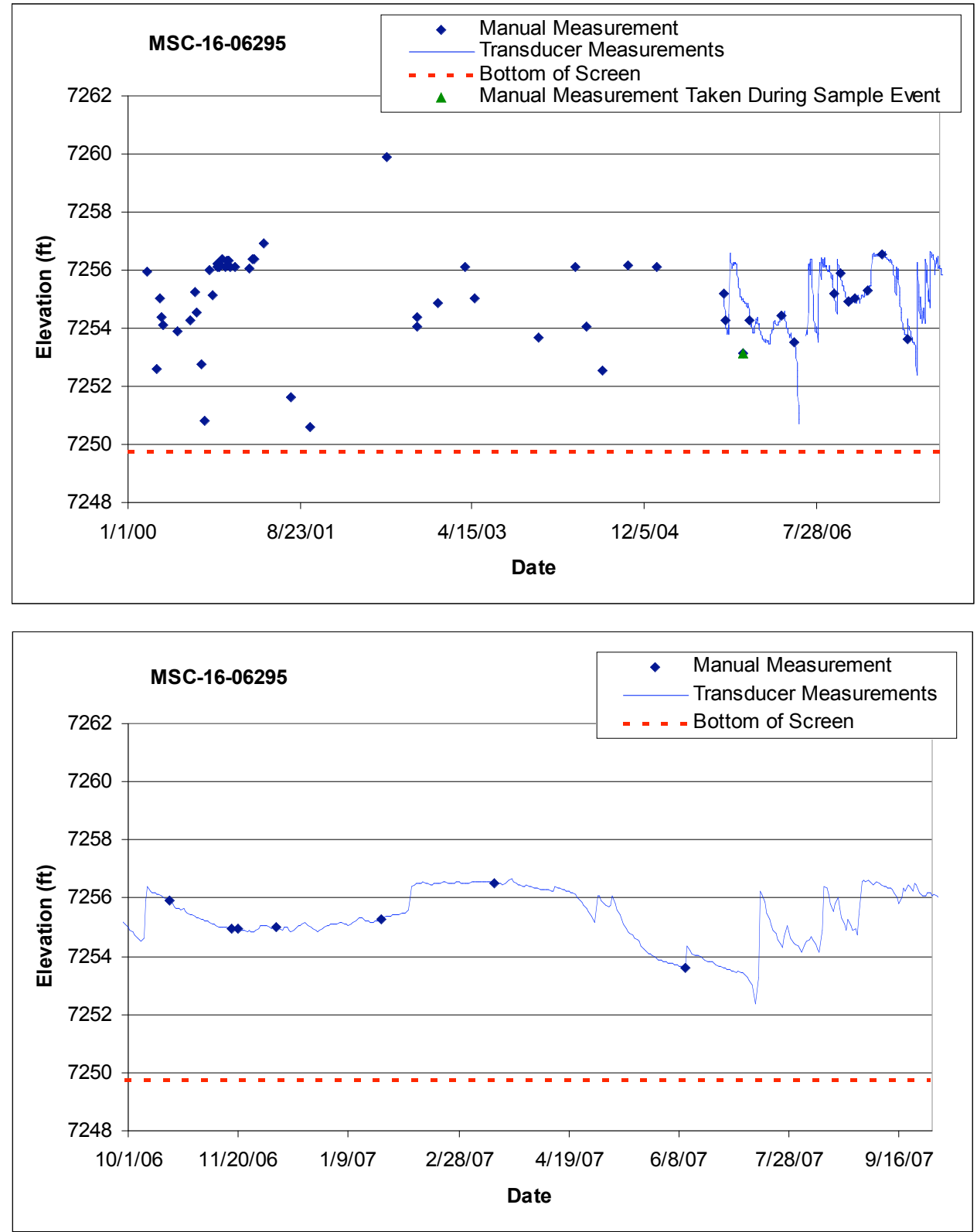


\subsection{MT-1}

Location: Middle Mortandad Canyon, approximately 0.12 miles east of MCO-7.

Period of Record: November 1, 1988, through September 30, 2007

Remarks: Bottom of screen elevation is $6752.6 \mathrm{ft}$.
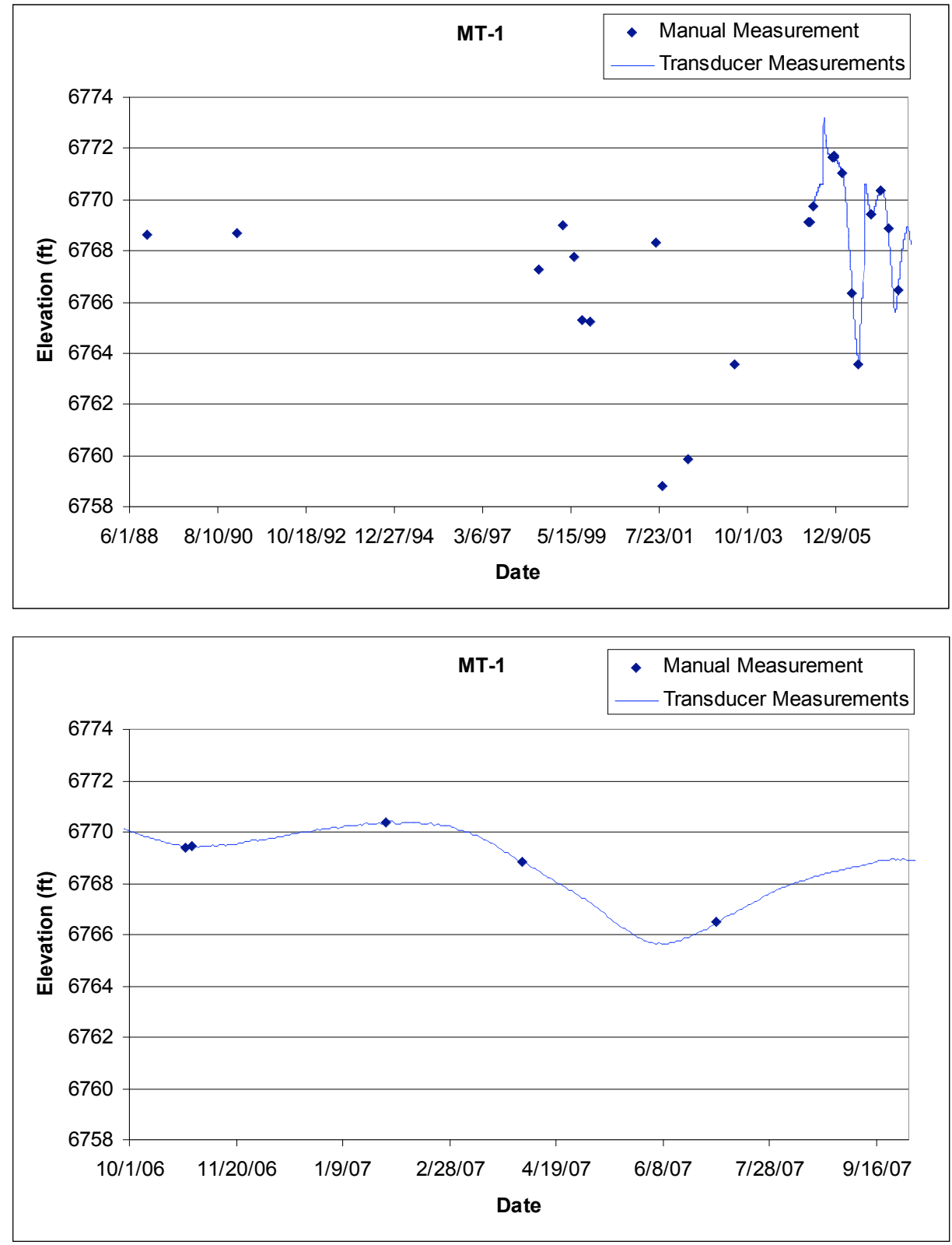


\subsection{MT-2}

Location: Middle Mortandad Canyon, down canyon of sediment traps, approximately 0.12 miles east of MT-1.

Period of Record: November 1, 1988, through September 30, 2007

Remarks: The transducer was above the pump until April 17, 2007, when the pump was removed from MT-2 and the transducer was lowered in the well. Transducer data before April 17, 2007 , do not represent water levels below the top of the pump, at an approximate elevation of $6749.3 \mathrm{ft}$.
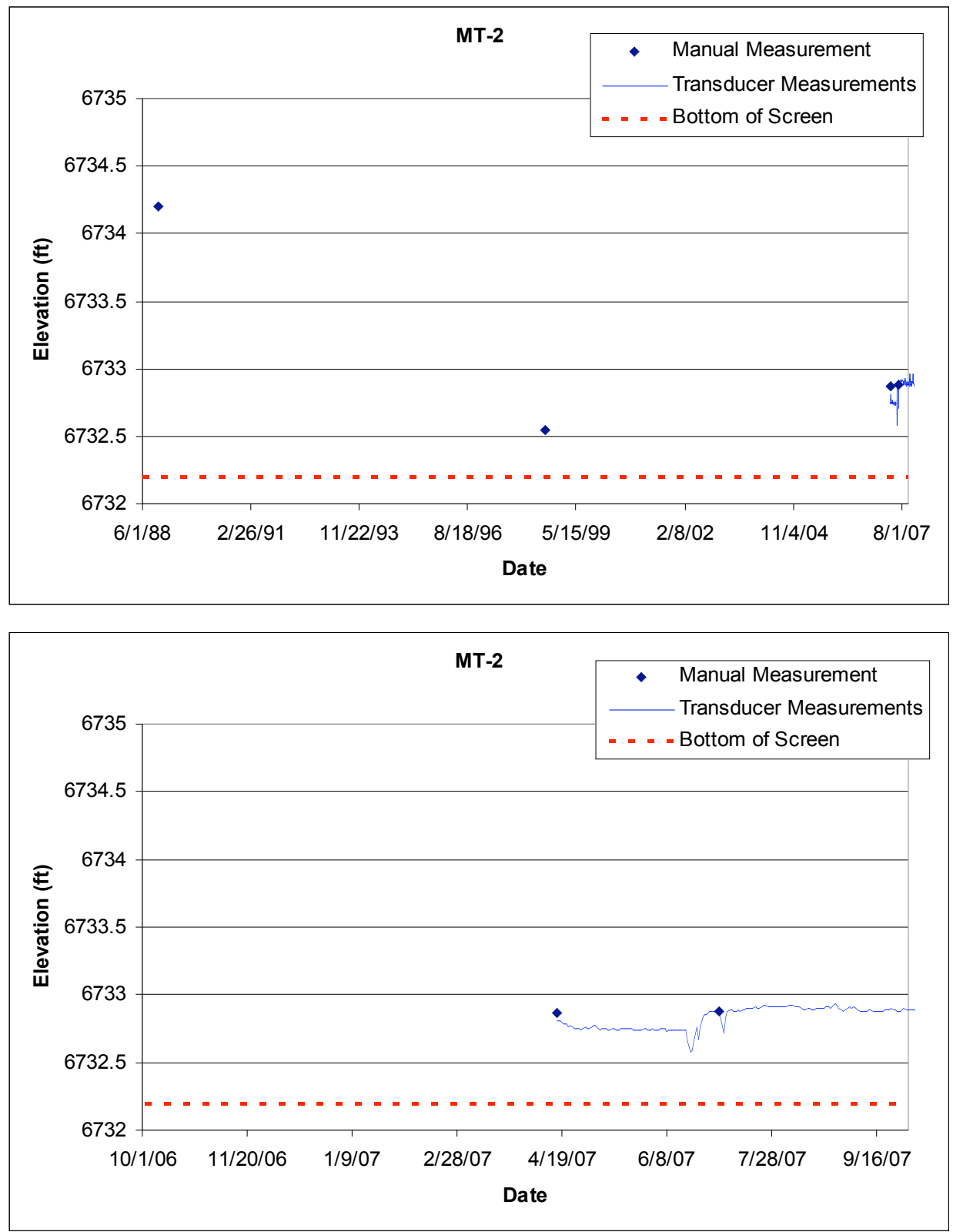


\subsection{MT-3}

Location: Middle Mortandad Canyon, down canyon of sediment traps, approximately 0.12 miles east of MT-1 and approximately $50 \mathrm{ft}$ north of MT-2.

Period of Record: November 1, 1988, through September 30, 2007

Remarks: Bottom of screen elevation is $6732.7 \mathrm{ft}$.
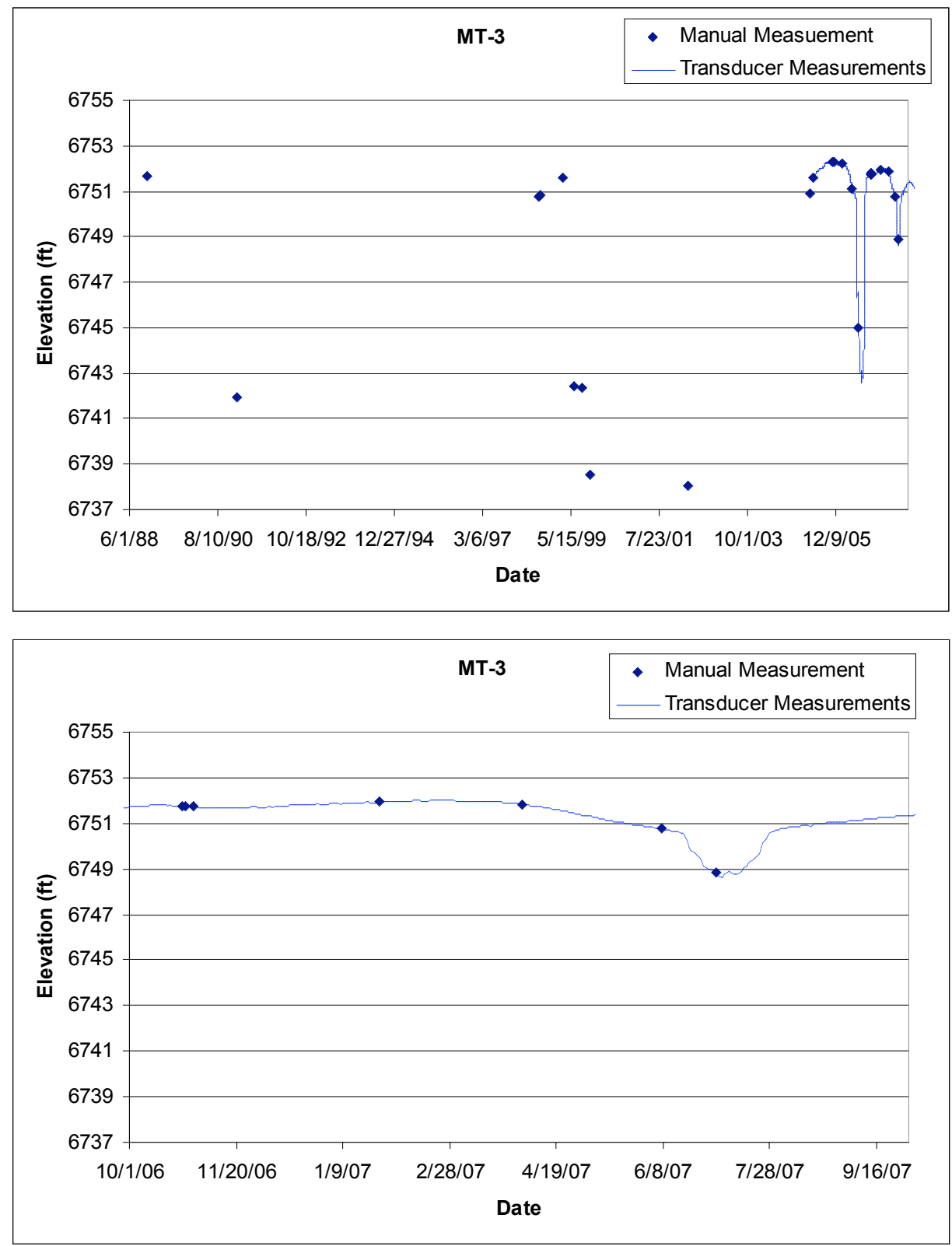


\subsection{MT-4}

Location: Middle Mortandad Canyon, down canyon of the sediment traps, approximately $525 \mathrm{ft}$ east of MT-3.

Period of Record: November 1, 1988, through September 30, 2007

Remarks: Bottom of screen elevation is $6719.59 \mathrm{ft}$.
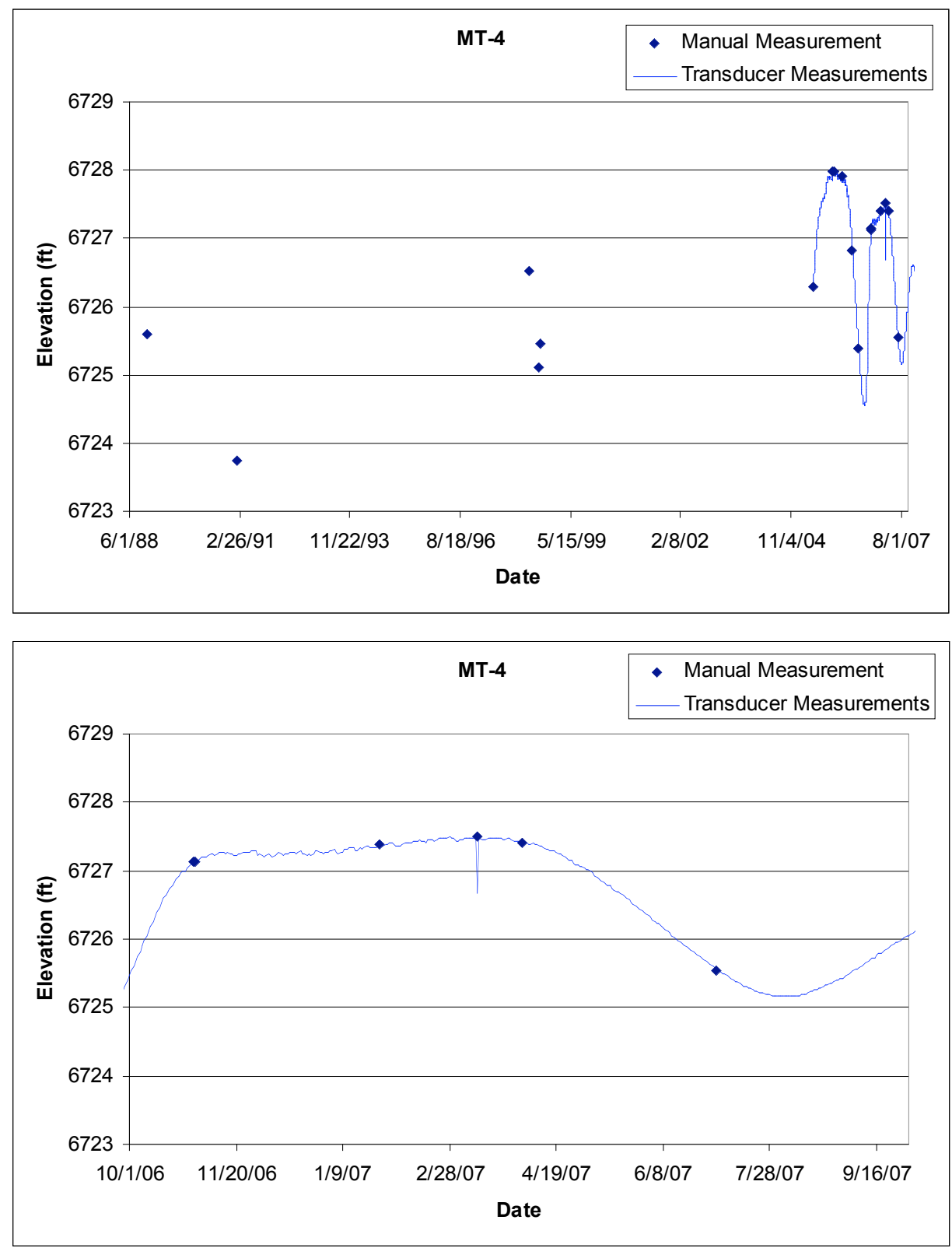


\subsection{PAO-1}

Location: Upper Pueblo Canyon, approximately $1000 \mathrm{ft}$ west of the confluence with Acid Canyon. Period of Record: October 29,1998, through September 30, 2007

Remarks: The transducer batteries failed on December 3, 2006, possibly due to cold conditions, and were replaced on February 27, 2007.
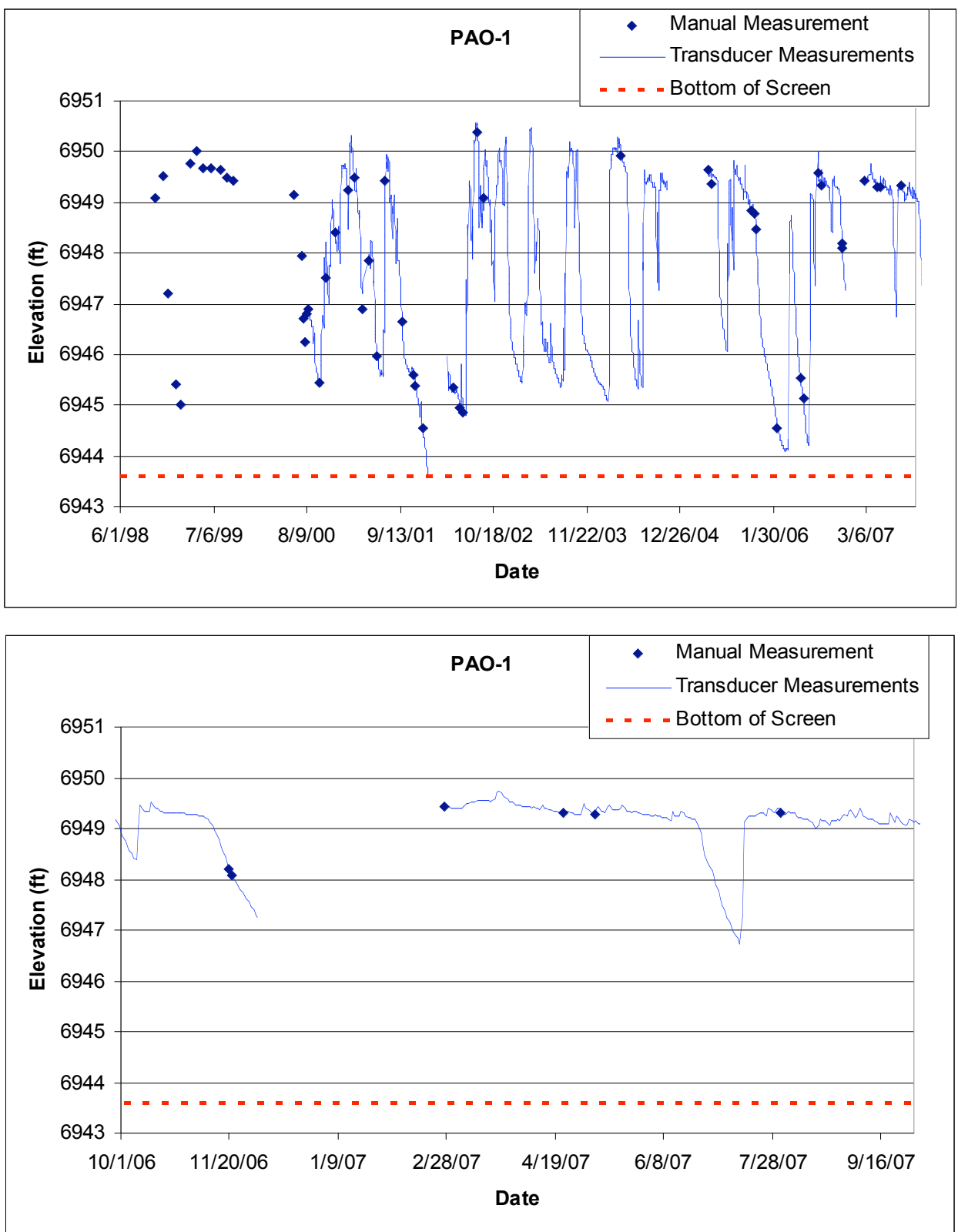


\subsection{PAO-2}

Location: Upper Pueblo Canyon, approximately $500 \mathrm{ft}$ east of the Acid Canyon confluence.

Period of Record: November 30, 1998, through September 30, 2007

Remarks: The water level dropped below the bottom of the screen November 15, 2006, to February 10, 2007, and July 5, 2007, to July 14, 2007.
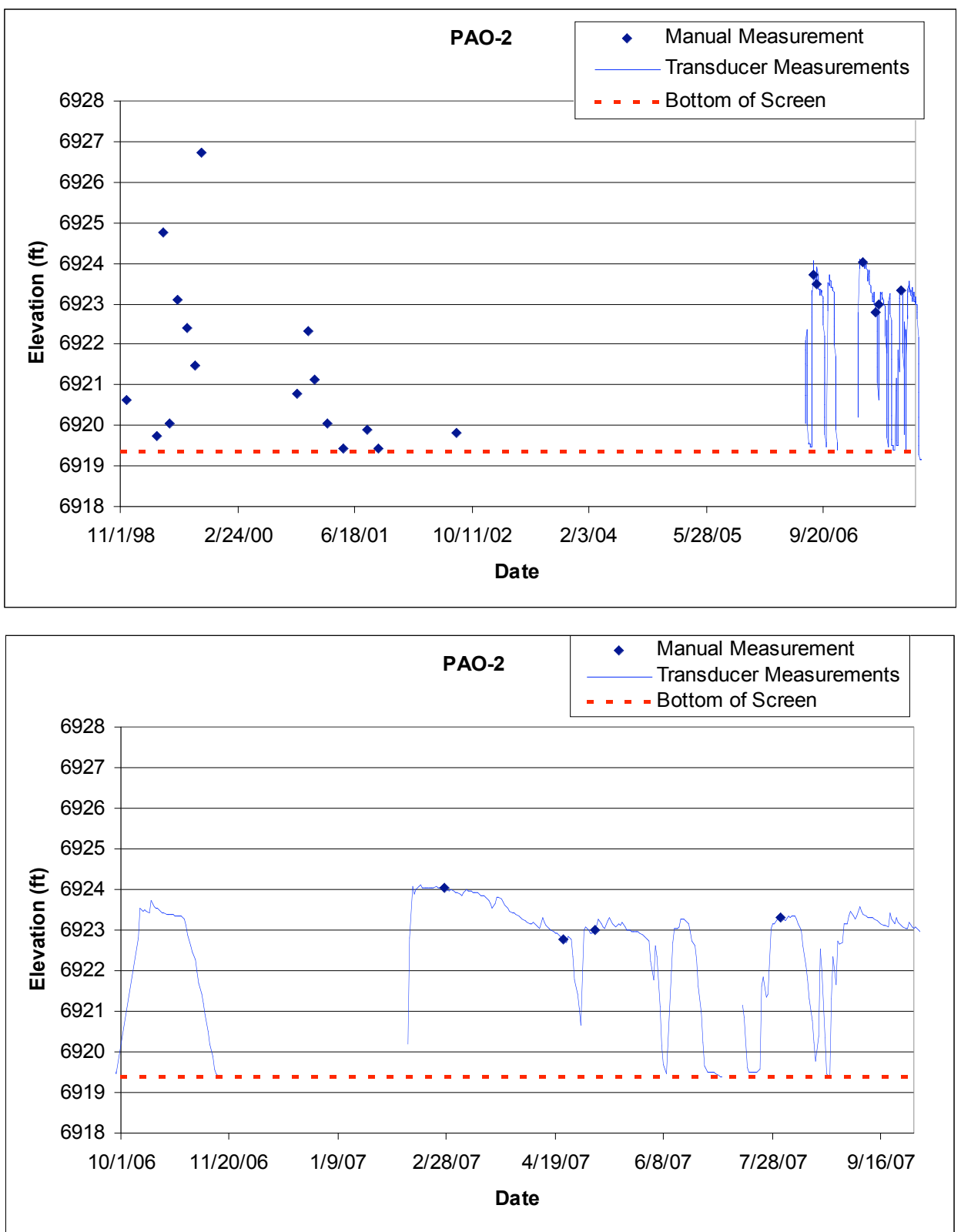


\subsection{PAO-4}

Location: Lower Pueblo Canyon, approximately $3100 \mathrm{ft}$ southeast of the old Los Alamos County Sewage Treatment Plant location.

Period of Record: July 24, 1997, through September 30, 2007

Remarks: The manual measurement taken on May 9, 2005, represents drawdown during a sampling event.
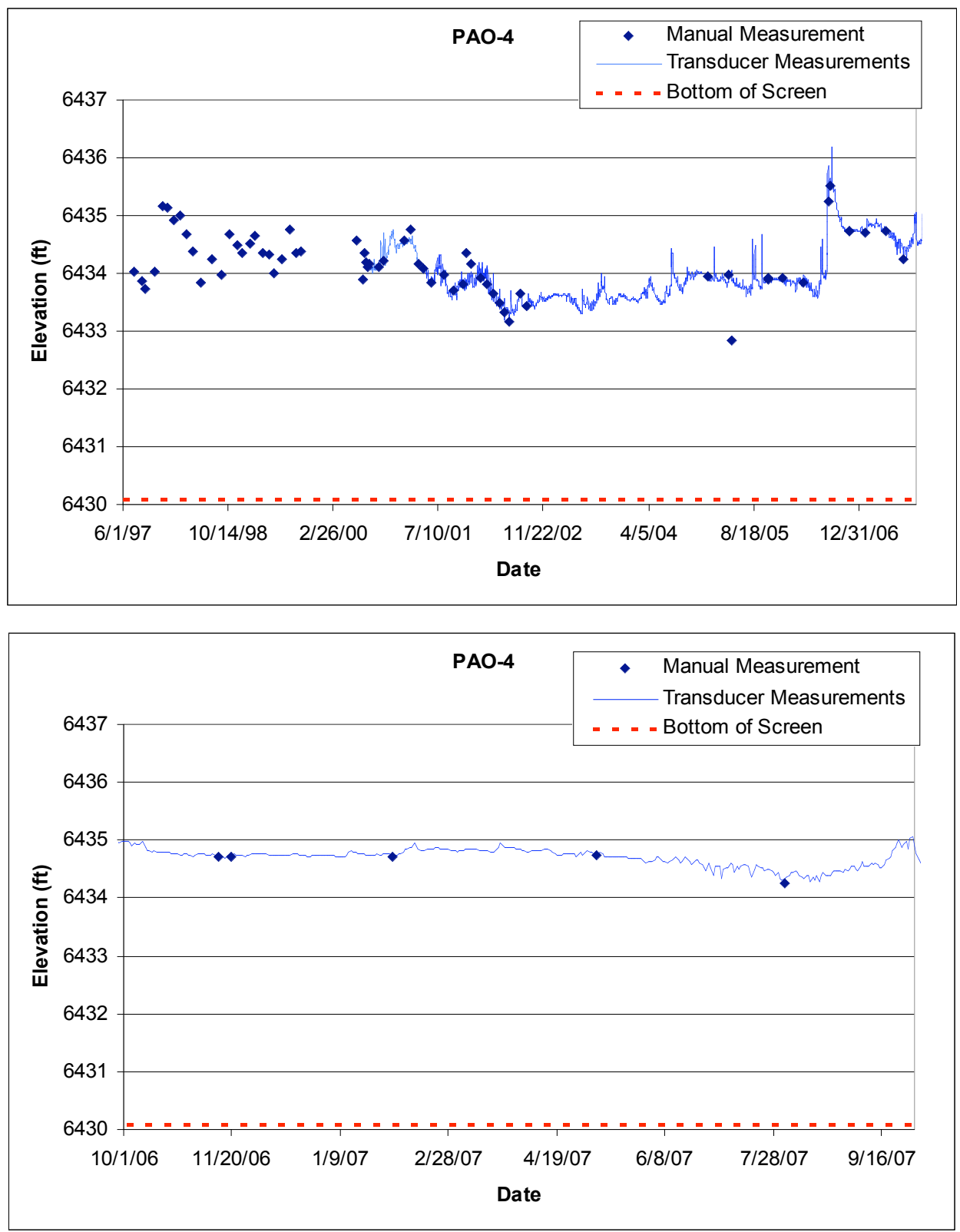


\subsection{PCO-1}

Location: Pajarito Canyon, approximately $200 \mathrm{ft}$ north of R-20.

Period of Record: June 11, 1985, through September 30, 2007

Remarks: Bottom of screen elevation is $6674.7 \mathrm{ft}$.
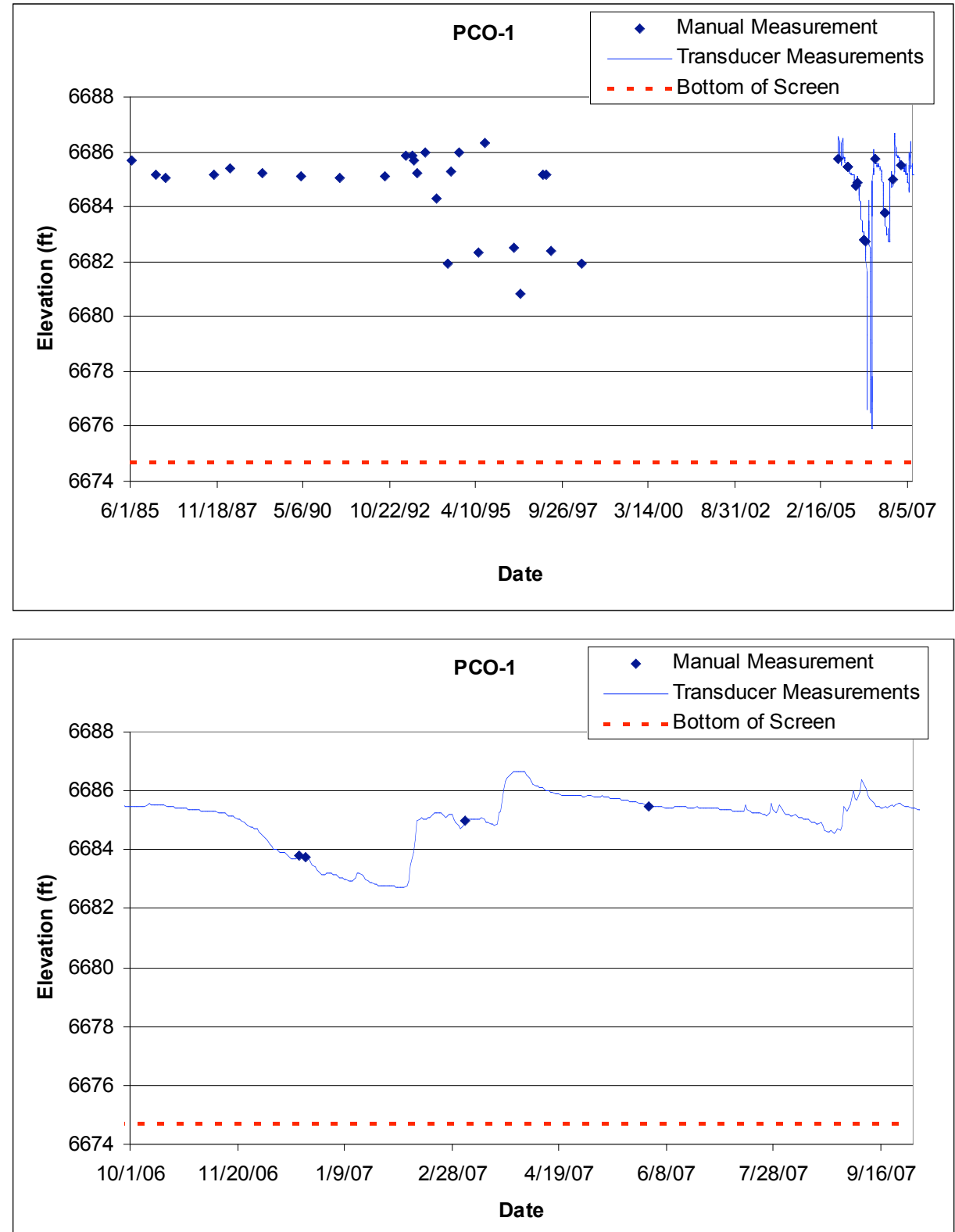


\subsection{PCO-2}

Location: Pajarito Canyon, approximately 0.1 miles east of R-32.

Period of Record: June 11, 1985, through September 30, 2007

Remarks: None.
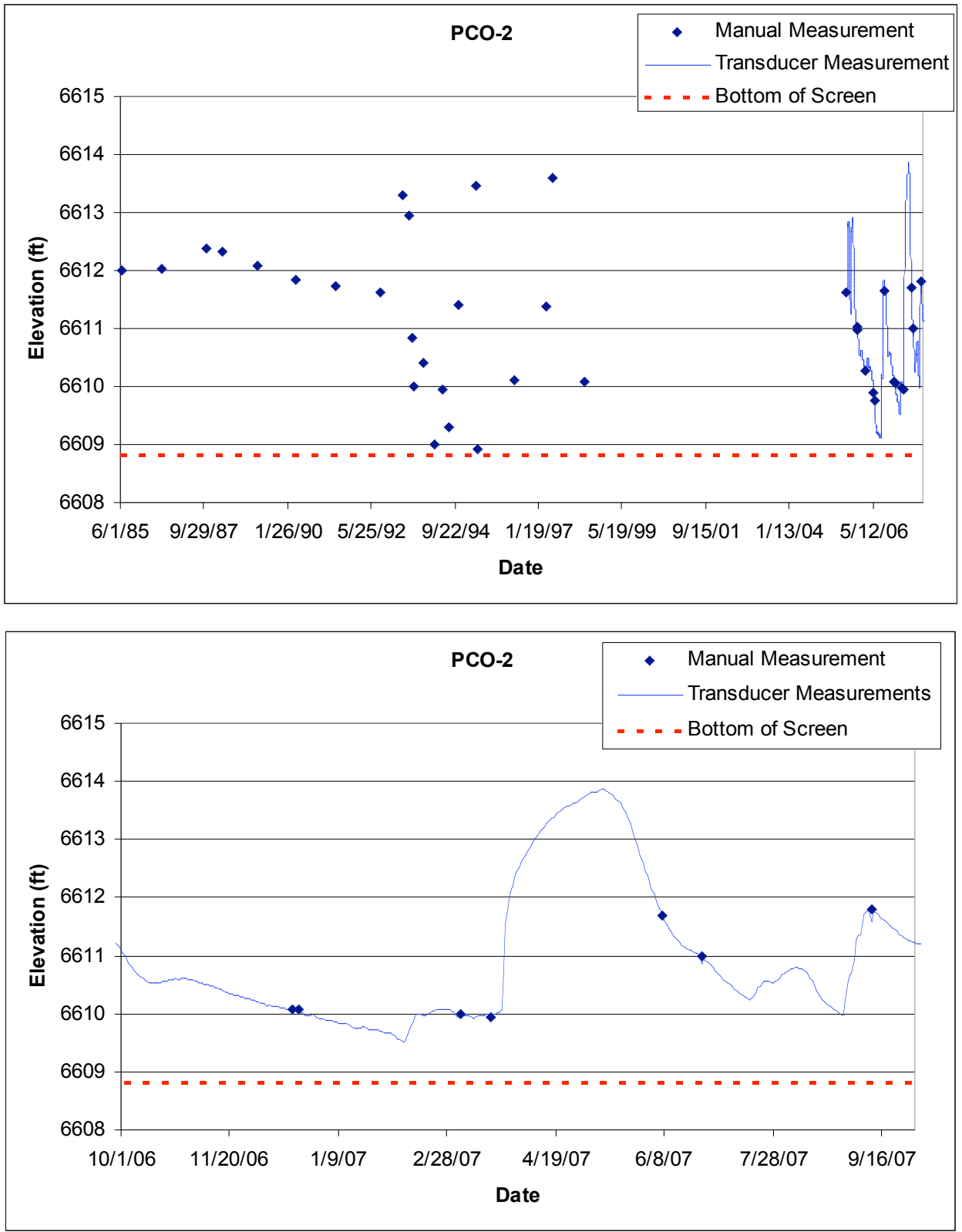


\subsection{PCO-3}

Location: Pajarito Canyon, approximately 1 mile east of R-32, in wetlands on the south side of the road.

Period of Record: June 11, 1985, through September 30, 2007

Remarks: Bottom of screen elevation is $6529.3 \mathrm{ft}$. The water level was below the bottom of the screen from July 2, 2007, to September 3, 2007.
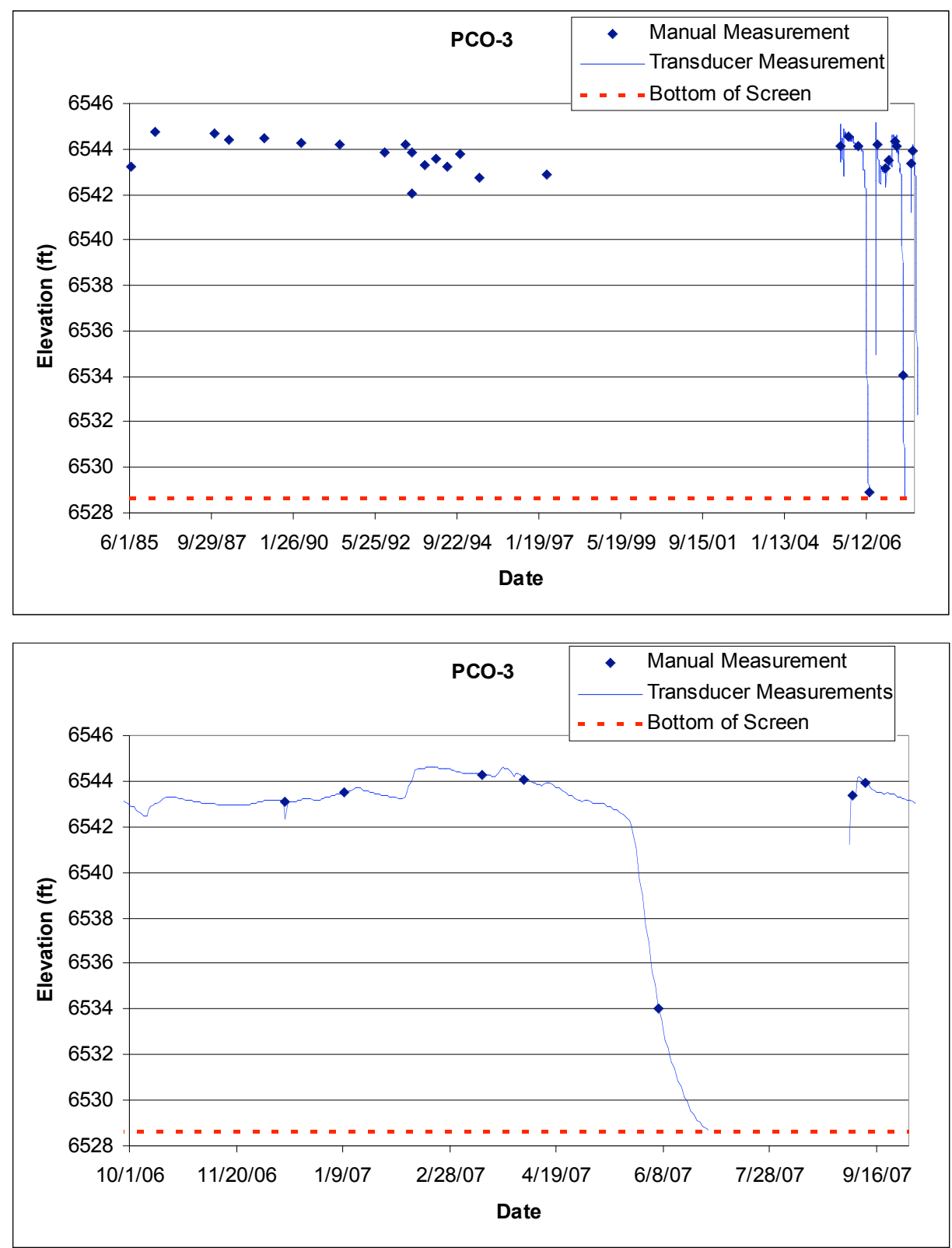


\subsection{SCA-1}

Location: Upper Sandia Canyon, in the wetlands approximately $350 \mathrm{ft}$ upstream from gaging station E123.

Period of Record: October 13, 2006, through September 30, 2007

Remarks: SCA-1 is a shallow alluvial well located in a wetland. A transducer was installed on October 13, 2006. The screen may be clogged with organic material inhibiting the equilibration of the water level.

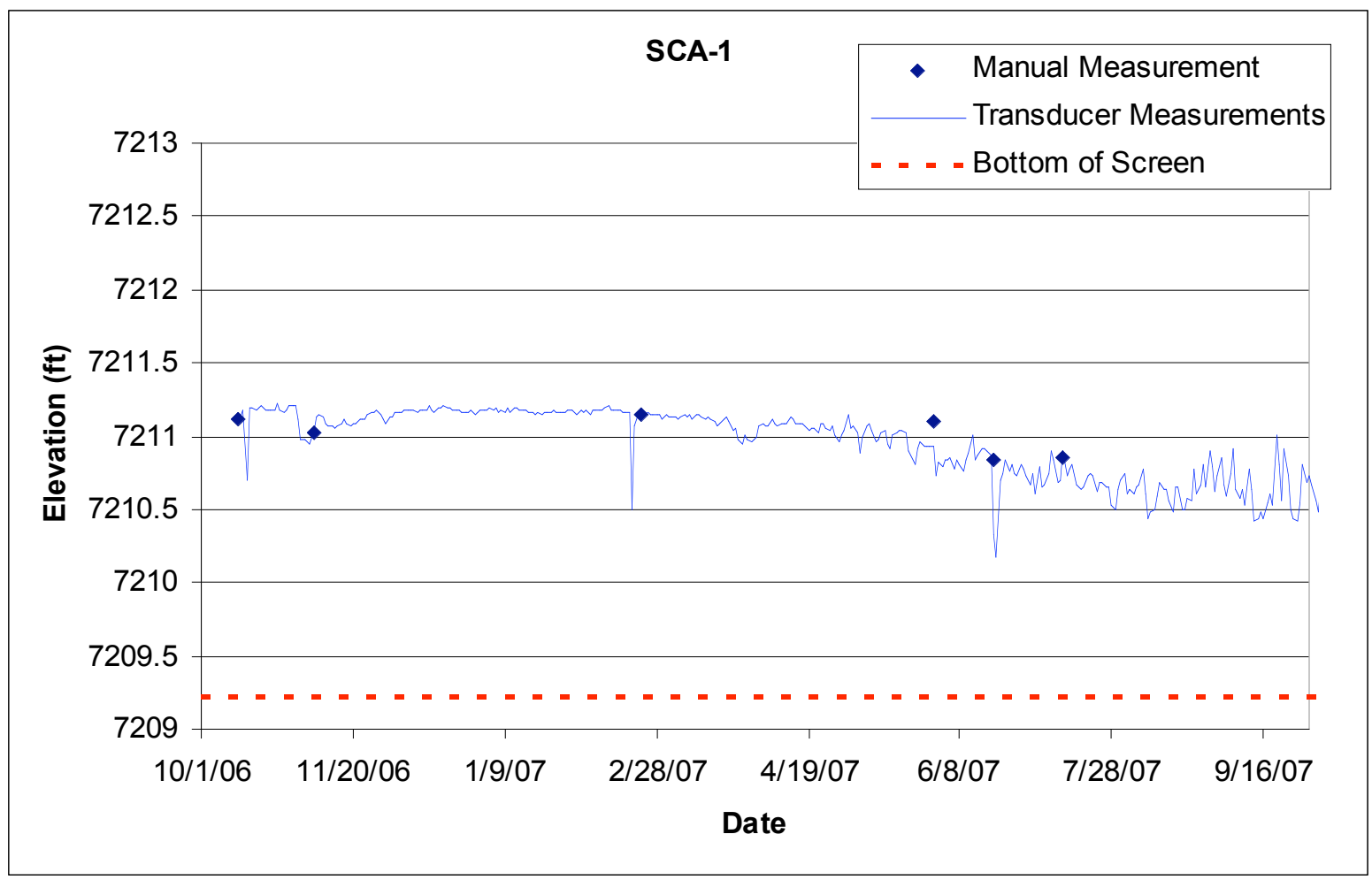




\subsection{SCA-2}

Location: Middle Sandia Canyon, approximately $700 \mathrm{ft}$ upstream of gaging station E124.

Period of Record: October 13, 2006, through September 30, 2007

Remarks: SCA-2 responds to the sewer treatment plant discharge in upper Sandia Canyon.

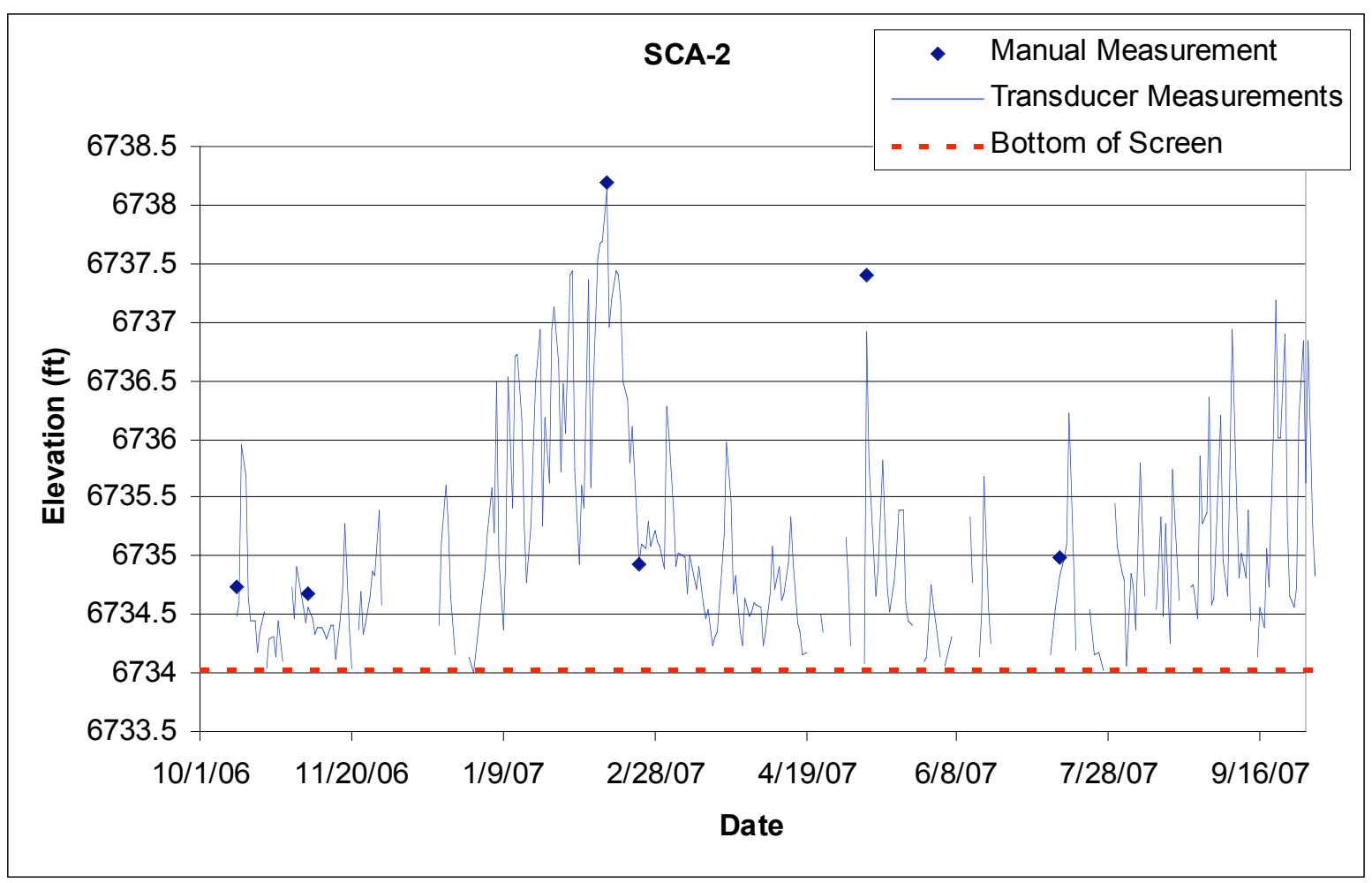




\subsection{SCA-3}

Location: Middle Sandia Canyon, approximately $700 \mathrm{ft}$ downstream of gaging station E124.

Period of Record: October 13, 2006, through November 30, 2007

Remarks: A transducer was installed in SCA-3 on October 13, 2006. The well has been dry or there has been water in the sump since installation of the transducer.

\begin{tabular}{|c|c|c|c|}
\hline Well Name & Date Time & Water Level (ft) & Comments \\
\hline SCA-3 & 10/13/2006 12:33:00 PM & 6690.77 & Water is in sump \\
\hline SCA-3 & 10/13/2006 12:41:00 PM & 6690.83 & Water is in sump \\
\hline SCA-3 & 11/6/2006 1:33:00 PM & 6690.82 & Water is in sump \\
\hline SCA-3 & 11/13/2006 10:12:00 AM & 6690.8 & Water is in sump \\
\hline SCA-3 & 2/12/2007 10:11:00 AM & & Dry \\
\hline SCA-3 & 2/23/2007 11:07:00 AM & 6690.82 & Water is in sump \\
\hline SCA-3 & 5/8/2007 8:35:00 AM & 6690.82 & Water is in the sump \\
\hline SCA-3 & 6/14/2007 11:45:00 AM & & Dry \\
\hline SCA-3 & 7/12/2007 10:34:00 AM & 6690.81 & Water is in the sump \\
\hline SCA-3 & 11/12/2007 3:05:00 PM & 6690.81 & Water is in sump \\
\hline SCA-3 & 11/30/2007 11:24:00 AM & 6690.77 & Data are not valid; water is in the sump \\
\hline
\end{tabular}




\subsection{SCA-4}

Location: Middle Sandia Canyon, approximately $700 \mathrm{ft}$ downstream from SCA-3.

Period of Record: October 3, 2006, through September 30, 2007

Remarks: The transducer was installed on October 3,2006 , above the top of the pump at an elevation of $6665.28 \mathrm{ft}$. The pump was removed on October 31, 2006, to allow for complete water level monitoring. The well was dry from January 3, 2007, to February 23, 2007.

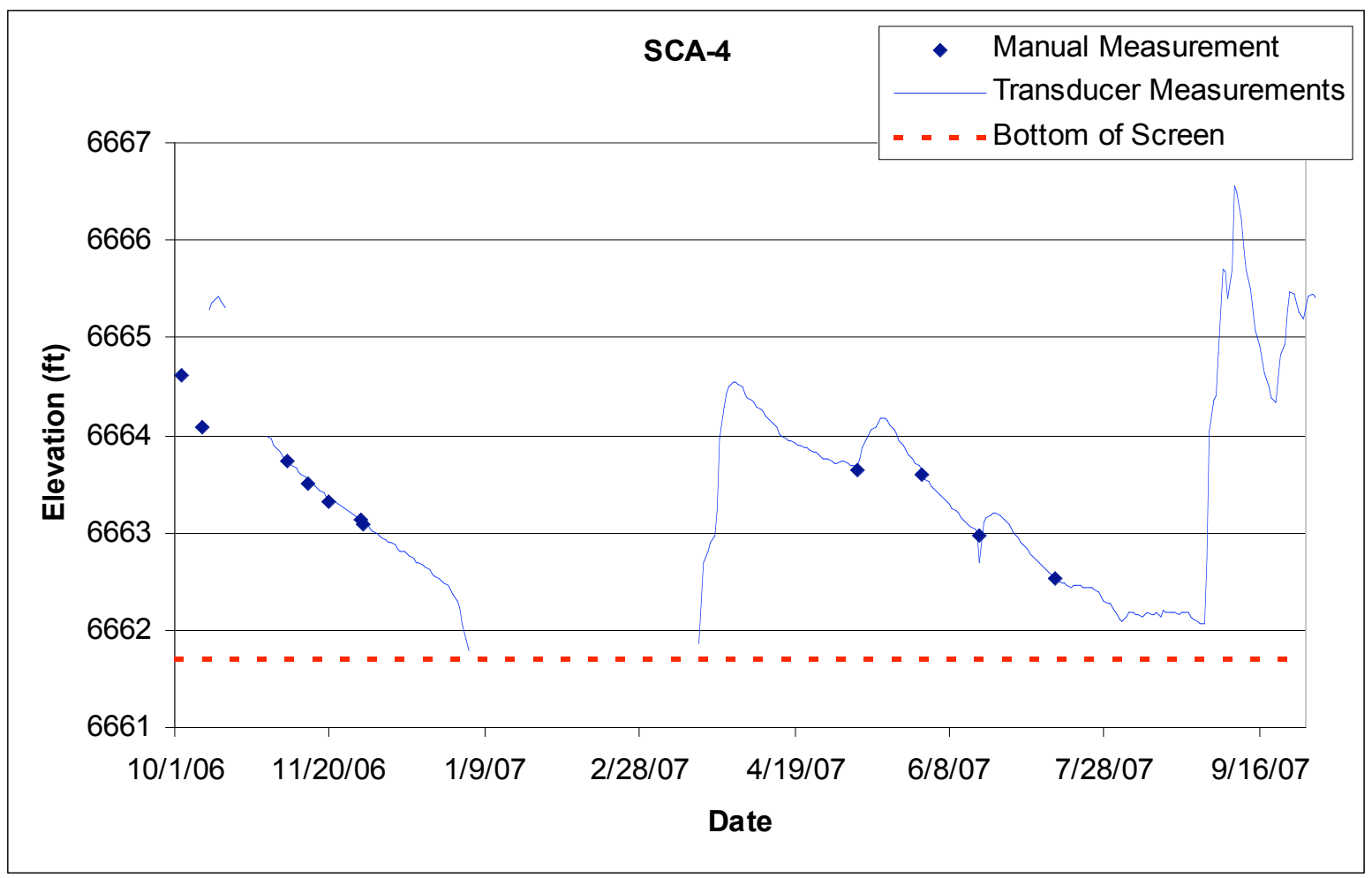




\subsection{SCA-5}

Location: Middle Sandia Canyon, approximately $650 \mathrm{ft}$ upstream from the firing range at TA-72 and about $325 \mathrm{ft}$ north of R-11.

Period of Record: October 3, 2006, through September 30, 2007

Remarks: The transducer is installed above the pump in the 2-in. casing. The transducer elevation is $6608.1 \mathrm{ft}$. The transducer data do not represent water levels below $6608.1 \mathrm{ft}$. Bottom of screen elevation is $6604.62 \mathrm{ft}$.

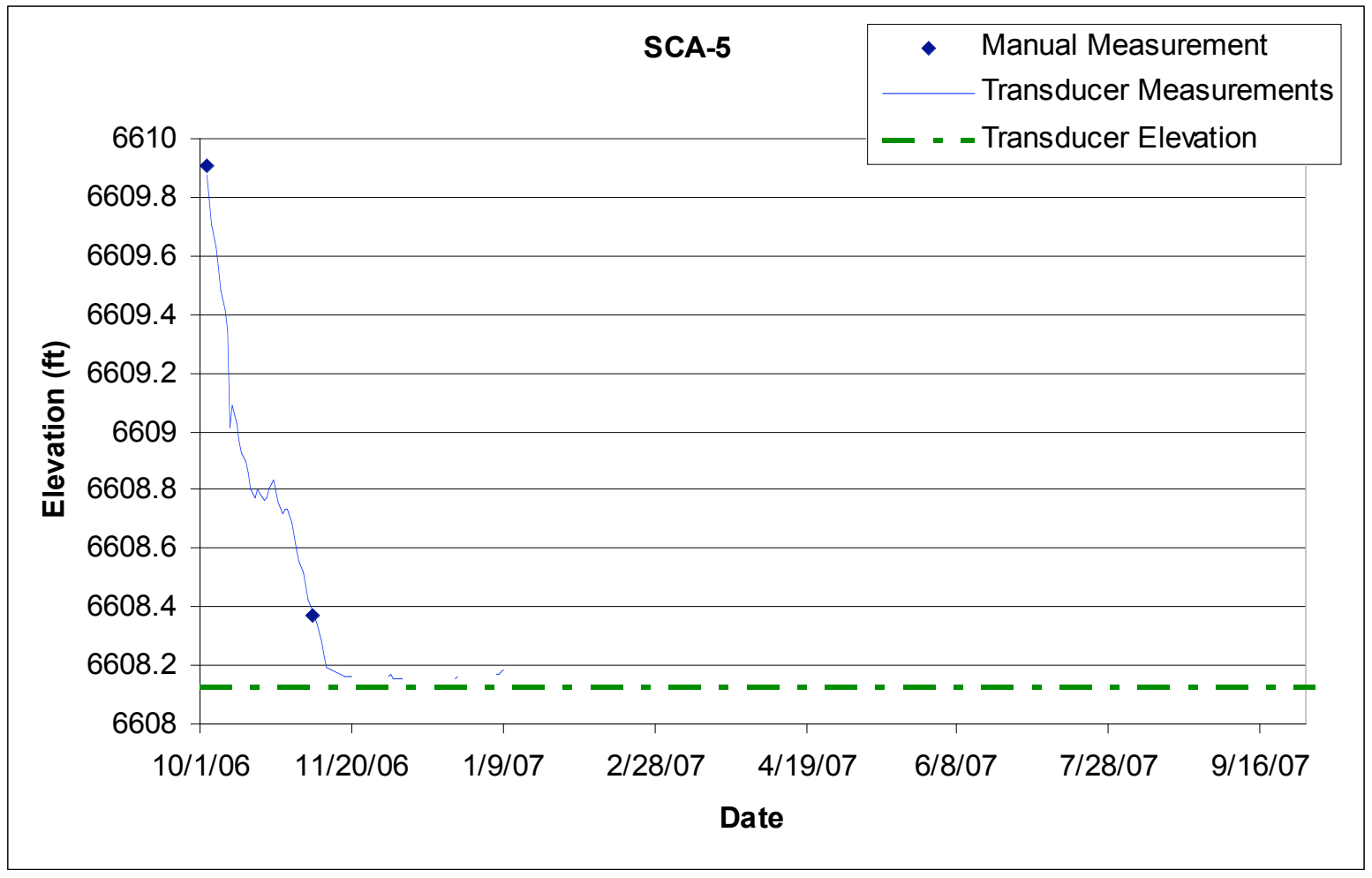




\subsection{SCO-1}

Location: Sandia Canyon, approximately 0.1 miles east of R-11.

Period of Record: June 7, 1997, through November 2, 2007

Remarks: No valid data; well has been dry during every measurement event.

\begin{tabular}{|c|c|c|c|}
\hline Well Name & Date Time & Water Level (ft) & Comments \\
\hline SCO-1 & 8/14/1989 12:00:00 PM & & Dry \\
\hline SCO-1 & 6/9/1997 12:00:00 PM & & Dry \\
\hline SCO-1 & 10/13/1997 12:00:00 PM & & Dry \\
\hline SCO-1 & 3/25/1998 12:00:00 PM & & Dry \\
\hline SCO-1 & 5/29/1998 12:00:00 PM & & Dry \\
\hline SCO-1 & 7/28/1998 12:00:00 PM & & Dry \\
\hline SCO-1 & 3/3/1999 12:00:00 PM & & Dry \\
\hline SCO-1 & 6/23/1999 12:00:00 PM & & Dry \\
\hline SCO-1 & 8/30/1999 12:00:00 PM & & Dry \\
\hline SCO-1 & 11/15/1999 12:00:00 PM & & Dry \\
\hline SCO-1 & 3/26/2000 12:00:00 PM & & Dry \\
\hline SCO-1 & 5/16/2000 12:00:00 PM & & Dry \\
\hline SCO-1 & 8/30/2000 12:00:00 PM & & Dry \\
\hline SCO-1 & 10/8/2000 12:00:00 PM & & Dry \\
\hline SCO-1 & 7/2/2001 12:00:00 PM & & Dry \\
\hline SCO-1 & 8/22/2001 12:00:00 PM & & Dry \\
\hline SCO-1 & 10/18/2001 12:00:00 PM & & Dry \\
\hline SCO-1 & 1/27/2002 12:00:00 PM & & Dry \\
\hline SCO-1 & 4/19/2002 12:00:00 PM & & Dry \\
\hline SCO-1 & 8/27/2002 12:00:00 PM & & Dry \\
\hline SCO-1 & 2/19/2003 12:00:00 PM & & Dry \\
\hline SCO-1 & 5/18/2003 12:00:00 PM & & Dry \\
\hline SCO-1 & 2/28/2005 1:00:00 PM & & Dry \\
\hline SCO-1 & 6/7/2005 12:00:00 PM & & Dry \\
\hline SCO-1 & 6/14/2005 1:40:00 PM & & Dry \\
\hline SCO-1 & 12/8/2005 10:30:00 AM & & Dry \\
\hline SCO-1 & 10/18/2005 8:08:00 AM & & Dry \\
\hline SCO-1 & 3/7/2006 9:36:00 AM & & Dry \\
\hline SCO-1 & 6/13/2006 8:32:00 AM & & Dry, $T D=20.44 \mathrm{ft}$ \\
\hline SCO-1 & 8/28/2006 3:15:00 PM & & Dry \\
\hline SCO-1 & 9/7/2006 9:05:00 AM & & Dry \\
\hline SCO-1 & 10/3/2006 8:35:00 AM & & Dry \\
\hline
\end{tabular}




\begin{tabular}{|l|r||l|l|}
\hline Well Name & \multicolumn{1}{|c|}{ Date Time } & Water Level (ft) & \multicolumn{1}{|c|}{ Comments } \\
\hline SCO-1 & \multicolumn{1}{|c|}{$12 / 8 / 2006$ 2:01:00 PM } & & Dry \\
\hline SCO-1 & $2 / 12 / 2007$ 10:44:00 AM & & Dry \\
\hline SCO-1 & $3 / 13 / 2007$ 12:17:00 PM & Dry \\
\hline SCO-1 & $6 / 7 / 2007$ 9:46:00 AM & Dry \\
\hline SCO-1 & $6 / 12 / 2007$ 7:20:00 AM & Dry \\
\hline SCO-1 & $9 / 5 / 20072: 37: 00$ PM & Dry \\
\hline SCO-1 & $11 / 12 / 2007$ 12:20:00 PM & Dry \\
\hline
\end{tabular}

\subsection{SCO-2}

Location: Sandia Canyon, approximately $300 \mathrm{ft}$ west of R-12.

Period of Record: June 9, 1997, through November 12, 2007

Remarks: No valid data; well has been dry during every measurement event.

\begin{tabular}{|c|c|c|c|}
\hline Well Name & Date Time & Water Level (ft) & Comments \\
\hline SCO-2 & 8/16/1989 12:00:00 PM & & Dry \\
\hline SCO-2 & 6/9/1997 12:00:00 PM & & Dry \\
\hline SCO-2 & 10/13/1997 12:00:00 PM & & Dry \\
\hline SCO-2 & 3/25/1998 12:00:00 PM & & Dry \\
\hline SCO-2 & 5/29/1998 12:00:00 PM & & Dry \\
\hline SCO-2 & 7/28/1998 12:00:00 PM & & Dry \\
\hline SCO-2 & 3/3/1999 12:00:00 PM & & Dry \\
\hline SCO-2 & 6/23/1999 12:00:00 PM & & Dry \\
\hline SCO-2 & 8/30/1999 12:00:00 PM & & Dry \\
\hline SCO-2 & 11/15/1999 12:00:00 PM & & Dry \\
\hline SCO-2 & 3/26/2000 12:00:00 PM & & Dry \\
\hline SCO-2 & 5/16/2000 12:00:00 PM & & Dry \\
\hline SCO-2 & 8/30/2000 12:00:00 PM & & Dry \\
\hline SCO-2 & 10/8/2000 12:00:00 PM & & Dry \\
\hline SCO-2 & 7/2/2001 12:00:00 PM & & Dry \\
\hline SCO-2 & 8/22/2001 12:00:00 PM & & Dry \\
\hline SCO-2 & 10/18/2001 12:00:00 PM & & Dry \\
\hline SCO-2 & 4/19/2002 12:00:00 PM & & Dry \\
\hline SCO-2 & 8/27/2002 12:00:00 PM & & Dry \\
\hline SCO-2 & 10/27/2002 12:00:00 PM & & Dry \\
\hline SCO-2 & 2/19/2003 12:00:00 PM & & Dry \\
\hline SCO-2 & 5/18/2003 12:00:00 PM & & Dry \\
\hline SCO-2 & 6/7/2005 12:00:00 PM & & Dry \\
\hline SCO-2 & 6/14/2005 1:30:00 PM & & Dry \\
\hline
\end{tabular}




\begin{tabular}{|c|c|c|c|}
\hline Well Name & Date Time & Water Level (ft) & Comments \\
\hline SCO-2 & 12/8/2005 10:00:00 AM & & Dry \\
\hline SCO-2 & 10/18/2005 7:40:00 AM & & Dry \\
\hline SCO-2 & 3/7/2006 9:55:00 AM & & Dry \\
\hline SCO-2 & 6/13/2006 8:14:00 AM & & Dry, $T D=19.16 \mathrm{ft}$ \\
\hline SCO-2 & 8/28/2006 3:00:00 PM & & Dry \\
\hline SCO-2 & 9/7/2006 8:48:00 AM & & Dry \\
\hline SCO-2 & 10/3/2006 8:50:00 AM & & Dry \\
\hline SCO-2 & 12/8/2006 1:50:00 PM & & Dry \\
\hline SCO-2 & 2/12/2007 11:22:00 AM & & Dry \\
\hline SCO-2 & 3/13/2007 12:32:00 PM & & Dry \\
\hline SCO-2 & 6/7/2007 9:11:00 AM & & Dry \\
\hline SCO-2 & 6/12/2007 7:35:00 AM & & Dry \\
\hline SCO-2 & 9/5/2007 2:26:00 PM & & Dry \\
\hline SCO-2 & 11/12/2007 12:05:00 PM & & Dry \\
\hline
\end{tabular}




\subsection{SCP-1abc}

Location: Middle Sandia Canyon, approximately $5 \mathrm{ft}$ west of SCA-4.

Period of Record: October 13, 2006, through September 30, 2007

Remarks: SCP-1abc is a triple-nested piezometer. The bottom of screen 1a elevation is $6664.90 \mathrm{ft}$, the bottom of screen $1 \mathrm{~b}$ elevation is $6663.34 \mathrm{ft}$, and the bottom of screen 1c elevation is $6661.54 \mathrm{ft}$.

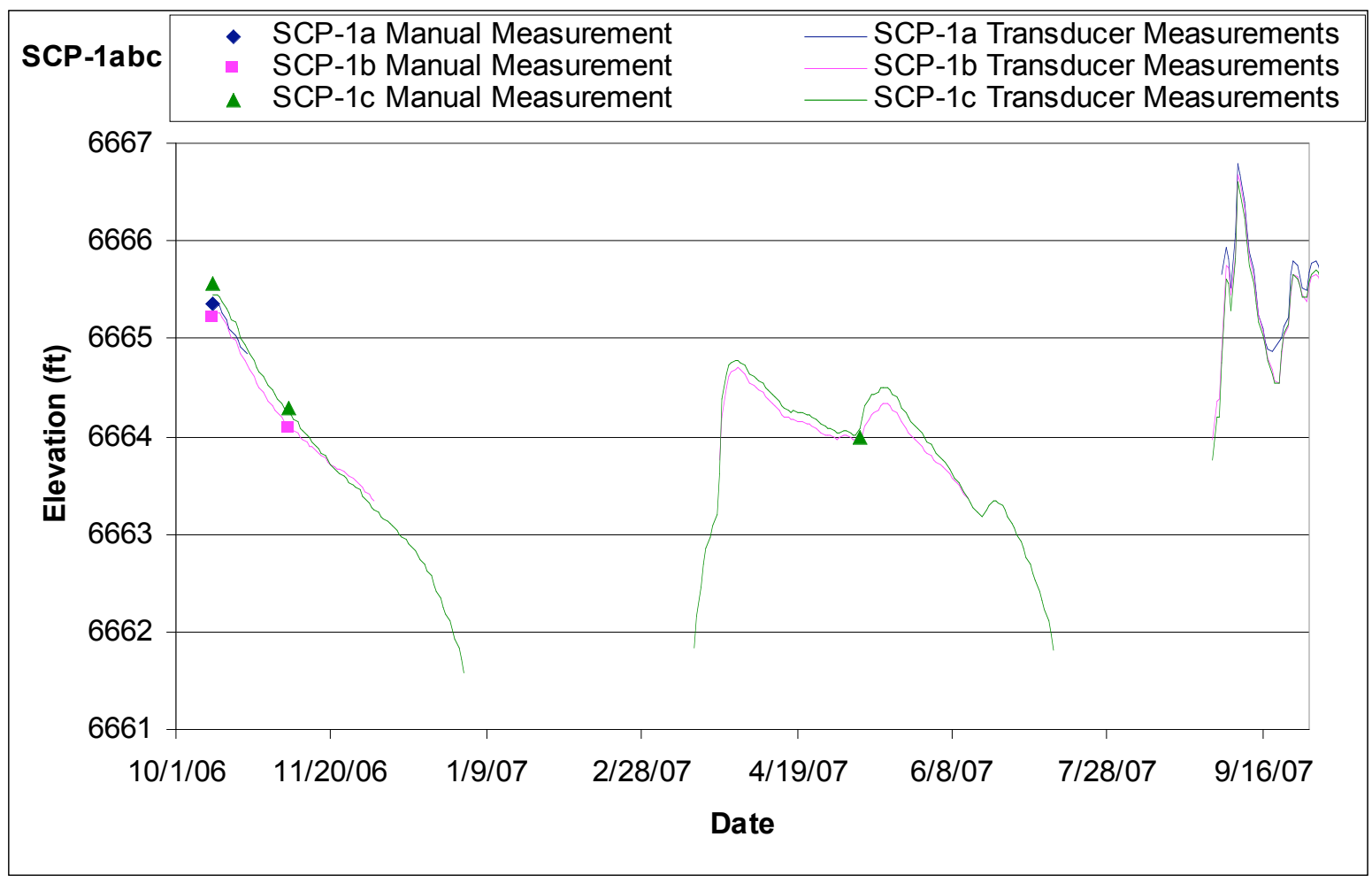




\subsection{SCP-2a}

Location: Middle Sandia Canyon, approximately $10 \mathrm{ft}$ east of SCA-3 and $5 \mathrm{ft}$ east of SCP-2b.

Period of Record: October 13, 2006, through September 30, 2007

Remarks: Screen bottom elevation is $6677.6 \mathrm{ft}$.
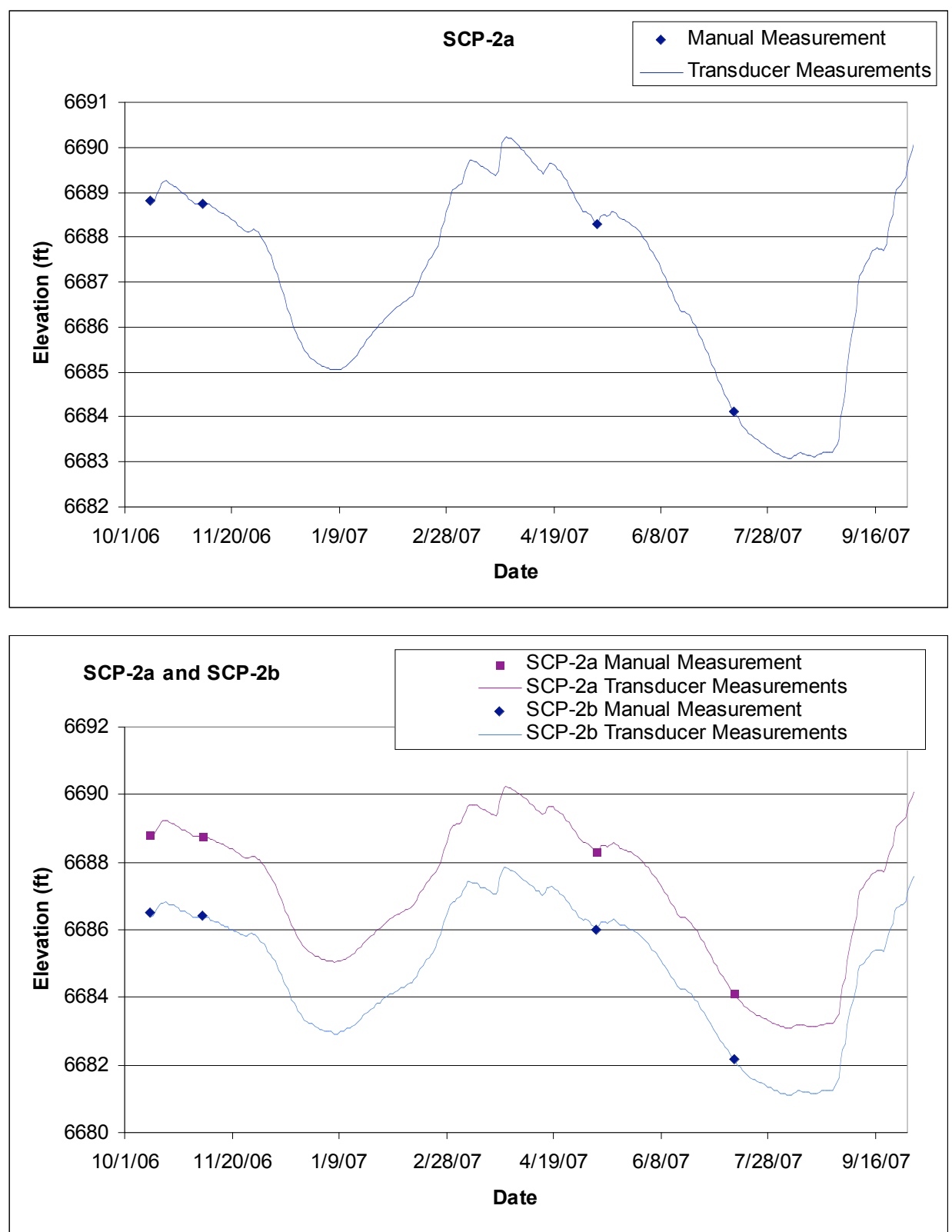


\subsection{SCP-2b}

Location: Middle Sandia Canyon, approximately $5 \mathrm{ft}$ east of SCA-3 and $5 \mathrm{ft}$ west of SCP-2a.

Period of Record: October 13, 2006, through September 30, 2007

Remarks: Bottom of screen elevation is $6672.5 \mathrm{ft}$.
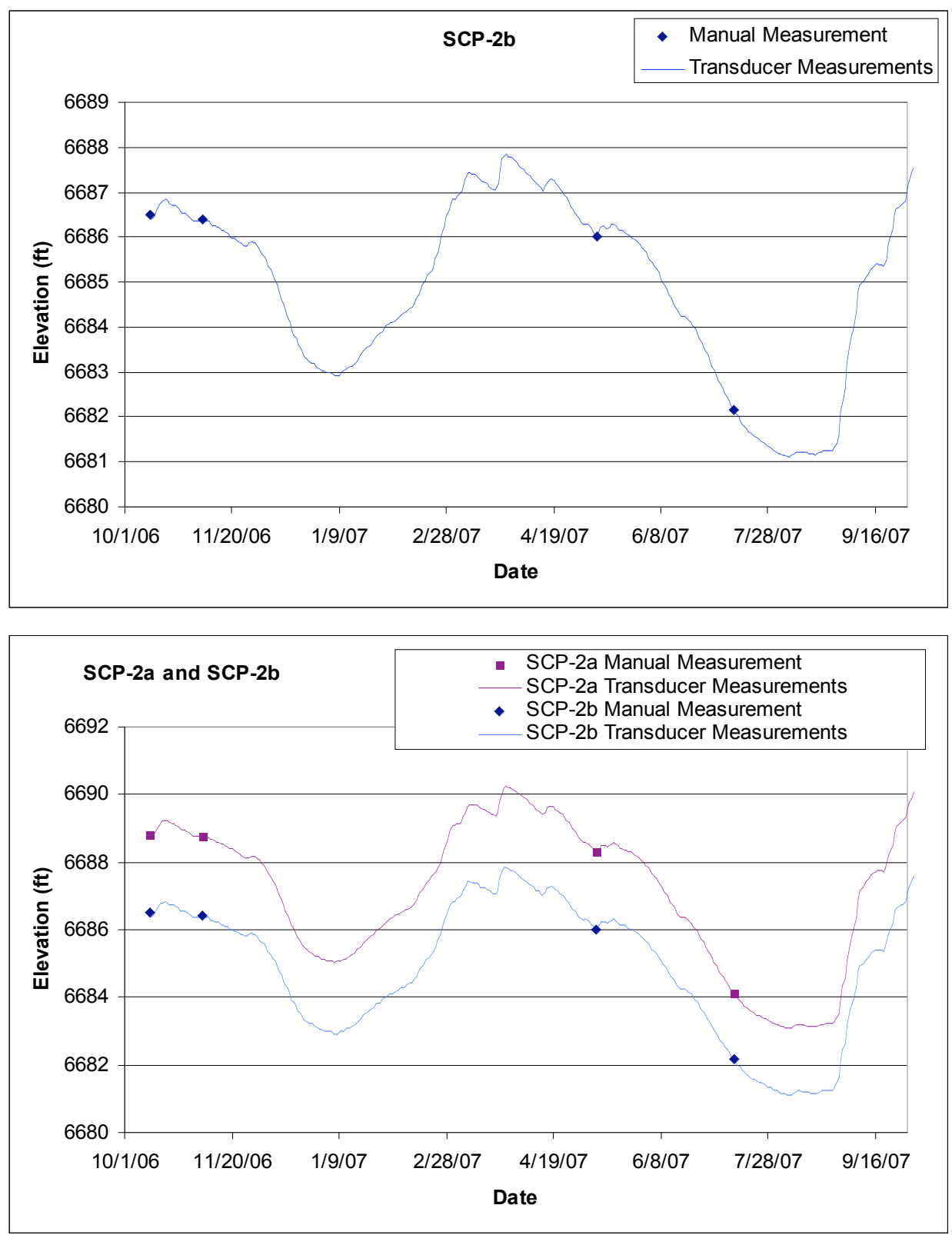


\subsection{TSCA-6}

Location: Ten Site Canyon, approximately $600 \mathrm{ft}$ west of Mortandad Canyon confluence.

Period of Record: April 18, 2005, through September 30, 2007

Remarks: TSCA-6 was dry from November 4, 2006, to the end of the period of record.
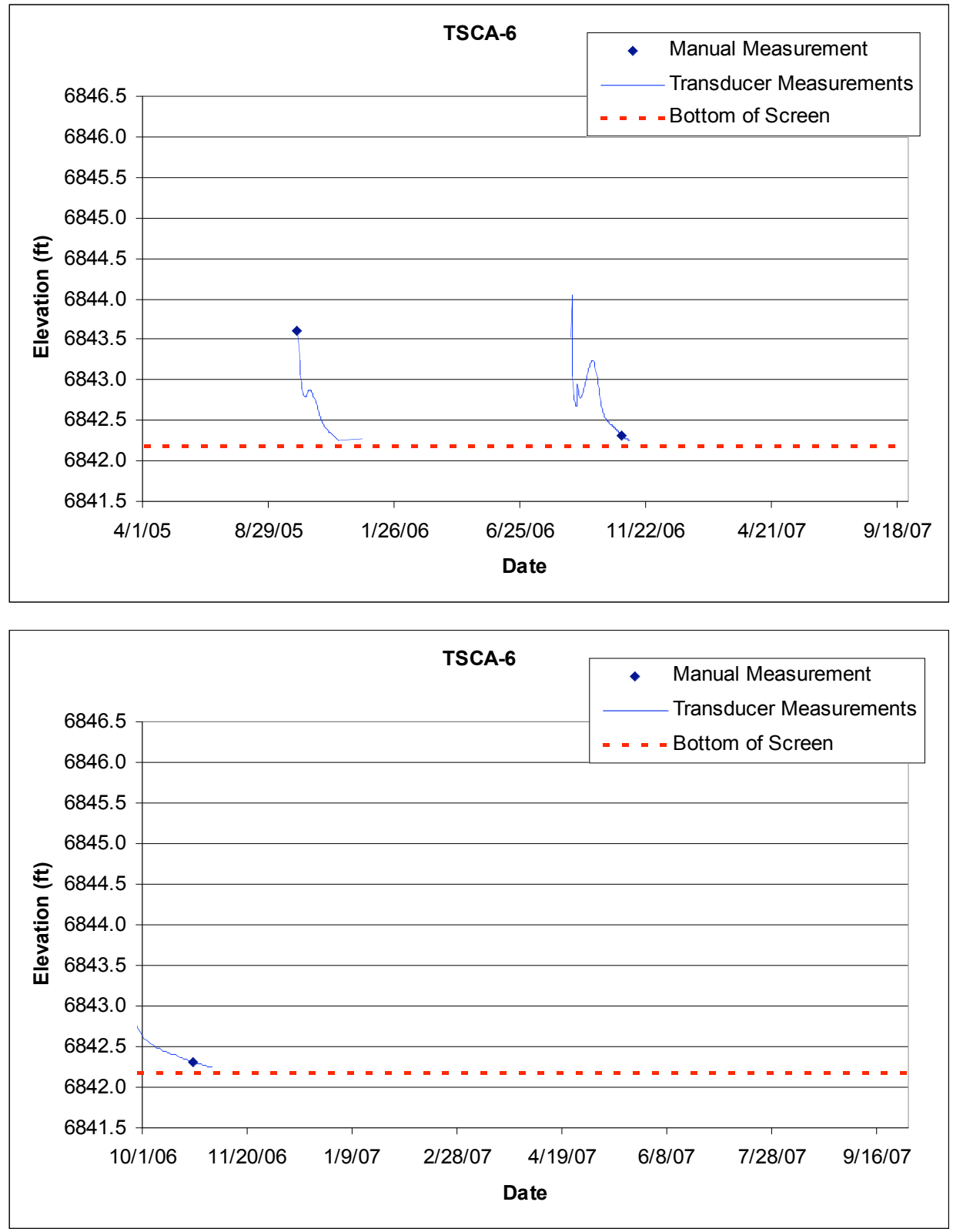


\subsection{TSWB-6}

Location: Ten Site Canyon, approximately $300 \mathrm{ft}$ west of Mortandad Canyon confluence.

Period of Record: January 9, 1995, through November 1, 2007

Remarks: No valid groundwater level data exist for TSWB-6. Well has been dry during every measurement event. A transducer has been installed since July 5, 2006.

\begin{tabular}{|c|c|c|c|}
\hline Well Name & Date Time & Water Level (ft)| & Comments \\
\hline TSWB-6 & $1 / 9 / 1995$ & & Dry \\
\hline TSWB-6 & 9/18/1995 & & Dry \\
\hline TSWB-6 & $11 / 13 / 1996$ & & Dry \\
\hline TSWB-6 & 6/25/1997 12:00:00 PM & & Dry \\
\hline TSWB-6 & 7/7/1997 12:00:00 PM & & Dry \\
\hline TSWB-6 & 2/9/2000 2:07:00 PM & & Dry \\
\hline TSWB-6 & 3/27/2000 12:00:00 PM & & Dry \\
\hline TSWB-6 & 6/23/2000 12:00:00 PM & & Dry \\
\hline TSWB-6 & 9/28/2000 12:00:00 PM & & Dry \\
\hline TSWB-6 & 11/15/2000 12:00:00 PM & & Dry \\
\hline TSWB-6 & 7/2/2001 12:00:00 PM & & Dry \\
\hline TSWB-6 & 8/22/2001 12:00:00 PM & & Dry \\
\hline TSWB-6 & 10/18/2001 12:00:00 PM & & Dry \\
\hline TSWB-6 & 4/17/2002 12:00:00 PM & & Dry \\
\hline TSWB-6 & 8/19/2002 12:00:00 PM & & Dry \\
\hline TSWB-6 & 11/14/2002 12:00:00 PM & & Dry \\
\hline TSWB-6 & 2/21/2003 12:00:00 PM & & Dry \\
\hline TSWB-6 & 6/11/2003 12:00:00 PM & & Dry \\
\hline TSWB-6 & 12/11/2000 12:00:00 PM & & Dry \\
\hline TSWB-6 & 6/11/2001 11:32:00 AM & & Dry \\
\hline TSWB-6 & 7/2/2002 9:58:00 AM & & Dry \\
\hline TSWB-6 & 12/14/2005 2:49:00 PM & & Dry \\
\hline TSWB-6 & 7/5/2006 8:30:00 AM & & Dry \\
\hline TSWB-6 & 7/10/2006 10:15:00 AM & & Dry \\
\hline TSWB-6 & 10/26/2006 11:14:00 AM & & Dry \\
\hline TSWB-6 & 9/26/2006 12:50:00 PM & & Dry \\
\hline TSWB-6 & 11/1/2006 11:42:00 AM & & Dry \\
\hline TSWB-6 & 1/30/2007 2:08:00 PM & & Dry \\
\hline TSWB-6 & $10 / 30 / 2006$ & & Dry \\
\hline TSWB-6 & 3/7/2007 8:30:00 AM & & Dry \\
\hline TSWB-6 & 4/16/2007 12:32:00 PM & & Dry \\
\hline TSWB-6 & 7/5/2007 1:09:00 PM & & Dry \\
\hline
\end{tabular}




\begin{tabular}{|l|c|l||l|}
\hline Well Name & Date Time & Water Level (ft) & Comments \\
\hline TSWB-6 & $11 / 1 / 2007$ 12:43:00 PM & & Well dry \\
\hline
\end{tabular}

\subsection{WCO-1}

Location: Water Canyon, near western border of TA-68.

Period of Record: October 31,1989, through October 17, 2007

Remarks: Intermittently dry, there are only two records indicating water in well.

\begin{tabular}{|c|c|c|c|}
\hline Well Name & Date Time & Water Level (ft) & Comments \\
\hline WCO-1 & 10/31/1989 12:00:00 PM & & Dry \\
\hline WCO-1 & 11/1/1989 12:00:00 PM & & Dry \\
\hline WCO-1 & 8/24/1990 12:00:00 PM & & Dry \\
\hline WCO-1 & 6/23/1997 12:00:00 PM & 6582.75 & \\
\hline WCO-1 & 10/13/1997 12:00:00 PM & & Dry \\
\hline WCO-1 & 3/25/1998 12:00:00 PM & & Dry \\
\hline WCO-1 & 5/29/1998 12:00:00 PM & 6582.75 & \\
\hline WCO-1 & 7/28/1998 12:00:00 PM & & Dry \\
\hline WCO-1 & 3/3/1999 12:00:00 PM & & Dry \\
\hline WCO-1 & 6/23/1999 12:00:00 PM & & Dry \\
\hline WCO-1 & 8/30/1999 12:00:00 PM & & Dry \\
\hline WCO-1 & 11/15/1999 12:00:00 PM & & Dry \\
\hline WCO-1 & 3/26/2000 12:00:00 PM & & Dry \\
\hline WCO-1 & 5/16/2000 12:00:00 PM & & Dry \\
\hline WCO-1 & 8/30/2000 12:00:00 PM & & Dry \\
\hline WCO-1 & 10/8/2000 12:00:00 PM & & Dry \\
\hline WCO-1 & 7/2/2001 12:00:00 PM & & Dry \\
\hline WCO-1 & $8 / 22 / 2001$ 12:00:00 PM & & Dry \\
\hline WCO-1 & 10/18/2001 12:00:00 PM & & Dry \\
\hline WCO-1 & 4/19/2002 12:00:00 PM & & Dry \\
\hline WCO-1 & 8/19/2002 12:00:00 PM & & Dry \\
\hline WCO-1 & 11/13/2002 12:00:00 PM & & Dry \\
\hline WCO-1 & 2/18/2003 12:00:00 PM & & Dry \\
\hline WCO-1 & 6/19/2003 12:00:00 PM & & Dry \\
\hline WCO-1 & 9/14/2005 12:30:00 PM & & Dry \\
\hline WCO-1 & 12/22/2005 2:30:00 PM & & Dry \\
\hline WCO-1 & 3/13/2006 11:11:00 AM & & Dry \\
\hline WCO-1 & 6/23/2006 12:10:00 PM & & Dry, $\mathrm{TD}=35.74 \mathrm{ft}$ \\
\hline WCO-1 & 9/13/2006 12:12:00 PM & & Dry \\
\hline WCO-1 & 12/15/2006 10:05:00 AM & & Dry \\
\hline WCO-1 & 1/24/2007 9:55:00 AM & & Dry \\
\hline
\end{tabular}




\begin{tabular}{|l|r||l|l|}
\hline Well Name & \multicolumn{1}{|c|}{ Date Time } & Water Level (ft) & \multicolumn{1}{|c|}{ Comments } \\
\hline WCO-1 & 3/15/2007 10:07:00 AM & Dry \\
\hline WCO-1 & $5 / 24 / 2007$ 8:54:00 AM & Dry \\
\hline WCO-1 & $6 / 6 / 2007$ 7:31:00 AM & Dry \\
\hline WCO-1 & $9 / 5 / 2007$ 12:49:00 PM & Dry \\
\hline WCO-1 & $10 / 17 / 2007$ 11:38:00 AM & Dry \\
\hline
\end{tabular}




\subsection{WCO-2}

Location: Water Canyon, about 0.9 miles west of gate 9 on SR-4.

Period of Record: October 26, 1989, through September 30, 2007

Remarks: Seasonally dry, a pressure transducer was installed on September 14, 2005.
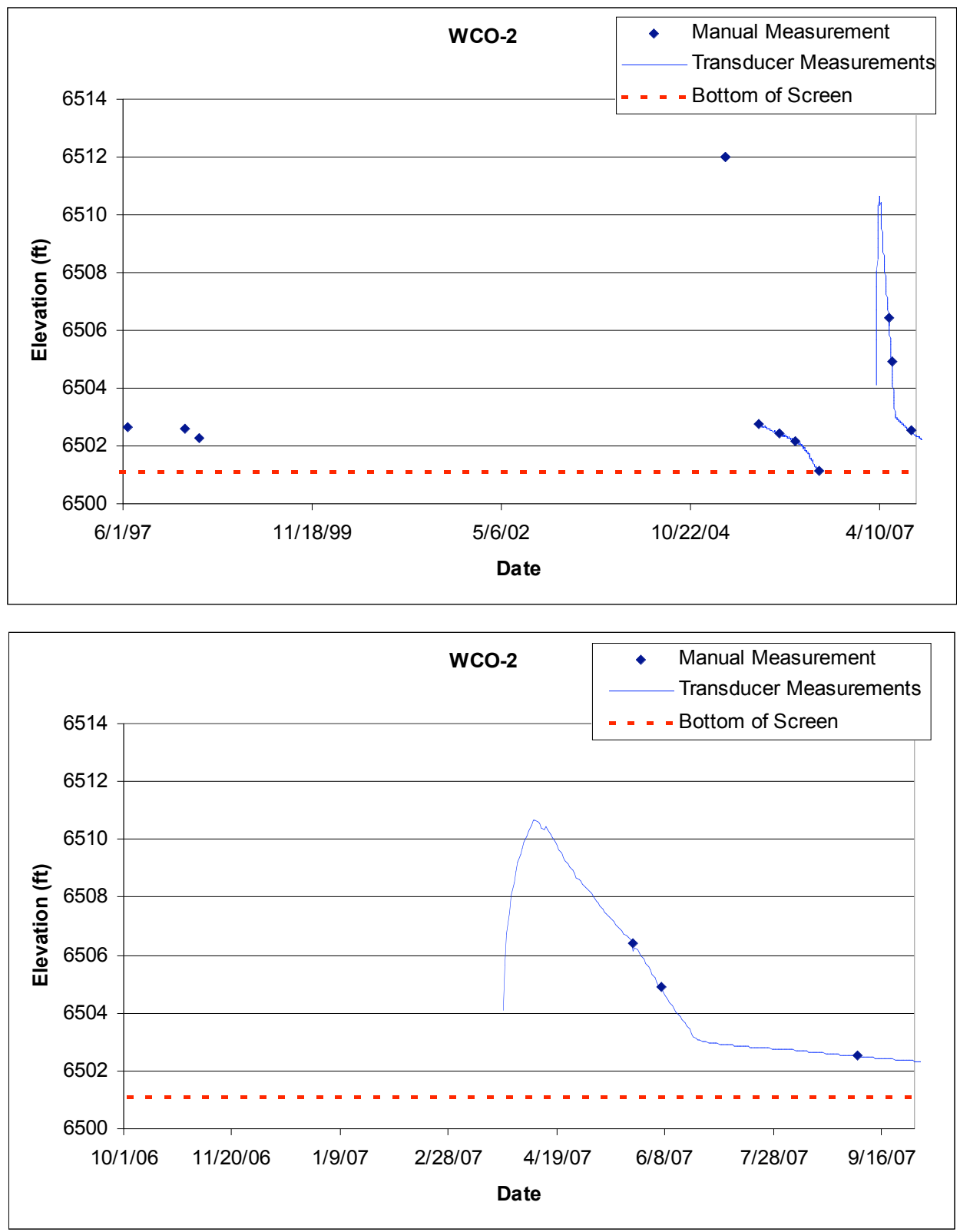


\subsection{WCO-3}

Location: Water Canyon, approximately 0.1 miles west of gate 9 on SR-4.

Period of Record: October 25, 1989, through October 17, 2007

Remarks: Often dry, only one record indicating water in well.

\begin{tabular}{|c|c|c|c|}
\hline Well Name & Date Time & Water Level (ft) & Comments \\
\hline WCO-3 & 10/25/1989 12:00:00 PM & & Dry \\
\hline WCO-3 & 8/24/1990 12:00:00 PM & & Dry \\
\hline WCO-3 & 6/23/1997 12:00:00 PM & 6424.6 & \\
\hline WCO-3 & 10/13/1997 12:00:00 PM & 6423.87 & \\
\hline WCO-3 & 3/25/1998 12:00:00 PM & & Dry \\
\hline WCO-3 & 5/29/1998 12:00:00 PM & & Dry \\
\hline WCO-3 & 7/28/1998 12:00:00 PM & & Dry \\
\hline WCO-3 & 3/3/1999 12:00:00 PM & & Dry \\
\hline WCO-3 & 6/23/1999 12:00:00 PM & & Dry \\
\hline WCO-3 & 8/30/1999 12:00:00 PM & & Dry \\
\hline WCO-3 & 11/15/1999 12:00:00 PM & & Dry \\
\hline WCO-3 & 3/26/2000 12:00:00 PM & & Dry \\
\hline WCO-3 & 5/16/2000 12:00:00 PM & & Dry \\
\hline WCO-3 & 8/30/2000 12:00:00 PM & & Dry \\
\hline WCO-3 & 10/8/2000 12:00:00 PM & & Dry \\
\hline WCO-3 & 7/2/2001 12:00:00 PM & & Dry \\
\hline WCO-3 & 8/22/2001 12:00:00 PM & & Dry \\
\hline WCO-3 & 10/18/2001 12:00:00 PM & & Dry \\
\hline WCO-3 & 4/19/2002 12:00:00 PM & & Dry \\
\hline WCO-3 & 8/19/2002 12:00:00 PM & & Dry \\
\hline WCO-3 & 11/13/2002 12:00:00 PM & & Dry \\
\hline WCO-3 & 2/18/2003 12:00:00 PM & & Dry \\
\hline WCO-3 & 6/19/2003 12:00:00 PM & & Dry \\
\hline WCO-3 & 9/14/2004 1:35:00 PM & & Dry \\
\hline WCO-3 & 12/22/2005 3:00:00 PM & & Dry \\
\hline WCO-3 & 3/13/2006 10:51:00 AM & & Dry \\
\hline WCO-3 & 6/23/2006 12:39:00 PM & & Dry, $T D=13.63 \mathrm{ft}$ \\
\hline WCO-3 & 9/13/2006 11:33:00 AM & & Dry \\
\hline WCO-3 & 12/15/2006 9:30:00 AM & & Dry \\
\hline WCO-3 & 1/24/2007 9:15:00 AM & & Dry \\
\hline WCO-3 & 3/15/2007 9:25:00 AM & & Dry \\
\hline WCO-3 & 5/24/2007 10:20:00 AM & & Dry \\
\hline
\end{tabular}




\begin{tabular}{|l|r||l|l|}
\hline Well Name & Date Time & Water Level (ft) & \multicolumn{1}{|c|}{ Comments } \\
\hline WCO-3 & 6/6/2007 8:00:00 AM & & Dry \\
\hline WCO-3 & $9 / 5 / 2007$ 12:26:00 PM & & Dry \\
\hline WCO-3 & $10 / 17 / 2007$ 11:50:00 AM & & Dry \\
\hline
\end{tabular}




\subsection{Groundwater Level Data from Water Supply Wells}

Table 6-1 lists the Los Alamos County water supply wells; all supply wells except G-1A and O-4 were monitored for groundwater levels in FY 2007. The table provides the well name, date of completion, well depth, surveyed location coordinates, ground surface elevation, and the screen top and bottom depths for each well. See Figure 3-1 for the locations of the wells.

The LANL GWLM Project integrated the water supply wells in the monitoring project beginning in FY 2007 with the cooperation of Los Alamos County Utility personnel. Recently obtained groundwater level data for the supply wells are provided in the following sections. Historical groundwater level data for the supply wells were most recently summarized by Koch and Rogers (2003) and in other preceding Water Supply Reports for Los Alamos.

Table 6-1. General Information for Los Alamos County Water Supply Wells

\begin{tabular}{|c|r|r|r|r|r|r|r|}
\hline $\begin{array}{c}\text { Well } \\
\text { Name }\end{array}$ & $\begin{array}{c}\text { Date } \\
\text { Completed }\end{array}$ & $\begin{array}{c}\text { Completed } \\
\text { Depth (ft) }\end{array}$ & $\begin{array}{c}\text { Easting } \\
\text { (ft) }\end{array}$ & $\begin{array}{c}\text { Northing } \\
\text { (ft) }\end{array}$ & $\begin{array}{c}\text { Surface } \\
\text { Elevation } \\
\text { (ft) }\end{array}$ & $\begin{array}{c}\text { Screen } \\
\text { Top } \\
\text { Depth (ft) }\end{array}$ & $\begin{array}{c}\text { Screen } \\
\text { Bottom } \\
\text { Depth (ft) }\end{array}$ \\
\hline G-1A & $12 / 15 / 1954$ & 1519 & 1655240.9 & 1784353.3 & 6014 & 272 & 1513 \\
\hline G-2A & $3 / 21 / 1998$ & 2000 & 1651973.8 & 1786166.3 & 6138 & 565 & 1980 \\
\hline G-3 & $8 / 25 / 1999$ & 1800 & 1651676.4 & 1786218.3 & 6139 & 441 & 1100 \\
\hline G-3A & $5 / 9 / 1998$ & 2000 & 1649661.5 & 1786585.3 & 6212 & 590 & 1980 \\
\hline G-4A & $4 / 1 / 1998$ & 2000 & 1647318.2 & 1787112.9 & 6299 & 655 & 1980 \\
\hline G-5A & $5 / 20 / 1998$ & 2000 & 1644877.2 & 1789636.0 & 6414 & 765 & 1980 \\
\hline O-1 & $8 / 1 / 1990$ & 2497 & 1649396.3 & 1772232.1 & 6396 & 1017 & 2477 \\
\hline O-4 & $3 / 1 / 1990$ & 2617 & 1637337.4 & 1772995.1 & 6627 & 1115 & 2596 \\
\hline PM-1 & $2 / 1 / 1965$ & 2499 & 1647734.3 & 1768112.1 & 6520 & 945 & 2479 \\
\hline PM-2 & $7 / 15 / 1965$ & 2300 & 1636697.5 & 1760406.4 & 6715 & 1004 & 2280 \\
\hline PM-3 & $11 / 1 / 1966$ & 2552 & 1642590.0 & 1769530.0 & 6610 & 956 & 2532 \\
\hline PM-4 & $8 / 15 / 1981$ & 2874 & 1635623.0 & 1764740.0 & 6920 & 1260 & 2854 \\
\hline PM-5 & $9 / 1 / 1982$ & 3092 & 1632110.0 & 1767790.0 & 7095 & 1440 & 3072 \\
\hline
\end{tabular}

All Los Alamos County water supply wells are powered by electric motors except for PM-4, which has a natural-gas-powered motor. The electric-powered wells are typically operated at night and on weekends when electricity rates are lower. Thus these wells usually cycle on and off daily, in contrast to PM-4, which usually runs continuously when in use, which is usually just during the summer months when water demand is highest. Thus, due to the operational characteristics of the electricpowered wells, the data displayed in the following sections for these wells are the maximum daily water level, or the "non-pumping" water level, and the minimum daily or "pumping" water level. The difference between the non-pumping and the pumping water level is the drawdown for each well. The data shown for the wells that aren't operated cyclically, which are PM-4 and O-1 (which hasn't been used in recent years), are mean daily water levels. 


\section{$6.1 \quad$ G-2A}

Location: G-2A is located in Guaje Canyon about $300 \mathrm{ft}$ east of monitoring well G-3.

Completion Type: Single completion in the Santa Fe Group.

Period of Record: Well completed in 1998, transducer installed December 2003; data through 2007. Remarks: The pumping and non-pumping water levels overlap depending on pumping stress to the aquifer. The drawdown is about $40 \mathrm{ft}$.

\begin{tabular}{|c|c|c|c|c|c|c|c|c|c|c|c|c|c|}
\hline \multicolumn{14}{|c|}{ G-2AConstruction Information } \\
\hline Zone & $\begin{array}{c}\text { Screen } \\
\text { Top } \\
\text { Depth (ft) }\end{array}$ & $\begin{array}{c}\text { Screen } \\
\text { Bottom } \\
\text { Depth } \\
\text { (ft) }\end{array}$ & $\begin{array}{c}\text { Screen } \\
\text { Top } \\
\text { Elev (ft) }\end{array}$ & $\begin{array}{l}\text { Screen } \\
\text { Bottom } \\
\text { Elev (ft) }\end{array}$ & $\begin{array}{l}\text { Screen } \\
\text { Length } \\
\text { (ft) }\end{array}$ & $\begin{array}{l}\text { Pump } \\
\text { Intake } \\
\text { Depth } \\
\text { (ft) }\end{array}$ & $\begin{array}{l}\text { Pump } \\
\text { Intake } \\
\text { Elevation } \\
\text { (ft) }\end{array}$ & $\begin{array}{c}\text { Depth to } \\
\text { Top of } \\
\text { Sump (ft) }\end{array}$ & $\begin{array}{l}\text { Top of } \\
\text { Sump } \\
\text { Elevation } \\
\text { (ft) }\end{array}$ & $\begin{array}{c}\text { Depth to } \\
\text { Sump } \\
\text { Bottom (ft) }\end{array}$ & $\begin{array}{l}\text { Sump } \\
\text { Length } \\
\text { (ft) }\end{array}$ & $\begin{array}{c}\text { Sump } \\
\text { Volume } \\
\text { (L) }\end{array}$ & Comment \\
\hline 1 & 565 & 1980 & 5573 & 4158 & 1415 & 540 & 5598 & 1980 & 4158 & 2000 & 20 & 444.8 & Supply Well \\
\hline
\end{tabular}

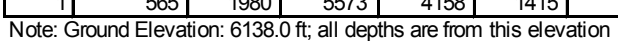

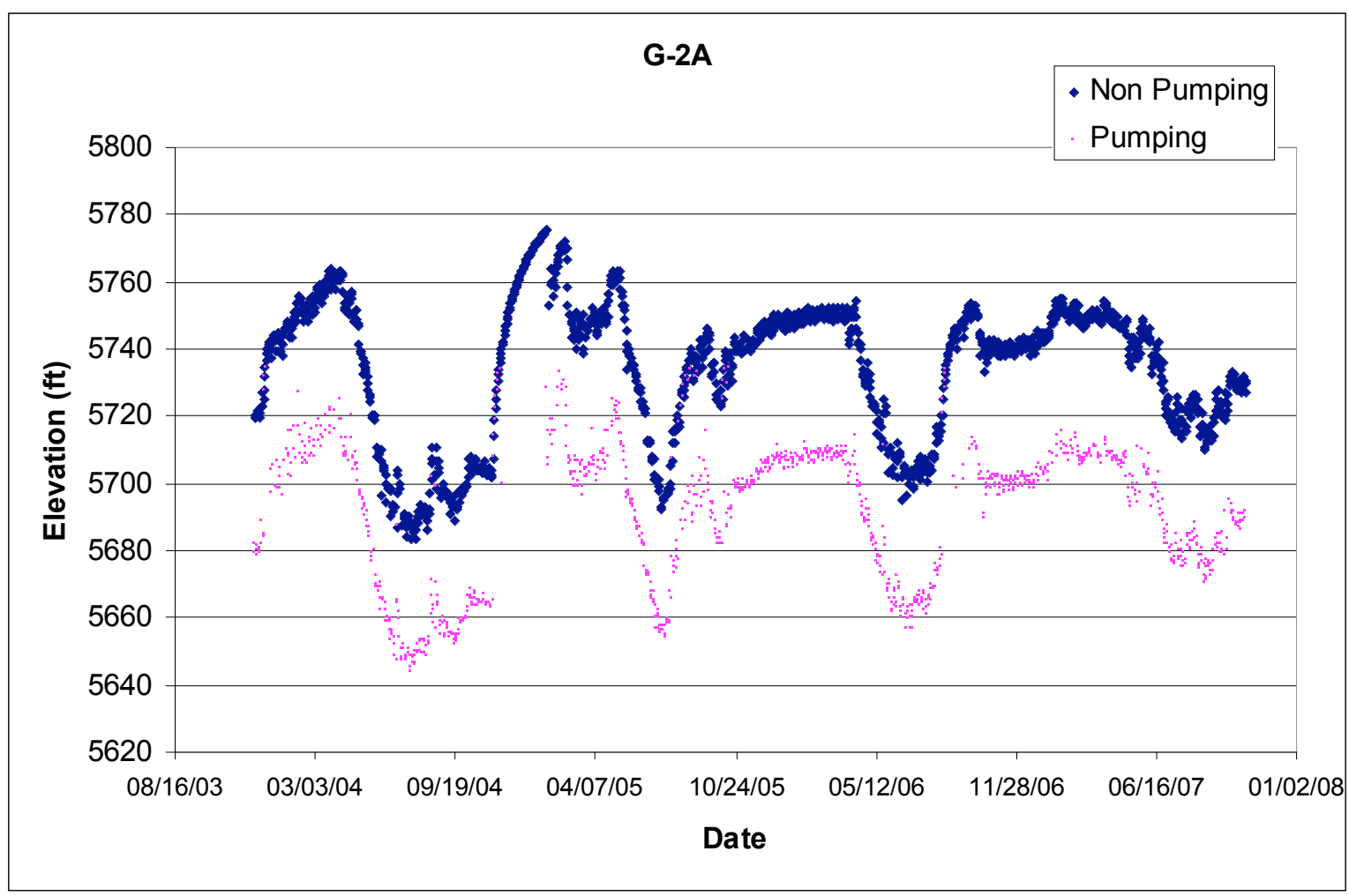




\subsection{G-3}

Location: G-3 is located in Guaje Canyon about $300 \mathrm{ft}$ west of supply well G-2A.

Completion Type: Single completion in the Santa Fe Group.

Period of Record: Well originally completed as a supply well in July 1951; plugged back to $1103 \mathrm{ft}$ and converted to a monitoring well in 1998, transducer installed June 2002; data through 2007.

Remarks: G-3 responds primarily to pumping at supply well G-2A. The aquifer in the Guaje well field fluctuates seasonally 40 to $70 \mathrm{ft}$ depending on pumping stresses.

\begin{tabular}{|c|c|c|c|c|c|c|c|c|c|c|c|c|c|}
\hline \multicolumn{14}{|c|}{ G-3 Construction Information } \\
\hline Zone & \begin{tabular}{|c} 
Screen \\
Top \\
Depth (ft)
\end{tabular} & $\begin{array}{l}\text { Screen } \\
\text { Bottom } \\
\text { Depth } \\
\text { (ft) }\end{array}$ & $\begin{array}{l}\text { Screen } \\
\text { Top } \\
\text { Elev (ft) }\end{array}$ & $\begin{array}{l}\text { Screen } \\
\text { Bottom } \\
\text { Elev (ft) }\end{array}$ & $\begin{array}{l}\text { Screen } \\
\text { Length } \\
\text { (ft) }\end{array}$ & $\begin{array}{l}\text { Pump } \\
\text { Intake } \\
\text { Depth } \\
\text { (ft) }\end{array}$ & $\begin{array}{l}\text { Pump } \\
\text { Intake } \\
\text { Elevation } \\
\text { (ft) }\end{array}$ & $\begin{array}{l}\text { Depth to } \\
\text { Top of } \\
\text { Sump (ft) }\end{array}$ & $\begin{array}{l}\text { Top of } \\
\text { Sump } \\
\text { Elevation } \\
\text { (ft) }\end{array}$ & $\begin{array}{l}\text { Depth to } \\
\text { Sump } \\
\text { Bottom (ft) }\end{array}$ & $\begin{array}{l}\text { Sump } \\
\text { Length } \\
\text { (ft) }\end{array}$ & $\begin{array}{l}\text { Sump } \\
\text { Volume } \\
\text { (L) }\end{array}$ & Comment \\
\hline 1 & 441 & 1100 & 5698 & 5039 & 659 & & & 1100 & 5039 & 1103 & 3.0 & 66.7 & Regional aquifer \\
\hline
\end{tabular}

Note: Ground Elevation: $6139.0 \mathrm{ft}$; all depths are from this elevation

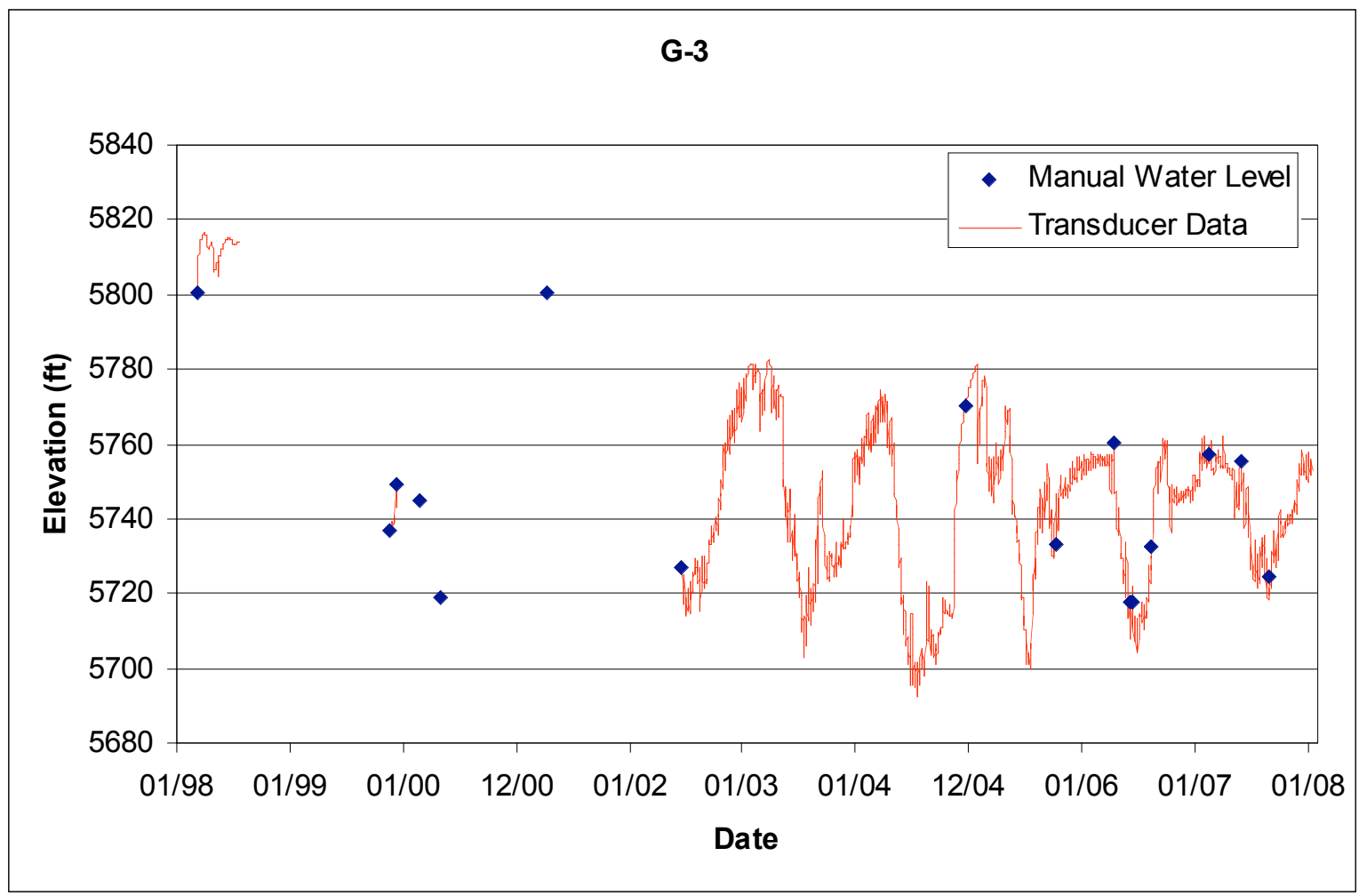

Note: mean daily water level values displayed 


\subsection{G-3A}

Location: G-3A is located in Guaje Canyon about 1.5 miles west of monitoring well G-3.

Completion Type: Single completion in the Santa Fe Group.

Period of Record: Well completed as a supply well in May 1998; transducer installed December 2003; intermittent data through 2007.

Remarks: Drawdown is 60 to $65 \mathrm{ft}$.

\begin{tabular}{|c|c|c|c|c|c|c|c|c|c|c|c|c|c|}
\hline \multicolumn{14}{|c|}{ G-3AConstruction Information } \\
\hline Zone & $\begin{array}{c}\text { Screen } \\
\text { Top } \\
\text { Depth (ft) }\end{array}$ & $\begin{array}{c}\text { Screen } \\
\text { Bottom } \\
\text { Depth } \\
\text { (ft) }\end{array}$ & $\begin{array}{c}\text { Screen } \\
\text { Top } \\
\text { Elev (ft) }\end{array}$ & $\begin{array}{l}\text { Screen } \\
\text { Bottom } \\
\text { Elev (ft) }\end{array}$ & $\begin{array}{c}\text { Screen } \\
\text { Length } \\
\text { (ft) }\end{array}$ & $\begin{array}{l}\text { Pump } \\
\text { Intake } \\
\text { Depth } \\
\text { (ft) }\end{array}$ & $\begin{array}{l}\text { Pump } \\
\text { Intake } \\
\text { Elevation } \\
\text { (ft) }\end{array}$ & $\begin{array}{c}\text { Depth to } \\
\text { Top of } \\
\text { Sump (ft) }\end{array}$ & $\begin{array}{l}\text { Top of } \\
\text { Sump } \\
\text { Elevation } \\
\text { (ft) }\end{array}$ & $\begin{array}{c}\text { Depth to } \\
\text { Sump } \\
\text { Bottom (ft) }\end{array}$ & $\begin{array}{c}\text { Sump } \\
\text { Length } \\
\text { (ft) }\end{array}$ & $\begin{array}{c}\text { Sump } \\
\text { Volume } \\
\text { (L) }\end{array}$ & Comment \\
\hline 1 & 590 & 1980 & 5622 & 4232 & 1390 & 560 & 5652 & 1980 & 4232 & 2000 & 20 & 853.7 & Supply Well \\
\hline
\end{tabular}

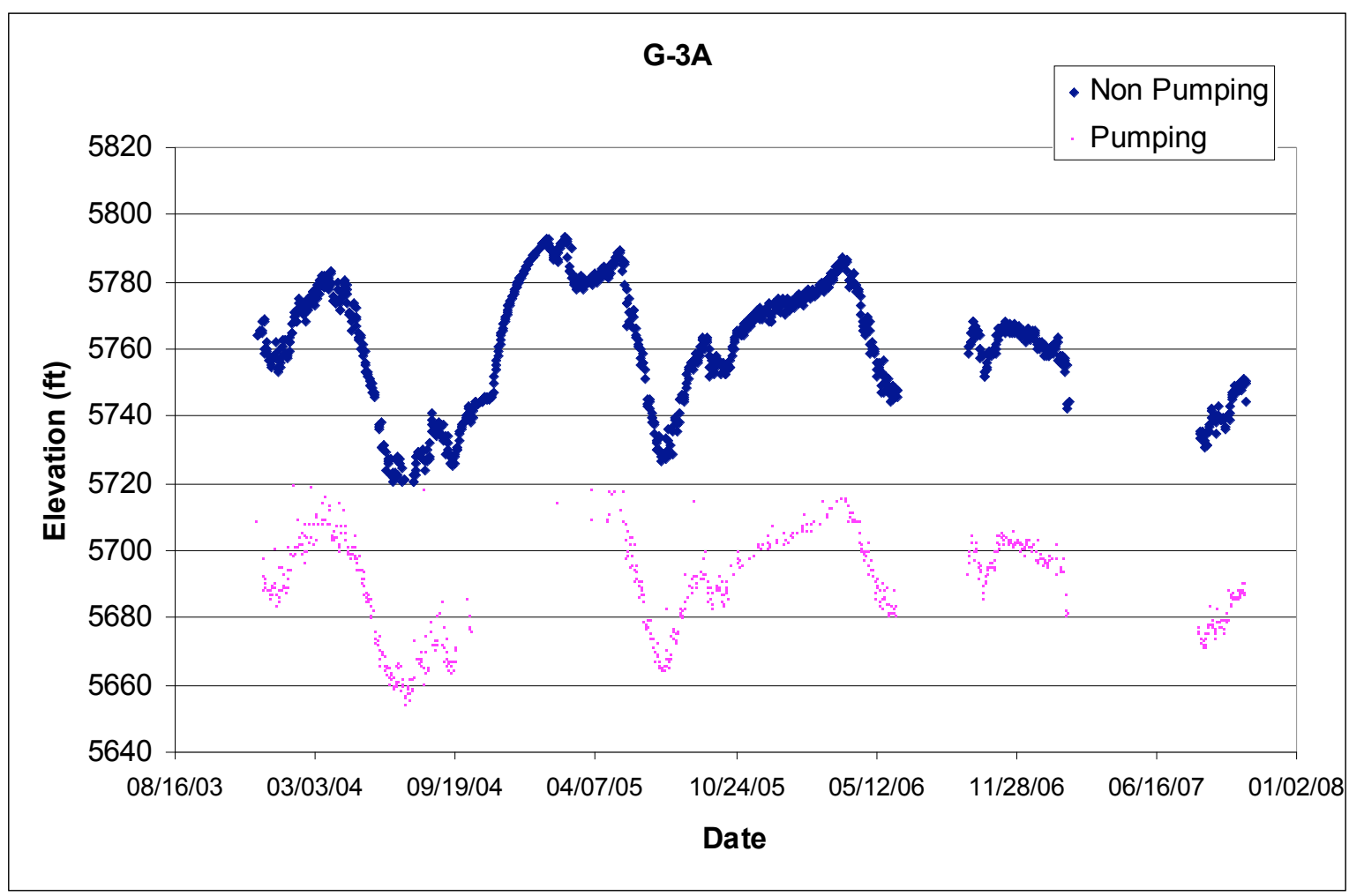




\section{$6.4 \quad$ G-4A}

Location: G-4A is located in lower Rendija Canyon near the confluence with Guaje Canyon and about 0.5 miles west of supply well G-3A.

Completion Type: Single completion in the Santa Fe Group.

Period of Record: Well completed as a supply well in April 1998; transducer installed December 2003; intermittent data through 2007.

Remarks: Drawdown is 80 to $85 \mathrm{ft}$.

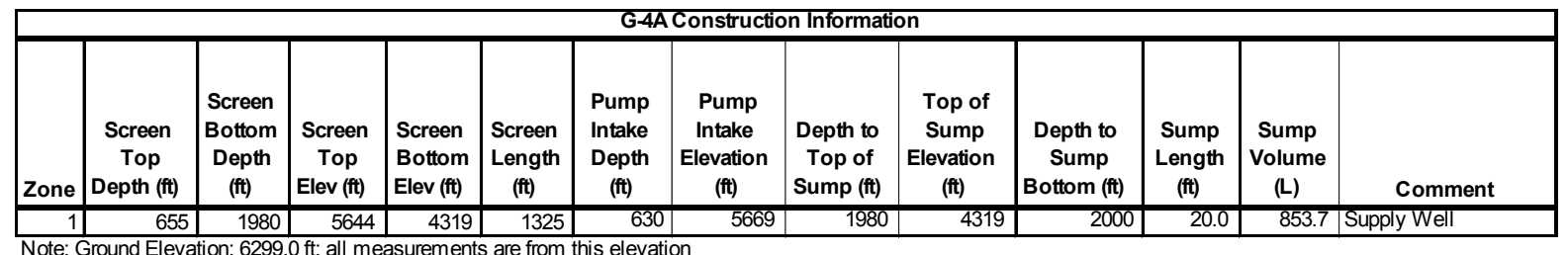

Note: Ground Elevation: $6299.0 \mathrm{ft}$; all measurements are from this elevation

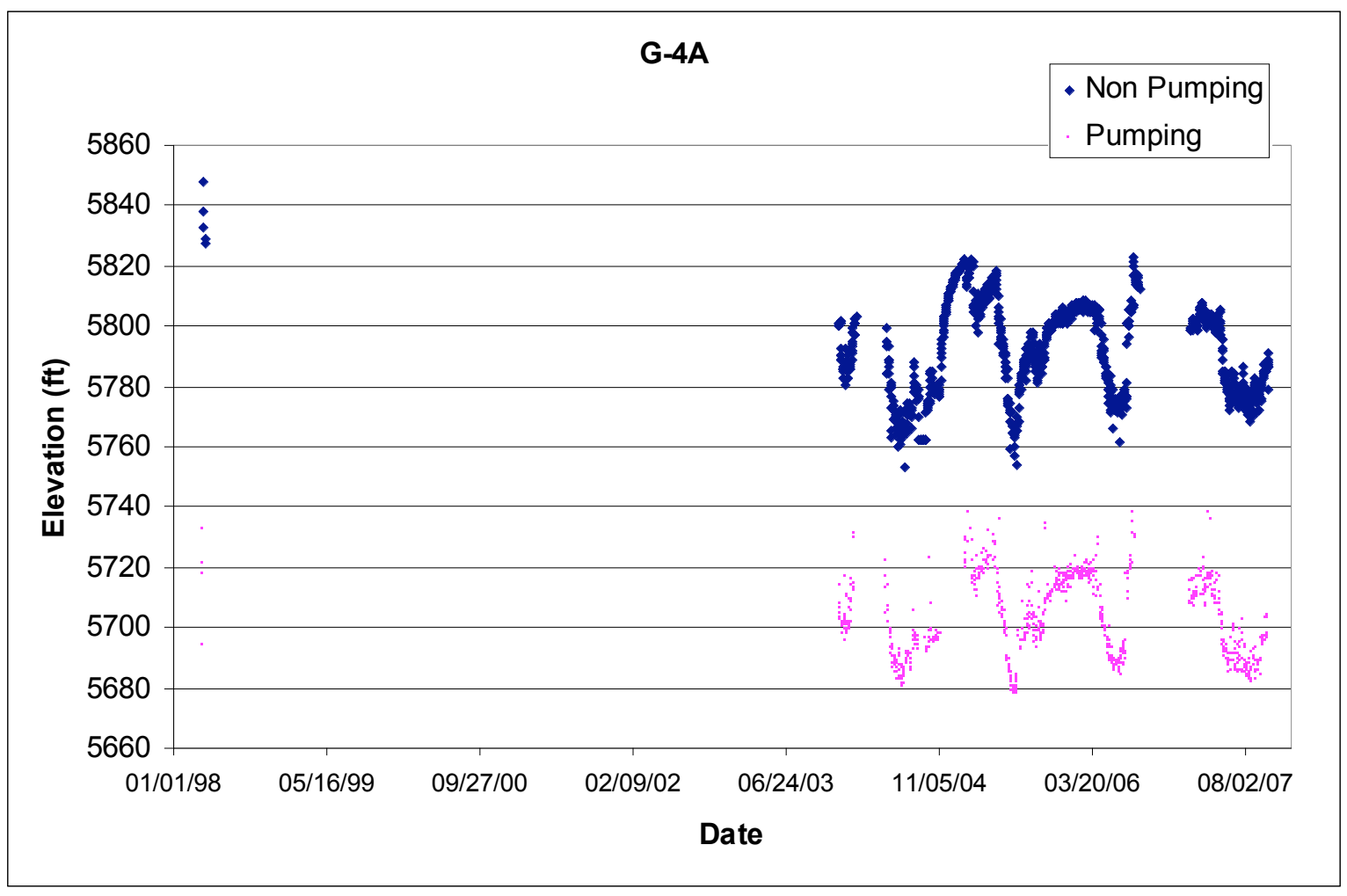




\section{$6.5 \quad$ G-5A}

Location: G-5A is located in Guaje Canyon upstream of Rendija Canyon and about 1.9 miles northwest of supply well G-4A.

Completion Type: Single completion in the Santa Fe Group.

Period of Record: Well completed as a supply well in May 1998; transducer installed January 2004; intermittent data through 2007.

Remarks: G-5A is not used on a regular basis. Drawdown is 140 to $150 \mathrm{ft}$.

\begin{tabular}{|c|c|c|c|c|c|c|c|c|c|c|c|c|c|}
\hline \multicolumn{14}{|c|}{ G-5AConstruction Information } \\
\hline Zone & $\begin{array}{c}\text { Screen } \\
\text { Top } \\
\text { Depth (ft) }\end{array}$ & $\begin{array}{c}\text { Screen } \\
\text { Bottom } \\
\text { Depth } \\
\text { (ft) }\end{array}$ & $\begin{array}{c}\text { Screen } \\
\text { Top } \\
\text { Elev (ft) }\end{array}$ & $\begin{array}{l}\text { Screen } \\
\text { Bottom } \\
\text { Elev (ft) }\end{array}$ & $\begin{array}{l}\text { Screen } \\
\text { Length } \\
\text { (ft) }\end{array}$ & $\begin{array}{l}\text { Pump } \\
\text { Intake } \\
\text { Depth } \\
\text { (ft) }\end{array}$ & $\begin{array}{l}\text { Pump } \\
\text { Intake } \\
\text { Elevation } \\
\text { (ft) }\end{array}$ & $\begin{array}{c}\text { Depth to } \\
\text { Top of } \\
\text { Sump (ft) }\end{array}$ & $\begin{array}{c}\text { Top of } \\
\text { Sump } \\
\text { Elevation } \\
\text { (ft) }\end{array}$ & $\begin{array}{c}\text { Depth to } \\
\text { Sump } \\
\text { Bottom (ft) }\end{array}$ & $\begin{array}{l}\text { Sump } \\
\text { Length } \\
\text { (ft) }\end{array}$ & $\begin{array}{c}\text { Sump } \\
\text { Volume } \\
\text { (L) }\end{array}$ & Comment \\
\hline 1 & 765 & 1980 & 5649 & 4434 & 1215 & 740 & 5674 & 1980 & 4434 & 2000 & 20 & 853.7 & Supply Well \\
\hline
\end{tabular}

Note: Ground Elevation: $6414.0 \mathrm{ft}$; all measurements are from this elevation

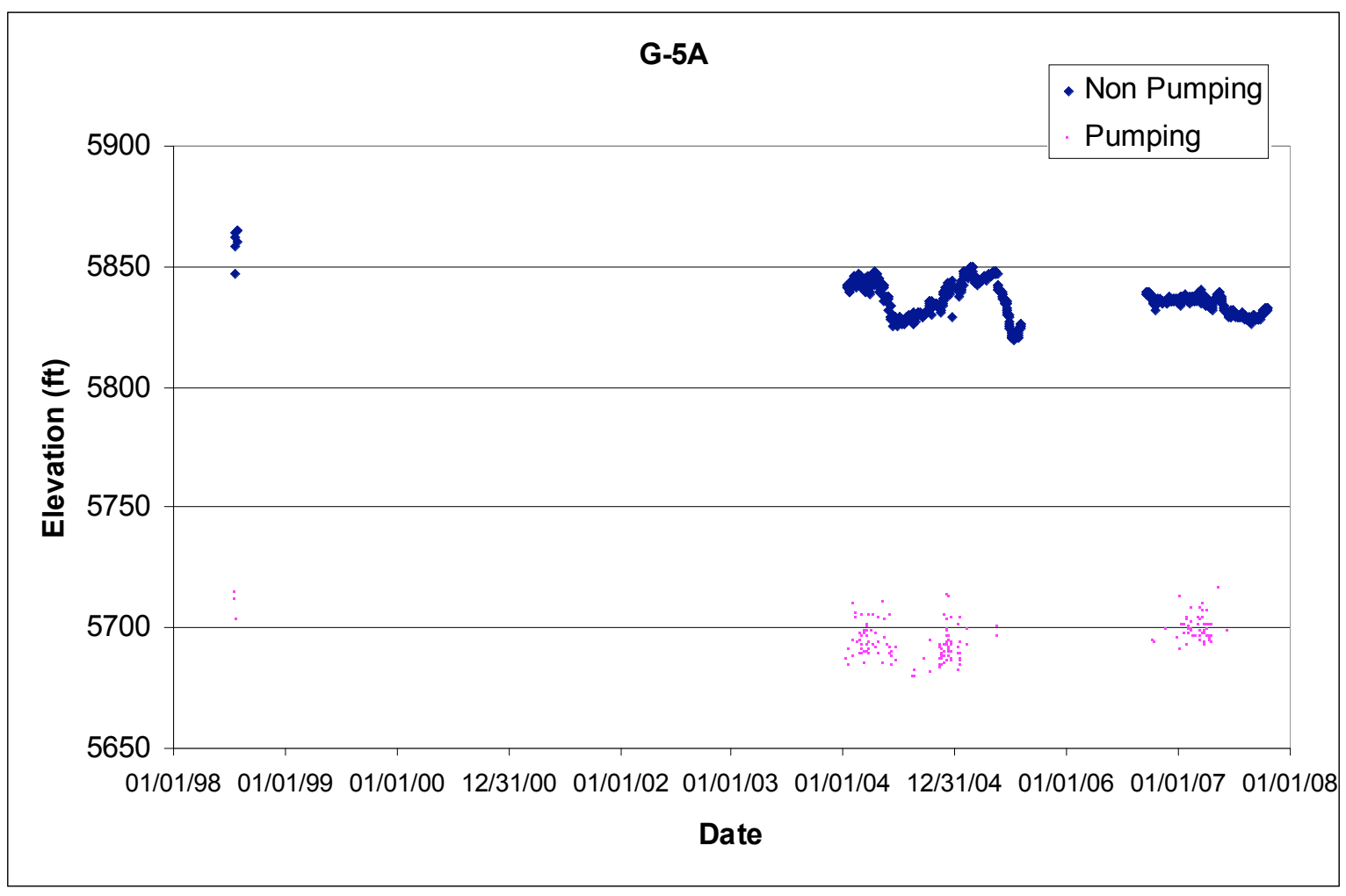




\section{$6.6 \quad 0-1$}

Location: O-1 is located in lower Pueblo Canyon about 0.5 miles downstream of monitoring well R-5. Completion Type: Single completion in the Santa Fe Group.

Period of Record: Well completed as a supply well in August 1990; transducer installed June 2007; data through 2007.

Remarks: O-1 has not been used on a regular basis except for quarterly groundwater sampling. Drawdown is about $100 \mathrm{ft}$. O-1 responds to pumping of supply well PM-1.

\begin{tabular}{|c|c|c|c|c|c|c|c|c|c|c|c|c|c|}
\hline \multicolumn{14}{|c|}{$0-1$ Construction Information } \\
\hline Zone & $\begin{array}{c}\text { Screen } \\
\text { Top } \\
\text { Depth (ft) }\end{array}$ & $\begin{array}{c}\text { Screen } \\
\text { Bottom } \\
\text { Depth } \\
\text { (ft) }\end{array}$ & $\begin{array}{c}\text { Screen } \\
\text { Top } \\
\text { Elev (ft) }\end{array}$ & $\begin{array}{l}\text { Screen } \\
\text { Bottom } \\
\text { Elev (ft) }\end{array}$ & $\begin{array}{c}\text { Screen } \\
\text { Length } \\
\text { (ft) }\end{array}$ & $\begin{array}{l}\text { Pump } \\
\text { Intake } \\
\text { Depth } \\
\text { (ft) }\end{array}$ & $\begin{array}{c}\text { Pump } \\
\text { Intake } \\
\text { Elevation } \\
\text { (ft) }\end{array}$ & $\begin{array}{c}\text { Depth to } \\
\text { Top of } \\
\text { Sump (ft) }\end{array}$ & $\begin{array}{c}\text { Top of } \\
\text { Sump } \\
\text { Elevation } \\
\text { (ft) }\end{array}$ & $\begin{array}{l}\text { Depth to } \\
\text { Sump } \\
\text { Bottom (ft) }\end{array}$ & $\begin{array}{l}\text { Sump } \\
\text { Length } \\
\text { (ft) }\end{array}$ & $\begin{array}{c}\text { Sump } \\
\text { Volume } \\
\text { (L) }\end{array}$ & Comment \\
\hline 1 & 1017 & 2477 & 5379 & 3919 & 1460 & 877 & 5519 & 2477 & 2477 & 2497 & 20 & 790.8 & Regional Aquife \\
\hline
\end{tabular}

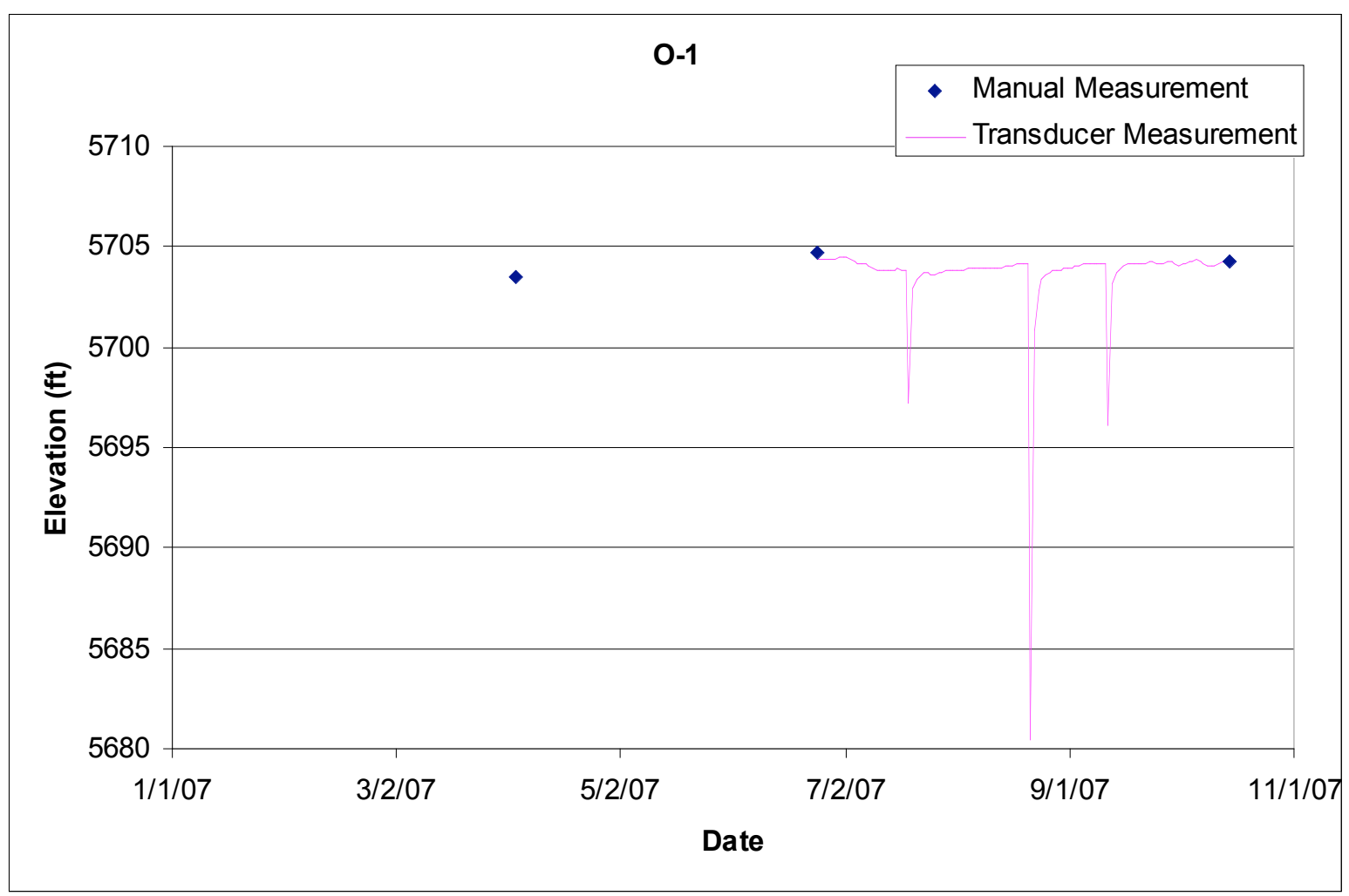

Note: Mean daily values shown 


\subsection{PM-1}

Location: PM-1 is located in lower Sandia Canyon near the eastern Laboratory boundary and about $360 \mathrm{ft}$ northeast of monitoring well R-12.

Completion Type: Single completion in the Santa Fe Group.

Period of Record: Well completed as a supply well in February 1965; transducer installed December 2006; data through 2007.

Remarks: Drawdown is about $30 \mathrm{ft}$.
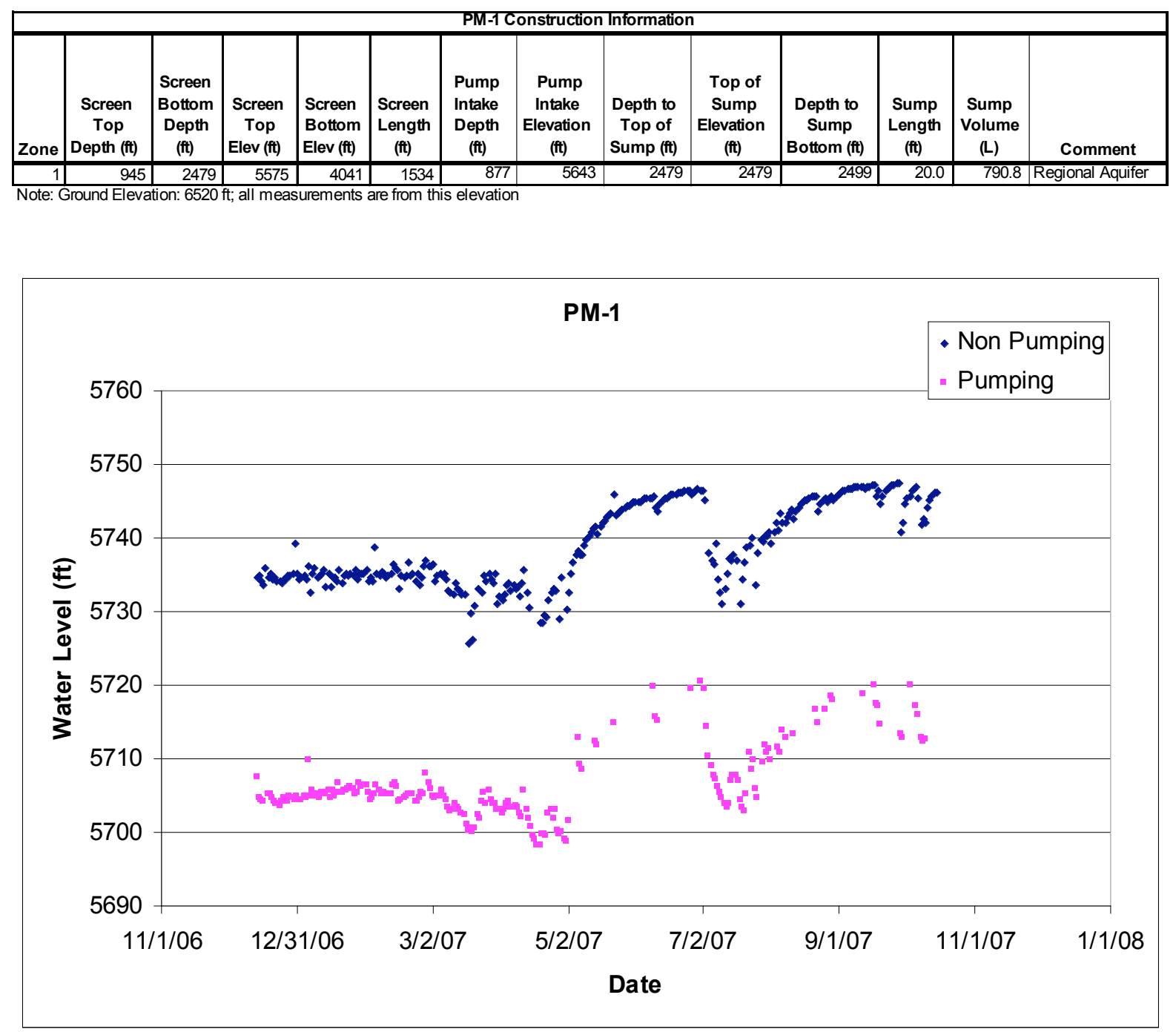


\section{$6.8 \quad \mathrm{PM}-2$}

Location: PM-2 is located in Pajarito Canyon about 0.25 miles west of monitoring well R-20.

Completion Type: Single completion in the Santa Fe Group.

Period of Record: Well completed as a supply well in July 1965; transducer installed December 2004; data to October 23, 2007. The transducer was removed in October 2007 during pump removal and well rehabilitation.

Remarks: Drawdown is about $70 \mathrm{ft}$. PM-2 responds to pumping at PM-4 (McLin 2006).

\begin{tabular}{|c|c|c|c|c|c|c|c|c|c|c|c|c|c|}
\hline \multicolumn{14}{|c|}{ PM-2 Construction Information } \\
\hline Zone & $\begin{array}{c}\text { Screen } \\
\text { Top } \\
\text { Depth (ft) }\end{array}$ & $\begin{array}{c}\text { Screen } \\
\text { Bottom } \\
\text { Depth } \\
\text { (ft) }\end{array}$ & $\begin{array}{c}\text { Screen } \\
\text { Top } \\
\text { Elev }(\mathrm{ft})\end{array}$ & $\begin{array}{l}\text { Screen } \\
\text { Bottom } \\
\text { Elev (ft) }\end{array}$ & $\begin{array}{c}\text { Screen } \\
\text { Length } \\
\text { (ft) }\end{array}$ & $\begin{array}{l}\text { Pump } \\
\text { Intake } \\
\text { Depth } \\
\text { (ft) }\end{array}$ & $\begin{array}{c}\text { Pump } \\
\text { Intake } \\
\text { Elevation } \\
\text { (ft) }\end{array}$ & $\begin{array}{l}\text { Depth to } \\
\text { Top of } \\
\text { Sump (ft) }\end{array}$ & $\begin{array}{c}\text { Top of } \\
\text { Sump } \\
\text { Elevation } \\
\text { (ft) }\end{array}$ & $\begin{array}{l}\text { Depth to } \\
\text { Sump } \\
\text { Bottom (ft) }\end{array}$ & $\begin{array}{l}\text { Sump } \\
\text { Length } \\
\text { (ft) }\end{array}$ & $\begin{array}{c}\text { Sump } \\
\text { Volume } \\
\text { (L) }\end{array}$ & Comment \\
\hline 1 & 1004 & 2280 & 5711 & 4435 & 1276 & 980 & 5735 & 2280 & 4435 & 2300 & 20.0 & 790.8 & Regional Aquifer \\
\hline
\end{tabular}

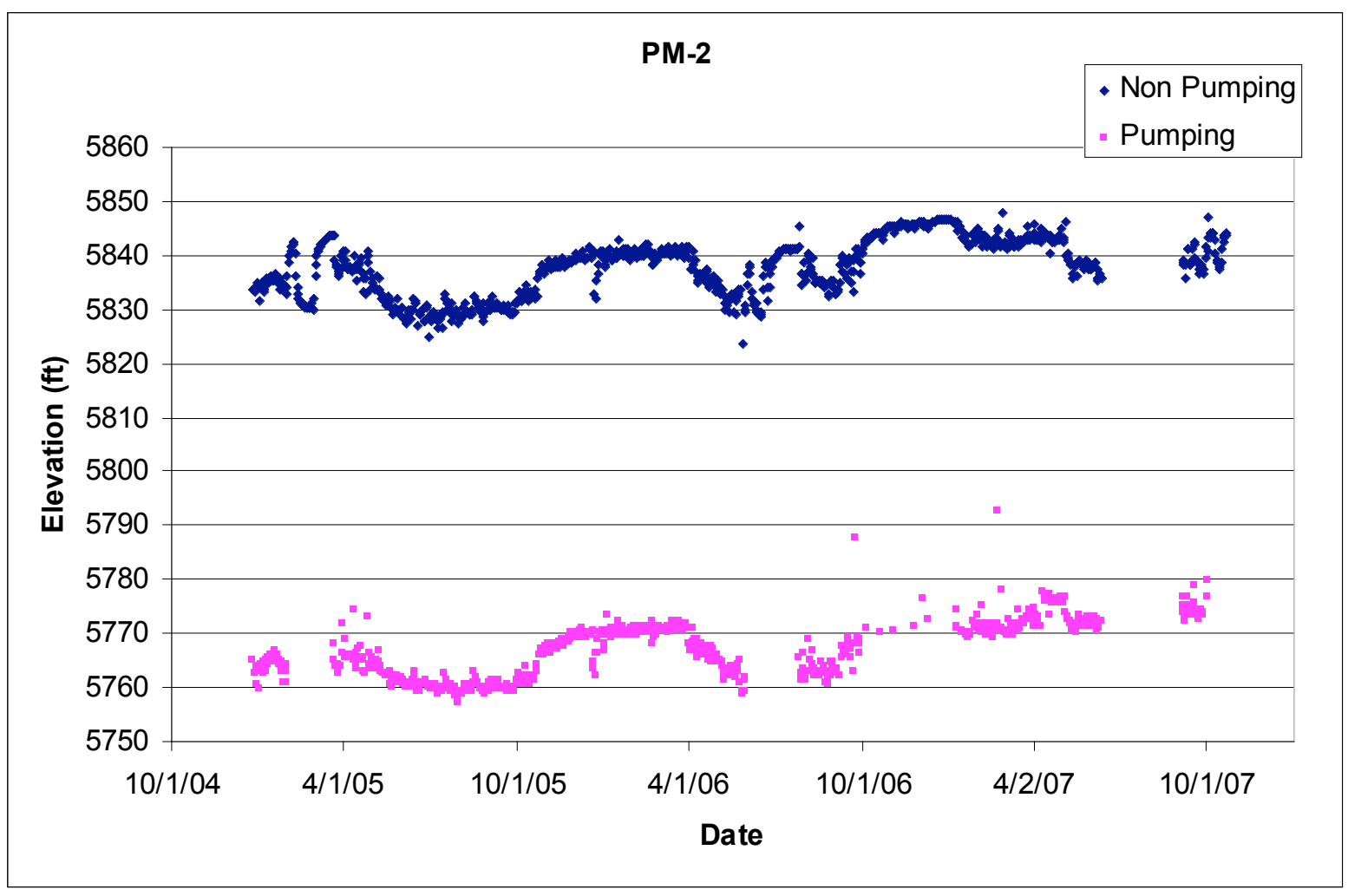




\section{$6.9 \quad$ PM-3}

Location: PM-3 is located in Sandia Canyon about 1 mile west of PM-1 and about $330 \mathrm{ft}$ northeast of monitoring well R-35a.

Completion Type: Single completion in the Santa Fe Group.

Period of Record: Well completed as a supply well in November 1966; transducer installed October 2006; data through 2007.

Remarks: Drawdown is about $27 \mathrm{ft}$. PM-3 responds to pumping at O-4.

\begin{tabular}{|c|c|c|c|c|c|c|c|c|c|c|c|c|c|}
\hline \multicolumn{14}{|c|}{ PM-3 Construction Information } \\
\hline Zone & $\begin{array}{c}\text { Screen } \\
\text { Top } \\
\text { Depth (ft) }\end{array}$ & $\begin{array}{l}\text { Screen } \\
\text { Bottom } \\
\text { Depth } \\
\text { (ft) }\end{array}$ & $\begin{array}{c}\text { Screen } \\
\text { Top } \\
\text { Elev (ft) }\end{array}$ & $\begin{array}{l}\text { Screen } \\
\text { Bottom } \\
\text { Elev (ft) }\end{array}$ & $\begin{array}{l}\text { Screen } \\
\text { Length } \\
\text { (ft) }\end{array}$ & $\begin{array}{l}\text { Pump } \\
\text { Intake } \\
\text { Depth } \\
\text { (ft) }\end{array}$ & $\begin{array}{l}\text { Pump } \\
\text { Intake } \\
\text { Elevation } \\
\text { (ft) }\end{array}$ & $\begin{array}{l}\text { Depth to } \\
\text { Top of } \\
\text { Sump (ft) }\end{array}$ & $\begin{array}{l}\text { Top of } \\
\text { Sump } \\
\text { Elevation } \\
\text { (ft) }\end{array}$ & $\begin{array}{l}\text { Depth to } \\
\text { Sump } \\
\text { Bottom (ft) }\end{array}$ & $\begin{array}{l}\text { Sump } \\
\text { Length } \\
\text { (ft) }\end{array}$ & $\begin{array}{c}\text { Sump } \\
\text { Volume } \\
\text { (L) }\end{array}$ & Comment \\
\hline 1 & 956 & 2532 & 5654 & 4078 & 1576 & 830 & 5780 & 2532 & 4078 & 2552 & 20 & 605.4 & Regional Aquifer \\
\hline
\end{tabular}

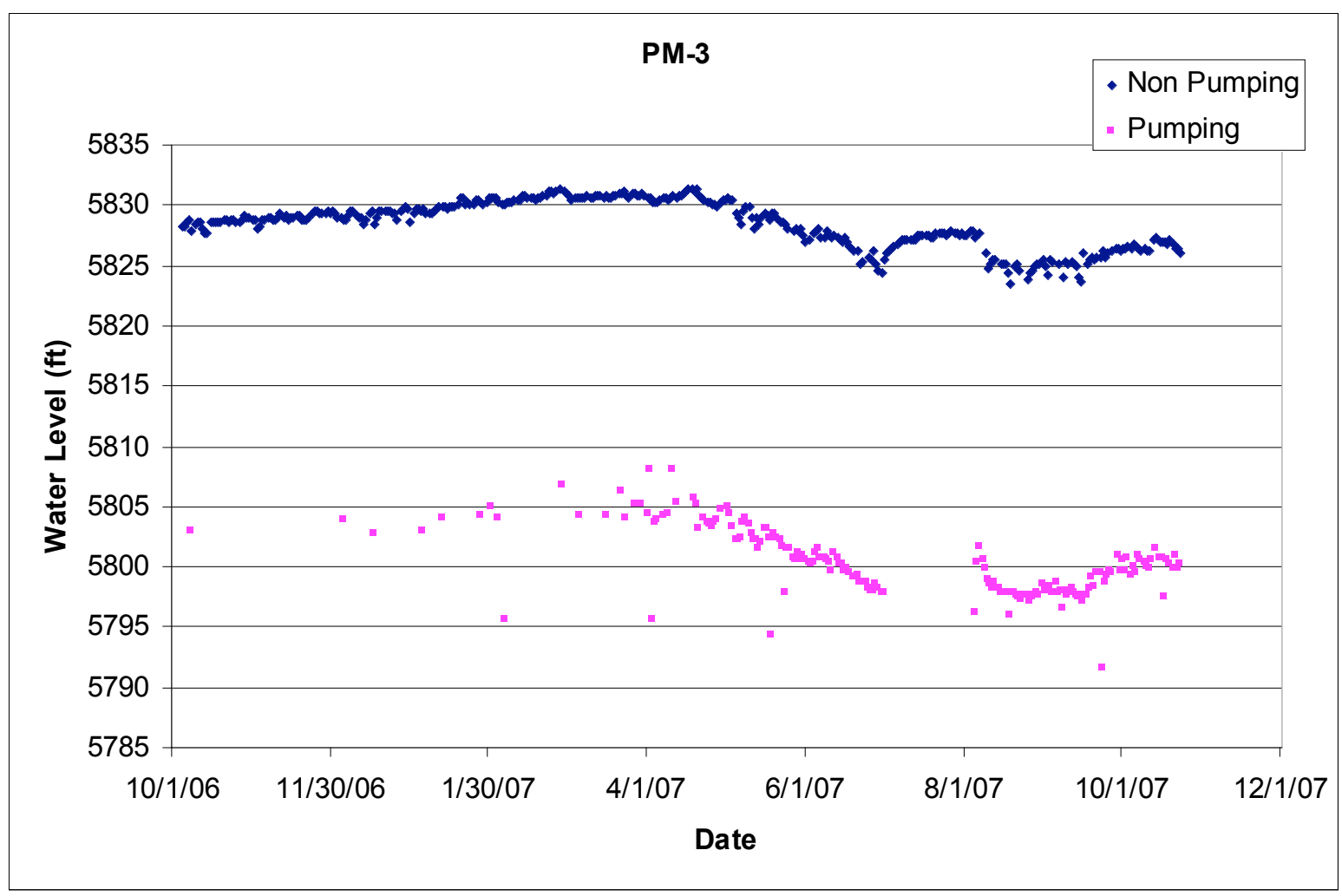




\subsection{PM-4}

Location: PM-4 is located on Mesita del Buey about mid way between supply wells PM-2 and PM-5.

The nearest monitoring well is R-15 in Mortandad Canyon, about 0.67 miles to the north.

Completion Type: Single completion in the Santa Fe Group.

Period of Record: Well completed as a supply well in August 1981; transducer installed August 2004.

The transducer failed in November 2006 and was replaced in April 2007; data through 2007.

Remarks: Well is powered by a natural gas motor and when used is operated continuously.

Drawdown in 2007 was about $40 \mathrm{ft}$. PM-4 responds to pumping at PM-2.

\begin{tabular}{|c|c|c|c|c|c|c|c|c|c|c|c|c|c|}
\hline \multicolumn{14}{|c|}{ PM-4 Construction Information } \\
\hline Zone & $\begin{array}{c}\text { Screen } \\
\text { Top } \\
\text { Depth (ft) }\end{array}$ & $\begin{array}{c}\text { Screen } \\
\text { Bottom } \\
\text { Depth } \\
\text { (ft) }\end{array}$ & $\begin{array}{c}\text { Screen } \\
\text { Top } \\
\text { Elev (ft) }\end{array}$ & $\begin{array}{l}\text { Screen } \\
\text { Bottom } \\
\text { Elev (ft) }\end{array}$ & $\begin{array}{l}\text { Screen } \\
\text { Length } \\
\text { (ft) }\end{array}$ & $\begin{array}{l}\text { Pump } \\
\text { Intake } \\
\text { Depth } \\
\text { (ft) }\end{array}$ & $\begin{array}{c}\text { Pump } \\
\text { Intake } \\
\text { Elevation } \\
\text { (ft) }\end{array}$ & $\begin{array}{c}\text { Depth to } \\
\text { Top of } \\
\text { Sump (ft) }\end{array}$ & $\begin{array}{c}\text { Top of } \\
\text { Sump } \\
\text { Elevation } \\
\text { (ft) }\end{array}$ & $\begin{array}{l}\text { Depth to } \\
\text { Sump } \\
\text { Bottom (ft) }\end{array}$ & $\begin{array}{l}\text { Sump } \\
\text { Length } \\
\text { (ft) }\end{array}$ & $\begin{array}{l}\text { Sump } \\
\text { Volume } \\
\text { (L) }\end{array}$ & Comment \\
\hline 1 & 1260 & 2854 & 5660 & 4066 & 1594 & 1210 & 5710 & 2854 & 4066 & 2874 & 20 & 790.8 & Regional Aquifer \\
\hline
\end{tabular}

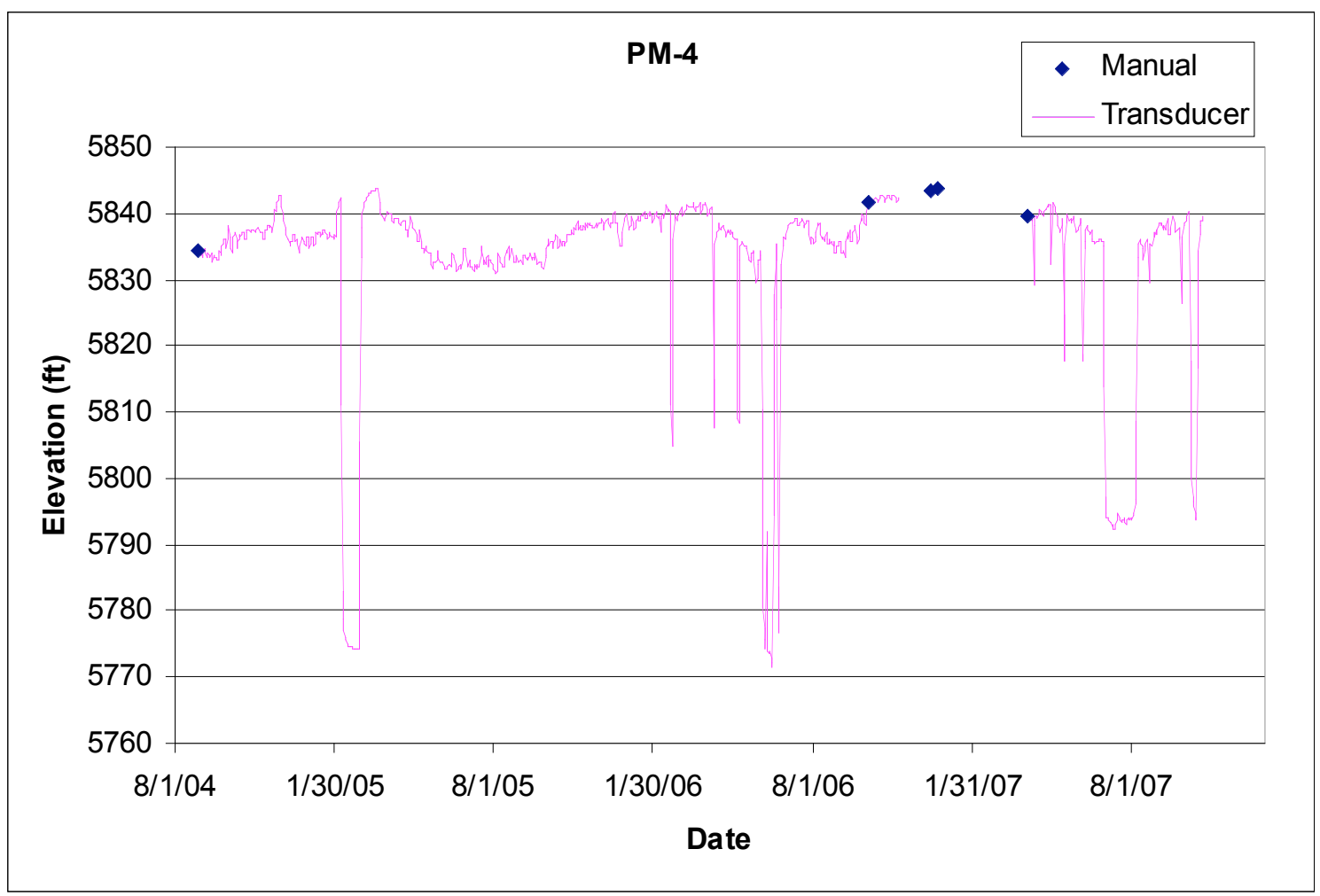

Note: mean daily water level values shown 


\subsection{PM-5}

Location: PM-5 is located on a mesa south of Ten Site and Mortandad canyons. The nearest monitoring well is R-33 in Ten Site Canyon about $1500 \mathrm{ft}$ to the northeast.

Completion Type: Single completion in the Santa Fe Group.

Period of Record: Well completed as a supply well in September 1982; transducer installed December 2004. The transducer failed in October 2006 and was replaced in April 2007; data through 2007.

Remarks: PM-5 responds to pumping PM-4. Drawdown is about $80 \mathrm{ft}$.

\begin{tabular}{|c|c|c|c|c|c|c|c|c|c|c|c|c|c|}
\hline \multicolumn{14}{|c|}{ PM-5 Construction Information } \\
\hline Zone & $\begin{array}{l}\text { Screen } \\
\text { Top } \\
\text { Depth (ft) }\end{array}$ & $\begin{array}{l}\text { Screen } \\
\text { Bottom } \\
\text { Depth } \\
\text { (ft) }\end{array}$ & $\begin{array}{l}\text { Screen } \\
\text { Top } \\
\text { Elev (ft) }\end{array}$ & $\begin{array}{l}\text { Screen } \\
\text { Bottom } \\
\text { Elev (ft) }\end{array}$ & $\begin{array}{l}\text { Screen } \\
\text { Length } \\
\text { (ft) }\end{array}$ & $\begin{array}{l}\text { Pump } \\
\text { Intake } \\
\text { Depth } \\
\text { (t) }\end{array}$ & $\begin{array}{l}\text { Pump } \\
\text { Intake } \\
\text { Elevation } \\
\text { (ft) }\end{array}$ & $\begin{array}{l}\text { Depth to } \\
\text { Top of } \\
\text { Sump (ft) }\end{array}$ & $\begin{array}{c}\text { Top of } \\
\text { Sump } \\
\text { Elevation } \\
\text { (ft) }\end{array}$ & $\begin{array}{l}\text { Depth to } \\
\text { Sump } \\
\text { Bottom (ft) }\end{array}$ & $\begin{array}{l}\text { Sump } \\
\text { Length } \\
\text { (ft) }\end{array}$ & $\begin{array}{l}\text { Sump } \\
\text { Volume } \\
\text { (L) }\end{array}$ & Comment \\
\hline 1 & 1440 & 3072 & 5655 & 4023 & 1632 & & 7095 & 3072 & 3072 & 3092 & 20 & 790.8 & egional Aquife \\
\hline
\end{tabular}

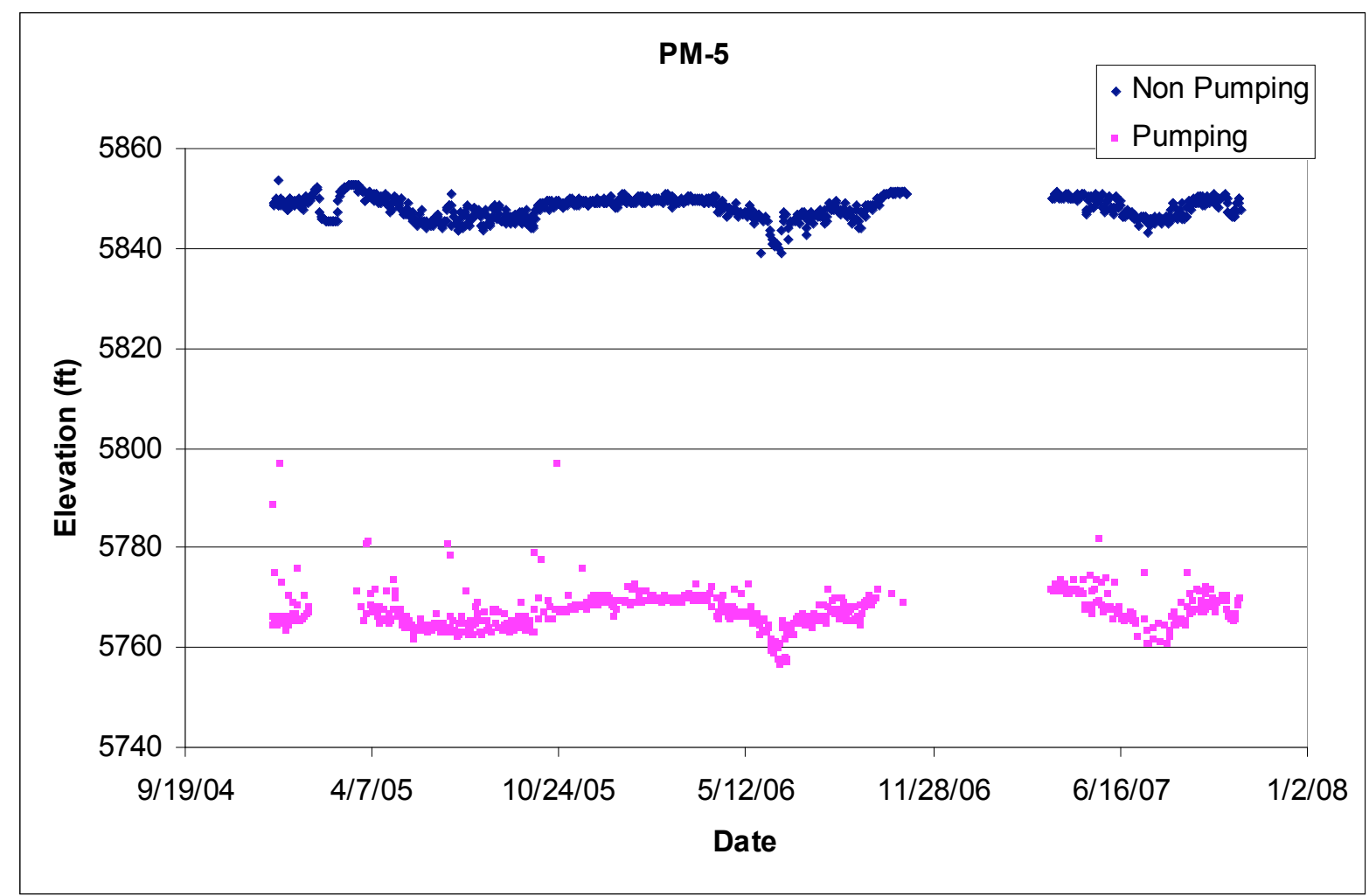




\subsection{Acknowledgments}

The authors would like to acknowledge all those whose work contributed to this report, especially the Water Stewardship Program Operations Team members who collected most of the groundwater level data: Consuelo Montoya and Ranee Onstott. Some data were also collected by the groundwater sampling team, Lisa Ansay, Alethea Banar, Jim Jordan, Zach Leonard, Max Maes, Bobbie Rappe, Jayson Romero, Rick Velasquez, and many others. Thanks to Armand Groffman, Matt Riggs, Mike Alexander, Tina Behr-Andres, Danny Katzman, and Tim Goering for programmatic support for the Groundwater Level Monitoring Project. David Rogers, Armand Groffman, and Tim Goering provided helpful reviews of the report. Thanks also to Hector Hinojosa for editing and compositional support.

\subsection{References and Bibliography}

The following reports and documents contain groundwater level data for wells at LANL.

Allen, S.A., and R.J. Koch, 2006, "Groundwater Level Status Report for 2005, Los Alamos National Laboratory," Los Alamos National Laboratory report LA-14292-PR, Los Alamos, New Mexico.

Allen, S.A., and R.J. Koch, 2007, "Groundwater Level Status Report for Fiscal Year 2006, Los Alamos National Laboratory," Los Alamos National Laboratory report LA-14331-PR, Los Alamos, New Mexico.

Ball, T., M. Everett, P. Longmire, D. Vaniman, W. Stone, D. Larssen, K. Greene, N. Clayton, and S. McLin, February 2002, "Characterization Well R-22 Completion Report," Los Alamos National Laboratory report LA-13893-MS, Los Alamos, New Mexico.

Broxton, D.E., R. Warren, D. Vaniman, B. Newman, A. Crowder, M. Everett, R. Gilkeson, P. Longmire, J. Marin, W. Stone, S. McLin, and D. Rogers, May 2001, "Characterization Well R-12 Completion Report," Los Alamos National Laboratory report LA-13822-MS, Los Alamos, New Mexico.

Broxton, D., R. Gilkeson, P. Longmire, J. Marin, R. Warren, D. Vaniman, A. Crowder, B. Newman, B. Lowry, D. Rogers, W. Stone, S. McLin, G. WoldeGabriel, D. Daymon, and D. Wycoff, May 2001, "Characterization Well R-9 Completion Report," Los Alamos National Laboratory report LA-13742MS, Los Alamos, New Mexico.

Broxton, D., D. Vaniman, W. Stone, S. McLin, J. Marin, R. Koch, R. Warren, P. Longmire, D. Rogers, and N. Tapia, May 2001, "Characterization Well R-19 Completion Report," Los Alamos National Laboratory report LA-13823-MS, Los Alamos, New Mexico.

Broxton, D., R. Warren, P. Longmire, R. Gilkeson, S. Johnson, D. Rogers, W. Stone, B. Newman, M. Everett, D. Vaniman, S. McLin, J. Skalski, and D. Larssen, March 2002, "Characterization Well R-25 Completion Report," Los Alamos National Laboratory report LA-13909-MS, Los Alamos, New Mexico.

Gray, R.N., November 2001, "Report on Alluvial Well Completions 1994-2001," Prepared for the Environmental Restoration Project, Los Alamos National Laboratory, 56 pages with appendices.

Keating, E., and R. Kelley, 2005, "Groundwater Elevation Contour Map of the Top of the Regional Aquifer in the Vicinity of LANL," Los Alamos National Laboratory report LA-UR-05-0455, Los Alamos, New Mexico.

Kleinfelder, March 19, 2004, "Final Completion Report, Characterization Well R-11 Los Alamos National Laboratory, Los Alamos, New Mexico," Project No. 37151. Prepared for the United States Department of Energy and the National Nuclear Security Administration through the United States Army Corps of Engineers, Sacramento District. Prepared by Kleinfelder, Inc., 8300 Jefferson NE, Ste B, Albuquerque, NM 87113, 27 pages, with appendices. 
Kleinfelder, March 23, 2004, "Final Completion Report, Characterization Well R-4, Los Alamos National Laboratory, Los Alamos, New Mexico," Project No. 37151/7.12. Prepared for the United States Department of Energy and the National Nuclear Security Administration through the United States Army Corps of Engineers, Sacramento District. Prepared by Kleinfelder, Inc., 8300 Jefferson NE, Ste B, Albuquerque, NM 87113, 29 pages, with appendices.

Kleinfelder, April 5, 2004, "Final, Well R-2 Completion Report, Los Alamos National Laboratory, Los Alamos, New Mexico," Project No. 37151/Task 11, Document No. ALB04RP001. Prepared for the United States Department of Energy and the National Nuclear Security Administration through the United States Army Corps of Engineers, Sacramento District. Prepared by Kleinfelder, Inc., 8300 Jefferson NE, Ste B, Albuquerque, NM 87113, 17 pages, with appendices.

Kleinfelder, April 19, 2004, "Final Well R-1 Completion Report, Los Alamos National Laboratory, Los Alamos, New Mexico," Project No. 37151/17.12. Prepared for the United States Department of Energy and the National Nuclear Security Administration through the United States Army Corps of Engineers, Sacramento District. Prepared by Kleinfelder, Inc., 8300 Jefferson NE, Ste B, Albuquerque, NM 87113, 20 pages, with appendices.

Kleinfelder, April 28, 2004, "Final Well R-28 Completion Report, Los Alamos National Laboratory, Los Alamos, New Mexico," Project No. 37151/16.12. Prepared for the United States Department of Energy and the National Nuclear Security Administration through the United States Army Corps of Engineers, Sacramento District. Prepared by Kleinfelder, Inc., 8300 Jefferson NE, Ste B, Albuquerque, NM 87113, 22 pages, with appendices.

Kleinfelder, May 7, 2004, "Final, Well CdV-16-1(i) Completion Report, Los Alamos National Laboratory, Los Alamos, New Mexico," Project No. 37151/9.12. Prepared for the United States Department of Energy and the National Nuclear Security Administration through the United States Army Corps of Engineers, Sacramento District. Prepared by Kleinfelder, Inc., 8300 Jefferson NE, Ste B, Albuquerque, NM 87113, 22 pages, with appendices.

Kleinfelder, May 14, 2004, "Final, Well CdV-16-2(i) Completion Report, Los Alamos National Laboratory, Los Alamos, New Mexico," Project No. 37151/10.12. Prepared for the United States Department of Energy and the National Nuclear Security Administration through the United States Army Corps of Engineers, Sacramento District. Prepared by Kleinfelder, Inc., 8300 Jefferson NE, Ste B, Albuquerque, NM 87113, 20 pages, with appendices.

Kleinfelder, May 18, 2004, "Final, Borehole CdV-16-3(i) Completion Report, Los Alamos National Laboratory, Los Alamos, New Mexico," Project No. 37151/11.12. Prepared for the United States Department of Energy and the National Nuclear Security Administration through the United States Army Corps of Engineers, Sacramento District. Prepared by Kleinfelder, Inc., 8300 Jefferson NE, Ste B, Albuquerque, NM 87113, 17 pages, with appendices.

Kleinfelder, November 2004, "Final Completion Report, Characterization Well R-34, Los Alamos National Laboratory, Los Alamos, New Mexico," Project No. 37151. Prepared for the United States Department of Energy and the National Nuclear Security Administration through the United States Army Corps of Engineers, Sacramento District. Prepared by Kleinfelder, Inc., 8300 Jefferson NE, Ste B, Albuquerque, NM 87113, 27 pages, with appendices.

Kleinfelder, January 2006, "Final Completion Report, Characterization Wells R-10a/R-10, Los Alamos National Laboratory, Los Alamos, New Mexico," Project No. 49436. Prepared for the United States Department of Energy and the National Nuclear Security Administration through the United States Army Corps of Engineers, Sacramento District. Prepared by Kleinfelder, Inc., 8300 Jefferson NE, Ste B, Albuquerque, NM 87113, 32 pages, with appendices. 
Kleinfelder, January 2006, "Final Completion Report, Intermediate Well LAOI-7, Los Alamos National Laboratory, Los Alamos, New Mexico," Project No. 49436. Prepared for the United States Department of Energy and the National Nuclear Security Administration through the United States Army Corps of Engineers, Sacramento District. Prepared by Kleinfelder, Inc., 8300 Jefferson NE, Ste B, Albuquerque, NM 87113, 32 pages, with appendices. Kleinfelder 2006a.

Koch, R.J., and D.B. Rogers, 2003, "Water Supply at Los Alamos, 1998-2001," Los Alamos National Laboratory report LA-13985-PR, Los Alamos, New Mexico, 28 pp.

Koch, R.J., D.B. Rogers, N.J. Tapia, and S.G. McLin, 2004, "Manual and Transducer Groundwater Levels from Test Wells at Los Alamos National Laboratory, 1992-2003," Los Alamos National Laboratory report LA-14132, Los Alamos, New Mexico, 36 pp.

Kopp, H.W., A.J. Crowder, M.C. Everett, D.T. Vaniman, D.D. Hickmott, W.J. Stone, N. Clayton, S.G. Pearson, and D.E. Larssen, April 2002, "Well CdV-R-15-3 Completion Report," Los Alamos National Laboratory report LA-13906-MS, Los Alamos, New Mexico.

LANL (Los Alamos National Laboratory), May 22, 1998, "Hydrogeologic Workplan," Los Alamos, New Mexico.

LANL (Los Alamos National Laboratory), September 2003, "Phase III RFI Report for SWMU 16021(c)-99," Los Alamos National Laboratory report LA-UR-03-5248, Los Alamos, New Mexico.

LANL (Los Alamos National Laboratory), April 2004, "Los Alamos and Pueblo Canyons Investigation Report," Los Alamos National Laboratory report LA-UR-04-2714, Los Alamos, New Mexico.

LANL (Los Alamos National Laboratory), January 27, 2005, "Groundwater Level Data Submittal to NMED," Los Alamos National Laboratory report LA-UR-05-0457, Los Alamos, New Mexico.

LANL (Los Alamos National Laboratory), March 2005, "Groundwater Level Monitoring Plan and Field Implementation Plan for the Groundwater Level Monitoring Project, 2005," draft unpublished monitoring plan, Water Quality and Hydrology Group, Environmental Stewardship Division, Los Alamos National Laboratory, Los Alamos, New Mexico.

LANL (Los Alamos National Laboratory), June 2006, "2006 Hydrogeologic Site Atlas," Los Alamos National Laboratory report LA-UR-06-3058, Los Alamos, New Mexico.

LANL (Los Alamos National Laboratory), 2006, "Quality Assurance Project Plan for the Groundwater Level Monitoring Project," Environmental Remediation and Support Services quality document EPERSS-WSP-1003, available online at http://erinternal.lanl.gov/procedures/water procedures.shtml.

LANL (Los Alamos National Laboratory), March 2007, "General Facility Information," Los Alamos National Laboratory report LA-UR-07-1837, Los Alamos, New Mexico.

LANL (Los Alamos National Laboratory), 2007, "Completion Report for Regional Aquifer Wells R-35a and R-35b," Los Alamos National Laboratory report LA-UR-07-5324, Los Alamos, New Mexico.

Longmire, P., D. Broxton, W. Stone, B. Newman, R. Gilkeson, J. Marin, D. Vaniman, D. Counce, D. Rogers, R. Hull, S. McLin, and R. Warren, May 2001, "Characterization Well R-15 Completion Report," Los Alamos National Laboratory report LA-13749-MS, Los Alamos, New Mexico.

McLin, S.G., September 1996, "Analysis of Water Level Fluctuations in Pajarito Plateau Wells," in New Mexico Geological Society Guidebook, 47th Field Conference, Jemez Mountains Region, New Mexico, pp. 421-426. 
McLin, S.G., W.D. Purtymun, and M.N. Maes, April 1997, "Water Supply at Los Alamos during 1995," Los Alamos National Laboratory report LA-13216-PR, Los Alamos, New Mexico.

McLin, S.G., W.D. Purtymun, and M.N. Maes, December 1998, "Water Supply at Los Alamos during 1997," Los Alamos National Laboratory report LA-13548-PR, Los Alamos, New Mexico.

McLin, S.G., July 2005, "Analyses of the PM-2 Aquifer Test Using Multiple Observation Wells," Los Alamos National Laboratory report LA-14225-MS, Los Alamos, New Mexico.

McLin, S.G., January 2006, "Analyses of the PM-4 Aquifer Test Using Multiple Observation Wells," Los Alamos National Laboratory report LA-14252-MS, Los Alamos, New Mexico.

Nylander, C., T. Ball, K. Bitner, K. Henning, E. Keating, P. Longmire, B. Robinson, D. Rogers, W. Stone, and D. Vaniman, April 2002, "Groundwater Annual Status Report for Fiscal Year 2001," Los Alamos National Laboratory report LA-13931-SR, Los Alamos, New Mexico.

Purtymun, W.D., and J.W. Herceg, 1972, "Summary of the Los Alamos Municipal Well-Field Characteristics, 1947-1971," Los Alamos Scientific Laboratory report LA-5040-MS, Los Alamos, New Mexico.

Purtymun, W.D., and J.W. Herceg, 1976, "Water Supply at Los Alamos during 1975," Los Alamos Scientific Laboratory report LA-6461-MS, Los Alamos, New Mexico.

Purtymun, W.D., 1984, "Hydrologic Characteristics of the Main Aquifer in the Los Alamos Area: Development of Groundwater Supplies," Los Alamos National Laboratory report LA-9957-MS, Los Alamos, New Mexico.

Purtymun, W.D., and A.K. Stoker, 1988, "Current Status of Wells and Future Water Supply," Los Alamos National Laboratory report LA-11332-MS, Los Alamos, New Mexico.

Purtymun, W.D., 1995, "Geologic and Hydrologic Records of Observation Wells, Test Holes, Test Wells, Supply Wells, Springs, and Surface Water Stations in the Los Alamos Area," Los Alamos National Laboratory report LA-12883-MS, Los Alamos, New Mexico.

Rogers, D.B., and R.J. Koch, 2005, "Revised Preliminary Map of Annual Water Level Decline Rate at LANL," Los Alamos National Laboratory report LA-UR-05-4456, Los Alamos, New Mexico.

Shomaker \& Associates, January 1999, "Well Report: Construction and Testing, Guaje Replacement Wells GR-1, GR-2, GR-3, and GR-4, Santa Fe County, New Mexico," John Shomaker \& Associates, Inc., report prepared for University of California, Los Alamos National Laboratory, Los Alamos, New Mexico, and Chavez-Grieves Consulting Engineers, Inc., Albuquerque, New Mexico.

Stone, W.J., D.T. Vaniman, P. Longmire, D.E. Broxton, M.C. Everett, R. Lawrence, and D.E. Larssen, April, 2002, "Characterization Well R-7 Completion Report," Los Alamos National Laboratory report LA-13932-MS, Los Alamos, New Mexico.

Vaniman, D., J. Marin, W. Stone, B. Newman, P. Longmire, N. Clayton, R. Lewis, R. Koch, S. McLin, G. WoldeGabriel, D. Counce, D. Rogers, R. Warren, E. Kluk, S. Chipera, D. Larssen, and W. Kopp, March 2002, "Characterization Well R-31 Completion Report," Los Alamos National Laboratory report LA-13910-MS, Los Alamos, New Mexico. 
Appendix A

Mean Annual Water Level at the Surface of the Regional Aquifer in LANL Monitoring Wells for 2007

\begin{tabular}{|c|c|c|c|c|}
\hline Well Name & $\begin{array}{c}\text { Top of } \\
\text { Regional } \\
\text { Aquifer (ft) }\end{array}$ & $\begin{array}{l}\text { No. of } \\
\text { Data } \\
\text { Values }\end{array}$ & $\begin{array}{c}\text { Std. Dev. } \\
\text { (ft) }\end{array}$ & $\begin{array}{c}\text { Last Data } \\
\text { Date }\end{array}$ \\
\hline CDV-R-15-3 & 6019.7 & 6306 & 0.04 & $10 / 18 / 07$ \\
\hline CDV-R-37-2 & 6137.1 & 8045 & 0.04 & $12 / 31 / 07$ \\
\hline G-3 & 5743.9 & 8757 & 11.66 & $12 / 31 / 07$ \\
\hline $\mathrm{R}-1$ & 5878.7 & 7954 & 0.48 & $11 / 28 / 07$ \\
\hline $\mathrm{R}-10 \mathrm{a}$ & 5740.4 & 8250 & 1.58 & $11 / 30 / 07$ \\
\hline R-11 & 5838.0 & 10251 & 0.37 & $12 / 4 / 07$ \\
\hline$R-12$ & No data & & & \\
\hline$R-13$ & 5836.0 & 10090 & 0.33 & $11 / 27 / 07$ \\
\hline $\mathrm{R}-14$ & 5883.4 & 7106 & 0.46 & $12 / 5 / 07$ \\
\hline $\mathrm{R}-15$ & 5850.5 & 7953 & 1.03 & $11 / 28 / 07$ \\
\hline R-16r & 5692.6 & 8000 & 0.19 & $11 / 30 / 07$ \\
\hline R-17 & 5884.7 & 6782 & 0.32 & $10 / 17 / 07$ \\
\hline $\mathrm{R}-18$ & 6117.0 & 6795 & 0.20 & $10 / 11 / 07$ \\
\hline $\mathrm{R}-19$ & 5887.5 & 3797 & 0.10 & $7 / 17 / 07$ \\
\hline R-2 & 5871.1 & 8757 & 0.30 & $12 / 31 / 07$ \\
\hline $\mathrm{R}-20$ & No data & & & \\
\hline $\mathrm{R}-21$ & 5854.2 & 7617 & 0.67 & $11 / 25 / 07$ \\
\hline $\mathrm{R}-22$ & 5762.2 & 7551 & 0.13 & $12 / 20 / 07$ \\
\hline $\mathrm{R}-23$ & 5697.6 & 6803 & 0.16 & $10 / 11 / 07$ \\
\hline R-24 & 5830.0 & 8757 & 2.03 & $12 / 31 / 07$ \\
\hline R-25 & 6234.4 & 6698 & 1.30 & $12 / 31 / 07$ \\
\hline R-26 & 6539.4 & 5024 & 2.28 & $10 / 18 / 07$ \\
\hline $\mathrm{R}-27$ & 5898.1 & 8755 & 0.21 & $12 / 31 / 07$ \\
\hline $\mathrm{R}-28$ & 5838.4 & 12032 & 0.41 & $12 / 4 / 07$ \\
\hline R-31 & 5827.7 & 8216 & 0.13 & $12 / 31 / 07$ \\
\hline R-32 & 5858.8 & 5413 & 0.09 & $8 / 31 / 07$ \\
\hline R-33 & No data & & & \\
\hline R-34 & 5834.8 & 8000 & 0.38 & $11 / 30 / 07$ \\
\hline$R-35 b$ & 5837.1 & 6243 & 0.14 & $12 / 31 / 07$ \\
\hline R-4 & 5831.5 & 8738 & 0.96 & $12 / 31 / 07$ \\
\hline $\mathrm{R}-5$ & 5767.2 & 9911 & 0.17 & $12 / 31 / 07$ \\
\hline$\overline{R-6}$ & 5838.9 & 8437 & 0.64 & $12 / 18 / 07$ \\
\hline R-7 & 5877.8 & 8277 & 0.12 & $12 / 18 / 07$ \\
\hline $\mathrm{R}-8$ & 5853.5 & 9286 & 0.95 & $12 / 31 / 07$ \\
\hline R-9 & 5691.7 & 10598 & 0.16 & $12 / 18 / 07$ \\
\hline Test Well 8 & 5875.0 & 7952 & 0.37 & $11 / 28 / 07$ \\
\hline Test Well DT-10 & 5918.8 & 8336 & 0.11 & $12 / 31 / 07$ \\
\hline Test Well DT-5A & 5958.1 & 8758 & 0.25 & $12 / 31 / 07$ \\
\hline Test Well DT-9 & 5915.2 & 8756 & 0.11 & $12 / 31 / 07$ \\
\hline
\end{tabular}




\section{Appendix B \\ Summary of Transient Responses to Supply Well Pumping in LANL Monitoring Wells}

\begin{tabular}{|c|c|c|c|c|c|c|c|c|c|c|c|}
\hline Well & Screen & $\begin{array}{l}\text { Seasonal } \\
\text { Response }\end{array}$ & Guaje & Otowi 1 & Otowi 4 & PM-1 & PM-2 & PM-3 & PM-4 & PM-5 & Comment \\
\hline CdV-R-15-3 & 4 & Yes & $\mathrm{NE}$ & $\mathrm{NE}$ & $\mathrm{NE}$ & $\mathrm{NE}$ & No & $\mathrm{NE}$ & No & No & Seasonal responæ notrelated to pumping \\
\hline$\overline{C d V-R-15-3}$ & 5 & Yes & $\overline{\mathrm{NE}}$ & $\overline{\mathrm{NE}}$ & $\mathrm{NE}$ & $\mathrm{NE}$ & No & $\overline{\mathrm{NE}}$ & No & No & Seasonal response notrelated to pumping \\
\hline CdV-R-15-3 & 6 & Yes & $\mathrm{NE}$ & $\overline{\mathrm{NE}}$ & $\mathrm{NE}$ & $\mathrm{NE}$ & No & $\overline{\mathrm{NE}}$ & No & Possible & Seasonal responæ notrelated to pumping \\
\hline CdV-R-37-2 & 2 & Yes & $\mathrm{NE}$ & $\mathrm{NE}$ & $\mathrm{NE}$ & $\mathrm{NE}$ & No & $\mathrm{NE}$ & No & No & Seasonal response notrelated to pumping \\
\hline CdV-R-37-2 & 3 & Yes & $\mathrm{NE}$ & $\mathrm{NE}$ & $\mathrm{NE}$ & $\mathrm{NE}$ & No & $\mathrm{NE}$ & No & No & Seasonal responæe notrelated to pumping \\
\hline CdV-R-37-2 & 4 & Yes & $\mathrm{NE}$ & $\mathrm{NE}$ & $\mathrm{NE}$ & $\mathrm{NE}$ & No & $\mathrm{NE}$ & No & No & Seasonal responæe notrelated to pumping \\
\hline G-3 & Single & Yes & $\overline{Y e s}$ & $\mathrm{NE}$ & $\mathrm{NE}$ & $\mathrm{NE}$ & $\mathrm{NE}$ & $\mathrm{NE}$ & $\mathrm{NE}$ & $\mathrm{NE}$ & Guaje well field monitoring well \\
\hline $\mathrm{R}-1$ & Single & Yes & $\mathrm{NE}$ & $\mathrm{NE}$ & Possible & $\mathrm{NE}$ & No & No & Possible & Yes & Primarily respondsto PM-5 \\
\hline$\overline{\mathrm{R}-2}$ & Single & No & No & $\mathrm{NE}$ & No & $\mathrm{NE}$ & $\mathrm{NE}$ & $\mathrm{NE}$ & $\mathrm{NE}$ & $\mathrm{NE}$ & Gradual decline of about $0.5 f t y r$ \\
\hline $\mathrm{R}-4$ & Single & Yes & Possible & No & Possible & $\mathrm{NE}$ & $\mathrm{NE}$ & Yes & $\mathrm{NE}$ & $\mathrm{NE}$ & Seasonal response butnot to a specific well \\
\hline $\mathrm{R}-5$ & 3 & No & No & No & No & No & $\mathrm{NE}$ & No & $\mathrm{NE}$ & $\mathrm{NE}$ & Gradual decline ofabout $0.6 \mathrm{ft} / \mathrm{r}$ \\
\hline R-5 & 4 & Yes & No & Possible & No & Yes & $\mathrm{NE}$ & No & $\mathrm{NE}$ & $\mathrm{NE}$ & Seasonal response butnot to a specific well \\
\hline $\mathrm{R}-6$ & Single & Yes & No & No & Possible & $\mathrm{NE}$ & $\mathrm{NE}$ & Yes & $\mathrm{NE}$ & No & Seasonal responæe butnot to a specific well \\
\hline R-7 & 3 & No & No & No & No & $\mathrm{NE}$ & $\mathrm{NE}$ & No & $\mathrm{NE}$ & $\mathrm{NE}$ & Gradual decline of about $0.5 \mathrm{ft} / \mathrm{rr}$ \\
\hline R-8 & 1 & Yes & No & $\mathrm{NE}$ & Possible & No & $\mathrm{NE}$ & Yes & $\mathrm{NE}$ & $\mathrm{NE}$ & Respondsprimarily to pumping atPM-3 \\
\hline$\overline{\mathrm{R}-8}$ & 2 & Yes & No & $\overline{\mathrm{NE}}$ & Possible & No & $\overline{\mathrm{NE}}$ & Yes & $\overline{\mathrm{NE}}$ & $\mathrm{NE}$ & Respondsprimarily to pumping atPM-3 \\
\hline R-9 & Single & Yes & No & No & $\mathrm{NE}$ & No & $\mathrm{NE}$ & No & $\mathrm{NE}$ & $\mathrm{NE}$ & Gradual decline of about $0.4 \mathrm{rthyr}$ \\
\hline $\mathrm{R}-10$ & 1 & ID & $\mathrm{NE}$ & $\mathrm{NE}$ & $\mathrm{NE}$ & Yes & $\mathrm{NE}$ & $\mathrm{NE}$ & $\mathrm{NE}$ & $\mathrm{NE}$ & Respondsprimarily to PM-1 \\
\hline $\mathrm{R}-10$ & 2 & $\mathbb{I D}$ & ID & ID & $\mathbb{I D}$ & $\mathbb{D}$ & $\mathbb{I D}$ & $\mathbb{I D}$ & $\mathbb{I D}$ & ID & Nowater level data as of $01 / 08$ \\
\hline R-10a & Single & No & $\mathrm{NE}$ & $\mathrm{NE}$ & $\mathrm{NE}$ & No & $\mathrm{NE}$ & No & $\mathrm{NE}$ & $\mathrm{NE}$ & No apparentresponঙ to pumping \\
\hline$\overline{R-11}$ & Single & Yes & $\mathrm{NE}$ & $\mathrm{NE}$ & No & $\mathrm{NE}$ & Possible & No & No & Possible & Seasonal responæ butnot to a specific well \\
\hline $\mathrm{R}-12$ & 3 & No & No & No & No & No & $\mathrm{NE}$ & No & No & No & No apparentresponæe to nearby well PM-1 \\
\hline $\mathrm{R}-13$ & Single & Yes & $\mathrm{NE}$ & $\mathrm{NE}$ & No & No & Possible & No & Yes & Possible & Seasonal responæe butnotto a specific well \\
\hline$\overline{R-14}$ & 1 & Yes & $\overline{\mathrm{NE}}$ & $\overline{\mathrm{NE}}$ & Possible & $\overline{\mathrm{NE}}$ & Possible & $\overline{\mathrm{NE}}$ & No & Yes & Respondsprimarily to PM-5 \\
\hline R-14 & 2 & Yes & $\mathrm{NE}$ & $\mathrm{NE}$ & Possible & $\mathrm{NE}$ & Possible & $\mathrm{NE}$ & No & Yes & Respondsprimarily to PM-5 \\
\hline$\overline{R-15}$ & Single & Yes & $\overline{\mathrm{NE}}$ & $\overline{\mathrm{NE}}$ & Possible & $\overline{\mathrm{NE}}$ & No & No & Yes & $\overline{Y e s}$ & Respondsprimarily to pumping atPM-4 and PM-5 \\
\hline $\mathrm{R}-16$ & 2 & No & $\mathrm{NE}$ & $\mathrm{NE}$ & NE & No & No & $\mathrm{NE}$ & NE & $\mathrm{NE}$ & No apparentresponse to Buckman pumping \\
\hline $\mathrm{R}-16$ & 3 & No & $\mathrm{NE}$ & $\mathrm{NE}$ & $\mathrm{NE}$ & No & No & $\mathrm{NE}$ & $\mathrm{NE}$ & $\mathrm{NE}$ & No apparentresponse to Buckman pumping \\
\hline $\mathrm{R}-16$ & 4 & No & $\mathrm{NE}$ & $\mathrm{NE}$ & $\mathrm{NE}$ & No & No & $\mathrm{NE}$ & NE & $\mathrm{NE}$ & No apparentresponse to Buckman pumping \\
\hline$R-16 r$ & Single & No & No & No & No & No & No & No & No & No & No apparentresponse to Buckman pumping \\
\hline $\mathrm{R}-17$ & 1 & Yes & $\mathrm{NE}$ & $\mathrm{NE}$ & $\mathrm{NE}$ & $\mathrm{NE}$ & No & $\mathrm{NE}$ & No & No & Seasonal response butnot to a specific well \\
\hline $\mathrm{R}-17$ & 2 & Yes & $\mathrm{NE}$ & $\mathrm{NE}$ & $\mathrm{NE}$ & $\mathrm{NE}$ & Possible & $\mathrm{NE}$ & Possible & Yes & Respondsprimarily to pumping atPM-5 \\
\hline$\overline{R-18}$ & Single & No & $\overline{\mathrm{NE}}$ & $\overline{\mathrm{NE}}$ & $\overline{\mathrm{NE}}$ & $\overline{\mathrm{NE}}$ & No & $\overline{\mathrm{NE}}$ & No & No & No apparentresponæ to pumping \\
\hline $\mathrm{R}-19$ & 3 & Yes & $\mathrm{NE}$ & $\mathrm{NE}$ & $\mathrm{NE}$ & $\mathrm{NE}$ & Possible & $\mathrm{NE}$ & No & Possible & Muted respons \\
\hline$\overline{R-19}$ & 4 & Yes & $\overline{\mathrm{NE}}$ & $\mathrm{NE}$ & $\mathrm{NE}$ & $\overline{\mathrm{NE}}$ & Yes & $\mathrm{NE}$ & Yes & Possible & Respondsprimarily to PM-2 \\
\hline $\mathrm{R}-19$ & 5 & Yes & $\mathrm{NE}$ & $\mathrm{NE}$ & $\mathrm{NE}$ & $\mathrm{NE}$ & Yes & $\mathrm{NE}$ & Yes & Possible & Respondsprimarily to PM-2 \\
\hline$\overline{R-19}$ & 6 & Yes & $\overline{\mathrm{NE}}$ & $\mathrm{NE}$ & $\mathrm{NE}$ & $\mathrm{NE}$ & Yes & $\overline{\mathrm{NE}}$ & Yes & Possible & Respondsprimarily to PM-2 \\
\hline$\overline{R-19}$ & 7 & Yes & $\mathrm{NE}$ & $\mathrm{NE}$ & $\mathrm{NE}$ & $\overline{\mathrm{NE}}$ & Yes & $\overline{\mathrm{NE}}$ & Yes & Possible & Respondsprimarily to PM-2 \\
\hline R-20 & 1 & Yes & $\mathrm{NE}$ & $\mathrm{NE}$ & $\mathrm{NE}$ & $\mathrm{NE}$ & Yes & $\mathrm{NE}$ & Yes & $\mathrm{NE}$ & Highly muted responঙ \\
\hline$\overline{\mathrm{R}-20}$ & 2 & Yes & $\mathrm{NE}$ & $\mathrm{NE}$ & $\overline{\mathrm{NE}}$ & $\mathrm{NE}$ & Yes & $\mathrm{NE}$ & Yes & $\overline{\mathrm{NE}}$ & Muted response \\
\hline R-20 & 3 & Yes & $\mathrm{NE}$ & $\mathrm{NE}$ & $\mathrm{NE}$ & $\mathrm{NE}$ & Yes & $\mathrm{NE}$ & Yes & $\mathrm{NE}$ & Respondsprimarily to PM-2 butalso to PM-4 \\
\hline$\overline{\mathrm{R}-21}$ & Single & Yes & $\mathrm{NE}$ & $\mathrm{NE}$ & $\overline{\mathrm{NE}}$ & No & Yes & No & Possible & $\overline{\mathrm{NE}}$ & Seasonal responæe butnotto a specific well \\
\hline R-22 & 1 & Yes & $\mathrm{NE}$ & $\mathrm{NE}$ & $\mathrm{NE}$ & No & No & No & No & $\mathrm{NE}$ & Seasonal response butnotto a specific well \\
\hline $\mathrm{R}-22$ & 2 & Yes & $\overline{\mathrm{NE}}$ & $\mathrm{NE}$ & $\mathrm{NE}$ & No & No & No & No & $\mathrm{NE}$ & Seasonal response butnotto a specific well \\
\hline$\overline{R-22}$ & 3 & Yes & $\overline{\mathrm{NE}}$ & $\overline{\mathrm{NE}}$ & $\mathrm{NE}$ & No & No & No & No & $\overline{\mathrm{NE}}$ & Seasonal responæe butnotto a specific well \\
\hline $\mathrm{R}-22$ & 4 & Yes & $\mathrm{NE}$ & $\mathrm{NE}$ & $\mathrm{NE}$ & No & No & No & No & $\mathrm{NE}$ & Seasonal responæe butnotto a specific well \\
\hline$\overline{\mathrm{R}-22}$ & 5 & Yes & $\overline{\mathrm{NE}}$ & $\overline{\mathrm{NE}}$ & $\mathrm{NE}$ & No & No & No & No & $\mathrm{NE}$ & Seasonal response butnotto a specific well \\
\hline R-23 & Single & No & NE & $\mathrm{NE}$ & NE & No & No & $\mathrm{NE}$ & $\mathrm{NE}$ & $\mathrm{NE}$ & No apparentrespons to pumping \\
\hline$\overline{\mathrm{R}-24}$ & Single & Yes & Possible & No & Possible & No & $\overline{\mathrm{NE}}$ & Yes & $\mathrm{NE}$ & $\mathrm{NE}$ & Respondsprimarily to pumping atPM-3 \\
\hline R-25 & 5 & No & $\mathrm{NE}$ & $\mathrm{NE}$ & $\mathrm{NE}$ & $\mathrm{NE}$ & No & No & No & No & No apparentresponæe to pumping \\
\hline $\mathrm{R}-25$ & 6 & No & $\mathrm{NE}$ & $\mathrm{NE}$ & $\mathrm{NE}$ & $\mathrm{NE}$ & No & No & No & No & No apparentresponse to pumping \\
\hline$\overline{R-25}$ & 7 & No & $\overline{\mathrm{NE}}$ & $\overline{\mathrm{NE}}$ & $\overline{\mathrm{NE}}$ & $\overline{N E}$ & No & No & No & No & No apparentresponæe to pumping \\
\hline R-25 & 8 & No & $\mathrm{NE}$ & NE & NE & $\mathrm{NE}$ & No & No & No & No & No apparentresponæe to pumping \\
\hline$\overline{R-26}$ & 2 & No & $\overline{\mathrm{NE}}$ & $\overline{\mathrm{NE}}$ & $\mathrm{NE}$ & $\overline{\mathrm{NE}}$ & No & No & No & No & No apparentrespons to pumping \\
\hline R-27 & Single & Yes & $\mathrm{NE}$ & $\mathrm{NE}$ & $\mathrm{NE}$ & $\mathrm{NE}$ & $\mathrm{NE}$ & $\mathrm{NE}$ & $\mathrm{NE}$ & $\mathrm{NE}$ & Seasonal responæe butnotto a specific well \\
\hline$\overline{\mathrm{R}-28}$ & Single & Yes & $\overline{\mathrm{NE}}$ & $\overline{\mathrm{NE}}$ & $\overline{\mathrm{NE}}$ & $\overline{\mathrm{NE}}$ & Possible & No & Yes & Possible & Seasonal response butnotto a specific well \\
\hline R-31 & 2 & No & NE & NE & NE & No & No & No & No & NE & No apparentresponse to pumping \\
\hline$\overline{R-31}$ & 3 & No & $\mathrm{NE}$ & $\mathrm{NE}$ & $\mathrm{NE}$ & No & No & No & No & $\mathrm{NE}$ & No apparentrespons to pumping \\
\hline$\overline{R-31}$ & 4 & No & $\mathrm{NE}$ & $\mathrm{NE}$ & $\mathrm{NE}$ & No & Possible & No & No & $\mathrm{NE}$ & Limited data for evaluation \\
\hline $\mathrm{R}-31$ & 5 & Yes & $\mathrm{NE}$ & $\mathrm{NE}$ & $\mathrm{NE}$ & No & Yes & No & Possible & $\mathrm{NE}$ & Appearsto respond seasonally likePM-2 \\
\hline $\bar{R}-32$ & 1 & No & $\mathrm{NE}$ & $\mathrm{NE}$ & $\mathrm{NE}$ & $\mathrm{NE}$ & No & No & No & $\mathrm{NE}$ & No apparentresponæe to pumping \\
\hline $\mathrm{R}-32$ & 2 & Yes & $\mathrm{NE}$ & $\mathrm{NE}$ & $\mathrm{NE}$ & No & Yes & No & Yes & $\mathrm{NE}$ & Respondsprimarily to pumping atPM-2 andPM-4 \\
\hline R-32 & 3 & Yes & $\mathrm{NE}$ & $\mathrm{NE}$ & $\mathrm{NE}$ & No & Yes & No & Yes & $\mathrm{NE}$ & Respondsprimarily to pumping atPM-2 and PM-4 \\
\hline R-33 & 1 & No & $\mathrm{NE}$ & $\mathrm{NE}$ & $\mathrm{NE}$ & $\mathrm{NE}$ & $\mathrm{NE}$ & $\mathrm{NE}$ & $\mathrm{NE}$ & $\mathrm{NE}$ & Limited data for evaluation \\
\hline $\mathrm{R}-33$ & 2 & Yes & $\mathrm{NE}$ & $\mathrm{NE}$ & $\mathrm{NE}$ & $\mathrm{NE}$ & $\mathrm{NE}$ & No & Yes & $\overline{Y e s}$ & Respondsprimarily to pumping atPM-5 \\
\hline$\overline{\mathrm{R}-34}$ & Single & Yes & $\mathrm{NE}$ & $\mathrm{NE}$ & $\mathrm{NE}$ & No & No & No & No & $\mathrm{NE}$ & Seasonal response butnotto a specific well \\
\hline R-35a & Single & Yes & $\mathrm{NE}$ & $\mathrm{NE}$ & Yes & $\mathrm{NE}$ & $\mathrm{NE}$ & Yes & $\mathrm{NE}$ & $\mathrm{NE}$ & Respondsprimarily to nearby supply well PM-3 \\
\hline$\overline{R-35 b}$ & Single & $\mathbb{D}$ & $\mathrm{NE}$ & $\mathrm{NE}$ & $\mathrm{NE}$ & $\mathrm{NE}$ & $\mathrm{NE}$ & No & $\mathrm{NE}$ & $\overline{\mathrm{NE}}$ & Insufficientdata to evaluate \\
\hline TW-1 & Single & No & No & No & $\mathrm{NE}$ & No & $\mathrm{NE}$ & $\mathrm{NE}$ & $\mathrm{NE}$ & $\mathrm{NE}$ & Water level impacted by surface water near well \\
\hline$\overline{T W}-2$ & Single & Yes & $\mathrm{NE}$ & $\mathrm{NE}$ & $\mathrm{NE}$ & $\mathrm{NE}$ & $\mathrm{NE}$ & $\mathrm{NE}$ & $\overline{\mathrm{NE}}$ & $\overline{\mathrm{NE}}$ & No recentvalid water level data \\
\hline TW-3 & Single & Yes & No & $\mathrm{NE}$ & No & $\mathrm{NE}$ & $\mathrm{NE}$ & No & $\mathrm{NE}$ & $\mathrm{NE}$ & Gradual decline of about $0.8 \mathrm{fthyr}$ \\
\hline TW-4 & Single & Yes & No & NE & No & $\mathrm{NE}$ & NE & NE & $\mathrm{NE}$ & $\mathrm{NE}$ & Seasonalliuctuationsnotrelated to pumping \\
\hline
\end{tabular}


This report has been reproduced directly from the best available copy. It is available electronically on the Web (http://www.doe.gov/bridge).

Copies are available for sale to U.S. Department of Energy employees and contractors from:

Office of Scientific and Technical Information

P.O. Box 62

Oak Ridge, TN 37831

(865) 576-8401

Copies are available for sale to the public from: National Technical Information Service

U.S. Department of Commerce

5285 Port Royal Road

Springfield, VA 22161

(800) 553-6847 
- Los Alamos NATIONAL LABORATORY

EST.1943 